\title{
A STUDY OF PARTICLE PRODUCTION IN PROTON INDUCED COLLISIONS USING THE MIPP DETECTOR AT FERMILAB
}

\author{
A THESIS \\ Submitted to the \\ FACULTY OF SCIENCE \\ PANJAB UNIVERSITY, CHANDIGARH \\ for the degree of
}

DOCTOR OF PHILOSOPHY

2015

SONAM MAHAJAN

CENTRE OF ADVANCED STUDY IN PHYSICS

PANJAB UNIVERSITY

CHANDIGARH 
Dedicated to my family 


\section{Acknowledgments}

Finally, the time has come when I can thank and acknowledge all the people who helped me to make this thesis possible. In the first place, I am deeply grateful to my supervisors, Dr. Vipin Bhatnagar and Prof. Brajesh C. Choudhary for their patient guidance, encouragement and advice through out my research. Without them, this thesis would have never come into existence. Thanks to them for giving me the oppurtunity to work on the MIPP experiment. They always encouraged me to discuss my work with various collaborators in the experiment. Without their supervision and continuous support, I would not have been able to accomplish this research work. I express my deepest gratitude to MIPP spokesperson Dr. Rajendran Raja who supervised me through out my research work at Fermilab. He provided me unflinching encouragement and support in various ways. His dedication to the experiment and to his work has always been an inspiration to me. Although he is no longer with us, he will always be remembered.

Special thanks to Dr. Durga Rajaram for his guidance and support from the early stage of my research. He helped me a lot in understanding the details of the MIPP experiment and shaping up my analyses. He was always there when I was stuck. Thanks to Dr. Turgun Nigmanov for his support, many fruitful discussions and always being ready to help. Many thanks to Dr. Holger Meyer for his valuable suggestions in both the analyses. He helped me a lot to give PID analysis a good shape and was always ready to answer my questions. Thanks to Dr. Jon Paley for his valuable suggestions and 
answering my questions. Thanks to Dr. Nick Solomey, Dr. Carl Rosenfeld, Dr. Win Baker and Prof. Sanjib Mishra for their suggestions. Thanks to all of you for asking me questions during the MIPP meetings and making me think more deeply. I have learned a great deal working with all of you. In addition, I would like to thank all the members of the MIPP Collaboration including physicists, engineers and technicians who worked hard, day and night, to make it successful. None of the work presented in this thesis would have been possible without their dedicated efforts. I want to thank my fellow grad students Arun, Sourabh and Amandeep for all the fruitful discussions.

I am thankful to Chairman, Department of Physics, Panjab University for providing adequate facilities to work in the department. Many thanks and regards go in particular to Prof. J. M. Kohli, Prof. J. B. Singh, Prof. S. Beri and Prof. M. Kaur for their continuous support and efforts to provide us adequate facility in PUHEP lab. I would also like to thank the technical, computing and purchase section staff at Panjab University.

I would like to thank Dr. Shekhar Mishra for his support and encouragement. Thanks to all the members of Indian Institutions and Fermilab Collaboration (IIFC) for their support.

I am deeply thankful to all my friends and fellow grad students in PUHEP lab; Monika, Nishu di, Archana di, Manuk di, Monika di, Sunil, Suneel, Supreet, Anil, Lovedeep di, Prabhdeep di, Inderpal, Vishal, Ritu, Richa, Bhawan, Kanishka, Ruchi, Genius, Aman, Raman, Ankita, Deepika, Daisy, Jyoti, Priyanka, Ridhi, Anter, Anwinder, Manisha and Sandeep who were always present for helping me out from all kind of problems with their suggestions, discussions and creating enjoyable environment in the lab. I would especially like to thank all my friends whom I met at Fermilab during my four years of stay; Jyoti di, Dileep, Arun, Ruchika di, Sumit, Pratima di, Suvadeep, Shilpee di, Amit, Ashish, Anju Bhabhi, Avdesh, Ekta Bhabhi, Neelam Bhabhi, Alpana di, Farah, Neha, Vijay, Arun, Sadia di, Saima, Casey, 
Seema di, Supriya di, Manbir, Kuldeep, Deepthi, Sudha di, Navaneeth, Nitish, Nitin, Siva, Pavan, Phil and Abhimanyu. Thank you for the wonderful memories. I would also like to thank my friends whom I met at Mata Gujri Hostel at Panjab University; Rajvir, Pooja and Geeta. Thank you for your care and wonderful times. Many thanks to Reena Ma'am for her love and support. Special thanks to my friends Pooja and Sunita for their support.

I express my gratitude to Selitha Raja for all the emotional and practical support. Thanks to Venktesh Sir and his family for warm memories at Fermilab. I am thankful to Cathryn and Cindy for making my stay comfortable. Thank you for your care and affection. I would also like to thank Housing Office staff at Fermilab for my awesome stay at Aspen East. Thank you Cheryl, Jack, Jackie and Terri for your care and giving me warm memories.

Finally I am immensely grateful to my family. My deepest gratitude goes to my parents for their unconditional love and encouragement. This thesis would have been simply impossible without their support. My heartiest regards to my Grandparents for showering their warm blessings on me through out my life. Although they are not with me, they are always remembered in my heart. I simply thank my brother Sahil for his constant support in all phases of my life. Thank you for always being there for me. Special thanks to Asha Auntyji for all the spiritual, emotional and practical support.

Last but not least, thanks to God for giving me strength through all the tests in my life. You have made my life more bountiful. May your name be exalted, honored, and glorified.

This research work has been supported by University Grants Commission (UGC) and Fermilab under IIFC. I am thankful to all of them for their confidence in me.

Dated:

Sonam Mahajan

Chandigarh 


\section{Abstract}

The Main Injector Particle Production (MIPP) experiment is a fixed target hadron production experiment at Fermilab. MIPP is a high acceptance spectrometer which provides excellent charged particle identification using Time Projection Chamber (TPC), Time of Flight (ToF), multicell Cherenkov (Ckov), ring imaging Cherenkov (RICH) detectors, and Calorimeter for neutrons. The MIPP experiment is designed to measure particle production in interactions of $120 \mathrm{GeV} / \mathrm{c}$ primary protons from the Main Injector and secondary beams of $\pi^{ \pm}, \mathrm{K}^{ \pm}$, p and $\overline{\mathrm{p}}$ from 5 to $90 \mathrm{GeV} / \mathrm{c}$ on nuclear targets which include $\mathrm{H}, \mathrm{Be}, \mathrm{C}, \mathrm{Bi}$ and $\mathrm{U}$, and a dedicated run with the NuMI target. The goal of the experiment is to measure hadron production cross sections or yields using these beams and targets. These hadronic interaction data can have a direct impact on the detailed understanding of the neutrino fluxes of several accelerator-based neutrino experiments like MINOS, MINER $\nu \mathrm{A}, \mathrm{NO} \nu \mathrm{A}$ and ELBNF. In this thesis, we present inelastic cross section measurements for 58 and $85 \mathrm{GeV} / \mathrm{c} \mathrm{p}+\mathrm{p}$ interactions, and 58 and $120 \mathrm{GeV} / \mathrm{c} \mathrm{p}+\mathrm{Be}, \mathrm{p}+\mathrm{C}$ and $\mathrm{p}+\mathrm{Bi}$ interactions. A new method is described to account for the low multiplicity inefficiencies in the interaction trigger using KNO scaling. Inelastic cross sections as a function of multiplicity are also presented for $\mathrm{p}+\mathrm{H}$ and $\mathrm{p}+\mathrm{C}$ interactions at different beam momenta. The MIPP data are compared with the Monte Carlo predictions and existing data. We also describe an algorithm to identify charged particles $\left(\pi^{ \pm} / \mathrm{p} / \overline{\mathrm{p}}\right.$ etc. $)$, and present the inclusive charged pion production cross sections in bins of true momentum using 58 and $120 \mathrm{GeV} / \mathrm{c}$ p $+\mathrm{C}$ interactions for both the data and Monte Carlo. 


\section{Contents}

List of Tables $\quad$ xii

List of Figures $\quad$ xiv

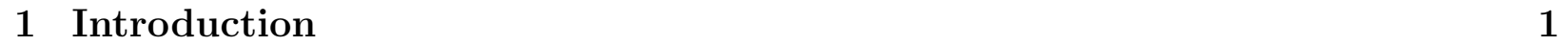

1.1 The Standard Model . . . . . . . . . . . . . . . . . . . . . . . . . 1

1.1 .1 Matter particles . . . . . . . . . . . . . . . . . 1

1.1 .2 Forces and carrier particles . . . . . . . . . . . . . . . . 3

1.2 Strong interactions and QCD . . . . . . . . . . . . . . . . . 5

1.3 Soft Hadronic Physics . . . . . . . . . . . . . . . . . . . . . . . . 6

1.4 Need for a Hadron Production Experiment . . . . . . . . . . . . . . . . . . . 7

1.5 Particle Production and Neutrino Experiments . . . . . . . . . . . . . . . . 7

1.6 The MIPP Experiment at Fermilab . . . . . . . . . . . . . . . . . . . . . . 10

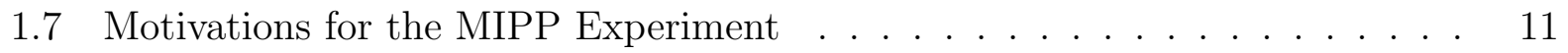

1.7 .1 Neutrino Flux Predictions . . . . . . . . . . . . . . . . . . . . . . . . 12

1.7 .2 Hadronic Shower Simulation . . . . . . . . . . . . . . . . . . . . . 12

1.7 .3 Study of non-perturbative QCD . . . . . . . . . . . . . . . . . . . . 12

1.7 .4 Scaling Law of Hadronic Fragmentation . . . . . . . . . . . . . . . . . 13

1.8 Other Hadron Production Experiments . . . . . . . . . . . . . . . . . . . . . 14

1.9 Outline of the thesis . . . . . . . . . . . . . . . . . . . . . . . . . . . . 14

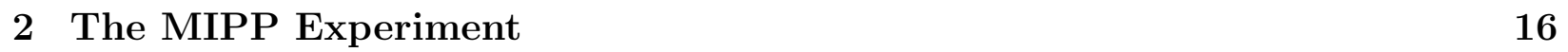

2.1 Fermilab Accelerator Complex . . . . . . . . . . . . . . . . . . . . . 16

2.2 MIPP Beamline . . . . . . . . . . . . . . . . . . . . . . . . 18

2.2 .1 Primary Beamline . . . . . . . . . . . . . . . . . . . . 18

2.2 .2 Secondary Beamline . . . . . . . . . . . . . . . . . . . 20

2.3 MIPP Spectrometer . . . . . . . . . . . . . . . . . . . . . 20

2.4 Tracking . . . . . . . . . . . . . . . . . . . . . . . . . . 21 
2.4 .1 Drift Chambers . . . . . . . . . . . . . . . . . . . 21

2.4 .2 Multiwire Proportional Chambers . . . . . . . . . . . . . . . . . . . . 22

2.4 .3 Time Projection Chamber . . . . . . . . . . . . . . . . . . . . . . . . 23

2.5 Particle Identification $\ldots \ldots \ldots$. . . . . . . . . . . . . . . . . . . . . . . . . . . . 24

2.5 .1 Beam Cherenkov . . . . . . . . . . . . . . . . . . . . 24

2.5.2 Threshold Cherenkov . . . . . . . . . . . . . . . . . . . . 26

2.5 .3 Beam Time of Flight . . . . . . . . . . . . . . . . . . . . . . . . . . . 27

2.5 .4 Time of Flight Wall . . . . . . . . . . . . . . . . . . . . . . . . . . . . 28

2.5 .5 Ring Imaging Cherenkov Detector . . . . . . . . . . . . . . . . . . . . 29

2.5 .6 Electromagnetic Calorimeter . . . . . . . . . . . . . . . . . . . . . 30

2.5.7 Hadron Calorimeter . . . . . . . . . . . . . . . . . . . . . . . . . . . 31

2.6 Analysis Magnets . . . . . . . . . . . . . . . . . . . . . . . . . . . 33

2.6 .1 Jolly Green Giant . . . . . . . . . . . . . . . . . . . . . . . . . . . . . 33

2.6 .2 Rosie . . . . . . . . . . . . . . . . . . . . . . . . . . 33

2.6 .3 Ziptrack field mapping . . . . . . . . . . . . . . . . . . . . . 34

2.7 Targets . . . . . . . . . . . . . . . . . . . . . . . 34

2.7 .1 Nuclear targets . . . . . . . . . . . . . . . . . . . 36

2.7 .2 Cryogenic target . . . . . . . . . . . . . . . . 36

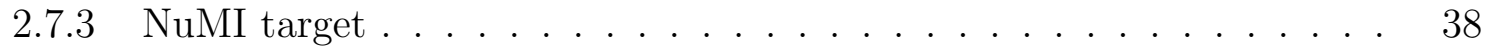

2.8 Trigger System . . . . . . . . . . . . . . . . . . . . . . 38

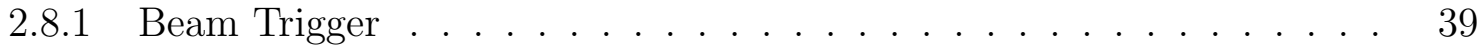

2.8 .2 Beam Particle Identification . . . . . . . . . . . . . . . . . . . . . 40

2.8 .3 Beam Trigger Purity . . . . . . . . . . . . . . . . . . . . . . . 41

2.8 .4 Interaction Trigger . . . . . . . . . . . . . . . . . . . . . . . . . . . 42

2.8 .5 End of Spill and Calibration Triggers . . . . . . . . . . . . . . . . . . 43

2.8 .6 Master Trigger . . . . . . . . . . . . . . . . . . . . . . . . . 44

2.9 Data Acquisition System . . . . . . . . . . . . . . . . . . . . . . . . . 45

2.9 .1 Slow Controls . . . . . . . . . . . . . . . . . . . . . . . . . . 48

2.10 Event Reconstruction . . . . . . . . . . . . . . . . . . . . . . . . . . . 49

2.10 .1 Track Reconstruction . . . . . . . . . . . . . . . . . . . . . . 50

2.10 .2 Vertex Reconstruction . . . . . . . . . . . . . . . . . 52

2.11 Monte Carlo Simulation . . . . . . . . . . . . . . . . . . . . . . . . . 54

3 Event Selection and Monte Carlo Corrections 56

3.1 Cross section Terminology . . . . . . . . . . . . . . . . . . . . 56

3.2 Event selection requirements . . . . . . . . . . . . . . . . . . . . 57 
3.2 .1 Good beam event selection . . . . . . . . . . . . . . . . . . . 57

3.2 .2 Good event selection . . . . . . . . . . . . . . . . . . . 63

3.2 .3 Good track selection . . . . . . . . . . . . . . . . . . . 71

3.3 Beam Flux Calculation . . . . . . . . . . . . . . . . . . . . . . . . . . 73

3.4 Interaction Trigger Efficiency . . . . . . . . . . . . . . . . . . . . 75

3.5 KNO-based technique to get trigger efficiency $\ldots \ldots \ldots$. . . . . . . . . 76

3.5.1 Get the KNO true multiplicity probability distribution . . . . . . . . 77

3.5.2 Get the predicted observed probability distribution . . . . . . . . . . 80

3.5.3 Get the observed multiplicity distribution . . . . . . . . . . . . . . . 80

3.5 .4 Minimization Scheme . . . . . . . . . . . . . . . . . . . . . . . 82

3.6 Corrections from Monte Carlo . . . . . . . . . . . . . . . . . . . . . . . . . . 90

3.6.1 Trigger efficiency . . . . . . . . . . . . . . . . . . . . . . . . . . 92

3.6 .2 Cut efficiency . . . . . . . . . . . . . . . . . . . . . . . . . . 92

$3.6 .3 \quad$ Geometrical acceptance $\times$ Reconstruction efficiency . . . . . . . . . . . 92

\begin{tabular}{lll}
\hline Pion Identification & 100
\end{tabular}

4.1 Particle Identification . . . . . . . . . . . . . . . . . . . . . . . . . 100

$4.1 .1 \mathrm{TPC} \mathrm{PID} \ldots \ldots \ldots \ldots \ldots \ldots$

4.1 .2 RICH PID . . . . . . . . . . . . . . . . . . . . . . . . . 101

4.2 PID algorithm . . . . . . . . . . . . . . . . . . . . . . . . . . . 102

4.2 .1 TPC likelihood calculations . . . . . . . . . . . . . . . . . . . . 104

4.2 .2 RICH likelihood calculations . . . . . . . . . . . . . . . . . . . . 114

4.2 .3 Why ToF is not used for PID in this analysis? . . . . . . . . . . . . . 120

$4.2 .4 \quad$ Global likelihoods and the problems associated with them . . . . . . 120

4.3 Target-out subtraction . . . . . . . . . . . . . . . . . . . . 120

4.4 Pion momentum spectrum . . . . . . . . . . . . . . . . . . . . . . . . . 121

4.5 Unfolding of the data pion momentum spectrum . . . . . . . . . . . . . . . . 122

4.6 Corrections in bins of true momentum . . . . . . . . . . . . . . . . . . . . . 126

$4.6 .1 \quad$ Trigger efficiency $\ldots \ldots \ldots \ldots$. . . . . . . . . . . . . . . . . 128

4.6 .2 Combined cut efficiency . . . . . . . . . . . . . . . . . . . . 129

$4.6 .3 \quad$ Geometrical acceptance $\times$ Reconstruction efficiency $\left(\mathcal{A} \epsilon_{\mathcal{R}}\right)$. . . . . . . 129

$4.6 .4 \quad$ Pion identification efficiency . . . . . . . . . . . . . . . . . . . . . . . 129

$\begin{array}{lll}5 & \text { Results } & 135\end{array}$

5.1 Inelastic cross sections . . . . . . . . . . . . . . . . . . . . . . . . 135

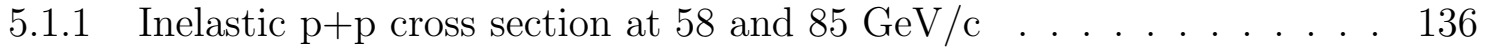

$5.1 .2 \quad$ Inelastic $\mathrm{p}+\mathrm{C}$ cross section at 58 and $120 \mathrm{GeV} / \mathrm{c}$. . . . . . . . . . . 136 
5.1 .3 Inelastic cross sections for Be and Bi targets . . . . . . . . . . . . . . 138

5.1 .4 Systematic Errors . . . . . . . . . . . . . . . . . . . . . . . . 138

5.1 .5 Inelastic cross section vs target atomic weight . . . . . . . . . . . . . 146

$5.2 \mathrm{LH}_{2}$ and carbon multiplicities . . . . . . . . . . . . . . . . . . . . 148

5.3 Charged particle production cross sections as a function of momentum . . . 150

5.4 Inclusive charged pion production cross sections . . . . . . . . . . . . . 156

5.4 .1 Cross sections using $58 \mathrm{GeV} / \mathrm{c} \mathrm{p}+\mathrm{C}$ interactions . . . . . . . . . . . . 157

5.4 .2 Cross sections using $120 \mathrm{GeV} / \mathrm{c} \mathrm{p}+\mathrm{C}$ interactions . . . . . . . . . . . 161

5.4 .3 Systematic Errors . . . . . . . . . . . . . . . . 163

5.4 .4 Comparison of data with MC . . . . . . . . . . . . . . . . . 167

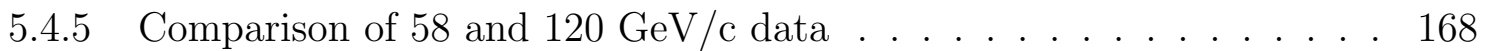

\begin{tabular}{lll}
\hline & Summary and Outlook & 173
\end{tabular}

6.1 Experimental results . . . . . . . . . . . . . . . . . . . 173

6.2 Implications of these results . . . . . . . . . . . . . . . 176

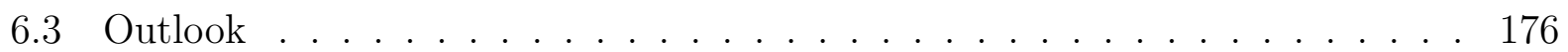

\begin{tabular}{ll}
\hline Bibliography & 177
\end{tabular}

\begin{tabular}{ll}
\hline List of Publications & 189
\end{tabular} 


\section{List of Tables}

1.1 The three generations of matter and their basic properties. . . . . . . . . . . 3

1.2 Fundamental forces included in Standard Model and their properties. . . . . 4

$1.3 \quad$ Data collected in MIPP using various beams and targets. . . . . . . . . . . . 11

2.1 Hadron calorimeter specifications. . . . . . . . . . . . . . . . . . 32

2.2 Size of the Ziptrack grids used to map the MIPP analysis magnet fields. . . . 34

2.3 The list of thin targets and their properties. . . . . . . . . . . . . . . . 37

$3.1 \quad$ Beam flux numbers before applying the beam selection cuts. . . . . . . . . . 73

3.2 Beam selection cut efficiencies for $\mathrm{LH}_{2}$ target. . . . . . . . . . . . . . . . . . 74

3.3 Beam selection cut efficiencies for carbon target. . . . . . . . . . . . . . . . . 74

3.4 Beam flux numbers after applying the beam selection cuts. . . . . . . . . . . 75

3.5 The average trigger and cut efficiencies, and $\mathcal{A} \epsilon_{\mathcal{R}}$ used for the inelastic cross section calculation for $\mathrm{p}+\mathrm{p}$ interactions at $58 \mathrm{GeV} / \mathrm{c}$. . . . . . . . . . . . . . 93

$3.6 \quad$ The average trigger and cut efficiencies, and $\mathcal{A} \epsilon_{\mathcal{R}}$ used for the inelastic cross section calculation for $\mathrm{p}+\mathrm{p}$ interactions at $85 \mathrm{GeV} / \mathrm{c}$. . . . . . . . . . . . . . 94

3.7 The average trigger and cut efficiencies, and $\mathcal{A} \epsilon_{\mathcal{R}}$ used for the inelastic cross section calculation for $\mathrm{p}+\mathrm{C}$ interactions at $58 \mathrm{GeV} / \mathrm{c}$. . . . . . . . . . . . . . 94

3.8 The average trigger and cut efficiencies, and $\mathcal{A} \epsilon_{\mathcal{R}}$ used for the inelastic cross section calculation for $\mathrm{p}+\mathrm{C}$ interactions at $120 \mathrm{GeV} / \mathrm{c} . \quad$. . . . . . . . . . 95

3.9 The average trigger and cut efficiencies, and $\mathcal{A} \epsilon_{\mathcal{R}}$ used for the inelastic cross section calculation for $\mathrm{p}+\mathrm{Be}$ interactions at $58 \mathrm{GeV} / \mathrm{c}$. . . . . . . . . . . . . 95

3.10 The average trigger and cut efficiencies, and $\mathcal{A} \epsilon_{\mathcal{R}}$ used for the inelastic cross section calculation for $\mathrm{p}+\mathrm{Be}$ interactions at $120 \mathrm{GeV} / \mathrm{c}$. . . . . . . . . . . . 96

3.11 The average trigger and cut efficiencies, and $\mathcal{A} \epsilon_{\mathcal{R}}$ used for the inelastic cross section calculation for $\mathrm{p}+\mathrm{Bi}$ interactions at $58 \mathrm{GeV} / \mathrm{c}$. . . . . . . . . . . . . . 96

3.12 The average trigger and cut efficiencies, and $\mathcal{A} \epsilon_{\mathcal{R}}$ used for the inelastic cross section calculation for $\mathrm{p}+\mathrm{Bi}$ interactions at $120 \mathrm{GeV} / \mathrm{c}$. . . . . . . . . . . . . 97 
5.1 The density, thickness, $\mathrm{A}$ and $\mathrm{n}_{t}$ for different targets used. . . . . . . . . . 136

$5.2 \mathrm{~N}_{\text {beam }}, \mathrm{N}_{\text {int }}$ and $\sigma_{\text {inel }}$ for $\mathrm{p}+\mathrm{p}$ interactions at 58 and $85 \mathrm{GeV} / \mathrm{c}$. . . . . . . . . 137

$5.3 \quad \mathrm{~N}_{\text {beam }}, \mathrm{N}_{\text {int }}$ and $\sigma_{\text {inel }}$ for $\mathrm{p}+\mathrm{C}$ interactions at 58 and $120 \mathrm{GeV} / \mathrm{c}$. . . . . . . . 137

$5.4 \mathrm{~N}_{\text {beam }}, \mathrm{N}_{\text {int }}$ and $\sigma_{\text {inel }}$ for $\mathrm{p}+$ Be interactions at 58 and $120 \mathrm{GeV} / \mathrm{c}$. . . . . . 138

$5.5 \mathrm{~N}_{\text {beam }}, \mathrm{N}_{\text {int }}$ and $\sigma_{\text {inel }}$ for $\mathrm{p}+\mathrm{Bi}$ interactions at 58 and $120 \mathrm{GeV} / \mathrm{c}$. . . . . . . 139

$5.6 \quad$ Inelastic cross sections for various targets at different beam momenta. . . . . 143

5.7 Inelastic cross sections for various targets at different beam momenta. . . . . 144

5.8 The fit parameters for the data using 58 and $120 \mathrm{GeV} / \mathrm{c}$ proton beams. . . . 147

5.9 The fit parameters for the MC using 58 and $120 \mathrm{GeV} / \mathrm{c}$ proton beams. . . . 147

5.10 Total charged particle production cross sections for $\mathrm{p}+\mathrm{C}$ interactions at 58

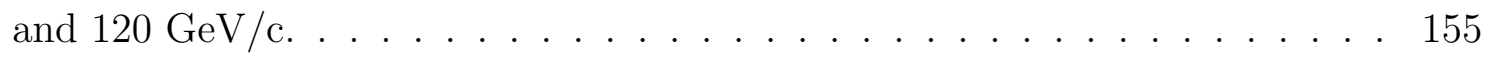

5.11 Inclusive $\pi^{ \pm}$production cross sections for $\mathrm{p}+\mathrm{C}$ interactions at 58 and 120 $\mathrm{GeV} / \mathrm{c} . \ldots \ldots \ldots \ldots$. . . . . . . . . . . . . . 166

5.12 Comparison of the data and MC inclusive $\pi^{ \pm}$production cross sections for the two momentum ranges using 58 and $120 \mathrm{GeV} / \mathrm{c} \mathrm{p}+\mathrm{C}$ interactions. . . . . 170 


\section{List of Figures}

1.1 The Standard Model of elementary particles. . . . . . . . . . . . . . . 2

1.2 Spectrum of pions in MINOS near and far detectors. . . . . . . . . . 8

1.3 Uncertainties in neutrino flux prediction. . . . . . . . . . . . . 9

1.4 MINOS neutrino flux from kaon decays. $\ldots \ldots \ldots \ldots$

$2.1 \quad$ Schematic of the Fermilab accelerator complex. $\ldots \ldots \ldots \ldots$

2.2 (a) Isometric and (b) plan views of the MIPP beamline. . . . . . . . . . . . 19

2.3 The layout of the MIPP experiment. . . . . . . . . . . . . . . 21

2.4 Schematic view of the TPC volume and pad plane. . . . . . . . . . . 25

2.5 Schematic view of the Cherenkov counter. . . . . . . . . . . . . . 27

2.6 Schematic of T00 and T01 construction. . . . . . . . . . . . 28

2.7 Schematic side view of the RICH detector. . . . . . . . . . . . . . 30

2.8 Schematic of the calorimeters. . . . . . . . . . . . . . . . . . . 32

2.9 Projections of the JGG field map. . . . . . . . . . . . . . . . . 35

2.10 Projections of the Rosie field map. . . . . . . . . . . . . . . . . 35

2.11 The target wheel used in the MIPP experiment. . . . . . . . . . . . . 37

2.12 Schematic of the NuMI target design. . . . . . . . . . . . . . . . 39

2.13 Trigger purity as measured by the RICH. . . . . . . . . . . . . . 42

2.14 Overall plan view of the interaction trigger counter. . . . . . . . . . . . 44

2.15 Schematic of data acquisition setup. . . . . . . . . . . . . . . 46

2.16 Event display showing both the reconstructed TPC as well as global tracks for a $120 \mathrm{GeV} / \mathrm{c}$ proton-carbon event. . . . . . . . . . . . . . 53

2.17 The distribution of the fitted $\mathrm{Z}$ vertex from different targets. . . . . . . . 55

$3.1 \quad$ Multiplicity distribution for $\mathrm{p}+\mathrm{p}$ data $\ldots \ldots \ldots \ldots \ldots \ldots$

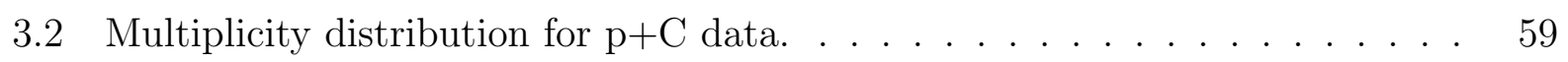

3.3 Beam track time distribution for $\mathrm{p}+\mathrm{p}$ data $\ldots \ldots \ldots$. . . . . . . 60

3.4 Beam track time distribution for $\mathrm{p}+\mathrm{C}$ data $\ldots \ldots \ldots \ldots$ 
3.5 Longitudinal vertex distribution for empty cryogenic target. . . . . . . . . . 61

3.6 Transverse vertex distributions for empty cryogenic target. . . . . . . . . . . 62

3.7 Transverse beam position distribution for $58 \mathrm{GeV} / \mathrm{c} \mathrm{p}+\mathrm{p}$ data. . . . . . . . . 62

3.8 Beam position with respect to target center in $\mathrm{X}$ direction for $\mathrm{p}+\mathrm{C}$ data. . . 63

3.9 Beam position with respect to target center in $\mathrm{Y}$ direction for $\mathrm{p}+\mathrm{C}$ data. . . 64

3.10 Beam radius distribution for $\mathrm{p}+\mathrm{C}$ data. . . . . . . . . . . . . . . . 64

3.11 Effect of beam radius cut on transverse beam position distribution. . . . . . 65

3.12 Effect of beam radius cut on transverse beam position distribution. . . . . . 65

3.13 Distribution of sum of momenta of charged tracks per event for $\mathrm{p}+\mathrm{p}$ data. $\quad$. 67

3.14 Distribution of sum of momenta of charged tracks per event for $\mathrm{p}+\mathrm{C}$ data. $\quad 67$

3.15 Longitudinal vertex distribution for $\mathrm{p}+\mathrm{p}$ data. . . . . . . . . . . . . . . 69

3.16 Longitudinal vertex distribution for $\mathrm{p}+\mathrm{C}$ data.... .70

3.17 Effect of tranverse beam and vertex cuts on transverse vertex distribution. . $\quad 70$

3.18 Effect of tranverse beam and vertex cuts on longitudinal vertex distribution. 71

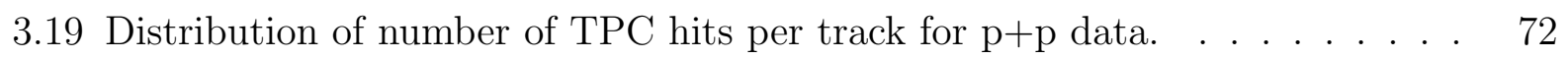

3.20 Distribution of number of TPC hits per track for $\mathrm{p}+\mathrm{C}$ data. . . . . . . . . . 72

3.21 Relative difference between two beam fluxes vs subrun numbers. . . . . . . . 76

3.22 The trigger efficiencies in bins of tracks passing through the scintillator for 58

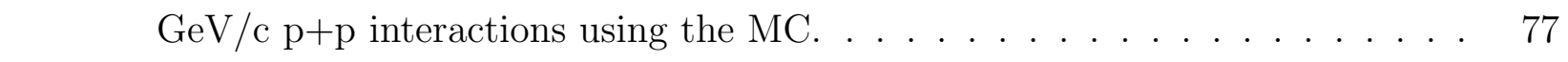

3.23 KNO scaling curve. . . . . . . . . . . . . . . . . . . . . . . 78

3.24 The KNO true probability distribution for $\mathrm{p}+\mathrm{p}$ interactions at $58 \mathrm{GeV} / \mathrm{c}$. . . $\quad 79$

3.25 The fits to the total and elastic cross sections as a function of lab momentum from the PDG. . . . . . . . . . . . . . . . . . . . . 80

3.26 The probability distributions for obtaining observed multiplicity, given a true multiplicity $\mathrm{n}_{t}$ for $\mathrm{p}+\mathrm{p}$ interactions at $58 \mathrm{GeV} / \mathrm{c} . \quad$. . . . . . . . . . . . . . 81

3.27 The predicted observed probability distribution for $\mathrm{p}+\mathrm{p}$ interactions at 58

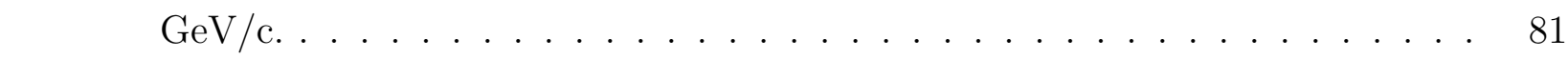

3.28 The observed multiplicity distribution for $\mathrm{p}+\mathrm{p}$ interactions at $58 \mathrm{GeV} / \mathrm{c}$ with the interaction trigger applied. . . . . . . . . . . . . . . . . 82

3.29 KNO fit results for $\mathrm{p}+\mathrm{p}$ interactions at $58 \mathrm{GeV} / \mathrm{c}$. . . . . . . . . . . . . . . . 84

$3.30 \mathrm{KNO}$ fit results for $\mathrm{p}+\mathrm{p}$ interactions at $58 \mathrm{GeV} / \mathrm{c}$. . . . . . . . . . . . . . 85

3.31 Comparison of DPMJET and KNO multiplicity probabilities for liquid hydrogen target. . . . . . . . . . . . . . . . . . . 85

3.32 The KNO true probability distribution for $\mathrm{p}+\mathrm{p}$ interactions at $85 \mathrm{GeV} / \mathrm{c}$. . . 86

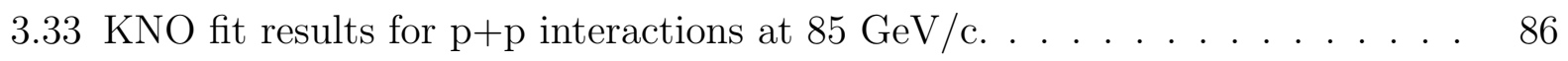

$3.34 \mathrm{KNO}$ true probability distribution for $58 \mathrm{GeV} / \mathrm{c} \mathrm{p}+\mathrm{C}$ interactions. . . . . . . 88 
3.35 The probability distributions for obtaining observed multiplicity, given a true multiplicity $\mathrm{n}_{t}$ for $\mathrm{p}+\mathrm{C}$ interactions at $58 \mathrm{GeV} / \mathrm{c}$. . . . . . . . . . . . . . 89

3.36 The predicted observed probability distribution and the observed multiplicity distribution for $58 \mathrm{GeV} / \mathrm{c} \mathrm{p}+\mathrm{C}$ interactions. . . . . . . . . . . . . . . . . . . 89

$3.37 \mathrm{KNO}$ fit results for $\mathrm{p}+\mathrm{C}$ interactions at $58 \mathrm{GeV} / \mathrm{c}$. . . . . . . . . . . . . 90

$3.38 \mathrm{KNO}$ true probability distribution for $120 \mathrm{GeV} / \mathrm{c} \mathrm{p}+\mathrm{C}$ interactions. . . . . . 91

3.39 KNO fit results for $\mathrm{p}+\mathrm{C}$ interactions at $120 \mathrm{GeV} / \mathrm{c}$. . . . . . . . . . . . . . 91

3.40 Trigger efficiencies in bins of momentum. . . . . . . . . . . . . . . . . . . . 97

3.41 Multiplicity cut efficiencies in bins of momentum. . . . . . . . . . . . . . . . 98

3.42 Combined cut efficiencies in bins of momentum. . . . . . . . . . . . . . . . . 98

$3.43 \mathcal{A} \epsilon_{\mathcal{R}}$ in bins of momentum. . . . . . . . . . . . . . . . . . . . . . . 99

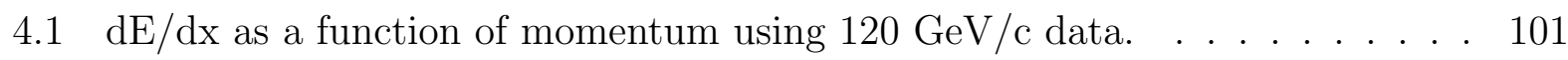

4.2 RICH ring radius as a function of momentum. . . . . . . . . . . . . . . 103

4.3 Momentum spectra for $120 \mathrm{GeV} / \mathrm{c} \mathrm{p}+\mathrm{C}$ data and MC. . . . . . . . . . . . . 104

$4.4 \mathrm{dE} / \mathrm{dx}$ as a function of momentum for $120 \mathrm{GeV} / \mathrm{c} \mathrm{p}+\mathrm{C}$ data and MC. . . . . 105

4.5 Mean offsets as a function of momentum and ntpchits for $58 \mathrm{GeV} / \mathrm{c}$ p+C data. 106

4.6 Mean offsets as a function of momentum and ntpchits for $120 \mathrm{GeV} / \mathrm{c}$ p+C data. 107

$4.7 \quad$ Fitted resolutions as a function of momentum and ntpchits for $58 \mathrm{GeV} / \mathrm{c} \mathrm{p}+\mathrm{C}$ data. . . . . . . . . . . . . . . . . . . 107

4.8 Fitted resolutions as a function of momentum and ntpchits for $120 \mathrm{GeV} / \mathrm{c}$ p+C data. . . . . . . . . . . . . . . . . . 108

4.9 Gaussion fits to difference of observed and predicted dE/dx for data. . . . . 109

4.10 Gaussion fits to difference of observed and predicted $\mathrm{dE} / \mathrm{dx}$ for data.. .109

4.11 Gaussion fits to difference of observed and predicted $\mathrm{dE} / \mathrm{dx}$ for data. . . . . 110

$4.12 \mathrm{dE} / \mathrm{dx}$ distributions for different momentum slices for data. . . . . . . . . . . 110

$4.13 \mathrm{dE} / \mathrm{dx}$ distributions for different momentum slices for data. . . . . . . . . . . 111

$4.14 \mathrm{dE} / \mathrm{dx}$ distributions for different momentum slices for data. . . . . . . . . . . 111

4.15 Likelihood distributions for 58 and $120 \mathrm{GeV} / \mathrm{c}$ p+C data using TPC. . . . . 112

4.16 Likelihoods as a function of momentum for 58 and $120 \mathrm{GeV} / \mathrm{c}$ p $+\mathrm{C}$ data using

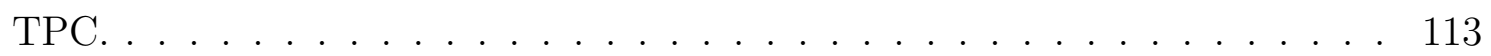

4.17 Weight distributions for 58 and $120 \mathrm{GeV} / \mathrm{c} \mathrm{p}+\mathrm{C}$ data using TPC. . . . . . . 113

4.18 Weights as a function of momentum for 58 and $120 \mathrm{GeV} / \mathrm{c} \mathrm{p}+\mathrm{C}$ data using

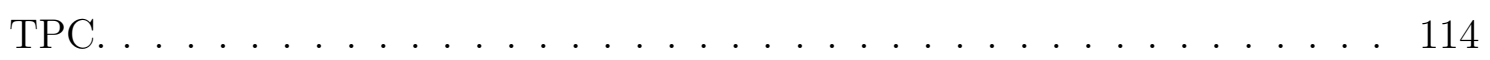

4.19 Number of hits/track and $\chi^{2}$ of the radius fit in RICH for $58 \mathrm{GeV} / \mathrm{c}$ p+C data. 115 
4.20 Number of hits/track and $\chi^{2}$ of the radius fit in $\mathrm{RICH}$ for $120 \mathrm{GeV} / \mathrm{c} \mathrm{p}+\mathrm{C}$ data. . . . . . . . . . . . . . . . . . . . 115

4.21 No ring track probability computed using the MIPP MC for $120 \mathrm{GeV} / \mathrm{c} \mathrm{p}+\mathrm{C}$ interactions. . . . . . . . . . . . . . . . . . . . 116

4.22 No ring track probability computed using the MIPP MC for $58 \mathrm{GeV} / \mathrm{c} \mathrm{p}+\mathrm{C}$ interactions. . . . . . . . . . . . . . . . . . . . 117

4.23 Likelihood distributions for 58 and $120 \mathrm{GeV} / \mathrm{c} \mathrm{p}+\mathrm{C}$ data using RICH. . . . . 118

4.24 Likelihoods as a function of momentum for 58 and $120 \mathrm{GeV} / \mathrm{c}$ p+C data using RICH. . . . . . . . . . . . . . . . . . . . . . . . . . 118

4.25 Weights for 58 and $120 \mathrm{GeV} / \mathrm{c} \mathrm{p}+\mathrm{C}$ data using RICH. . . . . . . . . . . . . 119

4.26 Weights as a function of momentum 58 and $120 \mathrm{GeV} / \mathrm{c}$ p+C data using RICH.119

4.27 Fits to the $\mathrm{Z}$ vertex distributions for lower and higher momentum ranges. . . 122

4.28 Momentum spectrum after target-out subtraction for momentum range $0.3-$ $1 \mathrm{GeV} / \mathrm{c}$ using TPC and $58 \mathrm{GeV} / \mathrm{c} \mathrm{p}+\mathrm{C}$ data . . . . . . . . . . . . . . . . . 123

4.29 Momentum spectrum after target-out subtraction for momentum range $0.3-$

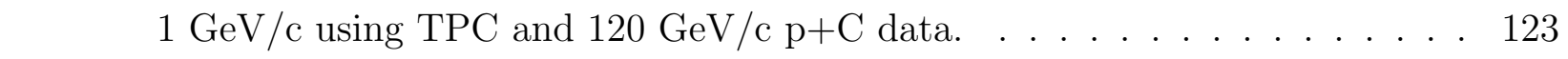

4.30 Momentum spectrum after target-out subtraction for momentum range 4.64 - $120 \mathrm{GeV} / \mathrm{c}$ using RICH and $58 \mathrm{GeV} / \mathrm{c} \mathrm{p}+\mathrm{C}$ data. . . . . . . . . . . . . . . 124

4.31 Momentum spectrum after target-out subtraction for momentum range 4.64

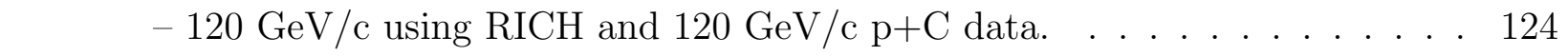

4.32 The K-matrix for the pion negatives for momentum range $0.3-1 \mathrm{GeV} / \mathrm{c}$ using TPC and $120 \mathrm{GeV} / \mathrm{c} \mathrm{p}+\mathrm{C}$ data. . . . . . . . . . . . . . . . . . 125

4.33 The K-matrix for the pion negatives for momentum range $4.64-120 \mathrm{GeV} / \mathrm{c}$ using RICH and $120 \mathrm{GeV} / \mathrm{c} \mathrm{p}+\mathrm{C}$ data. . . . . . . . . . . . . . . . . . . . . 125

4.34 Comparison of observed and predicted momentum distributions at minimum from TPC for momentum range $0.3-1 \mathrm{GeV} / \mathrm{c}$ using $58 \mathrm{GeV} / \mathrm{c} \mathrm{p}+\mathrm{C}$ data. .126

4.35 Comparison of observed and predicted momentum distributions at minimum

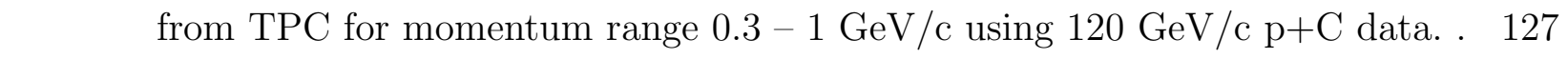

4.36 Comparison of observed and predicted momentum distributions at minimum from RICH for momentum range $4.64-60 \mathrm{GeV} / \mathrm{c}$ using $58 \mathrm{GeV} / \mathrm{c}$ p+C data. 127

4.37 Comparison of observed and predicted momentum distributions at minimum from RICH for momentum range $4.64-120 \mathrm{GeV} / \mathrm{c}$ using $120 \mathrm{GeV} / \mathrm{c}$ p+C data. 128

4.38 All the efficiencies in bins of true momentum superimposed for negative particles for the two momentum ranges using $58 \mathrm{GeV} / \mathrm{c} \mathrm{p}+\mathrm{C}$ MC. . . . . . . . . 130

4.39 All the efficiencies in bins of true momentum superimposed for positive particles for the two momentum ranges using $58 \mathrm{GeV} / \mathrm{c} \mathrm{p}+\mathrm{C} \mathrm{MC}$. . . . . . . . . 131 
4.40 All the efficiencies in bins of true momentum superimposed for negative particles for the two momentum ranges using $120 \mathrm{GeV} / \mathrm{c} \mathrm{p}+\mathrm{C} \mathrm{MC}$. . . . . . . . 131

4.41 All the efficiencies in bins of true momentum superimposed for positive particles for the two momentum ranges using $120 \mathrm{GeV} / \mathrm{c} \mathrm{p}+\mathrm{C} \mathrm{MC}$. . . . . . . . 132

4.42 Pion mis-identification rates using the TPC and $120 \mathrm{GeV} / \mathrm{c}$ p+C MC. . . . . 133

4.43 Pion mis-identification rates using the RICH and $120 \mathrm{GeV} / \mathrm{c} \mathrm{p}+\mathrm{C} \mathrm{MC}$. . . . 133

4.44 Predicted ring radii vs momenta superimposed for $\mathrm{e} / \pi / \mathrm{K} / \mathrm{p}$ for $\mathrm{MC}$. . . . . . 134

5.1 Narrow $\mathrm{Z}$ vertex selection for $\mathrm{p}+\mathrm{p}$ interactions at $85 \mathrm{GeV} / \mathrm{c}$. . . . . . . . . . 140

5.2 Ratio of data and MC multiplicities for 58 and $85 \mathrm{GeV} / \mathrm{c} \mathrm{p}+\mathrm{p}$ interactions. . 141

5.3 Ratio of data and MC multiplicities for 58 and $120 \mathrm{GeV} / \mathrm{c} \mathrm{p}+\mathrm{C}$ interactions. 141

5.4 Ratio of data and MC multiplicities for 58 and $120 \mathrm{GeV} / \mathrm{c} \mathrm{p}+$ Be interactions. 142

5.5 Ratio of data and MC multiplicities for 58 and $120 \mathrm{GeV} / \mathrm{c}$ p+Bi interactions. 142

5.6 Inelastic cross sections as a function of lab momentum for different targets. . 145

5.7 Inelastic cross sections vs target atomic weight for the data using 58 and 120 GeV/c proton beam. . . . . . . . . . . . . . . . . 146

$5.8 \quad$ Inelastic cross sections vs target atomic weight for the MC using 58 and 120 GeV/c proton beams. . . . . . . . . . . . . . . . . 147

$5.9 \quad$ Inelastic cross sections as a function of multiplicity for $\mathrm{LH}_{2}$ target. . . . . . . 149

5.10 Systematics from average multiplcity $<\mathrm{n}>$ for $\mathrm{LH}_{2}$ target. . . . . . . . . . . 149

5.11 Inelastic cross sections as a function of multiplicity for carbon target. . . . . 151

5.12 Systematics from average multiplcity $<\mathrm{n}>$ for carbon target. . . . . . . . . . 151

5.13 Track momentum distributions for 58 and $120 \mathrm{GeV} / \mathrm{c} \mathrm{p}+\mathrm{C}$ interactions. . . . 152

5.14 Uncorrected charged particle production cross sections in bins of momentum. 153

5.15 Corrected charged particle production cross sections in bins of momentum. . 153

5.16 Comparison of data and MC charged particle production cross sections in bins of momentum. . . . . . . . . . . . . . . . . . . . . 154

5.17 Comparison of nominal $\mathcal{A} \epsilon_{\mathcal{R}}$ and $\mathcal{A} \epsilon_{\mathcal{R}}$ using the reweighted MC in bins of momentum. . . . . . . . . . . . . . . . . . 155

5.18 Total systematic variations for charged particle production cross sections in bins of momentum. . . . . . . . . . . . . . . . . . . 156

5.19 Uncorrected inclusive $\pi^{ \pm}$production cross sections for momentum range 0.3 $-1 \mathrm{GeV} / \mathrm{c}$ using TPC for $58 \mathrm{GeV} / \mathrm{c} \mathrm{p}+\mathrm{C}$ interactions. . . . . . . . . . . . . 158

5.20 Corrected inclusive $\pi^{ \pm}$production cross sections for momentum range $0.3-1$ $\mathrm{GeV} / \mathrm{c}$ using TPC for $58 \mathrm{GeV} / \mathrm{c} \mathrm{p}+\mathrm{C}$ interactions. . . . . . . . . . . . . . . . 158 
5.21 Comparison of data inclusive $\pi^{ \pm}$production cross sections with the MC predictions for momentum range $0.3-1 \mathrm{GeV} / \mathrm{c}$ using TPC for $58 \mathrm{GeV} / \mathrm{c}$ p+C interactions. . . . . . . . . . . . . . . . . . . . . . 159

5.22 Uncorrected inclusive $\pi^{ \pm}$production cross sections for momentum range 4.64 - $60 \mathrm{GeV} / \mathrm{c}$ using RICH for $58 \mathrm{GeV} / \mathrm{c} \mathrm{p}+\mathrm{C}$ interactions. . . . . . . . . . . . 159

5.23 Corrected inclusive $\pi^{ \pm}$production cross sections for momentum range $4.64-$ $60 \mathrm{GeV} / \mathrm{c}$ using RICH for $58 \mathrm{GeV} / \mathrm{c} \mathrm{p}+\mathrm{C}$ interactions. . . . . . . . . . . . . 160

5.24 Comparison of data inclusive $\pi^{ \pm}$production cross sections with the MC predictions for momentum range $4.64-60 \mathrm{GeV} / \mathrm{c}$ using $\mathrm{RICH}$ for $58 \mathrm{GeV} / \mathrm{c} \mathrm{p}+\mathrm{C}$ interactions. . . . . . . . . . . . . . . . . 160

5.25 Uncorrected inclusive $\pi^{ \pm}$production cross sections for momentum range 0.3

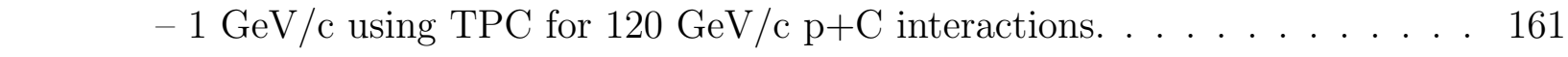

5.26 Corrected inclusive $\pi^{ \pm}$production cross sections for momentum range $0.3-1$ $\mathrm{GeV} / \mathrm{c}$ using TPC for $120 \mathrm{GeV} / \mathrm{c} \mathrm{p}+\mathrm{C}$ interactions. . . . . . . . . . . . . . . 162

5.27 Comparison of data inclusive $\pi^{ \pm}$production cross sections with the MC predictions for momentum range $0.3-1 \mathrm{GeV} / \mathrm{c}$ using TPC for $120 \mathrm{GeV} / \mathrm{c} \mathrm{p}+\mathrm{C}$ interactions. . . . . . . . . . . . . . . . . . . . . 162

5.28 Uncorrected inclusive $\pi^{ \pm}$production cross sections for momentum range 4.64

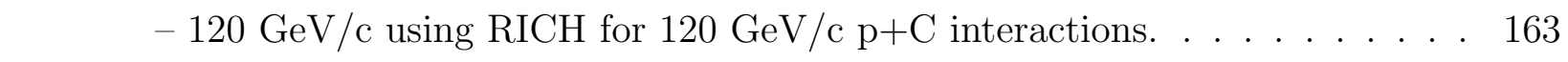

5.29 Corrected inclusive $\pi^{ \pm}$production cross sections for momentum range 4.64 $120 \mathrm{GeV} / \mathrm{c}$ using RICH for $120 \mathrm{GeV} / \mathrm{c} \mathrm{p}+\mathrm{C}$ interactions. . . . . . . . . . . . 164

5.30 Comparison of data inclusive $\pi^{ \pm}$production cross sections with the MC pre\begin{tabular}{|c|}
\hline dictions for momentum range $4.64-120 \mathrm{GeV} / \mathrm{c}$ using $\mathrm{RICH}$ for $120 \mathrm{GeV} / \mathrm{c}$ \\
\hline
\end{tabular} p+C interactions. . . . . . . . . . . . . . . . . . 164

5.31 Comparison of nominal acceptance and the acceptance using the reweighted MC in bins of true momentum for the momentum ranges $0.3-1 \mathrm{GeV} / \mathrm{c}$ and $4.64-120 \mathrm{GeV} / \mathrm{c}$ using $120 \mathrm{GeV} / \mathrm{c} \mathrm{p}+\mathrm{C}$ interactions. . . . . . . . . . . . . . 165

5.32 Comparison of data inclusive $\pi^{ \pm}$production cross sections with the MC pre\begin{tabular}{|c|}
\hline dictions for momentum range $0.3-1 \mathrm{GeV} / \mathrm{c}$ with both the statistical and \\
\hline
\end{tabular} systematic errors plotted for the data for $58 \mathrm{GeV} / \mathrm{c} \mathrm{p}+\mathrm{C}$ interactions. . . . . 167

5.33 Comparison of data inclusive $\pi^{ \pm}$production cross sections with the MC predictions for momentum range $4.64-60 \mathrm{GeV} / \mathrm{c}$ with both the statistical and systematic errors plotted for the data for $58 \mathrm{GeV} / \mathrm{c} \mathrm{p}+\mathrm{C}$ interactions. . . . . 168

5.34 Comparison of data inclusive $\pi^{ \pm}$production cross sections with the MC predictions for momentum range $0.3-1 \mathrm{GeV} / \mathrm{c}$ with both the statistical and systematic errors plotted for the data for $120 \mathrm{GeV} / \mathrm{c} \mathrm{p}+\mathrm{C}$ interactions. . . . 169 
5.35 Comparison of data inclusive $\pi^{ \pm}$production cross sections with the MC predictions for momentum range $4.64-120 \mathrm{GeV} / \mathrm{c}$ with both the statistical and systematic errors plotted for the data for $120 \mathrm{GeV} / \mathrm{c} \mathrm{p}+\mathrm{C}$ interactions. . . . 169

5.36 Comparison of data and MC inclusive $\pi^{ \pm}$production cross sections from TPC and RICH shown on a single plot for $58 \mathrm{GeV} / \mathrm{c} \mathrm{p}+\mathrm{C}$ interactions. . . . . . . 171

5.37 Comparison of data and MC inclusive $\pi^{ \pm}$production cross sections from TPC and RICH shown on a single plot for $120 \mathrm{GeV} / \mathrm{c} \mathrm{p}+\mathrm{C}$ interactions. . . . . . 171

5.38 Comparison of inclusive $\pi^{ \pm}$production cross sections from TPC and RICH for 58 and $120 \mathrm{GeV} / \mathrm{c} \mathrm{p}+\mathrm{C}$ interactions. . . . . . . . . . . . . . . . . . . . . 172 


\section{Chapter 1}

\section{Introduction}

\subsection{The Standard Model}

The main object of interest of Particle Physics, also known as High Energy Physics is the study of the structure of the matter, the subatomic particles. The Standard Model of Particle Physics [1, 2, 3, 4, 5] is a theory of elementary particles and their interactions. Developed in the early 1970s, it has successfully explained almost all of the High Energy Physics experimental data and precisely predicted a wide variety of phenomena. Over time and through many experiments, the Standard Model has become established as a well-tested physics theory.

\subsubsection{Matter particles}

The Standard Model assumes existence of twelve elementary particles, which, together with their antiparticles, compose all known matter, and force carriers - bosons (explained in the next section). These are six leptons (electron, muon, tau and their corresponding neutrinos) and six quarks ( $u p-\mathrm{u}$, down $-\mathrm{d}$, strange $-\mathrm{s}$, charm $-\mathrm{c}$, top $-\mathrm{t}$ and bottom $-\mathrm{b}$ ). Figure 1.1 presents the Standard Model of elementary particles, with the building blocks of matter i.e. leptons and quarks, force-carrier bosons, and finally the Higgs boson which is responsible for the mass of the particles. These are described in the next paragraphs.

The quarks and leptons are divided into three generations. The lightest and most stable particles make up the first generation, whereas the heavier and less stable particles belong to the second and third generations. All stable matter in the universe is made from particles that belong to the first generation; any heavier particles quickly decay to the next most stable level. The six quarks are paired in the three generations - the "up quark" and the "down quark" form the first generation, followed by the "charm quark" and the 


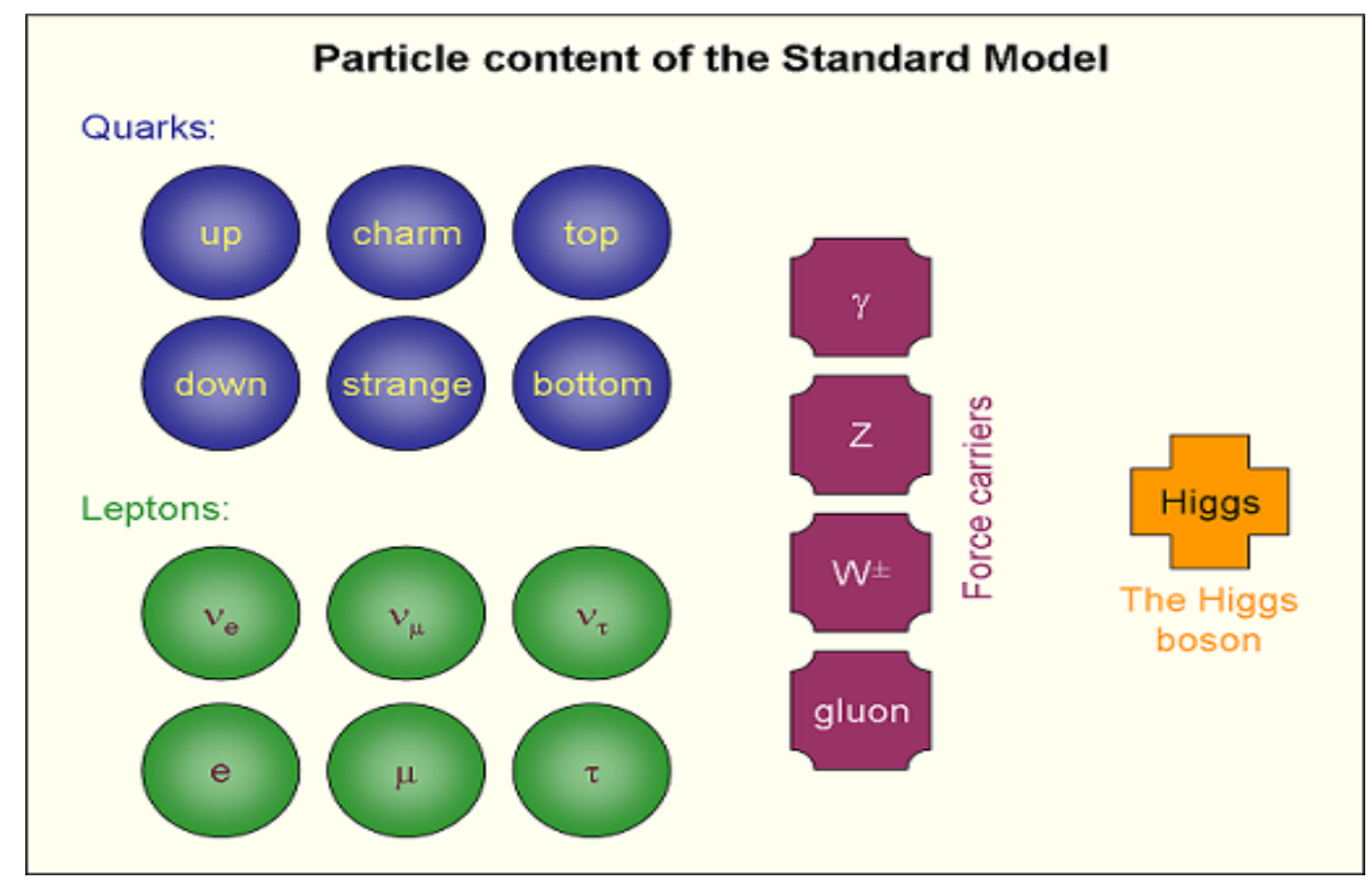

Figure 1.1: The Standard Model of elementary particles, with the three generations of matter, the force carriers - gauge bosons, and the Higgs boson [6].

"strange quark", then the "top quark" and the "bottom quark" forming the second and third generations, respectively. Quarks also come in three different "colors" (red, blue and green - RBG). Each quark can be in one of three color states. Anti-quarks can be in one of three anti-color states (anti-red, anti-blue or anti-green). The color quantum number is introduced to avoid violation of Pauli exclusion principle that all the fermions in a quantum state should not all have the same quantum numbers. The quarks only mix in such ways as to form objects with a color-neutral charge which is possible only by combining all colors RBG, all anti-colors $\bar{R} \bar{B} \bar{G}$, or a color and its anti-color, e.g. GG . Quarks carry fractional electrical charges of $+2 / 3 \mathrm{e}$ or $-1 / 3 \mathrm{e}$, with "-e" the charge of the electron. The six leptons are similarly arranged in three generations - the "electron" and the "electron neutrino", the "muon" and the "muon neutrino", and the "tau" and the "tau neutrino". The electron, the muon and the tau all have an electric charge and a sizeable mass, whereas the neutrinos are electrically neutral and have a very little mass. According to Standard Model, neutrino masses are taken to be zero. Neutrino oscillations show that neutrinos have mass which is very small [7].

Quarks are never observed as single particles but form bound states called hadrons. There are two groups of hadrons: particles, that consist of three quarks - baryons and those consisting of quark-antiquark pairs - mesons. Protons (made up of two up-quarks and one 
down-quark) and neutrons (made up of two down-quarks and one up-quark) are the most common examples of baryons. Pions $\left(\pi^{0}, \pi^{ \pm}\right)$and kaons $\left(\mathrm{K}^{ \pm}, \mathrm{K}^{0}, \bar{K}^{0}\right)$ are the most common types of mesons. The mesons are bosons which have integer values of the spin, therefore they obey Bose-Einstein statistics. Leptons, quarks and baryons, having fractional spin values, are fermions and hence obey Fermi-Dirac statistics and Pauli exclusion principle. While leptons exist as free particles, quarks do not. They are always confined in hadrons.

Table 1.1 lists the three generations of elementary particles and their basic properties [8].

\begin{tabular}{|c|c|c|c|c|c|c|}
\hline \multirow{2}{*}{ Gen } & \multicolumn{3}{|c|}{ Leptons $\left(\right.$ spin $\left.=\frac{1}{2}\right)$} & \multicolumn{3}{c|}{ Quarks (spin = $\left.\frac{1}{2}\right)$} \\
\cline { 2 - 7 } & Flavor & Charge & Mass $\left(\mathrm{MeV} / \mathrm{c}^{2}\right)$ & Flavor & Charge & Mass $\left(\mathrm{MeV} / \mathrm{c}^{2}\right)$ \\
\hline \hline \multirow{2}{*}{1} & $\mathrm{e}$ & -1 & 0.511 & $\mathrm{u}$ & $+\frac{2}{3}$ & $2.3_{-0.5}^{+0.7}$ \\
& $\nu_{e}$ & 0 & $<2.25 \times 10^{-4}$ & $\mathrm{~d}$ & $-\frac{1}{3}$ & $4.8_{-0.3}^{+0.5}$ \\
\hline \multirow{2}{*}{2} & $\mu$ & -1 & 105.7 & $\mathrm{c}$ & $+\frac{2}{3}$ & $(1.275 \pm 0.025) \times 10^{3}$ \\
& $\nu_{\mu}$ & 0 & $<0.19$ & $\mathrm{~s}$ & $-\frac{1}{3}$ & $95 \pm 5$ \\
\hline \multirow{2}{*}{3} & $\tau$ & -1 & 1777 & $\mathrm{t}$ & $+\frac{2}{3}$ & $\begin{array}{c}(173.21 \pm 0.51 \pm 0.71) \times 10^{3} \\
(4.18 \pm 0.03) \times 10^{3}\end{array}$ \\
& $\nu_{\tau}$ & 0 & $<18.2$ & $\mathrm{~b}$ & $-\frac{1}{3}$ & \\
\hline
\end{tabular}

Table 1.1: The three generations of matter and their basic properties.

\subsubsection{Forces and carrier particles}

There are four fundamental forces at work in the universe: the strong force, the weak force, the electromagnetic force, and the gravitational force. They work over different ranges and have different strengths. Gravity is the weakest but it has an infinite range. The electromagnetic force also has infinite range but it is many times stronger than gravity. The weak and strong forces are effective only over a very short range and dominate only at the level of subatomic particles. Despite its name, the weak force is much stronger than gravity but it is indeed the weakest of the other three. The strong force, as the name suggests, is the strongest of all the four fundamental interactions.

Three of the fundamental forces result from the exchange of force-carrier particles, which belong to a broader group called "bosons". Particles of matter transfer discrete amounts of energy by exchanging bosons with each other. Each fundamental force has its own corresponding boson. Strong nuclear force is responsible for the quark confinement. Its force carriers are eight gauge bosons - gluons. Strong interactions are described by Quantum Chromodynamics (QCD) [5] - included in the Standard Model. Gluons also carry 
color charge like quarks. Electromagnetic force is 100 times weaker than the strong force. It affects all charged particles. Photons are its force carriers. This force is described by Quantum Electrodynamics (QED) [9]. Weak nuclear force affects all leptons, quarks and neutrinos. Its force carriers are heavy gauge bosons $-\mathrm{W}^{ \pm}$and $\mathrm{Z}^{0}$. That is why the interaction has a short range. The weak interaction is the only interaction known to violate $\mathrm{P}$ and $\mathrm{CP}$ symmetries, and change particles' flavor [4]. It is $10^{13}$ times weaker than the strong force. The weak and electromagnetic forces have been successfully unified to electroweak interactions [10]. Although not yet found, the "graviton" should be the corresponding force-carrying particle of gravity. The characteristics of all the three forces which are included in the Standard Model, are listed in table 1.2 .

\begin{tabular}{|c|c|c|c|c|c|c|}
\hline Force & $\begin{array}{c}\text { Mediating } \\
\text { boson }\end{array}$ & Charge & Spin & Mass $\left(\mathrm{GeV} / \mathrm{c}^{2}\right)$ & Range $(\mathrm{m})$ & Rel. Strength \\
\hline Strong & Gluon $(\mathrm{g})$ & 0 & 1 & 0 & $10^{-15}$ & $10^{38}$ \\
\hline EM & Photon $(\gamma)$ & 0 & 1 & 0 & $\infty$ & $10^{36}$ \\
\hline Weak & $\mathrm{W}^{ \pm}$ & \pm 1 & 1 & $80.385 \pm 0.015$ & $10^{-18}$ & $10^{25}$ \\
& $\mathrm{Z}^{0}$ & 0 & 1 & $\begin{array}{c}91.187 \pm 0.002 \\
{[11,[12}\end{array}$ & & \\
& & & & {$[1$,} & & \\
\hline
\end{tabular}

Table 1.2: Fundamental forces included in Standard Model and their properties.

The Standard Model includes the electromagnetic, strong and weak forces, and all their carrier particles, and explains well how these forces act on all of the matter particles. However, the most familiar force in our everyday lives, gravity, is not part of the Standard Model, as fitting gravity comfortably into this framework has proved to be a difficult challenge. The quantum theory used to describe the micro world, and the general theory of relativity used to describe the macro world, are difficult to fit into a single framework. No one has managed to make the two mathematically compatible in the context of the Standard Model. But luckily for Particle Physics, when it comes to the minuscule scale of particles, the effect of gravity is so weak as to be negligible. Only when matter is in bulk, at the scale of the human body or of the planets for example, does the effect of gravity dominate. So the Standard Model still works well despite its reluctant exclusion of one of the fundamental forces.

Even though the Standard Model is currently the best description of the subatomic world, but we know that it is incomplete because the theory incorporates only three out of the four fundamental forces, omitting gravity. There are also important questions that it does not answer, such as "What is dark matter and dark energy?", or "Why is there matter/antimatter asymmetry in the Universe", or "Why are there three generations of quarks and leptons with such a different mass scale?" and many more. 
On July 4, 2012, the other missing piece of Standard Model - scalar boson named as Higgs boson was announced to be discovered by the ATLAS [13] and CMS [14] experiments at CERN's [15] Large Hadron Collider (LHC) [16]. These experiments announced that they had each observed a new particle in the mass region around $125 \mathrm{GeV}$. The results from these two experiments are consistent, within uncertainties, with expectations for the Standard Model Higgs boson [17, 18].

The Nobel prize in physics was awarded jointly to François Englert and Peter Higgs on October 8, 2013, "for the theoretical discovery of a mechanism [19] that contributes to our understanding of the origin of mass of subatomic particles, and which was confirmed through the discovery of the predicted fundamental particle, by the ATLAS and CMS experiments at CERN's LHC".

\section{$1.2 \quad$ Strong interactions and QCD}

Till the middle of 20th century, over a hundred strongly interacting particles were discovered. In 1964, Gell-Mann and Zweig proposed that these particles are not elementary but they consist of smaller objects called quarks. Scattering of high-energy leptons from hadrons confirmed that there are point-like constituents in the hadrons identified with quarks and gluons - carriers of strong force. Quarks appear in six types or flavors - u, d, s, c, b, t as discussed in section 1.1 .

Quantum Chromodynamics (QCD) [5] is a theory of strong interaction. In QCD, the fundamental particles (quarks) interact via exchange of gluons. The gluons are eight color charged field quanta. The quarks are constituents of bound systems without net color charge. These systems are called hadrons. Forces between hadrons can be considered as residual color interactions in analogy to electron scattering (photon exchange) in Quantum Electrodynamics (QED). Unlike QED where photon doesn't couple to itself, the gluons which have color charge couple to each other. The gluon self-interaction leads to decrease in strong coupling constant $\alpha_{s}$ as momentum transfer $\mathrm{Q}^{2}$ increases. In $\mathrm{QCD}$, the effective coupling constant is given by:

$$
\alpha_{s}\left(Q^{2}\right)=\frac{4 \pi}{\left(11-\frac{2}{3} n_{f}\right) \ln \frac{Q^{2}}{\Lambda^{2}}},
$$

where $\mathrm{n}_{f}$ is the number of quarks flavors and $\mathrm{Q}$ is the magnitude of momentum transfer during the interaction. Here $\alpha_{s}$ depends on one parameter $\Lambda$ which is measured experimentally and determined to be $\Lambda \approx 200 \mathrm{MeV}$. This formula is perturbative and it breaks down 
for large couplings. Nevertheless the parameter $\Lambda$ is a useful measure for energy scale where the strong coupling constant becomes large. It is called the QCD scale parameter. If the number of flavors $\mathrm{n}_{f} \leq 16$ (we believe that $\mathrm{n}_{f}=6$ ) then $\alpha_{s}$ will decrease with increase of momentum transfer $\mathrm{Q}$ and $\alpha_{s} \rightarrow 0$ as $\mathrm{Q} \rightarrow \infty$ (this corresponds to small distances) which is known as asymptotic freedom. This property of coupling constant gives a possibility to use perturbative QCD at high-energy processes. This domain is known as hard hadronic physics. At low momentum transfer perturbative methods are not applicable. This domain is known as soft hadronic physics.

Calculation of low-energy processes require non-perturbative methods. This can be performed by numerical techniques, where continuous space-time points are replaced with discrete coordinates, the method is called lattice QCD.

\subsection{Soft Hadronic Physics}

Perturbative methods are not applicable in low momentum transfer processes, since the coupling constant $\alpha$ is too large. A number of phenomenological models have been developed in order to study soft hadronic processes. A more recent approach is based on the parton model, motivated by its success in lepton-hadron deep inelastic scattering. In general, the hadron-hadron interaction is assumed to proceed in two steps. In first step, a quark from one of the hadrons interacts with a quark from the other hadron. In the second step, the "hadron remainder" fragments into final-state hadrons. The fragmentation function is the same as in $\mathrm{e}^{+} \mathrm{e}^{-}$and lepton-hadron interactions. A complete review can be found in [20]. A good understanding of hadronic interactions at low momentum transfer is of key importance to build and tune hadronic interaction models, which are a main component of neutrino flux and cosmic ray simulation models.

Phenomenological models have a number of free parameters which have to be determined from the measured data. In the lack of quantitative theoretical predictions, an attempt is made to look at a model independent way of improving understanding of the underlying production mechanism. This requires to exploit all possible types of hadronic interactions, ranging from elementary hadron-hadron $(h+h)$ through hadron-nucleus $(h+A)$ to nucleus-nucleus $(\mathrm{A}+\mathrm{A})$ collisions, combined with a large phase space coverage and particle identification.

Multi-particle final states encountered in hadronic interaction form a multi-dimensional phase space. An inclusive single particle production covers only the simplest surface of the multi-dimensional phase space. 
In this thesis, an inclusive production of charged pions in $\mathrm{p}+\mathrm{C}$ collisions is examined:

$$
p+C \rightarrow \pi^{ \pm}+X .
$$

Hadron-nucleus interactions give an access to multiple hadronic collision processes. They also give an important link between $\mathrm{h}+\mathrm{h}$ and $\mathrm{A}+\mathrm{A}$ interactions. In fact any model independent attempt for understanding the latter processes has to be based on the reference to more elementary $\mathrm{h}+\mathrm{h}$ and $\mathrm{h}+\mathrm{A}$ interactions.

\subsection{Need for a Hadron Production Experiment}

As neutrino experiments enter a new domain of precision, important systematic errors due to poor knowledge of production cross sections for pions and kaons require more dedicated measurements for precise neutrino flux predictions. The main focus of these neutrino experiments is to detect the neutrinos, to see how they interact with the matter and to look for the neutrino oscillations. But we do not know precisely the neutrino fluxes and their production cross sections that is at which rate they are being produced. As an example, the acceleratorbased neutrinos are decay products of pions and kaons produced in the collisions of incident protons on a nuclear target. The major source of uncertainty in the accurate prediction of neutrino fluxes is the production of these primary hadrons. So, the hadroproduction data play an important role in the neutrino experiments.

\subsection{Particle Production and Neutrino Experiments}

Particle production is one of the classic phenomena studied by many experiments over the twentieth century. While many experiments focused on production of exotic particles, there were few high statistics measurements of inclusive production of pions, kaons and protons. Some cross sections were measured with bubble chambers, but most high statistics measurements were done with single arm spectrometers [21, 22, 23]. These detection techniques have specific limitations. In bubble chamber, separation between pions, kaons, and protons above a few hundred $\mathrm{MeV} / \mathrm{c}$ is not possible. Furthermore, the number of events that can be collected and analyzed is limited. Single-arm spectrometers have small kinematic acceptance region but they can collect large number of events. Apart from bubble chamber and singlearm spectrometer experiments, some data collected in colliding beam were used in this field, for example, the data on the rapidity distributions in the central region collected by UA5 experiment at the CERN's super anti-proton proton collider [24]. These experiments have 
explored only small fraction of the phase space and without sufficient precision needed these days. As a result, hadronic production models like MARS [25] and FLUKA [26] have limited data to be tuned with and in some cases give significantly different results. Figure 1.2 shows appropriately scaled data from single arm spectrometers superimposed on the spectrum of pions which produce neutrinos in far and near detectors of Main Injector Oscillation Search (MINOS) experiment (accelerated-based neutrino experiment) 27] in the NuMI (Neutrinos at the Main Injector) beamline [28]. The discreteness of the measurements from the single arm spectrometers in $\left(\mathrm{p}, \mathrm{p}_{T}\right)$ space can be seen in the plot. The single arm spectrometers have inherently more systematics than open geometry apparatus. This is because they must change geometry of the single arm frequently and make assumptions in calculating acceptances. On the other hand, open geometry experiments have less systematics and they sample the phase space uniformly and continuously. They can separate primary pion spectra from pions induced by the decay of kaons etc..

\section{Low Energy Beam}

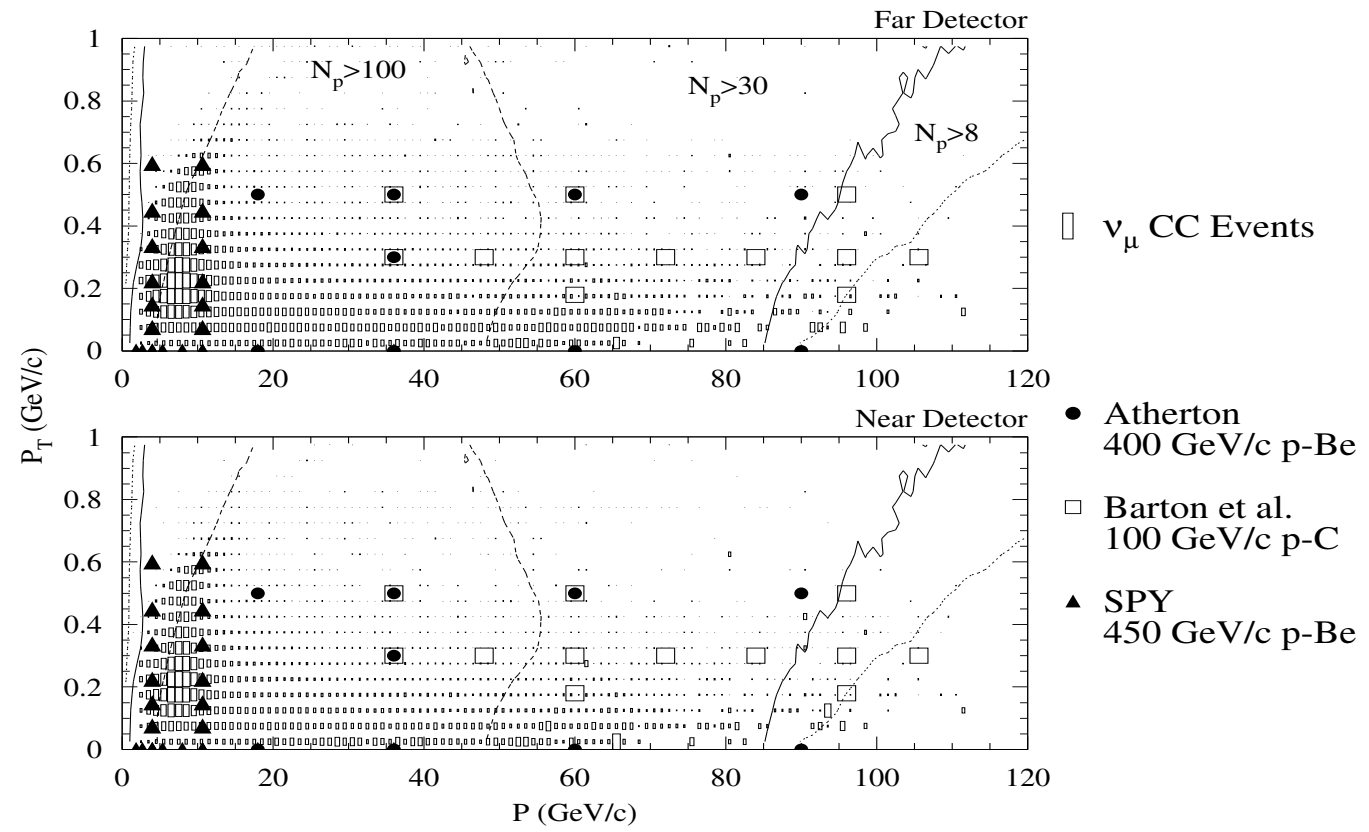

Figure 1.2: The plot shows the spectrum of pions which produce neutrinos in the MINOS far and near detectors. Superimposed are appropriately scaled data on hadron production obtained from single arm spectrometer measurements, which explains their discreteness in $\left(\mathrm{p}, \mathrm{p}_{T}\right)$ space [29].

Scarcity of hadronic production data affects every neutrino experiment [30, 31, 32, 33] as accelerator-made and atmospheric neutrinos come from the decay of pions and kaons produced in interactions of protons with matter. The uncertainties in particle production models 
largely cancel in comparison of the near and far detector data in case of a two-detector neutrino experiment, but calculation of neutrino flux with various hadronic production models differ by up to $30 \%$, shown in figure 1.3. As an example, in case of MINOS experiment, the near detector sees a line source of neutrinos and the far detector sees a point source of neutrinos, so these uncertainties do not cancel completely, and the near/far detector comparison depends on the hadronic production model. Figure 1.3(a) shows the predictions of the absolute neutrino rates in the MINOS near detector using four existing hadron production models [34]. The model predictions differ from the average by as much as $20 \%$ as a function of neutrino energy. Figure 1.3(b) shows the predictions of the ratio of the far to the near neutrino flux using the same four models. Again, there is considerable uncertainty in the predictions, which increases in the high energy tail of the spectrum.
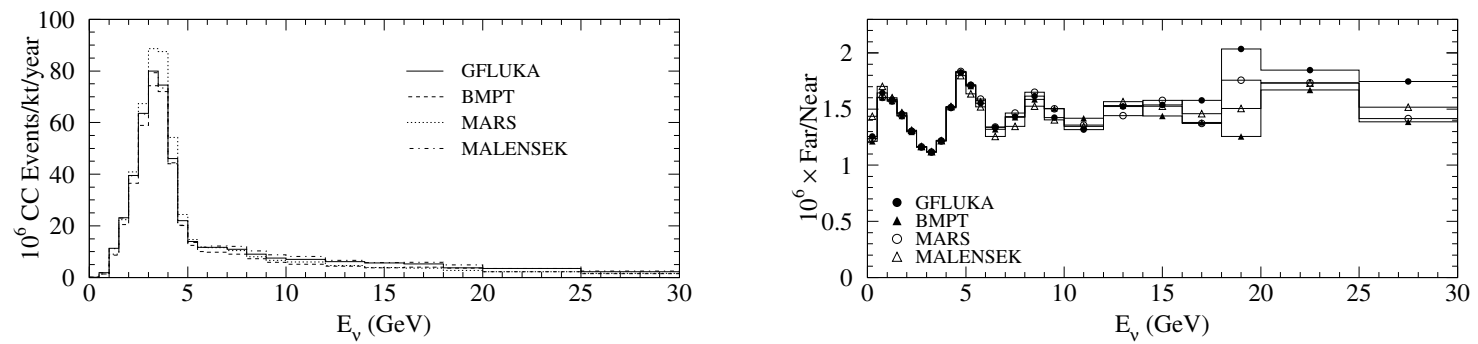

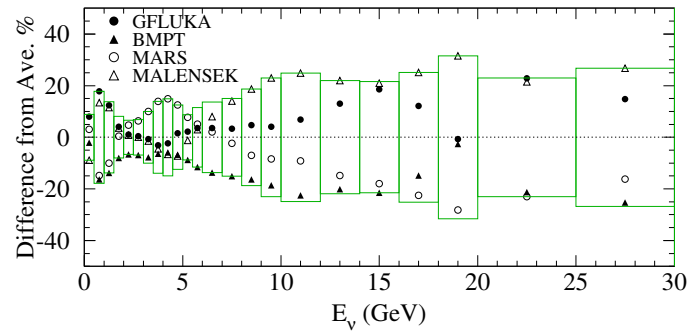

(a)

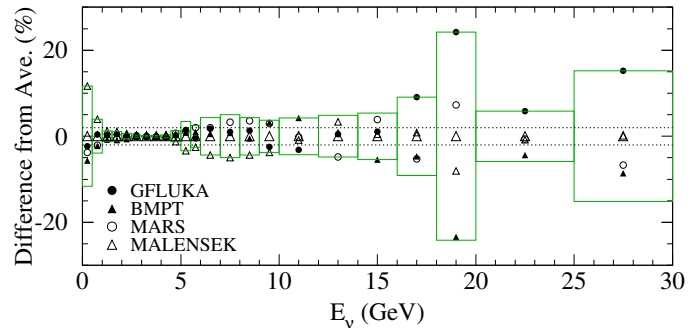

(b)

Figure 1.3: Uncertainties in neutrino flux calculation in NuMI beam. Since the MINOS near detector sees a line source of neutrino and far detector sees a point source, these uncertainties do not cancel for all momenta of interest. (a) Hadronic uncertainties contribute $15-20 \%$ to absolute rate uncertainty and (b) $2-10 \%$ uncertainties in far to near ratio [29]. Having better understanding of hadronic production can help to minimize the systematic error due to neutrino flux prediction.

No direct measurements of cross sections at the energy of NuMI proton beam existed until there were measurements on $\pi$-K ratios [35] and $\pi^{ \pm}$yields [36] from the NuMI target by MIPP [37]. The nearest data of proton on carbon were taken at $158 \mathrm{GeV} / \mathrm{c}$ by the NA49 experiment at CERN which published results of the measurement of charged pion production in proton-carbon collisions at $158 \mathrm{GeV} / \mathrm{c}$ [38], which is close to the NuMI beam 
of $120 \mathrm{GeV} / \mathrm{c}$ protons. However, the secondary and tertiary particle production on the NuMI target is significant, so if one is to tune Monte Carlo methods to better predict neutrino flux, it is essential to have the data of hadronic production of pions, kaons and protons on carbon at lower momenta. Moreover, large fraction of high energy neutrinos come from decay of charged kaons, shown in figure 1.4, so knowledge of kaon flux is necessary as well.
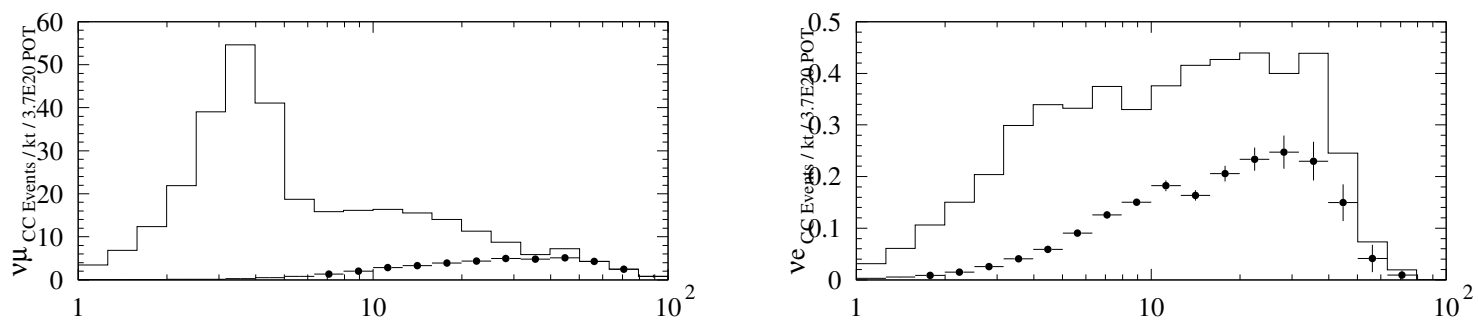

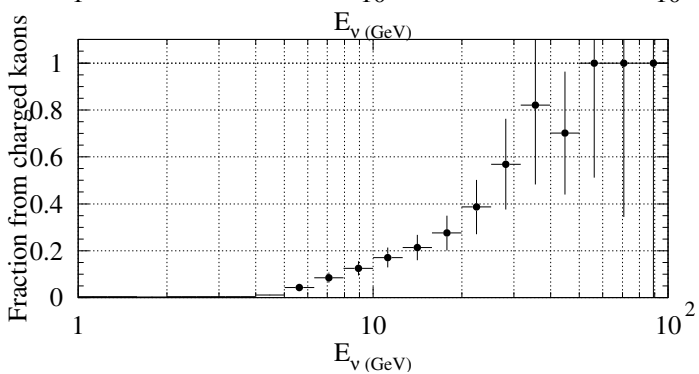

(a)

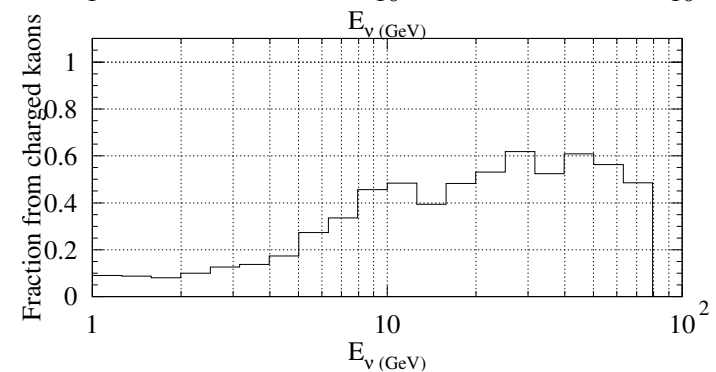

(b)

Figure 1.4: (a) MINOS muon neutrino and (b) electron neutrino fluxes (top) and the fraction of neutrinos produced in kaon decays (bottom) [29].

\subsection{The MIPP Experiment at Fermilab}

The Main Injector Particle Production (MIPP) experiment [39] is a fixed target hadron production experiment which was designed to measure the total charged particle production of $\pi^{ \pm}, \mathrm{K}^{ \pm}, \mathrm{p}$, and $\overline{\mathrm{p}}$ by tagged beams of charged pions and kaons, proton and anti-proton on nuclear targets. The MIPP experiment, located in the Meson Center beamline at Fermi National Accelerator Laboratory (FNAL) also known as Fermilab [40], took data from January 2005 to February 2006 and collected $\sim 18$ million events. It is an open geometry and full acceptance spectrometer. The experiment took data using primary beam of $120 \mathrm{GeV} / \mathrm{c}$ protons from the Main Injector and secondary beams from 5 to $90 \mathrm{GeV} / \mathrm{c}$ on various nuclear targets including beryllium, carbon, bismuth and uranium. These secondary beams were produced by striking the primary $120 \mathrm{GeV} / \mathrm{c}$ protons on a copper (primary) target. The 
list of data collected by the MIPP using various beams and targets is shown in table 1.3 . A time projection chamber (TPC) provides nearly complete coverage for the forward-going particles, and wire chambers allow measurement of momentum up to $120 \mathrm{GeV} / \mathrm{c}$. Particle identification is done for nearly all momenta of particles produced on the target using energy loss in the TPC, time of flight, threshold Cherenkov, and ring imaging Cherenkov (RICH) counters. An extensive description of the MIPP detectors is given in the next chapter.

\begin{tabular}{|c|c|c|c|}
\hline Beam & $\begin{array}{c}\text { Momentum } \\
(\mathrm{GeV} / \mathrm{c})\end{array}$ & Target & No. of events \\
\hline p's & 120 & NuMI & $2.1 \times 10^{6}$ \\
\hline$\pi$ 's, K's, p's & $120,60,35,20$ & $1-2 \% \lambda_{I} \mathrm{C}$ and Be & $3.2 \times 10^{6}$ \\
\hline$\pi$ 's, K's, p's & $85,60,20,5$ & $1.5 \% \lambda_{I} \mathrm{LH}_{2}$ & $7 \times 10^{6}$ \\
\hline$\pi$ 's, K's, p's & $120,60,35$ & $1 \% \lambda_{I} \mathrm{Bi}$ and $\mathrm{U}$ & $4 \times 10^{6}$ \\
\hline
\end{tabular}

Table 1.3: Data collected in MIPP using various beams and targets.

MIPP also collected data on a liquid hydrogen $\left(\mathrm{LH}_{2}\right)$ target. Multiplicity distributions and cross sections are very well known for liquid hydrogen from the bubble chamber data [41]. We can compare our data to these measurements for the validation purposes. In addition to taking data on liquid hydrogen and thin nuclear targets, the MIPP directly collected data on a target used in the NuMI facility at Fermilab. Thus it is possible to cross check the understanding of NuMI hadron flux by comparing the spectrum from the actual target to the model of secondary production by using the proton-carbon data at $120 \mathrm{GeV} / \mathrm{c}$ and hadron-carbon data at lower momenta. This improved understanding of hadronic flux can be useful to the NuMI-based experiments like MINOS, MINER $\nu$ A, NO $\nu$ A and ELBNF [30, 31, 42, 43, 44] since the systematic errors due to the hadron production rates will be reduced.

This thesis gives a detailed description of results obtained from data collected with the liquid hydrogen, beryllium, carbon and bismuth targets using proton beams at 58 and 85 $\mathrm{GeV} / \mathrm{c}$ for liquid hydrogen, and at 58 and $120 \mathrm{GeV} / \mathrm{c}$ for the other three targets. Inelastic cross sections have been measured for these data sets. Inclusive charged pion production cross sections have also been measured in bins of momentum for 58 and $120 \mathrm{GeV} / \mathrm{c}$ protoncarbon interactions.

\subsection{Motivations for the MIPP Experiment}

As already discussed in the previous sections, the hadroproduction data play an important role in the atmospheric and accelerator-based neutrino experiments. There are many other 
areas of Physics where the MIPP data could be useful. The primary motivations behind doing MIPP are as follows:

\subsubsection{Neutrino Flux Predictions}

The major source of uncertainty in the accurate prediction of neutrino fluxes for the neutrino and anti-neutrino cross section measurements by many NuMI-based experiments [45, 46, 47], is the production of the primary hadrons from which these neutrinos are produced. One of the goals of the MIPP experiment is to measure the hadron production yield off an actual NuMI target with $120 \mathrm{GeV} / \mathrm{c}$ protons from the Main Injector to within $5 \%$ in order to verify and validate the Monte Carlo calculations of the NuMI flux. The charged pion yields have been extracted from the MIPP NuMI data [36]. These data may be directly used to improve the calculation and the uncertainties on the calculation of the neutrino flux in the NuMI beam line.

\subsubsection{Hadronic Shower Simulation}

As already discussed in section 1.5, the Monte Carlo simulation programs like GEANT4, MARS, FLUKA, etc. model hadronic interactions based on available data. Most existing data are low statistics, with poor particle identification and sometimes contradictory. All neutrino flux problems (MINOS, MiniBooNE, K2K, T2K, NO $\nu$ A, MINER $\nu$ A) [45, 32, 48, 49, 50, 42, 46, 47], can be reduced to one problem: "the current insufficient state of hadronic shower simulators" [51]. MIPP has high statistics and low systematics data with 6 beam species. These data can be used in the simulation packages for better modeling of showers.

At a workshop on Hadronic Shower Simulations [52], experts in shower simulation codes from five major Monte Carlos (GEANT4, FLUKA, MARS, MCNPX, and PHITS) (and several less well-known ones) met and reviewed their code status and what needs to be done further to improve codes. It was acknowledged that more particle production data would help improve algorithms a great deal and there were calls for a rapid publication of existing MIPP data.

\subsubsection{Study of non-perturbative QCD}

The primary physics motivation behind MIPP is to restart the study of non-perturbative QCD interactions [53], which constitute over $99 \%$ of the strong interaction cross section. Perturbative QCD has made impressive progress. But it relies on structure functions for 
its calculations, which are non-perturbative and derived from data. The available data are of poor quality and old, and are not in easily accessible form. The TPC which is at the heart of the MIPP experiment represents the electronic equivalent of the bubble chamber with superior data acquisition rates. It also digitizes the charged tracks in three dimensions, obviating the need for track matching across stereo views. Coupled with the particle identification capability of MIPP, the data from MIPP would add significantly to our knowledge base of non-perturbative QCD.

\subsubsection{Scaling Law of Hadronic Fragmentation}

One of the primary goals of MIPP is to verify a general scaling law of inclusive particle production in question [54]. The law states that the ratio of a semi-inclusive cross section to an inclusive cross section involving the same particles is a function only of the missing mass squared $\left(\mathrm{M}^{2}\right)$ of the system and not of the other two Mandelstam variables $s$ and $t$, i.e. the center of mass energy squared and the momentum transfer squared respectively. Stated mathematically, the ratio

$$
\frac{f_{\text {subset }}(a+b \rightarrow c+X)}{f(a+b \rightarrow c+X)}=\frac{f_{\text {subset }}\left(M^{2}, s, t\right)}{f\left(M^{2}, s, t\right)}=\beta_{\text {subset }}\left(M^{2}\right)
$$

i.e. the ratio of two functions of three variables $\mathrm{M}^{2}, s$ and $t$, is only a function of one of the three variables which is $\mathrm{M}^{2}$. The physics behind the scaling law may be understood by considering inclusive cross sections as the analytic continuations of crossed three body interactions, which factorize into a production term that results in the formation of a short-lived fireball of mass $\mathrm{M}^{2}$, which subsequently decays into the subset in question. The formation is governed by s and t. The decay term is only a function of $\mathrm{M}^{2}$. It should be noted that the physics in question falls outside the scope of perturbative QCD and as such the scaling law is not currently derivable from QCD considerations.

This scaling relation has been shown to hold very well in a limited number of reactions [55]. The problem with existing data is that it is usually sparse as bubble chambers were being used. It is very difficult to test the law using existing data for $s$ independence, since only rarely has the same apparatus been used to study the same reaction at multiple energies. MIPP will test this scaling as a function of both $s$ and $t$ for various particle types a, $\mathrm{b}$, and c for beam energies between 5 and $120 \mathrm{GeV}$ to unprecedented statistical and systematic accuracy for 36 reactions. In addition to this, MIPP acquired high quality data in liquid hydrogen with excellent particle identification and statistics over a range of beam momenta, which should make possible a systematic study of exclusive reactions that is essential for 
testing any future theory of non-perturbative QCD. The existence of beams of different flavor and energies will be a great advantage in sorting out the flavor content of any new states seen. The other physics clients for MIPP data are nuclear and heavy ion physics groups who are interested in data from several targets.

\subsection{Other Hadron Production Experiments}

As already mentioned in the previous sections, results from the accelerator-based neutrino experiments are dominated by systematic uncertainties linked to the unsatisfactory prediction of the neutrino flux. Over the past years, several hadron production experiments have been conducted to provide the needed reference data for the neutrino experiments. These experiments have been conducted over a range of incident proton beam momenta from around 10 $\mathrm{GeV} / \mathrm{c}$ to $450 \mathrm{GeV} / \mathrm{c}$, using different target materials, mostly beryllium, aluminium, copper and lead [56].

The HARP experiment [57] at CERN-PS had taken data using beams of protons and charged pions in the momentum range from 1.5 to $15 \mathrm{GeV} / \mathrm{c}$ and nuclear target materials from hydrogen to lead, having thicknesses varying from 2 to $100 \% \lambda_{I}$. The results were subsequently applied to the final analysis of K2K [48] and MiniBooNE [58]. In K2K, the data from $12.9 \mathrm{GeV} / \mathrm{c}$ protons interacting on a thin aluminium target $\left(\lambda_{I}=5 \%\right)$ had a significant impact on the final disappearance analysis. It allowed a reduction of the dominant systematic error associated with the calculation of the far to near ratio from $5.1 \%$ to $2.9 \%$ and thus an increased K2K sensitivity to the oscillation signal [59]. The double-differential inelastic cross section for the production of positive pions from proton-beryllium interactions at $8.9 \mathrm{GeV} / \mathrm{c}[58$, had also contributed to the MiniBooNE results.

The NA61/SHINE [60] experiment at CERN-SPS measured the proton-carbon cross section at $31 \mathrm{GeV} / \mathrm{c}$ for the $\mathrm{T} 2 \mathrm{~K}$ experiment [61]. Using these measurements, the model dependence of the $\nu$ flux prediction is reduced down to $10 \%$ as compared to $40 \%$ in the standard approach.

If we compare the data from the MIPP experiment to the data available so far from the other hadron production experiments mentioned earlier, MIPP had also collected data using beams of anti-protons and charged kaons on nuclear targets and these data are not available in any other hadron production experiment.

\subsection{Outline of the thesis}

This thesis is organized as follows: 
Chapter 2 contains the description of the MIPP beamline, targets, and detectors used by the MIPP spectrometer. The MIPP trigger and data acquisition system, and the event reconstruction are also explained in this chapter.

Chapter 3 describes the detailed analysis procedure for the inelastic cross section measurements and the inclusive charged pion production cross section measurements. The event and track selection criteria, beam flux calculation, interaction trigger efficiency and the Monte Carlo corrections are discussed.

Chapter 4 discusses about the particle identification techniques used for identifying pions. The unfolding of the data pion momentum spectrum using the Monte Carlo is also described.

Chapter 5 presents the final results on inelastic cross sections for $\mathrm{p}+\mathrm{p}, \mathrm{p}+\mathrm{Be}, \mathrm{p}+\mathrm{C}$ and $\mathrm{p}+\mathrm{Bi}$ interactions at different beam momenta, and also the inclusive charged pion production cross sections in bins of momentum for 58 and $120 \mathrm{GeV} / \mathrm{c} \mathrm{p}+\mathrm{C}$ interactions.

Finally, Chapter 6 gives a summary of the results. 


\section{Chapter 2}

\section{The MIPP Experiment}

In this chapter, I describe the MIPP beamline and the detectors used by the MIPP spectrometer. The targets used in the experiment, the trigger and data acquisition system (DAQ), and the event reconstruction are also described.

\subsection{Fermilab Accelerator Complex}

The beam for the MIPP experiment is extracted from the Main Injector. The schematic of the Fermilab accelerator complex is shown in figure 2.1. A Cockcroft-Walton pre-accelerator, a linear accelerator (Linac) and a synchrotron (Booster) accelerate batches of protons up to $8 \mathrm{GeV} / \mathrm{c}$, and then the Main Injector RF cavities accelerate protons up to $119.7 \mathrm{GeV} / \mathrm{c}$. Up to 7 Booster batches can be injected into the Main Injector to be accelerated simultaneously. Protons for the MINOS experiment [27] or for $\bar{p}$ production for the Tevatron collider [62] were extracted in single turn. The MIPP spectrometer required resonant extraction out of the Main Injector to reduce the instantaneous rate. Proton beam extracted towards the Switchyard passes through the A-section of the Tevatron ring. At the Switchyard, it can be split between the Meson Test area, the Meson Center (MC) area and the beam dump. MIPP spectrometer is located in the MC7 hall.

The experiment was approved under the condition that it would not impact the accelerator program by more than $5 \%$. In practice that meant that we were not to slow down $\overline{\mathrm{p}}$ production by more than $5 \%$. At the beginning of the MIPP run, this translated into extracting all but one Booster batch to the $\overline{\mathrm{p}}$ production, and slowly extracting the last batch to MIPP. Since resonant extraction increased the length of the Main Injector time cycle, while the $\overline{\mathrm{p}}$ stack was small and the stacking rate high, MIPP received about six 600 ms spills per minute. Once the $\overline{\mathrm{p}}$ stack grew and stacking rate slowed down, MIPP was able to receive up to 15 spills per minute. 


\section{FERMILAB'S ACCELERATOR CHAIN}

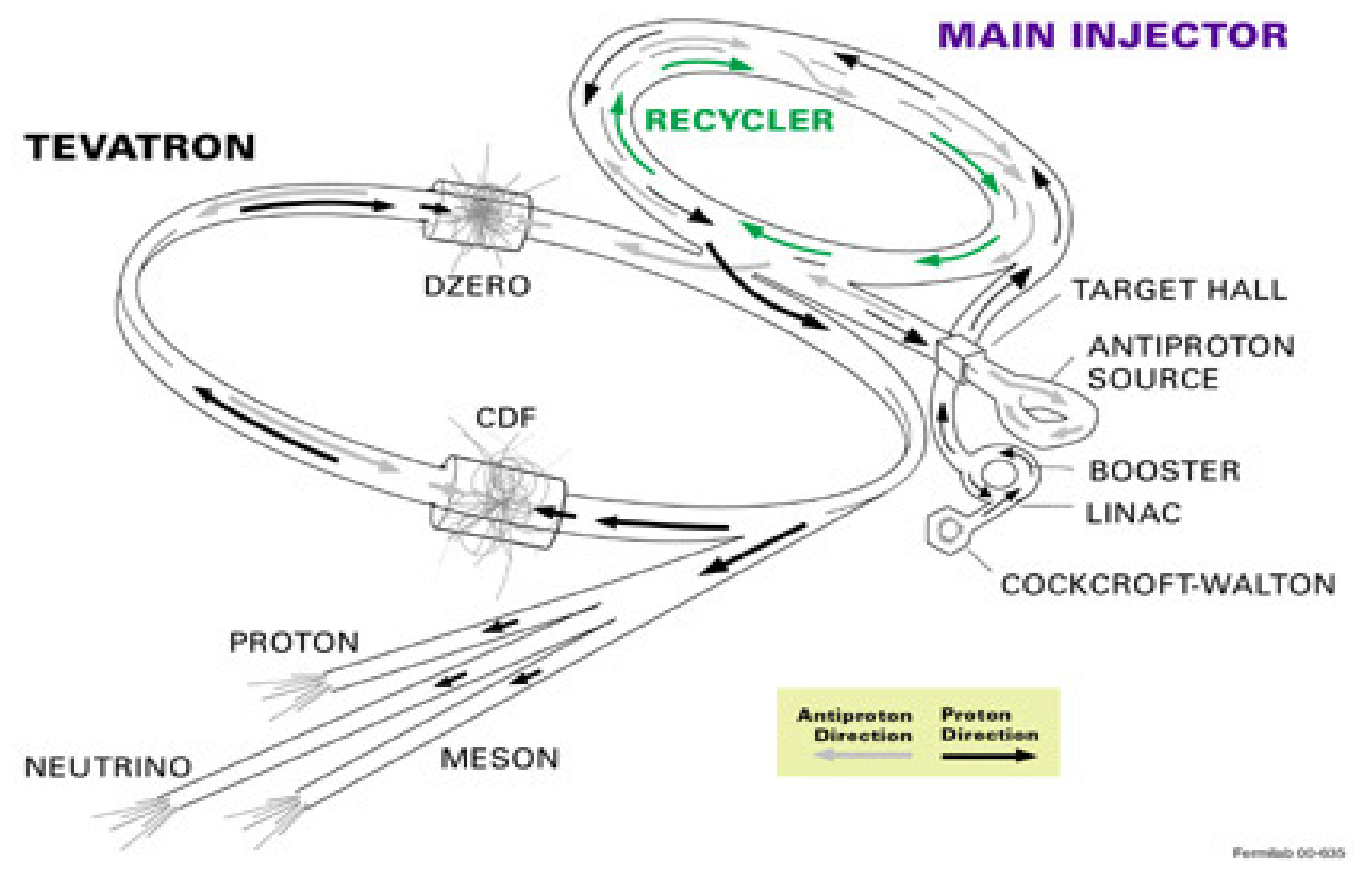

Figure 2.1: Schematic of the Fermilab accelerator complex. The MIPP experiment is located in the Meson Center beamline. 
In March 2005, the NuMI beamline [28] started receiving proton beam for the MINOS experiment, and in April 2005, the accelerator complex operation was changed so that NuMI and $\overline{\mathrm{p}}$ production co-existed with single turn extraction to each destination, and a 4 second spill was extracted to the Switchyard once every 2 minutes. MIPP operated under these conditions through February 2006.

\section{$2.2 \quad$ MIPP Beamline}

The MIPP beamline is operated in two distinct modes:

1. $120 \mathrm{GeV} / \mathrm{c}$ primary protons.

2. $5-90 \mathrm{GeV} / \mathrm{c}$ secondary particles produced on a copper (primary) target.

The MIPP primary and secondary beamlines are installed in the Meson Center (MC) area at Fermilab, beginning in enclosure 5 (MC5), and extending halfway through enclosure 7 (MC7). A picture of the physical layout of the MIPP beamline is given in figure 2.2 .

The primary beamline, primarily in MC5, focuses and collimates the $120 \mathrm{GeV} / \mathrm{c}$ protons. These collimators also control the primary beam flux. The secondary beam line, beginning in MC6, consists of two pairs of dipoles to bring the beam to the centerline of the spectrometer, the primary target, the momentum selection aperture, and associated focusing quadrupoles. Following the last quadrupole, at the beginning of $\mathrm{MC}$, three wire chambers track the secondary beam particles, and a time of fight system and pair of Cherenkov counters identify the beam particle species (explained later in this chapter).

\subsubsection{Primary Beamline}

The $120 \mathrm{GeV} / \mathrm{c}$ primary proton beam is directly extracted from the Main Injector. The minimum rate at which protons can be extracted from the Main Injector is about $10^{8}$ particles per second. Control over primary beam characteristics is established through a focusing quadrupole doublet and adjustable collimators located upstream of the primary target. Due to enclosure restrictions and data taking rates in the TPC (see section 2.9), intensity of the primary beam is attenuated to less than $10^{5}$ particles per second. By tuning the primary optics and the collimator aperture, the desired beam intensity is achieved.

During the NuMI target running period, it was necessary to reduce the beam intensity

to $10^{3}$ particles/spill, with a 4 -second spill occurring every two minutes from the Main Injector. This is because the NuMI target being 2 interaction lengths long, nearly $90 \%$ of 


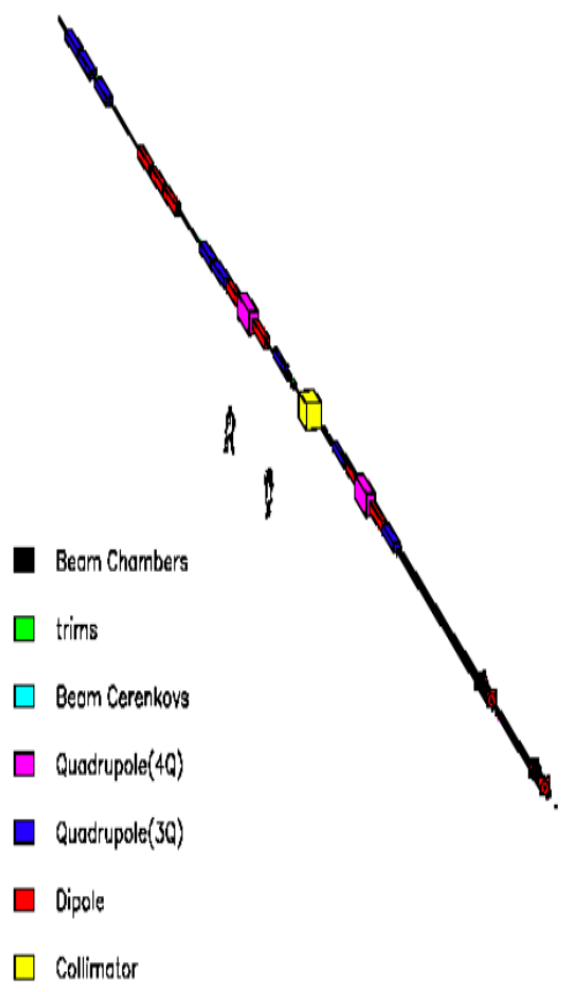

(a) Isometric view of the MIPP beamline. The secondary beamline is short (98 meters) permitting kaon fluxes down to $\approx 3 \mathrm{GeV} / \mathrm{c}$ in momentum.

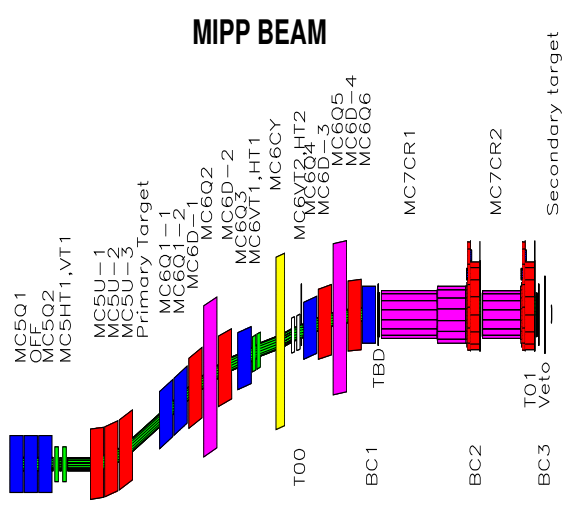

$$
\begin{aligned}
& \text { MC5Q1-2,MC6Q1-6--Quadrupoles } \\
& \text { MC5U1-3, MC6D1-3--Dipoles } \\
& \text { MC6CY -- Momentum width selection Collimator } \\
& \text { MCHT 1VT1 MC6HT2,VT2 --Trim Dipoles } \\
& \text { MC7CR1, MC7CR2 --Beam Cerenkovs } \\
& \text { BC1-3 --Beam Chambers } \\
& \text { TED, TOO, TO1 --time of flight/trigger counters }
\end{aligned}
$$

(b) Plan view of the MIPP beamline.

Figure 2.2: (a) Isometric and (b) plan views of the MIPP beamline. 
incident particles interact with the target, hence beam intensity has to be maintained at a lower rate so that the desired event rate can be achieved, thus avoiding pile-ups.

\subsubsection{Secondary Beamline}

At the entrance to the MC6 area immediately upstream of the MC7 hall, $120 \mathrm{GeV} / \mathrm{c}$ protons are focused onto a $0.5 \mathrm{~cm} \times 0.5 \mathrm{~cm} \times 20 \mathrm{~cm}$ copper block, located $97 \mathrm{~m}$ upstream of the experimental (secondary) target. The copper target is about 2 interaction lengths, hence a tenth of the primary beam does not interact and is dumped into a series of concrete blocks. The beam strikes the target at an angle of 1.3 degrees with respect to the horizontal axis. Two dipole magnets in the MC5 area upstream of the primary target bend proton beam towards the target and two other dipole magnets located downstream of the target focus the secondary beam produced at the target onto the beamline center.

The secondary beam consists of charged pions, kaons and protons in the momentum range of $5-90 \mathrm{GeV} / \mathrm{c}$. These secondary charged particles are struck on the experimental target. The scattering on the secondary target is the main focus of the experiment. The secondary beamline must accomodate both the demanded momentum resolution and the precision in particle identification. The former requires the momentum-dispersed beam size to be larger than the transverse beam size and the latter requires low divergence at the beam Cherenkov detectors. The four quadrupole magnets between the downstream dipoles are used to focus the secondary beam onto a jaw collimator, with a vertical aperture which can be varied. The strength of the two downstream dipole magnets determine the central momentum of the beam, and momentum spread, $d p / p$ is then set by varying the jaw aperture size. Finally, the secondary beam after the collimator is refocused onto the experimental target by three additional quadrupole magnets.

\section{$2.3 \quad$ MIPP Spectrometer}

The MIPP spectrometer, shown in figure 2.3, consists of a number of detectors which are capable of providing tracking and identification of charged particles. The beam chambers are used to track the beam particles. The experimental target is located a few centimeters upstream of the time projection chamber (TPC). The TPC allows the reconstruction of tracks in three dimensions and the momentum measurement of low momentum tracks. MIPP uses two magnets, Jolly Green Giant (JGG) and Rosie, which have vertical (y-axis) magnetic fields in opposite directions. The net effect of the two magnets is a transverse displacement of the track from the straight line in the $\mathrm{x}-\mathrm{z}$ plane. Position measurement in the four drift 
chambers (DC's) and two multiwire proportional chambers (PWC's) make it possible to measure the momentum of high momentum tracks. Charged particle identification is done by four different detectors: TPC, time of flight wall (ToF), threshold Cherenkov (Ckov), and ring imaging Cherenkov (RICH) counters, depending on momentum of the particle. Finally, electromagnetic and hadron calorimeters make it possible to identify electrons, gamma rays and neutrons. All these detectors are described in detail in the following sections.

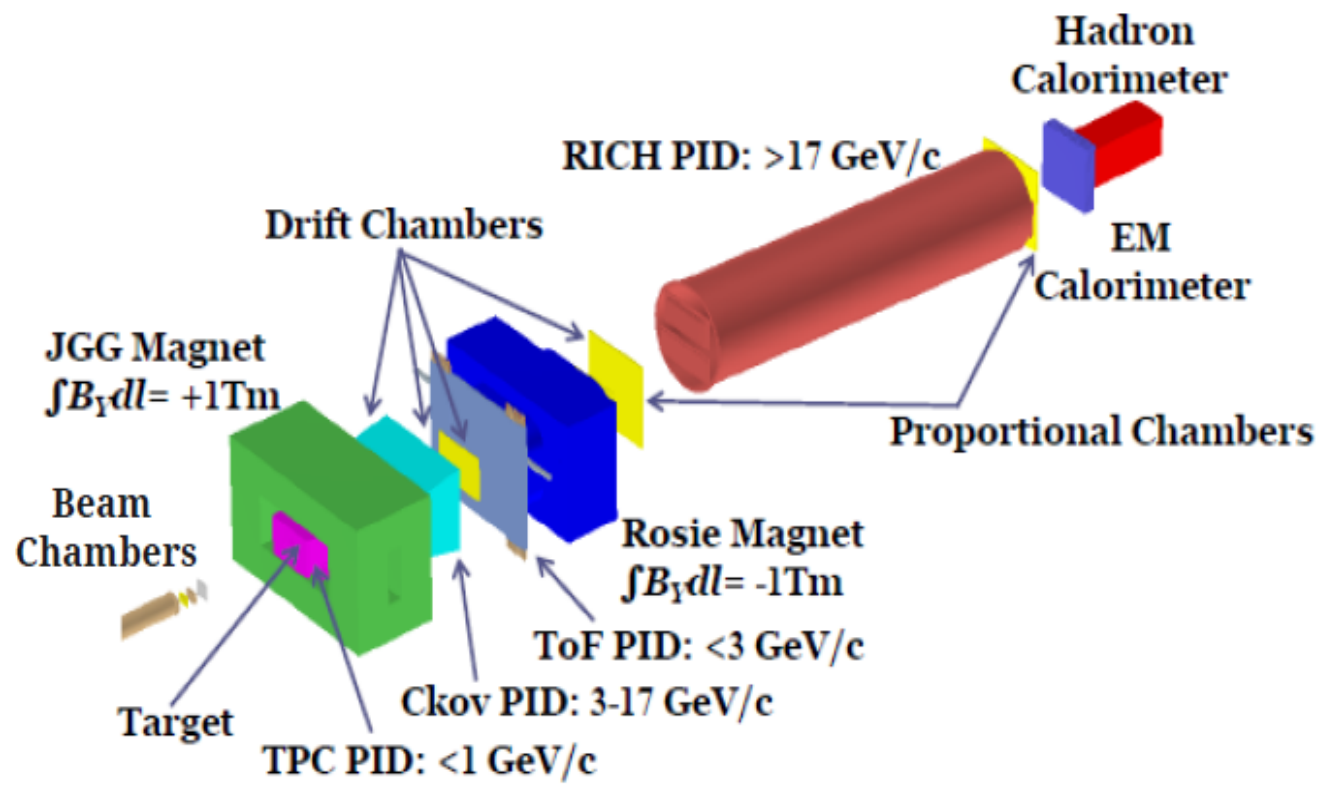

Figure 2.3: The layout of the MIPP experiment.

\subsection{Tracking}

The spectrometer provides data to reconstruct incident beam particle and particles coming out of an interaction in the target. Three small drift chambers called beam chambers upstream of the target (BC's) are able to reconstruct the incident particle trajectory. The TPC, four DC's, and two PWC's can reconstruct tracks coming from an interaction. They are described below.

\subsubsection{Drift Chambers}

MIPP uses three small drift chambers (BC1, $\mathrm{BC} 2, \mathrm{BC} 3)$ to measure incident beam particle trajectories and four larger drift chambers (DC1, DC2, DC3, DC4) located downstream of 
the target for tracking the particles coming from the interactions in the target. DC1 is located immediately in front of the Ckov, DC2 and DC3 are located in the gap between the Ckov and the ToF, and DC4 covers the downstream aperture of the Rosie magnet. The BC's and DC's were built by FNAL-E690 experiment and the BC's are described in detail in 663.

All drift chambers have four planes of wires with the wires oriented at an angle of \pm 21.60 degrees on planes 1 and 4 , and \pm 7.93 degrees on planes 2 and 3 with respect to the vertical direction.

The beam chambers are identical and have 160 wires per plane with $1.016 \mathrm{~mm}$ wire spacing. They have an active area of $15.24 \mathrm{~cm} \times 10.16 \mathrm{~cm}$ and are filled with a gas mixture of $\mathrm{Ar}, \mathrm{C}_{4} \mathrm{H}_{10}$ (isobutane) and Methylal in proportions of 82:15:3. The beam chambers provide $150 \mu \mathrm{m}$ resolution of position and $5 \mu \mathrm{rad}$ resolution of angle of the incoming particle, assuming negligible multiple scattering. In addition, the narrow $(1 \mathrm{~mm})$ wire spacing allows us to distinguish multiple particles, and the $1 \mu$ s range of the TDC's allows one to measure the time of the track with respect to the trigger signal.

DC1 has 512 wires per plane with $3.493 \mathrm{~mm}$ wire spacing. DC2, DC3 and DC4 have 512 wires on planes 2 and 3 but only 448 wires on planes 1 and 4 with $3.175 \mathrm{~mm}$ wire spacing. The active area of DC1 is $182.88 \mathrm{~cm} \times 121.92 \mathrm{~cm}$ while the active area of DC2, DC3, DC4 is $152.40 \mathrm{~cm} \times 101.60 \mathrm{~cm}$. The wire planes in the chambers are separated by a distance of $0.7 \mathrm{~cm}$. The DC's are filled with the gas mixture of Ar, $\mathrm{C}_{4} \mathrm{H}_{10}$, and Methylal in proportions of 69:28:3.

The signals from the planes of the chambers are amplified by 8-channel preamplifiers, discriminated by 32-channel Nevis discriminators, and the drift time is measured by a LeCroy 4290 TDC CAMAC system with 1 ns resolution. The LeCroy 4290 system consists of LeCroy 4291B 32-channel TDC CAMAC modules, organized in a total of 15 CAMAC crates with 20-22 modules per crate. Each crate is read out by LeCroy 4298 TDC crate controllers, in turn connected to LeCroy 4299 Databus Interfaces in conventional CAMAC crates. When triggered by the 4299 cards, the 4298 controllers zero suppress the data and send them out to be read out.

\subsubsection{Multiwire Proportional Chambers}

Two identical multiwire proportional chambers (PWC5 and PWC6) are placed upstream and downstream of the RICH detector to provide three dimensional trajectories of high momentum secondary charged particles. These chambers were constructed at CERN in the 1970's and were used in the NA24 experiment at CERN and in the SELEX experiment at FNAL where MIPP inherited them. Chamber construction is documented in [64]. 
The chambers have an active area of $2 \mathrm{~m} \times 2 \mathrm{~m}$ and a length of $40 \mathrm{~cm}$ along the beam direction (z-axis). Each chamber has four planes of wires. Each plane has 160 tungsten anode wires with $3 \mathrm{~mm}$ wire spacing. The anode wire planes are oriented in four different ways: vertical, horizontal, and at angles of 28.07 degrees with respect to the vertical direction. The gas mixture used in the chambers is $\mathrm{Ar} / \mathrm{CH}_{4} / \mathrm{CF}_{4}$ in proportions of 76.5:8.5:15.

The chambers are read out by the RMH electronics [65] designed at CERN in the 1970's. The signals from the anode wires are amplified by 32-channel preamplifiers and discriminated by a total of 160 32-channel RMH modules located in 8 CAMAC crates. Zerosuppressed readout consists of up to 4000 16-bit words indicating which channel of which module has a signal above threshold.

Unfortunately, only two of four planes in PWC5 could hold high voltage, which made the chamber less efficient and made it impossible to reduce combinatorial combinations of hit positions. However, these problems have a small effect on track reconstruction (explained later in this chapter).

\subsubsection{Time Projection Chamber}

One of the most important detectors in the MIPP experiment is the time projection chamber (TPC). It is used for three dimensional tracking. The TPC is also used for particle identification of charged particles having momentum up to $1 \mathrm{GeV} / \mathrm{c}$. With particle position measured at tens of points, the chamber is an excellent detector for pattern recognition of the tracks in an event. It was constructed in 1990 by BEVALAC facility at Lawrence Berkeley Laboratory for the EOS experiment [66, 67, 68, 69, 70, 71, 72]. Prior to being used by the MIPP experiment, it was used by the BNL-E895 and BNL-E910 experiments.

The TPC is located inside the JGG magnet just a few centimeters downstream of the experimental target. The trajectories of the charged particles coming from interactions in the target are bent according to their charge type $(+/-)$ in the presence of magnetic field. The active tracking region in the TPC is a rectangular box $1.5 \mathrm{~m}$ long in the beam direction, $96 \mathrm{~cm}$ wide in the bending direction and $75 \mathrm{~cm}$ high in the drift direction. The TPC volume is filled with $\mathrm{P} 10$ gas (90\% Ar with $10 \% \mathrm{CH}_{4}$ ). The working principle of the TPC is based on the ionization of the $\mathrm{P} 10$ gas when particles coming from interactions passed through the gas. The ionization electrons travel to the anode wire plane on the bottom of the chamber, and the positive ions drift to the cathode plane on the top of the chamber under a uniform electric field of $125 \mathrm{~V} / \mathrm{cm}$, parallel to JGG's magnetic field. The electrons pass through three wire planes while they drift to the bottom: gating grid, ground wire plane, and anode wire plane. At the bottom of the drift volume, in the region between ground and anode wires, an 
avalanche is created by the strong electric field $(>3 \mathrm{kV} / \mathrm{cm})$ near the anode wires, achieving gas gain of 3000. This avalanche creates an image charge on the readout copper pad plane $4 \mathrm{~mm}$ below the anode wire plane. In order to avoid unwanted drifts, a gating grid which is located slightly above the ground wires in the drift region is switched on when no event is triggered (see section 2.8). Schematic view of the TPC volume and pad plane with anode and ground wires, is shown in figure 2.4 .

The pad plane consists of 128 pad rows along the beam direction and 120 pad columns in the $\mathrm{x}$ direction. Each pad is $12 \mathrm{~mm}$ in length and $8 \mathrm{~mm}$ in width. The active pad plane area is $96 \mathrm{~cm}$ in width and $153.6 \mathrm{~cm}$ in length. The $\mathrm{x}$ and $\mathrm{z}$ positions of track hits are given by the pad plane and the y position is provided by drift time. The pad rows record a slice of ionization energy in z. Each pad is digitized by 12-bit flash ADC at $10 \mathrm{MHz}$. With drift velocity of about $5 \mathrm{~cm} / \mu \mathrm{s}$, this gives $5 \mathrm{~mm}$ sampling along the vertical. A typical minimum ionizing track would leave signal on about 3 pads in a given row, with the amplified signal spread over seven $100 \mathrm{~ns}$ time buckets. The large number of measurement points (up to 128 per track), yield sub-millimeter position resolution of the track position. The tracking in the magnetic field measures particle rigidity and multi-sampling of $\mathrm{dE} / \mathrm{dx}$ along the track provides particle identification.

The TPC electronics consist of sticks underneath the pad plane for preamplifying and shaping of the signals, ADC modules for digitizing, and dedicated VME boards connected to sticks by fiber optics for interface to Data Acquisition (DAQ) Processors (see section 2.9).

\subsection{Particle Identification}

The spectrometer allows one to identify the beam particle and the particles coming out of an interaction. Beam particles are identified using two differential Cherenkov counters: one for $\pi / \mathrm{K}$ and the other for $\mathrm{K} / \mathrm{p}$ separation, located upstream of the experimental target. The particles coming from the interactions are identified by four different detectors depending on their momentum. The TPC provides the particle identification for momentum up to 1 $\mathrm{GeV} / \mathrm{c}$, the ToF wall up to $3 \mathrm{GeV} / \mathrm{c}$, the Ckov up to $17 \mathrm{GeV} / \mathrm{c}$, and the RICH up to 120 $\mathrm{GeV} / \mathrm{c}$. All these detectors are described below.

\subsubsection{Beam Cherenkov}

Each beam Cherenkov (BCkov) counter consists of a long cylindrical radiator volume and a head at the downstream end, which contains two photomultiplier tubes (PMT's). The head 


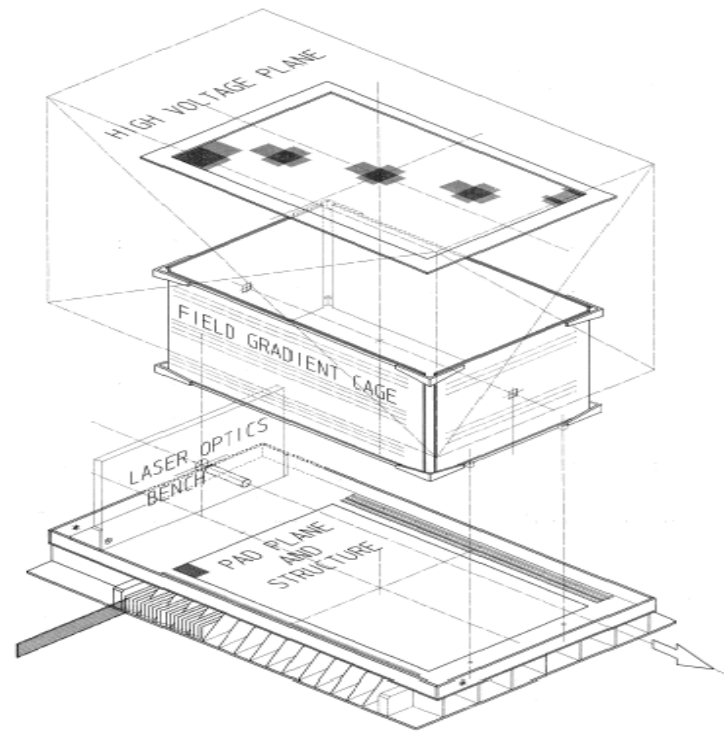

(a)

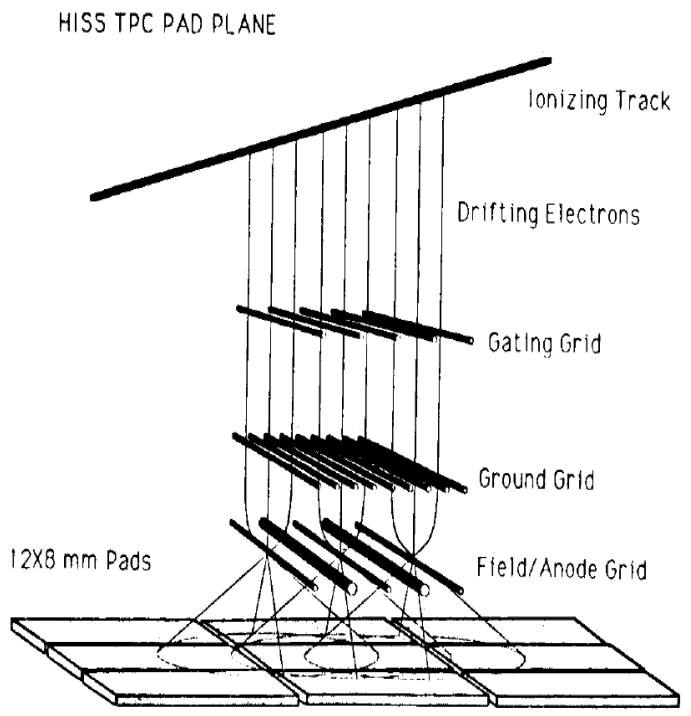

(b)

Figure 2.4: (a) The exploded view of the TPC with the outer case removed. The active volume is defined by the pad plane, field cage, and high voltage (cathode) plane. (b) The schematic view of the pad plane with anode and ground wires, taken from [68].

contains a focusing mirror which reflects the light onto a PMT covered by a second mirror with a hole. The aperture of the hole is chosen in such a way that Cherenkov light emitted at angles below the cutoff would hit the PMT behind the second mirror (inner PMT) whereas light emitted at larger angles would be reflected onto the outer PMT. The cutoff Cherenkov angles for the inner PMT's for the upstream and downstream counters are 5 and $7 \mathrm{mrad}$, respectively. These counters are located in between the three beam chambers $\mathrm{BC} 1, \mathrm{BC} 2$ and BC3 used to track the beam particles.

For each threshold Cherenkov detector, one particle is below threshold and the other is above. The lengths of the detectors are determined by the mass difference of the particles which need to be differentiated and the momentum at which the differentiation is made. The radiator volume lengths of the two counters are $22.9 \mathrm{~m}$ and $12.2 \mathrm{~m}$ so that statistically the same number of photo-electrons hit inner PMT's on both the counters.

Beam particles are identified using the counters by taking account of the fact that at the same momentum, particles with different masses emit Cherenkov light at different angles. To tag beam particles, the density in the upstream counter is chosen for each beam momentum in such a way that the pion Cherenkov light hits only the outer PMT, and the kaon light at the same momentum hits the inner PMT only, and protons don't not radiate. Similarly, the pressure in the downstream counter is set so that the proton Cherenkov light hits the inner 
PMT, but not the outer PMT. The outer PMT collects light emitted at angles less than 30 mrad. This is important since for momenta below $\approx 32 \mathrm{GeV} / \mathrm{c}$, the pion Cherenkov angle is so large that the photons emitted by pions are no longer collected on the outer PMT. This property is independent of the gas that filled the radiator volume. At $120 \mathrm{GeV} / \mathrm{c}$, the beam consists of protons only and beam particle identification is not needed. The 1st BCkov counter is evacuated to reduce multiple scattering in the beam and the 2nd BCkov counter is set to identify protons to reduce background in the beam.

One of the challenges for identification of incident particles is the large span of momenta $(5-90 \mathrm{GeV} / \mathrm{c})$. The counters are tuned to different beam momenta by establishing the desired gas density in each counter. The cylinders can only be pressurized to $\approx 1.5 \mathrm{~atm}$, therefore at low momenta, a heavy non-flammable gas has to be used in order to identify protons below $30 \mathrm{GeV} / \mathrm{c}$ and kaons below $20 \mathrm{GeV} / \mathrm{c}$. MIPP uses $\mathrm{C}_{4} \mathrm{~F}_{8} \mathrm{O}$ for this purpose. Nitrogen at different densities is used for all higher momenta. However, at $5 \mathrm{GeV} / \mathrm{c}$, even the heavy gas is not dense enough for kaons or protons to radiate. But at that momentum, we can take advantage of the measurable difference in the time of flight of the three particle species between the front of the beamline and the experimental target.

\subsubsection{Threshold Cherenkov}

The threshold Ckov detector is a highly segmented Cherenkov counter presenting a minimum amount of material to traversing particles. The counter was designed to provide particle identification using full pulse-height information for BNL-E766 experiment, and was used subsequently for FNAL-E690, BNL-E910, and BNL-E895 experiments. The Cherenkov counter is located immediately downstream of the first drift chamber and is followed by the second and third drift chambers. It is used to separate secondary charged particles in the momentum region $3-17 \mathrm{GeV} / \mathrm{c}$. The radiator gas used in the counter is $\mathrm{C}_{4} \mathrm{~F}_{10}$ with a density of $10.12 \mathrm{~g} / \mathrm{l}$ (i.e. atmospheric pressure at room temperature) and an index of refraction of $\eta_{0}=1.00153$, corresponding to thresholds of $2.6 \mathrm{GeV} / \mathrm{c}$ for pions, $8 \mathrm{GeV} / \mathrm{c}$ for kaons, and $17 \mathrm{GeV} / \mathrm{c}$ for protons.

The counter box is $2.8 \mathrm{~m}$ wide, $3.3 \mathrm{~m}$ high and $1.1 \mathrm{~m}$ long. The detector, shown in figure 2.5, consists of a thin Kevlar front window with active area $55 " \times 77$ ", an inner gas radiator volume, and a mosaic of 96 primary mirrors which reflect the radiated light onto a set of conical secondary mirrors enveloping 96 photomultiplier tubes. The 96 PMT's at the top and the bottom of the counter are individually matched to the mirrors. The alignment of the mirrors is done in such a way that Cherenkov light is reflected onto only one PMT if particles originated at a certain focal length in front of the Cherenkov counter. The charge of 
the PMT's is digitized using LeCroy 4300 ADC modules, and LeCroy 3377 multi-hit TDC's are used to record the hit times of the signals.

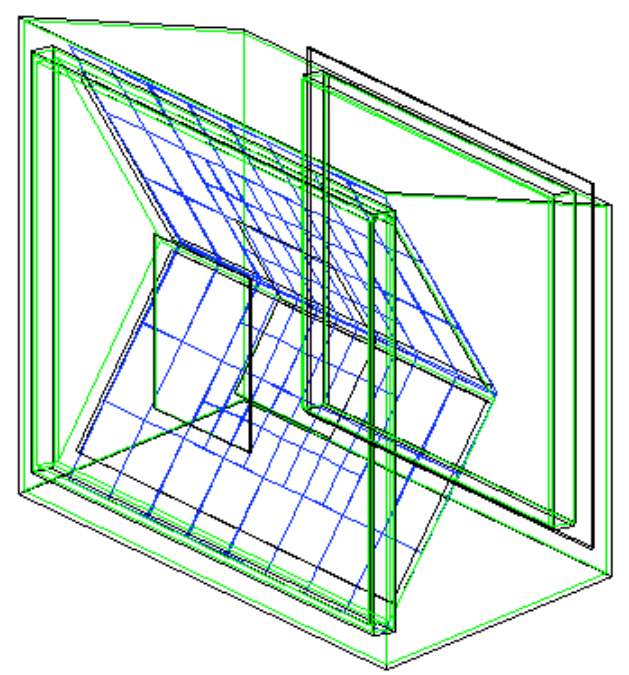

Figure 2.5: Schematic view of the Cherenkov counter.

\subsubsection{Beam Time of Flight}

The beam time of flight detectors consist of T01, TBD, and T00 scintillator counters which are placed between upstream of the experimental target and downstream of the secondary beamline collimators. The purposes of the scintillator counters are forming beam trigger signals, resolving pileups, providing a reference start time for ToF wall, and identifying incoming beam particles at momentum of $5 \mathrm{GeV} / \mathrm{c}$.

Each counter consists of a square piece of scintillator and four Hamamatsu R5900U PMT's, one on each side of the scintillator. Figure 2.6 shows schematic of the inside of the counters. The initial two counters T00 and T01 are equipped with light guides, which improve timing resolution. TBD counter, which is added at a later time to help form the beam trigger doesn't not have the light guides, as we are not planning to use it for measuring the time of flight of beam particles.

The temporal coincidence of TBD and T01 signals is used to form the beam trigger. The experimental trigger will be further discussed in the later section of this chapter.

Two sets of CAMAC TDC's measure PMT hit times with respect to the experimental trigger time:

1. LeCroy 3377 with $0.5 \mathrm{~ns}$ resolution and up to 16 hits in $512 \mathrm{~ns}$ window. 
2. LeCroy 2229 with 30 ns resolution and only 1 hit in 60 ns window.

The first set of TDC's help to determine presence of pileup (multiple incident particles in one event), while the second set of TDC's are used to reconstruct particle flight time and serve as the reference time for the ToF wall.

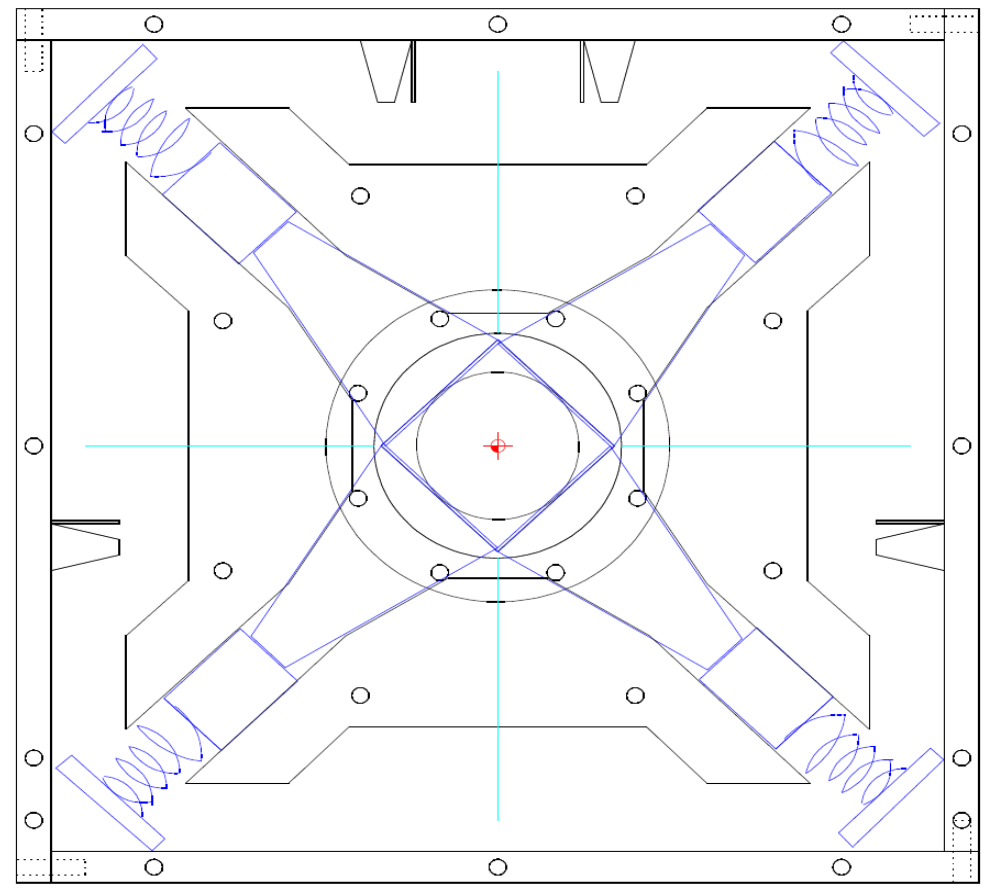

Figure 2.6: Schematic of T00 and T01 construction. Light from the scintillator is collected onto four PMT's by light guides. Phototubes are held in place by springs. TBD assembly is similar, except no light guides are used.

\subsubsection{Time of Flight Wall}

The time of flight wall (ToF) detector is located upstream of the Rosie magnet. The ToF wall consists of $543-\mathrm{m}$ long vertical scintillator bars, and its horizontal width is $3.5 \mathrm{~m}$. Forty $5 \mathrm{~cm} \times 5 \mathrm{~cm}$ bars cover the central $2 \mathrm{~m}$ spanning the Rosie aperture, and seven $10 \mathrm{~cm} \times$ $10 \mathrm{~cm}$ bars flank the central bars on either side. The top and bottom ends of the bars have PMT's in order to collect light.

At most, one particle track hits the given bar. Beam counters are used for the reference time of the beam particles. That is, ToF is triggered when the hit happens at the target position. Then, as a particle crosses one of the ToF bars, the PMT's collect scintillation light that provides a stop signal for the TDC. The velocity of the particle is calculated from the track length and the time of flight. ToF is also able to measure particle momentum from 
curvature in tracking. Measuring the $\beta$ value and the momentum of the particle, the mass of the particle can be computed which provides us particle identification. ToF identifies produced charged particles having momentum up to $3 \mathrm{GeV} / \mathrm{c}$.

The PMT's used in the ToF system are Hamamatsu R5900U, identical to the ones used in the beam counters. The signals from the PMT's are split into an ADC and TDC, the TDC's having a time-width of 30 or 50 ps. The timing resolution of the ToF system varies by scintillator bar, but is typically $\sim 300 \mathrm{ps}$. Such resolution allows for nearly $3 \sigma$ separation of $\pi$ and p out to about $2.5 \mathrm{GeV} / \mathrm{c}$, and $3 \sigma$ separation of $\pi$ and $\mathrm{K}$ out to about $1.5 \mathrm{GeV} / \mathrm{c}$.

\subsubsection{Ring Imaging Cherenkov Detector}

The ring imaging Cherenkov (RICH) detector was built for the SELEX experiment [73, 74, 75] in the 1990's. It is placed downstream of the Rosie magnet. The detector is a $10 \mathrm{~m}$ long cylindrical vessel with a radius of $1.17 \mathrm{~m}$ and a wall thickness of 0.5 inches. The end flanges are made of 1.5 inch thick aluminium with provisions for thin beam windows and a photomultiplier tube holder plate.

In MIPP, the radiator volume is filled with $\mathrm{CO}_{2}$ gas to a total pressure of $\approx 1.03 \mathrm{~atm}$. Over the course of a running period lasting for about a year, the oxygen level was kept at 1000 parts per million. The volume of $\mathrm{CO}_{2}$ was slowly leaking over time, so $\mathrm{CO}_{2}$ was added on a weekly basis to keep the pressure variation to within 1\%. The RICH vessel is tilted off the horizontal axis by 2.4 degrees to make particles pass through the RICH front window. As a charged particle passes through the RICH volume, the Cherenkov light is emitted at certain angles. The mirror plane at the end of the vessel consists of an array of 16 hexagonally spherical mirrors. The mirrors are mounted in an array which is approximately $2.4 \mathrm{~m}$ wide and $1.2 \mathrm{~m}$ high, fixed individually to a flat, low mass honeycomb panel of 1 inch thickness with a 3-point kinematic mount. The average radius of mirrors is $19.8 \mathrm{~m}$ with RMS spread of less than $3 \mathrm{~cm}$. These mirrors reflect the light onto an array of 1 inch PMT's located at the upstream end above the beam window. The schematic side view of the RICH detector is shown in figure 2.7 .

Prior to the run, mirrors are aligned with laser by adjusting the mirror angles so that their foci are at the center of the PMT array. The image of the Cherenkov light at the PMT's has a ring shape, and since the particles with the same momentum but different masses create rings with different radii, measuring the ring radius provides identification of the charged particles travelling through the RICH. The RICH can identify charged particles having momentum up to $120 \mathrm{GeV} / \mathrm{c}$.

A holder plate supports the PMT's in a hexagonally close-packed array of $89 \times 32$ 


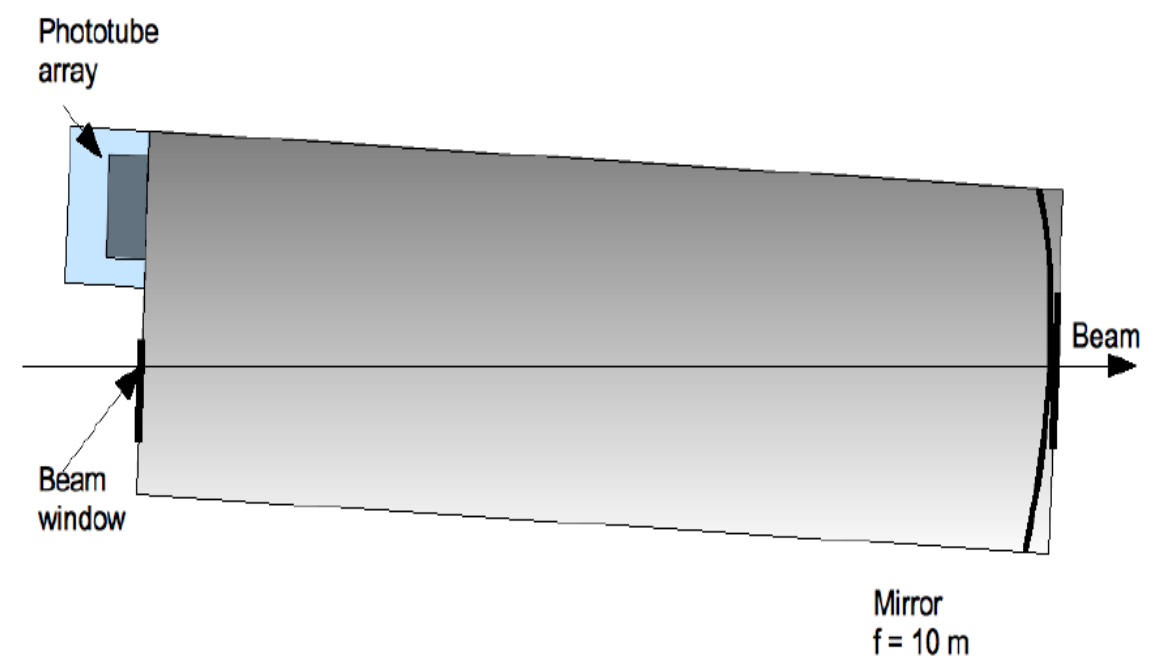

Figure 2.7: Schematic side view of the RICH detector.

tubes. The plate separates the array from radiator volume with 2848 individually glued quartz windows and holds aluminized mylar cones for each phototube. Essentially $100 \%$ of the reflected Cherenkov light that reaches the front of the vessel gets to the phototubes.

Two different types of PMT's are used in the RICH. One is the Hamamatsu R-760 with a quartz window allowing photon detection down to $170 \mathrm{~nm}$, and the other one is the FEU-60 with a glass window coated with a wavelength shifter so as to reach same wavelength range as that of the Hamamatsu tubes. The efficiency of the FEU-60 PMT is only about $42 \%$ but it is 10 times cheaper than the Hamamatsu PMT.

The front-end electronics originally used in the SELEX experiment is completely replaced. The electronics prototype is produced at Fermilab, and the production boards are fabricated and tested at Harvard University. The readout electronics consist of front-end cards and custom VME boards. The signals from the PMT's are read out by 89 32-channel front-end cards. Each channel in a card contains a differential amplifier and an analog comparator. The discriminated signals are then sent to VME boards.

\subsubsection{Electromagnetic Calorimeter}

The Electromagnetic calorimeter (EMCAL) is mounted at the downstream end of the MIPP spectrometer immediately before the Hadron calorimeter to detect forward going high momentum photons and measure their energies. The active volume of the calorimeter consists of 10 planes of alternating horizontal and vertical proportional wire chambers sandwiched between $15 \mathrm{~mm}$ thick lead plates. Each active plane consists of eight 8 inch wide and 1 inch 
thick wire chambers. These chambers are filled with a gas mixture of $76.5 \% \mathrm{Ar}, 8.5 \% \mathrm{CH}_{4}$, and $15 \% \mathrm{CF} 4$. The total thickness of EMCAL is $\approx 10$ radiation lengths. The active volume is $162 \times 162 \times 31 \mathrm{~cm}^{3}$.

The proportional chambers are constructed from aluminum extrusions with anode wires strung through the center of each chamber. The anode wires are $162 \mathrm{~cm}$ long and $25 \mu \mathrm{m}$ in diameter, and the wire-to-wire spacing is $2.54 \mathrm{~cm}$.

There are 8 chambers and 64 wires in each plane. These horizontal and vertical wire planes are stacked alternately to measure $\mathrm{x}$ and y positions. The operating voltage for the anode wires is $+2.3 \mathrm{kV}$ and is chosen with the intent of maximizing dynamic range while at the same time providing sensitivity for the detection of minimum ionizing radiation. Pulses from the wires are shaped and amplified after which they are digitized by ADC's and read out through a custom CAMAC module.

\subsubsection{Hadron Calorimeter}

The Hadron calorimeter (HCAL) is the last detector in the MIPP spectrometer located directly behind the EMCAL and used to identify the forward going neutrons and measure their energies. The calorimeter also serves as the experiment's beam dump. It was previously used by the HyperCP [76] experiment. The calorimeter specifications are given in table 2.1 .

The calorimeter is mounted on a stand with jacks that allow limited vertical movement. It is composed of 64 layers of $24.1 \mathrm{~mm}$ thick iron plates interspersed with $5 \mathrm{~mm}$ thick scintillators as the active medium, giving a sampling fraction of $3.5 \%$ and a total thickness of 9.6 interaction lengths and 88.5 radiation lengths. The active volume of the calorimeter is $0.99 \mathrm{~m}$ in width, $0.98 \mathrm{~m}$ in height, and $2.4 \mathrm{~m}$ in length. It is meant to determine the energy of hadrons by summing energy deposition of the resulting hadronic shower initiated in the iron sheets that comprise the majority of the interaction lengths of the detector. Figure 2.8 shows schematic of the construction of the EMCAL and HCAL.

For readout purposes, the HCAL is subdivided into four longitudinal and two lateral sections, for a total of eight cells that are read out with wavelength shifting fibers spaced $30 \mathrm{~mm}$ apart. Fibers from each cell are bundled into a single two-inch Hamamatsu R329-02 photomultiplier tube. The pulse heights are flash digitized in custom built CAMAC 14-bit ADC modules with a $75 \mathrm{fC}$ least count. 


\begin{tabular}{|l|l|}
\hline Type & Sampling (Fe:scintillator, 5:1) \\
Composition & $24.1 \mathrm{~mm}$ Fe, 5.0 mm PS scintillator \\
Layer depth & $36.93 \mathrm{~mm}$ \\
Number of layers & 64 \\
Size $(\mathrm{x} \times \mathrm{y} \times \mathrm{z})$ & $0.990 \times 0.980 \times 2.388 \mathrm{~m}^{3}$ \\
Mass & $12667 \mathrm{~kg}$ \\
Total cells & 8 \\
Fiber diameter & $2 \mathrm{~mm}$ \\
Fiber separation & $30 \mathrm{~mm}$ \\
Fibers per cell & $16 \times 16=256$ \\
Total fibers & $8 \times 256=2048$ \\
Interaction length & $2.40 \lambda_{I}$ per cell, $9.62 \lambda_{I}$ total \\
Radiation length & $22.1 \mathrm{X}_{0}$ per cell, $88.5 \mathrm{X}_{0}$ total \\
Sampling fraction & $3.54 \%$ \\
\hline
\end{tabular}

Table 2.1: Hadron calorimeter specifications.

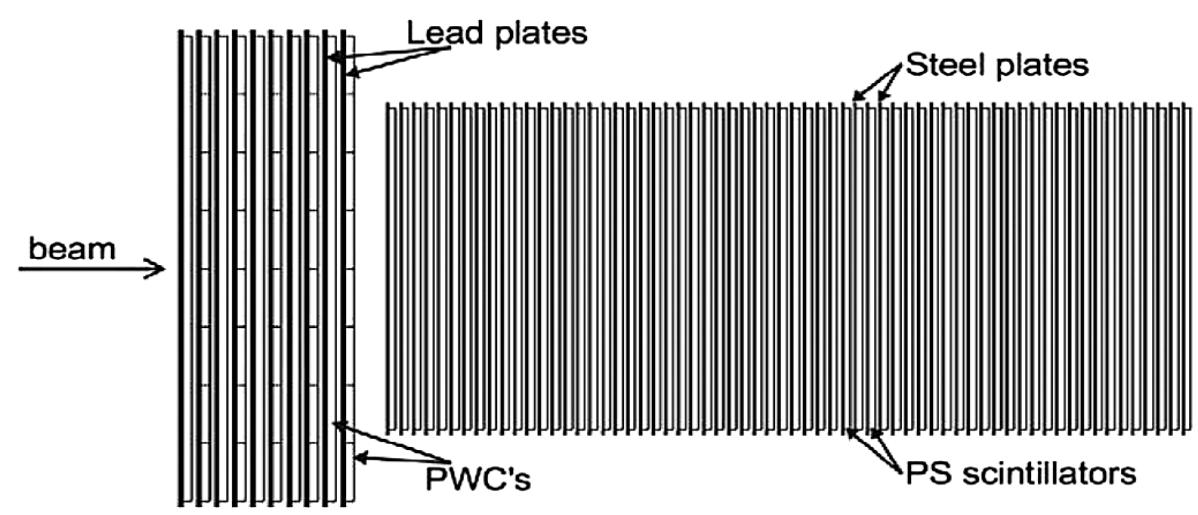

Figure 2.8: Schematic of the calorimeters. 


\subsection{Analysis Magnets}

MIPP uses two magnets for momentum measurement: JGG and Rosie. The JGG analysis magnet surrounds the target and the TPC detector to be used for measuring momentum of the low momentum charged particles. The Rosie magnet is placed between the ToF counter and the last drift chamber DC4, and is used for high momentum measurements. For safety, magnetic fields are set so that the kick angles are about equal and opposite $\left(\int B_{y} d l \approx 1\right.$ $\mathrm{Tm}$ ). This way regardless of momentum, beam is absorbed by the beam dump.

While the JGG has a magnetic field of $0.7 \mathrm{~T}$ in $+\mathrm{y}$ direction, the Rosie has a magnetic field of $0.6 \mathrm{~T}$ in $-\mathrm{y}$ direction. When the produced particles pass through the TPC volume, the JGG affects charged particles by its magnetic field and all the charged particles are bent according to their charge type. After that, the Rosie magnet works as a focusing magnet to bend particles back along the beam axis. In this way, the particles don't miss the downstream detectors which results into a good acceptance of the spectrometer.

Each magnet is equipped with two Group3 Hall probes, each near the center at the top and bottom of the aperture. These Hall probes and the power supply current readback are monitored throughout the run to ensure magnetic field stability. Both magnets use water cooled copper conductors and are powered by a series of two $500 \mathrm{~kW}$ Transrex DC magnet power supplies built in the early 1970's. The two magnets are described below.

\subsubsection{Jolly Green Giant}

The JGG magnet was originally built at Harvard in the 1960's. It consists of two sets of flat approximately rectangular coils surrounding the pole pieces above and below the aperture, and the yoke. Its total weight is 250 tons. The aperture of JGG is $125 \mathrm{~cm}$ high, $262 \mathrm{~cm}$ wide and $221 \mathrm{~cm}$ deep, which is sufficiently large for the TPC to fit in. This coil geometry results in large $\mathrm{B}_{x}$ and $\mathrm{B}_{z}$ components of the magnetic field as compared to the desired vertical field $\mathrm{B}_{y}$. The non-uniform magnetic field of JGG causes up to $7 \mathrm{~cm}$ distortion in particle trajectories in the TPC. Therefore, distortion correction is applied to the TPC hit positions during the reconstruction stage to obtain true hit positions.

\subsubsection{Rosie}

The Rosie magnet is constructed to provide a more uniform magnetic field than the JGG. But unfortunately, its aperture is a few centimeters small for the TPC to fit in. The saddle coils provide field-terminating current sheets at the sides of the magnet aperture. Mirror plates increase field homogeneity near the transition regions at the upstream and downstream ends 
of the aperture.

\subsubsection{Ziptrack field mapping}

Both magnets by design have eight identical octants. The real magnetic fields are only approximately symmetric in the octants due to manufacturing tolerances. As these imperfections can not be modeled, it is very important in the data analysis to have an accurate measured magnetic field map.

The magnetic field of JGG and Rosie was measured with the Ziptrack apparatus after the magnets were installed in the experimental hall and before the detectors were moved in. The Ziptrack system moved a cart with Hall probes along a beam through the magnet aperture. The beam was supported upstream and downstream of the magnet by manipulators that allowed the beam to move horizontally and vertically. Thus the system could position the Hall probes anywhere inside the magnet aperture as well as upstream or downstream of the aperture. Motion of the manipulators and the cart, and readout of the Hall probes were computer controlled. The fields were mapped on a grid with two inch grid-spacing in all three directions in a box with dimensions given in table 2.2. The upstream and downstream limits were chosen to obtain a good map of the fringe fields upstream of JGG and between the magnets. The position readout of the Hall probes was sufficiently accurate, however the exact rotation of the probe was later inferred from comparison of data from the TPC and the wire chambers. The field maps for JGG and Rosie magnets are shown in figures 2.9 and 2.10, respectively. Complete field maps are used to compute trajectories of particles in the non-uniform field and to compute trajectories of electron drift in the TPC.

\begin{tabular}{|l|l|l|l|l|l|l|}
\hline Magnet & $\mathbf{x}_{\min }$ & $\mathbf{x}_{\max }$ & $\mathbf{y}_{\min }$ & $\mathbf{y}_{\max }$ & $\mathbf{z}_{\min }$ & $\mathbf{z}_{\max }$ \\
\hline JGG & -25 & 25 & -11 & 11 & -60 & 40 \\
Rosie & -16 & 16 & -8 & 7 & -70 & 57 \\
\hline
\end{tabular}

Table 2.2: Size of the Ziptrack grids used to map the MIPP analysis magnet fields. The coordinates are given in grid positions with respect to the center of the magnet.

\subsection{Targets}

The several goals of the MIPP experiment require the use of three target systems - a copy of the meter-long NuMI target to understand the particle source terms for that neutrino experiment, a cryogenic liquid hydrogen target for the purest studies of elementary particle 
Field map projection at $\mathrm{z}=0$

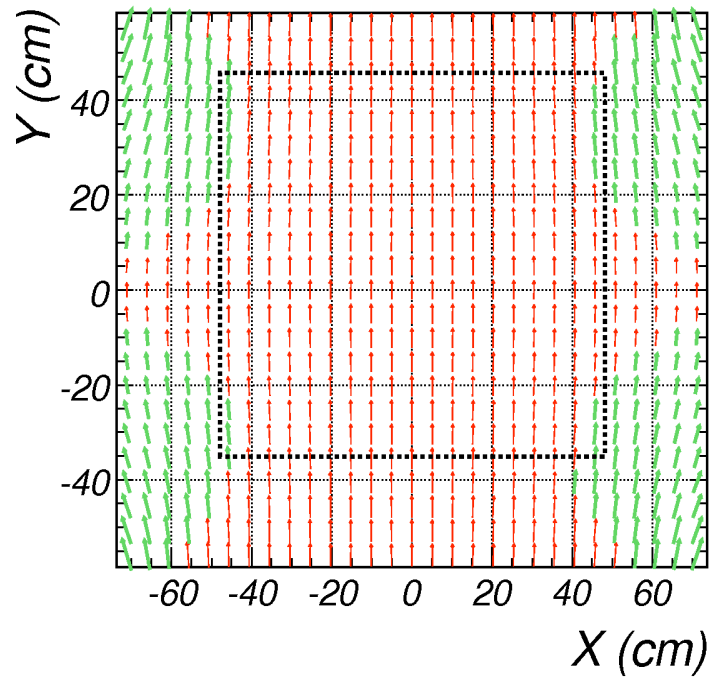

(a)
Field map projection at $x=0$

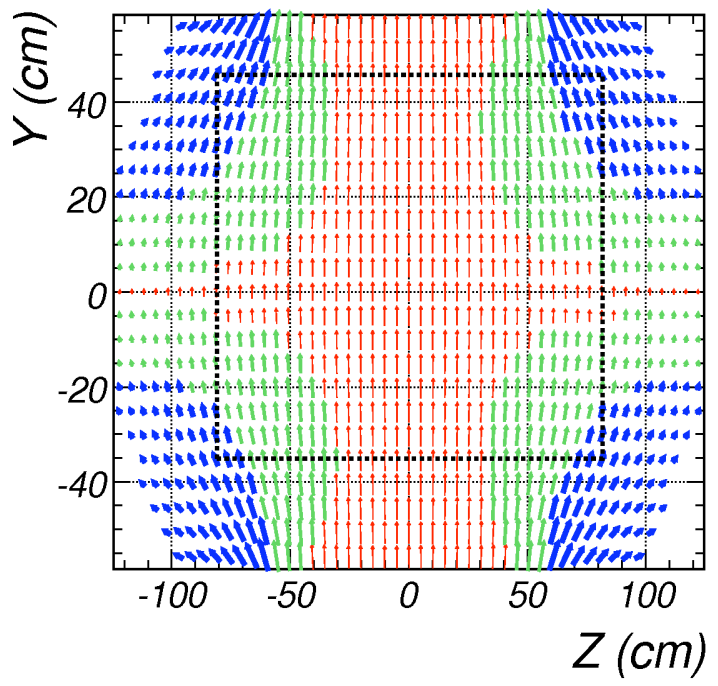

(b)

Figure 2.9: Projections of the JGG field map in slices along the center of the magnet. Regions of non-uniform field are highlighted with thicker arrows where $\mathrm{B}_{i} / \mathrm{B}_{y}>0.1$ and thickest arrows where $\mathrm{B}_{i} / \mathrm{B}_{y}>0.5$. The dashed line shows the boundary of the TPC drift volume.

Field map projection at $\mathrm{z}=0$

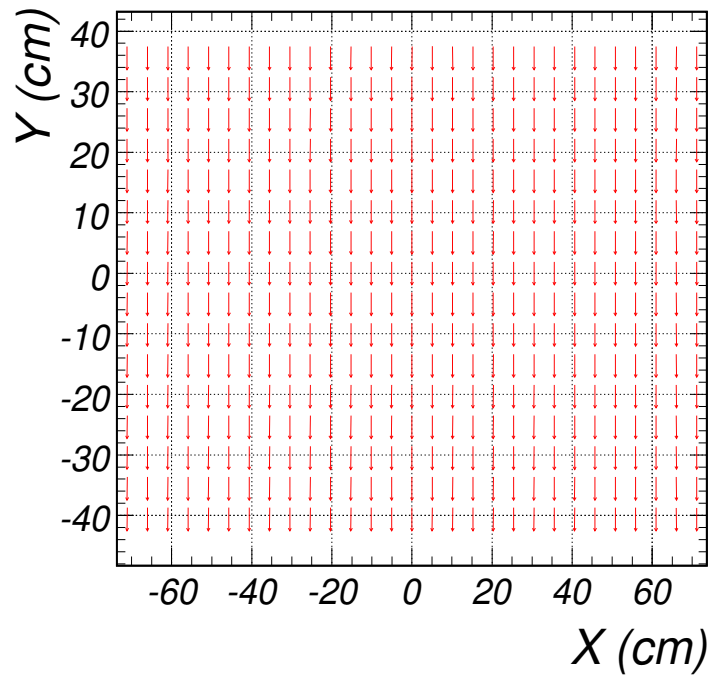

(a)
Field map projection at $\mathrm{x}=0$

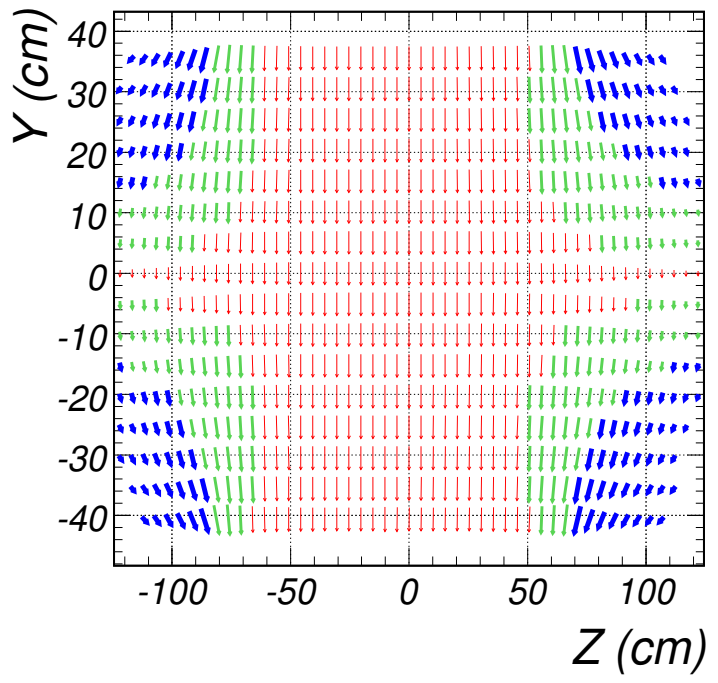

(b)

Figure 2.10: Projections of the Rosie field map in the same volume as that in figure 2.9. using the same legend for thickness of arrows. 
production, and an array of thin samples of complex nuclei for studies of hadron production and propagation in nuclei.

\subsubsection{Nuclear targets}

Since the TPC is able to analyze charged hadron ejectiles down to low energies, our samples of nuclear targets are thin, about 1\% interaction length. For the heavy Bismuth case, this is about $30 \%$ of a radiation length. Nuclei are chosen to be approximately uniformly distributed in $\log \mathrm{A}$, and are single or two isotope nuclei only, so as to avoid effects of wide neutron excesses. In general, spherical nuclei are chosen, with an emphasis on nuclei used in previous hadronic reaction studies or of particular interest to our collaboration. In order to maximize the TPC acceptance, the samples are placed with their downstream faces within a few $\mathrm{mm}$ of the entrance window. The back edges of the samples are at a fixed distance from the window, known to within $2 \mathrm{~mm}$, with z-thicknesses of the samples ranging from 0.17 to $4.0 \mathrm{~cm}$. Areal densities and uniformities of the samples are known to within $1 \%$.

An eight-position aluminium circular wheel with $20 \mathrm{~cm}$ diameter holds the samples, each $5 \mathrm{~cm}$ in diameter. The target wheel was built by the University of Colorado and taken to Fermilab unassembled. The target wheel is installed a few centimeters upstream of the front face of the TPC. Out of eight positions of the wheel, two positions are vacant. Runs on empty target positions are carried out for background studies. The targets mounted are beryllium, carbon $1 \% \lambda_{I}$, carbon $2 \% \lambda_{I}$, bismuth and uranium. The target wheel is shown in figure 2.11 .

Runs are also taken with a $2 \%$ carbon target to check on target attenuation effects. Studying the carbon target is useful for understanding particle production with the NuMI target. The atomic weights of the nuclear targets are between 9 and 238 which allows MIPP to investigate nuclear interactions over a wide range of atomic weights. The other main properties of the nuclear targets are shown in table 2.3. The wheel is not centered on the beam, since the target holes are off-axis. The wheel is turned by an ACNET (Fermilab Accelerator Control NETwork) controlled motor mounted to the TPC table upstream of the TPC, outside of the magnetic field of the JGG magnet. This allows targets to be changed without accessing the MIPP experimental hall.

\subsubsection{Cryogenic target}

The cryogenic target used at the MIPP is filled with liquid hydrogen and controlled through the iFix/APACS system, which generally controls the pressure and temperature of the gas systems. The target cell is approximately a cylinder of $3.8 \mathrm{~cm}$ diameter and $14 \mathrm{~cm}$ length 


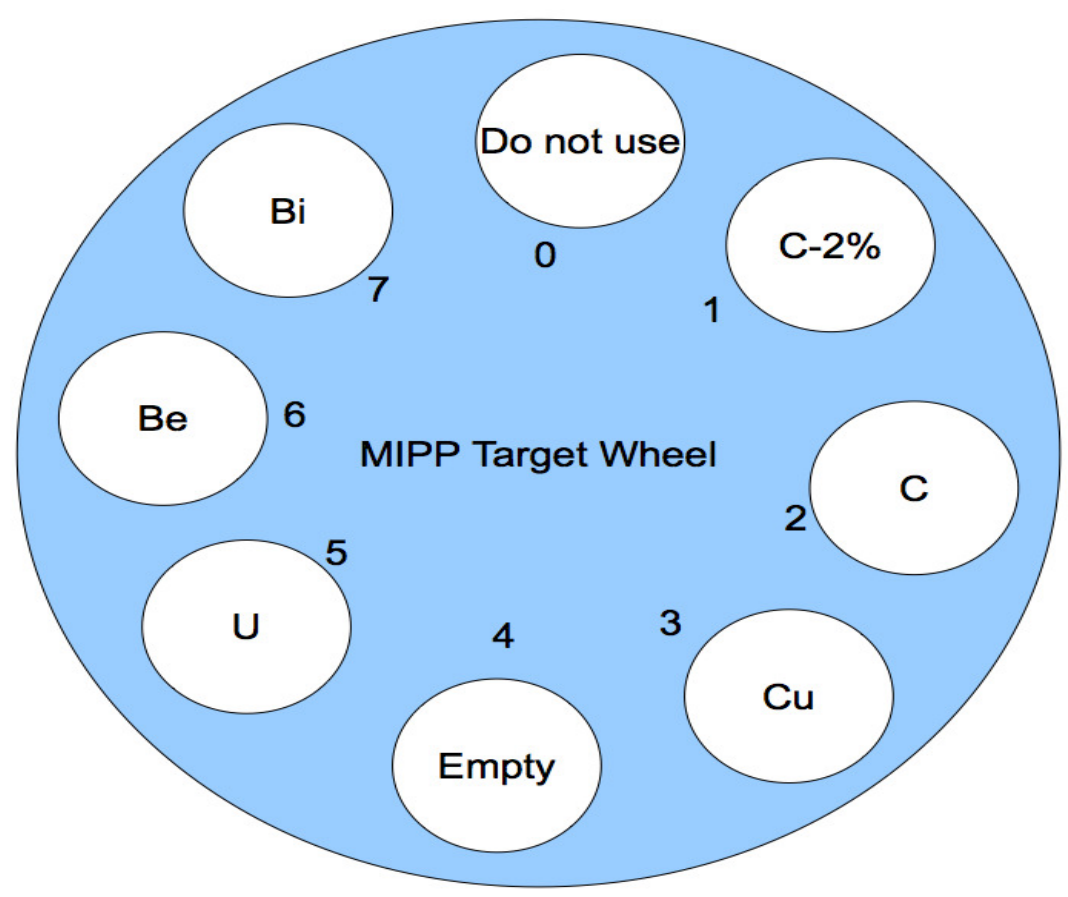

Figure 2.11: The thin targets used in the MIPP experiment are installed in a rotating wheel.

\begin{tabular}{|l|l|l|l|l|l|}
\hline Target & Mass(gm) & Thickness(cm) & $\begin{array}{l}\text { Areal } \\
\text { density }\left(\mathbf{g m} / \mathbf{c m}^{2}\right)\end{array}$ & $\begin{array}{l}\text { Interaction } \\
\text { length(\%) }\end{array}$ & $\begin{array}{l}\text { Radiation } \\
\text { length(\%) }\end{array}$ \\
\hline Beryllium & 14.4 & 0.399 & 0.710 & 0.94 & 1.1 \\
\hline Carbon 1\% & 16.6 & 0.498 & 0.819 & 0.94 & 2 \\
\hline Carbon $2 \%$ & 34 & 1.003 & 1.667 & 1.94 & 3.9 \\
\hline Bismuth & 34.25 & 0.173 & 1.69 & 0.87 & 27 \\
\hline Uranium & 38 & 0.1 & 1.875 & & \\
\hline
\end{tabular}

Table 2.3: The list of thin targets and their properties. 
with a cell volume of 1.6 liters. There are caps bulged out on both ends of the target, so that they can hold the pressure. The caps are not flat and the exact target length is a function of the radius. The total cryo-target system holds 0.2 liters of liquid hydrogen during operation. The temperature and the pressure of the target are monitored continuously. Although there are some operational fluctuations, the average volume density of the target can be estimated as $0.070 \mathrm{~g} / \mathrm{cm}^{3}$, so the areal density is around $1 \mathrm{~g} / \mathrm{cm}^{2}$.

Empty target runs are taken to obtain background spectra, without moving the cell.

\subsubsection{NuMI target}

MIPP uses a spare NuMI target from the MINOS experiment. The target is made of graphite with a density of $1.78 \mathrm{~g} / \mathrm{cm}^{3}$. The target core consists of 47 graphite segments, which are placed vertically and soldered to two stainless steel cooling pipes. Each segment is $0.64 \mathrm{~cm}$ wide, $2.0 \mathrm{~cm}$ tall and $1.36 \mathrm{~cm}$ long, with a gap of $0.67 \mathrm{~cm}$ in between the target segments. The gaps between the segments allow most of the produced pions and kaons to leave the target before interacting and avoid contact of heated segments. The target core is inserted into an aluminium cylinder casing which has a diameter of $30 \mathrm{~mm}$ and thickness of $0.4 \mathrm{~mm}$. The total length of the casing is $1.2 \mathrm{~m}$.

The target canister is filled with helium gas to provide heat transfer from the aluminium casing to the cooling water pipes. A $48^{\text {th }}$ target segment is installed at the upstream end of the target segments horizontally to provide a cross check of the position of the target. The schematic of the NuMI target design [77] is shown in figure 2.12. The detailed information about the NuMI target can be found in [78, 79.

The NuMI target is 2 interaction lengths. As a result, $\sim 90 \%$ of the incoming protons interact within the target, thus reducing the backgrounds created by non-interacting protons and maximizing meson production.

The pair of stainless steel cooling tubes was designed to remove the heat deposited in the target in the MINOS experiment. Since the beam intensity during MIPP NuMI running period was much lower than that in MINOS, no water was circulated in these tubes. This is taken into account in the MIPP Monte Carlo simulation which is described in a later section of this chapter.

\subsection{Trigger System}

MIPP uses a single-level experimental trigger in two stages - the first stage of the trigger decides on the species of the particle and the second stage on whether or not the particle 


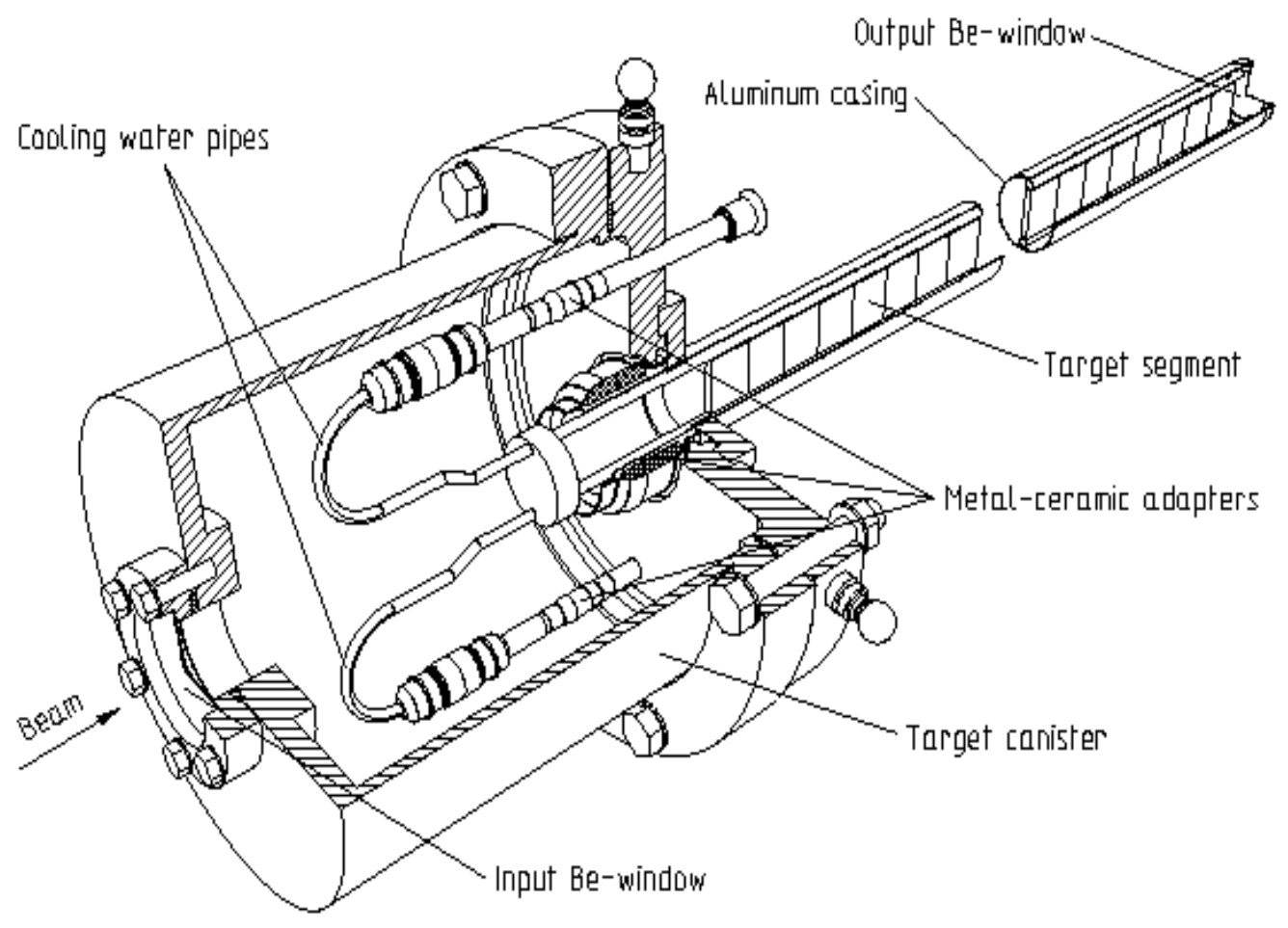

Figure 2.12: Schematic of the NuMI target design.

interacts in the target. Thus, 7 physics trigger bits are typically in use: untagged beam, three particle species, and three particle species with interaction. Each trigger bit is prescaled differently before forming a global OR in order to achieve approximately $20 \%$ minimum bias and $80 \%$ interaction trigger mix. These scaling factors are set to achieve equal amounts of protons, kaons, and pions.

Since the trigger has only a single level, a decision has to be made in the shortest time possible so as not to degrade the resolution of the ToF system. In the final implementation, the trigger is formed about $250 \mathrm{~ns}$ after the incident particle passes through the target.

\subsubsection{Beam Trigger}

In the MIPP trigger system, beam particles (BEAM) are defined by the coincidence of the TBD and T01 scintillation counters in the MIPP beamline and the anti-coincidence of the BVeto counter.

$$
\mathrm{BEAM}=\mathrm{TBD} \cdot \mathrm{T} 01 \cdot \overline{B V e t o}
$$

The triggers from the TBD and T01 counters are formed by the coincidence of three of the four discriminated PMT signals. 
The BVeto counter is a $35 \mathrm{~cm} \times 40 \mathrm{~cm}$ scintillator paddle with a $4.32 \mathrm{~cm}$ diameter hole which is mounted on an aluminum plate $140 \mathrm{~cm}$ upstream of the experimental target, close to the T01 counter. This counter is used to limit the effect of beam halo coming from the primary target in secondary beam configurations. Two PMT's are attached to the sides of the veto scintillator through light guides, and the logical AND of these PMT's forms an $80 \mathrm{~ns}$ veto signal for rejecting events with particles outside of the hole.

\subsubsection{Beam Particle Identification}

Beam particle identification (PID) is achieved by the two beam Cherenkov counters as described in the section 2.5. The beam PID for the different ranges of the incident beam momentum is described as follows:

\section{Momentum Above $20 \mathrm{GeV} / \mathrm{c}$}

At $20 \mathrm{GeV} / \mathrm{c}$ and above, the pressure in the beam Cherenkov counters can be tuned so that all charged particles radiate light. The four PMT's are abbreviated as UI for upstream inner, UO for upstream outer, DI for downstream inner, and DO for downstream outer. Under these operating conditions, the particle trigger bits are defined as:

- $\pi: \overline{U I} . \mathrm{UO}$

- K: UI. $\overline{U O}$

- p: DI. $\overline{D O}$

At $35 \mathrm{GeV} / \mathrm{c}$ and above, the pion and kaon Cherenkov angle in the downstream counter is below $30 \mathrm{mrad}$, so under those conditions, a more stringent trigger can be formed, based on all four PMT's:

- $\pi: \overline{U I} . \mathrm{UO} . \overline{D I} . \mathrm{DO}$

- K: UI. $\overline{U O} \cdot \overline{D I} \cdot \mathrm{DO}$

- p: $\overline{U I} \cdot \overline{U O} \cdot \mathrm{DI} \cdot \overline{D O}$

\section{$5 \mathrm{GeV} / \mathrm{c}$ Momentum}

Using the beam Cherenkov counters, it is not possible to tag protons and kaons with momentum below about $20 \mathrm{GeV} / \mathrm{c}$ and $11 \mathrm{GeV} / \mathrm{c}$, respectively, as a sufficiently high gas density can not be obtained. Even if it were possible to do that, the gas density would have been so 
large that multiple scattering in the counters would degrade the information of position and direction of the incoming particle. Instead, at $5 \mathrm{GeV} / \mathrm{c}$, we rely on particle time of flight to separate the kaons and protons offline.

For the purpose of the trigger, densities of both the beam Cherenkov counters are tuned to radiate on pions, muons, and electrons. Then proton plus kaon trigger is the absence of signal in all beam Cherenkov PMT's. Both negative and positive beams of this momentum are primarily $(\approx 90 \%$ ) composed of pions, with similar fractions of protons and kaons, so it is acceptable to accumulate equal fractions of protons and kaons, as long as they can be distinguished offline.

The PID trigger signals are combined in coincidence with the beam trigger logic as described earlier in such a manner as to preserve the leading edge of the resulting signal as a time reference that remains definite with respect to the TBD initial crossing. The final copies of the "Beam with PID" are given the physics designations $\pi$-PID, K-PID and p-PID. These signals are regenerated as standard NIM level outputs with fixed widths of less than $16 \mathrm{~ns}$ to preserve the single bucket timing determinism required by the system. The total transit time from initial beam trigger formation to output formation of the PID triggers is $37 \mathrm{~ns}$.

\subsubsection{Beam Trigger Purity}

The absolute purities of each particle species tagged are independently verified through offline reconstruction of the Cherenkov ring radii left in the $\mathrm{RICH}$ counter as a function of incident beam particles (non-interactions) [80]. The full spectrum of calculated purities as a function of incident beam momentum is shown in figure 2.13. The trigger purities for pions, protons in the positive polarity run configuration are verified to exceed the $97 \%$ design goal across the momentum range of 5 to $85 \mathrm{GeV} / \mathrm{c}$. The tagged purity of kaons in the positive polarity are measured to range from $>85 \%$ at momenta below $50 \mathrm{GeV} / \mathrm{c}$, down to a purity of $80 \%$ at beam momenta above $60 \mathrm{GeV} / \mathrm{c}$ where the total kaon fraction in the beam is less than $1.8 \%$. In the negative running configuration, trigger PID purities are above $97 \%$ for pions and kaons, and anti-protons in the high momentum region. In the low momentum region the purity of the anti-proton is highly contaminated by false trigger positives coming from the divergence in the beam tune. The resulting purity for extremely rare anti-proton portion of the beam at $-20 \mathrm{GeV} / \mathrm{c}$ is measured at $20 \%$, while for the $\mathrm{K}^{-}$population the purity in this configuration remains near $88 \%$. 


\section{Trigger purity determined by $\mathrm{RICH}$ ring radii of beam particles}

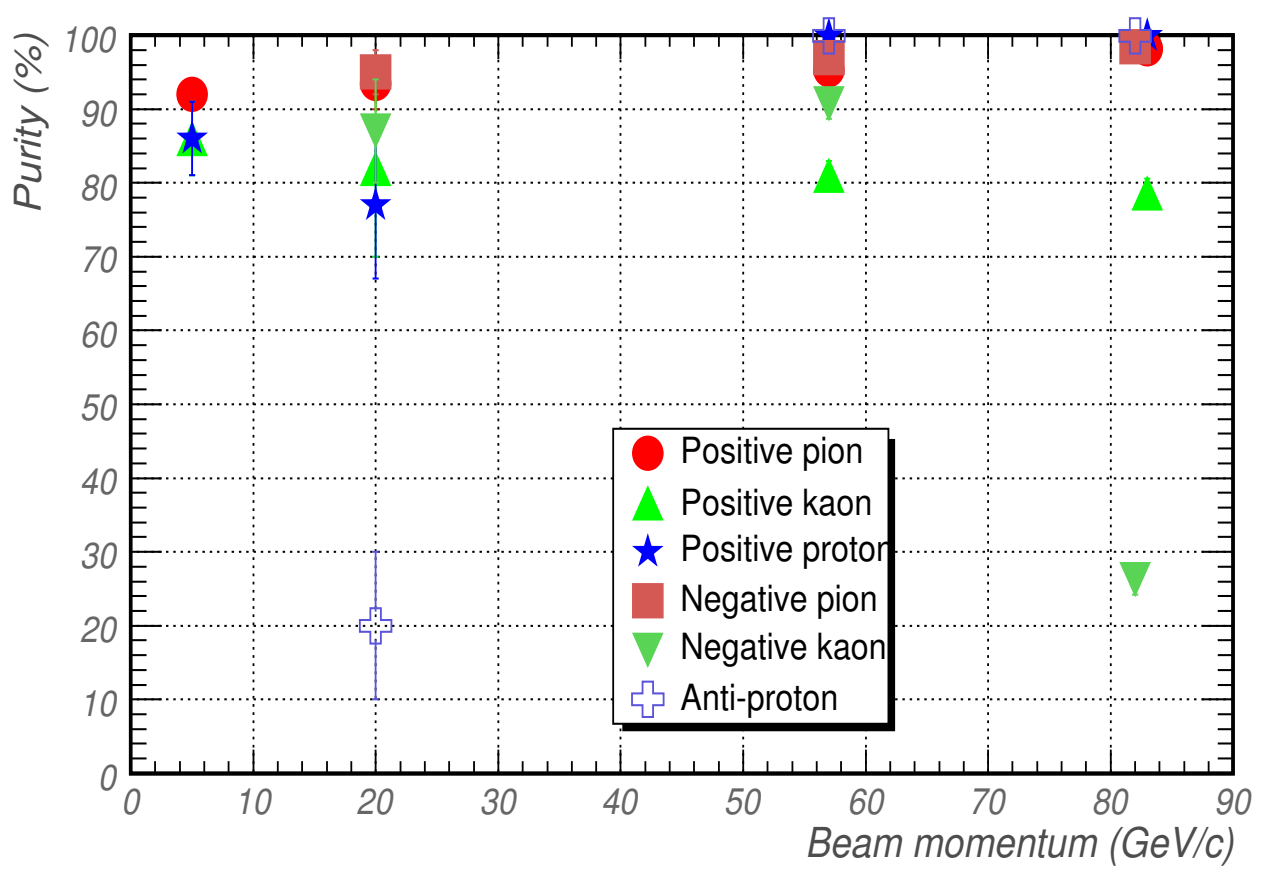

Figure 2.13: Trigger purity as measured by the RICH.

\subsubsection{Interaction Trigger}

Initially, the MIPP interaction trigger was based on the multiplicity of the first drift chamber DC1, requiring 2 or more wire clusters in at least three of the four planes. This trigger has the following problems associated with it:

1. The large chamber area makes it highly susceptible to poor beam conditions,

2. The chamber is sometimes noisy because of low discriminator thresholds which reduces the purity of the trigger,

3. The low efficiency for two-particle final states where one particle is soft i.e. with very low momentum.

To address the problems of DC1 interaction trigger, a scintillator-based interaction trigger is built. Both the triggers are explained below.

\section{DC1 Trigger}

The preamplifier cards, each serving 8 wires, are mounted on the drift chamber. Four preamplifier cards are connected to one discriminator. Each discriminator outputs $32 \mathrm{ECL}$ 
signals, one per wire, and 4 ECL OR signals, one for each cluster of 8 wires. The cluster OR signals are sent to majority logic units (LeCroy 4532 CAMAC module) to create a multiplicity interaction trigger, called iDC. The trigger fires if there are at least 2 different clusters in at least 3 out of 4 planes.

\section{Scintillator Interaction Trigger}

The scintillator interaction trigger (Scint) [81] is much simpler than the iDC. An overall plan view of the interaction trigger is shown in figure 2.14. A $3.175 \mathrm{~mm}$ thick $5.40 \mathrm{~cm} \times 7.62 \mathrm{~cm}$ piece of plastic scintillator is placed $\approx 1.5 \mathrm{~cm}$ downstream of the experimental target with its shorter edge horizontal and its taller edge vertical. It is sandwiched between two aluminum brackets at the top and bottom, and two aluminized Mylar sides. The scintillator has a density of $1.032 \mathrm{~g} / \mathrm{cm}^{3}$ and its thickness corresponds to about $0.4 \%$ interaction length. 191 clear fibers with $1.2 \mathrm{~mm}$ diameter collect light from the two shorter sides of the scintillator. The other ends of the fibers are closely packed onto the face of a Burle 83054H PMT. By safety rules, in order to operate the trigger with the liquid hydrogen target, all high voltages (i.e. PMT) have to be at least $3 \mathrm{~m}$ away from the target, hence calling for rather long fibers. Even with $3 \mathrm{~m}$ of fiber, the counter gets about 73 photoelectrons per minimum ionizing particle (mip).

The PMT charge is amplified with a LeCroy 612 AM photomultiplier amplifier module, creating two copies of the signal. One copy goes directly to an ADC and the second copy goes to an analog splitter. The outputs of the splitter are discriminated with two different thresholds, creating two digital signals - SciHi $(\approx 3 \mathrm{mip})$ and SciLo $(\approx 1.5 \mathrm{mip})$. The problem with the SciLo signal is that the Landau tail of the single-particle energy deposition dominates the count rate from the $1 \%$ interaction length target.

\subsubsection{End of Spill and Calibration Triggers}

Each accelerator beam spill is followed by an end of spill trigger and a few pulser calibration triggers. Upon receipt of the end of spill trigger, the detectors are not triggered, and only the following actions are taken:

1. Scalers for the entire spill are read out and cleared;

2. A record is written to the database with the number of different triggers recorded in the spill;

3. Buffered data are transferred from the PowerPCs to the DAQ server (explained in the next section). 


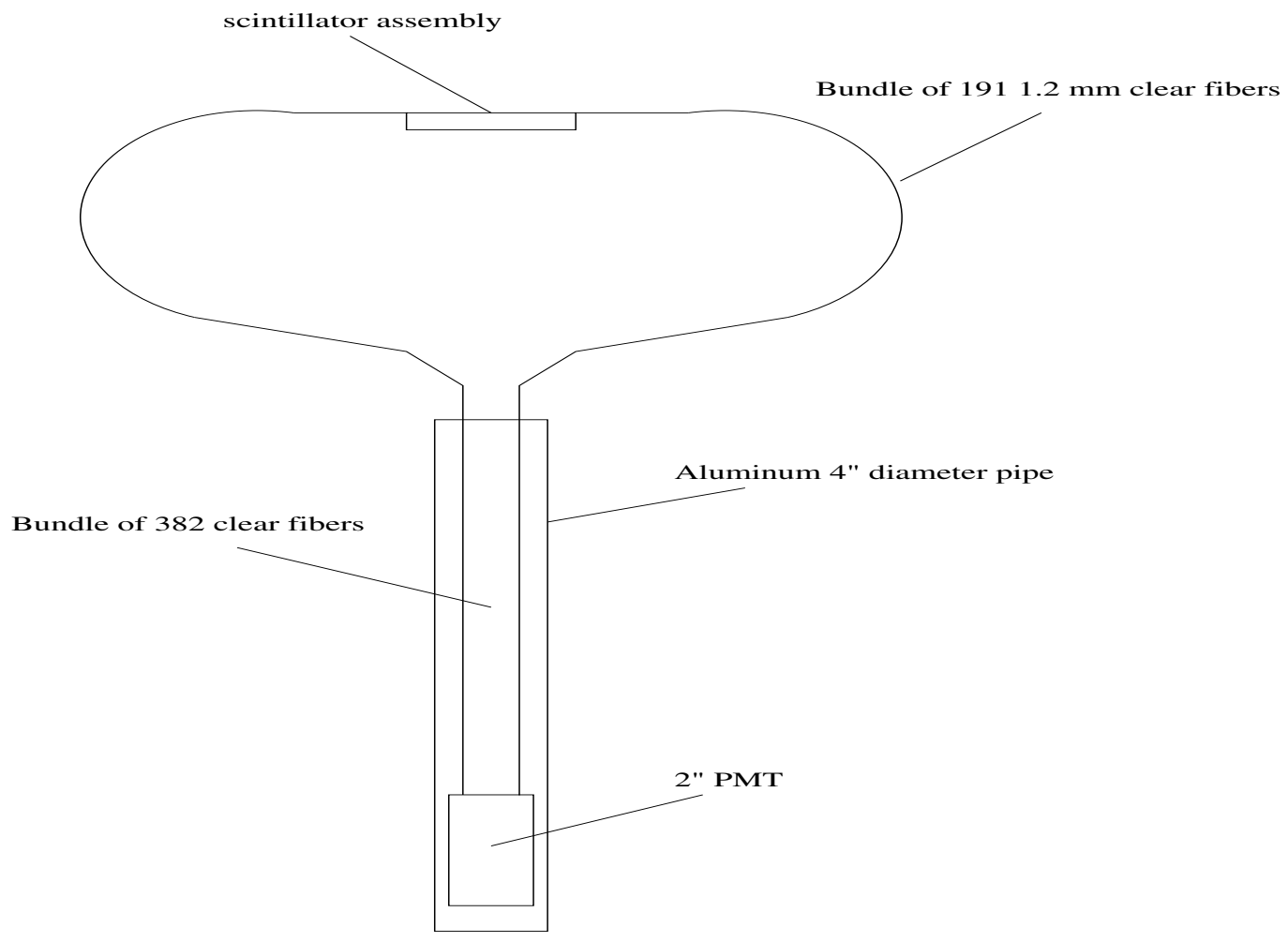

Figure 2.14: Overall plan view of the interaction trigger counter.

On calibration triggers, the detectors are triggered by a pulse generator. These triggers are used to calculate pedestals and identify hot channels.

\subsubsection{Master Trigger}

A master trigger signal is formed from the various triggers listed above and distributed to the detector readouts. The occurences of all these triggers are counted for each beam spill in ungated and gated, unprescaled and prescaled scalers.

The sixteen individual trigger bits are passed through FNAL PD-22 prescaler module to provide $1 / \mathrm{N}$ divide down prescaling where $\mathrm{N}$ is prescale value. The prescale values are optimized to provide roughly equal bandwidth to each of the particle species interaction triggers. This optimization is performed at each incident beam momentum and tuned where the minority species are always set to a baseline prescale of 1 . In the event where the available trigger bandwidth is not fully utilized by an even $(1 / 3,1 / 3,1 / 3)$ mix of $\mathrm{K}, \pi$ and $\mathrm{p}$ triggers, such as is the case in negative running where both the $\mathrm{K}^{-}$and $\bar{p}$ rates are extremely low, the available bandwidth is filled by reducing the prescales on first and second minority species, and then on the dominating species. The full list of triggers is balanced in this manner 
to achieve target rates for the total interacted beam sample as well as sufficient samples of PID-biased and minimum-biased triggers to calculate the required trigger normalizations and biases. Under nominal run conditions, the fraction of minimum-biased beam and PID triggers is set as not to exceed a total of $20 \%$ of the total physics bandwidth.

\subsection{Data Acquisition System}

The MIPP data acquisition system (DAQ) is designed to read out all detectors at a minimum rate of $60 \mathrm{~Hz}$ with $100 \mathrm{kB}$ average event size (both set by the TPC) for a beam delivered in $1 \mathrm{~s}$ spills, 20 spills per minute, with an overall throughput of $2 \mathrm{MB} / \mathrm{s}$. The DAQ is able to keep up with the actual data rate, which does not exceed fifteen $600 \mathrm{~ms}$ spills per minute.

Figure 2.15 shows the overall schematic of the data acquisition system. The complete data acquisition system consists of:

- a DAQ server with two network cards, which is connected to the public net and to the private subnet,

- Six VME PowerPC's (PPC's), connected to the DAQ server through 100 Mbit network with the ability to access the VME and CAMAC readout electronics and send data to the server,

- Six VIrGen cards built to generate VME interrupts,

- Two CBD 8210 VME CAMAC readout cards,

- An online monitoring machine, which enables monitoring of data in real time,

- A High Voltage machine, which is used to control and monitor all PMT and TPC high voltages (wire chamber high voltages are manually controlled),

- A database server, which runs a PostgreSQL database where all online information is stored.

Events are read out by a set of 6 PowerPC's running Linux - four dedicated to TPC read out and two more to read out all other detectors. These machines have $350 \mathrm{MHz}$ processors and $256 \mathrm{MB}$ of memory. One PowerPC is used to read out each quadrant of the TPC, and the remaining two are used to read out the RICH VME electronics and all CAMAC modules through two CBD 8210 CAMAC branch drivers. Each has a VME Interrupt Generator (VIrGen) board for handling VME interrupts. These boards are designed at FNAL and built at Harvard University. The DAQ system is configured to expect two types of interrupts: one 


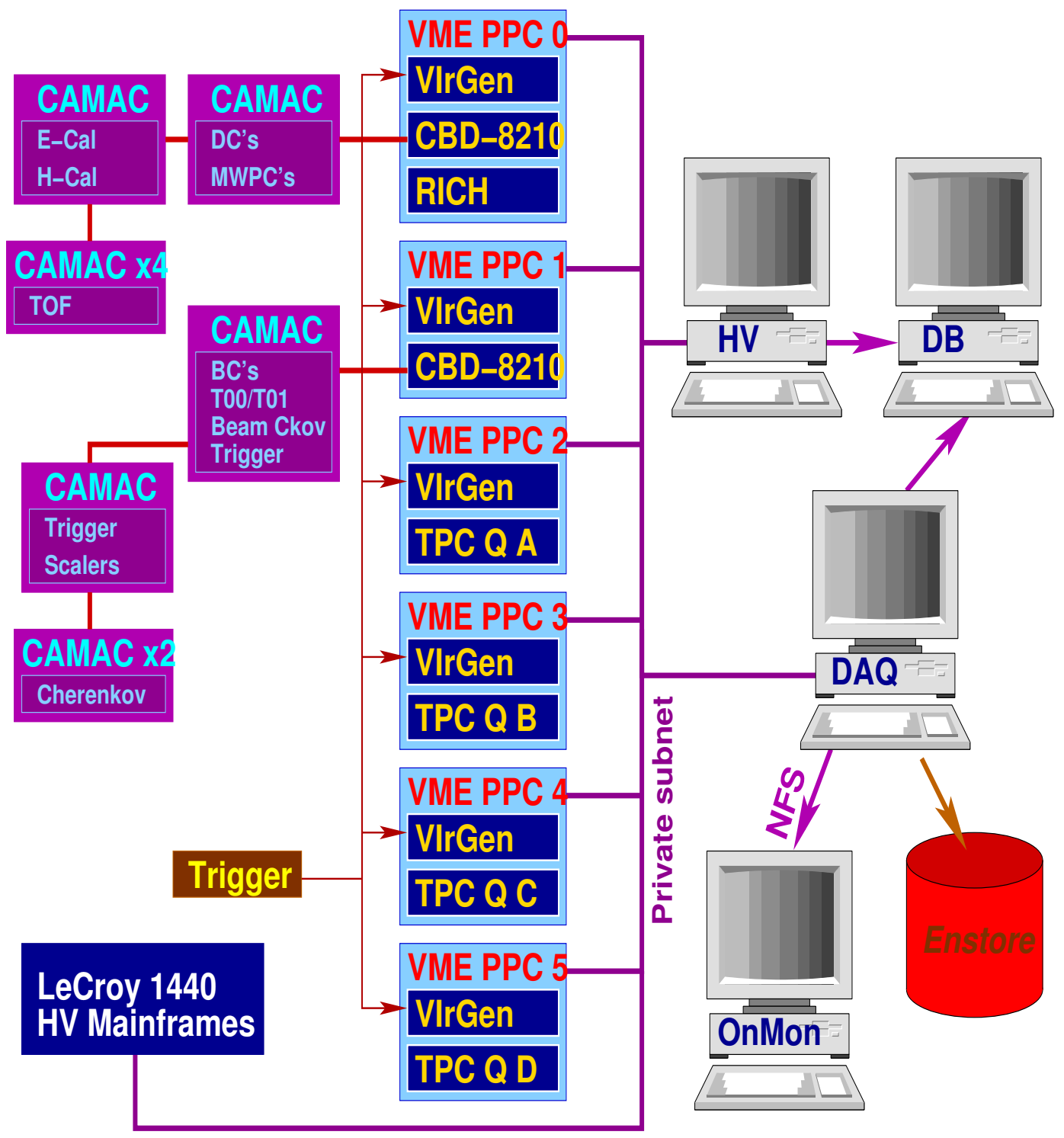

Figure 2.15: Schematic of data acquisition setup. Of the six VME PowerPC's, four are dedicated to control and read out of the TPC quadrants, and the other two read out the rest of the detectors, mainly CAMAC electronics. Read out processes are triggered through VME interrupts delivered by VIrGen boards. High voltage mainframes connected to private subnet as well, are controlled and monitored by HV machine. Detector data are written out to dedicated files, and online monitoring data are stored in a PostgreSQL database. Online monitoring is done by exporting data disks through NFS. Finally, data are regularly shipped to the FNAL robot tape storage (Enstore). 
for the data trigger, and second for end of spill, for which only scalers are read out in the trigger process and all other processes send empty data messages.

A dedicated I/O library, MippIo, is written to store data in a structured byte-packing format organized into blocks. A MIPP raw data file consists of a file header block followed by any number of event blocks and concluded with an end of file block. Event blocks contain detector blocks consisted of data as it is read from VME or CAMAC with no pre-processing done on the PPC's.

All processes are controlled from the E907 DAQ PC by a run control daemon. This process maintains the overall state of the DAQ system, processes operator requests to start and stop data acquisition, and responds to exceptions and errors in the system. Communication to and from run control is handled by a message passing system implemented through stream sockets, allowing maximal flexibility and expansion of the DAQ system. A number of different messages are implemented each having a 20-byte header containing message id, its number, size, second, and microsecond when the message is packed. Upon start-up, run control would open a socket to listen to incoming connections and would initiate an infinite loop which polls all known sockets for activity. New connections are initiated to start the event builder and the MIPP daq daemon, and to open new graphical user interfaces. Since the number of connections is small (typically 8) and the response time to nearly all messages does not involve much CPU time, this simple architecture works very well. At the start of each run, run control would read slow control devices (explained later in this section), record the run conditions to a database, and send start messages to the event builder and MIPP daq daemon on each PC followed by the start run command. At the end of each run, a stop run message would be sent to each daemon, to the event builder, and final event statistics would be recorded in the database.

Events are assembled by an event builder provided by the FNAL Computing Division. Upon receiving subevents for each detector and each DAQ daemon, it combines them according to trigger time stamps before writing to disk. The event builder also divides long runs into subruns of $1 \mathrm{~GB}$ or less to facilitate subsequent file handling on the batch farm. With the exception of the TPC which is divided into quadrants, each detector has a single detector process running on a PowerPC that would read and buffer data for every trigger, send data to event builder on every end of spill trigger. Each process has two threads, one for each interrupt type.

Run configuration information is written to a Postgres database via a dbLogger interface so that executables running on the PowerPC's can add and modify entries in the database. The logger daemon, running on the DAQ server, listens to incoming connections from the PowerPC's and passes SQL commands to the Postgres server without parsing them. 
The main use for dbLogger is to store the number of spills and triggers recorded in a given run.

Online monitoring runs on a dedicated computer which has the data disks mounted through NFS. The program scans disks for new files, continually updating histograms which helps shift workers identify any problems with the data. The online monitoring software is written in ROOT [82, and histograms for each run are saved for subsequent comparisons.

\subsubsection{Slow Controls}

The environment in the experimental hall as well as settings and readbacks of devices are controlled and continually monitored by the FNAL APACS system. Examples of data recorded by APACS are gas pressures, hall temperatures, magnet current settings, and high voltage settings.

MIPP uses two different systems to monitor and control the devices. They are explained below.

\section{iFix}

The Intellusion iFix system is used as a front-end for APACS control. It is used to:

1. Read air and gas pressures, multiple temperature sensors, humidity sensor;

2. Control the pressure in beam Cherenkov counters;

3. Control the flow of gas through threshold Cherenkov counter;

4. Act as an interlock for the RICH cooling system;

5. Monitor chamber gas flow and mixing;

6. Monitor and control cryogenic target information.

An XML-RPC server provided by the FNAL Beam Division was running on the Windows $\mathrm{PC}$ where iFix was running to provide a way to store variables to the Postgres database and for DAQ to find out the status of the cryogenic target.

\section{ACNET}

ACNET is Accelerator Control NETwork developed at and supported by Fermilab. It is used to:

1. Control and monitor secondary beamline settings for dipole and quadrupole magnets; 
2. Control momentum collimator slit width;

3. Monitor beam profiles;

4. Monitor spill structure;

5. Control and monitor analysis magnet currents;

6. Monitor Hall probes installed in the apertures of analysis magnets;

7. Control and monitor RICH high voltage supplies;

8. Control beam Cherenkov mirror positions;

9. Monitor wire chamber and electromagnetic calorimeter voltages and currents;

10. Monitor spill intensity;

11. Control the target wheel position;

12. Monitor hall and target temperature information.

Similar to iFix, the XML-RPC server is used to control devices and record their state into the database.

\section{High Voltage}

High voltages on all the PMT's and the TPC anodes are controlled through two LeCroy 1440 systems. Once high voltages are set, the system continually cycles through all known channels, storing the readback voltage to the database and verifying that no trips have occured. This is especially important for TPC anodes which do occasionally trip. Prompt detection of trips ensures that as little data as possible is lost.

A more detailed discussion of the MIPP DAQ can be found in [80].

\subsection{Event Reconstruction}

In this section we discuss event reconstruction of the data collected in the MIPP experiment. The event reconstruction is divided into two steps - first is track reconstruction and second is vertex reconstruction. Both of these are described below. 


\subsubsection{Track Reconstruction}

Secondary charged particle trajectories are reconstructed using reconstructed hits in the TPC, drift chambers (DC's) and proportional wire chambers (PWC's). The reconstruction is done in stages. Helical TPC track segments are first formed, followed by the formation of straight line track segments using hits from the DC's and PWC's. Next, the TPC track segments are refitted using the map of the JGG magnet and matched to the DC and PWC track segments. A fit of the trajectory is performed with all hit information obtained from the matched track segments. All the steps involved in the track reconstruction are described below.

\section{Time Projection Chamber}

The TPC dominates the event size and is the most complex detector to reconstruct. The reconstruction algorithm is adopted from the algorithm used by the BNL E910 [83]. The TPC data are formed into 3D-spacepoint hits in ( $\mathrm{x}, \mathrm{y}, \mathrm{z})$. To first order, $\mathrm{x}$ and $\mathrm{z}$ positions are determined by the position of the readout-pad on the bottom plane of the detector, and the y position is determined from the timing information of the readout-pad. The extent of the ionized electron cloud is typically $\sim 3 \mathrm{~cm}$. TPC hits are formed from 2D clusters of contiguous pad hits in each pad-row (slizes of $z$, along the direction of the beam). Peaks in the charge-weighted time distribution of each pad in each cluster are separated and fitted to a Gamma function. The peak time is set to the most-probable value from the Gamma function fit, $\mathrm{t}_{m p v}$. Peaks which are within two TPC pad widths $(1.6 \mathrm{~cm})$ are grouped together to form a "hit", and the hit x position and time of the hit are determined according to:

$$
x_{h i t}=\sum_{i} Q_{i} x_{i, p a d}, \quad t_{h i t}=\sum_{i} Q_{i} t_{i, m p v}
$$

where $\mathrm{i}$ is the $\mathrm{i}^{\text {th }}$ pad in the cluster, $\mathrm{Q}_{i}$ is the integrated charge from the Gamma fit of the peak in pad $\mathrm{i}, \mathrm{x}_{i, p a d}$ is the $\mathrm{x}$-center of pad $\mathrm{i}$, and $\mathrm{t}_{i, m p v}$ is the most-probable value for the peak in pad $i$.

TPC track segments are formed by first searching for at least three contiguous TPC hits in any direction beginning in the downstream end of the gas volume where the density of hits is the lowest. The track segment is then projected backward and forward assuming a helical trajectory, picking up more hits that fall within an acceptable distance from the predicted position. As hits are added to the track segment, the track segment is refitted improving the quality of the predicted trajectory. The details of the TPC track reconstruction algorithm can be found in [84]. 


\section{Wire Chambers}

The MIPP wire chambers are grouped into 3 triplets (BC123, DC123, DC4/PWC56) with the two magnets i.e. JGG and Rosie separating the triplets. The curvature due to magnetic field is negligible between the magnets as well as before JGG and after Rosie, so one can reconstruct a straight line segment in each group. Once the segments are found, it is simple to test whether a combination of the DC123 segment with the DC4/PWC56 segment appears to form a reasonable track candidate with momentum obtained from the Rosie kick. Finally, if the track candidate momentum is similar to the beam momentum, one can check whether combining the candidate with the $\mathrm{BC} 123$ segment would give a reasonable 9-chamber track. The steps involved in the formation of the chamber track candidates are described below.

- Wire clusters: A wire cluster is defined as either one wire or a group of contiguous wires. In the DC's, wires are clustered only if their hit times are similar and the cluster size is limited to two wires. In PWC's, there is no limit on the cluster size. After finding clusters, the next step is to form wire crosses.

- Wire crosses: Wire crosses are the combination of the wire clusters from different planes in a chamber. Initially, two cluster combinations from different planes are obtained and intersection of the clusters is calculated. Then, the clusters in the other planes are added to the cross if their positions are consistent with the intersection of the initial two cluster combination.

- Track segments: The straight line track segment search is similar to the wire cross finding. For that, in each triplet, all possible two wire cross combinations from different chambers are found. The crosses which are not compatible in time are thrown out. Acceptable combinations are fitted to a 3D straight line and the position in the other chamber is predicted. The crosses which are compatible in time with the first two crosses and whose positions are consistent with the predicted position are added to the straight line segment.

- Chamber track candidates: Once all track segments are found, 6-chamber track candidates are formed by combining the segments in the DC123 and DC4/PWC56 triplets. The positions of the segments in both chamber groups are extended to the center of the Rosie magnet, and they are matched if their $\mathrm{x}$ and $\mathrm{y}$ positions are consistent. Matching segments are defined as 6-chamber track candidates. The track candidate is fitted with the template track fitting method. The $\chi^{2}$ of the fit determines if the track is to be accepted or not. The template track fitting has five parameters for 
a given track: q/p (charge/momentum), $\mathrm{x}$ and $\mathrm{y}$ position, and the angles $\mathrm{dx} / \mathrm{dz}$ and $\mathrm{dy} / \mathrm{dz}$.

- The final step of the algorithm is to attempt to match the track with the BC123 track segment. Obviously wrong candidates are filtered out by requiring that the charge of the candidate matches the charge of the beam particles in the run, and that the momentum be at least 0.8 of the beam momentum. The decision on whether to keep the track candidate as a 6-chamber or a 9-chamber object is based on the $\chi^{2}$ of the resulting track.

\section{Global Tracking}

The aim of global tracking was:

1. To refit the TPC tracks using the measured JGG magnetic field map;

2. To combine the TPC tracks with the chamber track candidates, track segments, or stand-alone wire clusters.

Global tracks i.e. the tracks including TPC and chamber information, are formed by first attempting to match the TPC tracks to the chamber track candidates at DC1. If a matching candidate is not found, then a matching DC123 segment is searched for. If a segment is not found, then the nearest wire clusters in DC1, DC2, and DC3 are searched for. The track is refitted when the new information is added to the track. If the predicted track position at a chamber is outside of the active area of the chamber, then no attempt is made to extend the track further. For tracks that are extended to DC3, the process continues to the downstream chambers, first searching for matching DC4/PWC56 track segments and then descending to the level of wire clusters if it is necessary.

An event display showing both the reconstructed TPC as well as global tracks for a $120 \mathrm{GeV} / \mathrm{c}$ proton-carbon event is shown in figure 2.16. Blue circles and stars indicate wire chamber clusters and crosses, respectively.

\subsubsection{Vertex Reconstruction}

Primary and secondary vertex identification is essential for the data analysis. Vertex position

tells us where the track came from. In addition when we do a vertex-constrained fit, the track momentum and position resolution are improved.

The vertex reconstruction is split into two parts - vertex finding and vertex-constrained track fitting. The algorithm used is based on a deterministic annealing filter (DAF). Using 


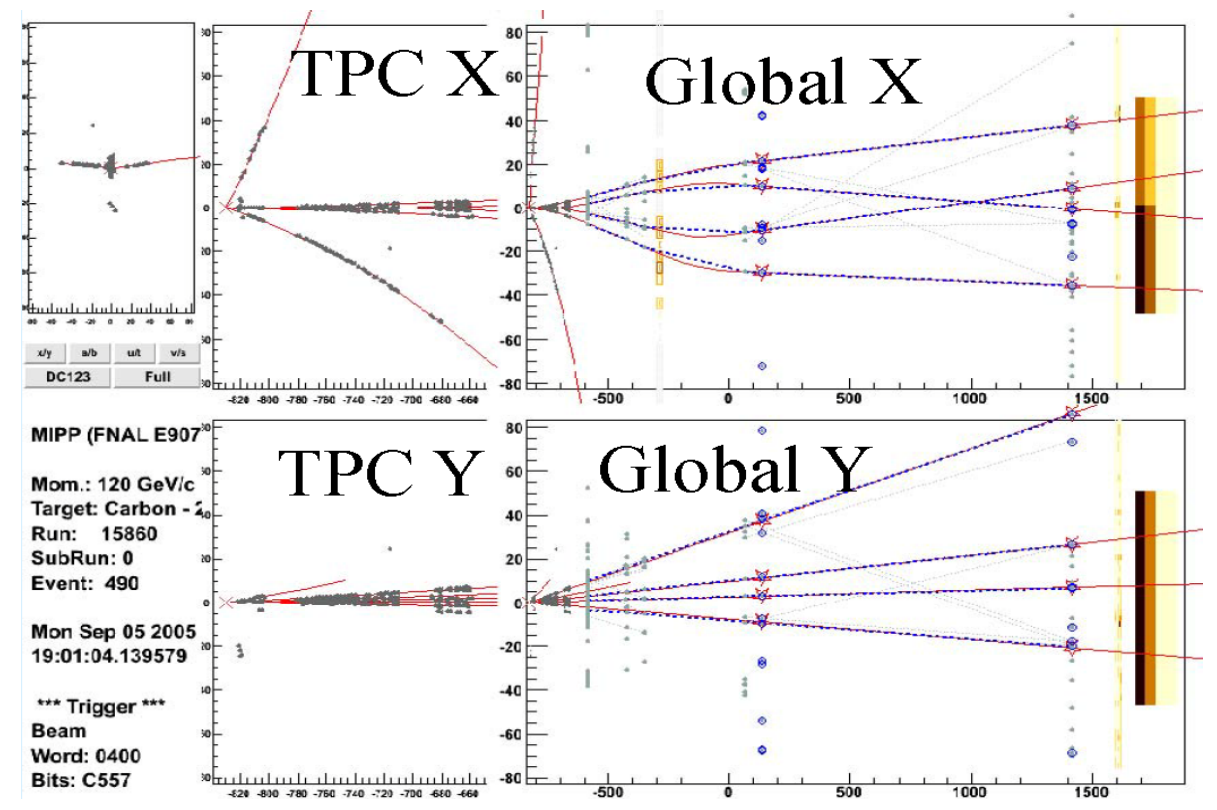

Figure 2.16: Event display showing both the reconstructed TPC as well as global tracks for a $120 \mathrm{GeV} / \mathrm{c}$ proton-carbon event.

MINUIT [85], we can fit for the point $(\mathrm{x}, \mathrm{y}, \mathrm{z})$ which minimizes the weighted distance squared to all tracks in the event i.e.

$$
D^{2}=w_{x}\left(x-x_{t}\right)^{2}+w_{y}\left(y-y_{t}\right)^{2}+w_{z}\left(z-z_{t}\right)^{2} .
$$

where $\mathrm{w}_{x}, \mathrm{w}_{y}$ and $\mathrm{w}_{z}$ are the weights that corresponded to errors on the measurements in $\mathrm{x}, \mathrm{y}$ and $\mathrm{z}$ direction, respectively, and $\left(\mathrm{x}_{t}, \mathrm{y}_{t}, \mathrm{z}_{t}\right)$ is the point on the track to which we are going to minimize the weighted distance squared from the point $(\mathrm{x}, \mathrm{y}, \mathrm{z})$.

The filter helps in the selection of the tracks associated with the vertex by reducing the weight with

$$
w_{D A F}=\frac{1}{1+\exp \left(\frac{D_{i}^{2}-D_{c}^{2}}{2 T_{D A F}}\right)},
$$

where $\mathrm{D}_{i}$ is the distance to the track from the vertex, $\mathrm{D}_{c}$ is the critical distance of the filter which is taken to be $7 \mathrm{~cm}$, and $\mathrm{T}_{D A F}$ is the temperature. As the temperature is decreased, the weights are reduced while the weights of the tracks with $\mathrm{D}_{i}^{2}<\mathrm{D}_{c}^{2}$ are not affected. The temperature used range from $5000 \mathrm{~cm}^{2}$ at the first iteration to $0.1 \mathrm{~cm}^{2}$ at the final iteration. As the iterations converge on a vertex position, this procedure gradually reduces the weights of tracks that do not belong to the vertex, to near zero. Once the final temperature is reached, a vertex is formed at the point $(\mathrm{x}, \mathrm{y}, \mathrm{z})$ and all tracks with weights larger than $10^{-6}$ are assigned to that vertex. The tracks which are included into the vertex are excluded from 
the available track list and the process is repeated from the start using the remaining tracks in the event until all tracks are assigned to a vertex.

In the events where the only two tracks are the incoming beam track measured by the BC's and the same track measured by the TPC and chambers, we find that when the temperature is lowered to $25 \mathrm{~cm}^{2}$, it is necessary to repeat the procedure at the same temperature so that the fitted vertex position would prefer to land in the middle of the two tracks rather than excluding one of them completely.

Finally, a vertex-constrained fit is performed using all tracks associated to each vertex. This fit simultaneously determines the vertex position and refits the particle trajectories such that all associated tracks originate from that vertex.

The details of the tracking and vertex algorithm can be found in [80].

\section{Limitations of Vertex Fitting}

The vertex finder clusters tracks within $\pm 3 \mathrm{~cm}$ of the vertex position. This means that nearby secondary vertices might get erroneously pulled into the primary vertex. The two problems that arise are the pull that the incorrect tracks would have on the vertex position, and the incorrect conclusion that the extra particles come from the vertex rather than from a neutral decay or re-interaction.

The resolution of the longitudinal $(\mathrm{Z})$ vertex is $\sim 6 \mathrm{~mm}$. Figure 2.17 shows the distribution of the fitted $\mathrm{Z}$ vertex from carbon, beryllium and bismuth targets, and the target-out configuration.

\subsection{Monte Carlo Simulation}

Monte Carlo (MC) simulation is necessary to measure the performance of the reconstruction. All the corrections including interaction trigger efficiency, particle identification (PID) efficiency and detector acceptance used in this analysis are calculated using the MC.

The MIPP MC simulation package is based on GEANT 3.21 [86]. DPMJET-3.0 [87] and FLUKA-06 [26, 88] are used to model the particle production and particles are propagated through the spectrometer by GEANT creating hits with idealized energy deposition. The GEANT hits are stored into the ROOT event tree and converted into digits (mock raw data) using the MIPP digitization packages. The details about the digitization of the hits from all the detectors can be found in [80].

DPMJET is used for the event generation in case of liquid hydrogen target only. For all the other targets, event generation is done using FLUKA. 


\section{Vertex $\mathbf{Z}$ distribution}

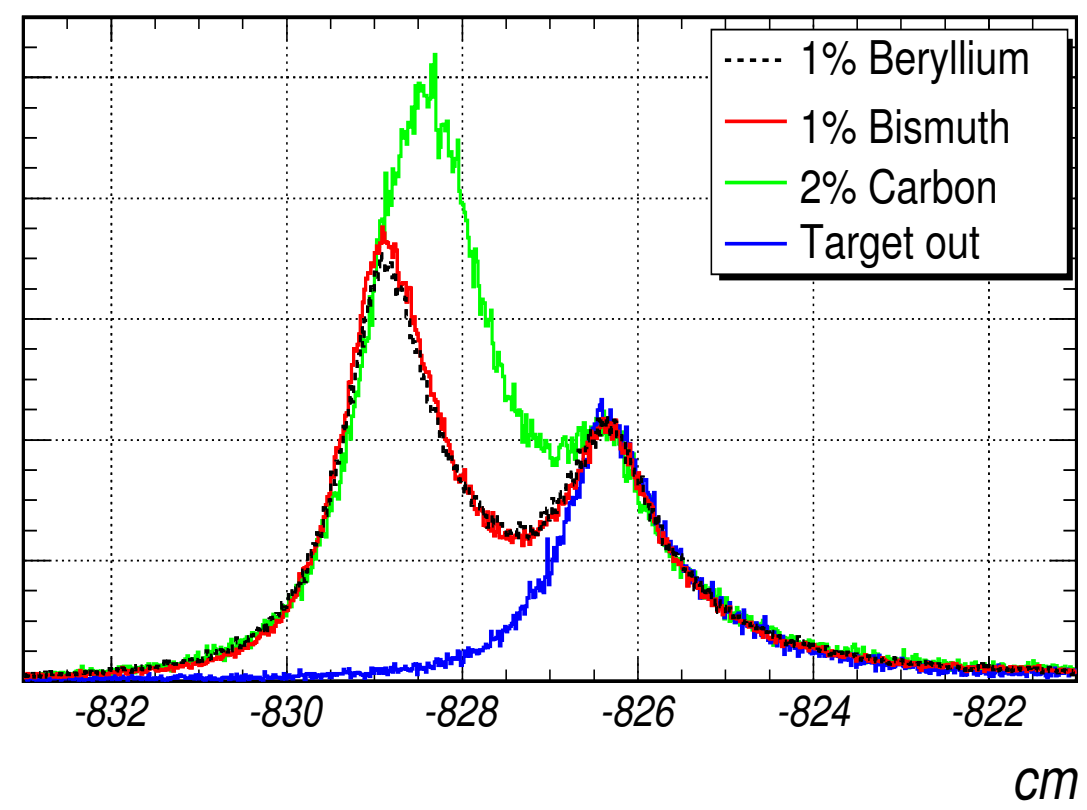

Figure 2.17: The distribution of the fitted $\mathrm{Z}$ vertex from three different targets i.e. carbon, beryllium and bismuth, and the target-out configuration. The histograms are normalized to have the same scintillator peak height. 


\section{Chapter 3}

\section{Event Selection and Monte Carlo Corrections}

This chapter describes the event and track selection criteria which are applied in order to obtain a clean sample of events and tracks for which inelastic cross section and inclusive pion production measurements are done. The beam flux measurements and Monte Carlo corrections are also described.

\subsection{Cross section Terminology}

Cross section represents the probability of an interaction between a beam particle and a target.

In $\mathrm{p}+\mathrm{p}$ interaction the total cross section $\sigma_{t o t}$ is given by the sum:

$$
\sigma_{t o t}=\sigma_{e l}+\sigma_{\text {inel }}
$$

$\sigma_{e l}$ is the total elastic cross section where projectile and target particles are emitted without any change in their c.m.s energies and quantum numbers but in different directions, and $\sigma_{\text {inel }}$ is the total inelastic cross section where projectile and/or target particles are emitted with different momentum and/or quantum numbers.

In $\mathrm{p}+\mathrm{A}$ interaction $\sigma_{t o t}$ is defined as:

$$
\sigma_{\text {tot }}=\sigma_{e l}+\sigma_{\text {inel }}+\sigma_{\text {quasi }}
$$

Here in contrast to $\mathrm{p}+\mathrm{p}$ an additional member appears $\sigma_{\text {quasi }}$, corresponding to quasi-elastic scattering which is a subset of the inelastic cross section. It corresponds to scattering of 
incoming proton on individual nucleons in the nucleus as $\sigma_{e l}$ is the scattering of the proton from the whole nucleus. The $\mathrm{p}+\mathrm{C}$ total inelastic cross section was measured in several experiments [89, 90, 91], however in most of the cases $\sigma_{\text {quasi }}$ was not taken into account. In the MIPP measurement, the $\sigma_{\text {quasi }}$ part is taken into account in the calculation of inelastic cross section.

In case of inclusive particle production cross section, the production rate of some final state particles is determined. We are measuring the inclusive charged pion production cross sections for $\mathrm{p}+\mathrm{C}$ interactions i.e.

$$
\begin{aligned}
& p+C \rightarrow \pi^{+}+X, \\
& p+C \rightarrow \pi^{-}+X .
\end{aligned}
$$

\subsection{Event selection requirements}

A sample of "good" events is selected which means that each event should pass some requirements [92]. The selection creteria for both the liquid hydrogen $\left(\mathrm{LH}_{2}\right)$ and thin targets is described below. Only one thin target i.e. carbon (C) is discussed here, selection creteria for other thin targets i.e. beryllium (Be) and bismuth (Bi) is the same as that for carbon.

\subsubsection{Good beam event selection}

"Good" beam events are selected using the following requirements:

- Selection of events with single beam track

- Selection of events having $\leq 30$ tracks

- Selection of events with $\Sigma n$ BeamCrossings $\leq 4$ to reject unreconstructed second beam track

- Cut on beam track time

- Cut on transverse beam position

\section{Selection of events with single beam track}

There must be only one beam track incident on the target so that the initial state is well determined. The events with single beam track are selected. 


\section{Selection of events having $\leq 30$ tracks}

To select clean events, the multiplicity per event should be $\leq 30$. Figures 3.1(a) and 3.1(b) show the multiplicity distributions for 58 and $85 \mathrm{GeV} / \mathrm{c} \mathrm{p}+\mathrm{p}$ interactions, and figures 3.2(a) and $3.2(\mathrm{~b})$ show these distributions for 58 and $120 \mathrm{GeV} / \mathrm{c} \mathrm{p}+\mathrm{C}$ interactions. The red line shows the cut used i.e. the events in the region after red line are rejected. In these figures, the "+" sign before the beam momenta of 58 and $85 \mathrm{GeV} / \mathrm{c}$ represents that we have used the positive (proton) beams. We have both positive as well as negative beams available at the secondary beam momenta, on the other hand, we only have positive beam at $120 \mathrm{GeV} / \mathrm{c}$ beam momentum i.e. primary proton beam as discussed in section 1.6. We will use this notation through out the thesis.

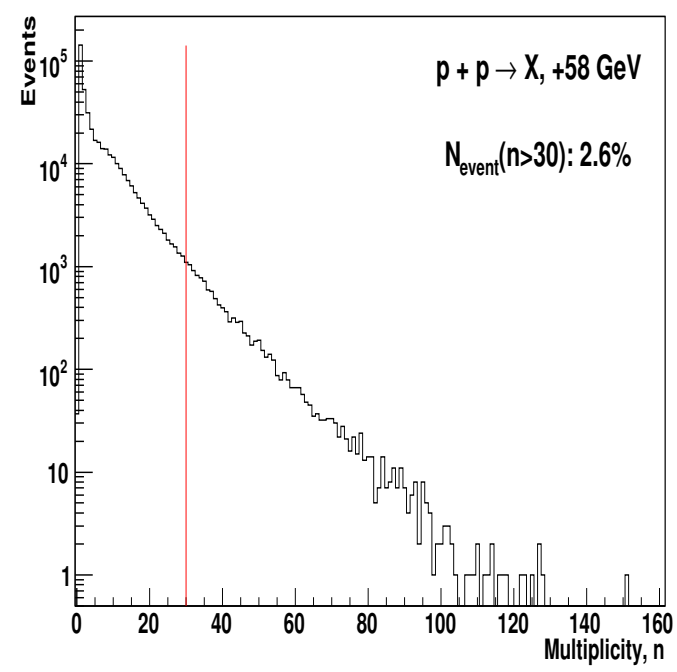

(a)

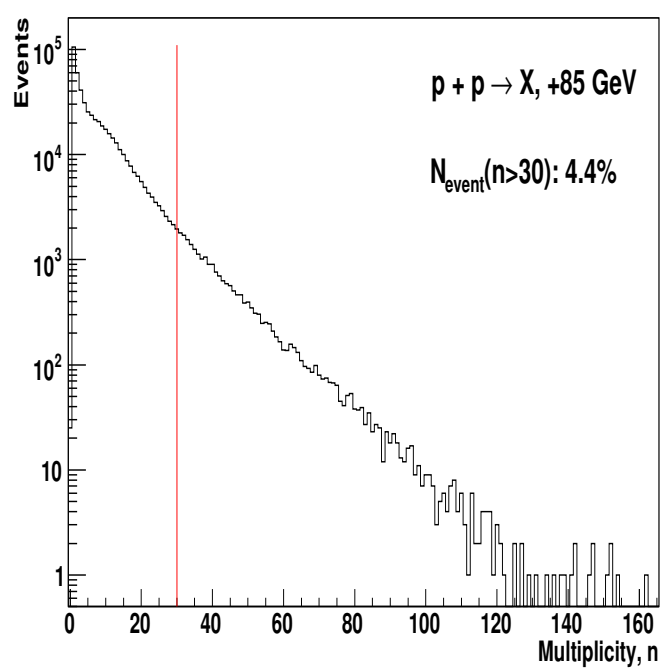

(b)

Figure 3.1: Multiplicity distribution for (a) 58 and (b) $85 \mathrm{GeV} / \mathrm{c} \mathrm{p}+\mathrm{p}$ interactions. The red line shows the cut used i.e. the events in the region after red line are rejected.

\section{Cut on इnBeamCrossings}

Sum of number of beam crossings in the three beam chambers described in section 2.4 i.e. $\Sigma n$ BeamCrossings should be $\leq 4$. This cut is used to reject unreconstructed second beam track. 


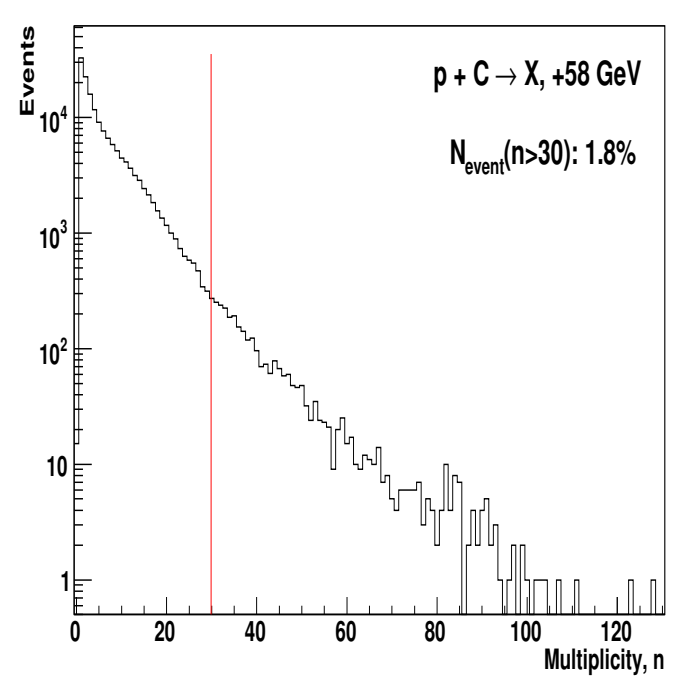

(a)

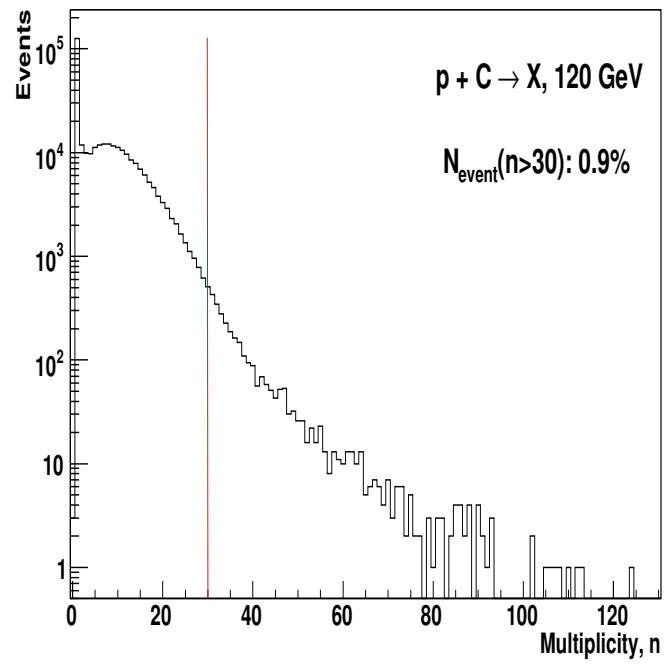

(b)

Figure 3.2: Multiplicity distribution for (a) 58 and (b) $120 \mathrm{GeV} / \mathrm{c} \mathrm{p}+\mathrm{C}$ interactions. The red line shows the cut used i.e. the events in the region after red line are rejected.

\section{Beam track time cut}

Beam track time distributions for 58 and $85 \mathrm{GeV} / \mathrm{c}$ proton interactions with $\mathrm{LH}_{2}$ and empty cryogenic targets described in section 2.7.2 are shown in figures 3.3(a) and 3.3(b), and distributions for 58 and $120 \mathrm{GeV} / \mathrm{c}$ proton interactions with carbon and empty targets described in section 2.7.1 are shown in figures $3.4(\mathrm{a})$ and 3.4(b). The plots clearly show the presence of buckets having separation of $19 \mathrm{~ns}$ and half width of $9.5 \mathrm{~ns}$. The buckets closest to the central bucket are at -12 ns and at 24 ns. Hence we set the beam track time cut between -2 ns and 15 ns.

\section{Transverse beam position cut}

The $\mathrm{LH}_{2}$ target is in the form of cylinderical tube which is surrounded by transport pipes and aluminium ring which supports the tube. Transverse beam position cut is made to make sure that the beam spot is consistent with the target dimensions so that the interactions are within the target only. The empty cryogenic target data is used to make this cut.

Figure 3.5 shows the distribution of longitudinal $(\mathrm{Z})$ vertex position with respect to target center for empty cryogenic target using pion, kaon and proton interaction triggers described in section 2.8.4 at all available energies i.e. $+20,-20,+58,-58,+85$ and $-85 \mathrm{GeV}$. The peaks in the $\mathrm{Z}$ regions $-80 \mathrm{~cm}<Z_{v t x}-Z_{t g t}<-60 \mathrm{~cm},-8 \mathrm{~cm}<Z_{v t x}-Z_{t g t}<3 \mathrm{~cm}$ and 3 


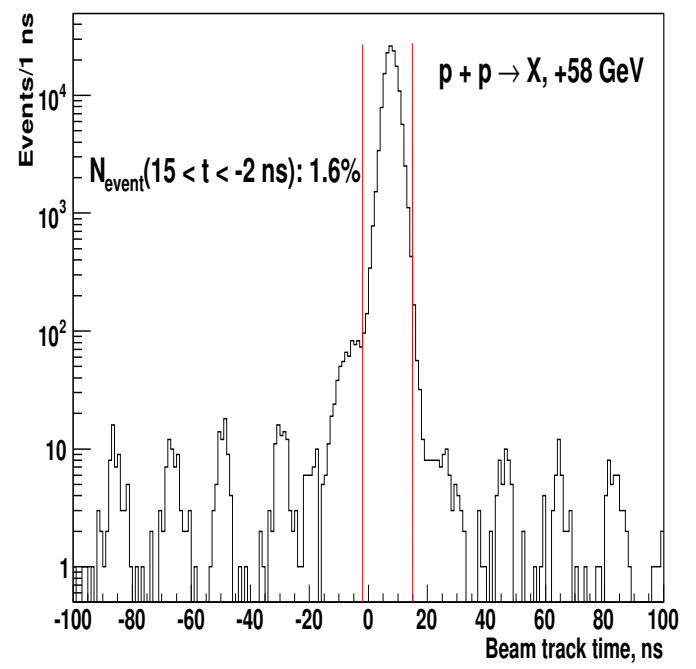

(a)

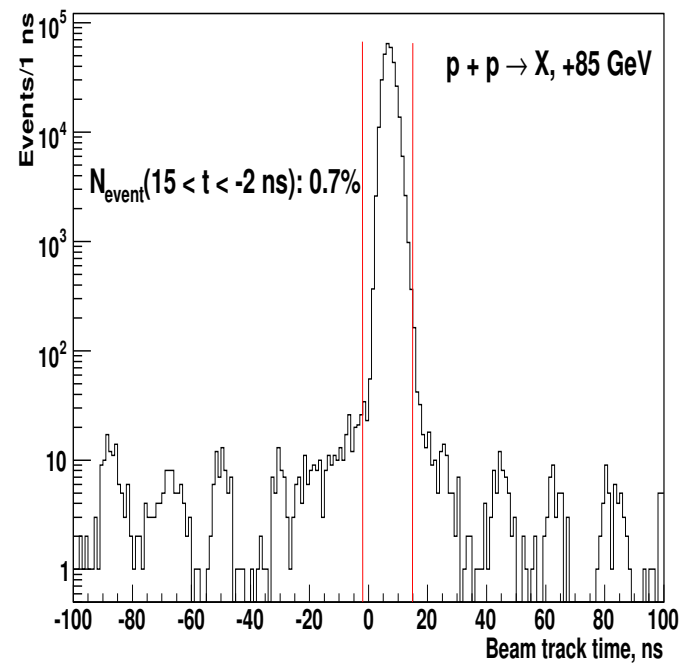

(b)

Figure 3.3: The beam track time distribution for (a) 58 and (b) $85 \mathrm{GeV} / \mathrm{c}$ proton incident on $\mathrm{LH}_{2}$ and empty cryogenic targets. The red lines show the selected beam track time cut i.e. $-2<t<15$ ns.

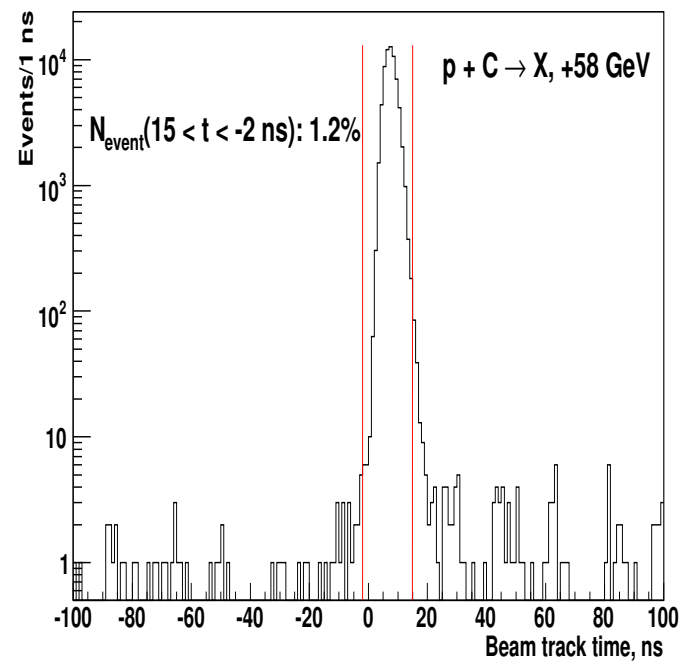

(a)

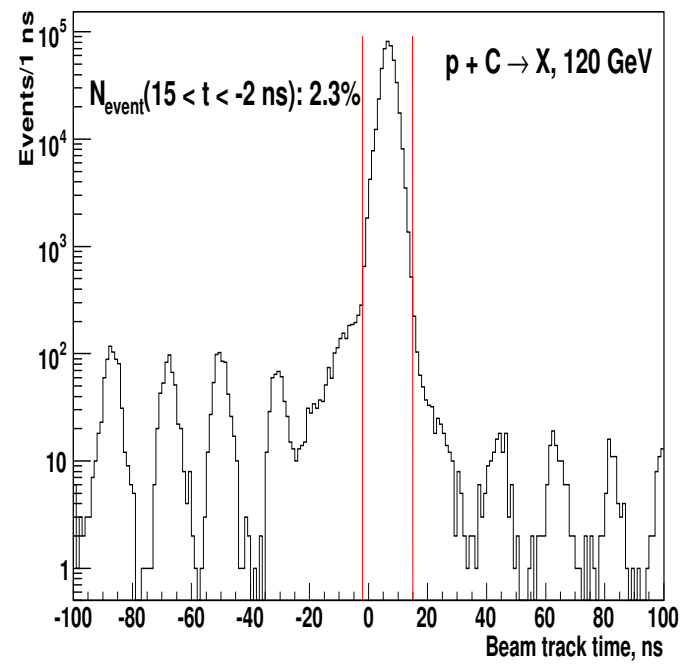

(b)

Figure 3.4: The beam track time distribution for (a) 58 and (b) $120 \mathrm{GeV} / \mathrm{c}$ proton incident on carbon and empty targets. The red lines show the selected beam track time cut i.e. $-2<$ $t<15 \mathrm{~ns}$. 
$\mathrm{cm}<Z_{v t x}-Z_{t g t}<10 \mathrm{~cm}$ are due to the interactions with $\mathrm{LH}_{2}$ transport pipes, aluminium ring, and scintillator which is placed just $\sim 1.5 \mathrm{~cm}$ downstream of the target, respectively.

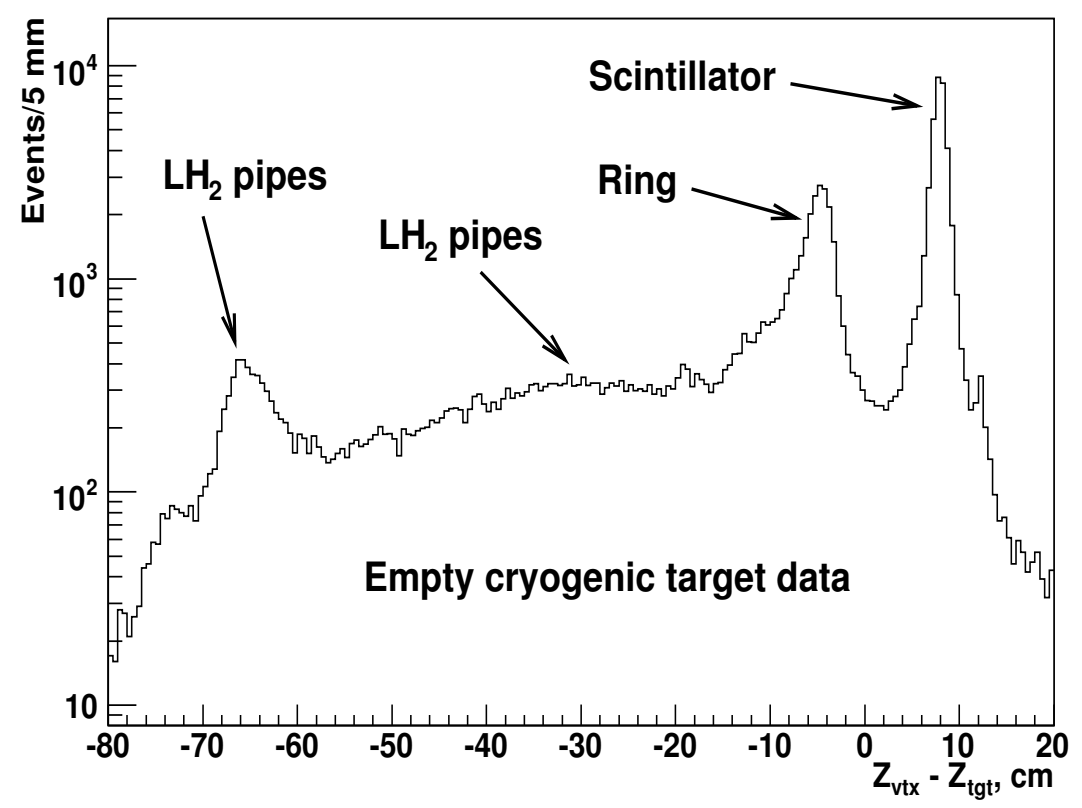

Figure 3.5: The longitudinal vertex distribution for empty cryogenic target using pion, kaon and proton interaction triggers at all available energies $+20,-20,+58,-58,+85$ and $-85 \mathrm{GeV}$.

Figure 3.6 shows the distributions of the transverse vertex position with respect to target center i.e. $\mathrm{Y}_{v t x}-\mathrm{Y}_{t g t}$ vs $\mathrm{X}_{v t x}-\mathrm{X}_{t g t}$ for empty cryogenic target using pion, kaon and proton interaction triggers at all available energies i.e. $+20,-20,+58,-58,+85$ and -85 $\mathrm{GeV}$. Figure 3.6(a) shows the $\mathrm{Z}$ vertex region $-8 \mathrm{~cm}<Z_{v t x}-Z_{t g t}<3 \mathrm{~cm}$ and indicates the interactions with the supporting aluminium ring. The red ellipse shows the interactions within the $\mathrm{LH}_{2}$ volume. Figure 3.6(b) shows the $\mathrm{Z}$ vertex region $-80 \mathrm{~cm}<Z_{v t x}-Z_{t g t}<$ $-60 \mathrm{~cm}$ and indicates that the interactions with $\mathrm{LH}_{2}$ transport pipes are entering the $\mathrm{LH}_{2}$ volume. These interactions are shown in the region common to the red and blue ellipses. We select the transverse beam position cut by selecting the interactions within the red ellipse and subtracting the region common to the red and blue ellipses from the red ellipse i.e. the interactions with the $\mathrm{LH}_{2}$ ring and pipes are rejected.

Figures 3.7(a) and 3.7(b) show the distributions of the transverse beam position with respect to target center i.e. $\mathrm{Y}_{\text {beam }}-\mathrm{Y}_{\text {tgt }} \mathrm{vs} \mathrm{X}_{\text {beam }}-\mathrm{X}_{\text {tgt }}$ before and after applying the cut on transverse beam position for $\mathrm{p}+\mathrm{p}$ interactions at $58 \mathrm{GeV} / \mathrm{c}$. The figure 3.7(b) shows that the beam spot is consistent with the target dimensions (section 2.7).

Unlike $\mathrm{LH}_{2}$ target, carbon target is in the form of a thin disc. For the cut on the 


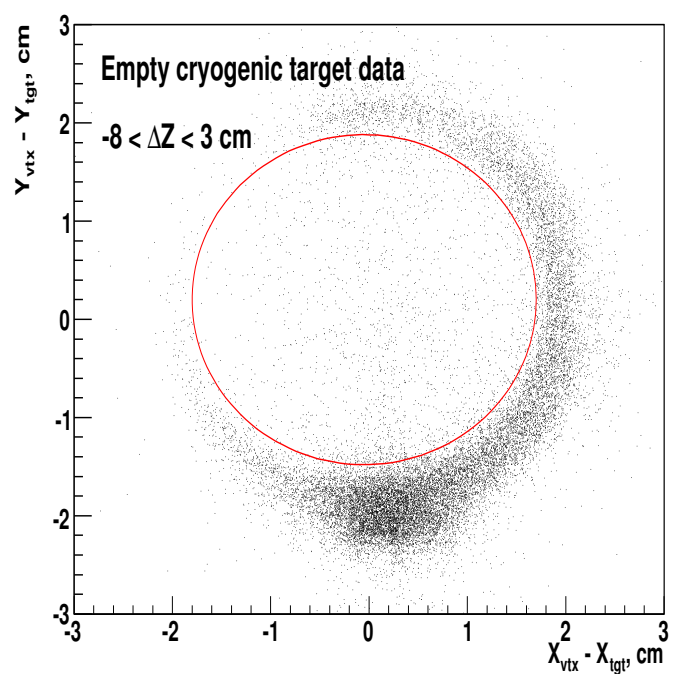

(a)

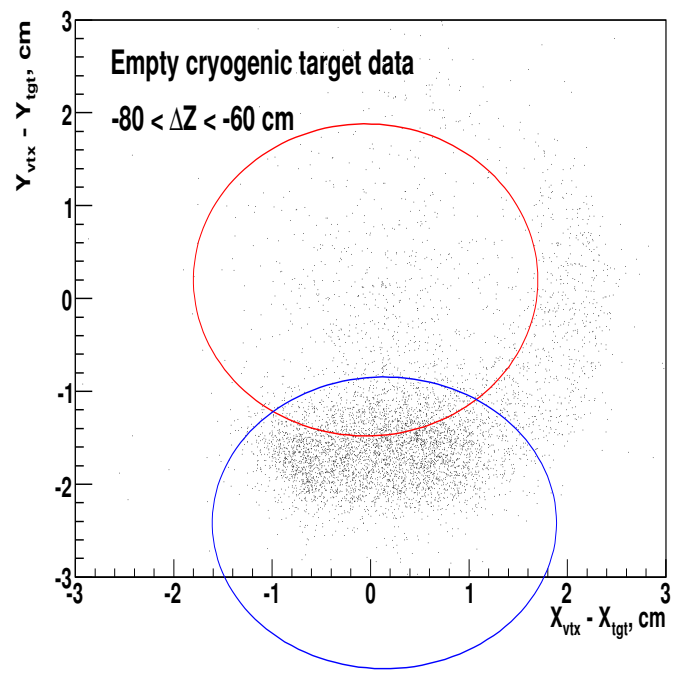

(b)

Figure 3.6: The distribution of the transverse vertex position with respect to target center i.e. $\mathrm{Y}_{v t x}-\mathrm{Y}_{t g t}$ vs $\mathrm{X}_{v t x}-\mathrm{X}_{t g t}$ for empty cryogenic target (a) showing the $\mathrm{Z}$ vertex region: -8 $\mathrm{cm}<Z_{v t x}-Z_{t g t}<3 \mathrm{~cm}$, and (b) showing the $\mathrm{Z}$ vertex region: $-80 \mathrm{~cm}<Z_{v t x}-Z_{t g t}<-60$ $\mathrm{cm}$.

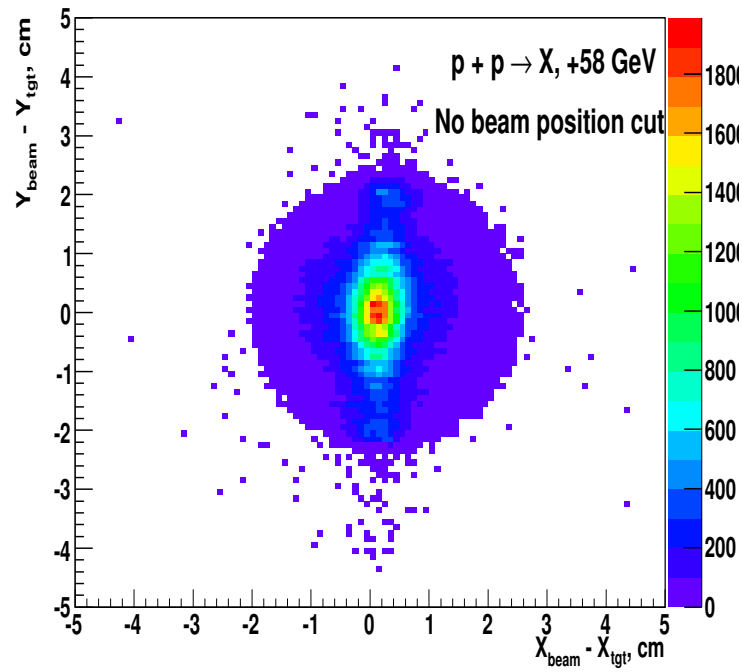

(a)

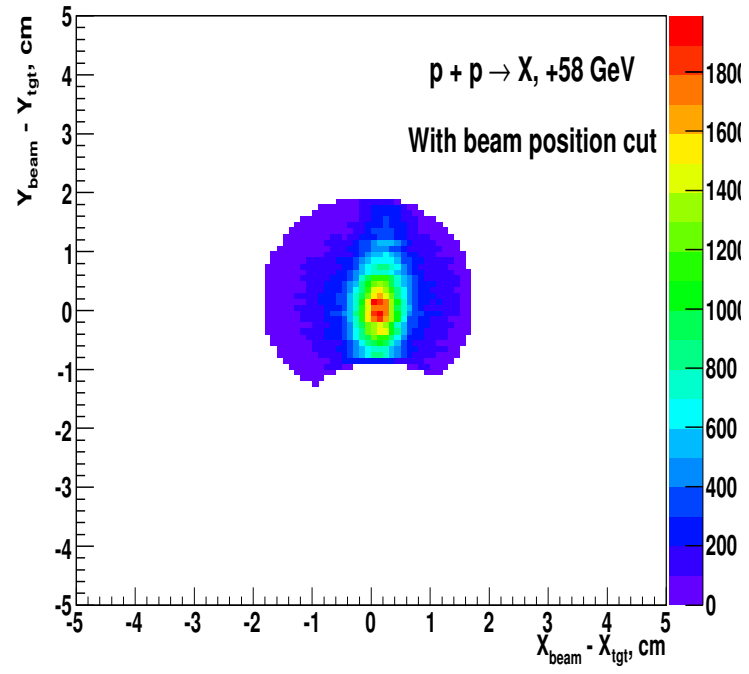

(b)

Figure 3.7: The distribution of the transverse beam position with respect to target center i.e. $\mathrm{Y}_{\text {beam }}-\mathrm{Y}_{\text {tgt }}$ vs $\mathrm{X}_{\text {beam }}-\mathrm{X}_{\text {tgt }}$ (a) before and (b) after applying the cut on transverse beam position for $\mathrm{p}+\mathrm{p}$ interactions at $58 \mathrm{GeV} / \mathrm{c}$. 
transverse beam position for carbon target, we select a cut on beam radius. The beam radius should be $\leq 2 \mathrm{~cm}$ and $\leq 0.8 \mathrm{~cm}$, in case of 58 and $120 \mathrm{GeV} / \mathrm{c}$ beams, respectively.

The distributions of the beam positions with respect to target center in $\mathrm{X}$ and $\mathrm{Y}$ direction, and the beam radius for 58 and $120 \mathrm{GeV} / \mathrm{c} \mathrm{p}+\mathrm{C}$ interactions are shown in figures $3.8,3.9$ and 3.10 , respectively.

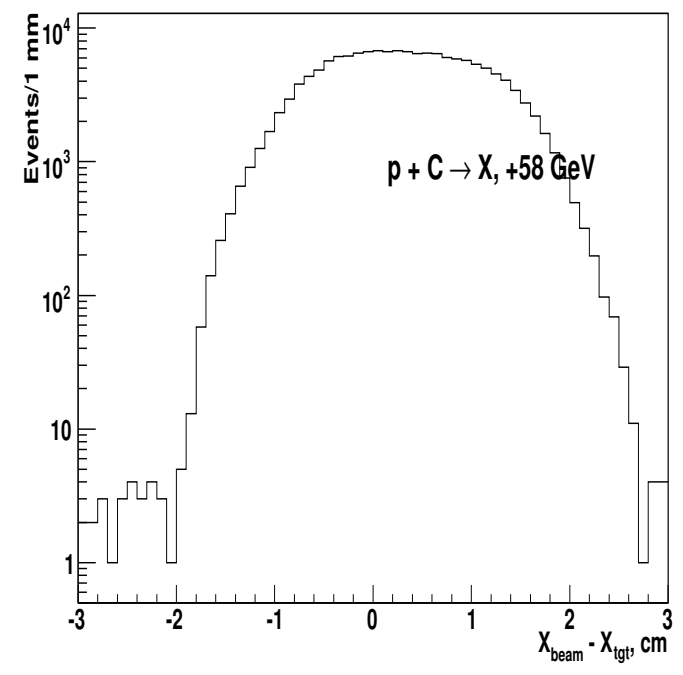

(a)

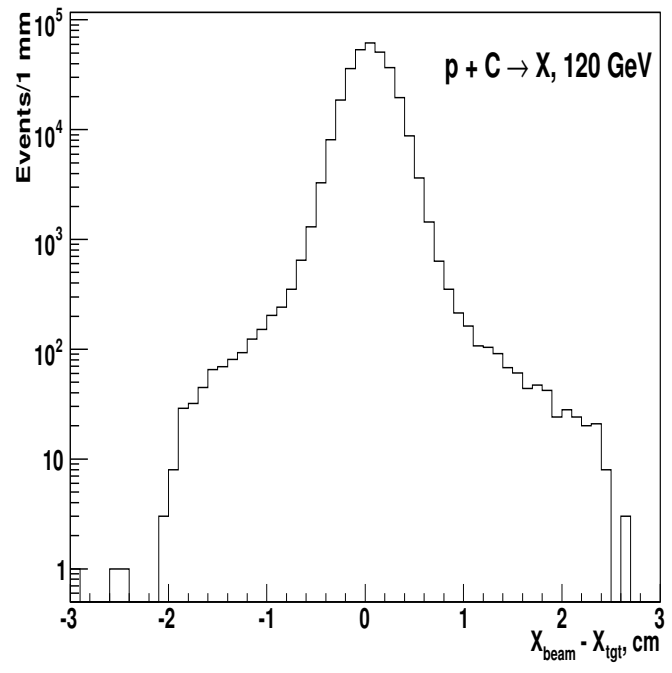

(b)

Figure 3.8: The beam position with respect to target center in $\mathrm{X}$ direction for (a) 58 and (b) $120 \mathrm{GeV} / \mathrm{c} \mathrm{p}+\mathrm{C}$ interactions.

Figures 3.11 and 3.12 show the transverse beam position distribution i.e. $\mathrm{Y}_{\text {beam }}-\mathrm{Y}_{\text {tgt }}$ vs $\mathrm{X}_{\text {beam }}-\mathrm{X}_{\text {tgt }}$ before and after applying the cut on beam radius for $\mathrm{p}+\mathrm{C}$ interactions at 58 and $120 \mathrm{GeV} / \mathrm{c}$, respectively. The figures 3.11(b) and 3.12(b) show that the beam spot is consistent with the target dimensions (section 2.7) after applying the beam radius cut. The $120 \mathrm{GeV} / \mathrm{c}$ beam is narrower as compared to $58 \mathrm{GeV} / \mathrm{c}$ beam. The beam spot is circular in shape for carbon target unlike $\mathrm{LH}_{2}$ target where the beam spot is elliptical.

\subsubsection{Good event selection}

The interactions are selected by requiring interaction trigger described in section 2.8.4. $80 \%$ of the MIPP data were collected with the interaction trigger required. This set of the data is used for this analysis. The interaction trigger used in MIPP is scintillator-based. There should be at least 3 charged tracks for the scintillator to fire i.e. for the trigger to be ON. Taking account of MIPP DAQ rate (section 2.9), the rate at which beam particles and 


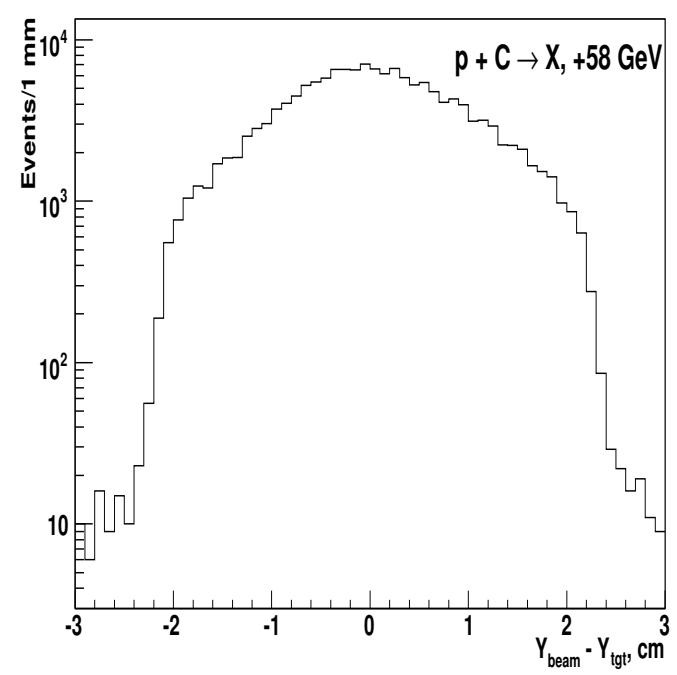

(a)

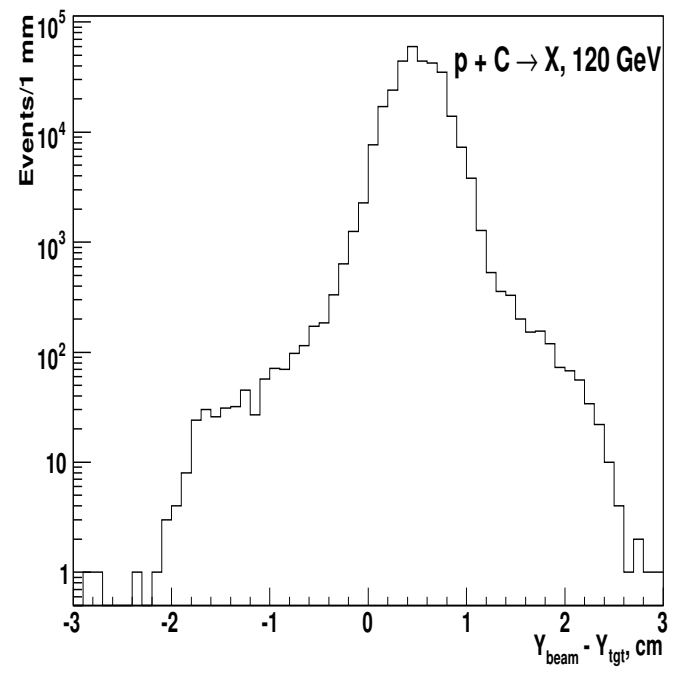

(b)

Figure 3.9: The beam position with respect to target center in $\mathrm{Y}$ direction for (a) 58 and (b) $120 \mathrm{GeV} / \mathrm{c} \mathrm{p}+\mathrm{C}$ interactions.

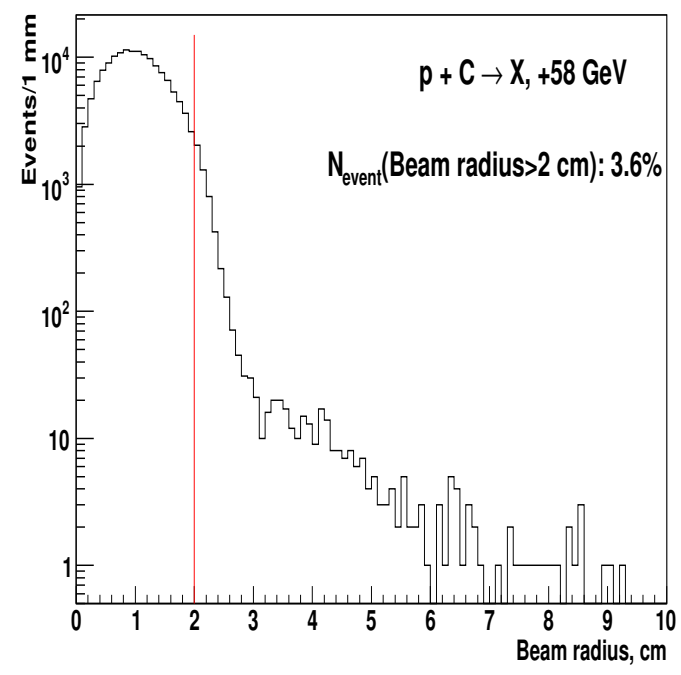

(a)

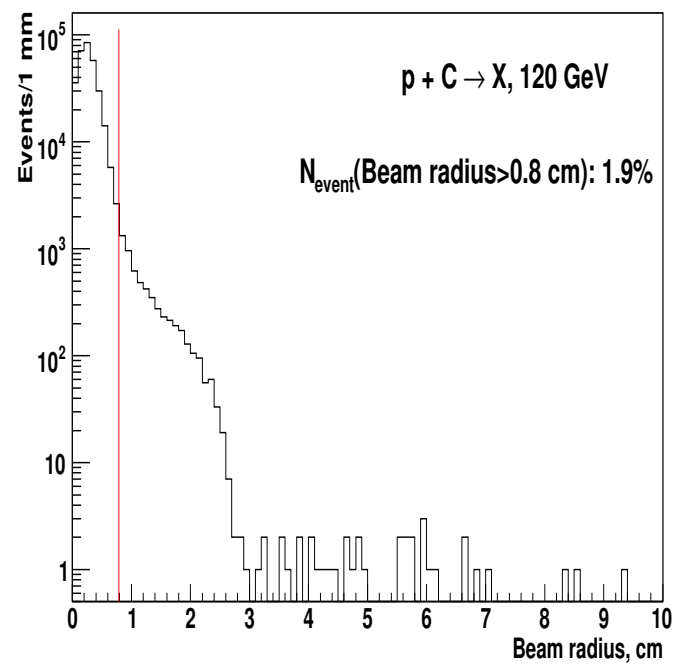

(b)

Figure 3.10: The beam radius distribution for (a) 58 and (b) $120 \mathrm{GeV} / \mathrm{c} \mathrm{p}+\mathrm{C}$ interactions. The red line shows the cut used i.e. the events in the region after red line are rejected. 


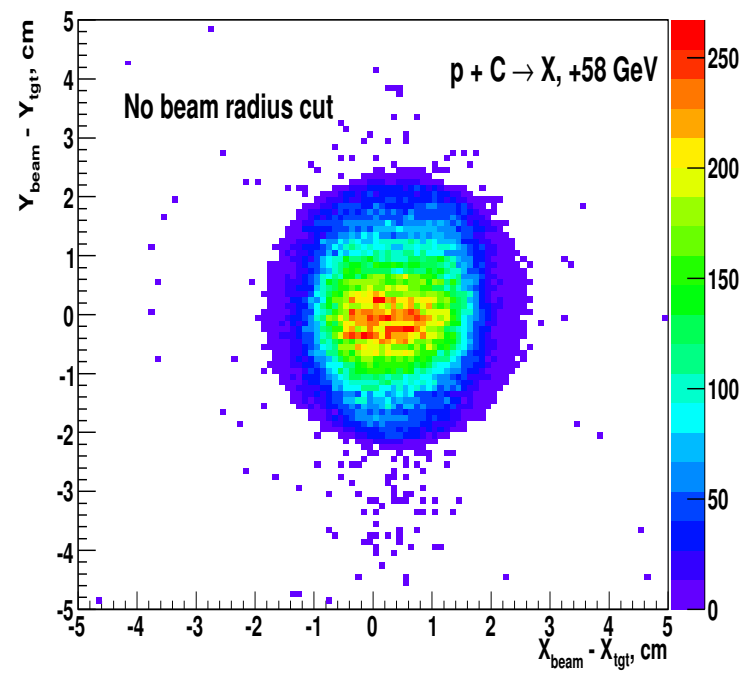

(a)

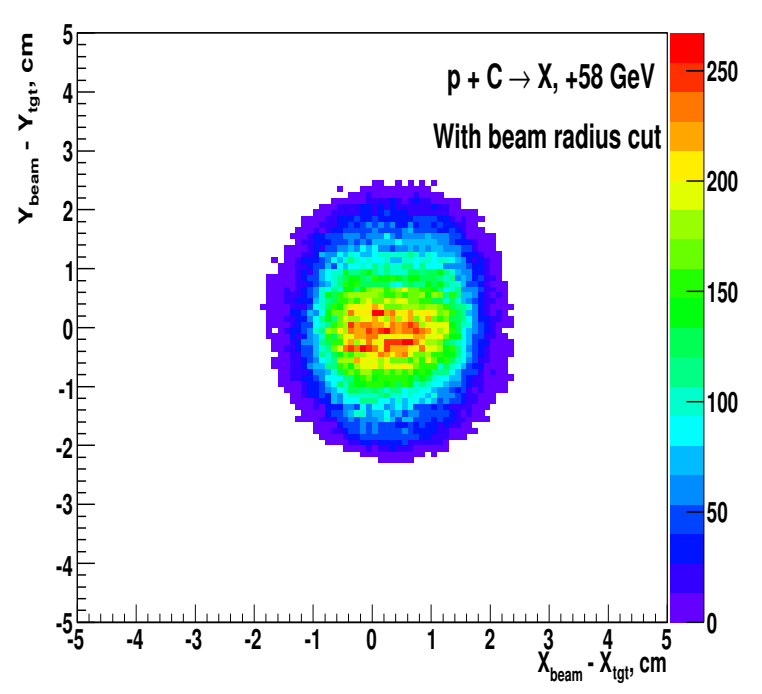

(b)

Figure 3.11: The transverse beam position distribution i.e. $\mathrm{Y}_{\text {beam }}-\mathrm{Y}_{\text {tgt }}$ vs $\mathrm{X}_{\text {beam }}-\mathrm{X}_{\text {tgt }}$ for $58 \mathrm{GeV} / \mathrm{c} \mathrm{p}+\mathrm{C}$ interactions (a) before and (b) after applying the cut on beam radius i.e. beam radius $\leq 2 \mathrm{~cm}$.

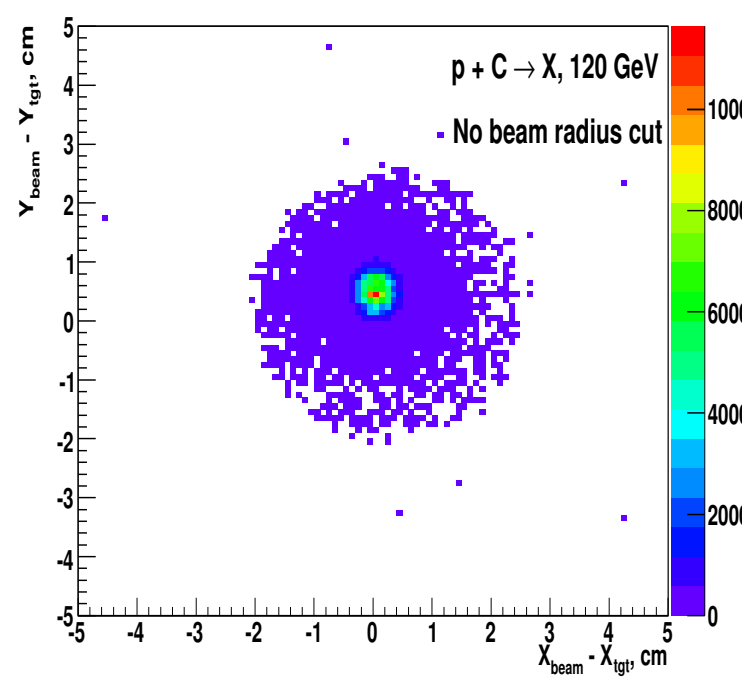

(a)

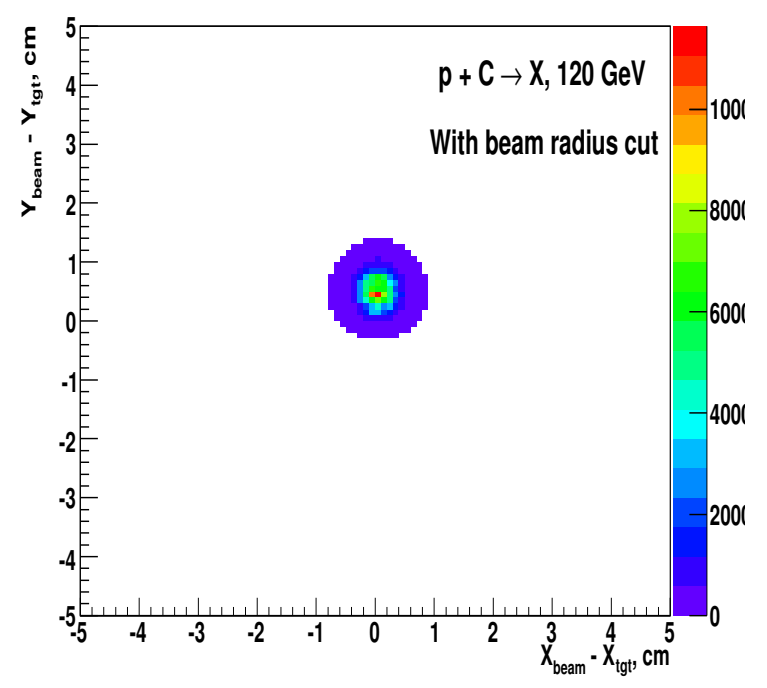

(b)

Figure 3.12: The transverse beam position distribution i.e. $\mathrm{Y}_{\text {beam }}-\mathrm{Y}_{\text {tgt }}$ vs $\mathrm{X}_{\text {beam }}-\mathrm{X}_{\text {tgt }}$ for $120 \mathrm{GeV} / \mathrm{c} \mathrm{p}+\mathrm{C}$ interactions (a) before and (b) after applying the cut on beam radius i.e. beam radius $\leq 0.8 \mathrm{~cm}$. 
interactions were recorded had to be prescaled (suppressed). So both the number of beam particles as well as interactions are scaled by the run-dependent prescale factor that was set during data taking.

Events with proton interaction trigger are selected. Primary interactions are selected by requiring primary vertices which are distinguished from the secondary vertices on the basis of indices of the vertices which are stored for each event.

For the selection of "good" events, following cuts are made:

\section{Cut on beam track index}

The beam track index should be $\geq 0$ to make sure that the selected vertex has an incoming beam track associated with it.

\section{Cut on sum of momenta of tracks in an event}

Figures 3.13(a) and 3.13(b) show the distributions of sum of momenta of charged tracks (emanating from primary vertex) in an event for 58 and $85 \mathrm{GeV} / \mathrm{c}$ proton interactions with $\mathrm{LH}_{2}$ and empty cryogenic targets, and figures 3.14(a) and 3.14(b) show these distributions for 58 and $120 \mathrm{GeV} / \mathrm{c}$ proton interactions with carbon and empty targets. From the plots it is clear that there is a small fraction of the events in which the sum of momenta of tracks is significantly higher than the beam momentum. These events are rejected. The red line shows the cut i.e. the events in the region after red line are rejected. The track momentum resolution is taken into account in making the cut.

\section{$\Delta p_{T}$ cut to reject straight through tracks}

To reject the straight through tracks i.e. uninteracted beam particles, we select a cut - the difference of vector sum of $\mathrm{p}_{T}$ 's of all the charged tracks in an event and $\mathrm{p}_{T}$ of the beam track should be $\geq 150 \mathrm{MeV}$.

\section{Track multiplicity cut}

By requiring interaction trigger which requires at least 3 charged tracks for the trigger to be ON, it should reject almost all the elastics but to make sure that we select inelastic events only, we select a cut - the track multiplicity per event after making all the track selection cuts (explained later in this section) should be $\geq 3$ i.e. rejection of one-prong and two-prong events. If we make this cut, we need to correct for any inelastic events rejected by this cut. 


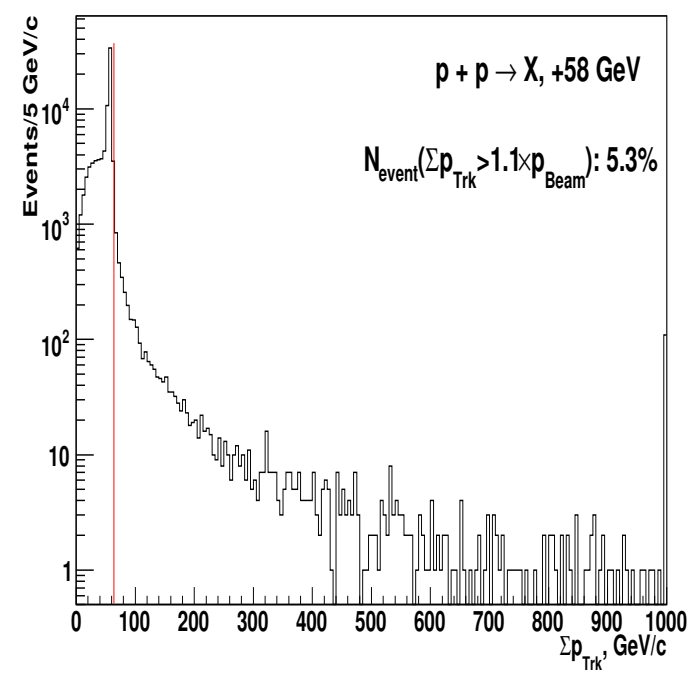

(a)

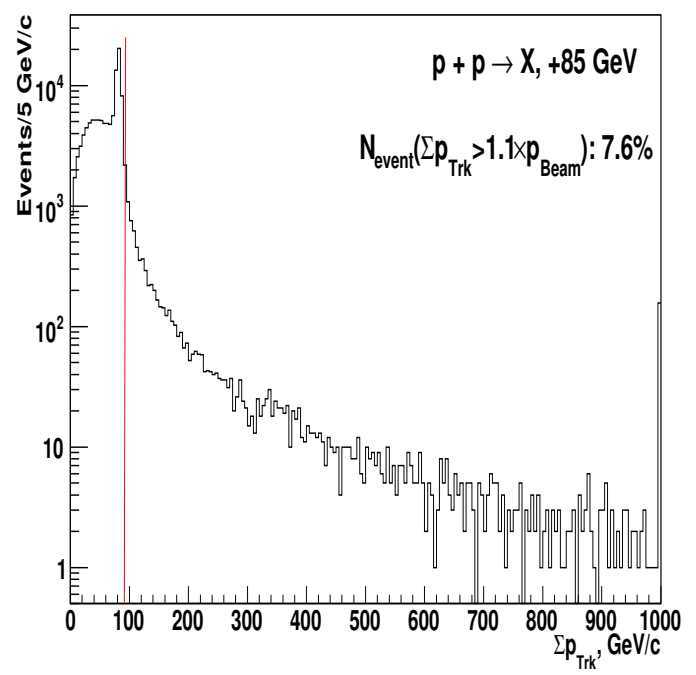

(b)

Figure 3.13: The distribution of sum of momenta of the charged tracks in an event for (a) 58 and (b) $85 \mathrm{GeV} / \mathrm{c}$ proton incident on $\mathrm{LH}_{2}$ and empty cryogenic targets.

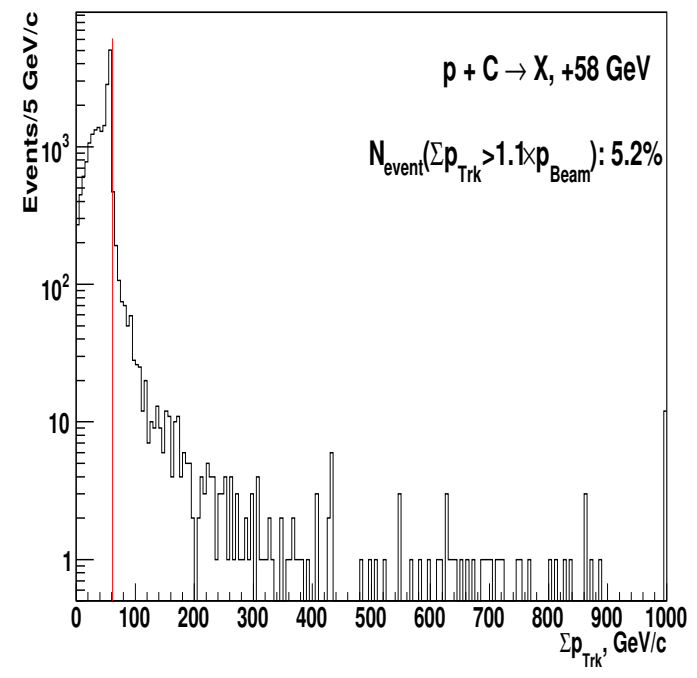

(a)

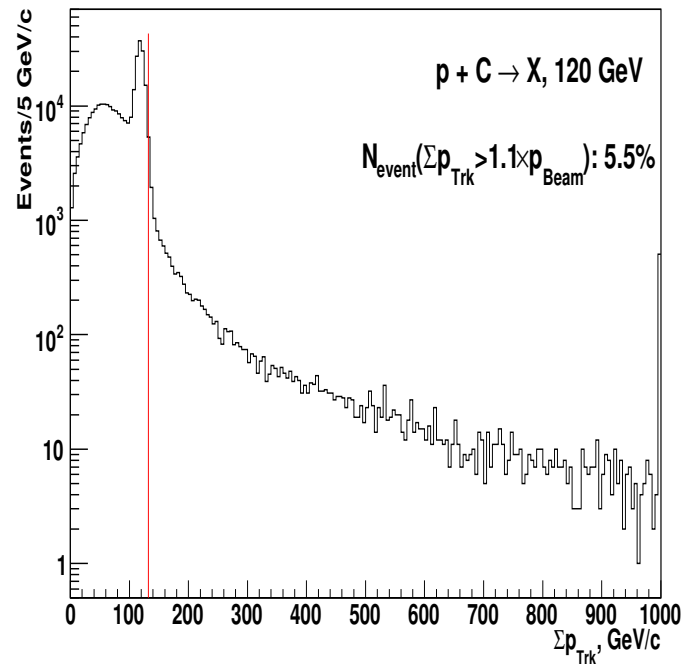

(b)

Figure 3.14: The distribution of sum of momenta of the charged tracks in an event for (a) 58 and (b) $120 \mathrm{GeV} / \mathrm{c}$ proton incident on carbon and empty targets. 


\section{Vertex Selection}

The cuts on both the transverse and longitudinal vertex positions are explained below.

\section{Cut on transverse vertex position:}

The cut on transverse vertex position for the $\mathrm{LH}_{2}$ target is selected in the same way as the cut on the transverse beam position which was described in the section 3.2.1 (see figure 3.6). The only difference is that the contribution from the blue ellipse is not subtracted from the red ellipse i.e. all the interactions within the red ellipse are selected.

For carbon target, the transverse vertex position cut is selected using a cut on vertex radius just like the transverse beam position cut is made using a cut on the beam radius. The vertex radius should be $\leq 2 \mathrm{~cm}$ so that the interactions are selected within the target only.

\section{Cut on longitudinal vertex position and empty target subtraction:}

Figures $3.15(\mathrm{a})$ and $3.15(\mathrm{~b})$ show the longitudinal vertex distributions for 58 and $85 \mathrm{GeV} / \mathrm{c}$ proton interactions with $\mathrm{LH}_{2}$ and empty cryogenic targets. The selected longitudinal vertex region i.e $-15 \mathrm{~cm}<Z_{v t x}-Z_{t g t}<15 \mathrm{~cm}$ is shown. The distribution for the empty cryogenic target is normalized to the same number of incident protons as those of $\mathrm{LH}_{2}$ target so that the interactions for the $\mathrm{LH}_{2}$ and empty cryogenic targets correspond to same number of incident protons. The peak in both the distributions at $\sim 7.5 \mathrm{~cm}$ is due to the interactions with the scintillator where as the flat region in the $\mathrm{LH}_{2}$ distribution is due to the interactions with the target. To reject the interactions with the scintillator, empty target subtraction is done. Before subtraction, the distribution for the empty cryogenic target is scaled by an extra scaling factor to match the heights of the scintillator peaks for $\mathrm{LH}_{2}$ and empty cryogenic targets. The $\mathrm{Z}$ vertex distribution after subtracting the empty target contribution from the $\mathrm{LH}_{2}$ distribution is also shown in the plots.

Figures 3.16(a) and 3.16(b) show the longitudinal vertex distributions for 58 and 120 $\mathrm{GeV} / \mathrm{c}$ proton interactions with carbon and empty targets. The selected longitudinal vertex region i.e $-4 \mathrm{~cm}<Z_{v t x}-Z_{t g t}<6 \mathrm{~cm}$ is shown. There are two peaks in the distribution for carbon target, one is due to the interactions with the target and the other is due to the interactions with the scintillator. There is only one peak in the distribution for empty target as there is no target, and interactions are with the scintillator only. The empty target distribution is scaled by an extra scale factor just like it is done in the case of empty cryogenic target. The procedure for getting this scale factor is explained later in section 4.3 . 
The $\mathrm{Z}$ vertex distribution after subtracting the empty target contribution from the carbon distribution is also shown in the plots. The $\mathrm{Z}$ distribution for carbon target is narrower as compared to that of $\mathrm{LH}_{2}$ target which has a wider distribution.

\section{Effect of transverse beam and vertex position cuts on transverse vertex dis-}

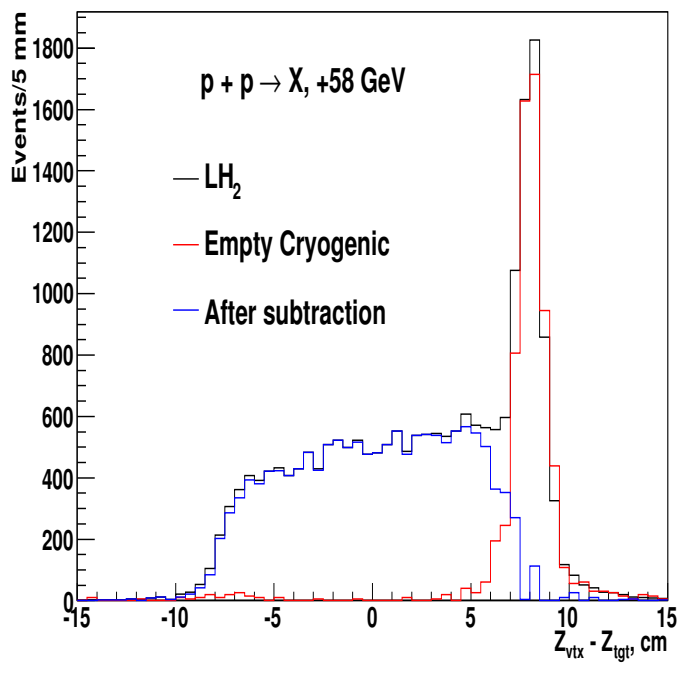

(a)

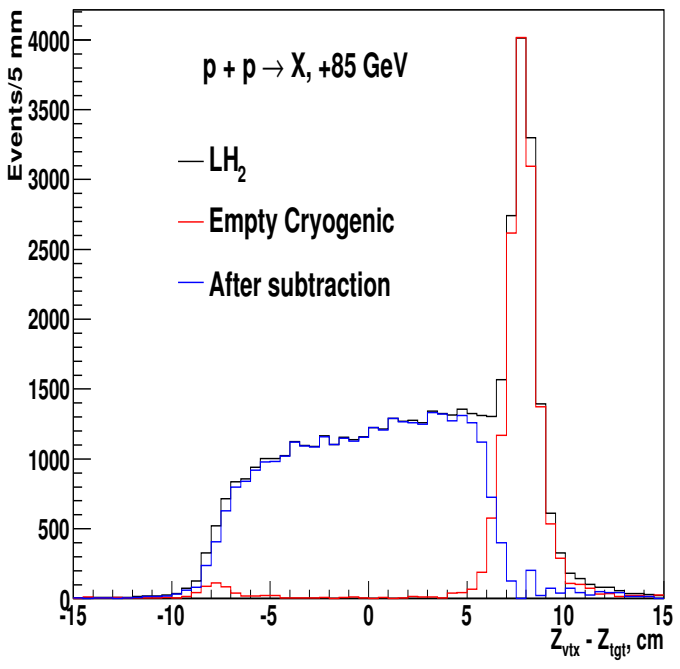

(b)

Figure 3.15: The longitudinal vertex distribution for (a) 58 and (b) $85 \mathrm{GeV} / \mathrm{c}$ proton interactions with $\mathrm{LH}_{2}$ (shown in black) and empty cryogenic (shown in red) targets. The blue distribution is after subtracting the empty target contribution.

tribution: Figures $3.17(\mathrm{a}), 3.17(\mathrm{~b})$ and $3.17(\mathrm{c})$ show the transverse vertex distributions i.e. $\mathrm{Y}_{v t x}-\mathrm{Y}_{t g t}$ vs $\mathrm{X}_{v t x}-\mathrm{X}_{t g t}$ for $58 \mathrm{GeV} / \mathrm{c}$ proton interactions with $\mathrm{LH}_{2}$ target without applying transverse beam and vertex position cuts, after applying transverse beam position cut, and after applying both the transverse beam as well as vertex position cuts, respectively.

Before applying transverse beam and vertex position cuts, the interactions occur outside the target region in both $\mathrm{X}$ and $\mathrm{Y}$ direction as shown in figure 3.17(a), The interactions in $-\mathrm{Y}$ region are due to the interactions with the $\mathrm{LH}_{2}$ pipes. After applying transverse beam position cut, the interactions in $-\mathrm{Y}$ region are mostly gone as shown in figure 3.17(b), The interactions from the other materials like $\mathrm{LH}_{2}$ ring etc. are also reduced. After applying both the transverse beam as well as vertex position cuts, the interactions lie in the region -2 to $2 \mathrm{~cm}$ in both $\mathrm{X}$ and $\mathrm{Y}$ direction as shown in figure 3.17(c). All the other interactions due to the $\mathrm{LH}_{2}$ supporting ring and transport pipes are gone after applying these cuts. 


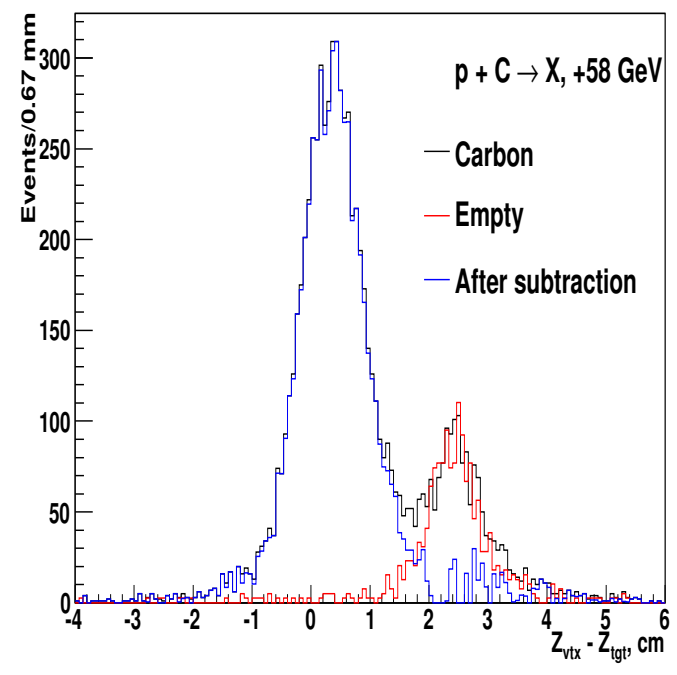

(a)

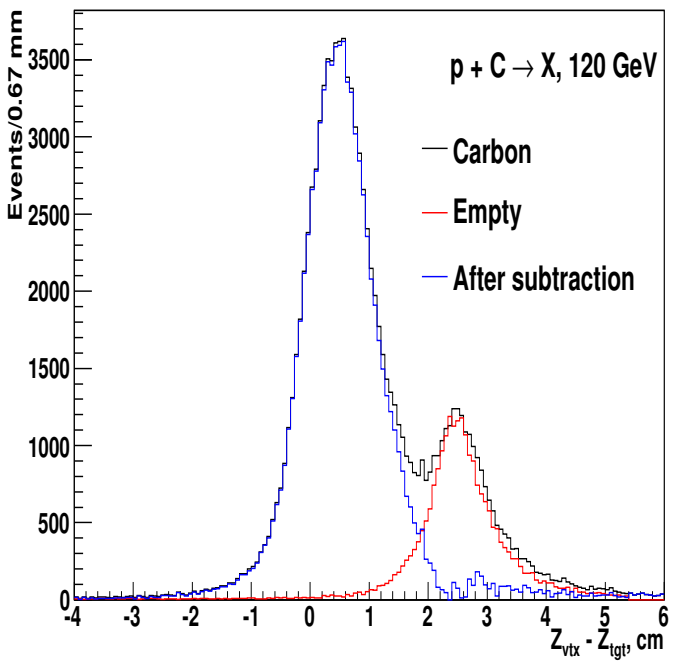

(b)

Figure 3.16: The longitudinal vertex distribution for (a) 58 and (b) $120 \mathrm{GeV} / \mathrm{c}$ proton interactions with carbon (shown in black) and empty (shown in red) targets. The blue distribution is after subtracting the empty target contribution.

\section{Effect of transverse beam and vertex position cuts on longitudinal vertex dis-}

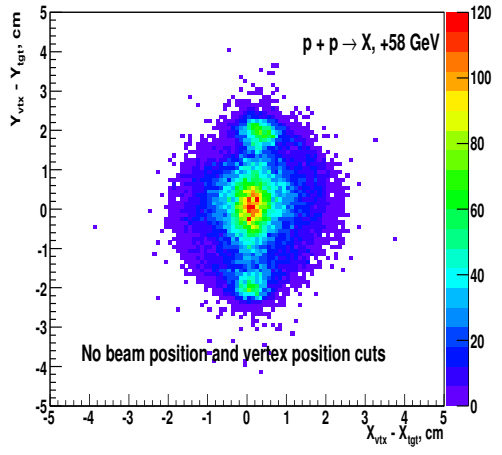

(a)

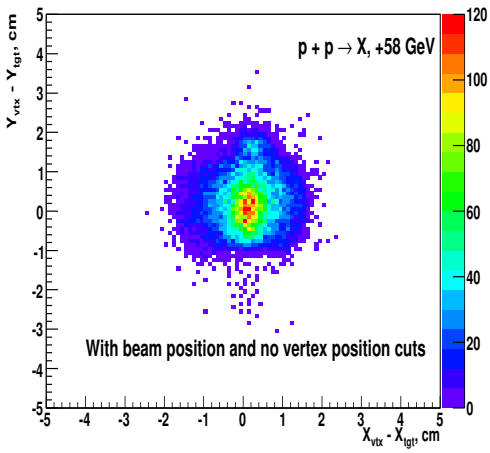

(b)

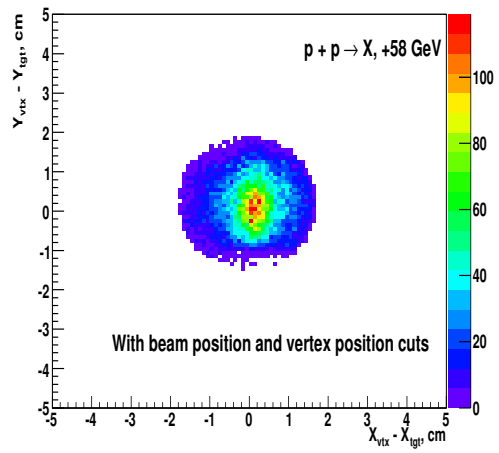

(c)

Figure 3.17: The transverse vertex distribution i.e. $\mathrm{Y}_{v t x}-\mathrm{Y}_{t g t}$ vs $\mathrm{X}_{v t x}-\mathrm{X}_{t g t}$ for $58 \mathrm{GeV} / \mathrm{c}$ $\mathrm{p}+\mathrm{p}$ interactions (a) without transverse beam and vertex position cuts, (b) after applying transverse beam position cut and (c) after applying both the transverse beam as well as vertex position cuts.

tribution: Figure 3.18 shows the longitudinal vertex distribution for $58 \mathrm{GeV} / \mathrm{c}$ proton interactions with $\mathrm{LH}_{2}$ target before and after applying the cuts on transverse beam and 
vertex positions. The peak in $\mathrm{Z}$ region due to the interactions with the $\mathrm{LH}_{2}$ supporting ring and transport pipes in black distribution is gone after applying the transverse beam and vertex position cuts as shown in red distribution.

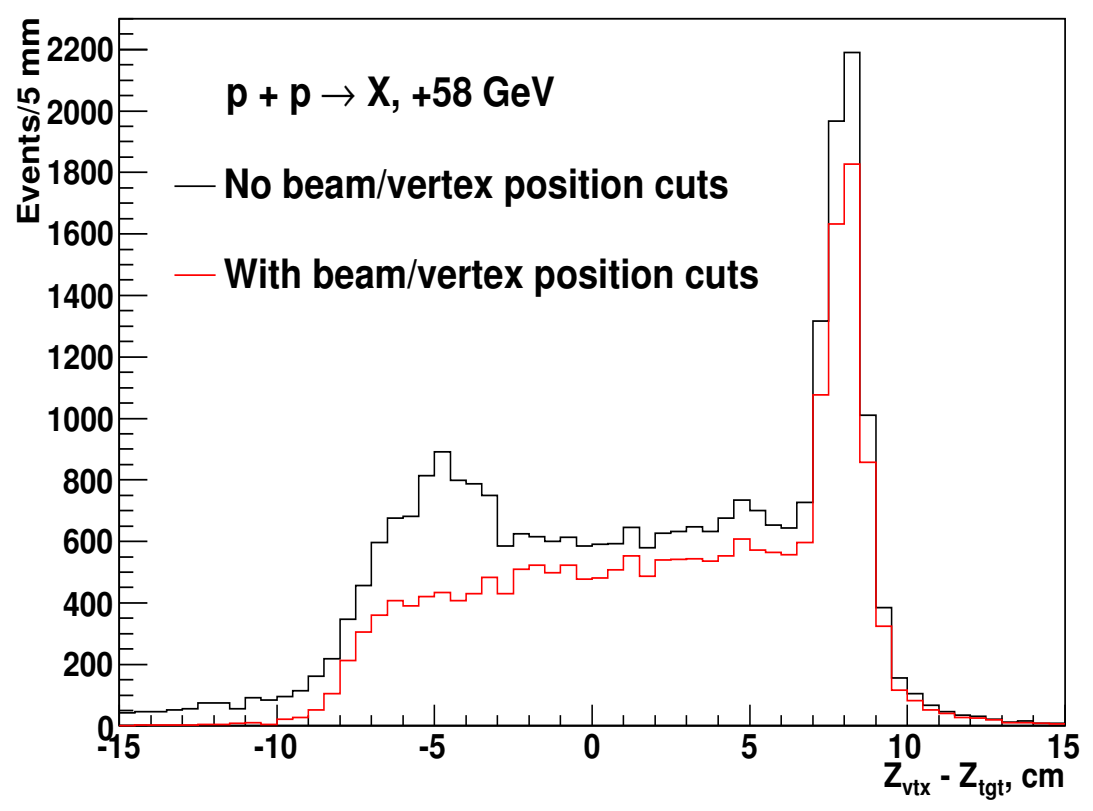

Figure 3.18: The longitudinal vertex distribution for $58 \mathrm{GeV} / \mathrm{c} \mathrm{p}+\mathrm{p}$ interactions without transverse beam and vertex position cuts (shown in black) and after applying transverse beam and vertex position cuts (shown in red).

\subsubsection{Good track selection}

"Good" tracks are selected by requiring the following cuts:

\section{Cut on number of TPC hits for a track}

Figures 3.19(a) and 3.19(b) show the distributions of number of TPC hits (ntpchit) per track for 58 and $85 \mathrm{GeV} / \mathrm{c}$ proton interactions with $\mathrm{LH}_{2}$ and empty cryogenic targets, and figures $3.20(\mathrm{a})$ and 3.20(b) show these distributions for 58 and $120 \mathrm{GeV} / \mathrm{c}$ proton interactions with carbon and empty targets. The selected cut is $20<$ ntpchit $<100$.

\section{Cut on momentum of tracks}

We reject the tracks having momentum less than $100 \mathrm{MeV}$ as the reconstruction is not good for low momentum tracks. 


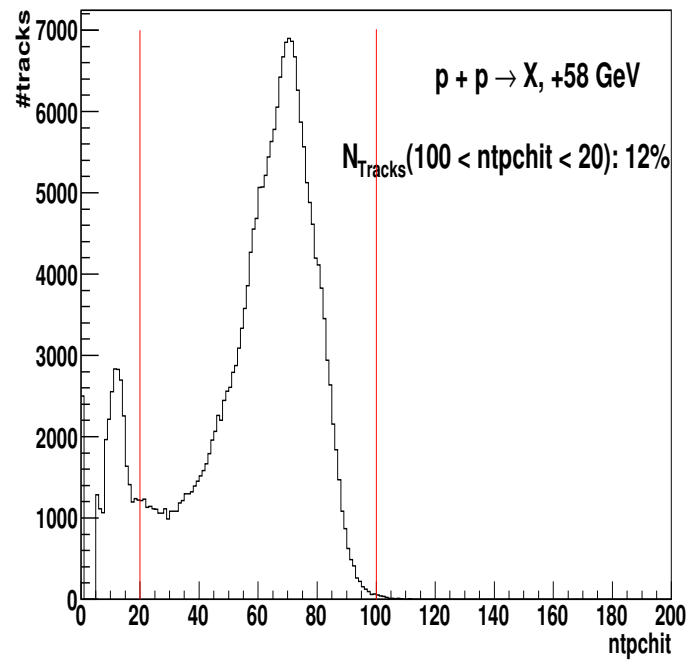

(a)

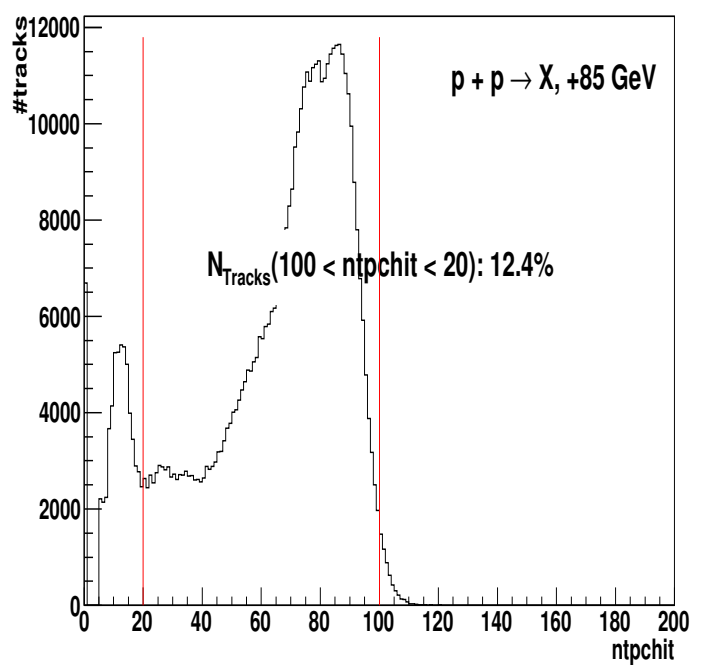

(b)

Figure 3.19: The distribution of number of TPC hits for a track for (a) 58 and (b) $85 \mathrm{GeV} / \mathrm{c}$ proton incident on $\mathrm{LH}_{2}$ and empty cryogenic targets. The red lines show the selected cut on the number of TPC hits i.e $20<$ ntpchit $<100$.

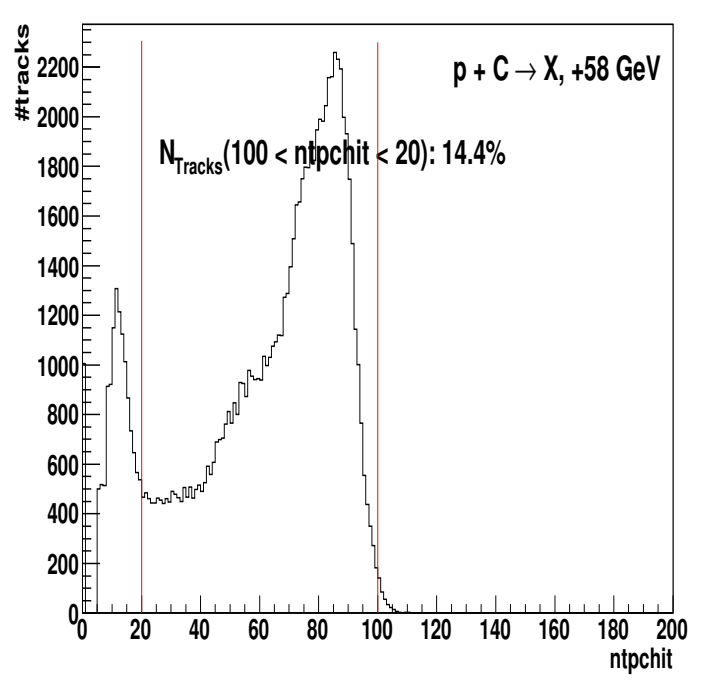

(a)

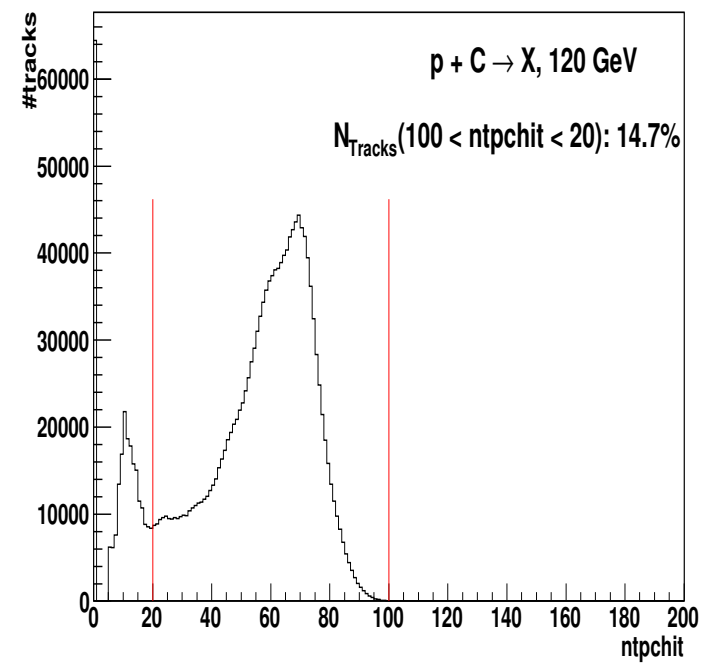

(b)

Figure 3.20: The distribution of number of TPC hits for a track for (a) 58 and (b) $120 \mathrm{GeV} / \mathrm{c}$ proton incident on carbon and empty targets. The red lines show the selected cut on the number of TPC hits i.e $20<$ ntpchit $<100$. 


\subsection{Beam Flux Calculation}

We have calculated the beam flux i.e. number of incident protons using two methods. One method makes use of direct scalers and the other makes use of prescalers. Direct scalers are those where all the beam particles were counted and on the other hand, the prescalers are those where the beam particles were prescaled (suppressed). Good beam events are selected by requiring the good beam event selection cuts as described in section 3.2 , and the beam flux is determined by counting the number of unbiased proton beam trigger events and applying the run-dependent prescale factor. The beam flux is also determined using the second method i.e. by counting the direct scalers. The beam flux numbers using the two methods for $\mathrm{LH}_{2}$, empty cryogenic, $\mathrm{C}, \mathrm{Be}, \mathrm{Bi}$ and empty targets using different proton beam momenta before applying the beam selection cuts are given in table 3.1. The relative differences between the beam fluxes using the two methods are also shown in this table.

For $120 \mathrm{GeV} / \mathrm{c}$ beam, for some runs, the direct scalar numbers are wrong. It can be seen from the direct scaler numbers for $120 \mathrm{GeV} / \mathrm{c}$ beam which are way high as compared to the prescaler numbers as shown in the table 3.1. Those runs are excluded for getting the relative difference between the beam fluxes using two methods.

\begin{tabular}{|l|l|l|l|l|}
\hline Target & $\begin{array}{l}\text { Beam momen- } \\
\text { tum, GeV/c }\end{array}$ & $\begin{array}{l}\text { Direct } \\
\text { scalers } \\
\left(\mathbf{N}_{\text {dirsc }}\right)\end{array}$ & $\begin{array}{l}\text { Prescalers } \\
\left(\mathbf{N}_{\text {prsc }}\right)\end{array}$ & $\begin{array}{l}\frac{N_{\text {prsc }}-N_{\text {dirsc }}}{(\mathbf{N})} \\
(\mathbf{\%})\end{array}$ \\
\hline $\mathrm{LH}_{2}$ & 58 & $5.49413 \mathrm{e}+07$ & $5.54254 \mathrm{e}+07$ & 0.88 \\
\hline Empty cryogenic & 58 & $1.18372 \mathrm{e}+07$ & $1.21574 \mathrm{e}+07$ & 2.70 \\
\hline $\mathrm{LH}_{2}$ & 85 & $9.54766 \mathrm{e}+07$ & $9.88646 \mathrm{e}+07$ & 3.55 \\
\hline Empty cryogenic & 85 & $2.70825 \mathrm{e}+07$ & $2.93814 \mathrm{e}+07$ & 8.49 \\
\hline Carbon & 58 & $1.51616 \mathrm{e}+07$ & $1.52692 \mathrm{e}+07$ & 0.71 \\
\hline Carbon & 120 & $8.56154 \mathrm{e}+07$ & $9.05006 \mathrm{e}+06$ & 4.49 \\
\hline Beryllium & 58 & $4.37406 \mathrm{e}+06$ & $4.3776 \mathrm{e}+06$ & 0.08 \\
\hline Beryllium & 120 & $2.64674 \mathrm{e}+08$ & $3.33796 \mathrm{e}+07$ & 4.84 \\
\hline Bismuth & 58 & $3.2646 \mathrm{e}+07$ & $3.35715 \mathrm{e}+07$ & 2.83 \\
\hline Bismuth & 120 & $2.45432 \mathrm{e}+08$ & $3.81832 \mathrm{e}+07$ & 4.54 \\
\hline Empty & 58 & $2.54402 \mathrm{e}+07$ & $2.61769 \mathrm{e}+07$ & 2.90 \\
\hline Empty & 120 & $5.47818 \mathrm{e}+07$ & $1.53211 \mathrm{e}+07$ & 8.41 \\
\hline
\end{tabular}

Table 3.1: Beam flux numbers using direct scalers and prescalers for $\mathrm{LH}_{2}$, empty cryogenic, carbon, beryllium, bismuth and empty targets using different proton beam momenta before appyling the beam selection cuts.

To get the beam flux numbers after applying the beam selection cuts for direct scalers, the efficiency of each beam selection cut is calculated using the prescalers and applied to the 
direct scalers which gives the direct scaler beam flux after applying the beam selection cuts. The efficiencies of the beam selection cuts for $\mathrm{LH}_{2}$ target using 58 and $85 \mathrm{GeV} / \mathrm{c}$ proton beams are given in table 3.2 , and the efficiencies for carbon target using 58 and $120 \mathrm{GeV} / \mathrm{c}$ proton beams are given in table 3.3 . The beam flux numbers using the two methods for $\mathrm{LH}_{2}$, empty cryogenic, $\mathrm{C}, \mathrm{Be}, \mathrm{Bi}$ and empty targets using different proton beam momenta after applying the beam selection cuts are given in table 3.4. Again the $120 \mathrm{GeV} / \mathrm{c}$ runs for which the direct scalars are wrong, are excluded for getting the relative difference between the beam fluxes using the two methods.

\begin{tabular}{|c|c|c|c|c|}
\hline Cut name & $\begin{array}{l}\mathbf{N}_{\text {beam }}\left(\mathrm{LH}_{2}\right. \\
+58 \mathrm{GeV})\end{array}$ & $\begin{array}{l}\text { Efficiency } \\
\left(\mathrm{LH}_{2} \quad+58\right. \\
\mathrm{GeV})\end{array}$ & $\begin{array}{l}\mathbf{N}_{\text {beam }}\left(\mathrm{LH}_{2}\right. \\
+85 \mathrm{GeV})\end{array}$ & $\begin{array}{l}\text { Efficiency } \\
\left(\mathrm{LH}_{2} \quad+85\right. \\
\mathrm{GeV})\end{array}$ \\
\hline Total beam events & $5.54254 \mathrm{e}+07$ & & $9.88646 \mathrm{e}+07$ & \\
\hline Single beam track & $4.23743 \mathrm{e}+07$ & 0.7645 & $7.1515 \mathrm{e}+07$ & 0.7234 \\
\hline Multiplicity $\leq 30$ & $4.17642 \mathrm{e}+07$ & 0.9856 & $7.01721 \mathrm{e}+07$ & 0.9812 \\
\hline Enbeamcrossings $\leq 4$ & $3.42869 \mathrm{e}+07$ & 0.8210 & $5.92233 \mathrm{e}+07$ & 0.8440 \\
\hline $\begin{array}{l}\text { Elliptical beam posi- } \\
\text { tion cut }\end{array}$ & $2.85359 \mathrm{e}+07$ & 0.8323 & $4.77601 \mathrm{e}+07$ & 0.8064 \\
\hline Beam track time cut & $2.84993 \mathrm{e}+07$ & 0.9987 & $4.77333 \mathrm{e}+07$ & 0.9994 \\
\hline
\end{tabular}

Table 3.2: The efficiencies of beam selection cuts applied to the direct scalers to get the beam flux numbers after applying the beam cuts for $\mathrm{LH}_{2}$ target at 58 and $85 \mathrm{GeV} / \mathrm{c}$ beam momenta.

\begin{tabular}{|c|c|c|c|c|}
\hline Cut name & $\begin{array}{ll}\mathbf{N}_{\text {beam }} & \text { (Car- } \\
\text { bon } & +58 \\
\text { GeV }) & \end{array}$ & $\begin{array}{l}\text { Efficiency } \\
\text { (Carbon } \\
+58 \mathrm{GeV} \text { ) }\end{array}$ & $\begin{array}{lr}\mathbf{N}_{\text {beam }} & \text { (Car- } \\
\text { bon } & \mathbf{1 2 0} \\
\mathrm{GeV}) & \end{array}$ & $\begin{array}{l}\text { Efficiency } \\
\text { (Carbon } \\
120 \mathrm{GeV})\end{array}$ \\
\hline Total beam events & $1.52692 \mathrm{e}+07$ & & $7.23344 \mathrm{e}+06$ & \\
\hline Single beam track & $1.21544 \mathrm{e}+07$ & 0.7960 & $6.07964 \mathrm{e}+06$ & 0.8405 \\
\hline Multiplicity $\leq 30$ & $1.209 \mathrm{e}+07$ & 0.9947 & $6.07613 \mathrm{e}+06$ & 0.9994 \\
\hline Enbeamcrossings $\leq 4$ & $9.95709 \mathrm{e}+06$ & 0.8236 & $5.15529 \mathrm{e}+06$ & 0.8485 \\
\hline Beam radius cut & $9.59822 \mathrm{e}+06$ & 0.9640 & $5.05459 \mathrm{e}+06$ & 0.9805 \\
\hline Beam track time cut & $9.59483 \mathrm{e}+06$ & 0.9996 & $5.04156 \mathrm{e}+06$ & 0.9974 \\
\hline
\end{tabular}

Table 3.3: The efficiencies of beam selection cuts applied to the direct scalers to get the beam flux numbers after applying the beam cuts for carbon target at 58 and $120 \mathrm{GeV} / \mathrm{c}$ beam momenta.

We have plotted the relative difference between the beam fluxes using the two methods before applying the beam selection cuts for the combined data set for 58,85 and $120 \mathrm{GeV} / \mathrm{c}$ proton beams, and $\mathrm{LH}_{2}$ and thin targets including $\mathrm{C}, \mathrm{Be}, \mathrm{Bi}$ and $\mathrm{U}$, and also empty cryogenic 


\begin{tabular}{|l|l|l|l|l|}
\hline Target & $\begin{array}{l}\text { Beam momen- } \\
\text { tum, GeV/c }\end{array}$ & $\begin{array}{l}\text { Direct } \\
\text { scalers } \\
\left(\mathbf{N}_{\text {dirsc }}\right)\end{array}$ & $\begin{array}{l}\text { Prescalers } \\
\left(\mathbf{N}_{\text {prsc }}\right)\end{array}$ & $\begin{array}{l}\frac{N_{\text {prsc }}-N_{\text {dirsc }}}{N_{\text {dirsc }}} \\
(\mathbf{\%})\end{array}$ \\
\hline $\mathrm{LH}_{2}$ & 58 & $2.82504 \mathrm{e}+07$ & $2.84993 \mathrm{e}+07$ & 0.88 \\
\hline Empty cryogenic & 58 & $5.76277 \mathrm{e}+06$ & $5.91865 \mathrm{e}+06$ & 2.70 \\
\hline $\mathrm{LH}_{2}$ & 85 & $4.60975 \mathrm{e}+07$ & $4.77333 \mathrm{e}+07$ & 3.55 \\
\hline Empty cryogenic & 85 & $1.34795 \mathrm{e}+07$ & $1.46237 \mathrm{e}+07$ & 8.49 \\
\hline Carbon & 58 & $9.52051 \mathrm{e}+06$ & $9.58806 \mathrm{e}+06$ & 0.71 \\
\hline Carbon & 120 & $5.96758 \mathrm{e}+07$ & $6.30809 \mathrm{e}+06$ & 4.49 \\
\hline Beryllium & 58 & $2.52024 \mathrm{e}+06$ & $2.52228 \mathrm{e}+06$ & 0.08 \\
\hline Beryllium & 120 & $1.76274 \mathrm{e}+08$ & $2.22309 \mathrm{e}+07$ & 4.84 \\
\hline Bismuth & 58 & $1.84219 \mathrm{e}+07$ & $1.89442 \mathrm{e}+07$ & 2.83 \\
\hline Bismuth & 120 & $1.5906 \mathrm{e}+08$ & $2.47459 \mathrm{e}+07$ & 4.54 \\
\hline Empty & 58 & $1.32248 \mathrm{e}+07$ & $1.36078 \mathrm{e}+07$ & 2.90 \\
\hline Empty & 120 & $3.46522 \mathrm{e}+07$ & $9.69134 \mathrm{e}+06$ & 8.41 \\
\hline
\end{tabular}

Table 3.4: Beam flux numbers using direct scalers and prescalers for $\mathrm{LH}_{2}$, empty cryogenic, carbon, beryllium, bismuth and empty targets using different proton beam momenta after appyling the beam selection cuts.

and empty targets. Figure 3.21(a) shows the relative difference as a function of subrun number and figure $3.21(\mathrm{~b})$ shows the Y-projection of the plot in figure 3.21(a). The overall difference between the two methods is $3.5 \%$.

\subsection{Interaction Trigger Efficiency}

MIPP uses a scintillator-based interaction trigger which requires at least 3 charged tracks for the scintillator to fire. This causes inefficiencies at low multiplicities and these need to be corrected. The trigger efficiencies are calculated in bins of tracks passing through the scintillator using the Monte Carlo $(\mathrm{MC})$ by requiring the scintillator to be fired. The efficiencies can also be calculated using unbiased beam triggers which are independent of the interaction triggers but the statistics is not enough, so that method is not used. For calculation of efficiencies, two histograms are made and the division of these two gives the efficiency. One is the distribution of tracks passing through the scintillator which forms the denominator, and the other is the distribution of tracks passing through the scintillator with the scintillator fired, and that forms the numerator. All the requirements including good beam selection cuts, track selection cuts, and the event selection cuts described in the section 3.2 are $\mathrm{ON}$ while making both these histograms.

The trigger efficiencies in bins of tracks passing through the scintillator for $58 \mathrm{GeV} / \mathrm{c}$ 


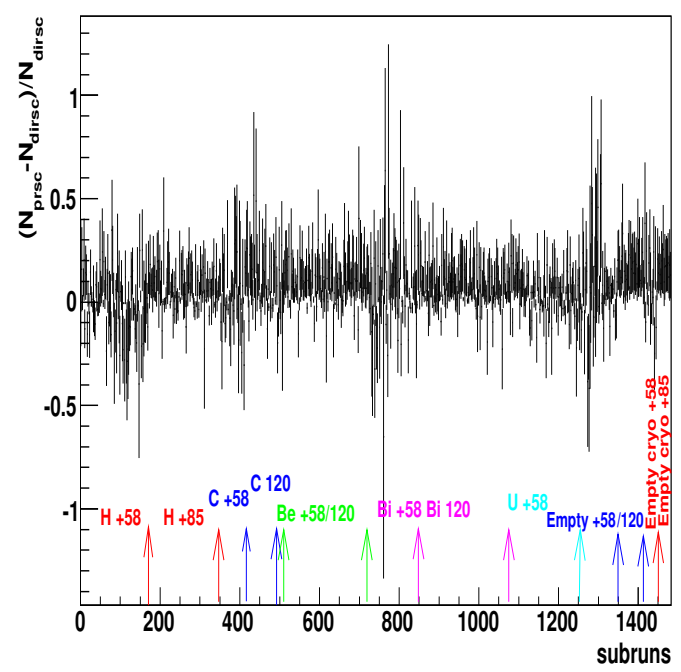

(a)

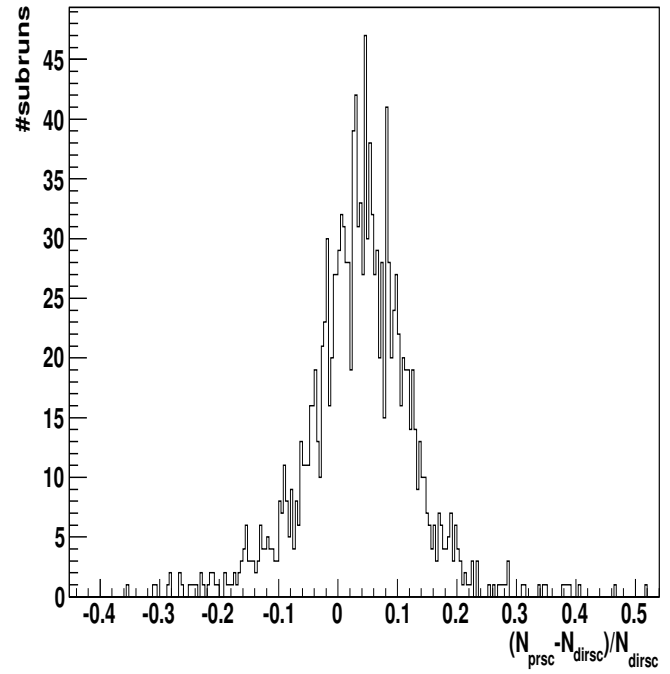

(b)

Figure 3.21: (a) Relative difference between beam fluxes using two methods vs subrun numbers for all the data sets combined. (b) Relative difference between beam fluxes using two methods for all the data sets combined. The overall difference between two methods is $3.5 \%$.

$\mathrm{p}+\mathrm{p}$ interactions using the reconstructed $\mathrm{MC}$ are shown in figure 3.22 . For multiplicities $>$ 3 , the efficiency becomes $\sim 95 \%$ and then becomes flat for the higher multiplicities.

We have also developed a technique which uses KNO scaling to get the trigger efficiencies. This technique is described in next section.

\subsection{KNO-based technique to get trigger efficiency}

In the beam momentum range of $50-300 \mathrm{GeV} / \mathrm{c}$, multiplicity distributions in $\mathrm{p}+\mathrm{p}$ interactions are known to scale according to the KNO scaling relations 93. The true multiplicity distributions are even number of charged particles $(2,4,6,8, \ldots)$. The observed multiplicity distributions span all multiplicities due to tracking inefficiency and acceptance of the TPC. Using a Monte Carlo such as DPMJET [87] and the full simulation of the TPC, and using the MIPP reconstruction algorithms, it is possible to obtain a relationship between the true and observed multiplicities in MIPP. This relationship is expressed as a K-matrix $\mathrm{K}\left(\mathrm{n}_{o} \mid n_{t}\right)$ which denotes the probability of obtaining observed multiplicity $\mathrm{n}_{o}$, given a true multiplicity $\mathrm{n}_{t}$. The observed multiplicity in this matrix is without the interaction trigger applied. In data, we only have observed multiplicities with the interaction trigger applied. The method used is to treat trigger efficiencies (as a function of the number of tracks going through the 


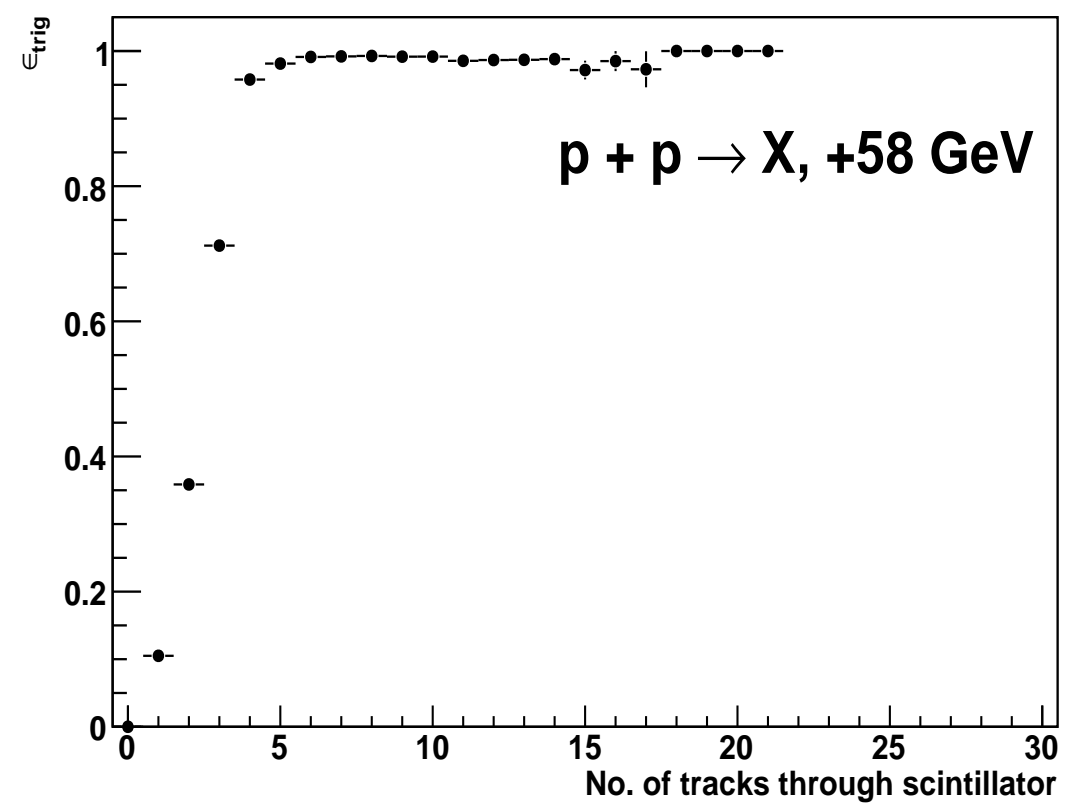

Figure 3.22: The trigger efficiencies in bins of tracks passing through the scintillator for 58 $\mathrm{GeV} / \mathrm{c} \mathrm{p}+\mathrm{p}$ interactions using the MC.

scintillator) as parameters to be determined by imposing the criterion that the true multiplicities are given by the KNO relation.

The steps of the fitting algorithm to get the KNO-based trigger efficiencies are described as follows:

\subsubsection{Get the KNO true multiplicity probability distribution}

KNO Scaling: KNO stands for Koba, Nielsen and Olesen, the three authors who put forward the hypothesis that at very high energies $\mathrm{s}$, the probability distributions $\mathrm{P}_{n}(\mathrm{~s})$ of producing $\mathrm{n}$ particles in a certain collision process should exhibit the scaling relation

$$
P_{n}(s)=\frac{1}{<n(s)>} \psi\left(\frac{n}{<n(s)>}\right)
$$

as $\mathrm{s} \rightarrow \infty$ with $<\mathrm{n}(\mathrm{s})>$ being the average multiplicity of secondaries at collision energy s [94]. According to KNO scaling hypothesis, if we rescale $\mathrm{P}_{n}(\mathrm{~s})$ measured at different energies via stretching (shrinking) the vertical (horizontal) axes by $\langle\mathrm{n}(\mathrm{s})\rangle$, these rescaled curves coincide with each other. That is, the multiplicity distributions become simple rescaled copies of the universal function $\psi(\mathrm{Z})$ depending only on the scaled multiplicity $\mathrm{Z}=\mathrm{n} /<\mathrm{n}(\mathrm{s})>$. $\psi(\mathrm{Z})$ is 
called KNO function. So the rescaled data points $\mathrm{P}_{n}(\mathrm{~s})$ measured at different energies $\mathrm{s}$ collapse onto the unique scaling curve $\psi(\mathrm{Z})$ shown in figure 3.23 .

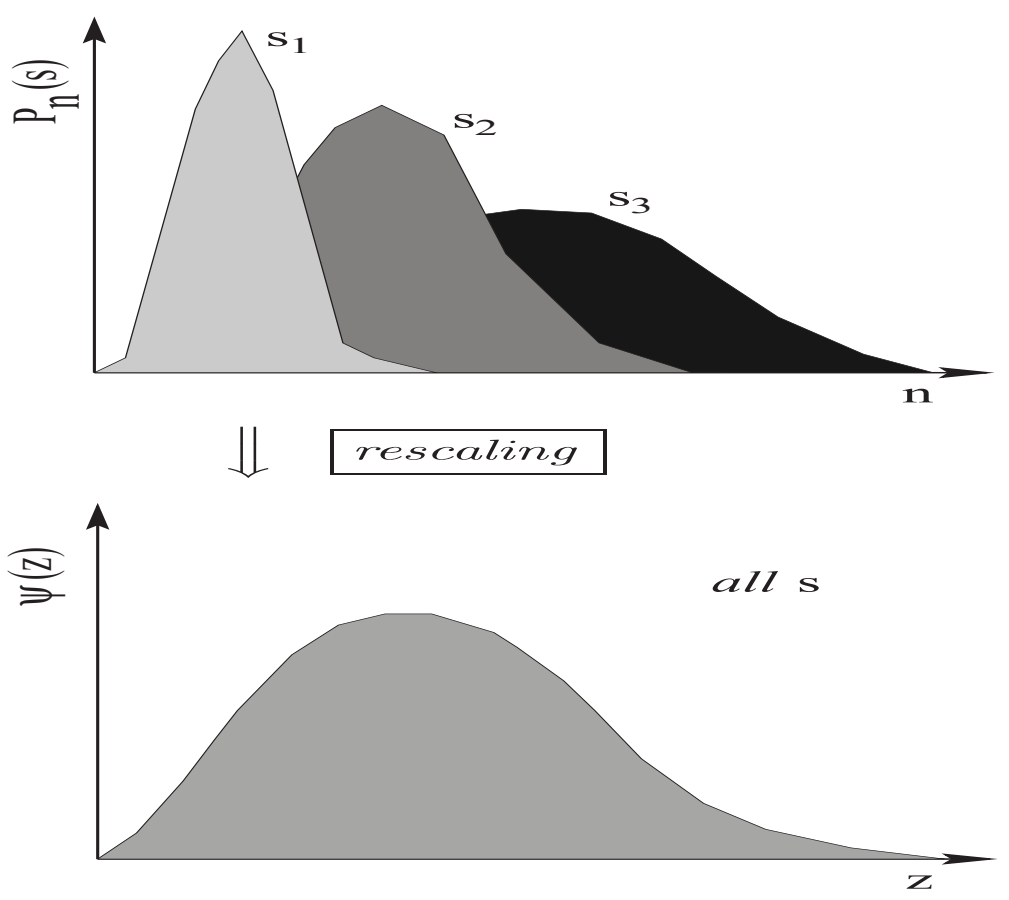

Figure 3.23: The KNO scaled data points $\mathrm{P}_{n}(\mathrm{~s})$ measured at different energies s collapse onto the unique scaling curve $\psi(\mathrm{Z})$.

For getting the KNO true multiplicity probability distribution for $\mathrm{p}+\mathrm{p}$ interactions, KNO function is used. The KNO function is written as $\psi(\mathrm{Z}=\mathrm{n} /<\mathrm{n}>)=\left(3.79 \mathrm{Z}+33.7 \mathrm{Z}^{3}\right.$ $\left.6.64 \mathrm{Z}^{5}+0.332 \mathrm{Z}^{7}\right) \mathrm{e}^{-3.04 Z}$. This function is a fit to the published data on $\mathrm{p}+\mathrm{p}$ collisions in momentum range $50-300 \mathrm{GeV} / \mathrm{c}$ [95]. $<\mathrm{n}>$ is calculated using the function $-2.9+1.79 \log (\mathrm{s})$, which is a fit to the average charged particle multiplicities per inelastic $\mathrm{p}+\mathrm{p}$ collision for all available data above $10 \mathrm{GeV} / \mathrm{c}$ [41]. $\mathrm{s}$ is calculated as $2 \times \mathrm{m}_{P} \times \mathrm{p}_{\text {Beam }}$ where $\mathrm{m}_{P}$ is the mass of proton $\left(0.938 \mathrm{GeV} / \mathrm{c}^{2}\right)$ and $\mathrm{p}_{\text {Beam }}$ is the momentum of the incident proton.

The KNO function is written as $<\mathrm{n}>\mathrm{P}_{n}$ (from equation 3.1). So using KNO function, the probabilities $\mathrm{P}_{n}$ 's are calculated. $\mathrm{P}_{n}$ can also be written as $\sigma_{n} / \sigma_{\text {inelastic }}$ where $\sigma_{n}$ is the partial cross section for the reaction $\mathrm{p}+\mathrm{p} \rightarrow \mathrm{n}$ charged particles, and $\sigma_{\text {inelastic }}$ is the total inelastic $\mathrm{p}+\mathrm{p}$ cross section. Here $\sigma_{2}$ does not include the elastic channel. The KNO true probabilities are normalized such that

$$
\sum_{k=2}^{k=2 N} P_{k}=1
$$


The KNO true probability distribution for $\mathrm{p}+\mathrm{p}$ interactions at $58 \mathrm{GeV} / \mathrm{c}$ is shown in figure 3.24 .

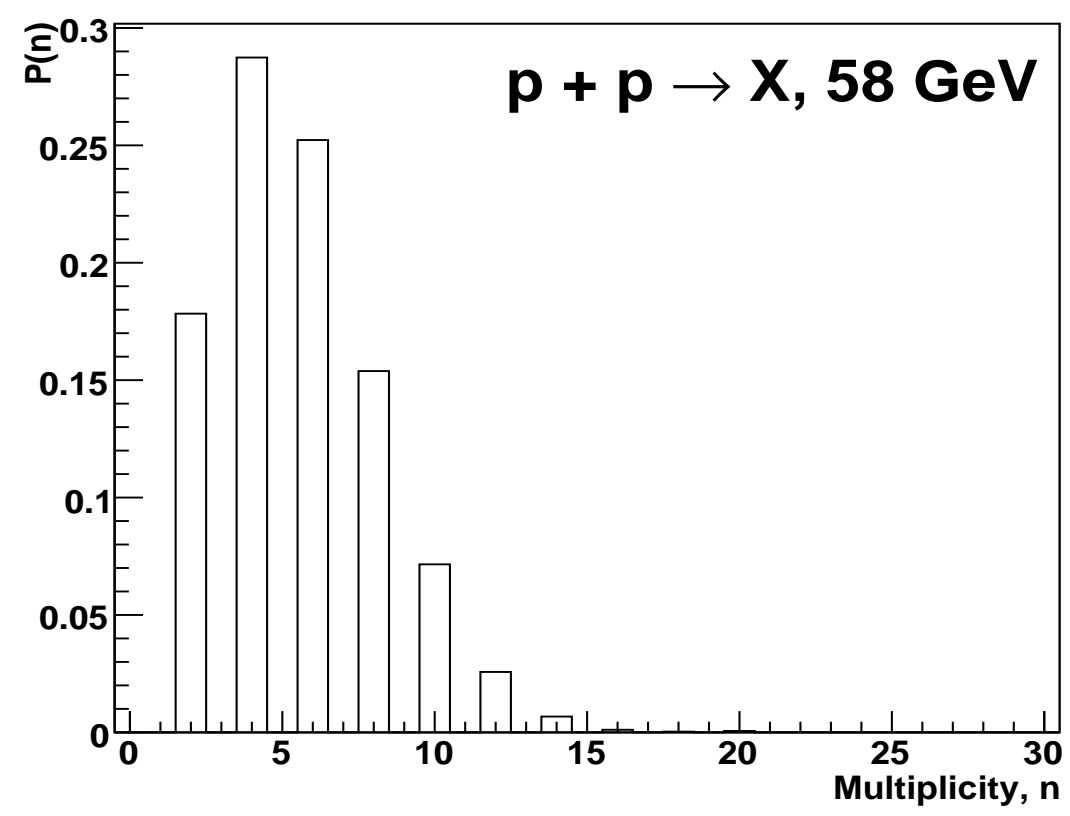

Figure 3.24: The KNO true probability distribution for $\mathrm{p}+\mathrm{p}$ interactions at $58 \mathrm{GeV} / \mathrm{c}$.

Model to get the cross section numbers for $\mathbf{p}+\mathbf{p}$ interaction: To get the total, inelastic and elastic cross section numbers for $\mathrm{p}+\mathrm{p}$ interaction at a particular beam momentum, the data files for the elastic and total cross section data are taken from the PDG [96]. A function of the form a $+\operatorname{blog}_{e}^{2}\left(\mathrm{p}_{L a b}\right)+\mathrm{c}\left(\mathrm{p}_{L a b}\right)^{d}$ is fitted to the distributions of total and elastic cross section vs lab momentum $\mathrm{p}_{\text {Lab }}[97$ in the range $5-200 \mathrm{GeV} / \mathrm{c}$. The function for the elastic cross section $10.93-0.8002 \log _{e}^{2}\left(\mathrm{p}_{\text {Lab }}\right)+0.9882\left(\mathrm{p}_{\text {Lab }}\right)^{0.5547}$ is subtracted from the function for the total cross section $40.6-0.6183 \log _{e}^{2}\left(\mathrm{p}_{\text {Lab }}\right)+0.7755\left(\mathrm{p}_{\text {Lab }}\right)^{0.5706}$ to get the function for the inelastic cross section. The value of the total, elastic and inelastic cross section at a particular beam momentum can be obtained from these three functions.

Figure 3.25 shows the fits to the total and elastic cross sections as a function of lab momentum from the PDG. The function for inelastic cross section obtained after subtracting the function for elastic cross section from the function for total cross section is also superimposed. 


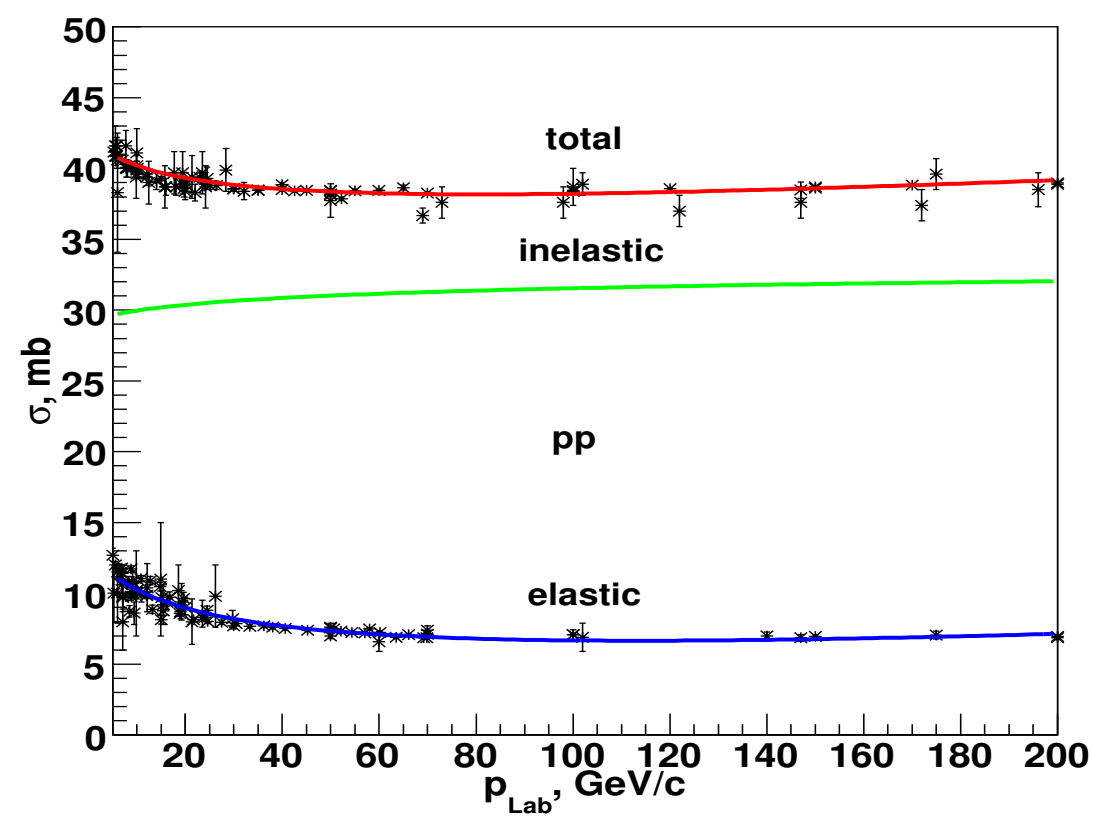

Figure 3.25: The fits to the total and elastic cross sections as a function of lab momentum from the PDG. The function for inelastic cross section obtained after subtracting the function for elastic cross section from the function for total cross section is also superimposed.

\subsubsection{Get the predicted observed probability distribution}

The predicted observed probability distribution is obtained by multiplying the K-matrix by the KNO true probability distribution. The K-matrix $K\left(\mathrm{n}_{o} \mid \mathrm{n}_{t}\right)$ denotes the probability of obtaining observed multiplicity $\mathrm{n}_{o}$, given a true multiplicity $\mathrm{n}_{t}$. The interaction trigger is not imposed while calculating the K-matrix. The probability distributions for obtaining observed multiplicity $\mathrm{n}_{o}(1,2,3,4, \ldots)$ for a given true multiplicity $\mathrm{n}_{t}(2,4,6,8, .$.$) and the$ predicted observed probability distribution obtained by multiplying the K-matrix and the KNO true probability distribution for $\mathrm{p}+\mathrm{p}$ interactions at $58 \mathrm{GeV} / \mathrm{c}$ are shown in figures 3.26 and 3.27, respectively.

\subsubsection{Get the observed multiplicity distribution}

The observed (data) multiplicity distribution for $\mathrm{p}+\mathrm{p}$ interactions at $58 \mathrm{GeV} / \mathrm{c}$ is shown in figure 3.28. These multiplicities are with the interaction trigger applied. 

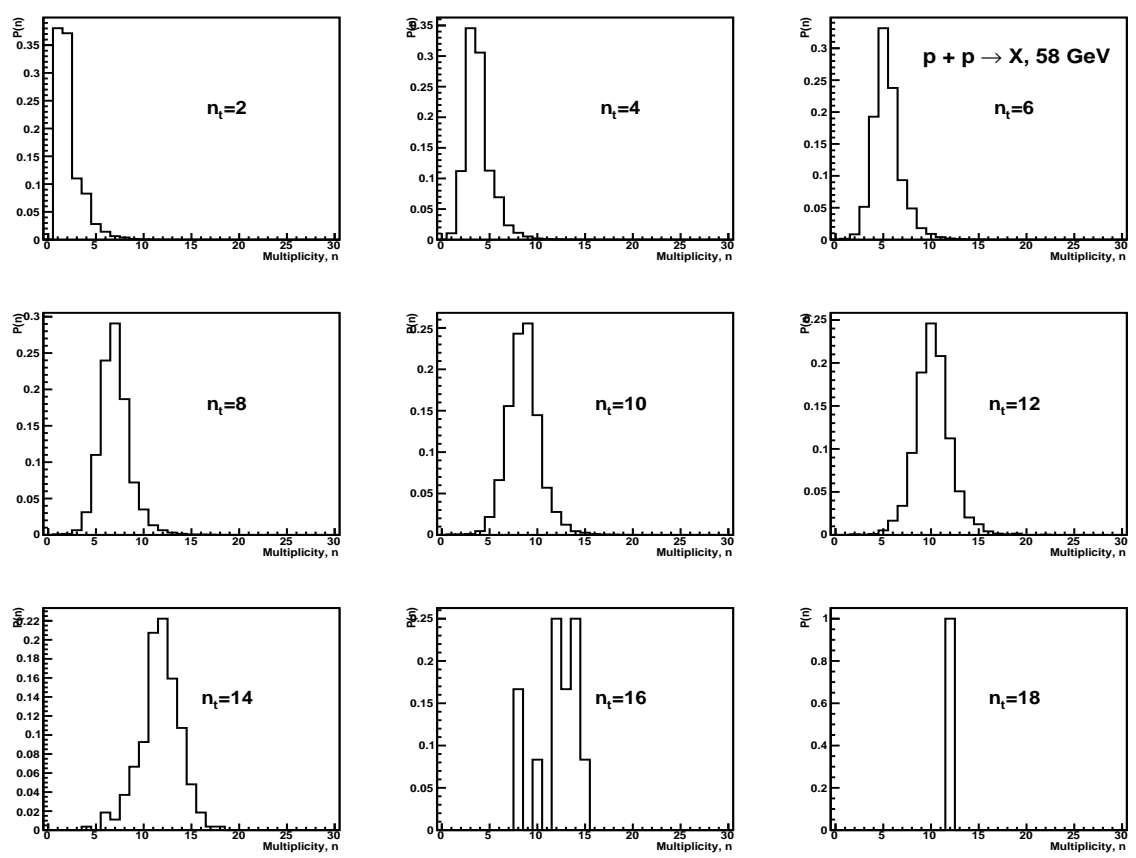

Figure 3.26: The probability distributions for obtaining observed multiplicity, given a true multiplicity $\mathrm{n}_{t}$ for $\mathrm{p}+\mathrm{p}$ interactions at $58 \mathrm{GeV} / \mathrm{c}$.

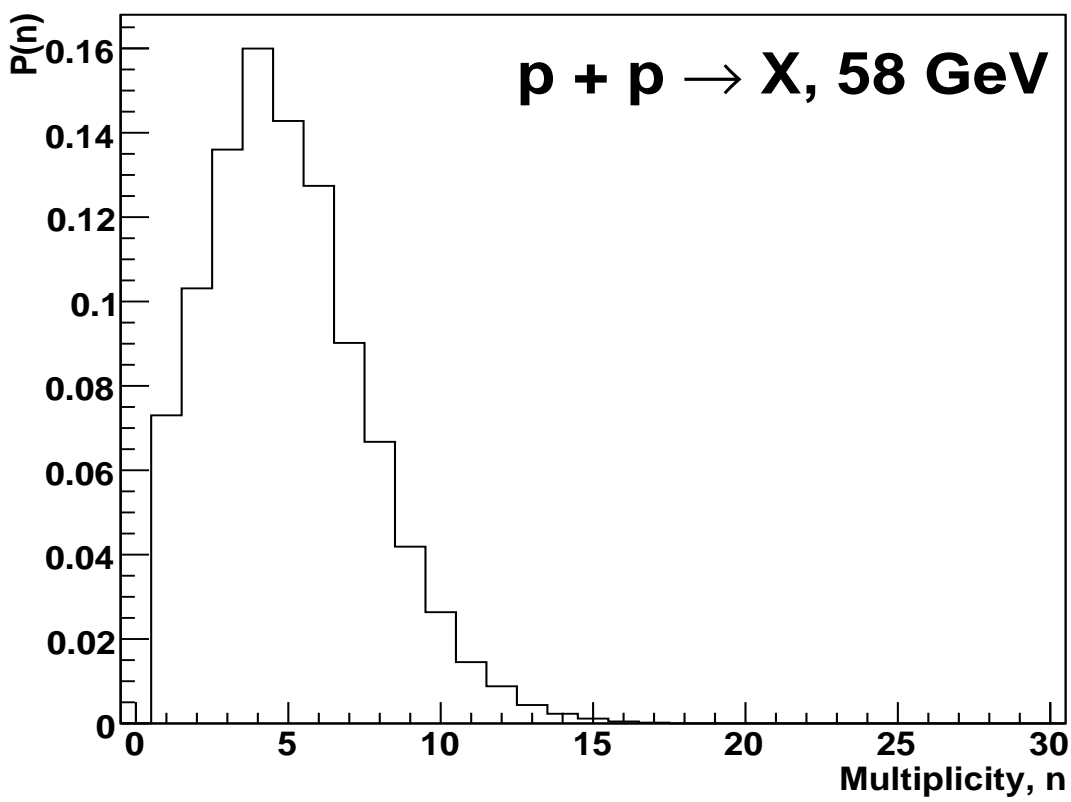

Figure 3.27: The predicted observed probability distribution obtained by multiplying the Kmatrix (figure 3.26) and KNO true probability distribution (figure 3.24) for $\mathrm{p}+\mathrm{p}$ interactions at $58 \mathrm{GeV} / \mathrm{c}$. 


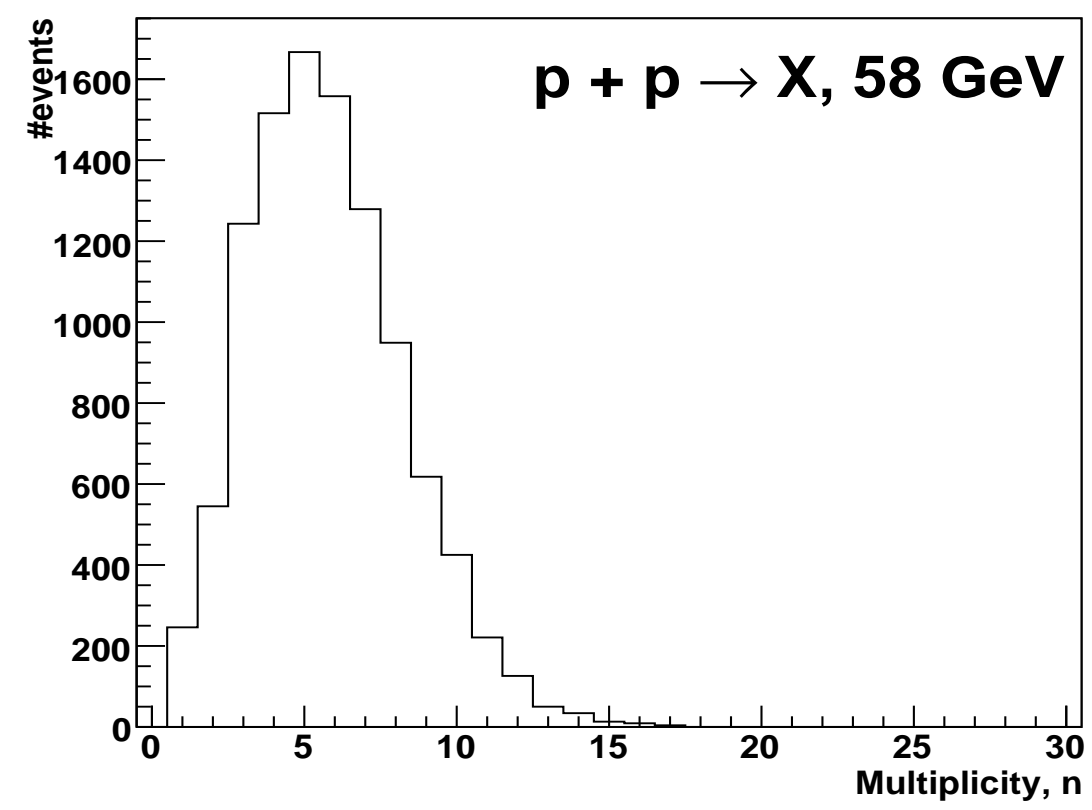

Figure 3.28: The observed multiplicity distribution for $\mathrm{p}+\mathrm{p}$ interactions at $58 \mathrm{GeV} / \mathrm{c}$ with the interaction trigger applied.

\subsubsection{Minimization Scheme}

The minimization scheme consists of two steps. At first step, a function of the form 1 $\mathrm{e}^{-a n-b n^{2}-c n^{3}}$ is fitted using MINUIT [85]. Here a, b, c are the parameters to be fitted and $\mathrm{n}$ is the number of tracks passing through the scintillator which is known event by event in the data. This function will give the trigger efficiency as a function of number of tracks passing through the scintillator. The observed distribution is fitted to the predicted observed distribution. The observed multiplicity distribution is corrected for the inefficiency due to the interaction trigger by dividing the observed number of events by the efficiency function at all the multiplicities at each iteration in the fit. This is done event by event since the number of tracks passing through the scintillator for a particular multiplicity varies from event to event. The efficiency function changes from iteration to iteration since the parameters a, b, c vary at each iteration.

At each iteration, the total number of events is obtained from the corrected observed distribution by summing over events at all the multiplicities. The predicted observed probability distribution is multiplied by this number to get the predicted observed multiplicity distribution so that the observed and predicted distributions are normalized to the same number of events. So, the predicted distribution also varies from iteration to iteration. 
The minimization function is a $\chi^{2}$ function given as

$$
\chi^{2}=\left(\frac{\text { observed }- \text { predicted }}{\sigma}\right)^{2}
$$

where "observed" is the observed number of events, "predicted" is the predicted observed number of events, and $\sigma$ is the error. The $\chi^{2}$ varies from iteration to iteration as the parameters of the efficiency function vary. At the minimum, the optimal trigger efficiency is obtained from the efficiency function that conforms to a KNO distribution of inelastic multiplicities. The comparison of observed and predicted multiplicity distributions at the minimum, and the efficiencies calculated using the fitted function $1-\mathrm{e}^{-0.12 n-0.058 n^{2}-0.05 n^{3}}$ for $58 \mathrm{GeV} / \mathrm{c} \mathrm{p}+\mathrm{p}$ interactions are shown in figure 3.29 . It is clear that the fit is not good. The error in the efficiency function is calculated as

$$
\operatorname{Var} F=\sum_{i, j}\left(\frac{\partial F}{\partial a_{i}}\right)\left(\frac{\partial F}{\partial a_{j}}\right) E_{i j}
$$

$\mathrm{E}_{i j}$ is the $3 \times 3$ error matrix and is obtained from the MINUIT output. The errors in the efficiencies are given as the square root of the following quantity:

$$
\begin{gathered}
\sigma_{f}^{2}=\left(\frac{\partial F}{\partial a_{1}}\right)^{2} a_{11}+\left(\frac{\partial F}{\partial a_{2}}\right)^{2} a_{22}+\left(\frac{\partial F}{\partial a_{3}}\right)^{2} a_{33}+2\left(\frac{\partial F}{\partial a_{1}}\right)\left(\frac{\partial F}{\partial a_{2}}\right) a_{12} \\
+2\left(\frac{\partial F}{\partial a_{2}}\right)\left(\frac{\partial F}{\partial a_{3}}\right) a_{23}+2\left(\frac{\partial F}{\partial a_{1}}\right)\left(\frac{\partial F}{\partial a_{3}}\right) a_{13}
\end{gathered}
$$

At the second step, up to multiplicity 6, the trigger efficiency parameters are fitted as a function of the number of tracks passing through the scintillator and after multiplicity 6 , the function which is known from step 1 is fitted to retain the constant behaviour of the efficiency at the higher multiplicities. So the total number of parameters to be fitted is 9 . The first 6 parameters are the efficiencies and the last 3 parameters are parameters of the function which will give us the efficiencies. The parameters of the function are fixed. The 6 efficiency parameters vary at each iteration.

An initial set of values for the 6 efficiency parameters in the fit are taken from figure 3.22 which shows the trigger efficiencies as a function of tracks passing through the scintillator for $58 \mathrm{GeV} / \mathrm{c} \mathrm{p}+\mathrm{p}$ interactions using the reconstructed MC. The observed distribution is fitted to the predicted distribution just like in step 1 to extract the efficiencies.

The comparison of the observed and predicted distributions at the minimum, and the comparison of the KNO-based efficiencies obtained from the fit with the MC efficiencies as a function of number of tracks through the scintillator for $58 \mathrm{GeV} / \mathrm{c} \mathrm{p}+\mathrm{p}$ interactions are shown in figure 3.30. The fit gets better after doing the step 2. The errors in the 


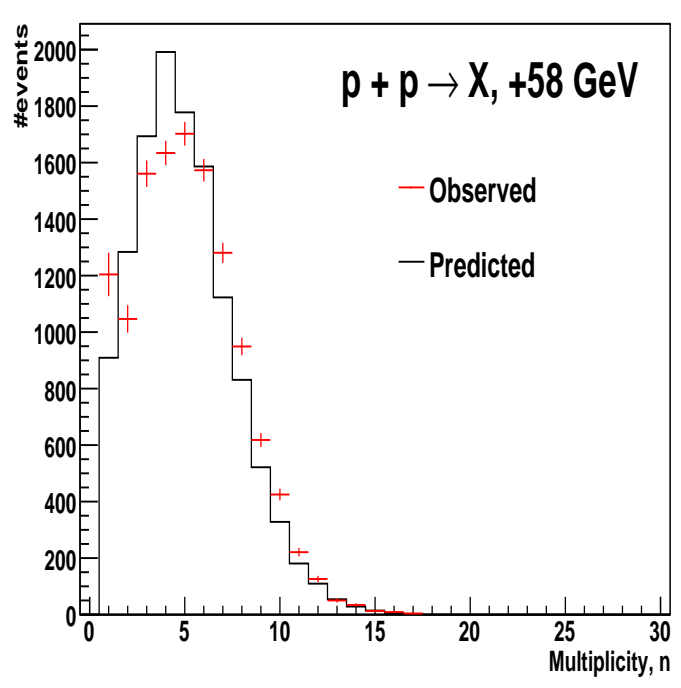

(a)

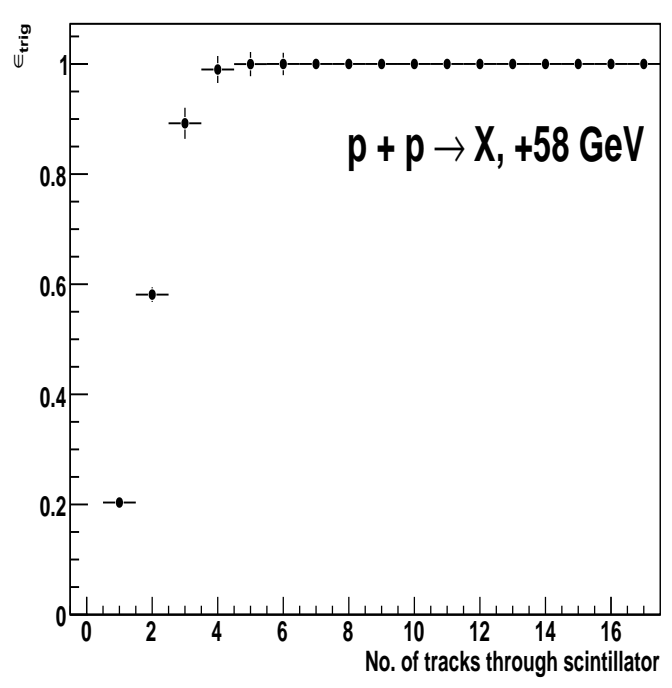

(b)

Figure 3.29: (a) Comparison of observed and predicted distributions at the minimum and (b) the trigger efficiencies obtained from the fitted function, for $58 \mathrm{GeV} / \mathrm{c} \mathrm{p}+\mathrm{p}$ interactions. The fit is not good.

efficiency parameters are obtained from the MINUIT output directly and the errors in the efficiency function are calculated using equation 3.3. A drop is observed at $n=4$ and $n=6$ in the KNO-based efficiency curve which is not reasonable as the trigger efficiency should be monotonically increasing with the number of tracks passing through the scintillator. From the studies, it is concluded that it is happening due to the differences in shapes of the multiplicities of the MC generator DPMJET and multiplicities from the KNO scaling function shown in figure 3.31. The unfolded MIPP data multiplicity probabilities using the MC K-matrix are also superimposed. From the plot it is clear that the DPMJET multiplicity shape completely disagrees with that of the MIPP data and the published data (KNO).

The KNO true probability distribution for $\mathrm{p}+\mathrm{p}$ interactions at $85 \mathrm{GeV} / \mathrm{c}$ is shown in figure 3.32, The comparison of observed and predicted distributions at the minimum, and the comparison of the KNO-based and MC efficiencies as a function of number of tracks through the scintillator for $85 \mathrm{GeV} / \mathrm{c} \mathrm{p}+\mathrm{p}$ interactions are shown in figure 3.33 . Again a drop is observed at $n=6$.

KNO-based trigger efficiencies for carbon target: The Koba-Nielsen-Olesen (KNO) scaling hypothesis [93] for $\mathrm{p}+\mathrm{p}$ interactions was verified by Slattery [95] for hydrogen bubble chamber data in the momentum range $50-300 \mathrm{GeV} / \mathrm{c}$. Subsequently attempts were made to 


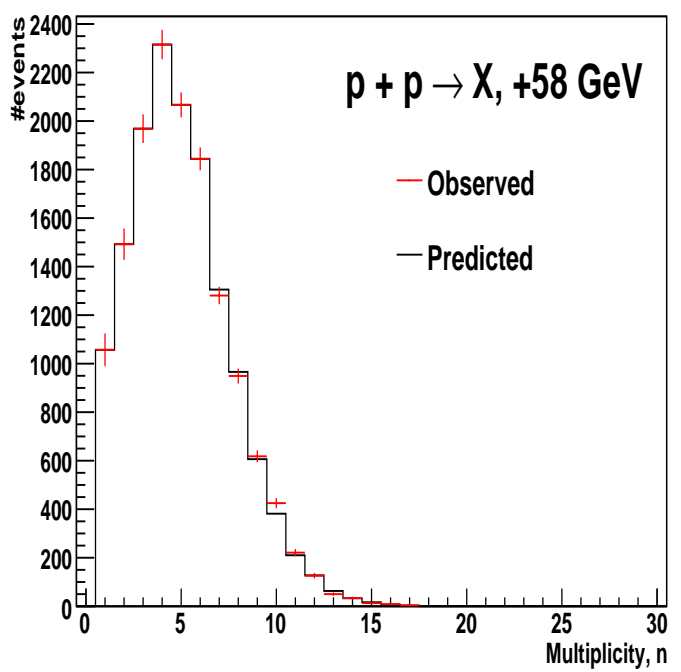

(a)

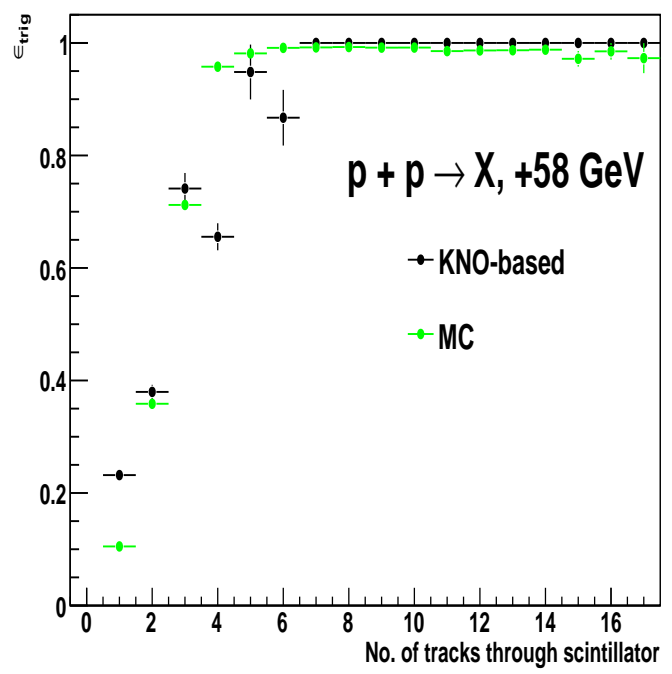

(b)

Figure 3.30: (a) Comparison of observed and predicted distributions at the minimum and (b) the KNO-based trigger efficiencies from the fit compared with the MC efficiencies, for 58 $\mathrm{GeV} / \mathrm{c} \mathrm{p}+\mathrm{p}$ interactions.

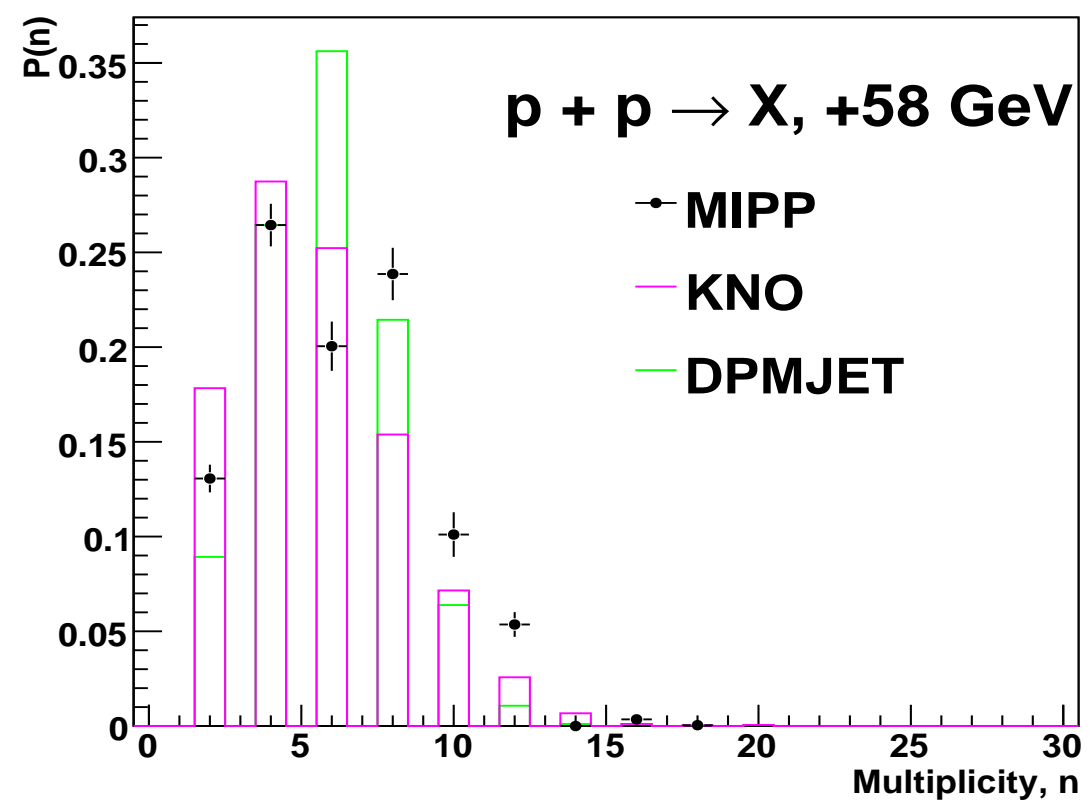

Figure 3.31: Comparison of DPMJET and KNO multiplicity probabilities for $58 \mathrm{GeV} / \mathrm{c} \mathrm{p}+\mathrm{p}$ interactions. The unfolded MIPP data multiplicity probabilities using the MC K-matrix are also superimposed. 


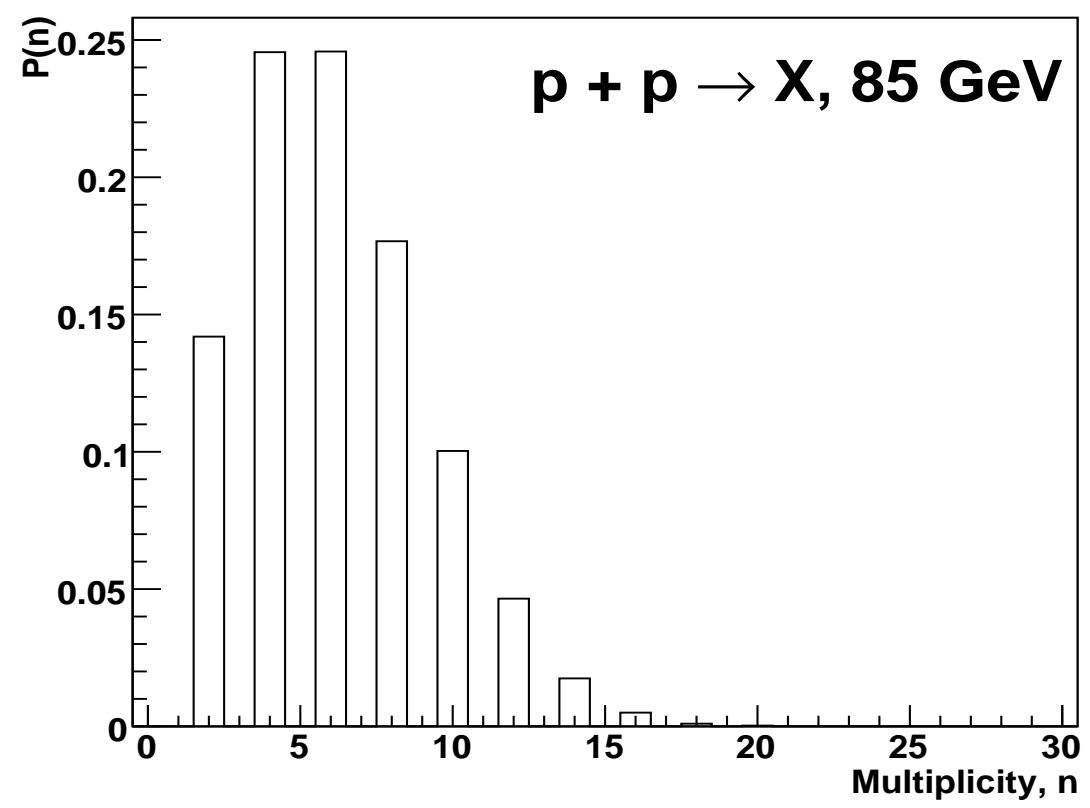

Figure 3.32: The KNO true probability distribution for $\mathrm{p}+\mathrm{p}$ interactions at $85 \mathrm{GeV} / \mathrm{c}$.

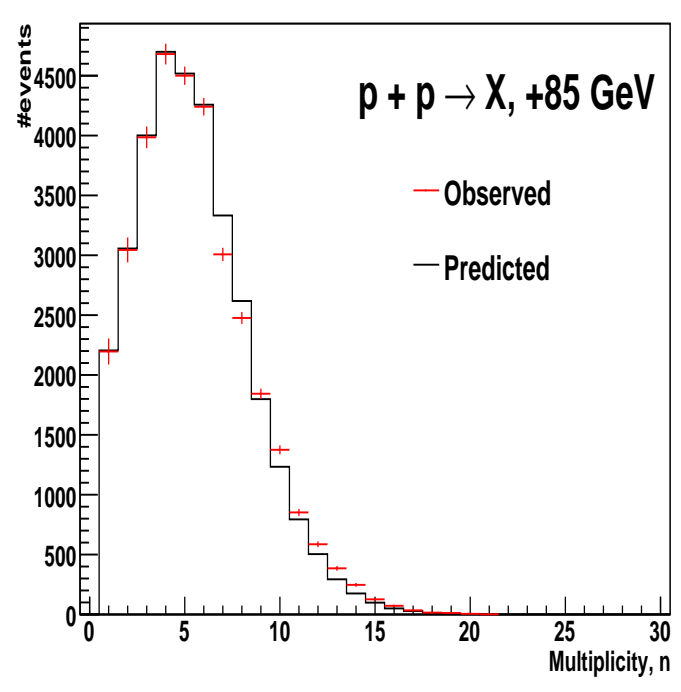

(a)

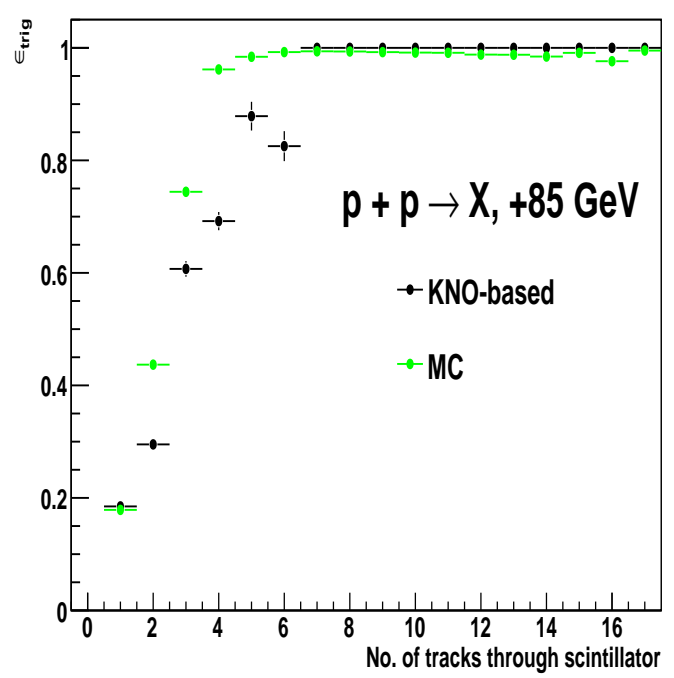

(b)

Figure 3.33: (a) Comparison of observed and predicted distributions at the minimum and (b) the KNO-based trigger efficiencies from the fit compared with the MC efficiencies, for 85 $\mathrm{GeV} / \mathrm{c} \mathrm{p}+\mathrm{p}$ interactions. 
test the validity of the scaling hypothesis in the case of proton-nucleus interactions. Martin et al. 98. found that for nuclear emulsion the parameters in the Slattery scaling function have to be modified in order to get a reasonable fit to the experimental data in the energy range $30-200 \mathrm{GeV} / \mathrm{c}$. Hébert et al. [99] pointed out that since emulsion is a composite target, it is not valid to reduce the multiplicity distribution to the form applicable to $\mathrm{p}+\mathrm{p}$ collisions. One should test the scaling hypothesis for a homogeneous target or, alternatively, use the $\mathrm{p}+\mathrm{p}$ scaling curve to deduce a multiplicity distribution for each component group of target nuclei in the emulsion and then combine the weighted distributions to obtain the distribution to be expected for the emulsion as a whole. This technique was originally applied to p-emulsion data at 200 and $300 \mathrm{GeV} / \mathrm{c}$ [99], using the Slattery scaling function which has the form

$$
\psi(Z)=\left(A Z+B Z^{3}+C Z^{5}+D Z^{7}\right) e^{E Z},
$$

where $\left.\mathrm{Z}=\mathrm{n}_{c h} /<\mathrm{n}_{c h}\right\rangle$. The quantity $\mathrm{n}_{c h}$ is the number of charged particles in the final state of a $\mathrm{p}+\mathrm{p}$ interaction. When applying the function to the case of proton-nucleus interactions in emulsion, $\mathrm{n}_{c h}$ was replaced by $\mathrm{n}_{s}$, where $\mathrm{n}_{s}$ is the shower-particle multiplicity. $\mathrm{n}_{s}$ is not the same as $\mathrm{n}_{c h}$, since only relativistic particles $(\beta \geq 0.7)$ are classified as shower particles. Slow secondary protons and some of the created pions are, therefore, not included. In the energy range $200-300 \mathrm{GeV}$, this approximation (of replacing $\mathrm{n}_{c h}$ by $\mathrm{n}_{s}$ in the scaling function) was found to be satisfactory, but it could not be used at lower energies.

For this reason, it was decided to test the KNO scaling hypothesis over a wider range of energies by using the scaling function of Buras et al. [100, which was shown to be applicable to $\mathrm{p}+\mathrm{p}$ interactions in the momentum range $5.5-300 \mathrm{GeV} / \mathrm{c}$. The scaling function used by Buras et al. in new scaling variable $\mathrm{Z}^{\prime}=(\mathrm{n}-\alpha) /(<\mathrm{n}>-\alpha)$ for extending the KNO scaling for $\mathrm{p}+\mathrm{p}$ collisions to low energies is given as follows:

$$
\psi^{\prime}\left(Z^{\prime}\right)=2.30\left(Z^{\prime}+0.142\right) \exp \left(-0.0586 Z^{\prime}-0.659 Z^{\prime 2}\right) .
$$

The value of $\alpha$ is 0.9 for $\mathrm{p}+\mathrm{p}$ collisions [100]. It was observed [101] that the scaling function of Buras et al. can be applicable to proton-nucleus interactions without any change in parameters (except for a factor of $1 / 2$ which accounts for the inclusion of odd values of the multiplicities) and the value of $\alpha$ is zero for this case. So, the KNO function for p-nucleus interactions can be written as:

$$
\psi(Z)=0.5 \times 2.30(Z+0.142) \exp \left(-0.0586 Z-0.659 Z^{2}\right)=<n_{s}>P(n)
$$

The value of average multiplicity $<\mathrm{n}_{s}>$ for $\mathrm{p}+\mathrm{C}$ interactions is taken from [101]. A function 
of the form $3.36 \log _{e}(0.072(\mathrm{p}+13.5))+1.24$ is fitted to the values of $<\mathrm{n}_{s}>$ for light group $(\mathrm{C}$, $\mathrm{N}, \mathrm{O}$ ) of target nuclei in emulsion at momenta (p) of 6.2, 22.5, 67, 200, and $300 \mathrm{GeV} / \mathrm{c}$. The value of $<\mathrm{n}_{s}>$ at $58 \mathrm{GeV} / \mathrm{c}$ is 7.0 , and at $120 \mathrm{GeV} / \mathrm{c}$ is 9.0 .

A correction to $\left\langle\mathrm{n}_{s}\right\rangle$ is needed as it does not include the slow secondary protons and some of the created pions. This correction is small and very close to the values of errors on $\left\langle\mathrm{n}_{s}\right\rangle$ [101, 102, 103]. Hence no corrections to $\left\langle\mathrm{n}_{s}\right\rangle$ values are applied.

The KNO true multiplicity probability distribution for $\mathrm{p}+\mathrm{C}$ interactions at $58 \mathrm{GeV} / \mathrm{c}$ is shown in figure 3.34. Unlike $\mathrm{LH}_{2}$ target, we have both the even and odd multiplicities in case of carbon. The step 1 and step 2 for getting the trigger efficiencies for $\mathrm{LH}_{2}$ target (explained earlier in this section) are followed. The K-matrix is shown in figure 3.35. The predicted observed probability distribution obtained by multiplying the K-matrix (figure 3.35) with the KNO true probability distribution (figure 3.34), and the observed multiplicity distribution are shown in figure 3.36 .

The comparison of observed and predicted distributions at the minimum, and the comparison of the KNO-based and MC efficiencies as a function of number of tracks through the scintillator for $58 \mathrm{GeV} / \mathrm{c} \mathrm{p}+\mathrm{C}$ interactions are shown in figure 3.37 .

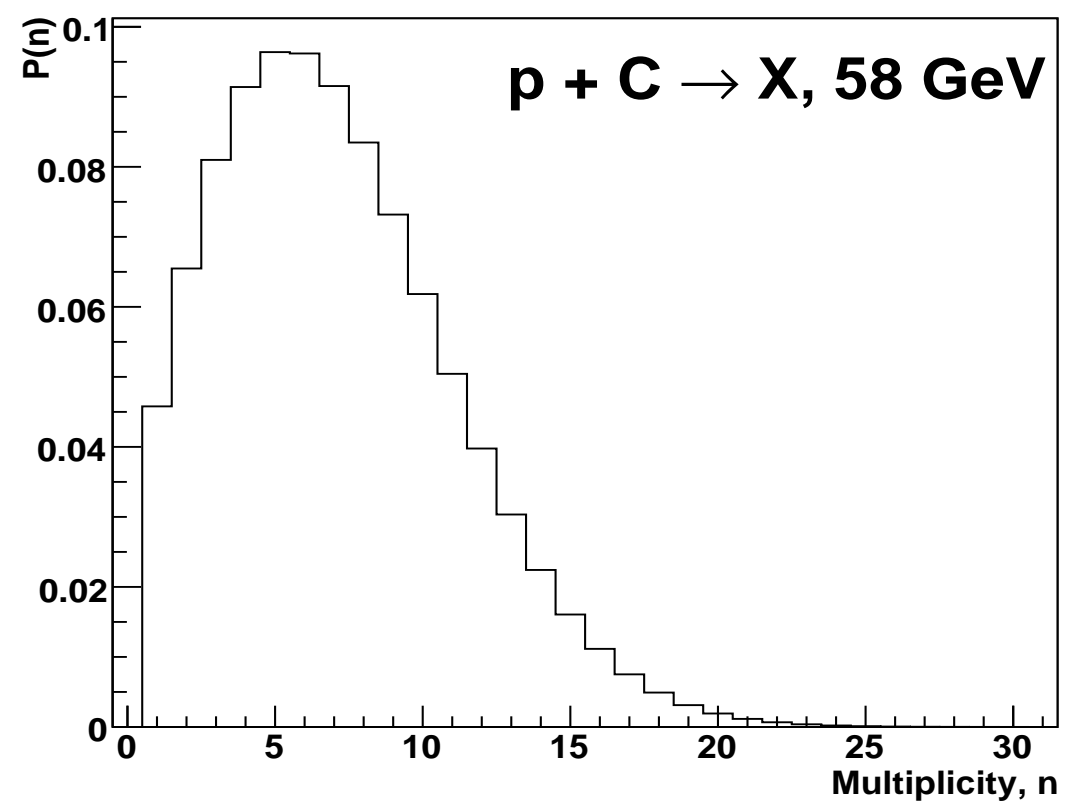

Figure 3.34: KNO true probability distribution for $58 \mathrm{GeV} / \mathrm{c} \mathrm{p}+\mathrm{C}$ interactions.

The KNO true probability distribution for $120 \mathrm{GeV} / \mathrm{c} \mathrm{p}+\mathrm{C}$ interactions is shown in figure 3.34. The comparison of observed and predicted distributions at the minimum, and 

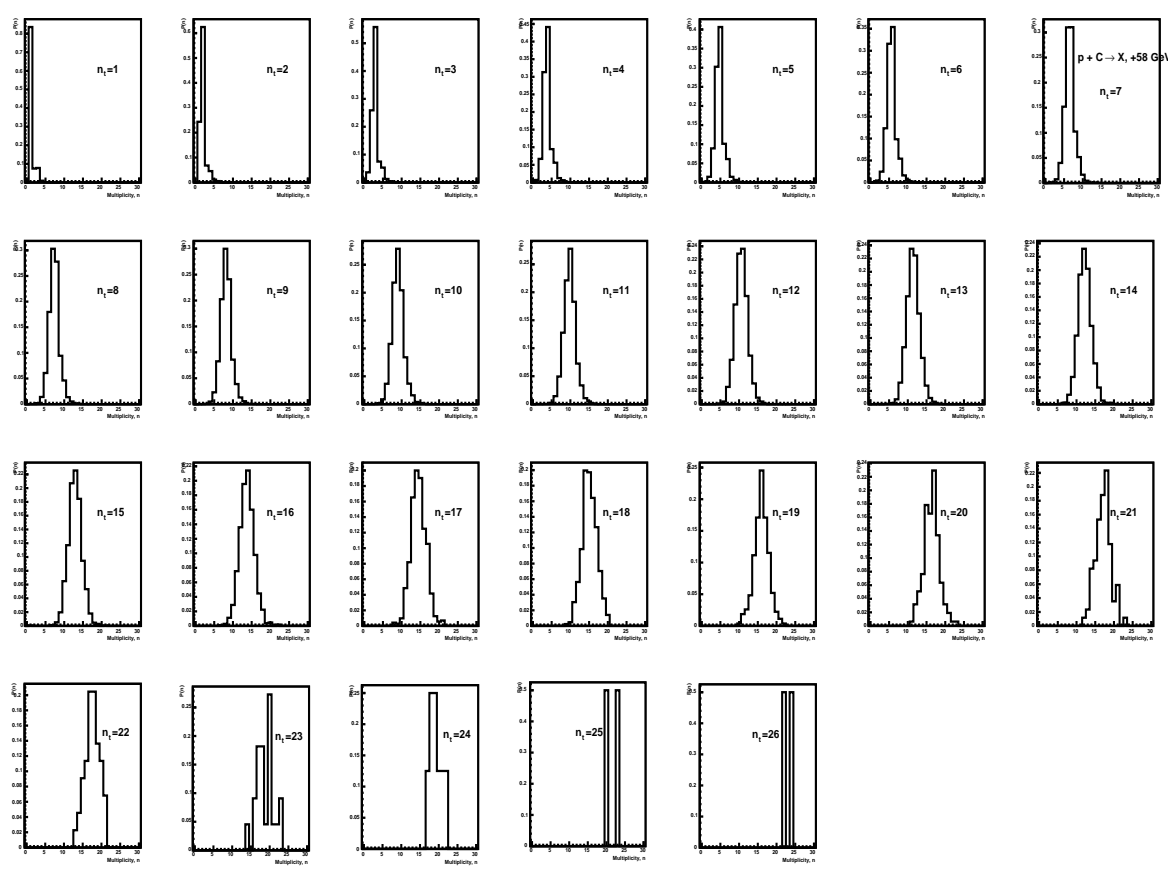

Figure 3.35: The probability distributions for obtaining observed multiplicity, given a true multiplicity $\mathrm{n}_{t}$ for $\mathrm{p}+\mathrm{C}$ interactions at $58 \mathrm{GeV} / \mathrm{c}$.

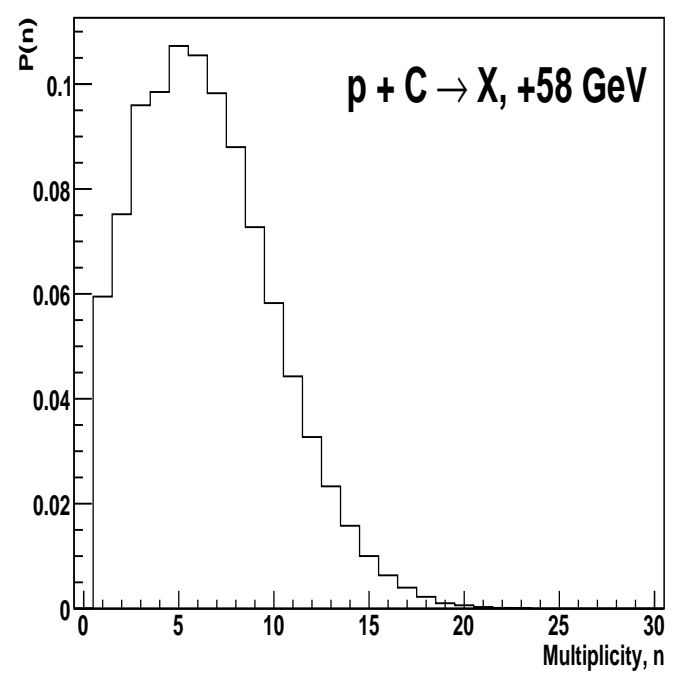

(a)

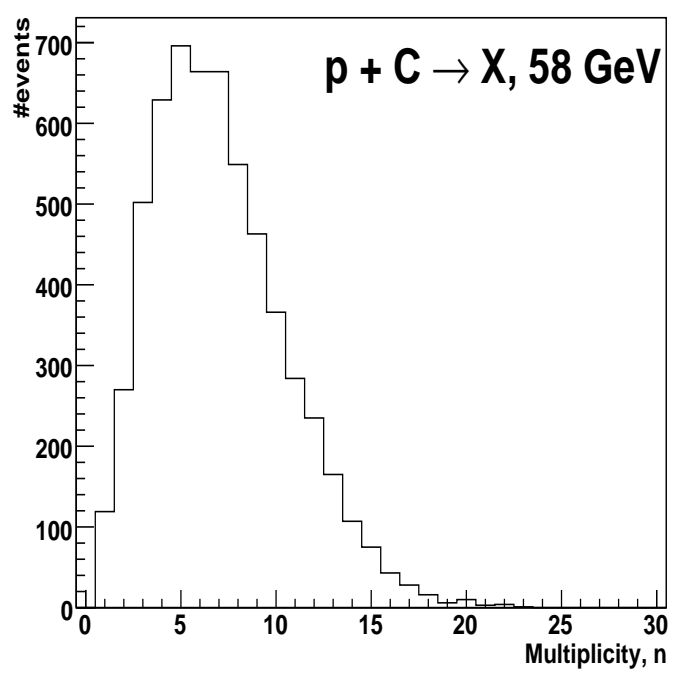

(b)

Figure 3.36: (a) The predicted observed probability distribution and (b) the observed multiplicity distribution, for $58 \mathrm{GeV} / \mathrm{c} \mathrm{p}+\mathrm{C}$ interactions. 


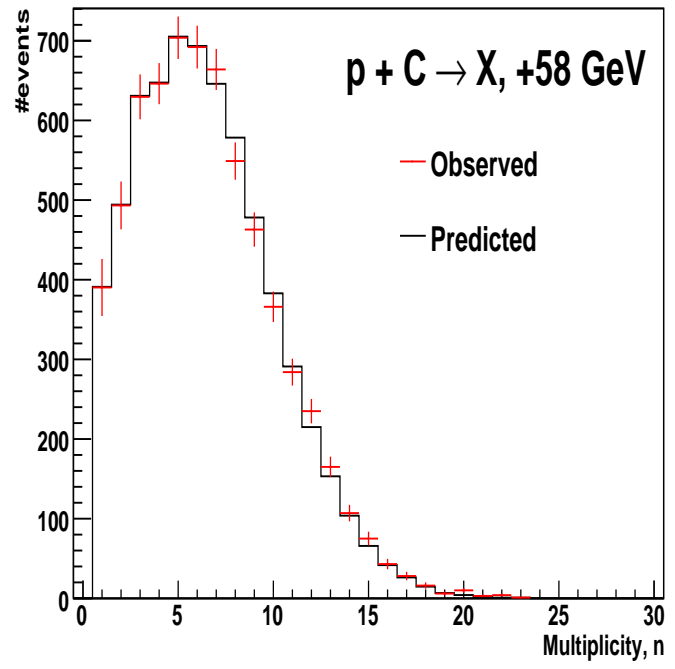

(a)

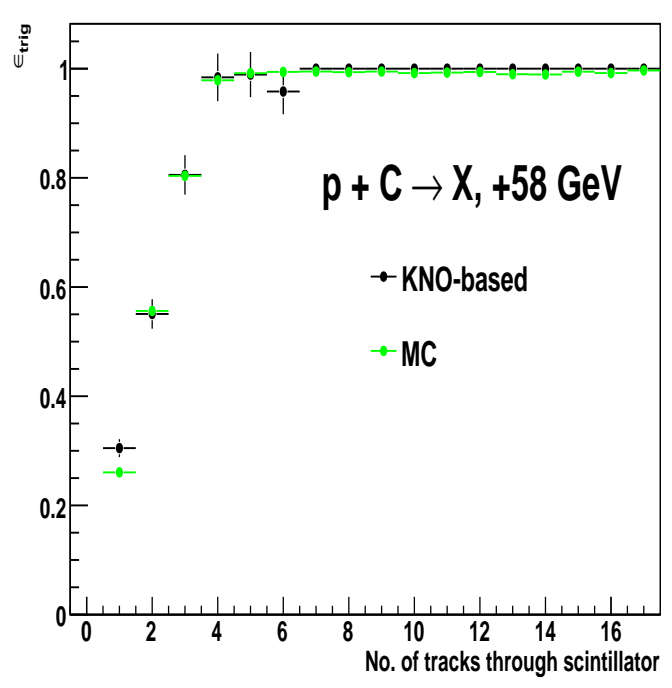

(b)

Figure 3.37: (a) Comparison of observed and predicted distributions at the minimum and (b) the KNO-based trigger efficiencies from the fit compared with the MC efficiencies, for 58 $\mathrm{GeV} / \mathrm{c} \mathrm{p}+\mathrm{C}$ interactions.

the comparison of the KNO-based and MC efficiencies as a function of number of tracks through the scintillator for $120 \mathrm{GeV} / \mathrm{c}$ p $+\mathrm{C}$ interactions are shown in figure 3.39 .

\subsection{Corrections from Monte Carlo}

All the event and track selection requirements explained in section 3.2 are ON in the analysis. When a cut is applied, there are inefficiencies caused due to it and these need to be corrected for. These corrections include interaction trigger and cut inefficiency corrections, geometrical acceptance corrections, and reconstruction inefficiency corrections, and all of these are calculated using the reconstructed MC. The average values of these efficiencies and acceptance are calculated for correcting the total inelastic cross sections using $\mathrm{LH}_{2}, \mathrm{C}$, Be and Bi targets at different beam momenta. All these corrections are also calculated in bins of reconstructed momentum for correcting charged particle production cross sections in bins of momentum for 58 and $120 \mathrm{GeV} / \mathrm{c}$ p $+\mathrm{C}$ interactions. All of these corrections are discussed below. 


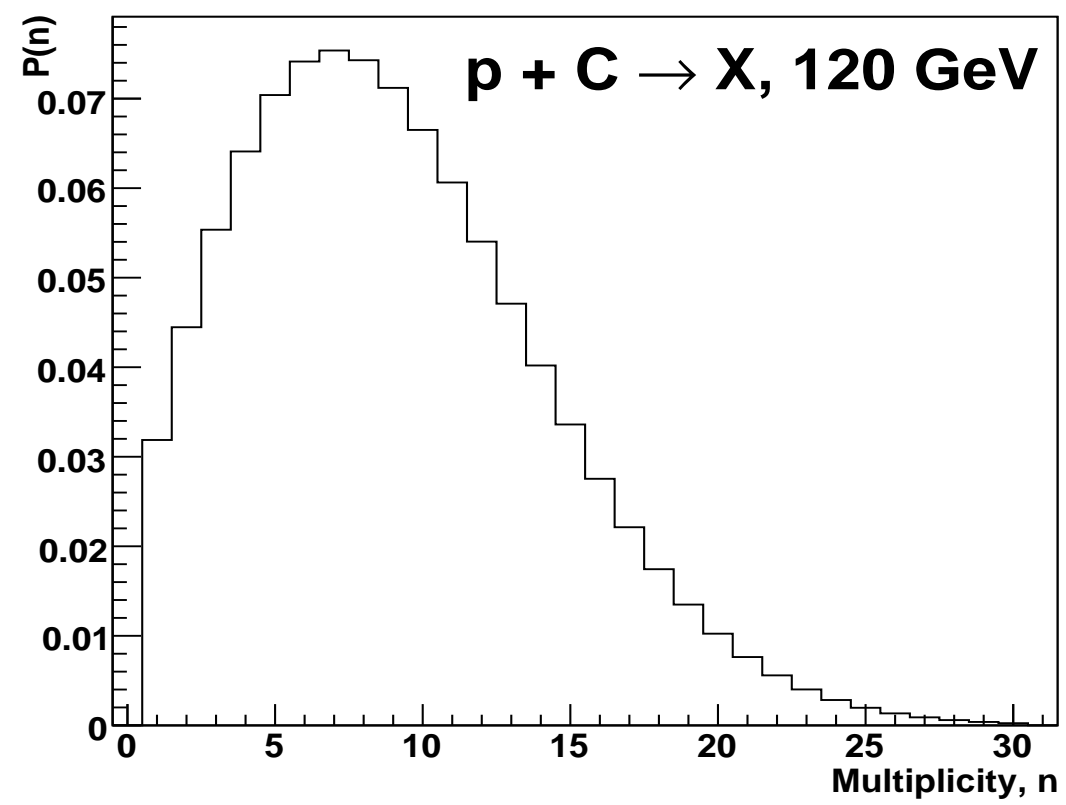

Figure 3.38: KNO true probability distribution for $120 \mathrm{GeV} / \mathrm{c}$ p $+\mathrm{C}$ interactions.

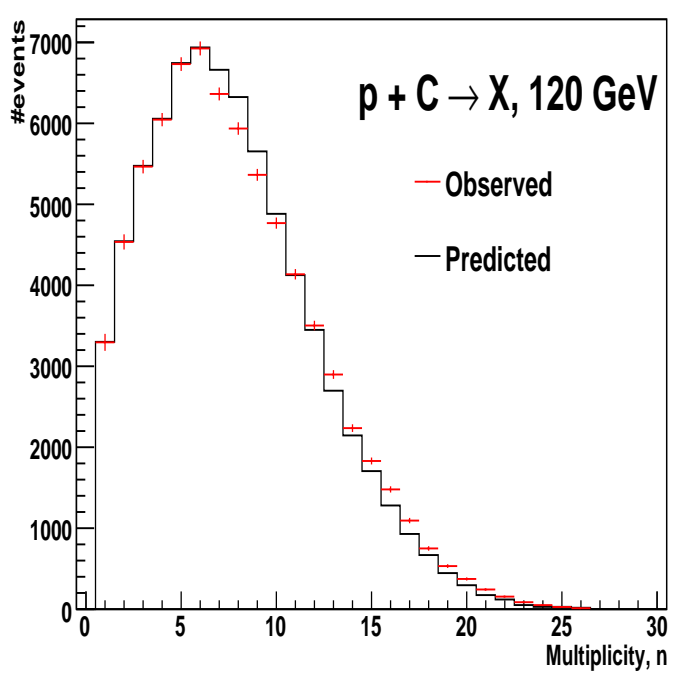

(a)

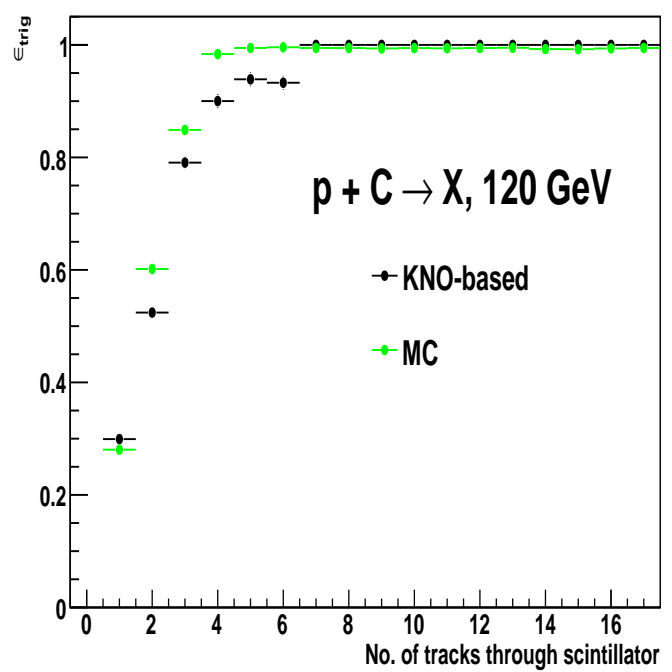

(b)

Figure 3.39: (a) Comparison of observed and predicted distributions at the minimum and (b) the KNO-based trigger efficiencies from the fit compared with the MC efficiencies, for $120 \mathrm{GeV} / \mathrm{c} \mathrm{p}+\mathrm{C}$ interactions. 


\subsubsection{Trigger efficiency}

The trigger efficiencies in bins of tracks passing through the scintillator were discussed in section 3.4. The average value of trigger efficiency is calculated by dividing the number of events where the scintillator is fired, by the total number of events. All the requirements including good beam selection cuts, track selection cuts and the event selection cuts are ON while counting both these numbers.

For the calculation of trigger efficiencies in bins of momentum, two histograms are made and division of these two gives the efficiency. One is the momentum spectrum of all the tracks which forms the denominator, and the other is the momentum spectrum of the tracks where the scintillator is fired, and that forms the numerator. While making these histograms, the requirements including good beam selection cuts, track selection cuts, and the event selection cuts are ON.

\subsubsection{Cut efficiency}

We might also loose some good events by requiring a cut. These need to be corrected for. The individual cut efficiencies for all the cuts applied in this analysis are calculated. These cuts are cut on beam track index, cut on sum of momenta of tracks per event, cut on track multiplicity, cuts on longitudinal and transverse vertex positions, and $\Delta p_{T}$ cut. The average value of a partcular cut efficiency is calculated by dividing the number of events after applying that cut by the number of events before applying that cut. The other requirements including good beam selection, interaction trigger and all the other cuts (mentioned above) are ON while counting both these numbers.

For cut efficiencies in bins of momentum, we have calculated combined cut efficiencies for all the cuts involved, except the multiplicity cut whose efficiency is calculated separately as this cut is correlated to the $\Delta \mathrm{p}_{T}$ cut. Out of the two histograms used in the calculation of combined cut efficiency in bins of momentum, one is the momentum spectrum of the tracks with cuts not applied which forms the denominator, and the other is the momentum spectrum of tracks with cuts applied, and that forms the numerator. While making these histograms, the other requirements including good beam selection cuts and interaction trigger are ON.

\subsubsection{Geometrical acceptance $\times$ Reconstruction efficiency}

Geometrical acceptance means whether a track makes it to the TPC or not. A track can miss the TPC because of its high angle $\left(\mathrm{p}_{T}\right)$. Reconstruction inefficiency is caused due to the tracks which could not be reconstructed. These tracks which didn't make it to the TPC and 
the tracks which made it to the TPC but for some reason couldn't be reconstructed, need to be corrected for. The reason for a track not being reconstructed can be - low momentum of the track, very low number of TPC hits due to the dead sections in the TPC, etc.

For the calculation of geometrical acceptance $\times$ reconstruction efficiency denoted as $\mathcal{A} \epsilon_{\mathcal{R}}$, we require that the true MC track (at the generation level) should have a matching reconstructed MC track. The average value of $\mathcal{A} \epsilon_{\mathcal{R}}$ is calculated by dividing the number of true MC tracks which have matched reconstructed MC tracks by the total number of true MC tracks.

For the calculation of $\mathcal{A} \epsilon_{\mathcal{R}}$ in bins of momentum, out of two histograms, one is the momentum spectrum of all the true MC tracks which forms the denominator, and the other is the momentum spectrum of the true MC tracks with matched reconstructed tracks, and that forms the numerator.

The average values of all the efficiencies described above are listed in tables 3.5 and 3.6 for $\mathrm{p}+\mathrm{p}$ interactions at 58 and $85 \mathrm{GeV} / \mathrm{c}$, respectively. The efficiencies for $\mathrm{p}+\mathrm{C}$ interactions at 58 and $120 \mathrm{GeV} / \mathrm{c}$ are listed in tables 3.7 and 3.8 , respectively.

\begin{tabular}{|c|c|c|c|}
\hline Cut name & $\begin{array}{l}\mathbf{N}_{\text {event }} \text { before } \\
\text { cut }\end{array}$ & $\mathbf{N}_{\text {event }}$ after cut & Efficiency \\
\hline Beam track index $\geq 0$ & 545472 & 543323 & $0.9961 \pm 0.000085$ \\
\hline$\sum p_{\text {trk }} \leq 1.1 \times p_{\text {beam }}$ & 559224 & 543323 & $0.9716 \pm 0.000222$ \\
\hline Multiplicity $\geq 3$ & 558884 & 543323 & $0.9722 \pm 0.000220$ \\
\hline $\mathrm{Z}$ vertex: $-15<\Delta \mathrm{Z}<15 \mathrm{~cm}$ & 544563 & 543323 & $0.9977 \pm 0.000065$ \\
\hline Elliptical XY vertex cut & 552604 & 543323 & $0.9832 \pm 0.000173$ \\
\hline $\begin{array}{l}\Delta p_{T}: \sum p_{T_{\text {trk }}}-p_{T_{\text {beam }}} \geq 150 \\
\mathrm{MeV}\end{array}$ & 596703 & 543323 & $0.9105 \pm 0.000369$ \\
\hline Trigger efficiency & 608784 & 543323 & $0.8925 \pm 0.000397$ \\
\hline $\mathcal{A} \epsilon_{\mathcal{R}}$ & 5536149 & 4932701 & $0.8910 \pm 0.000132$ \\
\hline
\end{tabular}

Table 3.5: The average trigger and cut efficiencies, and $\mathcal{A} \epsilon_{\mathcal{R}}$ used for the inelastic cross section calculation for $\mathrm{p}+\mathrm{p}$ interactions at $58 \mathrm{GeV} / \mathrm{c}$.

The efficiencies for $\mathrm{p}+\mathrm{Be}$ interactions at 58 and $120 \mathrm{GeV} / \mathrm{c}$ are listed in tables 3.9 and 3.10, respectively. The efficiencies for $\mathrm{p}+\mathrm{Bi}$ interactions at 58 and $120 \mathrm{GeV} / \mathrm{c}$ are listed in tables 3.11 and 3.12 , respectively.

The trigger efficiencies, multiplicity cut efficiencies and the combined cut efficiencies in bins of momentum for 58 and $120 \mathrm{GeV} / \mathrm{c} \mathrm{p}+\mathrm{C}$ interactions are shown in figures 3.40, 3.41 and 3.42 , respectively. $\mathcal{A} \epsilon_{\mathcal{R}}$ for 58 and $120 \mathrm{GeV} / \mathrm{c}$ p $+\mathrm{C}$ interactions are shown in figure 3.43 .

As the momentum increases, the trigger efficiency decreases since the track multiplicity is expected to decrease with the increasing momentum. The multiplicity cut efficiency 


\begin{tabular}{|l|l|l|l|}
\hline Cut name & $\begin{array}{l}\mathbf{N}_{\text {event }} \text { before } \\
\text { cut }\end{array}$ & $\mathbf{N}_{\text {event }}$ after cut & Efficiency \\
\hline Beam track index $\geq 0$ & $1.05242 \mathrm{e}+06$ & $1.04694 \mathrm{e}+06$ & $0.9948 \pm 0.000070$ \\
\hline$\sum p_{\text {trk }} \leq 1.1 \times p_{\text {beam }}$ & $1.0815 \mathrm{e}+06$ & $1.04694 \mathrm{e}+06$ & $0.9680 \pm 0.000169$ \\
\hline Multiplicity $\geq 3$ & $1.08321 \mathrm{e}+06$ & $1.04694 \mathrm{e}+06$ & $0.9665 \pm 0.000173$ \\
\hline $\mathrm{Z}$ vertex: $-15<\Delta \mathrm{Z}<15 \mathrm{~cm}$ & $1.0507 \mathrm{e}+06$ & $1.04694 \mathrm{e}+06$ & $0.9964 \pm 0.000058$ \\
\hline Elliptical XY vertex cut & $1.06621 \mathrm{e}+06$ & $1.04694 \mathrm{e}+06$ & $0.9819 \pm 0.000129$ \\
\hline $\begin{array}{l}\Delta p_{T}: \sum p_{T_{\text {trk }}}-p_{T_{\text {beam }}} \geq 150 \\
\text { MeV }\end{array}$ & $1.14409 \mathrm{e}+06$ & $1.04694 \mathrm{e}+06$ & $0.9151 \pm 0.000261$ \\
\hline Trigger efficiency & $1.13295 \mathrm{e}+06$ & $1.04694 \mathrm{e}+06$ & $0.9241 \pm 0.000249$ \\
\hline $\mathcal{A} \epsilon_{\mathcal{R}}$ & 11419593 & 10031589 & $0.8785 \pm 0.000097$ \\
\hline
\end{tabular}

Table 3.6: The average trigger and cut efficiencies, and $\mathcal{A} \epsilon_{\mathcal{R}}$ used for the inelastic cross section calculation for $\mathrm{p}+\mathrm{p}$ interactions at $85 \mathrm{GeV} / \mathrm{c}$.

\begin{tabular}{|c|c|c|c|}
\hline Cut name & $\begin{array}{l}\mathbf{N}_{\text {event }} \text { before } \\
\text { cut }\end{array}$ & $\mathbf{N}_{\text {event }}$ after cut & Efficiency \\
\hline Beam track index $\geq 0$ & 125410 & 124907 & $0.9960 \pm 0.000178$ \\
\hline$\sum p_{\text {trk }} \leq 1.1 \times p_{\text {beam }}$ & 128273 & 124907 & $0.9738 \pm 0.000446$ \\
\hline Multiplicity $\geq 3$ & 129774 & 124907 & $0.9625 \pm 0.000527$ \\
\hline $\mathrm{Z}$ vertex: $-4<\Delta \mathrm{Z}<6 \mathrm{~cm}$ & 125556 & 124907 & $0.9948 \pm 0.000202$ \\
\hline Vertex radius $\leq 2 \mathrm{~cm}$ & 126142 & 124907 & $0.9902 \pm 0.000277$ \\
\hline $\begin{array}{l}\Delta p_{T}: \sum p_{T_{\text {trk }}}-p_{T_{\text {beam }}} \geq 150 \\
\mathrm{MeV}\end{array}$ & 134474 & 124907 & $0.9289 \pm 0.000701$ \\
\hline Trigger efficiency & 129305 & 124907 & $0.9660 \pm 0.000504$ \\
\hline $\mathcal{A} \epsilon_{\mathcal{R}}$ & 1441741 & 1249636 & $0.8668 \pm 0.000283$ \\
\hline
\end{tabular}

Table 3.7: The average trigger and cut efficiencies, and $\mathcal{A} \epsilon_{\mathcal{R}}$ used for the inelastic cross section calculation for $\mathrm{p}+\mathrm{C}$ interactions at $58 \mathrm{GeV} / \mathrm{c}$. 


\begin{tabular}{|c|c|c|c|}
\hline Cut name & $\begin{array}{l}\mathbf{N}_{\text {event }} \text { before } \\
\text { cut }\end{array}$ & $\mathbf{N}_{\text {event }}$ after cut & Efficiency \\
\hline Beam track index $\geq 0$ & 183100 & 182343 & $0.9959 \pm 0.000150$ \\
\hline$\sum p_{\text {trk }} \leq 1.1 \times p_{\text {beam }}$ & 188470 & 182343 & $0.9675 \pm 0.000409$ \\
\hline Multiplicity $\geq 3$ & 188729 & 182343 & $0.9662 \pm 0.000416$ \\
\hline $\mathrm{Z}$ vertex: $-4<\Delta \mathrm{Z}<6 \mathrm{~cm}$ & 184024 & 182343 & $0.9909 \pm 0.000222$ \\
\hline Vertex radius $\leq 2 \mathrm{~cm}$ & 182344 & 182343 & $1.0 \pm 0.0000055$ \\
\hline $\begin{array}{l}\Delta_{\mathrm{MeV}}: \sum p_{T_{\text {trk }}}-p_{T_{\text {beam }}} \geq 150 \\
\end{array}$ & 194615 & 182343 & $0.9369 \pm 0.000551$ \\
\hline Trigger efficiency & 186067 & 182343 & $0.98 \pm 0.000325$ \\
\hline $\mathcal{A} \epsilon_{\mathcal{R}}$ & 2348391 & 2016908 & $0.8588 \pm 0.000227$ \\
\hline
\end{tabular}

Table 3.8: The average trigger and cut efficiencies, and $\mathcal{A} \epsilon_{\mathcal{R}}$ used for the inelastic cross section calculation for $\mathrm{p}+\mathrm{C}$ interactions at $120 \mathrm{GeV} / \mathrm{c}$.

\begin{tabular}{|c|c|c|c|}
\hline Cut name & $\begin{array}{l}\mathbf{N}_{\text {event }} \text { before } \\
\text { cut }\end{array}$ & $\mathbf{N}_{\text {event }}$ after cut & Efficiency \\
\hline Beam track index $\geq 0$ & 68558 & 68296 & $0.9962 \pm 0.000236$ \\
\hline$\sum p_{\text {trk }} \leq 1.1 \times p_{\text {beam }}$ & 70119 & 68296 & $0.9740 \pm 0.000601$ \\
\hline Multiplicity $\geq 3$ & 70794 & 68296 & $0.9647 \pm 0.000693$ \\
\hline $\mathrm{Z}$ vertex: $-4<\Delta \mathrm{Z}<6 \mathrm{~cm}$ & 68674 & 68296 & $0.9945 \pm 0.000282$ \\
\hline Vertex radius $\leq 2 \mathrm{~cm}$ & 68994 & 68296 & $0.9899 \pm 0.000381$ \\
\hline $\begin{array}{l}\Delta p_{T}: \sum p_{T_{\text {trk }}}-p_{T_{\text {beam }}} \geq 150 \\
\mathrm{MeV}\end{array}$ & 73773 & 68296 & $0.9258 \pm 0.000965$ \\
\hline Trigger efficiency & 71225 & 68296 & $0.9589 \pm 0.000744$ \\
\hline $\mathcal{A} \epsilon_{\mathcal{R}}$ & 752420 & 648081 & $0.8613 \pm 0.000398$ \\
\hline
\end{tabular}

Table 3.9: The average trigger and cut efficiencies, and $\mathcal{A} \epsilon_{\mathcal{R}}$ used for the inelastic cross section calculation for $\mathrm{p}+$ Be interactions at $58 \mathrm{GeV} / \mathrm{c}$. 


\begin{tabular}{|c|c|c|c|}
\hline Cut name & $\begin{array}{l}\mathbf{N}_{\text {event }} \text { before } \\
\text { cut }\end{array}$ & $\mathbf{N}_{\text {event }}$ after cut & Efficiency \\
\hline Beam track index $\geq 0$ & 55432 & 55158 & $0.9951 \pm 0.000298$ \\
\hline$\sum p_{\text {trk }} \leq 1.1 \times p_{\text {beam }}$ & 56876 & 55158 & $0.9698 \pm 0.000718$ \\
\hline Multiplicity $\geq 3$ & 57222 & 55158 & $0.9639 \pm 0.000779$ \\
\hline $\mathrm{Z}$ vertex: $-4<\Delta \mathrm{Z}<6 \mathrm{~cm}$ & 55663 & 55158 & $0.9909 \pm 0.000402$ \\
\hline Vertex radius $\leq 2 \mathrm{~cm}$ & 55158 & 55158 & $1.0 \pm 0.0$ \\
\hline $\begin{array}{l}\Delta p_{T}: \sum p_{T_{\text {trk }}}-p_{T_{\text {beam }}} \geq 150 \\
\mathrm{MeV}\end{array}$ & 58994 & 55158 & $0.9350 \pm 0.001015$ \\
\hline Trigger efficiency & 56370 & 55158 & $0.9785 \pm 0.000611$ \\
\hline $\mathcal{A} \epsilon_{\mathcal{R}}$ & 674170 & 571357 & $0.8475 \pm 0.000438$ \\
\hline
\end{tabular}

Table 3.10: The average trigger and cut efficiencies, and $\mathcal{A} \epsilon_{\mathcal{R}}$ used for the inelastic cross section calculation for $\mathrm{p}+\mathrm{Be}$ interactions at $120 \mathrm{GeV} / \mathrm{c}$.

\begin{tabular}{|c|c|c|c|}
\hline Cut name & $\begin{array}{l}\mathbf{N}_{\text {event }} \text { before } \\
\text { cut }\end{array}$ & $\mathbf{N}_{\text {event }}$ after cut & Efficiency \\
\hline Beam track index $\geq 0$ & 118895 & 118477 & $0.9965 \pm 0.000172$ \\
\hline$\sum p_{\text {trk }} \leq 1.1 \times p_{\text {beam }}$ & 121674 & 118477 & $0.9737 \pm 0.000459$ \\
\hline Multiplicity $\geq 3$ & 120225 & 118477 & $0.9855 \pm 0.000345$ \\
\hline $\mathrm{Z}$ vertex: $-4<\Delta \mathrm{Z}<6 \mathrm{~cm}$ & 118785 & 118477 & $0.9974 \pm 0.000148$ \\
\hline Vertex radius $\leq 2 \mathrm{~cm}$ & 119758 & 118477 & $0.9893 \pm 0.000297$ \\
\hline $\begin{array}{l}\Delta p_{T}: \sum p_{T_{\text {trk }}}-p_{T_{\text {beam }}} \geq 150 \\
\mathrm{MeV}\end{array}$ & 124496 & 118477 & $0.9517 \pm 0.000608$ \\
\hline Trigger efficiency & 120551 & 118477 & $0.9828 \pm 0.000375$ \\
\hline $\mathcal{A} \epsilon_{\mathcal{R}}$ & 2276457 & 1633576 & $0.7176 \pm 0.000298$ \\
\hline
\end{tabular}

Table 3.11: The average trigger and cut efficiencies, and $\mathcal{A} \epsilon_{\mathcal{R}}$ used for the inelastic cross section calculation for $\mathrm{p}+\mathrm{Bi}$ interactions at $58 \mathrm{GeV} / \mathrm{c}$. 


\begin{tabular}{|c|c|c|c|}
\hline Cut name & $\begin{array}{l}\mathbf{N}_{\text {event }} \text { before } \\
\text { cut }\end{array}$ & $\mathbf{N}_{\text {event }}$ after cut & Efficiency \\
\hline Beam track index $\geq 0$ & 49001 & 48830 & $0.9965 \pm 0.000266$ \\
\hline$\sum p_{\text {trk }} \leq 1.1 \times p_{\text {beam }}$ & 50468 & 48830 & $0.9675 \pm 0.000789$ \\
\hline Multiplicity $\geq 3$ & 49718 & 48830 & $0.9821 \pm 0.000594$ \\
\hline $\mathrm{Z}$ vertex: $-4<\Delta \mathrm{Z}<6 \mathrm{~cm}$ & 49074 & 48830 & $0.9950 \pm 0.000318$ \\
\hline Vertex radius $\leq 2 \mathrm{~cm}$ & 48831 & 48830 & $0.99998 \pm 0.000021$ \\
\hline $\begin{array}{l}\Delta p_{T}: \sum p_{T_{\text {trk }}}-p_{T_{\text {beam }}} \geq 150 \\
\mathrm{MeV}\end{array}$ & 51369 & 48830 & $0.9506 \pm 0.000956$ \\
\hline Trigger efficiency & 49408 & 48830 & $0.9883 \pm 0.000484$ \\
\hline $\mathcal{A} \epsilon_{\mathcal{R}}$ & 1287523 & 950315 & $0.7381 \pm 0.000387$ \\
\hline
\end{tabular}

Table 3.12: The average trigger and cut efficiencies, and $\mathcal{A} \epsilon_{\mathcal{R}}$ used for the inelastic cross section calculation for $\mathrm{p}+\mathrm{Bi}$ interactions at $120 \mathrm{GeV} / \mathrm{c}$.

decreases with momentum since low multiplicities are expected at the higher momenta. The combined cut efficiency decreases with the momentum. The dominating cut is the $\Delta p_{T}$ cut, the reason being we expect to have more beam-like particles at the higher momenta. $\mathcal{A} \epsilon_{\mathcal{R}}$ increases with momentum.

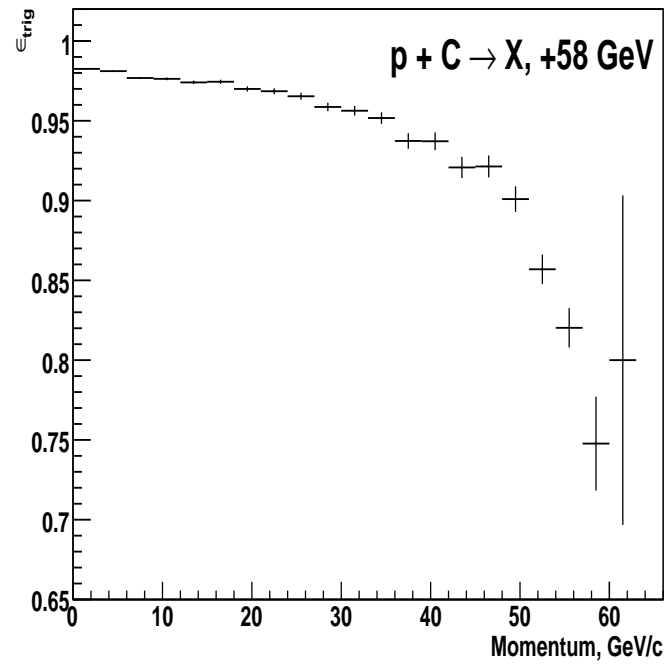

(a)

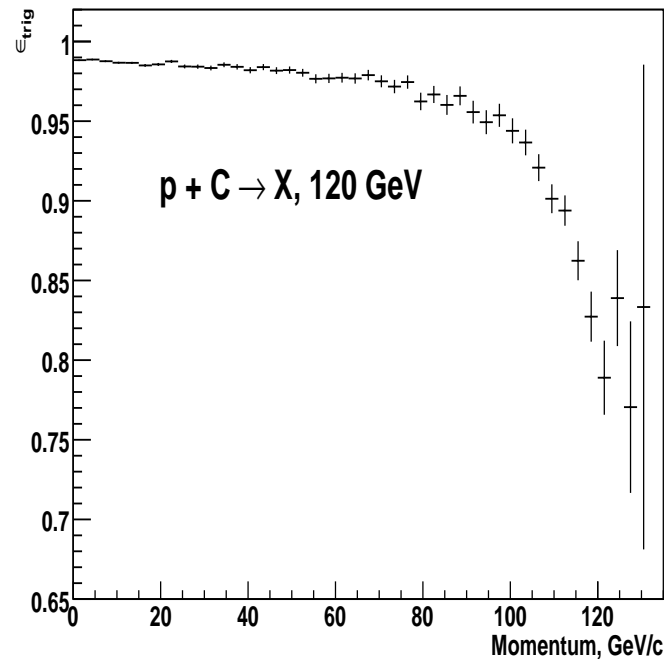

(b)

Figure 3.40: The trigger efficiencies in bins of momentum for (a) 58 and (b) $120 \mathrm{GeV} / \mathrm{c} \mathrm{p}+\mathrm{C}$ interactions. 


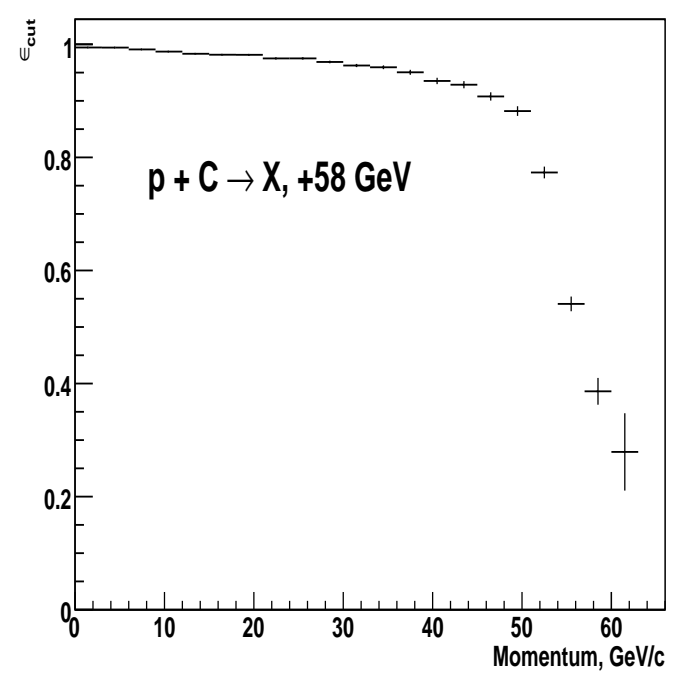

(a)

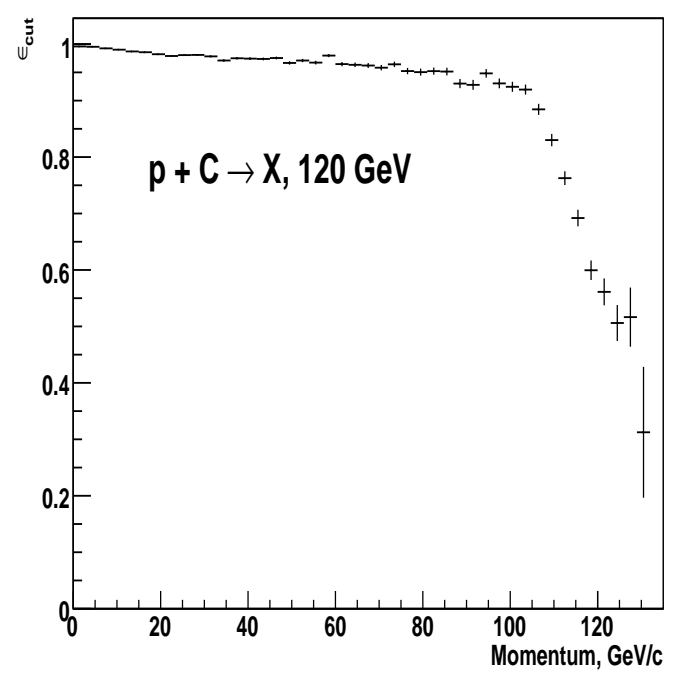

(b)

Figure 3.41: The multiplicity cut efficiencies in bins of momentum for (a) 58 and (b) 120 $\mathrm{GeV} / \mathrm{c} \mathrm{p}+\mathrm{C}$ interactions.

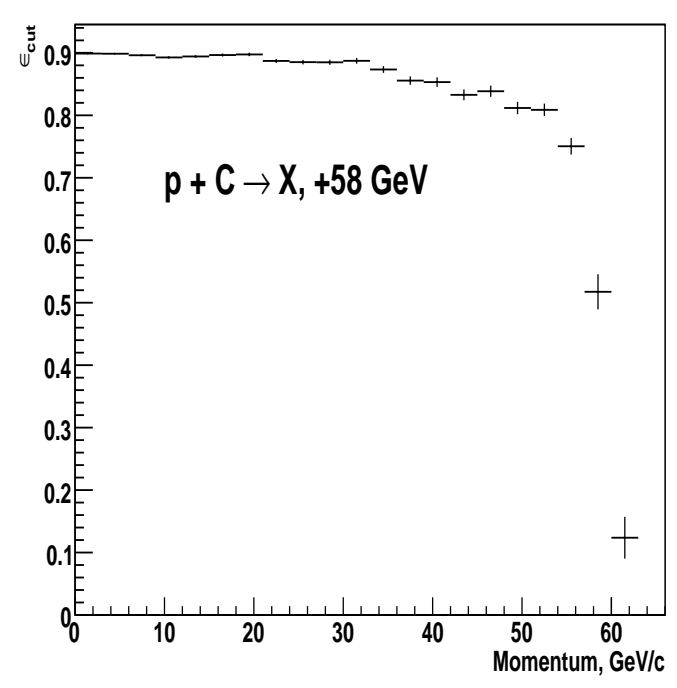

(a)

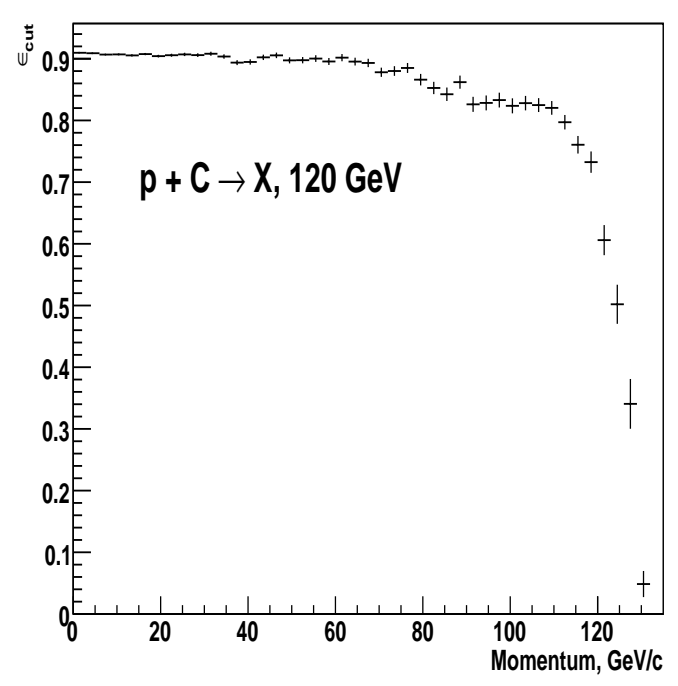

(b)

Figure 3.42: The combined cut efficiencies in bins of momentum for (a) 58 and (b) 120 $\mathrm{GeV} / \mathrm{c} \mathrm{p}+\mathrm{C}$ interactions. 


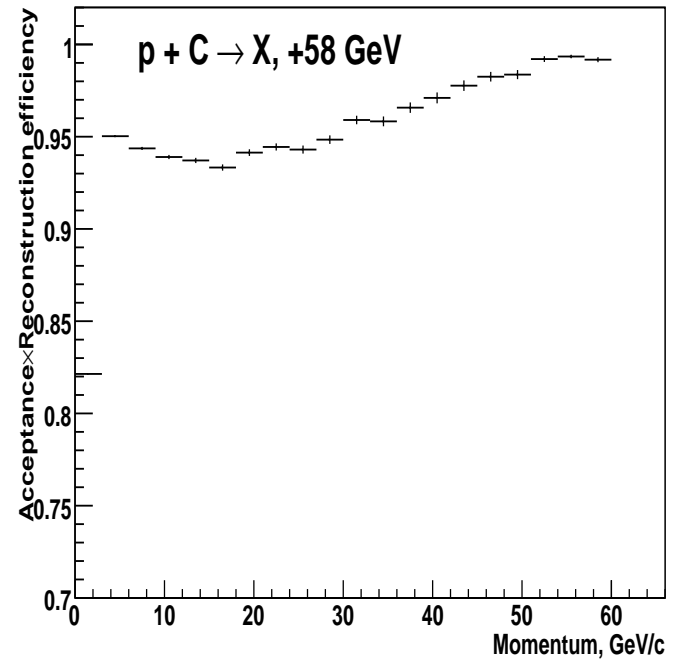

(a)

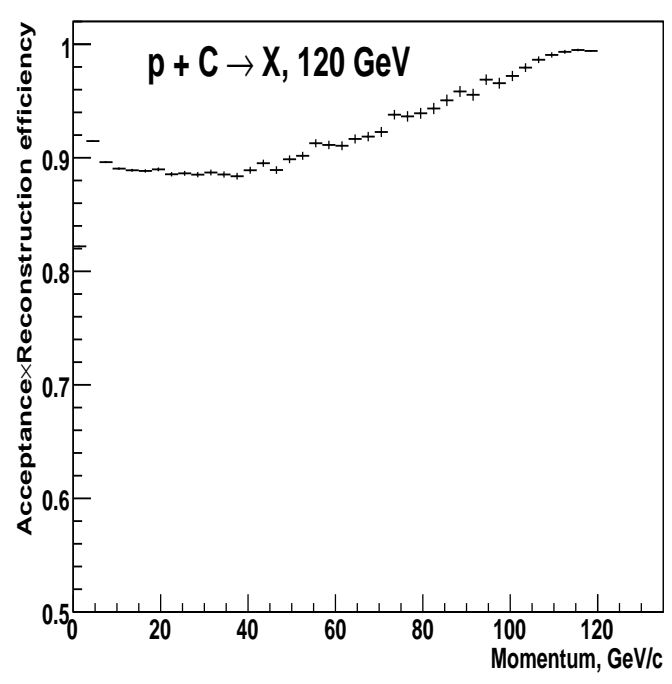

(b)

Figure 3.43: $\mathcal{A} \epsilon_{\mathcal{R}}$ in bins of momentum for (a) 58 and (b) $120 \mathrm{GeV} / \mathrm{c} \mathrm{p}+\mathrm{C}$ interactions. 


\section{Chapter 4}

\section{Pion Identification}

This chapter describes the techniques used to identify charged particles $\left(\pi^{ \pm} / \mathrm{p} / \overline{\mathrm{p}}\right.$ etc. $)$. The unfolding of the data pion momentum spectrum using the Monte Carlo is described. The Monte Carlo corrections in bins of true momentum are also described. The data sets used for this analysis are 58 and $120 \mathrm{GeV} / \mathrm{c}$ proton on carbon $(\mathrm{p}+\mathrm{C})$ interactions.

\subsection{Particle Identification}

Two detectors TPC and RICH 2 are used to do the particle identification (PID) [104]. The PID using these two detectors is described as follows:

\subsubsection{TPC PID}

The TPC particle identification is based on the specific energy loss ( $\mathrm{dE} / \mathrm{dx}$ ). The particles coming from interactions ionize the $\mathrm{P} 10$ gas $\left(90 \% \mathrm{Ar}\right.$ with $\left.10 \% \mathrm{CH}_{4}\right)$ when they pass through the TPC volume, and create free electrons. The energy loss of the particle due to the ionization depends on its velocity which allows for identification of the particle. The mean energy loss per unit length is given by the Bethe-Bloch function [105]:

$$
-\frac{d E}{d x}=K z^{2} \frac{Z}{A} \frac{1}{\beta^{2}}\left[\frac{1}{2} \ln \frac{2 m_{e} c^{2} \beta^{2} \gamma^{2} T_{\max }}{I^{2}}-\beta^{2}-\frac{\delta}{2}\right] .
$$

Here $\mathrm{T}_{\max }$ is the maximum kinetic energy which can be imparted to a free electron in a single collision, $\mathrm{z}$ is the charge of particle traversing medium, $\mathrm{Z}$ and $\mathrm{A}$ are the atomic number and atomic mass of the medium, $\mathrm{I}$ is the mean ionization energy, $\delta$ is a density effect correction to ionization energy loss, $\mathrm{K}=4 \pi \mathrm{N}_{A} \mathrm{r}_{e}^{2} \mathrm{~m}_{e} \mathrm{c}^{2}=0.307 \mathrm{MeV} \cdot \mathrm{g}^{-1} \cdot \mathrm{cm}^{2}, \mathrm{~m}_{e}$ is the electron mass, $\mathrm{r}_{e}$ is the classical electron radius and $\mathrm{N}_{A}$ is Avogadro number, $\beta$ is the velocity of the particle 
and $\gamma=1 / \sqrt{1-\beta^{2}}$.

Figure 4.1 shows the $\mathrm{dE} / \mathrm{dx}$ as a function of reconstructed momentum of TPC tracks using $120 \mathrm{GeV} / \mathrm{c}$ data on all nuclei. Superimposed are the Bethe-Bloch $\mathrm{dE} / \mathrm{dx}$ predictions for electrons, pions, kaons, protons, deuterons and tritons. From the plot it is clear that the TPC can very well distinguish between the different particles in the momentum region 0 $1 \mathrm{GeV} / \mathrm{c}$.

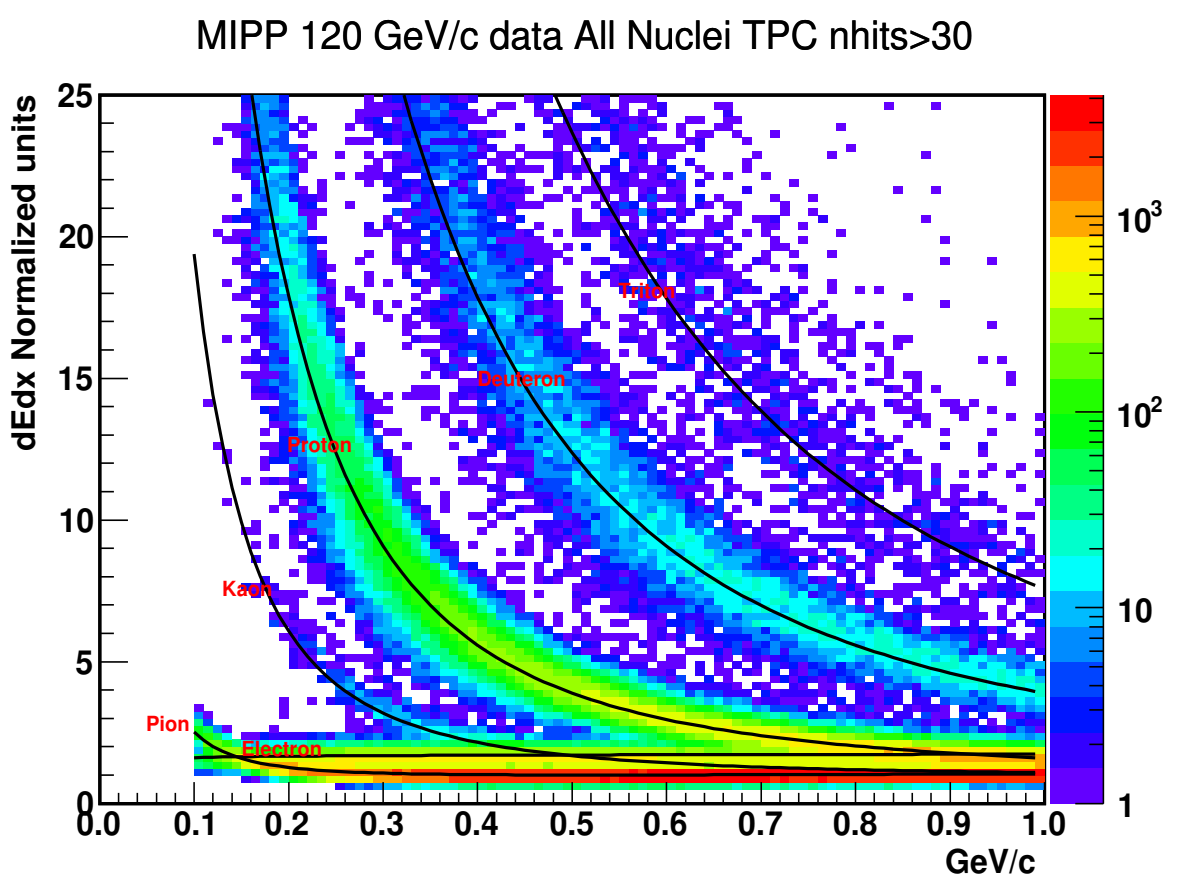

Figure 4.1: dE/dx as a function of reconstructed momentum of TPC tracks using $120 \mathrm{GeV} / \mathrm{c}$ data on all nuclei.

\subsubsection{RICH PID}

The RICH particle identification is based on the Cherenkov effect [106]. The Cherenkov effect occurs when the velocity of a charged particle traversing a medium exceeds the velocity of light in that medium $(\mathrm{c} / \mathrm{n})$, where $\mathrm{n}$ is the index of refraction for the medium. Excited atoms in the vicinity of the particle become polarized and coherently emit radiation at a characteristic angle $\theta$, which is determined by the velocity of the particle and the index of refraction of the medium, using the relation

$$
\cos \theta=1 / \beta n
$$


For a given index of refraction $\mathrm{n}$, no light is emitted below a threshold velocity $\beta_{t h r}=1 / \mathrm{n}$. As the particle velocity increases beyond $\beta_{t h r}$, the light is given off at larger and larger angles up to a maximum angle $\theta_{\max }=\cos ^{-1}(1 / \mathrm{n})$ which occurs for $\beta=1$. Cherenkov light is mainly in the visible and ultraviolet regions and is emitted along the surface of a cone defined by the angle $\theta$.

The RICH detector uses spherical mirrors to focus Cherenkov light onto an array of photomultiplier tubes (PMT's) situated at the focal plane. The light which is emitted as a cone, forms a ring when it reaches the PMT array. From the pattern of hit PMT's, this ring can then be reconstructed. Particles with the same momentum but different masses create rings with different radii, hence measuring the ring radius provides identification of the charged particles travelling through the RICH.

From the expression for the Cherenkov angle, equation 4.2, and the geometry of the $\mathrm{RICH}$, the radius of a ring can be related to $\mathrm{n}$, the refractive index of the radiator gas, and $\beta$ of the particle:

$$
R=F_{L} \sin ^{2} \theta=F_{L} \sqrt{n^{2} \beta^{2}-1}
$$

where $\mathrm{F}_{L}$ is the focal length of the mirror. Thus the reconstructed ring radius provides a measure of the Cherenkov angle.

Figure 4.2 shows the radii of secondary charged particles as a function of particle momentum in the MIPP RICH. Electrons give light in the RICH at practically all the momenta due to their small mass. The pions are above RICH threshold $4.64 \mathrm{GeV} / \mathrm{c}$, kaons above $16.53 \mathrm{GeV} / \mathrm{c}$ and protons above $31.42 \mathrm{GeV} / \mathrm{c}$. From the plot it is clear that the RICH can provide particle identification for momentum up to $120 \mathrm{GeV} / \mathrm{c}$.

\subsection{PID algorithm}

TPC and RICH detectors are used to do the PID. Four hypotheses electron, pion, kaon and proton (e, $\pi, \mathrm{K}$ and $\mathrm{p}$ ) denoted by "H" are considered. Due to finite resolutions in TPC $\mathrm{dE} / \mathrm{dx}$ and Poisson fluctuations in RICH, the particle ID does not result in a unique ID with 100\% certainty [107]. Thus the information from both the PID detectors is best represented in the form of likelihoods. So we use maximum likelihood technique to determine the particle spectra. The quantities $\mathrm{dE} / \mathrm{dx}$ and ring radii are measured for the TPC and $\mathrm{RICH}$, respectively. Both the detectors provide a likelihood for the track being one of four hypotheses i.e. e, $\pi, K$, p. Deuterons and higher mass nuclear fragments are not considered in this analysis. None of the two detectors distinguish between the charge of the particles so the analysis will proceed initially without charge distinction. The purpose of the analysis is not to absolutely 


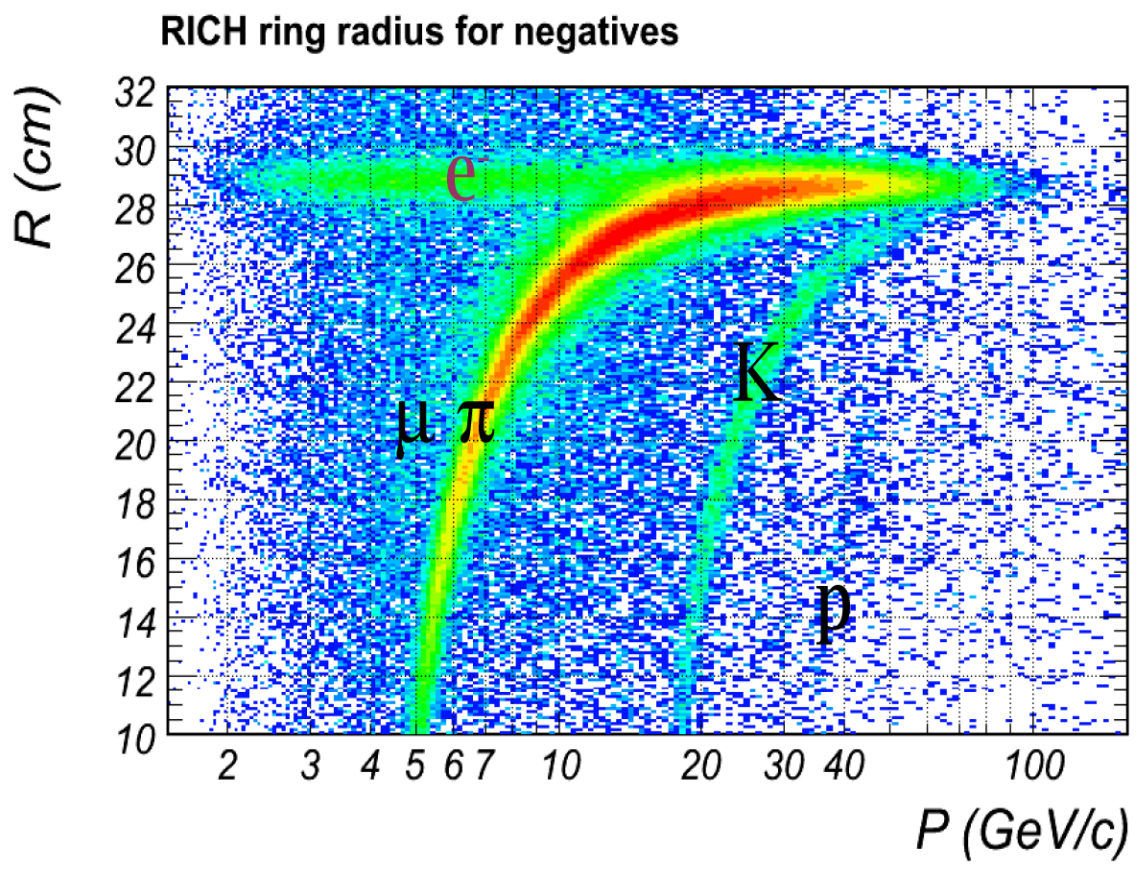

Figure 4.2: Ring radii of negatively charged particles as a function of particle momentum in the MIPP RICH.

determine the identity of a particle but to determine its maximum likelihood momentum spectrum.

\section{How the algorithm works?}

For both the detectors, a weight is calculated using the likelihood of observing the quantity $x(\mathrm{dE} / \mathrm{dx}$ for the TPC and ring radius for the $\mathrm{RICH})$ for a particular hypothesis and sum of the likelihoods of observing that quantity for all the hypotheses i.e.

$$
\text { Weight }=\frac{\text { Likelihood }}{\sum_{\mathrm{H}} \text { Likelihoods }} \text {. }
$$

This is used to weight the momentum spectra. Each track enters all hypothesis dependent plots with its hypothesis dependent weight. The aim is to obtain the momentum spectrum for the pion hypothesis.

Figure $4.3(\mathrm{a})$ shows the momentum spectrum of charged particles from the interactions of $120 \mathrm{GeV} / \mathrm{c}$ protons on $2 \%$ interaction length carbon target. The bump at $120 \mathrm{GeV} / \mathrm{c}$ is due to primary protons that punch through the target either without interacting or diffractively just before the end of the target with small number of secondary particles produced. Figure 
4.3(b) shows the momenta of tracks generated in the carbon target by the FLUKA Monte Carlo (MC) which has been through the MIPP GEANT3-based detector simulation program. The likelihood analysis of both the detectors is described as follows:

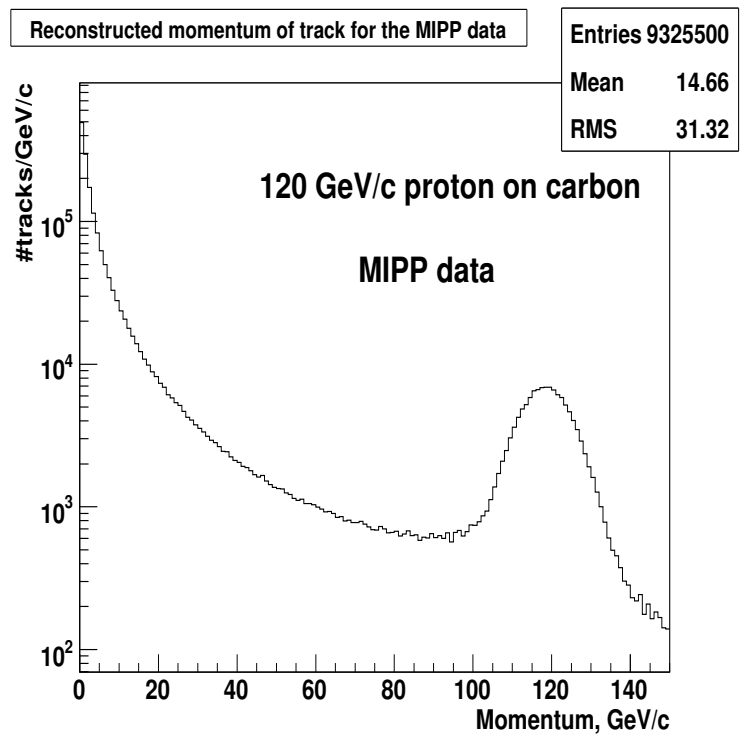

(a)

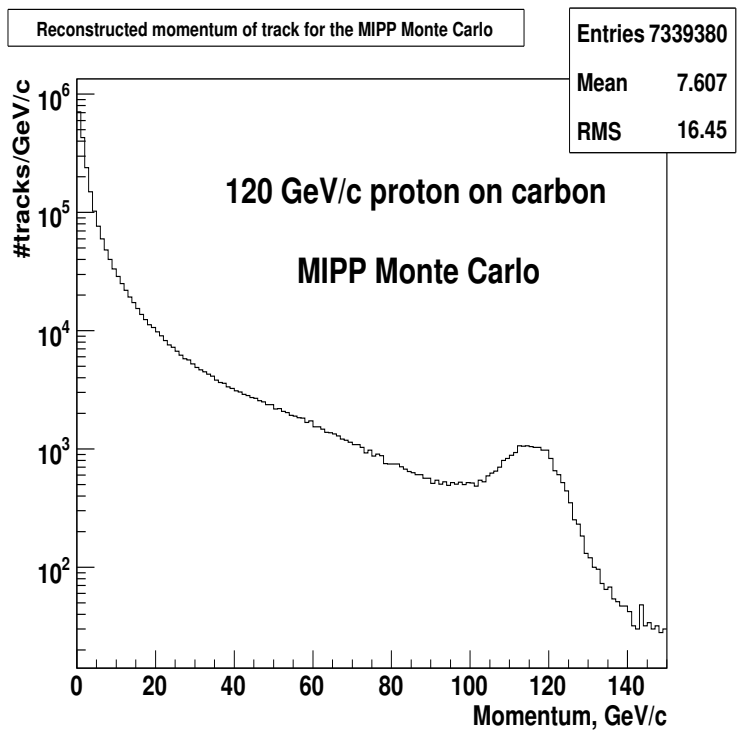

(b)

Figure 4.3: Momentum spectrum for (a) the MIPP data and (b) the MIPP Monte Carlo for $120 \mathrm{GeV} / \mathrm{c} \mathrm{p}+\mathrm{C}$ interactions.

\subsubsection{TPC likelihood calculations}

Figure 4.4(a) shows the $\mathrm{dE} / \mathrm{dx}$ distribution of particles in the data as a function of reconstructed momentum for $120 \mathrm{GeV} / \mathrm{c} \mathrm{p}+\mathrm{C}$ interactions. The curves are the Bethe-Bloch predictions for the hypotheses e, $\pi, \mathrm{K}$ and $\mathrm{p}$. The TPC data are adequately described by the predictions. Figure 4.4(b) shows the $\mathrm{dE} / \mathrm{dx}$ distribution as a function of momentum for the MC sample for $120 \mathrm{GeV} / \mathrm{c} \mathrm{p}+\mathrm{C}$ interactions. The theoretical model predicts the MC behavior adequately except for lower momentum protons where some discrepancy is observed. Similar discrepancy is observed in case of $58 \mathrm{GeV} / \mathrm{c} \mathrm{p}+\mathrm{C}$ interactions. Rather than attempt to tune the $\mathrm{MC}$ to make it match the predictions precisely, we will take into account the differences from the predictions in both the data and MC in a systematic way and use these offsets in the likelihood calculations. 


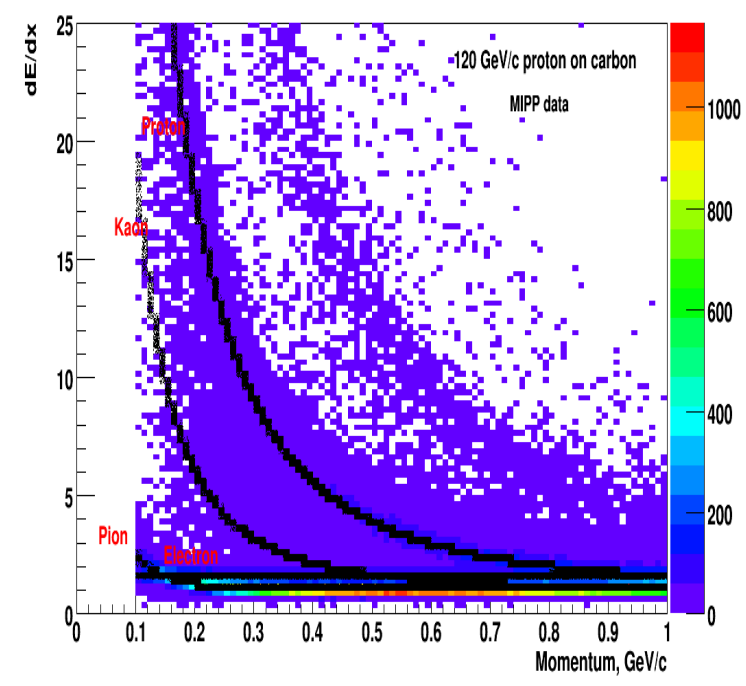

(a)

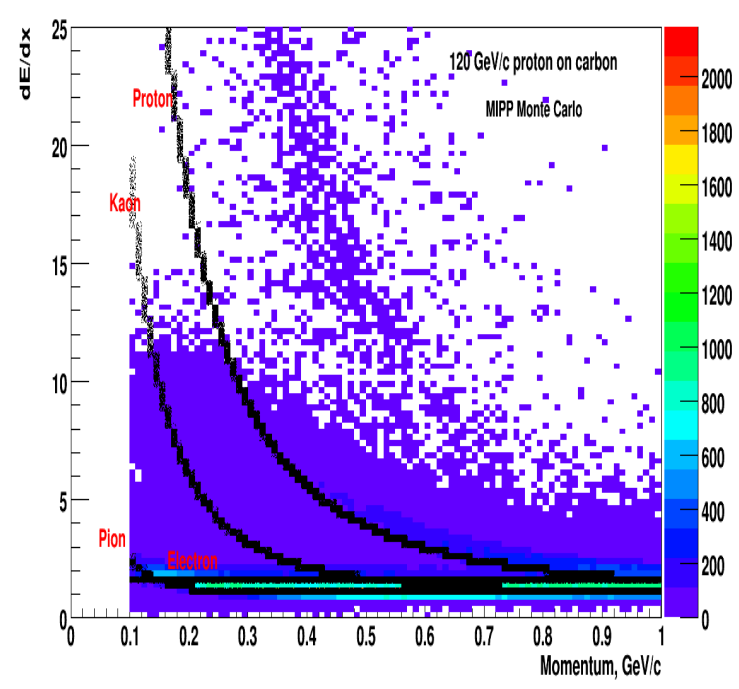

(b)

Figure 4.4: (a) dE/dx as a function of reconstructed momentum of TPC tracks for 120 $\mathrm{GeV} / \mathrm{c} \mathrm{p}+\mathrm{C}$ data. The curves are predictions for the mean $\mathrm{dE} / \mathrm{dx}$ values as a function of reconstructed momentum for the hypotheses e, $\pi, \mathrm{K}$ and p. (b) The same quantity simulated in MIPP Monte Carlo.

\section{$\mathrm{dE} / \mathrm{dx}$ mean offsets and resolutions}

Mean offsets and resolutions are calculated as a function of momentum (p) and number of TPC hits (ntpchits). Tracks with ntpchits below 20 are rejected. Only tracks with $\mathrm{p}<1.0$ $\mathrm{GeV} / \mathrm{c}$ are considered for this analysis. The mean offsets and resolutions are calculated for each hypothesis.

The mean offset for a given hypothesis defined as $<$ (Observed $\mathrm{dE} / \mathrm{dx}$ - Predicted pion $\mathrm{dE} / \mathrm{dx}$ ) $>$ i.e. average value of the quantity (Observed $\mathrm{dE} / \mathrm{dx}$ - Predicted pion $\mathrm{dE} / \mathrm{dx}$ ) (assuming all particles are pions) is determined by interpolating in the 2D LEGO plot in the variables $\mathrm{p}$ and ntpchits. The data sample for the kaons is too sparse to attempt to analyze on its own. Instead, the mean offsets for the pion and proton hypotheses are obtained and mass dependent weighting algorithm is used to obtain the mean offset for the kaon hypothesis. The mean offsets for e/ $\pi / \mathrm{K} / \mathrm{p}$ hypotheses for 58 and $120 \mathrm{GeV} / \mathrm{c} \mathrm{p}+\mathrm{C}$ data are shown in figures 4.5 and 4.6 , respectively.

The $\mathrm{dE} / \mathrm{dx}$ resolution " $\sigma$ " is parametrized as a function of the predicted $\mathrm{dE} / \mathrm{dx}$ "f" 

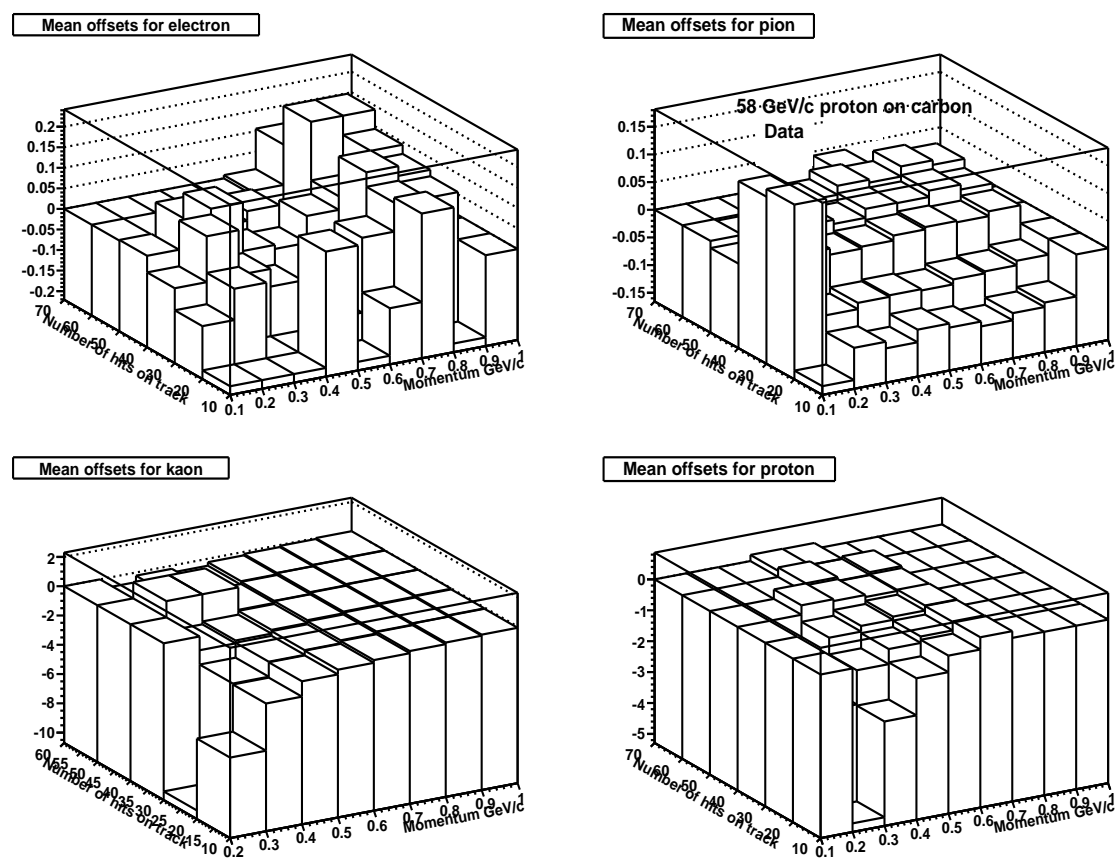

Figure 4.5: Mean offsets defined as $<($ Observed $\mathrm{dE} / \mathrm{dx}$ - Predicted pion $\mathrm{dE} / \mathrm{dx})>$ as a function of momentum and number of TPC hits for e $/ \pi / \mathrm{K} / \mathrm{p}$ hypotheses for $58 \mathrm{GeV} / \mathrm{c} \mathrm{p}+\mathrm{C}$ data.

using the following equation which is patterned after calorimeter resolutions:

$$
\left(\frac{\sigma}{f}\right)^{2}=\frac{C^{2}+\frac{S^{2}}{f}+\frac{N^{2}}{f^{2}}}{n}
$$

The quantities C, S, N are the constant, sampling and noise terms, and n denotes the number of hits in the TPC. The fitted resolutions i.e. sigmas/predicted $\mathrm{dE} / \mathrm{dx}$ for $\mathrm{e} / \pi / \mathrm{K} / \mathrm{p}$ hypotheses for 58 and $120 \mathrm{GeV} / \mathrm{c} \mathrm{p}+\mathrm{C}$ data are shown in figures 4.7 and 4.8 , respectively. The likelihood for any hypothesis is then calculated assuming a Gaussian with the resolution $\sigma$ and the mean offset from the predicted hypothesis $\mathrm{dE} / \mathrm{dx}$ value $\mathrm{f}$.

TPC issues at higher momenta: Large differences between the observed and predicted values of the $\mathrm{dE} / \mathrm{dx}$ are observed for higher momenta $(>\sim 10 \mathrm{GeV} / \mathrm{c})$ for both 58 and $120 \mathrm{GeV} / \mathrm{c} \mathrm{p}+\mathrm{C}$ data, and these are not understood. The plots of the differences between the observed and predicted $\mathrm{dE} / \mathrm{dx}$ values for different momentum slices for $120 \mathrm{GeV} / \mathrm{c} \mathrm{p}+\mathrm{C}$ data are shown in figures $4.9,4.10$ and 4.11 . The mean is shifted from " 0 " and moreover, a higly pronounced tail is seen on the positive end for the momenta $>\sim 10 \mathrm{GeV} / \mathrm{c}$, and these are not understood. So it is decided to use the TPC for the lower momenta only. Similar 

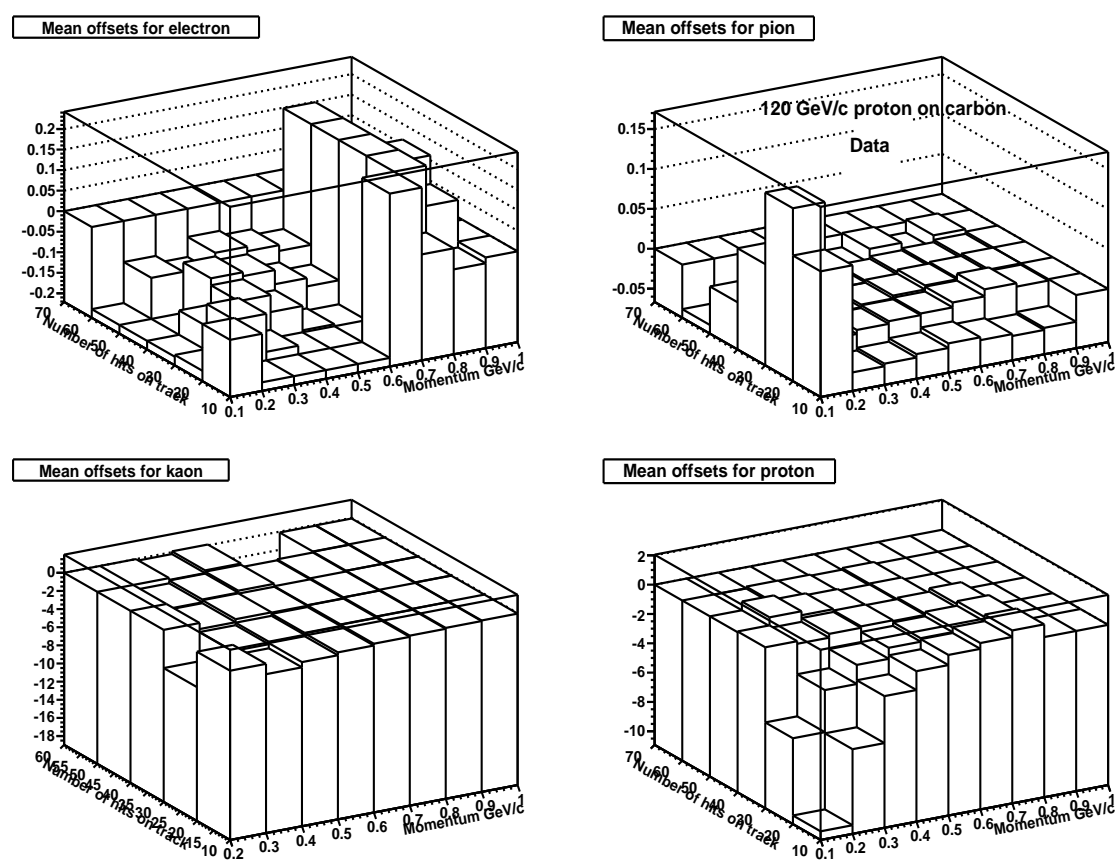

Figure 4.6: Mean offsets defined as $<($ Observed $\mathrm{dE} / \mathrm{dx}$ - Predicted pion $\mathrm{dE} / \mathrm{dx})>$ as a function of momentum and number of TPC hits for e/ $\pi / \mathrm{K} / \mathrm{p}$ hypotheses for $120 \mathrm{GeV} / \mathrm{c}$ $\mathrm{p}+\mathrm{C}$ data.
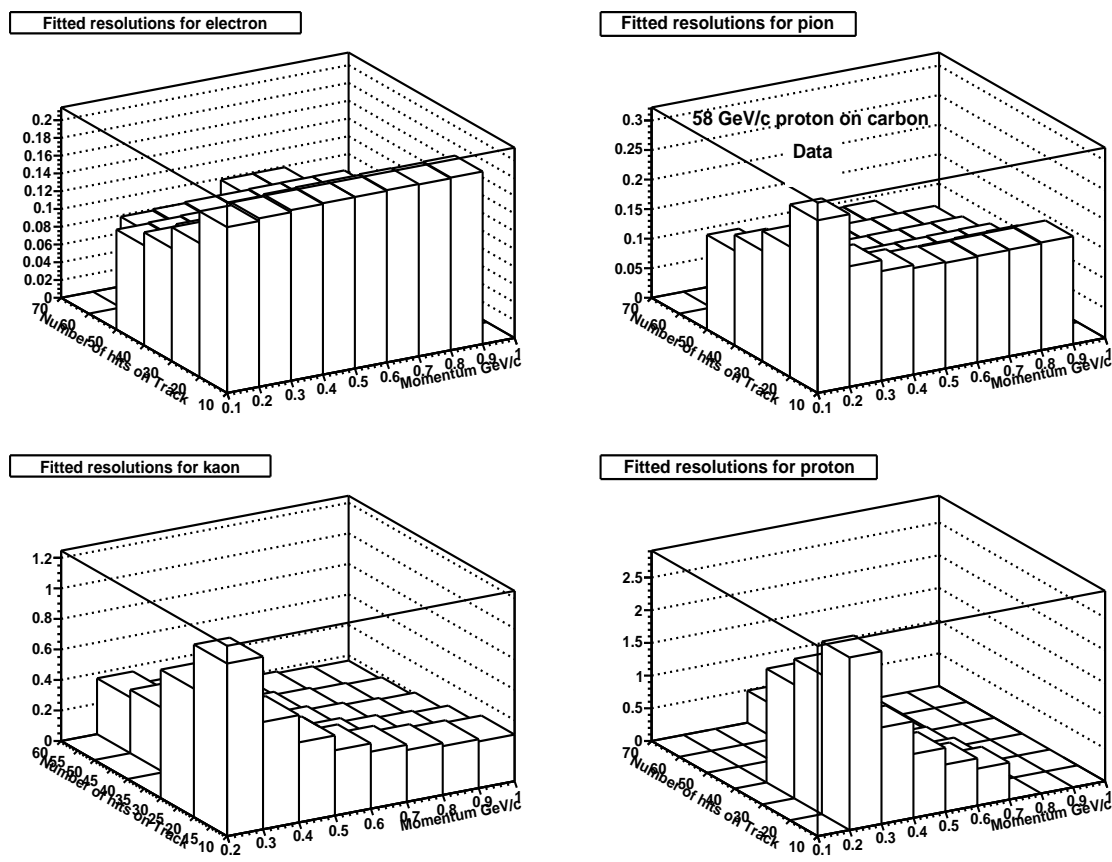

Figure 4.7: The fitted resolutions as a function of momentum and number of TPC hits for $\mathrm{e} / \pi / \mathrm{K} / \mathrm{p}$ hypotheses for $58 \mathrm{GeV} / \mathrm{c} \mathrm{p}+\mathrm{C}$ data. 

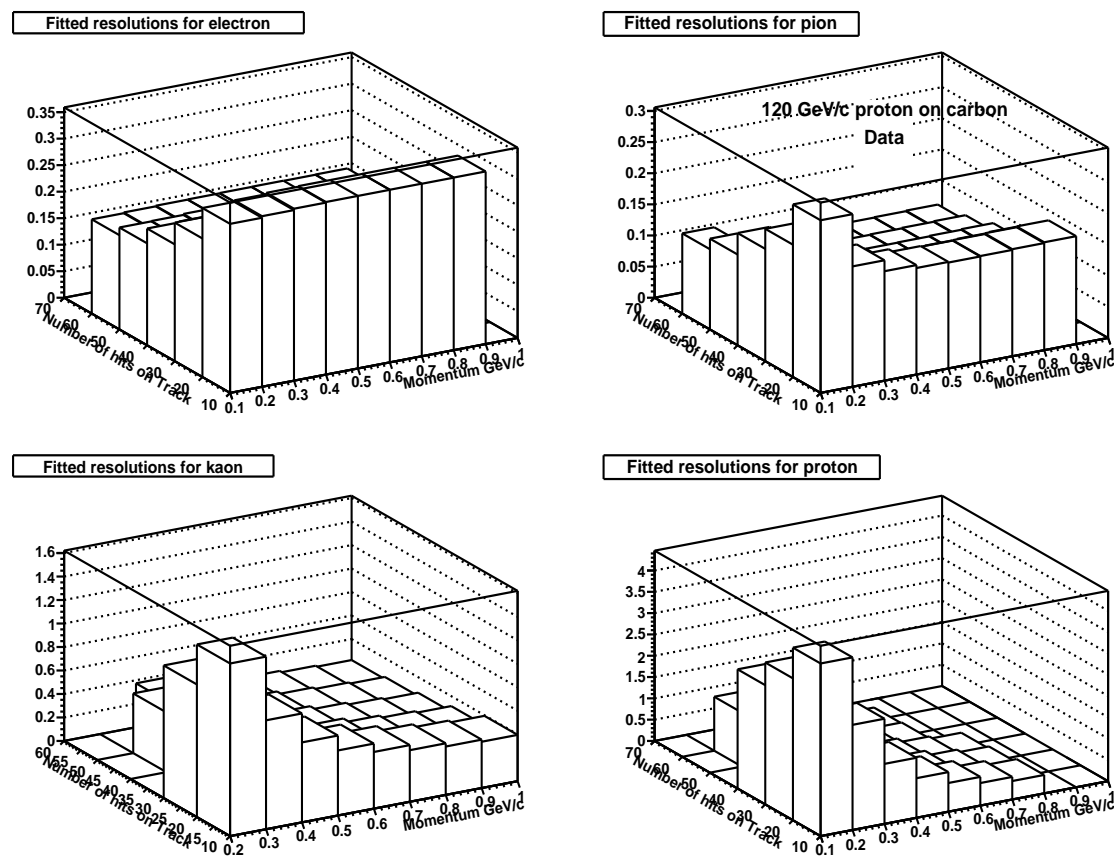

Figure 4.8: The fitted resolutions as a function of momentum and number of TPC hits for $\mathrm{e} / \pi / \mathrm{K} / \mathrm{p}$ hypotheses for $120 \mathrm{GeV} / \mathrm{c} \mathrm{p}+\mathrm{C}$ data.

differences were observed for the MC as well.

The TPC gives very good PID up to $\sim 1.1 \mathrm{GeV} / \mathrm{c}$ but after that, the $\mathrm{dE} / \mathrm{dx}$ curves for various particles start merging into each other, so the TPC doesn't give a good PID after $1.1 \mathrm{GeV} / \mathrm{c}$. The $\mathrm{dE} / \mathrm{dx}$ distributions for different momentum slices for $120 \mathrm{GeV} / \mathrm{c} \mathrm{p}+\mathrm{C}$ data are shown in figures 4.12 and 4.13 . Separate $\mathrm{dE} / \mathrm{dx}$ peaks corresponding to particles e, $\pi$, $\mathrm{K}$ and $\mathrm{p}$ (kaons are suppressed) can be seen for tracks having momenta up to $\sim 1.1 \mathrm{GeV} / \mathrm{c}$ but after that, the e/K/p's start merging into the pion $\mathrm{dE} / \mathrm{dx}$ peak. These $\mathrm{dE} / \mathrm{dx}$ peaks start separating out again at higher momenta $(>\sim 10 \mathrm{GeV} / \mathrm{c})$ as shown in figure 4.14 which shows that the TPC can separate particles at higher momenta. But, as it was mentioned earlier about the large discrepancies seen between observed and predicted $\mathrm{dE} / \mathrm{dx}$ values at the higher momenta, it is decided to use the TPC up to $1 \mathrm{GeV} / \mathrm{c}$ only, since after $1 \mathrm{GeV} / \mathrm{c}$ the $\mathrm{dE} / \mathrm{dx}$ curves for $\mathrm{e} / \pi / \mathrm{K} / \mathrm{p}$ 's start merging into each other. It is also decided to use the TPC for tracks with momenta $>0.3 \mathrm{GeV} / \mathrm{c}$ for the purpose of cross section measurements, since the momentum resolution of the lower momentum tracks is not so good.

The likelihood of obtaining a particular $\mathrm{dE} / \mathrm{dx}$ value is given by the following equation:

$$
\text { Likelihood }=\frac{1}{\sqrt{2 \pi} \sigma} \exp \left(\frac{-(\text { meas }- \text { pred })^{2}}{2 \sigma^{2}}\right),
$$



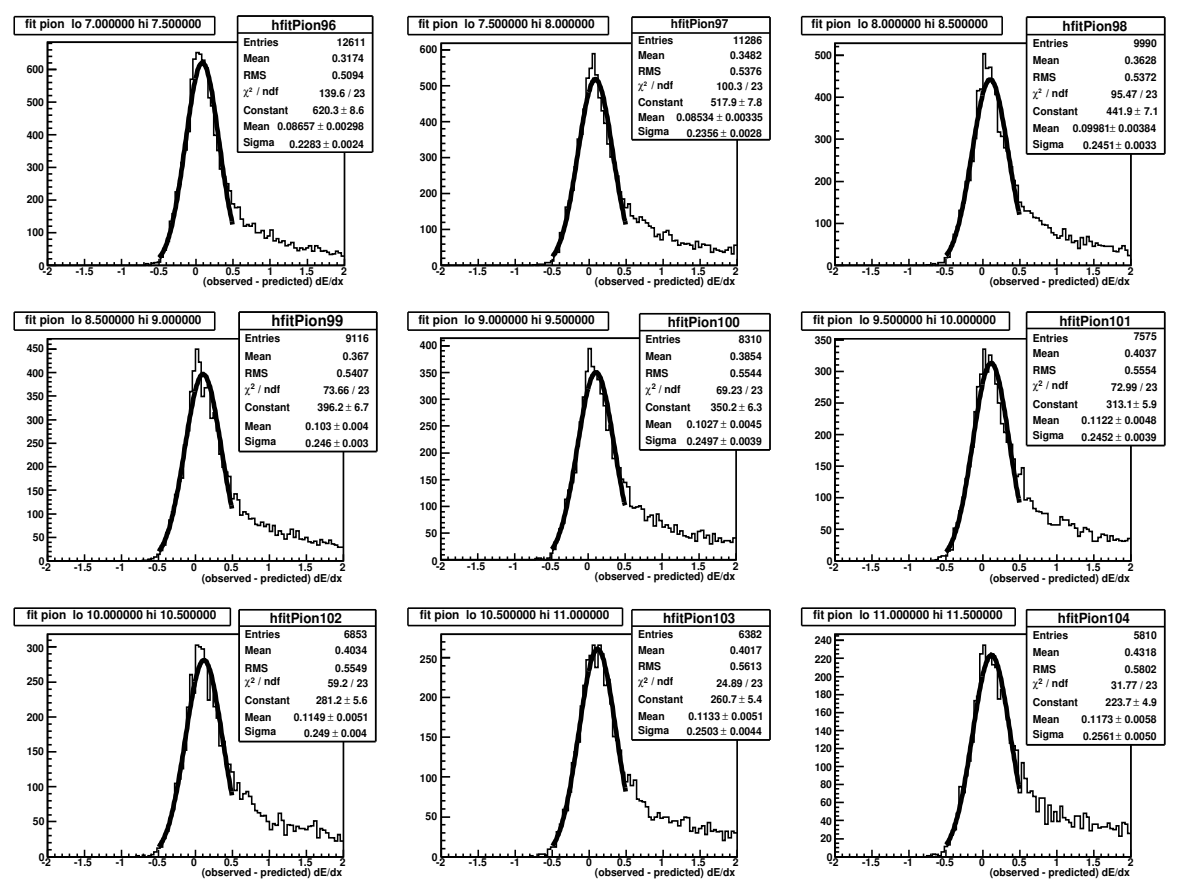

Figure 4.9: The differences between the observed and predicted $\mathrm{dE} / \mathrm{dx}$ values for different momentum slices fitted using a Gaussian function for $120 \mathrm{GeV} / \mathrm{c} \mathrm{p}+\mathrm{C}$ data.
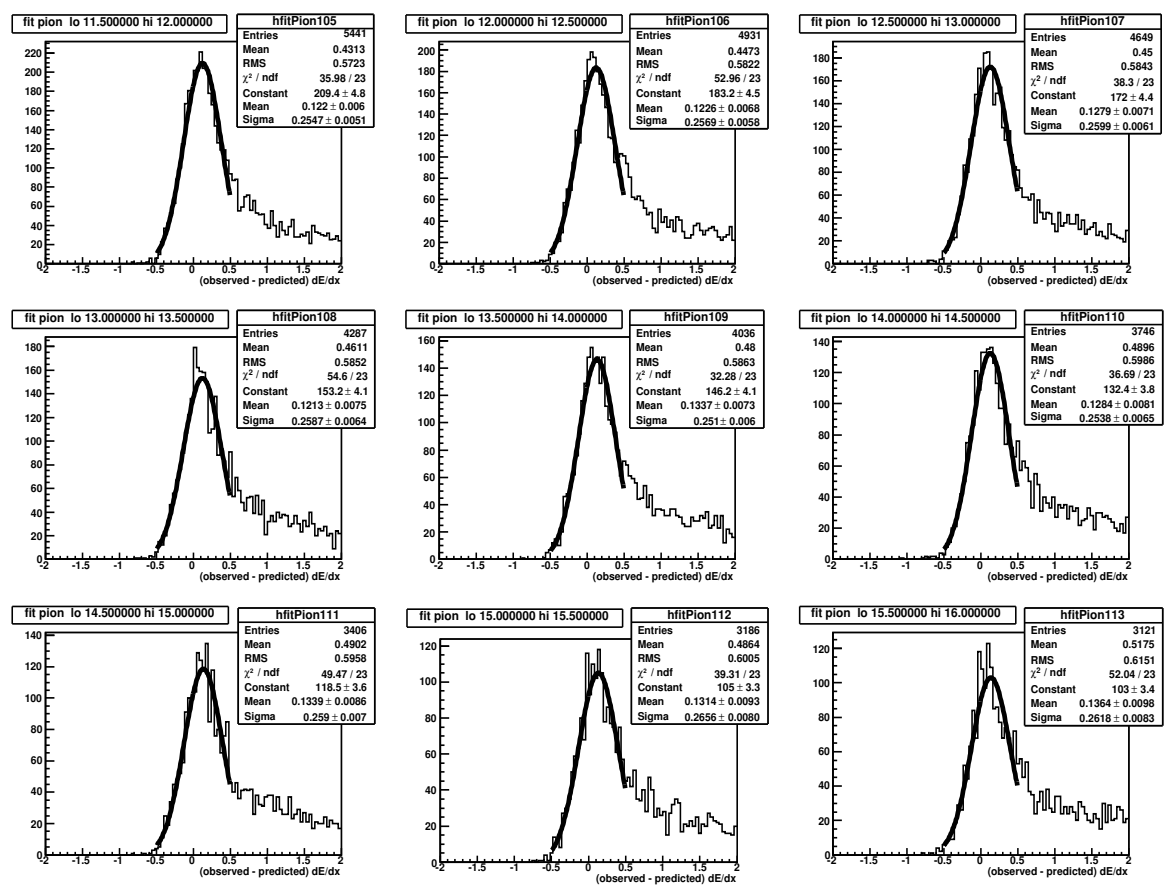

Figure 4.10: The differences between the observed and predicted $\mathrm{dE} / \mathrm{dx}$ values for different momentum slices fitted using a Gaussian function for $120 \mathrm{GeV} / \mathrm{c} \mathrm{p}+\mathrm{C}$ data. 

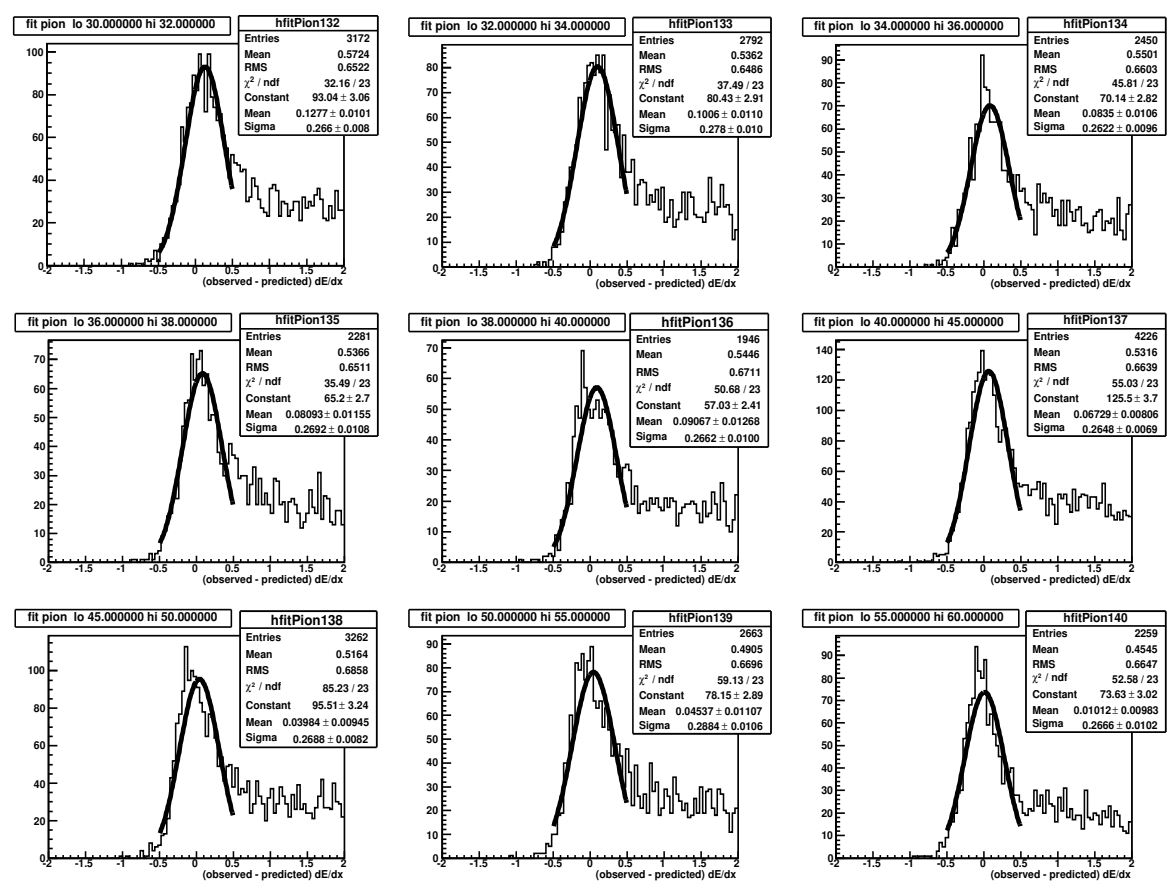

Figure 4.11: The differences between the observed and predicted $\mathrm{dE} / \mathrm{dx}$ values for different momentum slices fitted using a Gaussian function for $120 \mathrm{GeV} / \mathrm{c} \mathrm{p}+\mathrm{C}$ data.
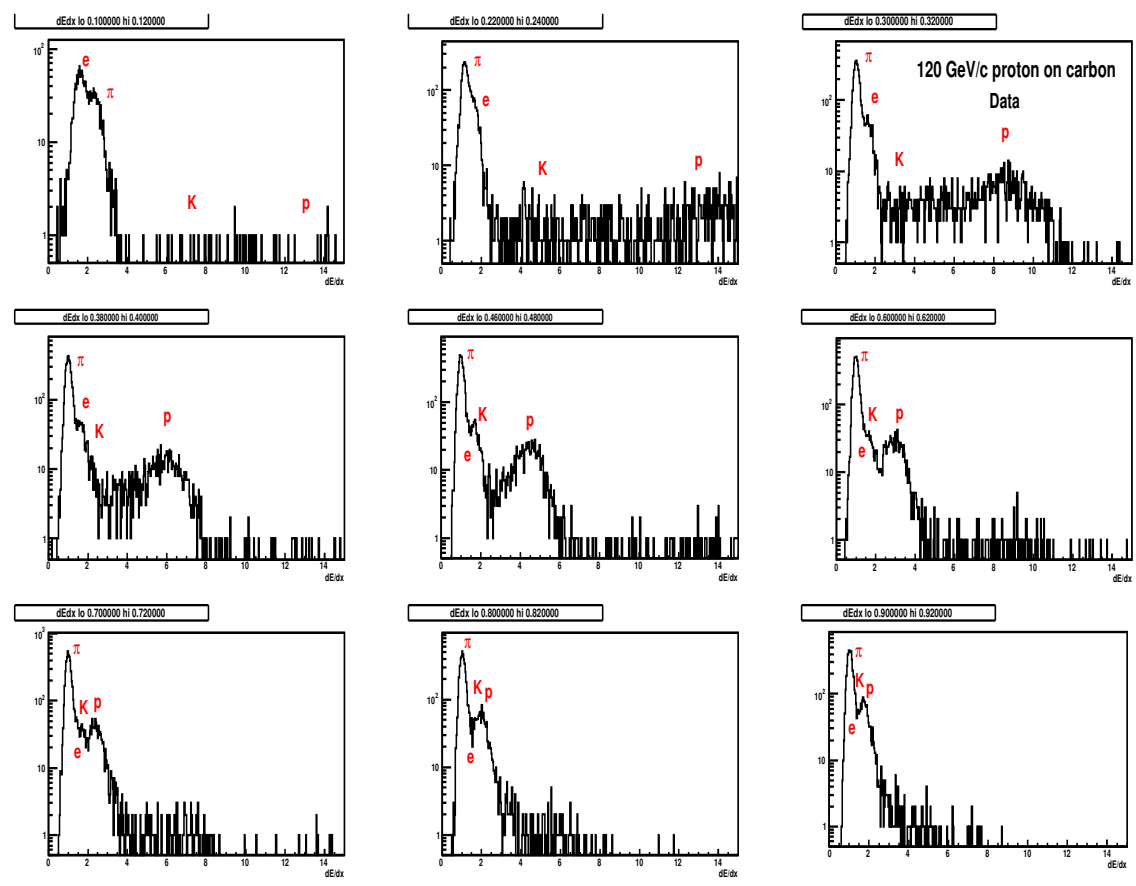

Figure 4.12: The $\mathrm{dE} / \mathrm{dx}$ distributions for different momentum slices for $120 \mathrm{GeV} / \mathrm{c} \mathrm{p}+\mathrm{C}$ data. Separate peaks for e $/ \pi / K / p$ 's (K's are suppressed) can be seen. The electrons and kaons merge after $\sim 0.4 \mathrm{GeV} / \mathrm{c}$. 

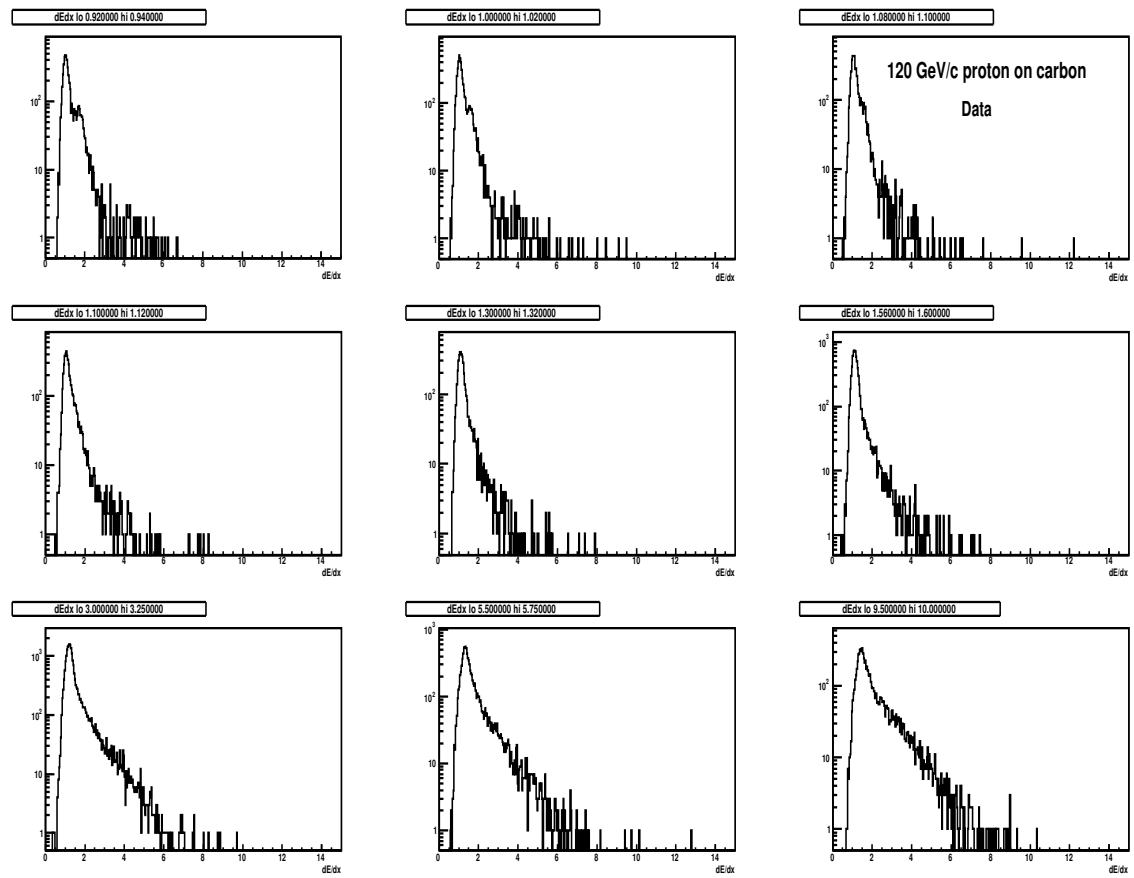

Figure 4.13: The $\mathrm{dE} / \mathrm{dx}$ distributions for different momentum slices for $120 \mathrm{GeV} / \mathrm{c} \mathrm{p}+\mathrm{C}$ data. The e/K/p's start merging into the pion $\mathrm{dE} / \mathrm{dx}$ peak after $\sim 1.1 \mathrm{GeV} / \mathrm{c}$.
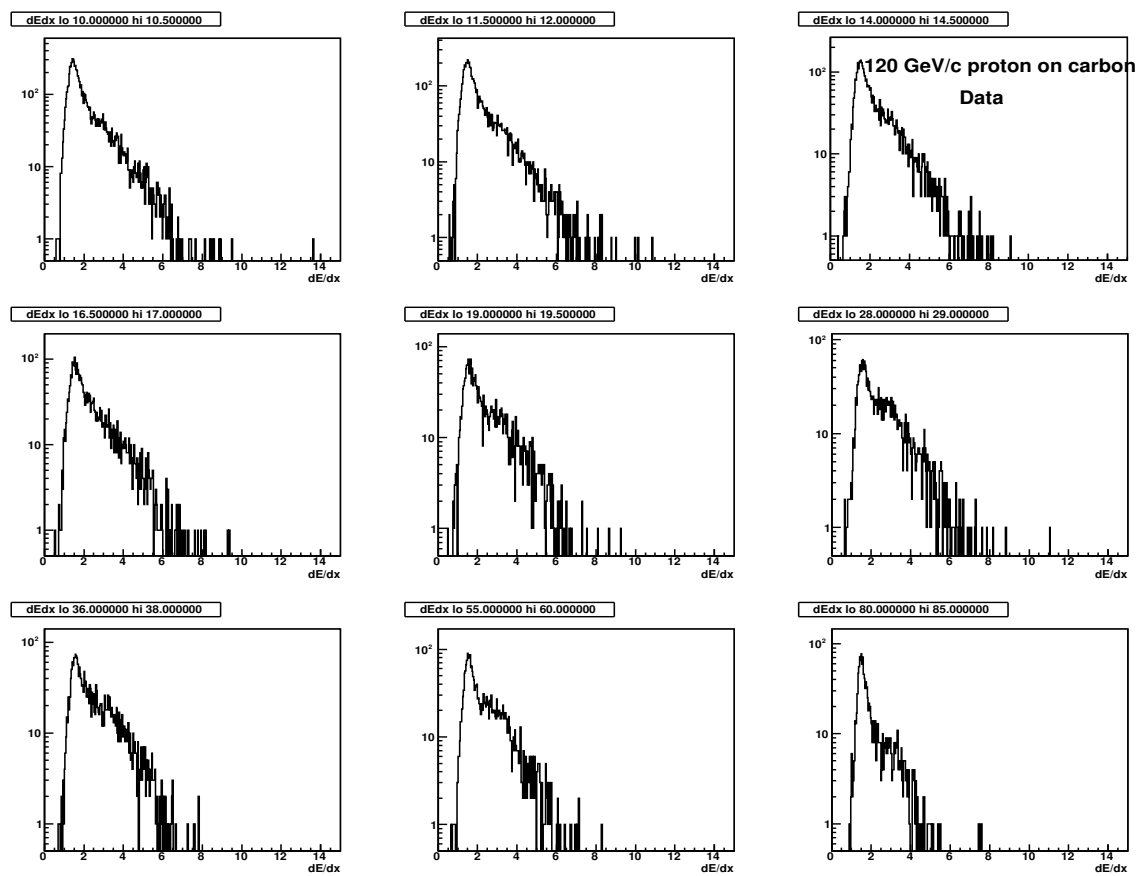

Figure 4.14: The $\mathrm{dE} / \mathrm{dx}$ distributions for different momentum slices for $120 \mathrm{GeV} / \mathrm{c} \mathrm{p}+\mathrm{C}$ data. After $\sim 10 \mathrm{GeV} / \mathrm{c}$, the peaks for various particles start separating out again. 
where "meas" and "pred" denote the measured and predicted $\mathrm{dE} / \mathrm{dx}$ values, and $\sigma$ denotes the resolution.

The likelihoods for all the hypotheses for 58 and $120 \mathrm{GeV} / \mathrm{c} \mathrm{p}+\mathrm{C}$ data using the TPC are shown in figures 4.15(a) and 4.15(b), respectively. The likelihoods as a function of momentum for 58 and $120 \mathrm{GeV} / \mathrm{c} \mathrm{p}+\mathrm{C}$ data are shown in figures 4.16(a) and 4.16(b), respectively.
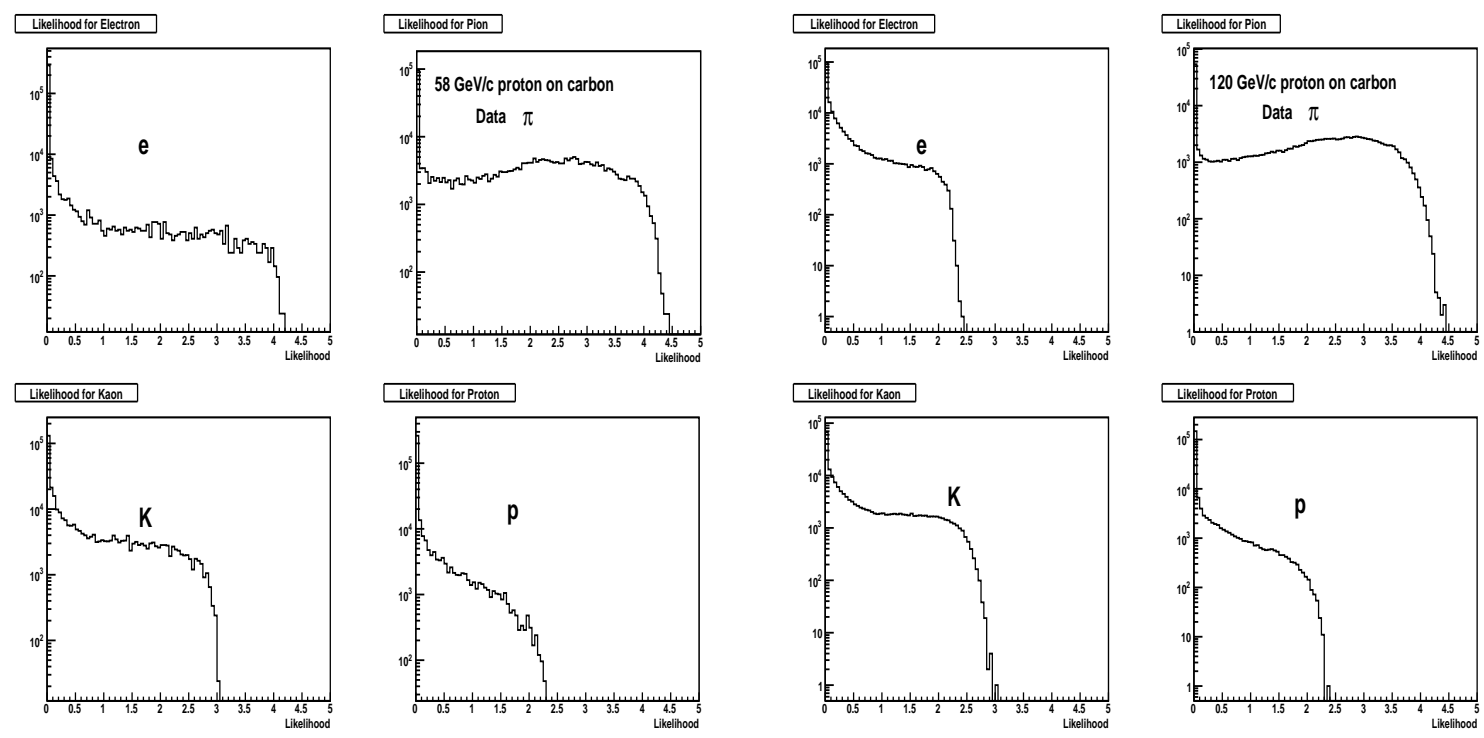

(a)

(b)

Figure 4.15: The likelihood distributions for all the hypotheses for (a) 58 and (b) $120 \mathrm{GeV} / \mathrm{c}$ $\mathrm{p}+\mathrm{C}$ data using TPC.

Weights are computed using the equation 4.2 . The weights for all the hypotheses for 58 and $120 \mathrm{GeV} / \mathrm{c} \mathrm{p}+\mathrm{C}$ data are shown in figures 4.17(a) and 4.17(b), respectively. The weights can lie between 0 and 1 . For a given track, the sum of the weights of all the hypotheses is always 1 . If the weight for a pion hypothesis is 0.7 , that means there are $70 \%$ chances that the particle can be a pion and $30 \%$ chances that the particle can either be a kaon or a proton or an electron. The weights as a function of momentum for 58 and $120 \mathrm{GeV} / \mathrm{c} \mathrm{p}+\mathrm{C}$ data are shown in figures 4.18(a) and 4.18(b), respectively. The weights for the kaon hypothesis are found to be increasing for tracks with momenta $>\sim 0.7 \mathrm{GeV} / \mathrm{c}$ and on the other hand, the pion hypothesis weights are decreasing. This is because of the reason that the $\mathrm{dE} / \mathrm{dx}$ curves for the kaons and pions start merging at these momenta. 

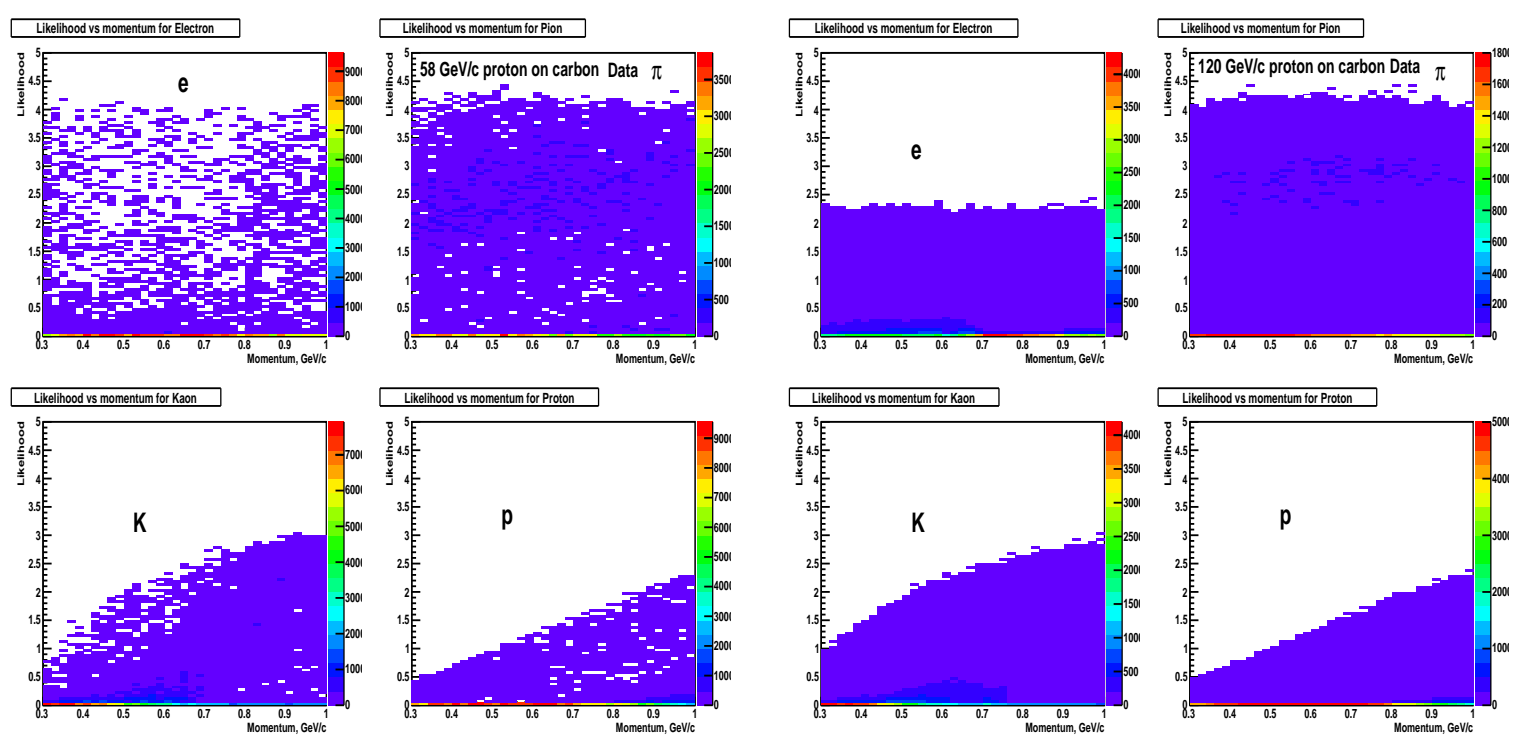

(a)
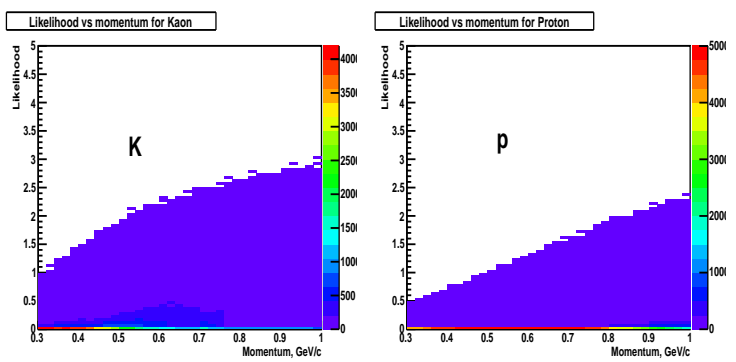

(b)

Figure 4.16: The likelihoods as a function of momentum for all the hypotheses for (a) 58 and (b) $120 \mathrm{GeV} / \mathrm{c} \mathrm{p}+\mathrm{C}$ data using TPC.
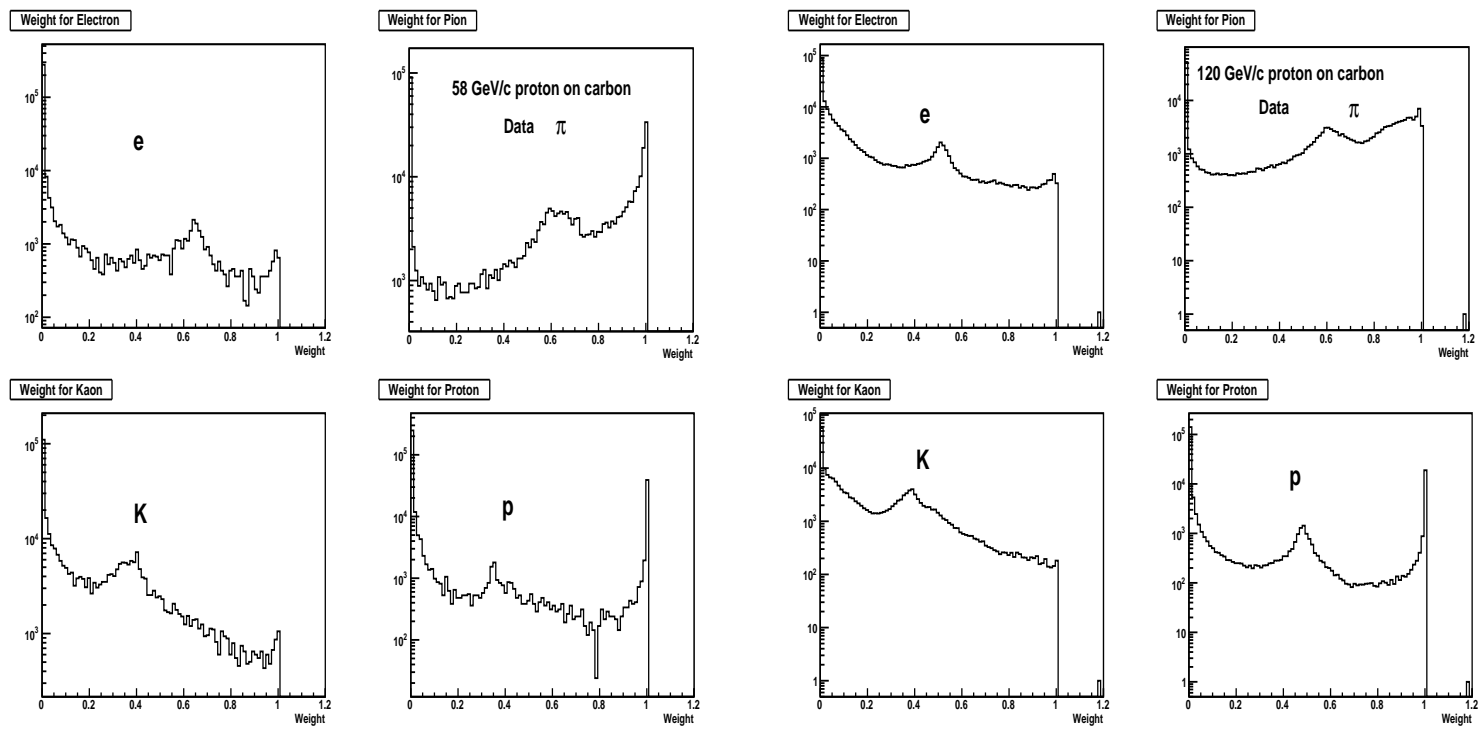

(a)

(b)

Figure 4.17: The weights for all the hypotheses for (a) 58 and (b) $120 \mathrm{GeV} / \mathrm{c}$ p+C data using TPC. 


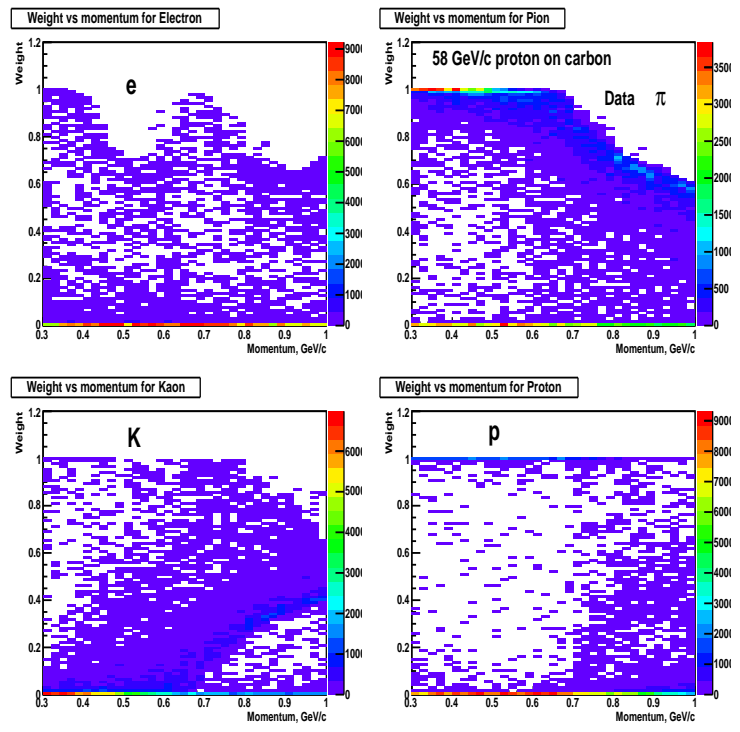

(a)
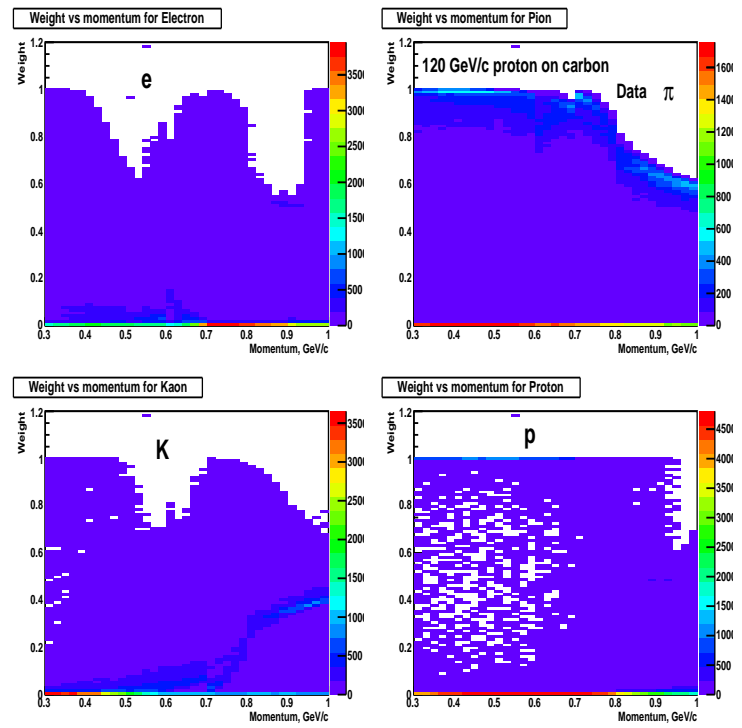

(b)

Figure 4.18: The weights as a function of momentum for all the hypotheses for (a) 58 and (b) $120 \mathrm{GeV} / \mathrm{c} \mathrm{p}+\mathrm{C}$ data using TPC.

\subsubsection{RICH likelihood calculations}

Since the RICH is able to identify pions only with momentum $\geq 4.64 \mathrm{GeV} / \mathrm{c}$, it is decided to use the RICH for the PID of the particles with momentum $\geq 4.64 \mathrm{GeV} / \mathrm{c}$. The likelihood from RICH radii is computed using a Gaussian with resolution $\sigma_{\text {radius }}$ obtained from the circle fitting procedure.

Good tracks in RICH are defined to be the tracks which have number of hits/track $\geq 6$, ring radius $>0$ and $0<\chi_{\text {radiusfit }}^{2}<999$. For tracks which don't satisfy these conditions, we use the probability for not finding a RICH ring from the MC (explained in the next section) as the likelihood. The number of hits/track and $\chi^{2}$ of the radius fit in RICH for 58 and 120 $\mathrm{GeV} / \mathrm{c} \mathrm{p}+\mathrm{C}$ data are shown in figures 4.19 and 4.20 , respectively.

The likelihood of obtaining a particular ring radius value is given by the following equation:

$$
\text { Likelihood }=\frac{1}{\sqrt{2 \pi} \sigma} \exp \left(\frac{-(\text { meas }- \text { pred })^{2}}{2 \sigma^{2}}\right),
$$

where "meas" and "pred" denote the measured and predicted ring radii, and $\sigma$ denotes the resolution.

Using the No Rings information: The RICH detector is such a powerful device that one can use absence of a ring to infer the nature of the particle, especially in the case of a proton. Figure 4.21 shows the probability of not finding a ring in the RICH detector for electrons, 


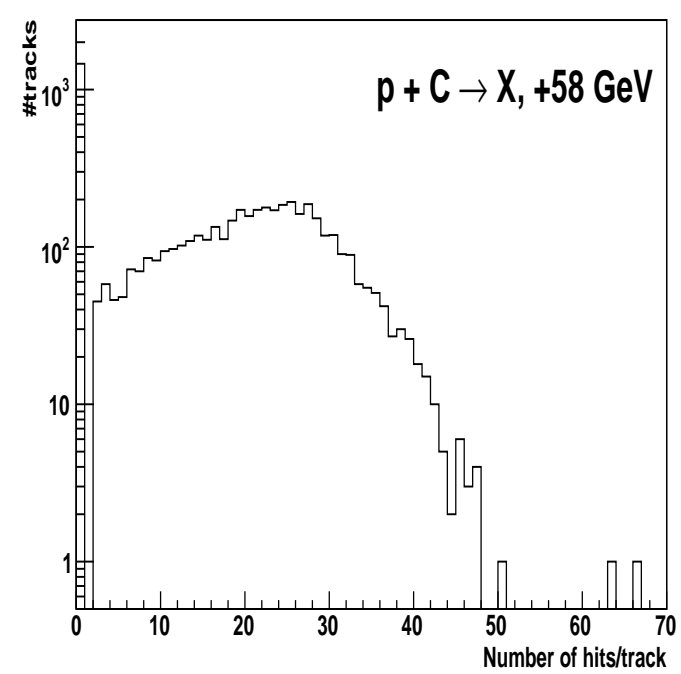

(a)

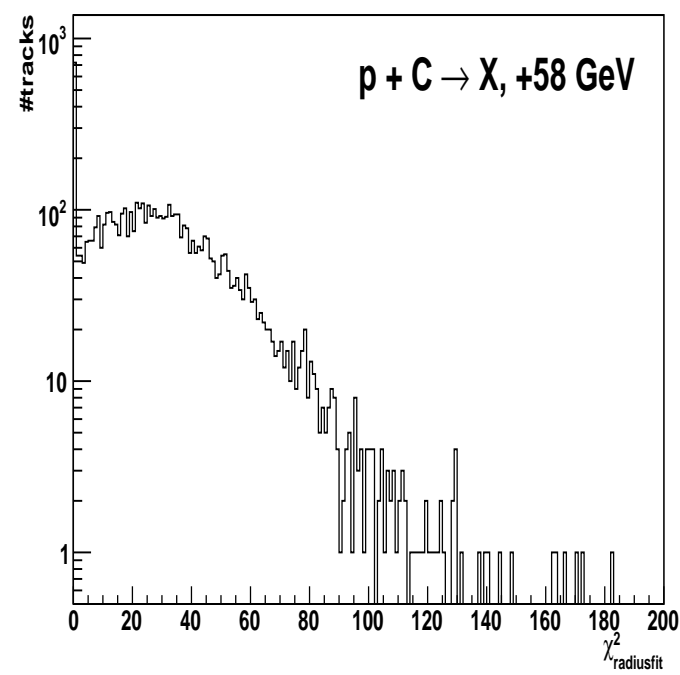

(b)

Figure 4.19: (a) Number of hits/track and (b) $\chi^{2}$ of the radius fit in RICH for $58 \mathrm{GeV} / \mathrm{c}$ $\mathrm{p}+\mathrm{C}$ data.

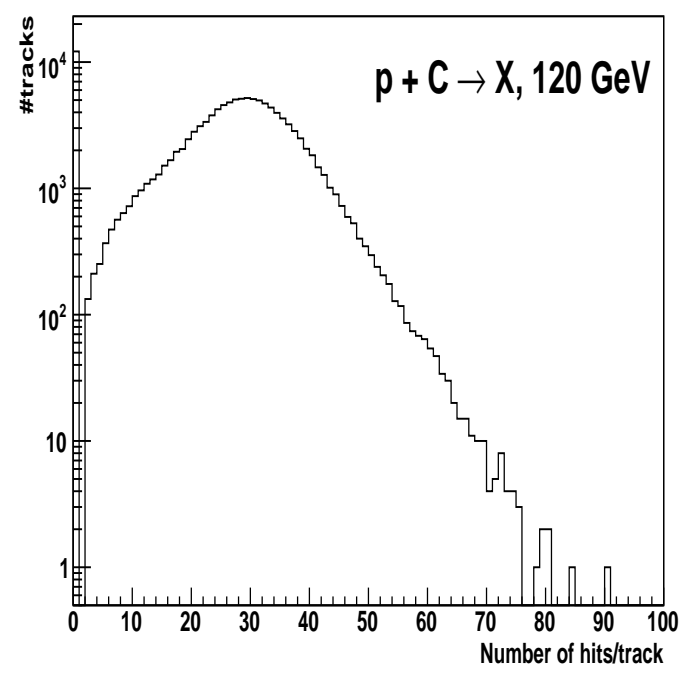

(a)

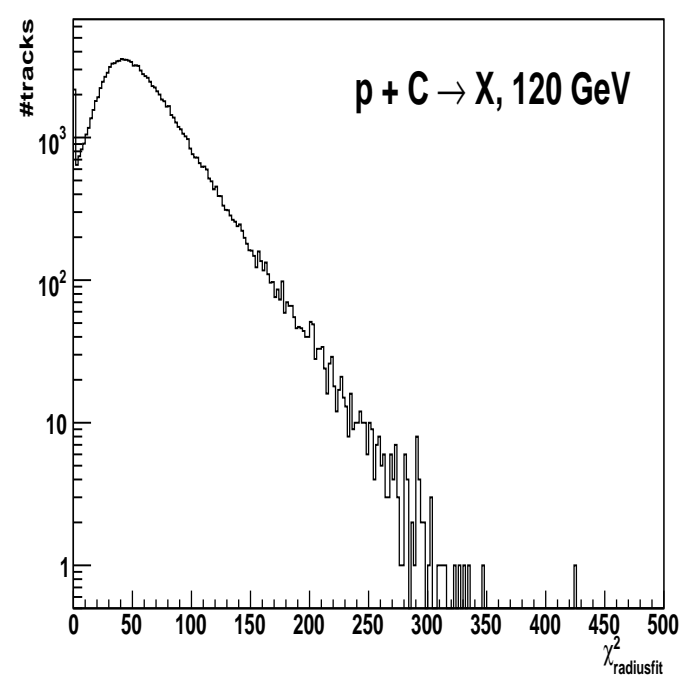

(b)

Figure 4.20: (a) Number of hits/track and (b) $\chi^{2}$ of the radius fit in RICH for $120 \mathrm{GeV} / \mathrm{c}$ $\mathrm{p}+\mathrm{C}$ data. 
pions, kaons and protons with momenta below $60 \mathrm{GeV} / \mathrm{c}$ computed using the MIPP MC for $120 \mathrm{GeV} / \mathrm{c} \mathrm{p}+\mathrm{C}$ interactions. Above $60 \mathrm{GeV} / \mathrm{c}$ momentum, one uses the values at 60 $\mathrm{GeV} / \mathrm{c}$ since the statistics for pions and kaons are limited above this momentum. The track must go through the RICH entrance and exit windows. Figure 4.22 shows the probability of not finding a ring using the MIPP MC for $58 \mathrm{GeV} / \mathrm{c} \mathrm{p}+\mathrm{C}$ interactions. Above $40 \mathrm{GeV} / \mathrm{c}$ momentum, the values at $40 \mathrm{GeV} / \mathrm{c}$ are used since the statistics for pions and kaons are limited above this momentum. For electrons, the values at 20 and $13 \mathrm{GeV} / \mathrm{c}$ are used for 120 and $58 \mathrm{GeV} / \mathrm{c}$ data, respectively for electrons with momenta greater than these momentum values since the statistics for electrons is poor above these momentum values. It can be seen that the particles exhibit threshold behavior at their appropriate thresholds with the probability of not having an associated ring decreasing from unity below threshold to 0.2 as the momentum goes above the threshold and the ring radius (and the emitted light) increases. We use this information to obtain additional particle identification likelihoods in the event where there is no ring associated with a track.

The likelihood disributions for all the hypotheses for 58 and $120 \mathrm{GeV} / \mathrm{c} \mathrm{p}+\mathrm{C}$ data are shown
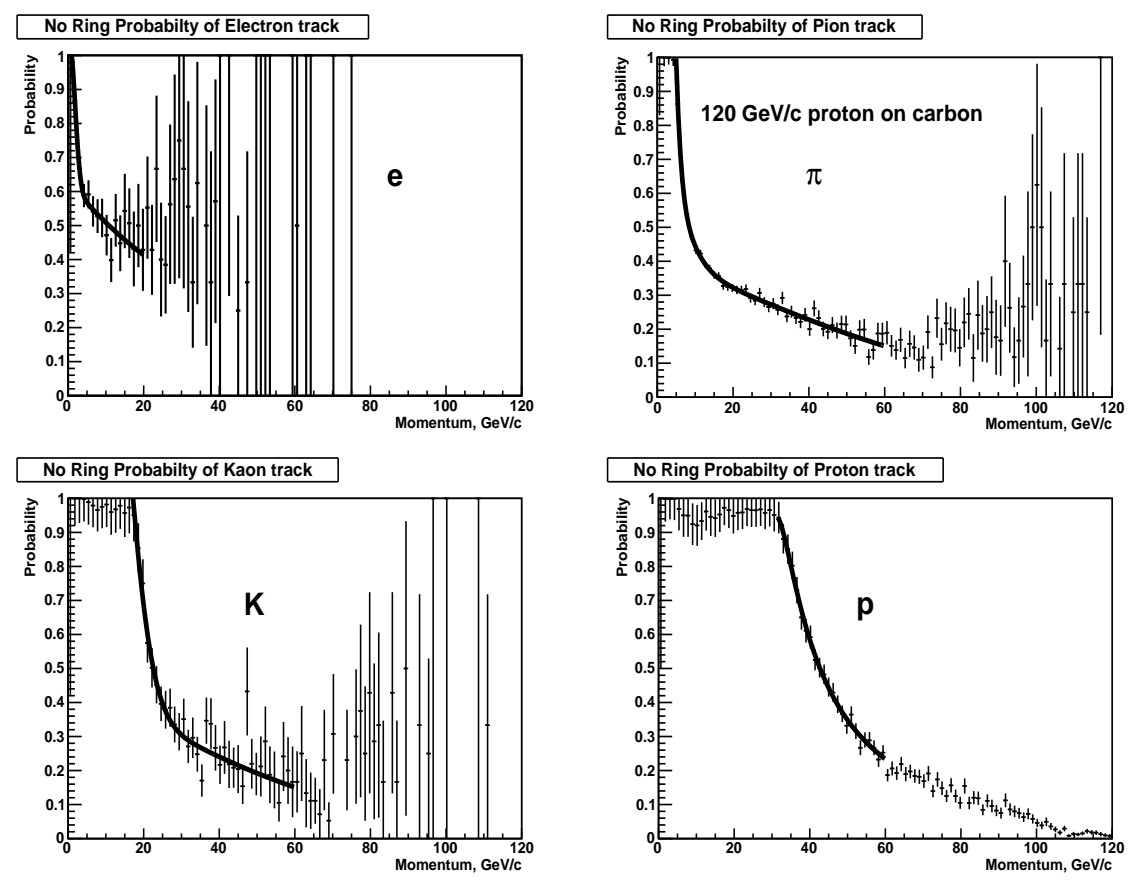

Figure 4.21: Probability of not finding a ring in the RICH for e $/ \pi / \mathrm{K} / \mathrm{p}$ tracks with momenta less than $60 \mathrm{GeV} / \mathrm{c}$ computed using the MIPP MC for $120 \mathrm{GeV} / \mathrm{c} \mathrm{p}+\mathrm{C}$ interactions.

in figures 4.23(a) and 4.23(b), respectively. The likelihoods as a function of momentum for 58 and $120 \mathrm{GeV} / \mathrm{c} \mathrm{p}+\mathrm{C}$ data are shown in figures 4.24(a) and 4.24(b), respectively. When the track momentum is greater than pion threshold but less than kaon or proton thresholds, 

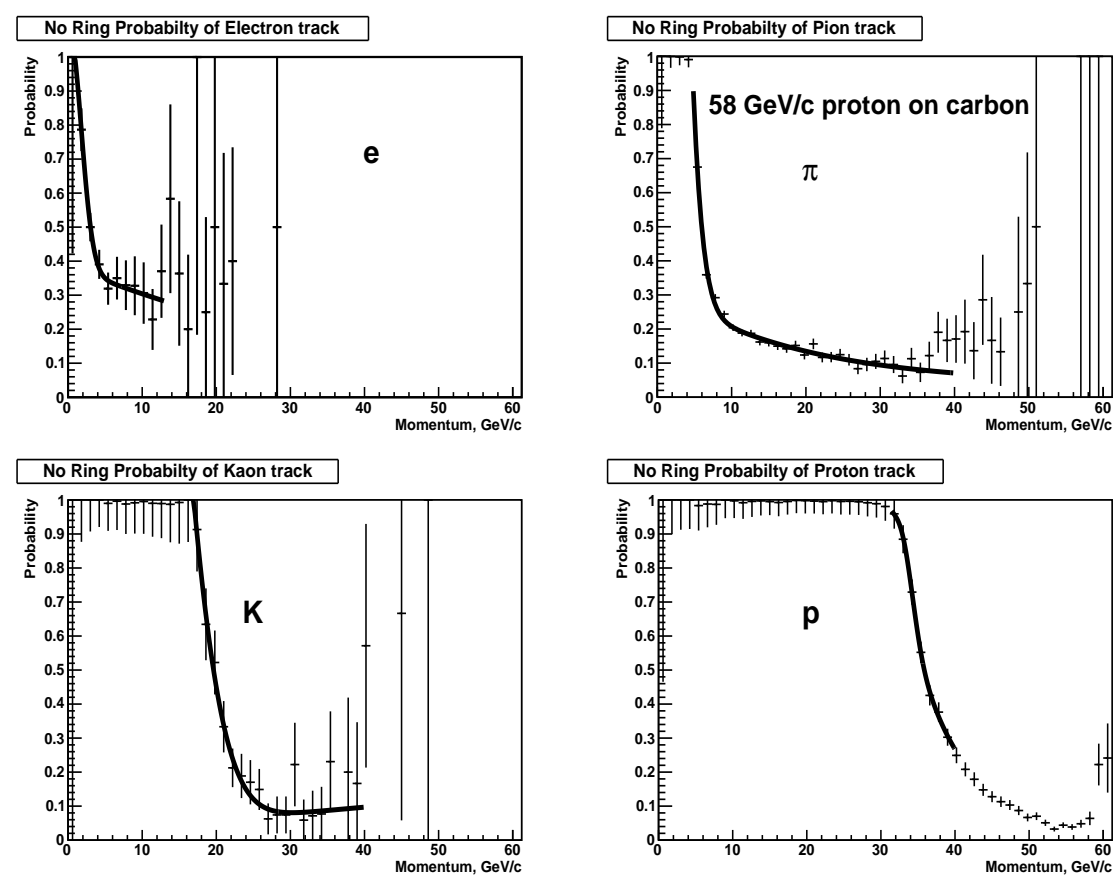

Figure 4.22: Probability of not finding a ring in the RICH for e $/ \pi / \mathrm{K} / \mathrm{p}$ tracks with momenta less than $40 \mathrm{GeV} / \mathrm{c}$ computed using the MIPP MC for $58 \mathrm{GeV} / \mathrm{c}$ p $+\mathrm{C}$ interactions.

and there is no ring associated with the track, then the possibility is that the particle can either be a kaon or a proton. In this case, we use the no ring probabilities shown in figures 4.21 and 4.22. In the likelihood distributions shown in figures 4.24(a) and 4.24(b), both the kaons and protons have likelihood of 1 up to their RICH thresholds which are 16.53 and $31.42 \mathrm{GeV} / \mathrm{c}$, respectively. Since the kaons and protons do not produce rings below their thresholds, the no ring probabilities are used in this case.

The weights for all the hypotheses for 58 and $120 \mathrm{GeV} / \mathrm{c} \mathrm{p}+\mathrm{C}$ data are shown in figures $4.25(\mathrm{a})$ and 4.25(b), respectively. The weights as a function of momentum for 58 and 120 $\mathrm{GeV} / \mathrm{c} \mathrm{p}+\mathrm{C}$ data are shown in figures 4.26(a) and 4.26(b), respectively. In case of kaons and protons, the weights are first increasing up to their thresholds, then decreasing after they pass their thresholds and then becoming flat. It is happening because when there is no ring for a particle with momentum greater than the pion threshold but below the kaon or proton thresholds, the probability for that particle to be a kaon or a proton is more as explained earlier. 

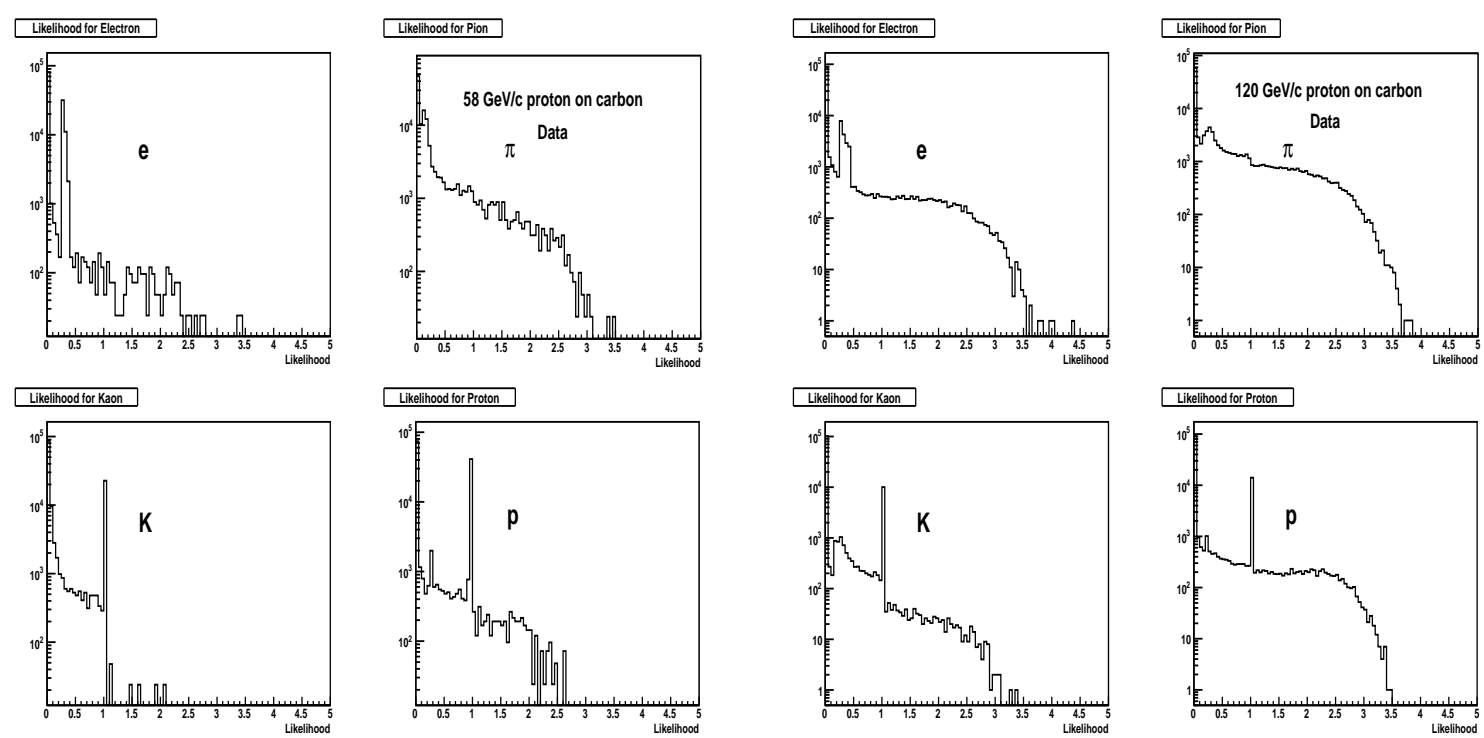

(a)

(b)

Figure 4.23: The likelihood distributions for all the hypotheses for (a) 58 and (b) $120 \mathrm{GeV} / \mathrm{c}$ $\mathrm{p}+\mathrm{C}$ data using $\mathrm{RICH}$.
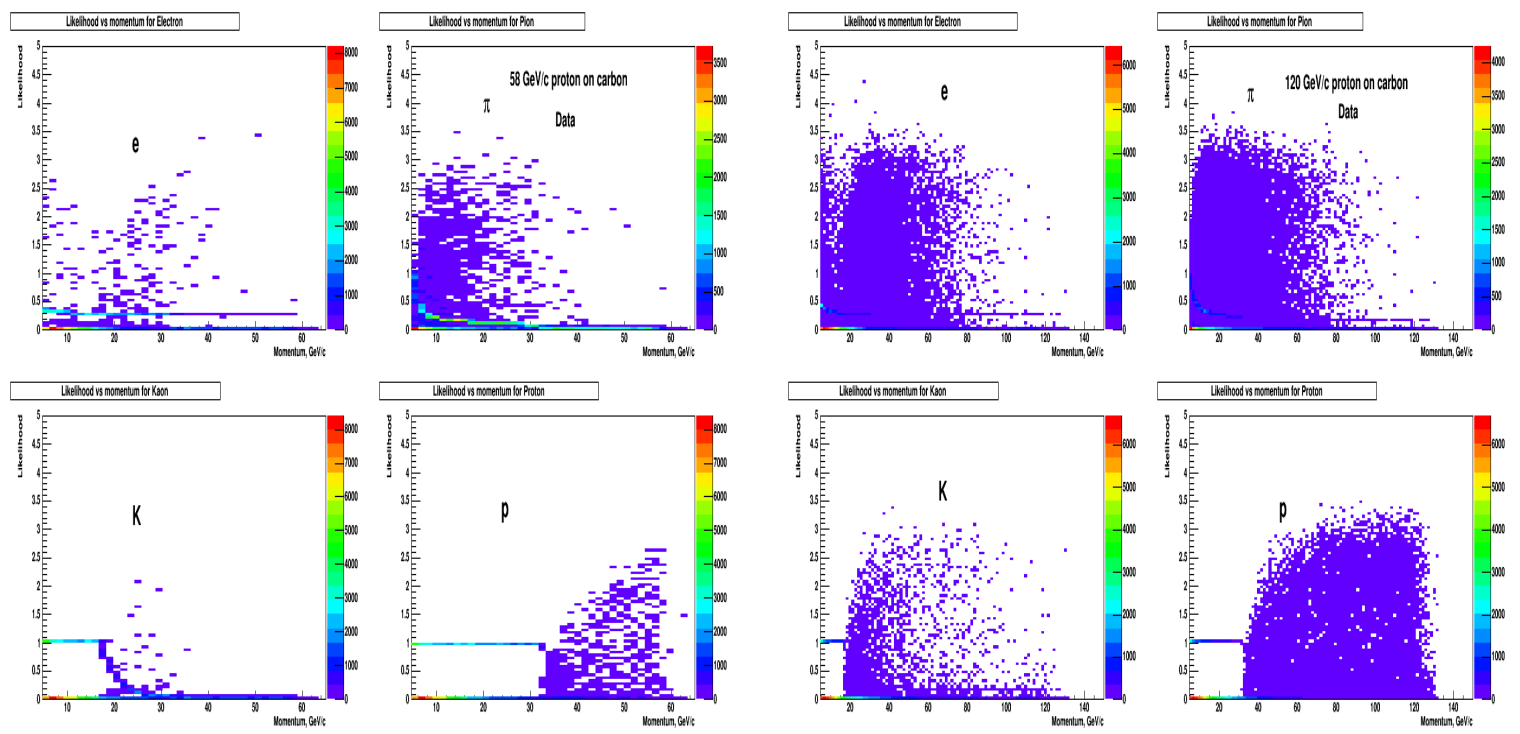

(a)

(b)

Figure 4.24: The likelihoods as a function of momentum for all the hypotheses for (a) 58 and (b) $120 \mathrm{GeV} / \mathrm{c} \mathrm{p}+\mathrm{C}$ data using $\mathrm{RICH}$. 

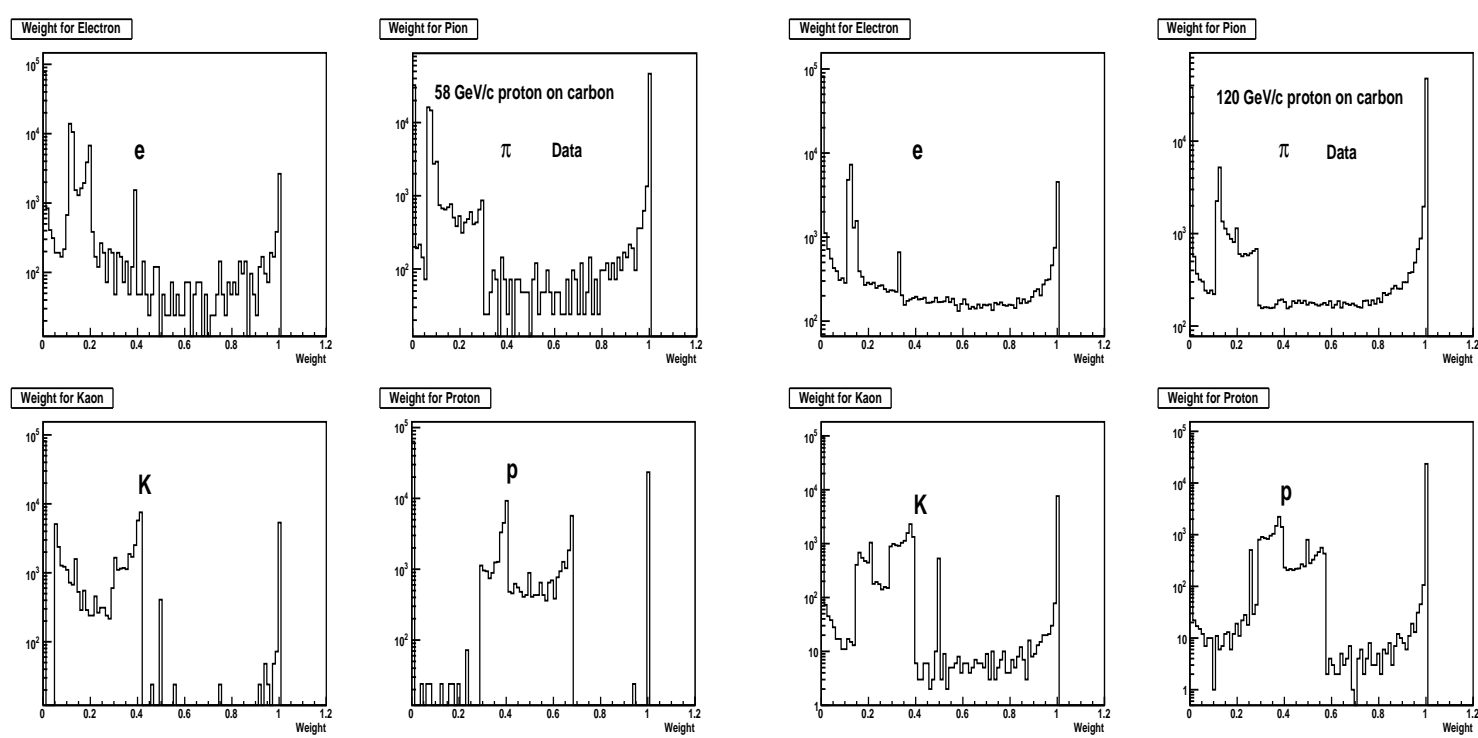

(a)

(b)

Figure 4.25: The weights for all the hypotheses for (a) 58 and (b) $120 \mathrm{GeV} / \mathrm{c} \mathrm{p}+\mathrm{C}$ data using RICH.
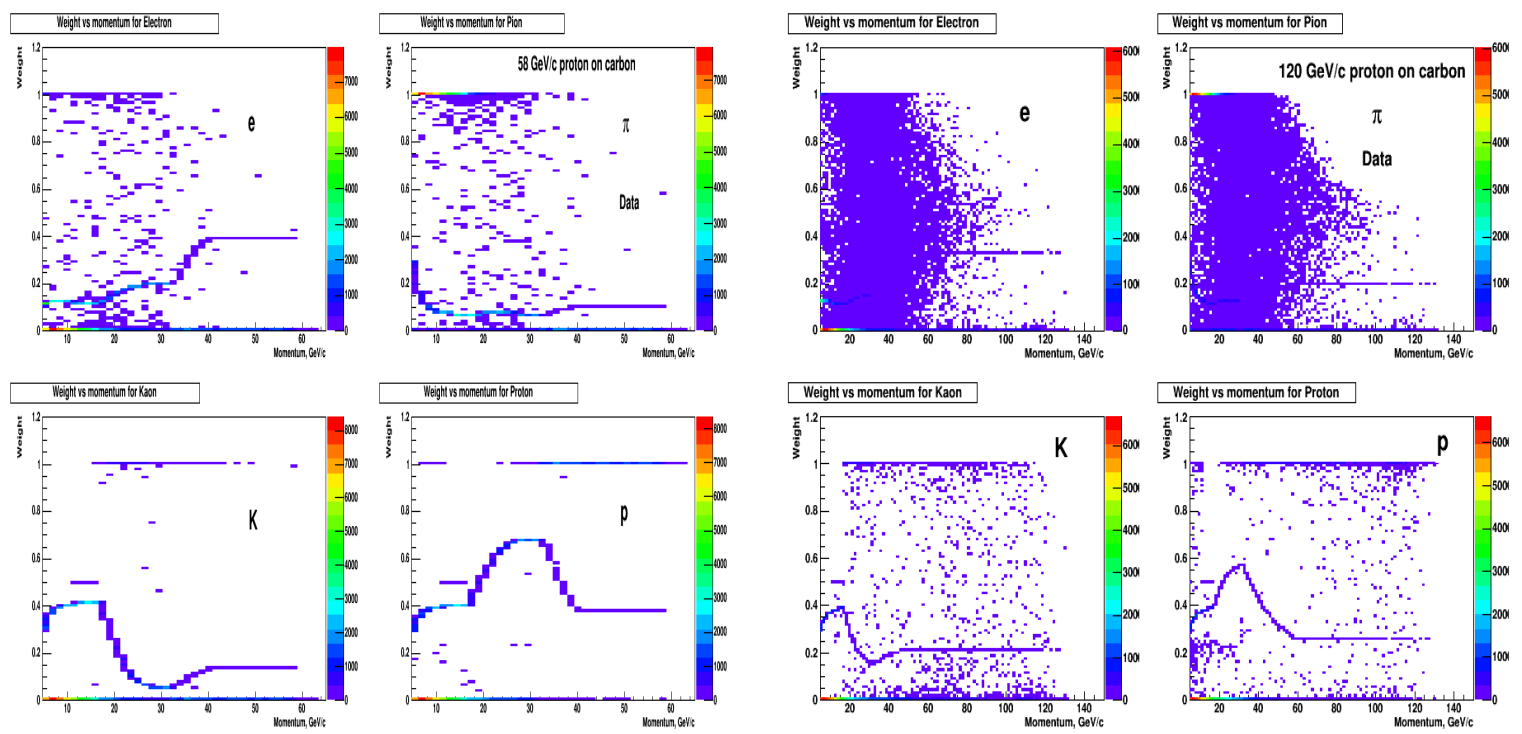

(a)

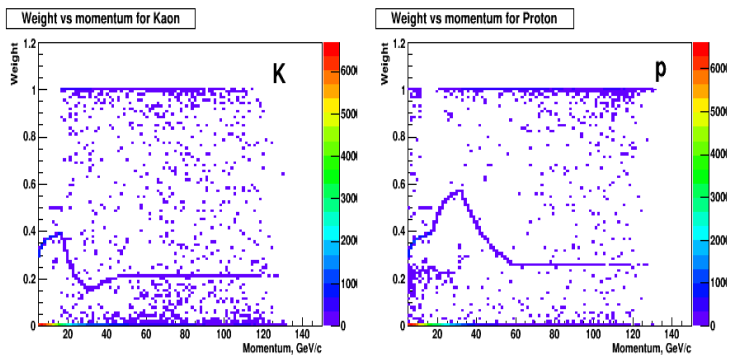

(b)

Figure 4.26: The weights as a function of momentum for all the hypotheses for (a) 58 and (b) $120 \mathrm{GeV} / \mathrm{c} \mathrm{p}+\mathrm{C}$ data using $\mathrm{RICH}$. 


\subsubsection{Why ToF is not used for PID in this analysis?}

The ToF (see section 2.5) can't separate the electrons and pions above $\sim 0.75 \mathrm{GeV} / \mathrm{c}$ within the resolution of the detector, though it can separate the pions and kaons up to $\sim 1.5 \mathrm{GeV} / \mathrm{c}$, and pions and protons up to $\sim 2.5 \mathrm{GeV} / \mathrm{c}$. Because the ToF can't do the electron-pion separation above $0.75 \mathrm{GeV} / \mathrm{c}$, we have decided not to use it for the PID.

\subsubsection{Global likelihoods and the problems associated with them}

Initially we were combining the likelihoods of all the three detectors i.e. TPC, ToF and RICH to obtain a global likelihood [108, 109] and ultimately obtain the PID. The problem in using this approach was that the likelihoods of the different detectors were not falling in the same range and if they were combined, that might lead to wrong values of the global likelihood. Proper normalizations of the individual detector likelihoods were required before combining them to form the global likelihood. Since we have now decided to use only two detectors and that also in separate momentum ranges i.e TPC in $0.3-1 \mathrm{GeV} / \mathrm{c}$ and RICH in $4.64-120 \mathrm{GeV} / \mathrm{c}(4.64 \mathrm{GeV} / \mathrm{c}$ is the RICH threshold for the pions, it's the relativistic momentum), combining them doesn't give us any extra information. The PID is obtained from the individual detectors in the two separate momentum ranges.

Inclusive charged pion production cross sections are calculated using the TPC for the momentum range $0.3-1 \mathrm{GeV} / \mathrm{c}$, and the $\mathrm{RICH}$ for momentum range $4.64-120 \mathrm{GeV} / \mathrm{c}$.

\subsection{Target-out subtraction}

MIPP uses a scintillator-based interaction trigger and this scintillator is placed $\sim 1.5 \mathrm{~cm}$ downstream of the experimental target. Since the scintillator is placed very close to the target, there is a possibility that the beam can also interact with the scintillator. MIPP had also taken data on empty target i.e. when the target was not there in the wheel as discussed in section 2.7.1. To reject the interactions with the scintillator, we subtract the target-out i.e. empty target data from the target-in i.e. $\mathrm{p}+\mathrm{C}$ data as described earlier in section 3.2.2. The target-out data is normalized to the same number of incident protons as those for target-in data before subtraction. After subtraction, only interactions with the target will be left. The heights of the scintillator peaks for the target-in and target-out data are not equal. The target-out data need to be scaled to match the heights of the scintillator peaks. To get

this extra scaling factor, the $\mathrm{Z}$ vertex distributions for the target-in and target-out data are fitted.

Figures $4.27(\mathrm{a})$ and $4.27(\mathrm{~b})$ show the fits to $\mathrm{Z}$ vertex distributions for the target-in 
and target-out data using $120 \mathrm{GeV} / \mathrm{c}$ proton beam for the lower momentum range i.e. 0.3 $-1 \mathrm{GeV} / \mathrm{c}$ and the higher momentum range i.e. $4.64-120 \mathrm{GeV} / \mathrm{c}$, respectively. The shape of the target-out data follows a Gaussian distribution with exponential tails on both sides. The target-out data is fitted to that function. Then, the target-in data is fitted to the combination of the two Gaussians with exponential tails: one representing the target peak and the other representing the scintillator peak. The mean and width of the second Gaussian in target-in Z fit are kept the same as the mean and width of the Gaussian fit to the targetout $\mathrm{Z}$ distribution. The amplitudes of scintillator peaks are calculated from the fits for both the target-in and target-out $Z$ vertex distributions, and it is found that there is difference in the amplitudes. The extra scaling factor is obtained by dividing the amplitude of the scintillator peak (second Gaussian) in the target-in fit by the amplitude of the scintillator peak (Gaussian) in the target-out fit.

The fit function is formulated as follows:

$$
f(z)= \begin{cases}A \exp \left(-\frac{1}{2}\left(\frac{z-z_{\text {mean }}}{\sigma_{z}}\right)^{2}\right)+\text { const. } & \text { if }\left|z-z_{\text {mean }}\right|<\sigma_{\text {cut }} \sigma_{z} \\ \text { Aexp }\left(\frac{1}{2} \sigma_{\text {cut }}^{2}-\frac{\mid z-z \text { mean } \mid}{\sigma_{z}} \sigma_{\text {cut }}\right)+\text { const. } & \text { if }\left|z-z_{\text {mean }}\right| \geq \sigma_{\text {cut }} \sigma_{z}\end{cases}
$$

where $\sigma_{c u t}$ determines how many $\sigma$ 's to the right and left, the function becomes exponential, $Z_{\text {mean }}$ is mean value of the Gaussian function. Similar procedure is followed for $58 \mathrm{GeV} / \mathrm{c}$ $\mathrm{p}+\mathrm{C}$ data.

\subsection{Pion momentum spectrum}

All the event and track selection requirements explained in the section 3.2 are $\mathrm{ON}$ in this analysis. The PID algorithm is run on the TPC and RICH data separately. The TPC is used for lower momentum range and the RICH for higher momentum range as described in the section 4.2. The pion momentum spectrum is obtained by scaling the total charged particle momentum spectrum by the pion hypothesis weight. In data, the PID algorithm is run on both the target-in i.e. carbon target and target-out i.e. empty target data, and pion momentum spectrum is obtained for both the data sets. The target-out contribution is subtracted from the target-in data to reject the interactions with the scintillator. The target-out data is normalized to the same number of incident protons as those of target-in data before subtraction. The extra scaling factor is also applied to the target-out data to match the scintillator peak heights for the target-in and target-out data. This is done for both the TPC and RICH data.

The momentum distributions for the pion negatives and positives for the target-in and 


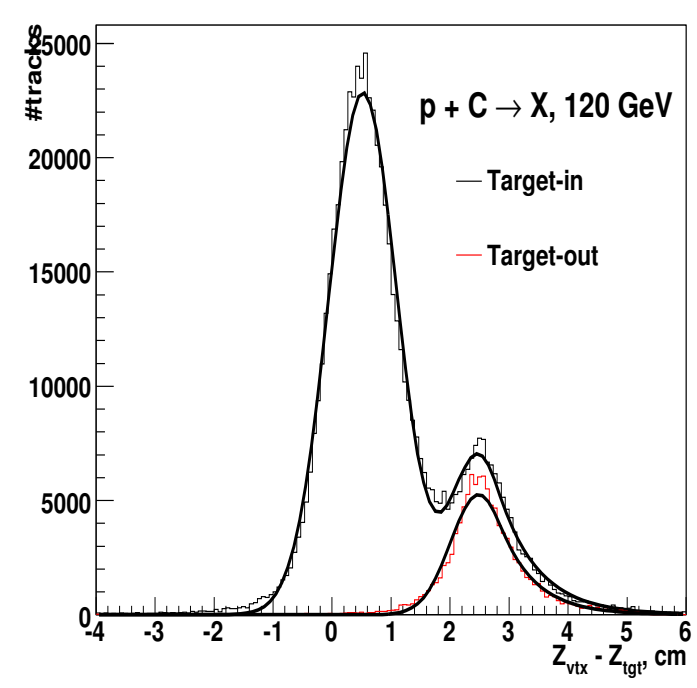

(a)

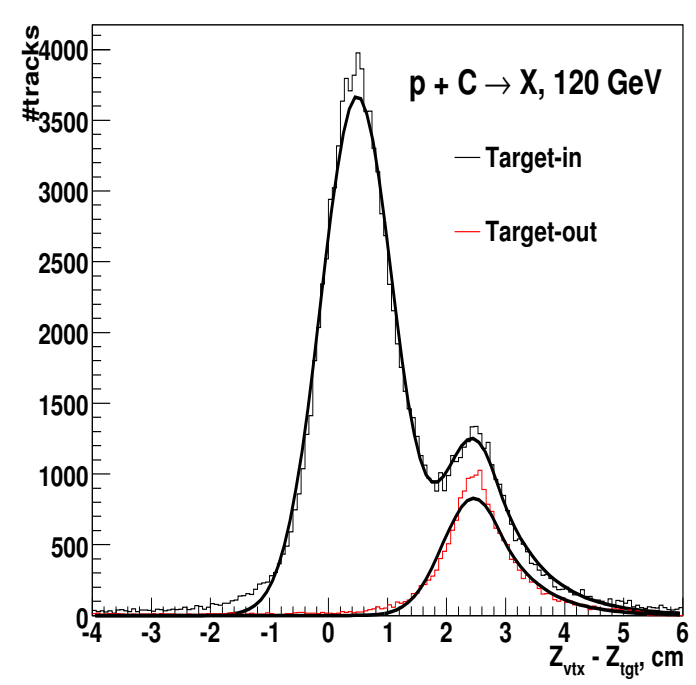

(b)

Figure 4.27: Fits to the $\mathrm{Z}$ vertex distributions for the target-in and target-out data for (a) lower momentum range i.e. $0-3-1 \mathrm{GeV} / \mathrm{c}$ and (b) higher momentum range i.e. $4.64-120$ $\mathrm{GeV} / \mathrm{c}$ using $120 \mathrm{GeV} / \mathrm{c} \mathrm{p}+\mathrm{C}$ data.

target-out data, and after target-out subtraction for momentum range $0.3-1 \mathrm{GeV} / \mathrm{c}$ using the TPC are shown in figures 4.28 and 4.29 using 58 and $120 \mathrm{GeV} / \mathrm{c}$ beams, respectively. The corresponding plots for momentum range $4.64-120 \mathrm{GeV} / \mathrm{c}$ using the RICH are shown in figures 4.30 and 4.31 .

\subsection{Unfolding of the data pion momentum spectrum}

The data pion momentum spectrum is unfolded using the Monte Carlo. In this method, a K-matrix $\mathrm{K}\left(\mathrm{n}_{r} \mid n_{t}\right)$ which denotes the probability of getting a reconstructed pion $\mathrm{n}_{r}$, for a given true pion $n_{t}$, is multiplied by the pion momentum parameters which are going to be fitted, to get the predicted pion momentum distribution. The K-matrix i.e. the probability of getting a reconstructed pion for a given true pion for the pion negatives for momentum range $0.3-1 \mathrm{GeV} / \mathrm{c}$ using the $\mathrm{TPC}$ and $120 \mathrm{GeV} / \mathrm{c} \mathrm{p}+\mathrm{C}$ data is shown in figure 4.32 . The corresponding plot using the $\mathrm{RICH}$ for momentum range $4.64-120 \mathrm{GeV} / \mathrm{c}$ is shown in figure 4.33. The observed (data) pion momentum distribution discussed in section 4.4 is fitted to the predicted distribution. The K-matrix is inverted and multiplied by the observed pion momentum spectrum to get the unfolded pion momentum spectrum for the data.

Figures 4.34 and 4.35 show the comparison of the observed and predicted distributions 


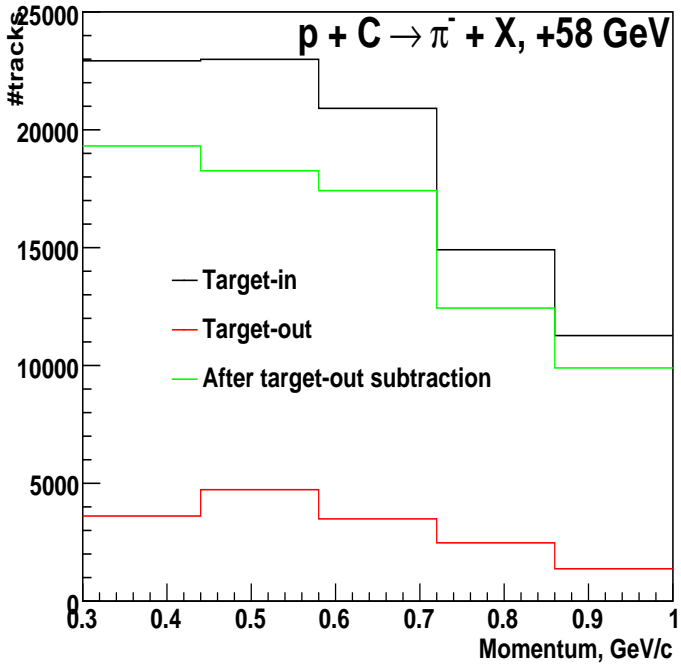

(a)

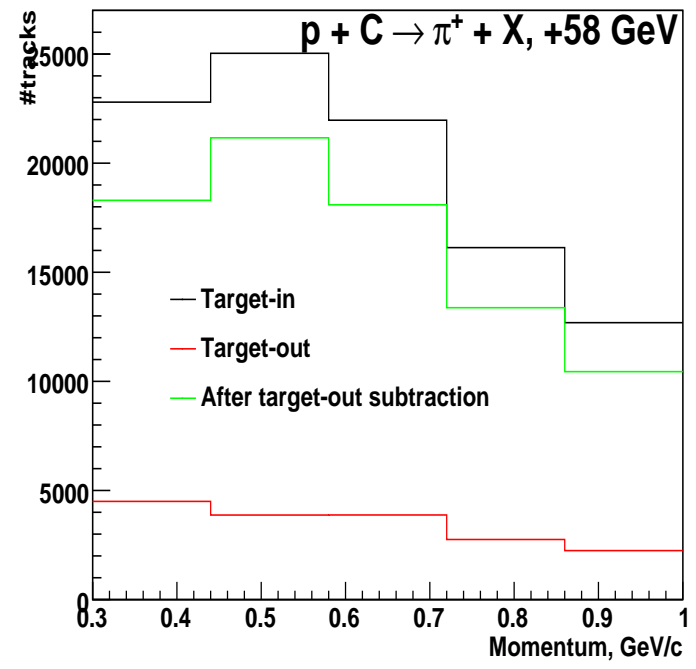

(b)

Figure 4.28: Momentum distributions for (a) pion negatives and (b) positives for target-in (shown in black) and target-out (shown in red) data for momentum range $0.3-1 \mathrm{GeV} / \mathrm{c}$ using TPC and $58 \mathrm{GeV} / \mathrm{c} \mathrm{p}+\mathrm{C}$ data. Momentum distribution after target-out subtraction (shown in green) is also shown.

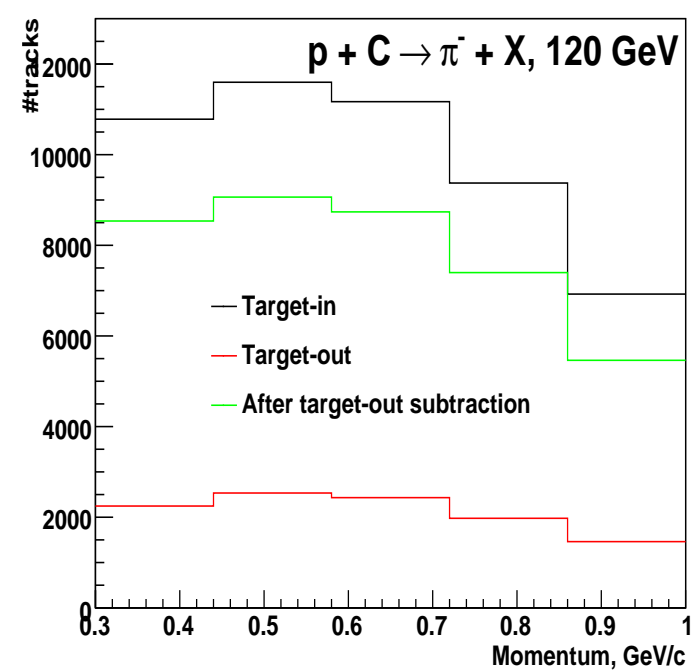

(a)

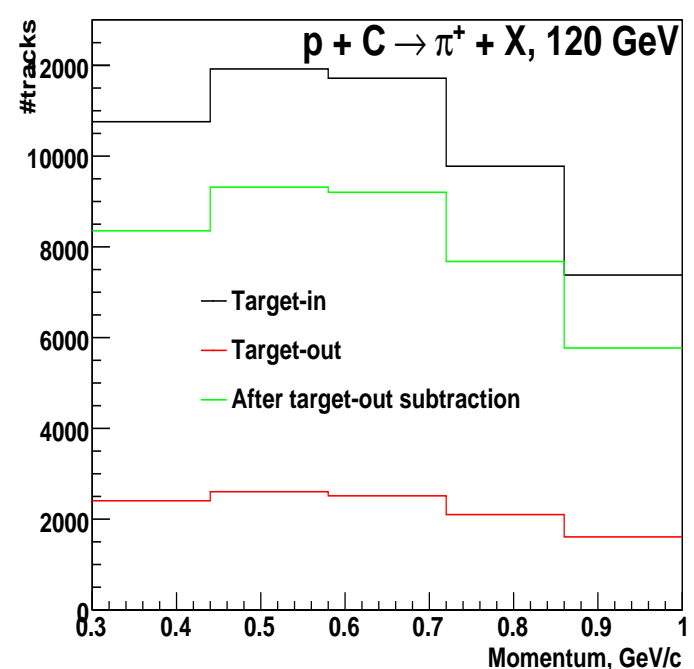

(b)

Figure 4.29: Momentum distributions for (a) pion negatives and (b) positives for target-in (shown in black) and target-out (shown in red) data for momentum range $0.3-1 \mathrm{GeV} / \mathrm{c}$ using TPC and $120 \mathrm{GeV} / \mathrm{c} \mathrm{p}+\mathrm{C}$ data. Momentum distribution after target-out subtraction (shown in green) is also shown. 


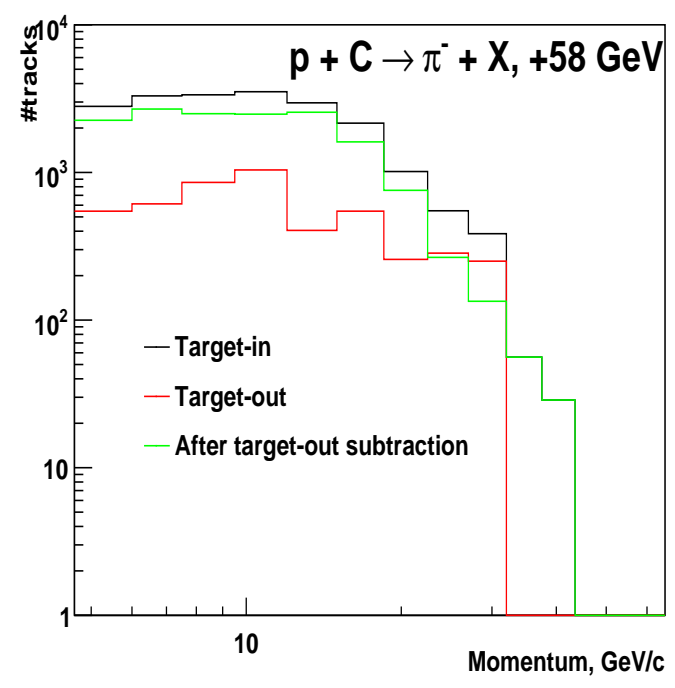

(a)

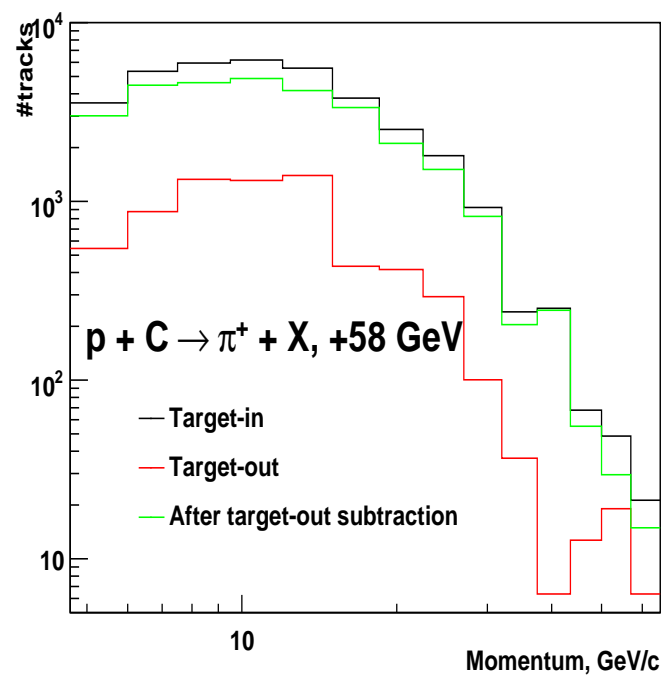

(b)

Figure 4.30: Momentum distributions for (a) pion negatives and (b) positives for target-in (shown in black) and target-out (shown in red) data for momentum range $4.64-120 \mathrm{GeV} / \mathrm{c}$ using $\mathrm{RICH}$ and $58 \mathrm{GeV} / \mathrm{c} \mathrm{p}+\mathrm{C}$ data. Momentum distribution after target-out subtraction (shown in green) is also shown.

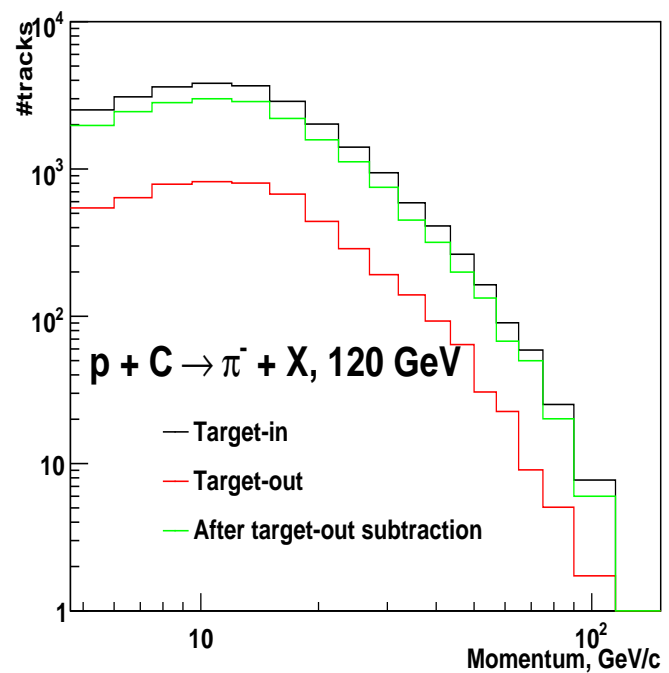

(a)

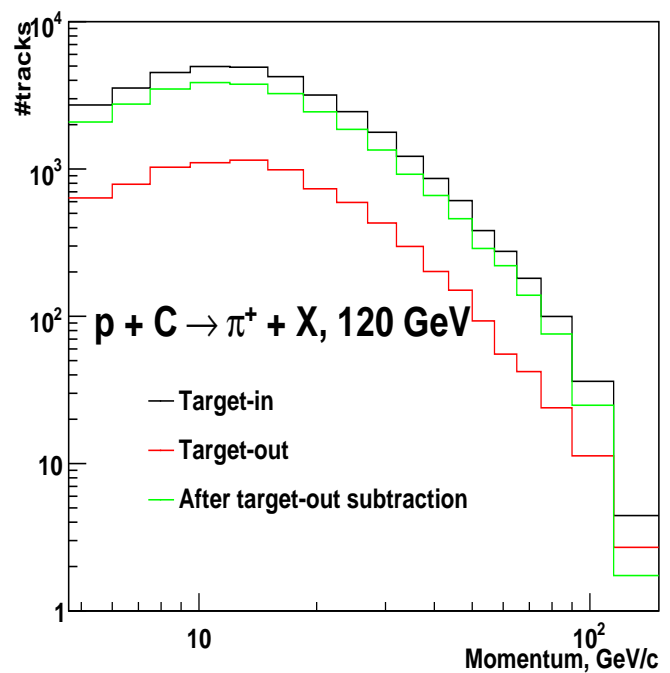

(b)

Figure 4.31: Momentum distributions for (a) pion negatives and (b) positives for target-in (shown in black) and target-out (shown in red) data for momentum range $4.64-120 \mathrm{GeV} / \mathrm{c}$ using RICH and $120 \mathrm{GeV} / \mathrm{c} \mathrm{p}+\mathrm{C}$ data. Momentum distribution after target-out subtraction (shown in green) is also shown. 

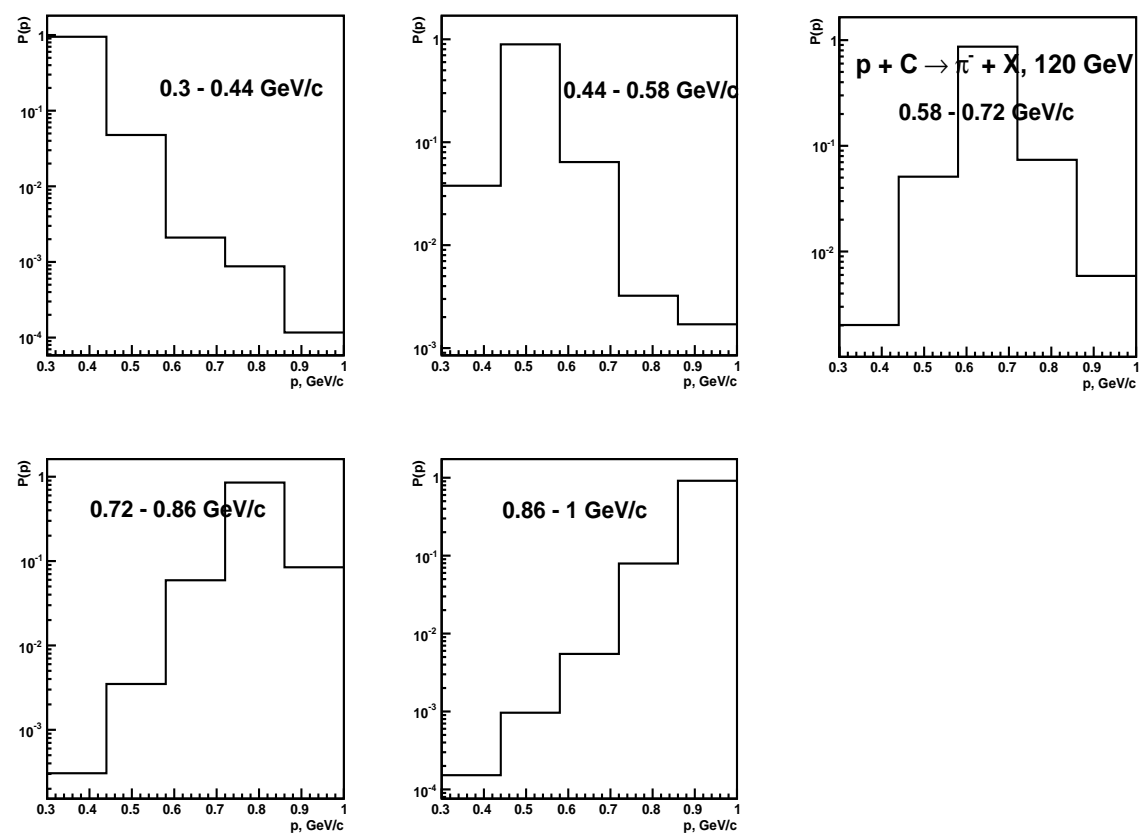

Figure 4.32: The probability of getting a reconstructed pion for a given true pion for the pion negatives for momentum range $0.3-1 \mathrm{GeV} / \mathrm{c}$ using $\mathrm{TPC}$ and $120 \mathrm{GeV} / \mathrm{c} \mathrm{p}+\mathrm{C}$ data.
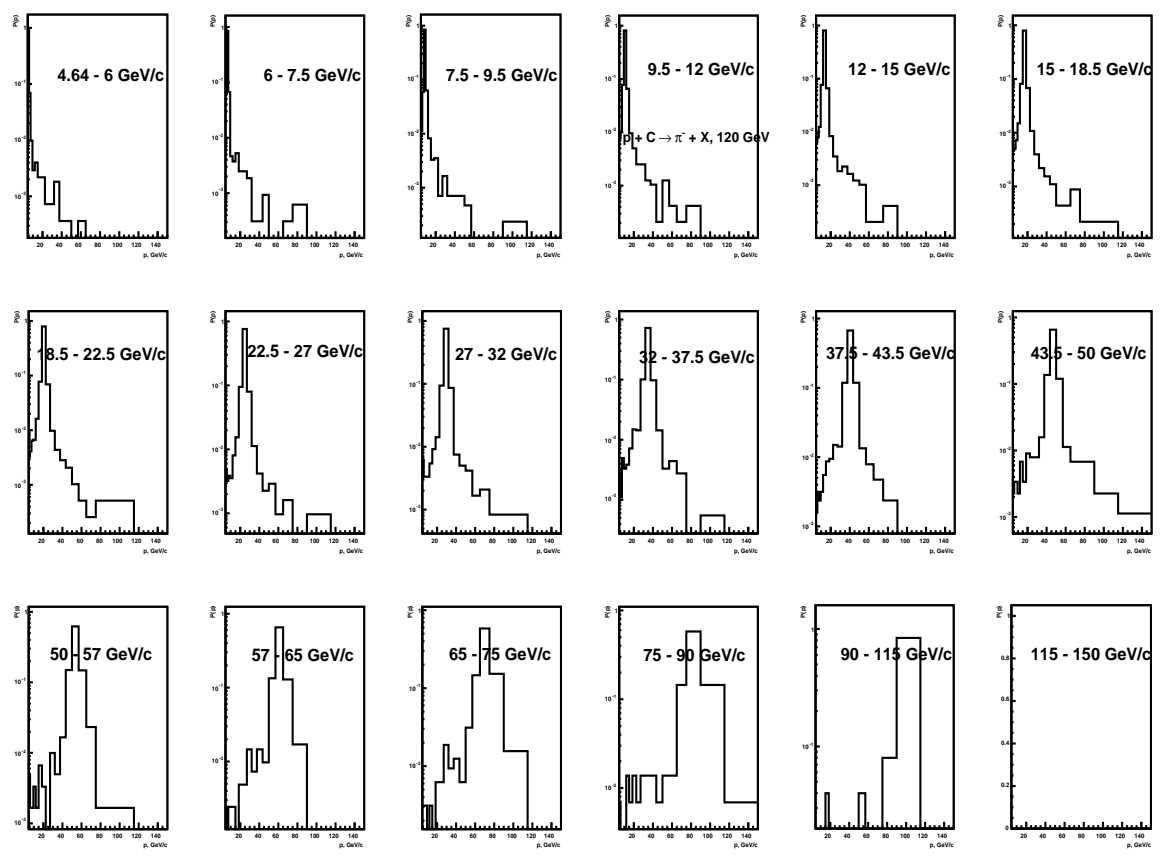

Figure 4.33: The probability of getting a reconstructed pion for a given true pion for the pion negatives for momentum range $4.64-120 \mathrm{GeV} / \mathrm{c}$ using $\mathrm{RICH}$ and $120 \mathrm{GeV} / \mathrm{c} \mathrm{p}+\mathrm{C}$ data. 
at the minimum for the pion negatives and positives using 58 and $120 \mathrm{GeV} / \mathrm{c} \mathrm{p}+\mathrm{C}$ data, respectively using the TPC for momentum range $0.3-1 \mathrm{GeV} / \mathrm{c}$. The corresponding plots using the RICH for momentum range $4.64-120 \mathrm{GeV} / \mathrm{c}$ are shown in figures 4.36 and 4.37 The fits are good i.e. the observed and predicted pion momentum distributions match well.

The unfolding is done in terms of momentum probabilities. The total number of pions is known in the data and these probabilities are multiplied by that number to get the momentum spectrum. This momentum spectrum is then converted into inclusive pion production cross section curve by dividing the number of pions in each momentum bin by the total number of beam particles i.e. incident protons, a constant quantity $\mathrm{n}_{t}$ which is number of target particles per $\mathrm{cm}^{2}$ depending on the target and a correction factor $\epsilon$ (explained later in section 5.1).

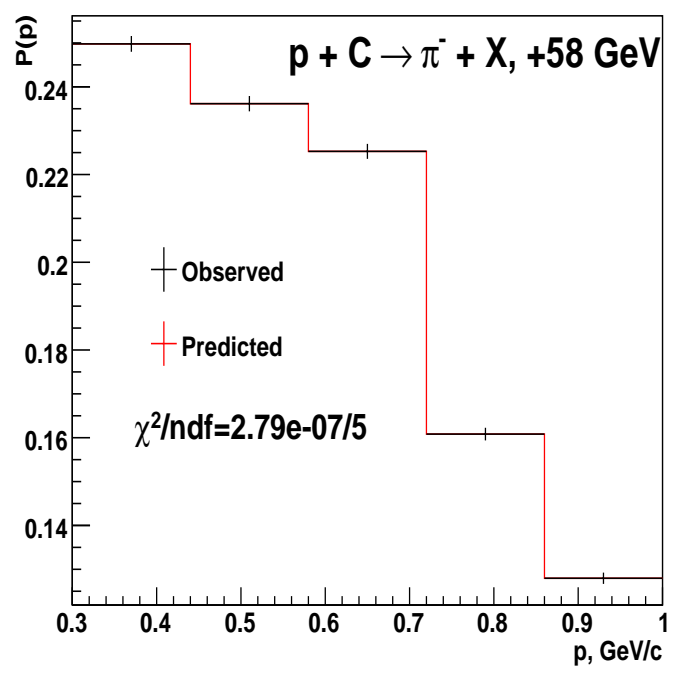

(a)

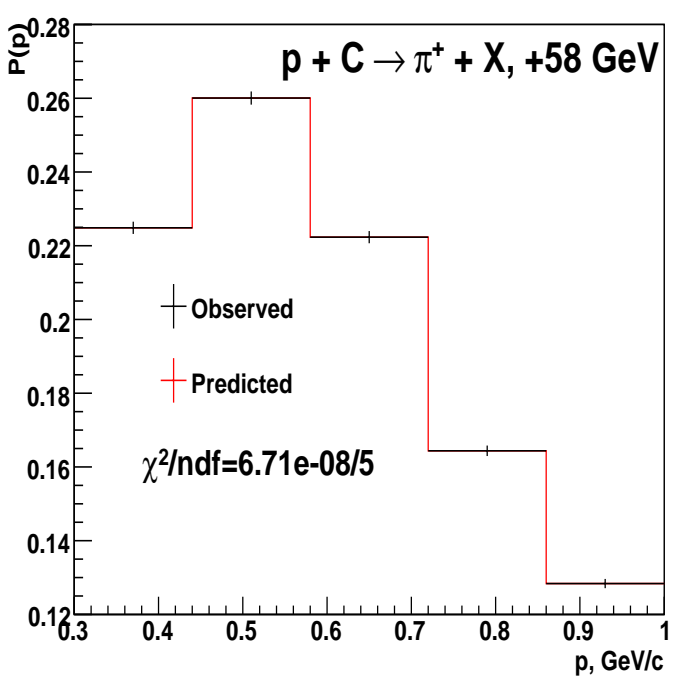

(b)

Figure 4.34: (a) The comparison of the observed and predicted distributions at the minimum for (a) pion negatives and (b) positives for momentum range $0.3-1 \mathrm{GeV} / \mathrm{c}$ using TPC and $58 \mathrm{GeV} / \mathrm{c} \mathrm{p}+\mathrm{C}$ data.

\subsection{Corrections in bins of true momentum}

All the event and track selection requirements explained in section 3.2 are $\mathrm{ON}$ in inclusive pion production analysis. Corrections are required because of the inefficiencies due to the interaction trigger and applied cuts, detector acceptances etc. The average trigger and cut efficiencies, and geometrical acceptance $\times$ reconstruction efficiencies, and all these efficiencies 


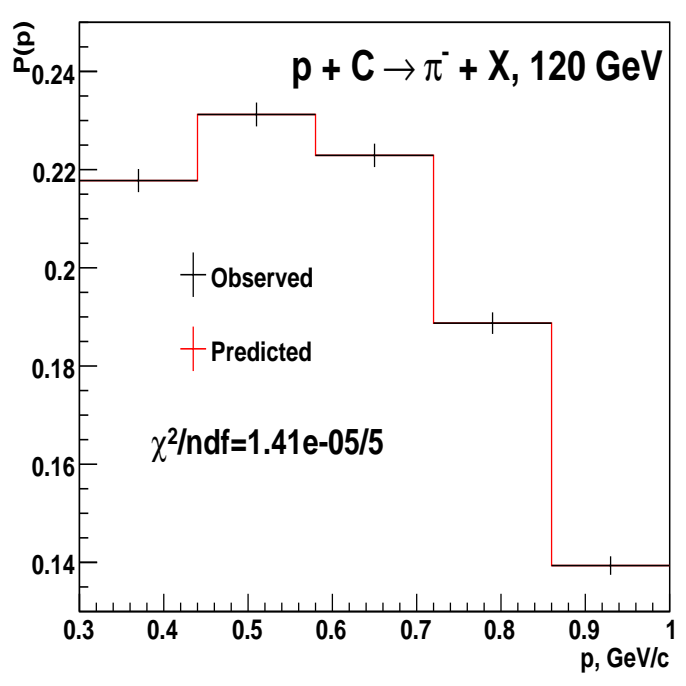

(a)

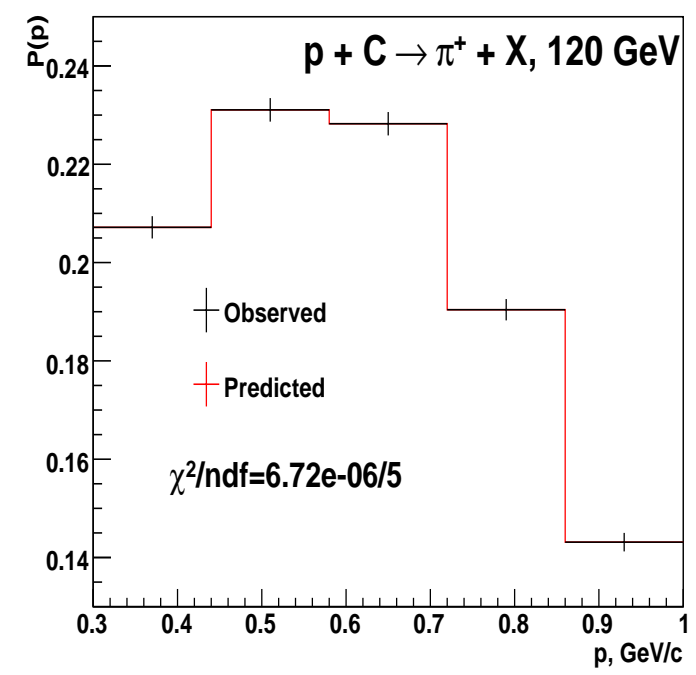

(b)

Figure 4.35: (a) The comparison of the observed and predicted distributions at the minimum for (a) pion negatives and (b) positives for momentum range $0.3-1 \mathrm{GeV} / \mathrm{c}$ using TPC and $120 \mathrm{GeV} / \mathrm{c} \mathrm{p}+\mathrm{C}$ data.

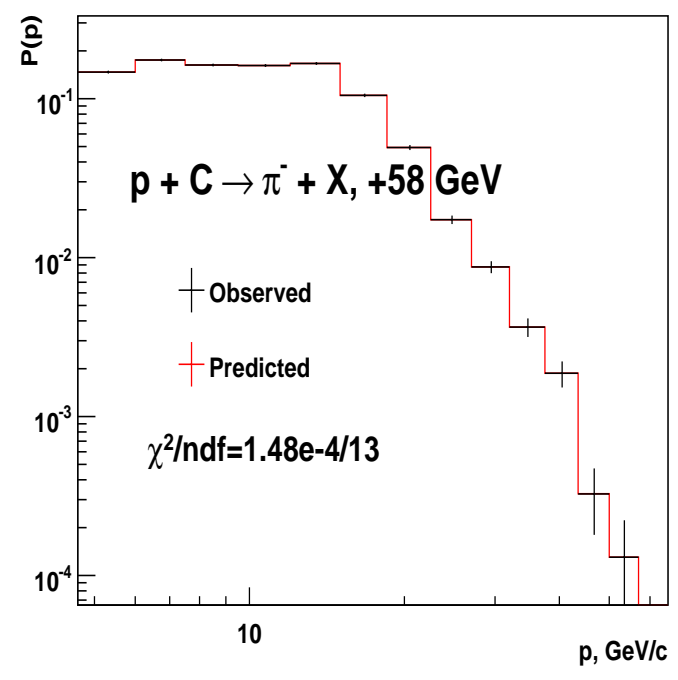

(a)

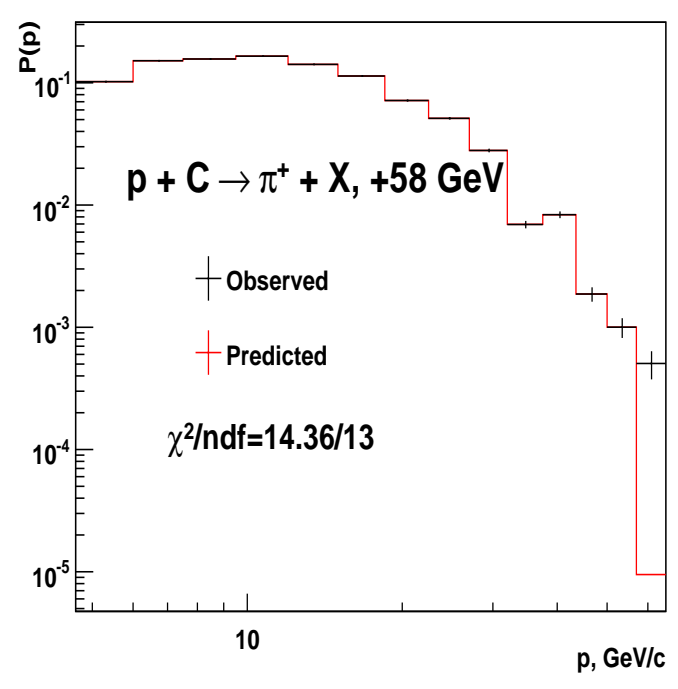

(b)

Figure 4.36: (a) The comparison of the observed and predicted distributions at the minimum for (a) pion negatives and (b) positives for momentum range $4.64-60 \mathrm{GeV} / \mathrm{c}$ using RICH and $58 \mathrm{GeV} / \mathrm{c} \mathrm{p}+\mathrm{C}$ data. 


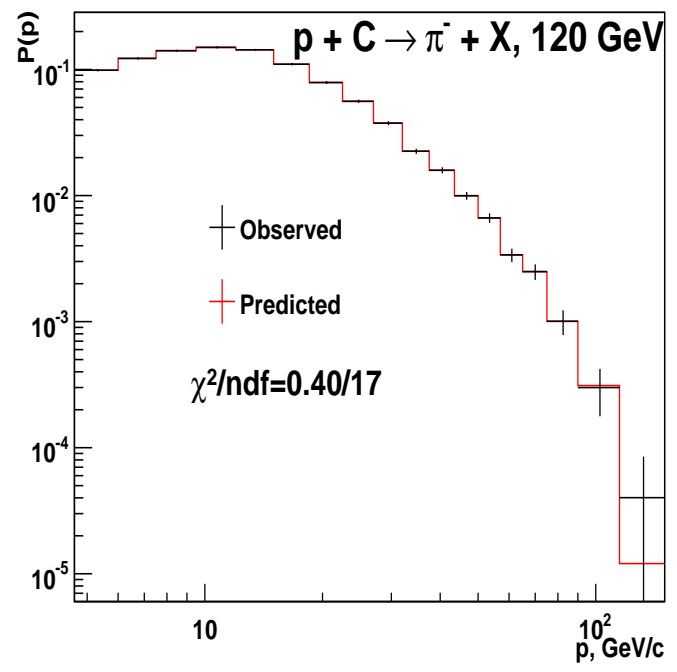

(a)

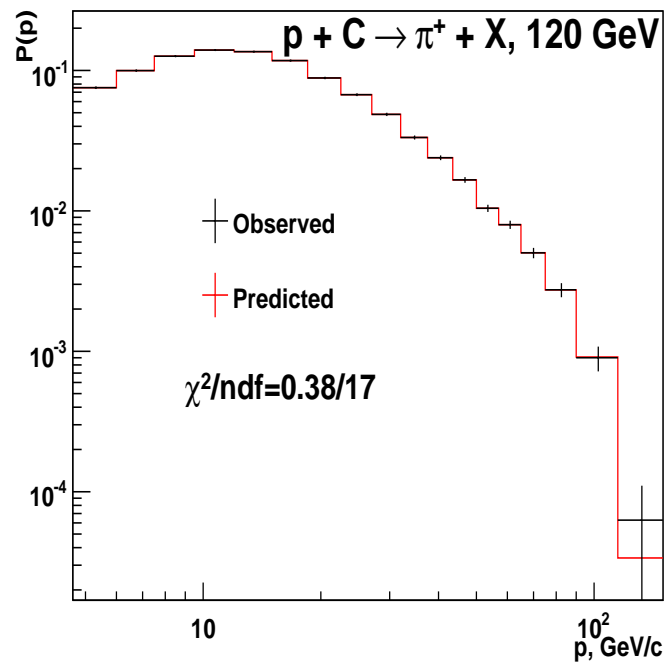

(b)

Figure 4.37: (a) The comparison of the observed and predicted distributions at the minimum for (a) pion negatives and (b) positives for momentum range $4.64-120 \mathrm{GeV} / \mathrm{c}$ using $\mathrm{RICH}$ and $120 \mathrm{GeV} / \mathrm{c} \mathrm{p}+\mathrm{C}$ data.

in bins of reconstructed momentum for correcting total inelastic cross sections and charged particle production cross sections in bins of momentum, respectively were presented in section 3.6.

There are inefficiencies caused by the particle identification also. The missing pions have to be corrected for. So the pion identification (PID) efficiency correction is also an important correction in the measurement of inclusive pion production cross sections. Since we are measuring these cross sections in bins of true momentum for two different momentum ranges i.e. $0.3-1 \mathrm{GeV} / \mathrm{c}$ and $4.64-120 \mathrm{GeV} / \mathrm{c}$, we have to calculate all the efficiencies in bins of true momentum for these two ranges. Also efficiencies are calculated for the negative and positive particles separately. All these efficiencies are discussed as follows:

\subsubsection{Trigger efficiency}

For the calculation of trigger efficiencies in bins of true momentum, two histograms are made and division of these two gives the efficiency. One is the true momentum spectrum of all the tracks which forms the denominator, and the other is the true momentum spectrum of the tracks with the scintillator fired, and that forms the numerator. While making these histograms, the requirements including good beam selection cuts, track selection cuts, and 
the event selection cuts are ON.

\subsubsection{Combined cut efficiency}

We have calculated combined cut efficiencies in bins of true momentum for all the cuts involved. These cuts are cut on beam track index, cut on sum of momenta of tracks per event, cuts on longitudinal and transverse vertex positions, and $\Delta p_{T}$ cut. Out of the two histograms used in the calculation of combined cut efficiency in bins of momentum, one is the true momentum spectrum of the tracks with all the mentioned cuts not applied which forms the denominator, and the other is the true momentum spectrum of the tracks with all these cuts applied, and that forms the numerator. While making these histograms, the other requirements including good beam selection cuts and interaction trigger are ON.

\subsubsection{Geometrical acceptance $\times$ Reconstruction efficiency $\left(\mathcal{A} \epsilon_{\mathcal{R}}\right)$}

Out of the two histograms used in the calculation of $\mathcal{A} \epsilon_{\mathcal{R}}$ (see section 3.6.3) in bins of true momentum, one is the true momentum spectrum of all the MC tracks which forms the denominator, and the other is the true momentum spectrum of the $\mathrm{MC}$ tracks with matched reconstructed tracks, and that forms the numerator. In case of higher momentum range i.e. $4.64-120 \mathrm{GeV} / \mathrm{c}$, the RICH detector acceptance is also taken into account.

\subsubsection{Pion identification efficiency}

For the calculation of pion identification (PID) efficiency, true pions (at generation level) are selected in the MC and corresponding matched reconstructed tracks are selected. Then the PID algorithm is run on these tracks. Out of the two histograms used in the calculation of the PID efficiency in bins of momentum, one is the true MC pion momentum spectrum which is the denominator, and the other is the true MC pion momentum spectrum scaled with the pion hypothesis weight, and that is the numerator. While making these histograms, all the requirements including good beam selection cuts, track selection cuts, and the event selection cuts are ON. The PID efficiency is basically defined as number of pions got reconstructed for a given number of true pions in a particular momentum bin.

All the efficiencies discussed above for the momentum ranges $0.3-1 \mathrm{GeV} / \mathrm{c}$ and $4.64-$ $120 \mathrm{GeV} / \mathrm{c}$ are superimposed in figures 4.38 and 4.39 for negative and positive particles, respectively using $58 \mathrm{GeV} / \mathrm{c} \mathrm{p}+\mathrm{C} \mathrm{MC}$. All the efficiencies using $120 \mathrm{GeV} / \mathrm{c} \mathrm{p}+\mathrm{C} \mathrm{MC}$ are superimposed in figures 4.40 and 4.41 . 
As the momentum increases, the trigger efficiency decreases since the track multiplicity is expected to decrease with the increasing momentum. The cut efficiency decreases with the momentum. The dominating cut is the $\Delta p_{T}$ cut, the reason being we expect to have more beam-like particles at the higher momenta. $\mathcal{A} \epsilon_{\mathcal{R}}$ increases with momentum. The PID efficiency drops at the higher momenta (see next section for more explanation).

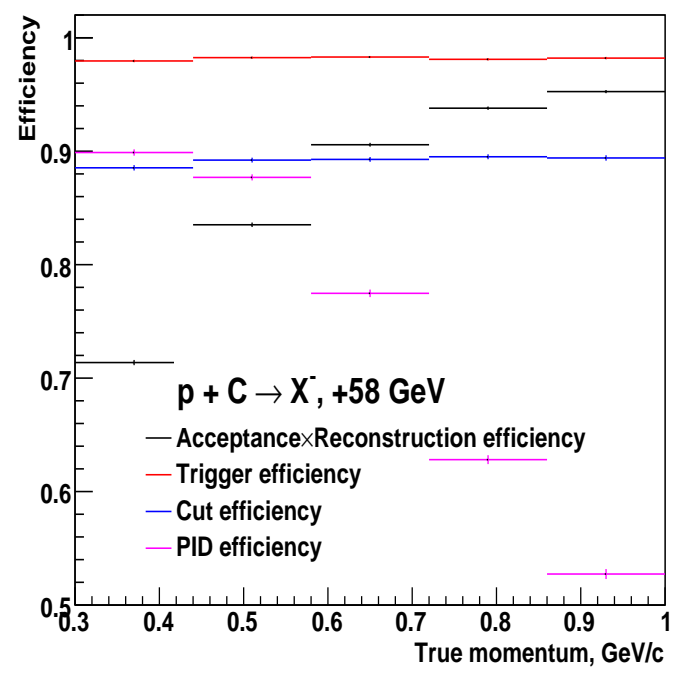

(a)

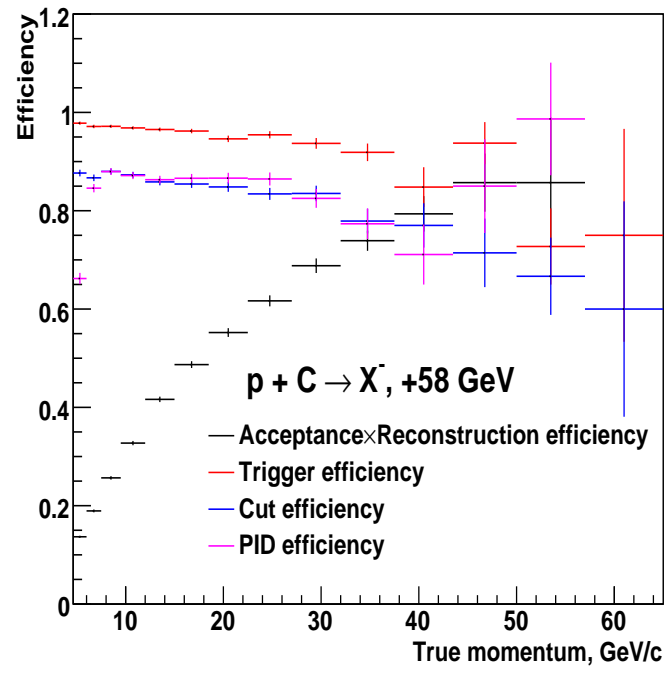

(b)

Figure 4.38: All the efficiencies in bins of true momentum superimposed for negative particles for momentum ranges (a) $0.3-1 \mathrm{GeV} / \mathrm{c}$ and (b) $4.64-60 \mathrm{GeV} / \mathrm{c}$ using $58 \mathrm{GeV} / \mathrm{c} \mathrm{p}+\mathrm{C} \mathrm{MC}$.

\section{Pion mis-identification rate}

We know that there is contamination from the other particles i.e. e/K/p's in both the detectors used for the PID i.e. TPC and RICH, and that is the reason the efficiencies are not $100 \%$. So we calculate the mis-identification rate for the pions i.e. for a given true MC pion, what is the probability for that particle to be identified as a particle other than pion.

The pion misidentification rates for the pion negatives and positives using the TPC and $\mathrm{RICH}$ detectors for $120 \mathrm{GeV} / \mathrm{c} \mathrm{p}+\mathrm{C} \mathrm{MC}$ are shown in figures 4.42 and 4.43 , respectively. In figure 4.42, out of the 4 plots, the top left is the pion identification efficiency itself, the top right is the pion mis-identification rate with electron hypothesis weight applied i.e. contamination from the electrons, the bottom left is the pion mis-identification rate with kaon hypothesis weight applied i.e. contamination from the kaons and the bottom right is the pion mis-identification rate with proton hypothesis weight applied i.e. contamination from 


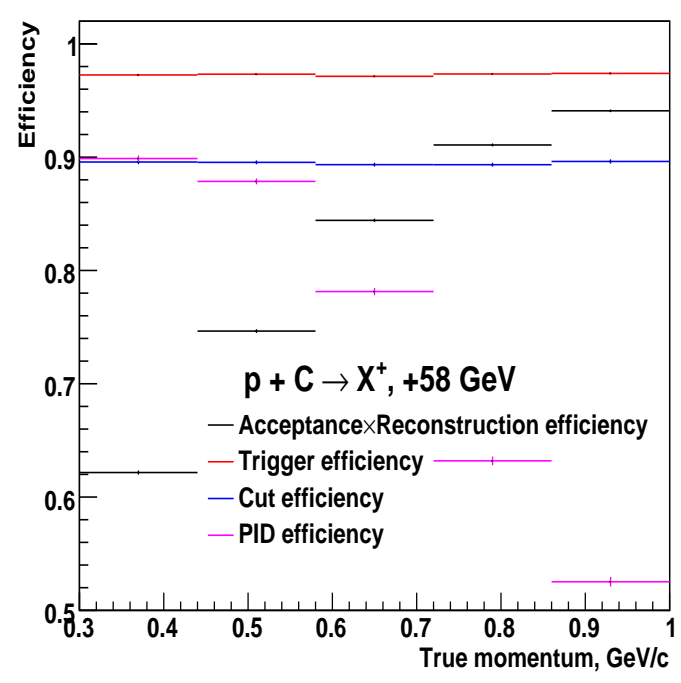

(a)

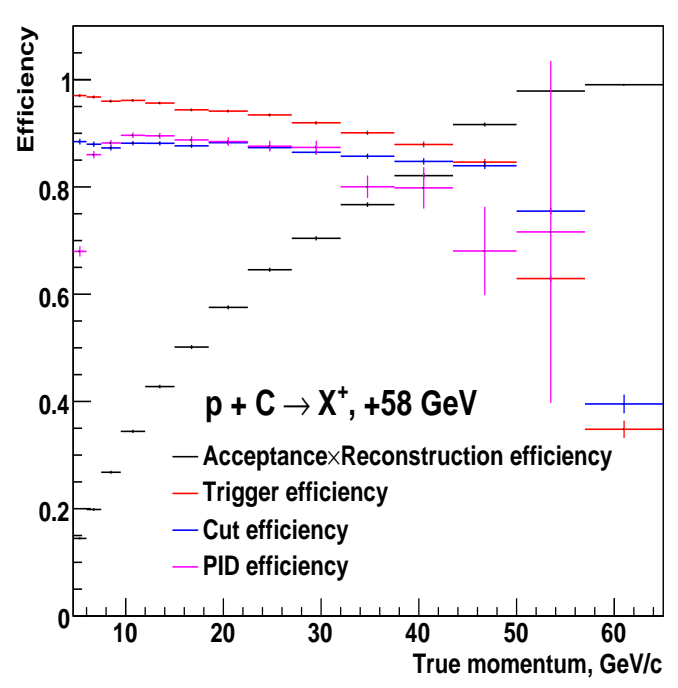

(b)

Figure 4.39: All the efficiencies in bins of true momentum superimposed for positive particles for momentum ranges (a) $0.3-1 \mathrm{GeV} / \mathrm{c}$ and (b) $4.64-60 \mathrm{GeV} / \mathrm{c}$ using $58 \mathrm{GeV} / \mathrm{c} \mathrm{p}+\mathrm{C} \mathrm{MC}$.

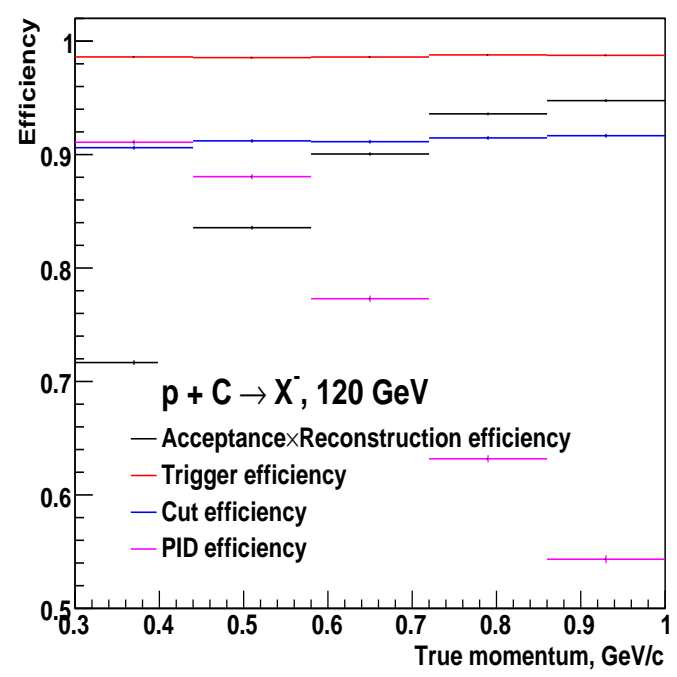

(a)

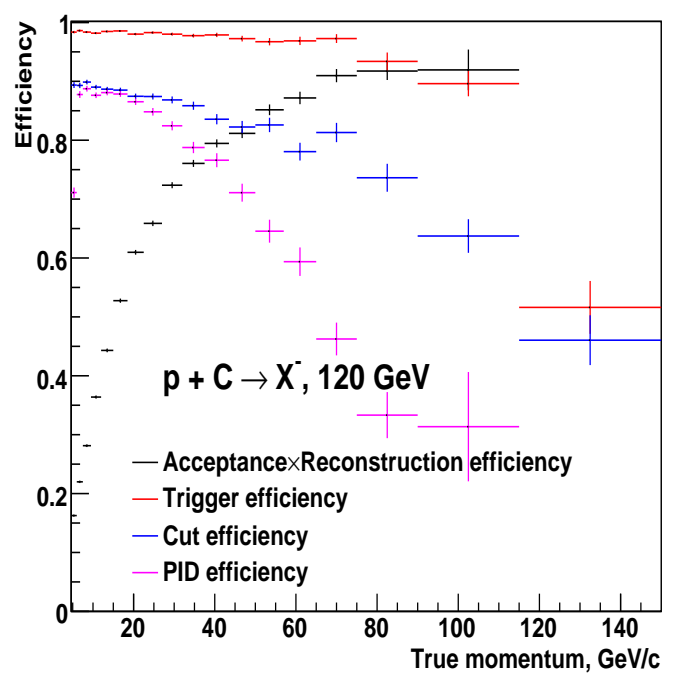

(b)

Figure 4.40: All the efficiencies in bins of true momentum superimposed for negative particles for momentum ranges (a) $0.3-1 \mathrm{GeV} / \mathrm{c}$ and (b) $4.64-120 \mathrm{GeV} / \mathrm{c}$ using $120 \mathrm{GeV} / \mathrm{c} \mathrm{p}+\mathrm{C}$ MC. 


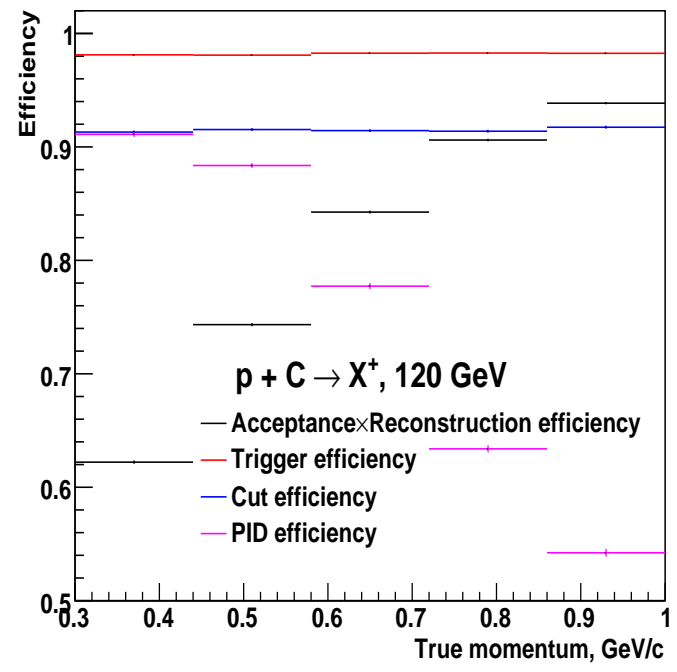

(a)

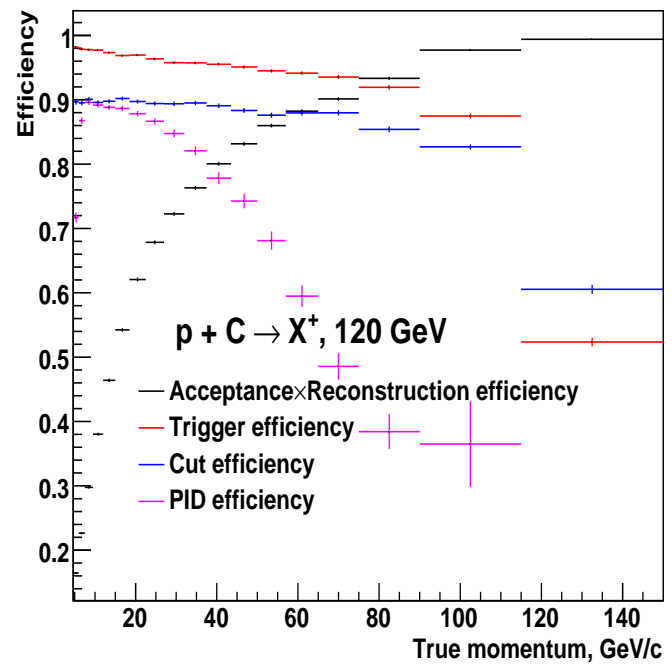

(b)

Figure 4.41: All the efficiencies in bins of true momentum superimposed for positive particles for momentum ranges (a) $0.3-1 \mathrm{GeV} / \mathrm{c}$ and (b) $4.64-120 \mathrm{GeV} / \mathrm{c}$ using $120 \mathrm{GeV} / \mathrm{c} \mathrm{p}+\mathrm{C}$ $\mathrm{MC}$.

the protons. The kaon weight increases while the electron weight decreases with momentum. The reason for the increasing kaon weights with momentum is that the $\mathrm{dE} / \mathrm{dx}$ curve for kaons merge with that of pions at the higher momenta as also mentioned earlier. From the plot it is clear that most of the contamination is from the electrons and kaons. The proton contamination is negligible as seen from the small weights assigned to them.

In figure 4.43 , the corresponding plots are shown using the RICH. The electron and kaon weights increase above $\sim 20 \mathrm{GeV} / \mathrm{c}$ and $\sim 50 \mathrm{GeV} / \mathrm{c}$, respectively which means that most of the contamination is from them. The contamination from the protons is negligible as seen from the small weights assigned to them.

The predicted ring radii as a funtion of momenta superimposed for all the hypotheses are shown in figure 4.44 for the $\mathrm{MC}$ sample with only true pions selected for $120 \mathrm{GeV} / \mathrm{c} \mathrm{p}+\mathrm{C}$ interactions. The radii curves of electrons and pions start merging above $\sim 20 \mathrm{GeV} / \mathrm{c}$, and the curves of pions and kaons start merging above $\sim 70 \mathrm{GeV} / \mathrm{c}$. Due to the close values of the ring radii, the likelihoods (weights) of these particles are also close that is why we see the lower pion identification efficiencies in these regions of momenta which means there are contaminations from the electrons and kaons. The radii curve for protons is not merging with that of pions which results in a negligible contamination from them. 

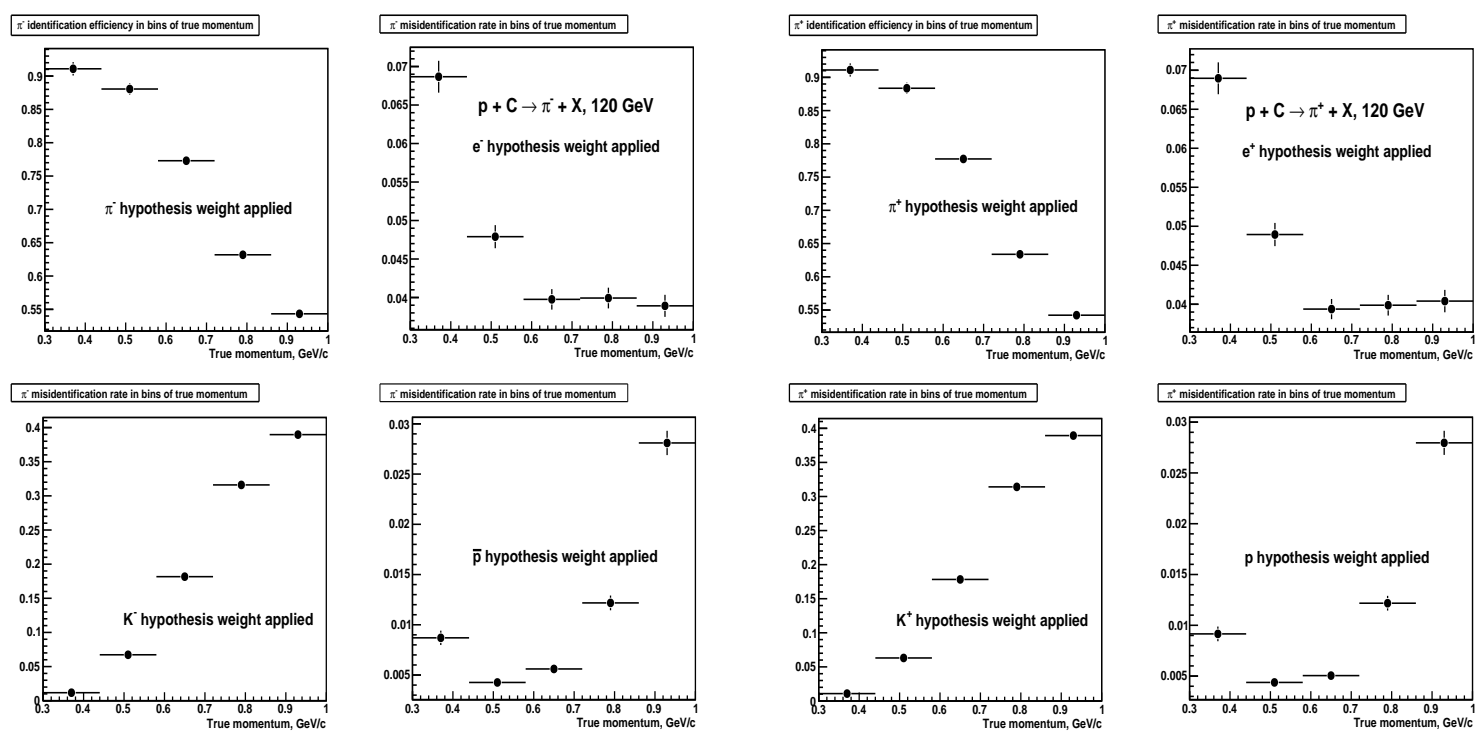

(a)

(b)

Figure 4.42: Pion mis-identification rate for (a) pion negatives and (b) pion positives using the $\mathrm{TPC}$ and $120 \mathrm{GeV} / \mathrm{c} \mathrm{p}+\mathrm{C} \mathrm{MC}$.
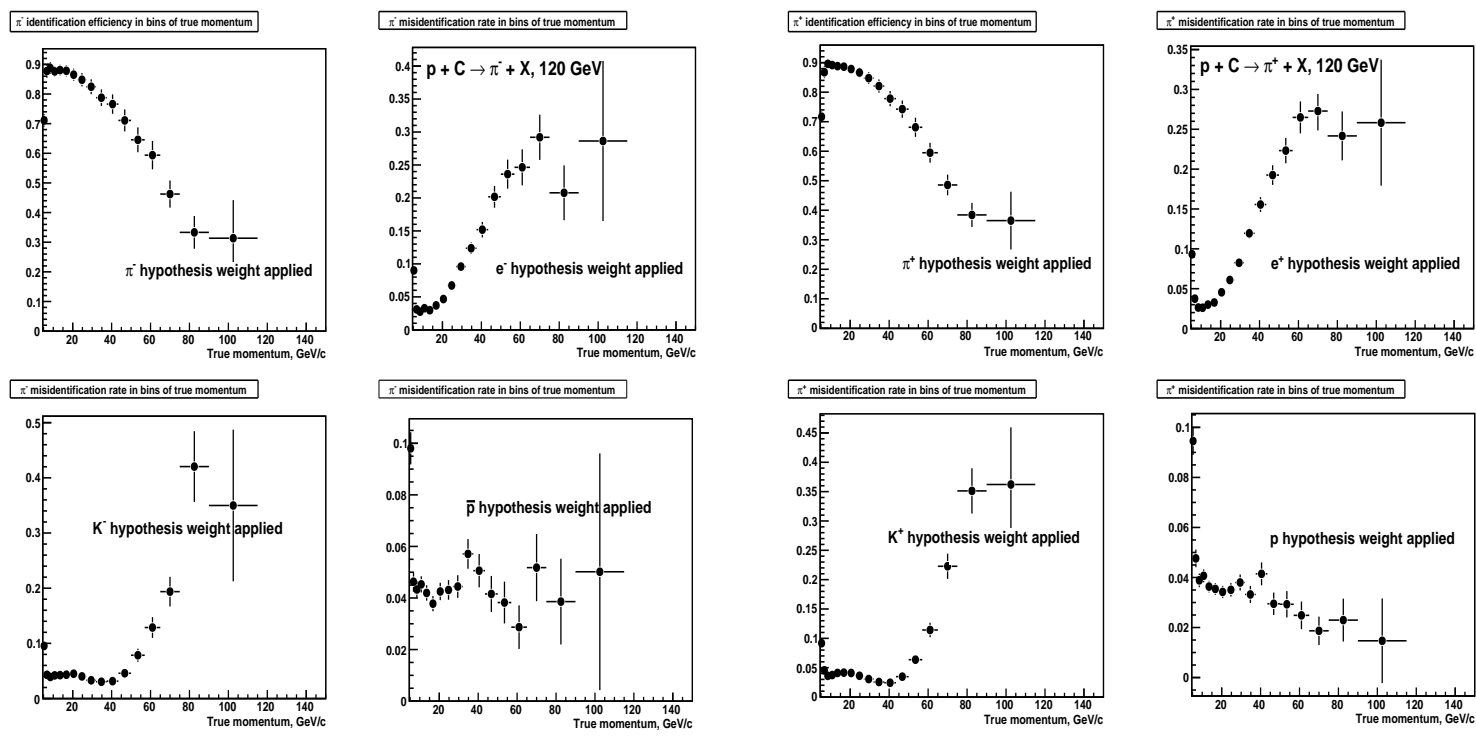

(a)
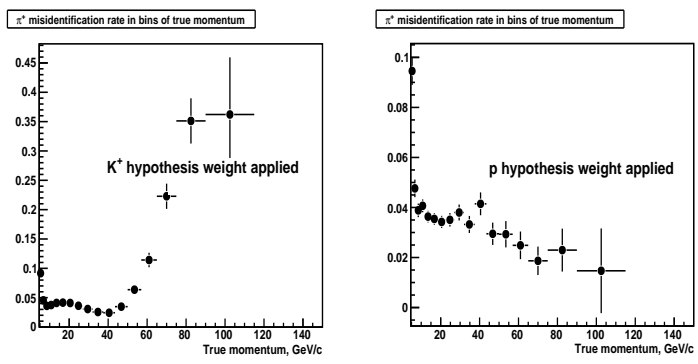

(b)

Figure 4.43: Pion mis-identification rate for (a) pion negatives and (b) pion positives using the $\mathrm{RICH}$ and $120 \mathrm{GeV} / \mathrm{c} \mathrm{p}+\mathrm{C} \mathrm{MC}$. 


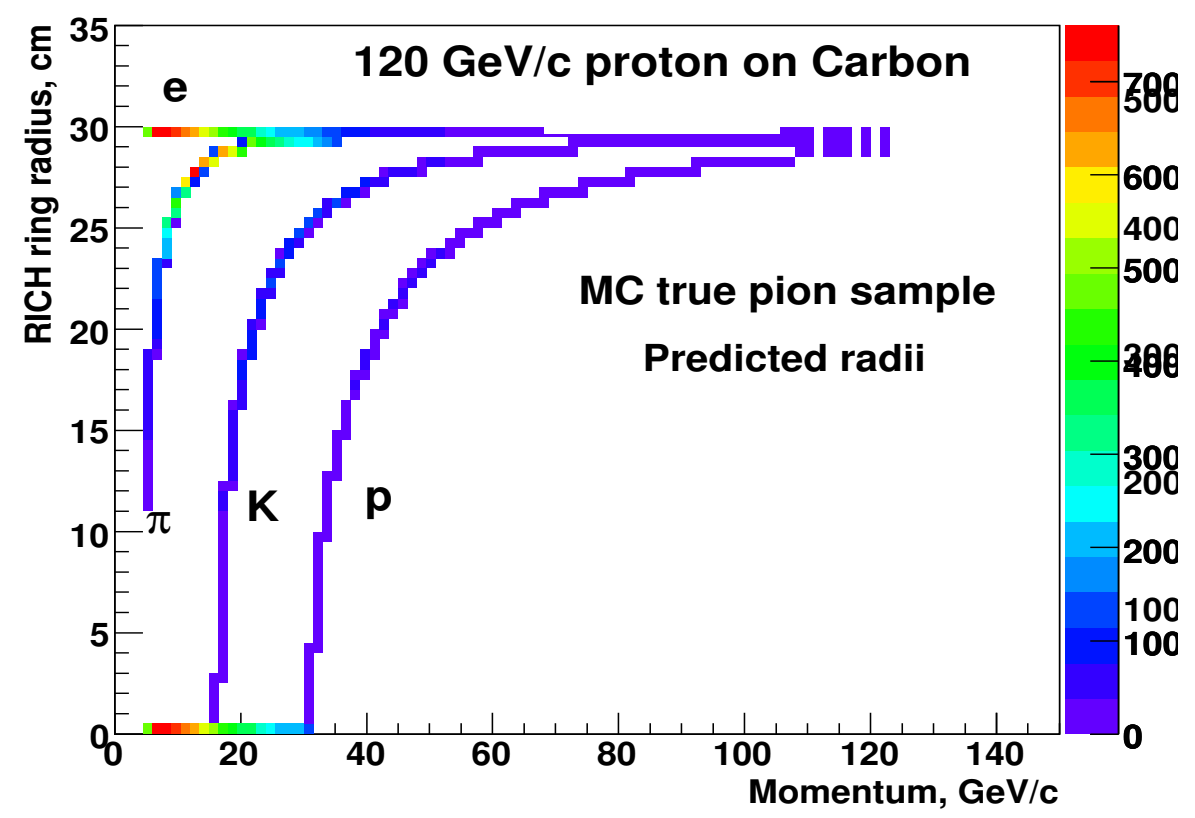

Figure 4.44: The predicted ring radii distributions as a funtion of momenta superimposed for all the hypotheses for the MC sample with only true pions selected for $120 \mathrm{GeV} / \mathrm{c} \mathrm{p}+\mathrm{C}$ interactions. 


\section{Chapter 5}

\section{Results}

In this chapter we present the results for inelastic cross section and inclusive charged pion production cross section measurements.

\subsection{Inelastic cross sections}

The data collected by MIPP consist of minimum-biased and interaction triggers. $80 \%$ of these data were collected with the interaction trigger required. The inelastic cross sections are calculated using the interaction triggers. All event selection cuts described in the section 3.2 are required during the selection of interactions. The cross sections are corrected using the corrections from the Monte Carlo as described in the section 3.6. The formula used in the cross section calculation is as follows:

$$
\sigma=\frac{N_{\text {int }}}{N_{\text {beam }} \times n_{t} \times \epsilon},
$$

where $\mathrm{N}_{\text {int }}$ is the number of interactions, $\mathrm{N}_{\text {beam }}$ is the number of beam particles i.e. incident protons, $\mathrm{n}_{t}$ is the number of target particles per $\mathrm{cm}^{2}$, and $\epsilon$ is the product of efficiencies. $\mathrm{N}_{\text {int }}$ is after subtracting the target-out (empty cryotarget/empty target) contribution from the target-in $\left(\mathrm{LH}_{2} / \mathrm{Be} / \mathrm{C} / \mathrm{Bi}\right)$ data. The target-out sample is normalized to the same number of incident protons as those for target-in sample so that the number of interactions for both the samples correspond to the same number of beam particles. The target-out data is scaled by an extra scale factor to match the scintillator peak heights for the target-in and target-out data as described in the section 3.2 .2 .

$\mathrm{n}_{t}$ is a target dependent quantity and is given by

$$
n_{t}=\frac{N_{A} \times \text { density } \times \text { thickness }}{A},
$$




\begin{tabular}{|l|l|l|l|l|}
\hline Target & $\begin{array}{l}\text { Atomic weight } \\
(\mathbf{g m} / \mathbf{m o l e})\end{array}$ & $\begin{array}{l}\text { Thickness } \\
(\mathbf{c m})\end{array}$ & $\begin{array}{l}\text { Density } \\
\left(\mathbf{g m} / \mathbf{c m}^{3}\right)\end{array}$ & $\mathbf{n}_{t} \times \mathbf{1 0}^{\mathbf{2 3}} \mathbf{( c m}^{-2} \mathbf{)}$ \\
\hline $\mathrm{LH}_{2}$ & 1.008 & 14 & 0.0708 & 5.922 \\
\hline Beryllium & 9.012 & 0.399 & 1.779 & 0.4746 \\
\hline Carbon & 12.011 & 1.003 & 1.672 & 0.8408 \\
\hline Bismuth & 209 & 0.173 & 9.769 & 0.04875 \\
\hline
\end{tabular}

Table 5.1: The density, thickness, $\mathrm{A}$ and $\mathrm{n}_{t}$ for different targets used.

where $\mathrm{N}_{A}$ is the Avogadro number and is equal to $6.0221 \times 10^{23} / \mathrm{mole}$, and $\mathrm{A}$ is the target atomic weight. The density, thickness, $\mathrm{A}$ and $\mathrm{n}_{t}$ for different targets used are shown in table 5.1 .

The cross section measurements for $\mathrm{LH}_{2}, \mathrm{C}, \mathrm{Be}$ and $\mathrm{Bi}$ targets using proton beam at different momenta are described as follows:

\subsubsection{Inelastic $\mathrm{p}+\mathrm{p}$ cross section at 58 and $85 \mathrm{GeV} / \mathrm{c}$}

The number of beam particles, number of inelastic interactions and cross section numbers for $\mathrm{LH}_{2}$ target using 58 and $85 \mathrm{GeV} / \mathrm{c}$ proton beams are listed in table 5.2. Both the uncorrected and corrected cross section numbers are given in this table along with statistical errors. The unscaled numbers are before applying prescaler and scaled numbers are after applying prescaler (see section 3.2.2). The statistical error is calculated using the number of events. For $58 \mathrm{GeV} / \mathrm{c}$ beam, the statistical error is $\sim 3 \%$, and for $85 \mathrm{GeV} / \mathrm{c}$ beam, the error is $\sim 2 \%$.

\subsubsection{Inelastic $\mathrm{p}+\mathrm{C}$ cross section at 58 and $120 \mathrm{GeV} / \mathrm{c}$}

The number of beam particles, number of inelastic interactions, and cross section numbers for carbon target at 58 and $120 \mathrm{GeV} / \mathrm{c}$ are listed in table 5.3. Both the uncorrected and corrected cross section numbers are given along with statistical errors. For $58 \mathrm{GeV} / \mathrm{c}$ beam, the statistical error is $\sim 5 \%$, and for $120 \mathrm{GeV} / \mathrm{c}$ beam, the error is $\sim 1 \%$.

The $\mathrm{p}+\mathrm{C}$ measurement at $120 \mathrm{GeV} / \mathrm{c}$ is found to be $\sim 20 \%$ lower than that at 58 $\mathrm{GeV} / \mathrm{c}$. A little rise in the cross section is expected as we go higher in beam momentum. Inelastic cross sections are also measured for other thin targets $\mathrm{Be}$ and $\mathrm{Bi}$ (presented in next section) where 58 and $120 \mathrm{GeV} / \mathrm{c}$ data are available, and similar discrepancy is observed. 


\begin{tabular}{|c|c|c|c|c|}
\hline & \multicolumn{2}{|c|}{$\mathrm{p}+\mathrm{p}, 58 \mathrm{GeV}$} & \multicolumn{2}{|c|}{$\mathrm{p}+\mathrm{p}, 85 \mathrm{GeV}$} \\
\hline & unscaled & scaled & unscaled & scaled \\
\hline $\mathrm{N}_{\text {beam }}$ target-in & 8682 & $2.84993 \mathrm{e}+07$ & 31366 & $4.77333 \mathrm{e}+07$ \\
\hline $\mathrm{N}_{\text {beam }}$ target-out & 1849 & $5.91865 \mathrm{e}+06$ & 9279 & $1.46237 \mathrm{e}+07$ \\
\hline $\mathrm{N}_{\text {int }}$ target-in & 20816 & 499584 & 48160 & 975590 \\
\hline $\mathrm{N}_{\text {int }}$ target-out & 1307 & $31368 \times 5.104=160105$ & 4054 & $85134 \times 3.721=316790$ \\
\hline $\begin{array}{l}\mathrm{N}_{\text {int }} \text { after target-out } \\
\text { subtraction }\end{array}$ & & $3 \overline{339479}$ & & 6558800 \\
\hline$\sigma_{\text {inel }}$ uncorrected $(\mathrm{mb})$ & & $20.27 \pm 0.62$ & & $23.43 \pm 0.41$ \\
\hline $\begin{array}{llr}\sigma_{\text {inel }} & \text { corrected } & \text { for } \\
\text { event } & \text { selection } & \text { cuts } \\
(\mathrm{mb}) & & \\
\end{array}$ & & $24.12 \pm 0.73$ & & $28.12 \pm 0.49$ \\
\hline $\begin{array}{l}\sigma_{\text {inel }} \text { corrected for trig- } \\
\text { ger }(\mathrm{mb})\end{array}$ & & $27.02 \pm 0.82$ & & $30.43 \pm 0.53$ \\
\hline $\begin{array}{l}\sigma_{\text {inel }} \text { corrected for } \mathcal{A} \epsilon_{\mathcal{R}} \\
(\mathrm{mb})\end{array}$ & & $30.33 \pm 0.92$ & & $34.64 \pm 0.60$ \\
\hline
\end{tabular}

Table 5.2: $\mathrm{N}_{\text {beam }}, \mathrm{N}_{\text {int }}$ and $\sigma_{\text {inel }}$ for $\mathrm{p}+\mathrm{p}$ interactions at 58 and $85 \mathrm{GeV} / \mathrm{c}$.

\begin{tabular}{|c|c|c|c|c|}
\hline & \multicolumn{2}{|c|}{$\mathrm{p}+\mathrm{C}, 58 \mathrm{GeV}$} & \multicolumn{2}{|c|}{$\mathrm{p}+\mathrm{C}, 120 \mathrm{GeV}$} \\
\hline & unscaled & scaled & unscales & scaled \\
\hline $\mathrm{N}_{\text {beam }}$ target-in & 1742 & $9.58806 \mathrm{e}+06$ & $\overline{c 12591}$ & 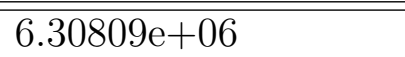 \\
\hline $\mathrm{N}_{\text {beam }}$ target-out & 934 & $5.00735 \mathrm{e}+06$ & 19344 & $9.69134 \mathrm{e}+06$ \\
\hline $\mathrm{N}_{\text {int }}$ target-in & 8151 & 195624 & 105750 & 105750 \\
\hline $\mathrm{N}_{\text {int }}$ target-out & 674 & $16176 \times 2.566=41504.8$ & 28719 & $28719 \times 0.814=23366.5$ \\
\hline $\begin{array}{l}\mathrm{N}_{\text {int }} \text { after target-out } \\
\text { subtraction }\end{array}$ & & 154119 & & 82383.5 \\
\hline$\sigma_{\text {inel }}$ uncorrected $(\mathrm{mb})$ & & $191.18 \pm 8.93$ & & $155.33 \pm 1.73$ \\
\hline $\begin{array}{llr}\sigma_{\text {inel }} & \text { corrected } & \text { for } \\
\text { event } & \text { selection } & \text { cuts } \\
(\mathrm{mb}) & & \\
\end{array}$ & & $223.81 \pm 10.45$ & & $179.72 \pm 2.0$ \\
\hline $\begin{array}{l}\sigma_{\text {inel }} \text { corrected for trig- } \\
\text { ger }(\mathrm{mb})\end{array}$ & & $231.69 \pm 10.82$ & & $183.39 \pm 2.04$ \\
\hline $\begin{array}{l}\sigma_{\text {inel }} \text { corrected for } \mathcal{A} \epsilon_{\mathcal{R}} \\
(\mathrm{mb})\end{array}$ & & $267.29 \pm 12.48$ & & $213.54 \pm 2.37$ \\
\hline
\end{tabular}

Table 5.3: $\mathrm{N}_{\text {beam }}, \mathrm{N}_{\text {int }}$ and $\sigma_{\text {inel }}$ for $\mathrm{p}+\mathrm{C}$ interactions at 58 and $120 \mathrm{GeV} / \mathrm{c}$. 


\subsubsection{Inelastic cross sections for $\mathrm{Be}$ and $\mathrm{Bi}$ targets}

The number of beam particles, number of inelastic interactions, and cross section numbers at 58 and $120 \mathrm{GeV} / \mathrm{c}$ for Be and Bi targets are listed in tables 5.4 and 5.5 , respectively. Both the uncorrected and corrected cross section numbers are given along with statistical errors.

\begin{tabular}{|c|c|c|c|c|}
\hline & \multicolumn{2}{|c|}{$\mathrm{p}+\mathrm{Be}, 58 \mathrm{GeV}$} & \multicolumn{2}{|c|}{$\mathrm{p}+\mathrm{Be}, 120 \mathrm{GeV}$} \\
\hline & unscaled & scaled & unscaled & scaled \\
\hline $\mathrm{N}_{\text {beam }}$ target-in & 452 & $2.52228 \mathrm{e}+06$ & 44400 & $2.22444 \mathrm{e}+07$ \\
\hline $\mathrm{N}_{\text {beam }}$ target-out & 934 & $5.00735 \mathrm{e}+06$ & 19344 & $9.69134 \mathrm{e}+06$ \\
\hline $\mathrm{N}_{\text {int }}$ target-in & 1204 & 28896 & 212496 & 212496 \\
\hline $\mathrm{N}_{\text {int }}$ target-out & 674 & $16176 \times 0.675=10918.5$ & 28719 & $28719 \times 2.571=73828.5$ \\
\hline $\begin{array}{l}\mathrm{N}_{\text {int }} \text { after target-out } \\
\text { subtraction }\end{array}$ & & 17977.5 & & 138667.5 \\
\hline$\sigma_{\text {inel }}$ uncorrected $(\mathrm{mb})$ & & $150.18 \pm 10.10$ & & $131.35 \pm 1.03$ \\
\hline $\begin{array}{lll}\sigma_{\text {inel }} & \text { corrected } & \text { for } \\
\text { event } & \text { selection } & \text { cuts } \\
(\mathrm{mb}) & & \\
\end{array}$ & & $176.04 \pm 11.84$ & & $152.41 \pm 1.20$ \\
\hline $\begin{array}{l}\sigma_{\text {inel }} \text { corrected for trig- } \\
\text { ger }(\mathrm{mb})\end{array}$ & & $183.58 \pm 12.35$ & & $155.76 \pm 1.23$ \\
\hline $\begin{array}{l}\sigma_{\text {inel }} \text { corrected for } \mathcal{A} \epsilon_{\mathcal{R}} \\
(\mathrm{mb})\end{array}$ & & $213.14 \pm 14.34$ & & $183.78 \pm 1.45$ \\
\hline
\end{tabular}

Table 5.4: $\mathrm{N}_{\text {beam }}, \mathrm{N}_{\text {int }}$ and $\sigma_{\text {inel }}$ for p+Be interactions at 58 and $120 \mathrm{GeV} / \mathrm{c}$.

\subsubsection{Systematic Errors}

For systematics, we take into account the contribution from beam flux, $\mathrm{Z}$ vertex selection, trigger inefficiency corrections, acceptance and target-out subtraction. All of these are discussed below:

- Beam flux: Beam flux systematics is estimated using the difference of two methods of beam flux calculation i.e. using beam prescalers and direct scalers. The calculation of beam flux using these two methods was discussed in the section 3.3 . The fractional differences between these two methods were shown in table 3.4. A conservative systematic error of $5 \%$ has been assigned due to beam flux. The beam flux is varied by $\pm 5 \%$ and the cross sections are recalculated. Difference of these cross sections from the nominal cross section gives the systematic error $( \pm)$ from the beam flux which is given in table 5.6 . 


\begin{tabular}{|c|c|c|c|c|}
\hline & \multicolumn{2}{|c|}{$\mathrm{p}+\mathrm{Bi}, 58 \mathrm{GeV}$} & \multicolumn{2}{|c|}{$\mathrm{p}+\mathrm{Bi}, 120 \mathrm{GeV}$} \\
\hline & unscaled & scaled & unscaled & scaled \\
\hline $\mathrm{N}_{\text {beam }}$ target-in & 6482 & $1.89442 \mathrm{e}+07$ & 49402 & $2.47504 \mathrm{e}+07$ \\
\hline $\mathrm{N}_{\text {beam }}$ target-out & 6390 & $1.36078 \mathrm{e}+07$ & 19344 & $9.69134 \mathrm{e}+06$ \\
\hline $\mathrm{N}_{\text {int }}$ target-in & 11387 & 189633 & 190120 & 190120 \\
\hline $\mathrm{N}_{\text {int }}$ target-out & 3405 & $46204 \times 1.211=55961.2$ & 28719 & $28719 \times 2.682=77011.8$ \\
\hline $\begin{array}{l}\mathrm{N}_{\text {int }} \text { after target-out } \\
\text { subtraction }\end{array}$ & & 1333672 & & 113108 \\
\hline$\sigma_{\text {inel }}$ uncorrected $(\mathrm{mb})$ & & $1447.4 \pm 33.50$ & & $937.43 \pm 7.28$ \\
\hline $\begin{array}{lll}\sigma_{\text {inel }} & \text { corrected } & \text { for } \\
\text { event } & \text { selection } & \text { cuts } \\
(\mathrm{mb}) & & \\
\end{array}$ & & $1611.88 \pm 37.31$ & & $1046.75 \pm 8.13$ \\
\hline $\begin{array}{l}\sigma_{\text {inel }} \text { corrected for trig- } \\
\text { ger }(\mathrm{mb})\end{array}$ & & $1640.09 \pm 37.96$ & & $1059.14 \pm 8.23$ \\
\hline $\begin{array}{l}\sigma_{\text {inel }} \text { corrected for } \mathcal{A} \epsilon_{\mathcal{R}} \\
(\mathrm{mb})\end{array}$ & & $2285.52 \pm 52.90$ & & $1434.95 \pm 11.14$ \\
\hline
\end{tabular}

Table 5.5: $\mathrm{N}_{\text {beam }}, \mathrm{N}_{\text {int }}$ and $\sigma_{\text {inel }}$ for $\mathrm{p}+\mathrm{Bi}$ interactions at 58 and $120 \mathrm{GeV} / \mathrm{c}$.

- $\mathbf{Z}$ vertex selection: To get an estimate of systematics from $Z$ vertex selection in case of $\mathrm{LH}_{2}$ target, a narrow $\mathrm{Z}$ selection is made in which case no target-out subtraction is needed, and the cross section is calculated. Difference of this cross section from the cross section using the nominal $\mathrm{Z}$ cut i.e. $-15<\mathrm{Z}_{v t x}-\mathrm{Z}_{t g t}<15 \mathrm{~cm}$ gives the systematic error from the $\mathrm{Z}$ vertex selection. The narrow $\mathrm{Z}$ selection cut used is $-6<\mathrm{Z}_{v t x}-\mathrm{Z}_{t g t}<$ $4 \mathrm{~cm}$. The narrow $\mathrm{Z}$ vertex selection for $85 \mathrm{GeV} / \mathrm{c} \mathrm{p}+\mathrm{p}$ interactions is shown in figure 5.1. A bump is observed in the $\mathrm{Z}$ region $-9<\mathrm{Z}_{v t x}-\mathrm{Z}_{t g t}<-6 \mathrm{~cm}$ that is why this region is not selected in making the narrow $\mathrm{Z}$ selection. From the studies done, it was found that this bump is due to the interactions with the $\mathrm{LH}_{2}$ transport pipes and ring.

To get an estimate of systematics from $\mathrm{Z}$ vertex selection in case of carbon target, the nominal $\mathrm{Z}$ vertex cut is varied by $\pm 3 \mathrm{~cm}$, more than the $\mathrm{Z}$ vertex resolution (see section 2.10.2. The nominal $\mathrm{Z}$ cut is $-4<\mathrm{Z}_{v t x}-\mathrm{Z}_{t g t}<6 \mathrm{~cm}$, after varying it by $+3 \mathrm{~cm}$, the cut is $-5.5<\mathrm{Z}_{v t x}-\mathrm{Z}_{t g t}<7.5 \mathrm{~cm}$, and after varying by $-3 \mathrm{~cm}$, the cut is $-2.5<\mathrm{Z}_{v t x}$ $\mathrm{Z}_{t g t}<4.5 \mathrm{~cm}$. The cross sections are recalculated using these new $\mathrm{Z}$ cuts. Difference of these cross sections from the nominal cross section gives the systematic error $( \pm)$ from the $\mathrm{Z}$ vertex selection which is given in table 5.6 .

- Interaction trigger: The cross section is corrected for the inefficiencies due to the interaction trigger. The trigger efficiency is calculated using the Monte Carlo. To get an estimate of systematics from this correction, the correction is varied by $\pm 10 \%$ and 


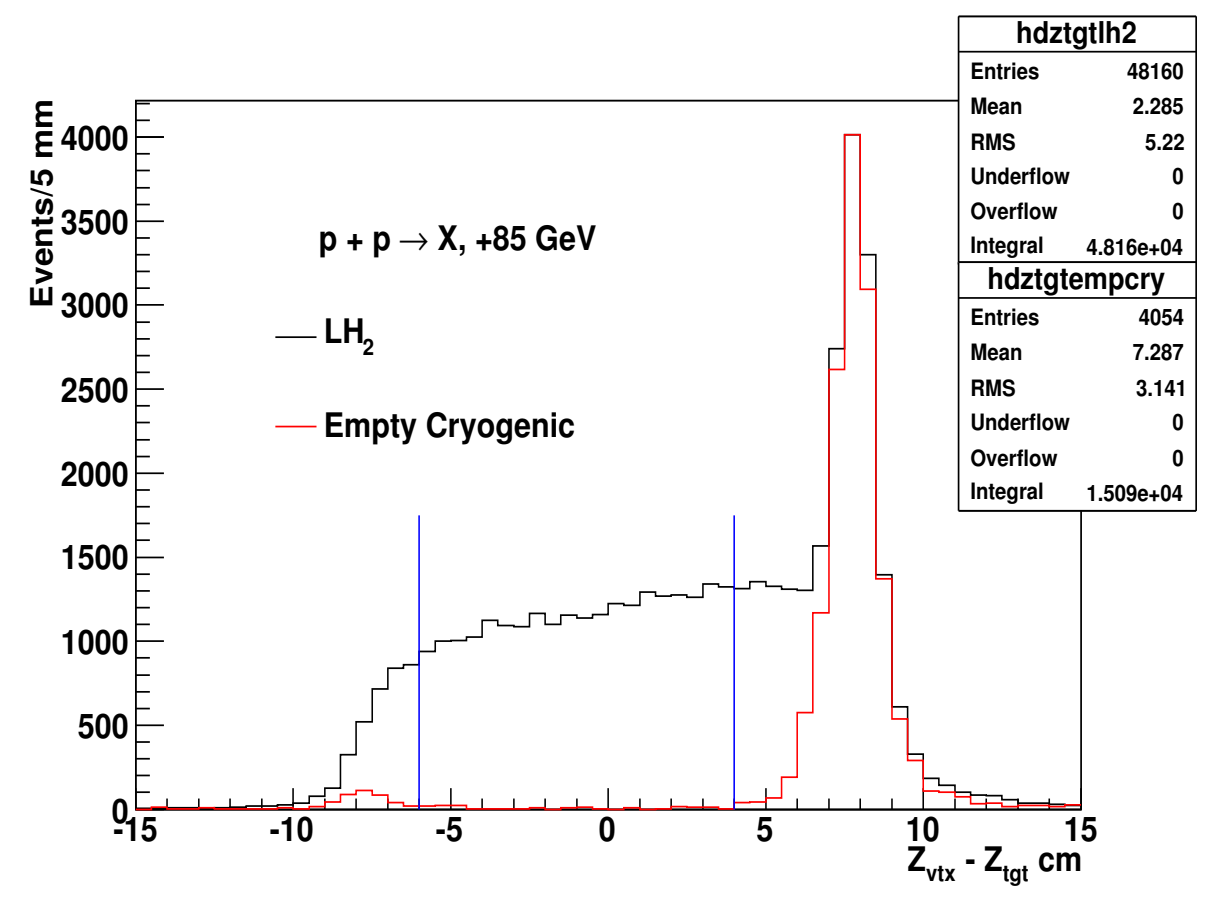

Figure 5.1: $\mathrm{Z}$ vertex distribution for $\mathrm{p}+\mathrm{p}$ interactions at $85 \mathrm{GeV} / \mathrm{c}$. The narrow $\mathrm{Z}$ cut used is shown by the blue lines i.e. $-6<\Delta Z<4 \mathrm{~cm}$.

the cross sections are recalculated. Difference of these cross sections from the nominal cross section gives the systematic error $( \pm)$ from trigger inefficiency corrections which is given in table 5.6 .

- Acceptance $\times$ reconstruction efficiency $\left(\mathcal{A} \epsilon_{\mathcal{R}}\right)$ : The improper modeling of particle yields in the Monte Carlo can arise a systematics from $\mathcal{A} \epsilon_{\mathcal{R}}$ corrections. To get the estimate of this systematics, ratio of data and MC multiplicities is calculated. The comparison of the data and $\mathrm{MC}$ multiplicities, and their ratio for 58 and $85 \mathrm{GeV} / \mathrm{c} \mathrm{p}+\mathrm{p}$ interactions are shown in figures $5.2(\mathrm{a})$ and $5.2(\mathrm{~b})$, respectively. The corresponding distributions for 58 and $120 \mathrm{GeV} / \mathrm{c} \mathrm{p}+\mathrm{C}, \mathrm{p}+\mathrm{Be}$ and $\mathrm{p}+\mathrm{Bi}$ interactions are shown in figures 5.3(a), 5.3(b), 5.4(a), 5.4(b), 5.5(a) and 5.5(b), Differences are observed between the data and MC multiplicities. The multiplicity ratio is applied to the MC to reweight it and the acceptance is recalculated. The cross section is recalculated using the new acceptance. Difference of this cross section from the nominal cross section gives the systematic error from $\mathcal{A} \epsilon_{\mathcal{R}}$ corrections which is given in table 5.6.

- Target-out subtraction: For getting an estimate of systematics from target-out subtraction, the extra scale factor which is used to match the scintillator peak heights is varied by $\pm 5 \%$, and the cross sections are recalculated. Difference of these cross 

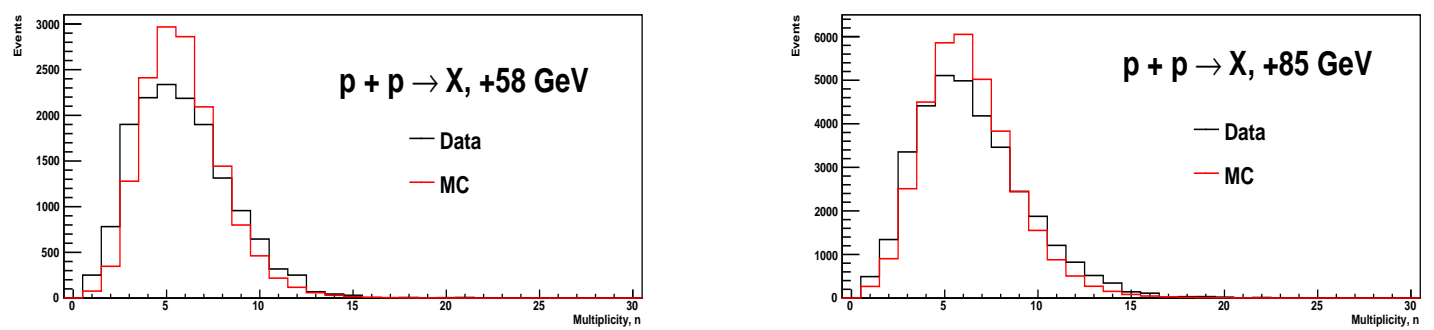

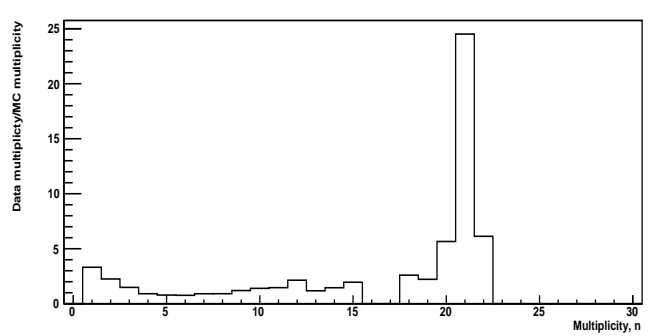

(a)

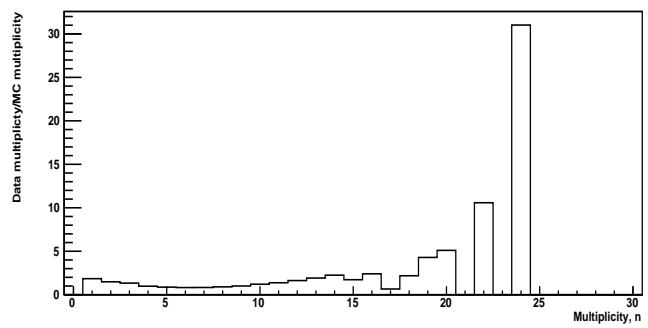

(b)

Figure 5.2: Comparison of data and MC multiplicities (top), and their ratio (bottom) for (a) 58 and (b) $85 \mathrm{GeV} / \mathrm{c} \mathrm{p}+\mathrm{p}$ interactions.
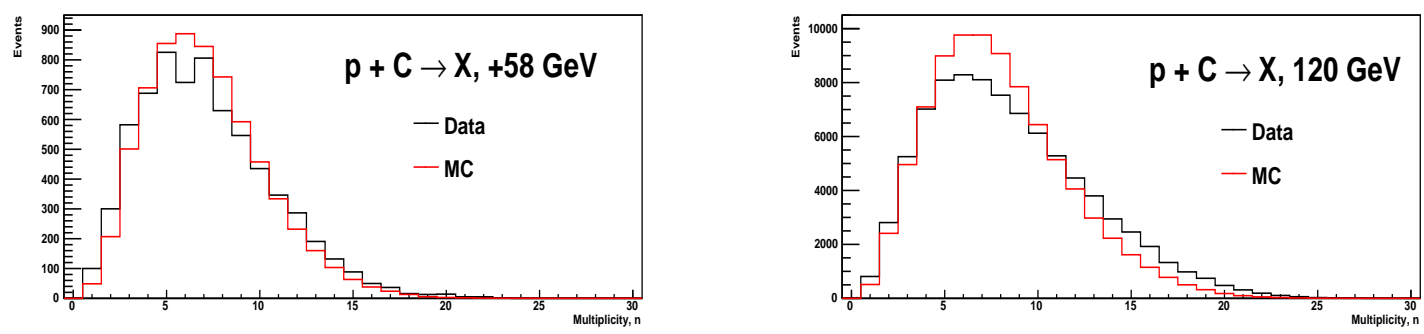

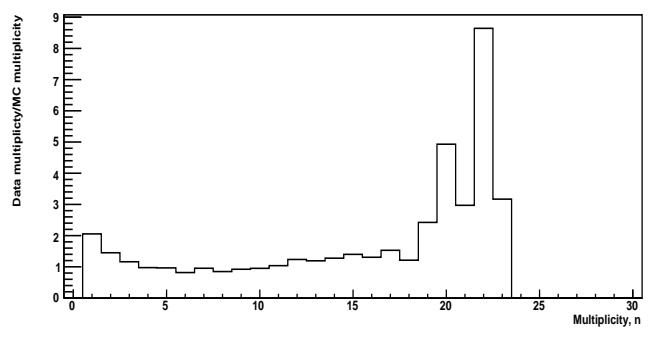

(a)

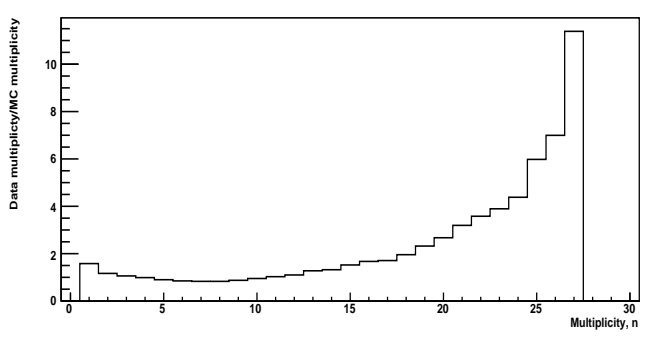

(b)

Figure 5.3: Comparison of data and MC multiplicities (top), and their ratio (bottom) for (a) 58 and (b) $120 \mathrm{GeV} / \mathrm{c} \mathrm{p}+\mathrm{C}$ interactions. 

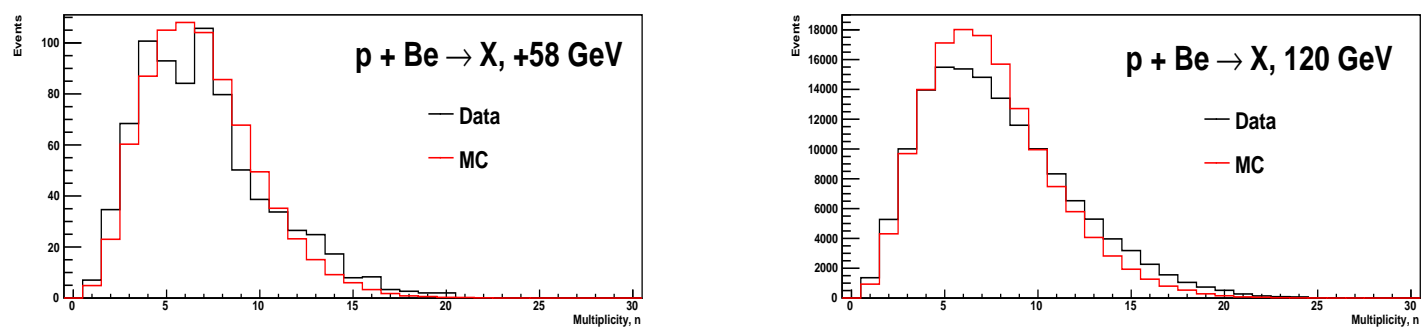

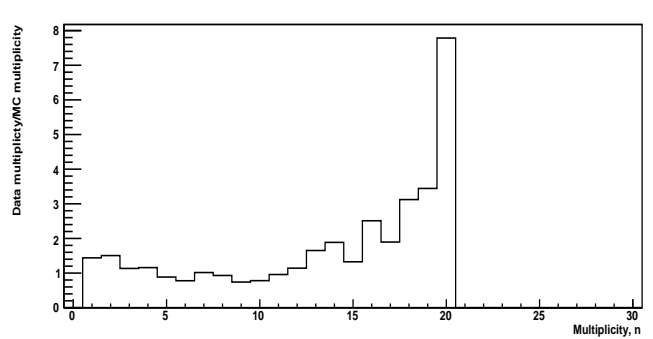

(a)

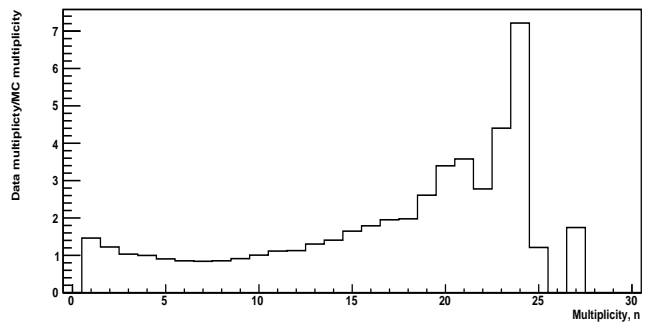

(b)

Figure 5.4: Comparison of data and MC multiplicities (top), and their ratio (bottom) for (a) 58 and (b) $120 \mathrm{GeV} / \mathrm{c} \mathrm{p}+$ Be interactions.
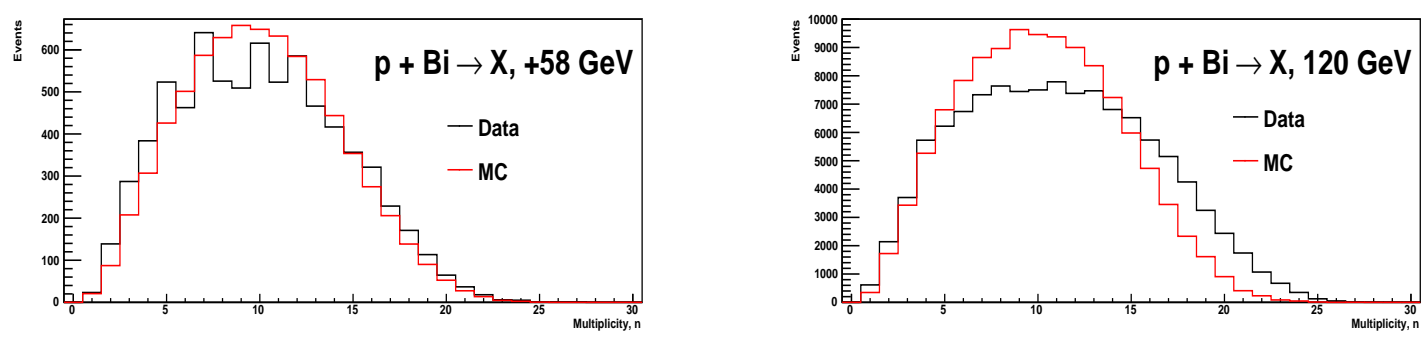

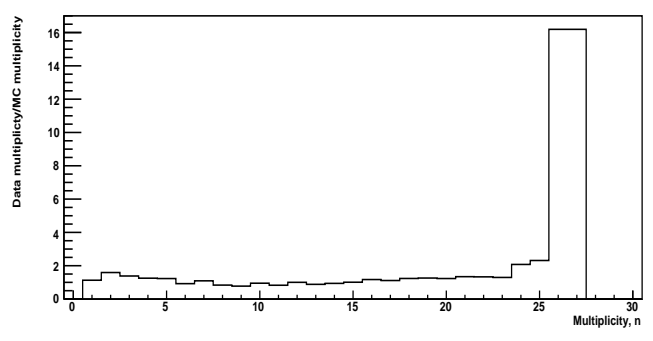

(a)

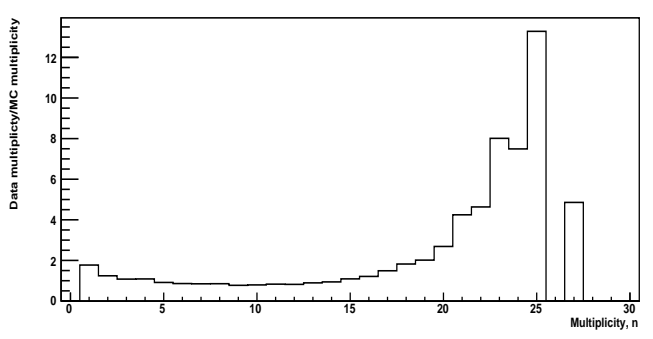

(b)

Figure 5.5: Comparison of data and MC multiplicities (top), and their ratio (bottom) for (a) 58 and (b) $120 \mathrm{GeV} / \mathrm{c} \mathrm{p}+\mathrm{Bi}$ interactions. 
sections from the nominal cross section gives the systematic error $( \pm)$ from the targetout subtraction which is given in table 5.6 .

\begin{tabular}{|c|c|c|c|c|c|c|c|c|}
\hline \multirow[b]{2}{*}{ Interaction } & \multirow[b]{2}{*}{$\sigma_{\text {inel }}(\mathrm{mb})$} & \multicolumn{7}{|c|}{ Errors (\%) } \\
\hline & & 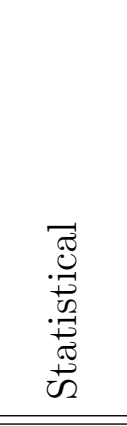 & 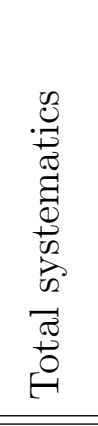 & 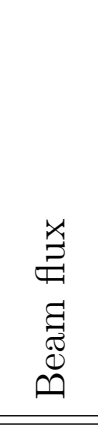 & 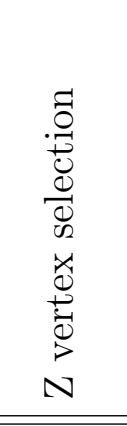 & 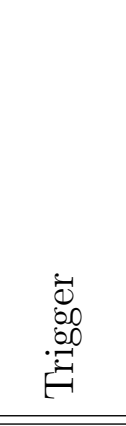 & 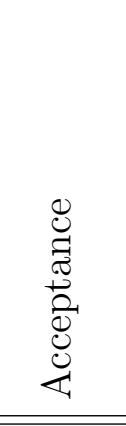 & 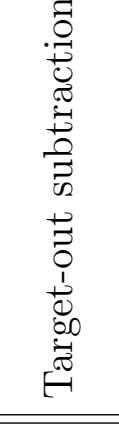 \\
\hline $\mathrm{p}+\mathrm{p}, 58 \mathrm{GeV}$ & $30.33_{-2.13}^{+2.02}$ & \pm 3.05 & $\begin{array}{l}+5.91 \\
-6.32 \\
\end{array}$ & & \pm 2.37 & \pm 1.08 & \pm 0.03 & \pm 2.35 \\
\hline $\mathrm{p}+\mathrm{p}, 85 \mathrm{GeV}$ & $34.64_{-2.18}^{+2.04}$ & \pm 1.73 & $\begin{array}{l}+5.63 \\
+6.06 \\
\end{array}$ & $\begin{array}{l}+4.76 \\
+5.26 \\
\end{array}$ & \pm 1.62 & \pm 0.76 & \pm 0.03 & \pm 2.42 \\
\hline $\mathrm{p}+\mathrm{Be}, 58 \mathrm{GeV}$ & $213.14_{-19.40}^{+18.93}$ & \pm 6.73 & $\begin{array}{l}+5.79 \\
-6.13\end{array}$ & $\begin{array}{l}+4.76 \\
-5.26\end{array}$ & $\begin{array}{l}+1.02 \\
-0.31 \\
\end{array}$ & \pm 0.41 & \pm 0.62 & \pm 3.04 \\
\hline $\mathrm{p}+\mathrm{Be}, 120 \mathrm{GeV}$ & $183.78_{-11.01}^{+10.24}$ & \pm 0.79 & $\begin{array}{l}+5.51 \\
{ }_{-5} .94 \\
\end{array}$ & $\begin{array}{l}+4.76 \\
{ }_{-5.26} \\
\end{array}$ & $\begin{array}{l}+0.33 \\
-0.25 \\
\end{array}$ & \pm 0.22 & \pm 0.66 & \pm 2.66 \\
\hline $\mathrm{p}+\mathrm{C}, 58 \mathrm{GeV}$ & $267.29_{-19.24}^{+18.31}$ & \pm 4.67 & $\begin{array}{l}+5.01 \\
-5.48\end{array}$ & $\begin{array}{l}+4.76 \\
-5.26\end{array}$ & $\begin{array}{l}+0.30 \\
-0.04\end{array}$ & \pm 0.34 & \pm 0.67 & \pm 1.35 \\
\hline $\mathrm{p}+\mathrm{C}, 120 \mathrm{GeV}$ & $213.54_{-12.11}^{+11.13}$ & \pm 1.11 & $\begin{array}{l}+5.09 \\
-5.56 \\
\end{array}$ & $\begin{array}{l}+4.76 \\
-5.26 \\
\end{array}$ & $\begin{array}{l}+0.29 \\
-0.30 \\
\end{array}$ & \pm 0.20 & \pm 1.07 & \pm 1.42 \\
\hline $\mathrm{p}+\mathrm{Bi}, 58 \mathrm{GeV}$ & $2285.52_{-173.47}^{+165.70}$ & \pm 2.31 & $\begin{array}{l}+6.87 \\
-7.23 \\
\end{array}$ & $\begin{array}{l}+4.76 \\
-5.26 \\
\end{array}$ & $\begin{array}{l}+0.39 \\
-0.52\end{array}$ & \pm 0.17 & \pm 4.47 & \pm 2.09 \\
\hline $\mathrm{p}+\mathrm{Bi}, 120 \mathrm{GeV}$ & $1434.95_{-98.44}^{+93.27}$ & \pm 0.78 & $\begin{array}{l}+6.45 \\
-6.82 \\
\end{array}$ & $\begin{array}{l}+4.76 \\
-5.26\end{array}$ & $\begin{array}{l}+0.28 \\
-0.14 \\
\end{array}$ & \pm 0.12 & \pm 2.69 & \pm 3.40 \\
\hline
\end{tabular}

Table 5.6: Inelastic cross sections for various targets at different beam momenta.

In table 5.6, we have given the inelastic cross sections with total combined (stat $\oplus^{1}$ pyst) errors for various data sets used in this analysis. The statistical and total systematic errors are also given. All the systematic errors explained earlier in this section are added in quadrature to get the total systematic error. The dominating systematic error is from the beam flux and it is $\sim 5 \%$. The systematic error is the dominating error for all the data sets except for data set of Be at beam momentum of $58 \mathrm{GeV} / \mathrm{c}$ where the statistical error is dominating.

The inelastic cross sections for $\mathrm{LH}_{2}, \mathrm{C}, \mathrm{Be}$ and Bi targets at different beam momenta using MIPP data along with statistical and systematic errors are given in table 5.7. Previous measurements are also given in this table.

Inelastic cross sections as a function of lab momentum along with statistical and systematic errors for different targets are shown in figure 5.1.4. The MC predictions and previous measurements are superimposed on the MIPP data.

\footnotetext{
1 " $\oplus$ " indicates addition in quadrature
} 


\begin{tabular}{|l|l|l|}
\hline Interaction & MIPP data (mb) & $\begin{array}{l}\text { Previous Measurements } \\
(\mathbf{m b})\end{array}$ \\
\hline $\mathrm{p}+\mathrm{p}, 58 \mathrm{GeV}$ & $30.33 \pm 0.92_{-1.92}^{+1.79}$ & $31.13 \pm 0.13 \pm 0.4[96]$ \\
\hline $\mathrm{p}+\mathrm{p}, 85 \mathrm{GeV}$ & $34.64 \pm 0.60_{-2.10}^{+1.95}$ & $31.42 \pm 0.13 \pm 0.5[96]$ \\
\hline $\mathrm{p}+\mathrm{Be}, 58 \mathrm{GeV}$ & $213.14 \pm 14.34_{-13.07}^{+12.34}$ & $216 \pm 2 \pm 2.16[90]$ \\
\hline $\mathrm{p}+\mathrm{Be}, 120 \mathrm{GeV}$ & $183.78 \pm 1.45_{-10.92}^{+10.13}$ & \\
\hline $\mathrm{p}+\mathrm{C}, 58 \mathrm{GeV}$ & $267.29 \pm 12.48_{-14.65}^{+13.39}$ & $252 \pm 4 \pm 2.52[90]$ \\
\hline $\mathrm{p}+\mathrm{C}, 120 \mathrm{GeV}$ & $213.54 \pm 2.37_{-11.87}^{+10.87}$ & $222 \pm 2.22 \pm 6.66[91]$ \\
\hline $\mathrm{p}+\mathrm{Bi}, 58 \mathrm{GeV}$ & $2285.52 \pm 52.90_{-165.24}^{+157.02}$ & \\
\hline $\mathrm{p}+\mathrm{Bi}, 120 \mathrm{GeV}$ & $1434.95 \pm 11.14_{-97.86}^{+92.55}$ & \\
\hline
\end{tabular}

Table 5.7: Inelastic cross sections for various targets at different beam momenta.

The inelastic $\mathrm{p}+\mathrm{p}$ cross section at $58 \mathrm{GeV} / \mathrm{c}$ is in a good agreement (within $5 \%$ ) with the PDG [96] and MC (DPMJET) [87] prediction. The $85 \mathrm{GeV} / \mathrm{c} \mathrm{p}+\mathrm{p}$ cross section is $\sim$ $10 \%$ higher than the PDG and MC prediction. The inelastic $\mathrm{p}+\mathrm{C}$ cross section at $58 \mathrm{GeV} / \mathrm{c}$ is consistent, within error bars, with the measurement of Denisov et al. [90] and 20\% higher than the measurement of Carroll et al. [91]. The MC (FLUKA) [26, 88] prediction is $\sim 10 \%$ lower than the data at $58 \mathrm{GeV} / \mathrm{c}$, while at $120 \mathrm{GeV} / \mathrm{c}$, the MC prediction is $\sim 12 \%$ higher than the data.

The inelastic $\mathrm{p}+\mathrm{Be}$ cross section at $58 \mathrm{GeV} / \mathrm{c}$ is consistent, within error bars, with the measurement of Denisov et al. [90] and the MC prediction is $\sim 9 \%$ lower than the data, while at $120 \mathrm{GeV} / \mathrm{c}$, the MC prediction is $\sim 6 \%$ higher than the data. For bismuth target, the MC prediction is $\sim 18 \%$ lower than the data at $58 \mathrm{GeV} / \mathrm{c}$, while $\sim 30 \%$ higher at 120 $\mathrm{GeV} / \mathrm{c}$. No previous measurement is available at $58 \mathrm{GeV} / \mathrm{c}$ for the bismuth target and also, at $120 \mathrm{GeV} / \mathrm{c}$ for all the three thin targets i.e. Be, $\mathrm{C}$ and $\mathrm{Bi}$.

The MC predictions are based on the PDG [110] for $\mathrm{p}+\mathrm{p}$ interaction, and a mixture of tabulated data and parameterized fits for $\mathrm{p}+\mathrm{A}$ interaction [26, 111, 112].

A little rise in the inelastic cross section is expected as we go higher in beam momentum. The inelastic cross section is increasing with the beam momentum in case of $\mathrm{LH}_{2}$ target. But in case of thin targets i.e. Be, $\mathrm{C}$ and $\mathrm{Bi}$, we have observed that the measurement at 120 $\mathrm{GeV} / \mathrm{c}$ is lower than that at $58 \mathrm{GeV} / \mathrm{c}$, and it is worse for the Bi target. A similar feature was observed in the previous analyses [113]. To find the reason for the discrepancy between the 58 and $120 \mathrm{GeV} / \mathrm{c}$ data for the thin targets, many studies were done [92] which include 

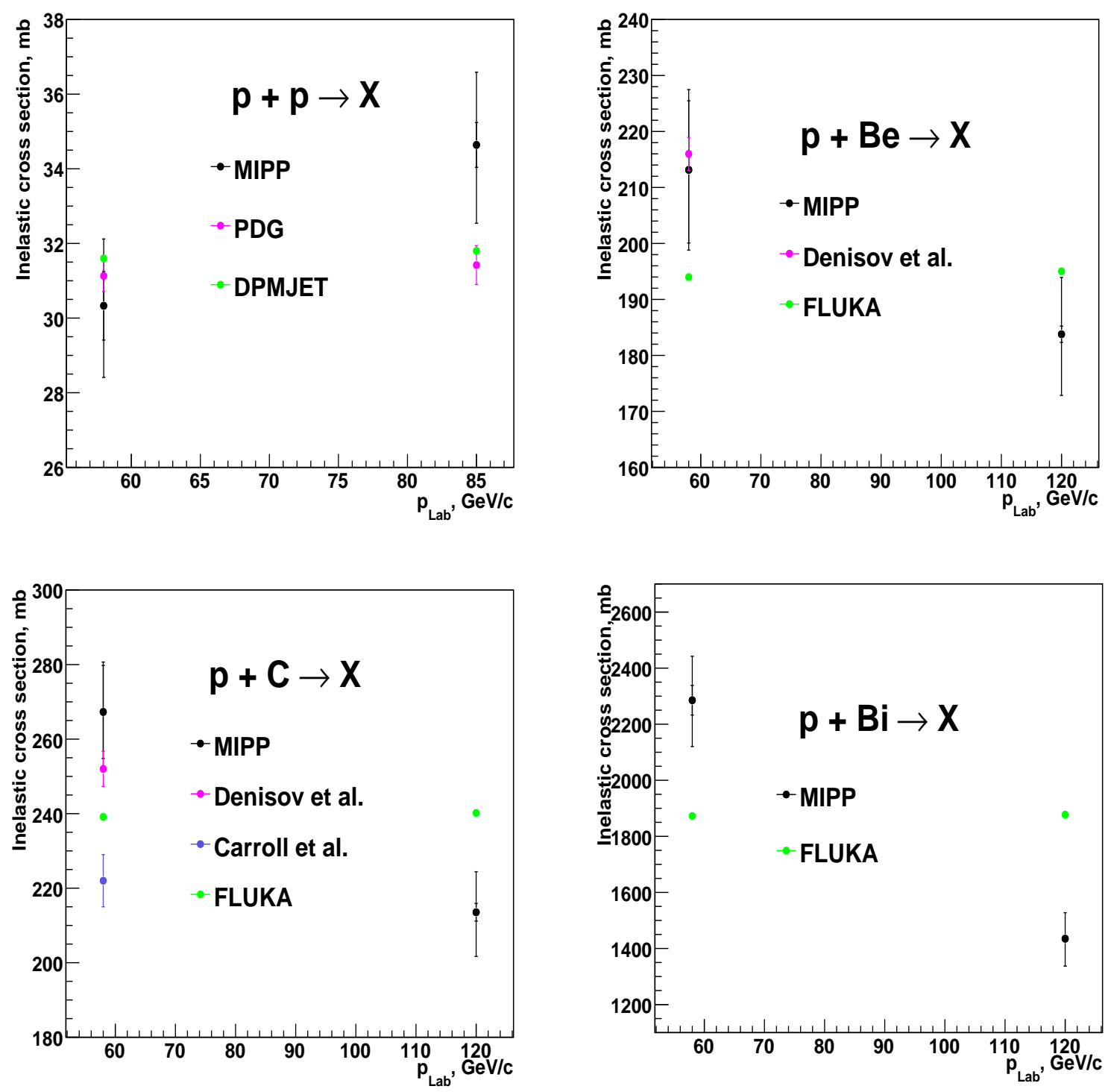

Figure 5.6: Inelastic cross sections as a function of lab momentum for different targets.

the comparison of the 58 and $120 \mathrm{GeV} / \mathrm{c}$ thin target data, the comparison of the data and reconstructed $\mathrm{MC}$ at 58 and $120 \mathrm{GeV} / \mathrm{c}$ since the corrections are calculated using the MC but nothing suspicious was found. The inelastic cross sections can also be calculated using the minimum-biased data i.e. the beam triggers which are independent of whether an interaction occurs or not. But there is not enough statistics at $58 \mathrm{GeV} / \mathrm{c}$. The $120 \mathrm{GeV} / \mathrm{c}$ minimumbiased data is sufficient to do the cross section calculation. The inelastic cross sections were calculated using the minimum-biased triggers for Be, $\mathrm{C}$ and Bi targets using $120 \mathrm{GeV} / \mathrm{c}$ proton beam but we couldn't get to the answer why the cross section at $120 \mathrm{GeV} / \mathrm{c}$ is lower 
than that at $58 \mathrm{GeV} / \mathrm{c}$. The cross sections were also cross checked using a narrow $\mathrm{Z}$ vertex selection where no empty target subtraction is needed but the same discrepancy was observed using this method as well.

\subsubsection{Inelastic cross section vs target atomic weight}

The inelastic cross sections using 58 and $120 \mathrm{GeV} / \mathrm{c}$ proton beams and different targets are plotted as a function of target atomic weight. Figures $5.7(\mathrm{a})$ and $5.7(\mathrm{~b})$ show the inelastic cross sections as a function of target atomic weight using 58 and $120 \mathrm{GeV} / \mathrm{c}$ proton beams for the data. The corresponding distributions for the MC are shown in figures 5.8(a) and $5.8(\mathrm{~b})$.

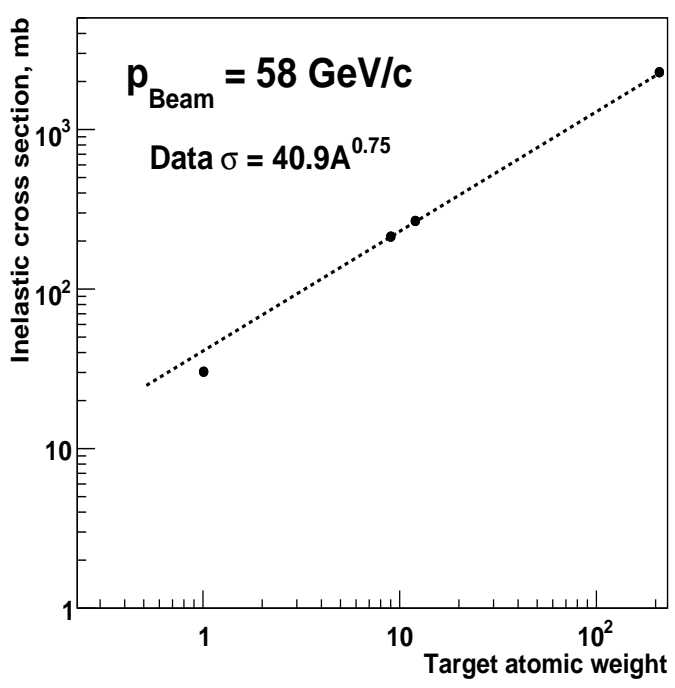

(a)

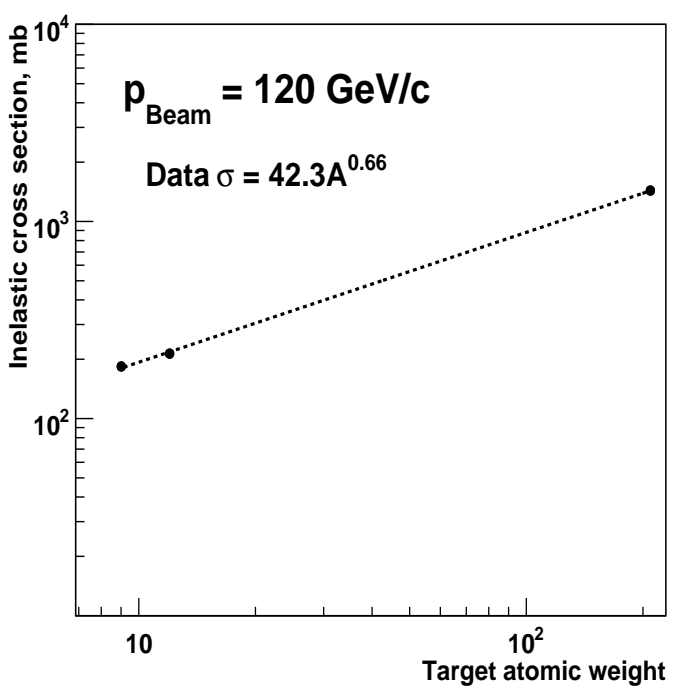

(b)

Figure 5.7: Inelastic cross sections vs target atomic weight for the data using (a) 58 and (b) $120 \mathrm{GeV} / \mathrm{c}$ proton beams.

The cross section points for both the data and $\mathrm{MC}$ are fitted using a function of the form $\mathrm{CA}^{\alpha}$ where $\mathrm{A}$ is the target atomic weight, $\mathrm{C}$ is the coefficient and $\alpha$ is the power. The cross section point for the $\mathrm{LH}_{2}$ target at $58 \mathrm{GeV} / \mathrm{c}$ is not included in the fit for both the data and MC. The fit parameters for the data using 58 and $120 \mathrm{GeV} / \mathrm{c}$ beams are shown in table 5.8, and the fit parameters for the $\mathrm{MC}$ are shown in table 5.9 . 


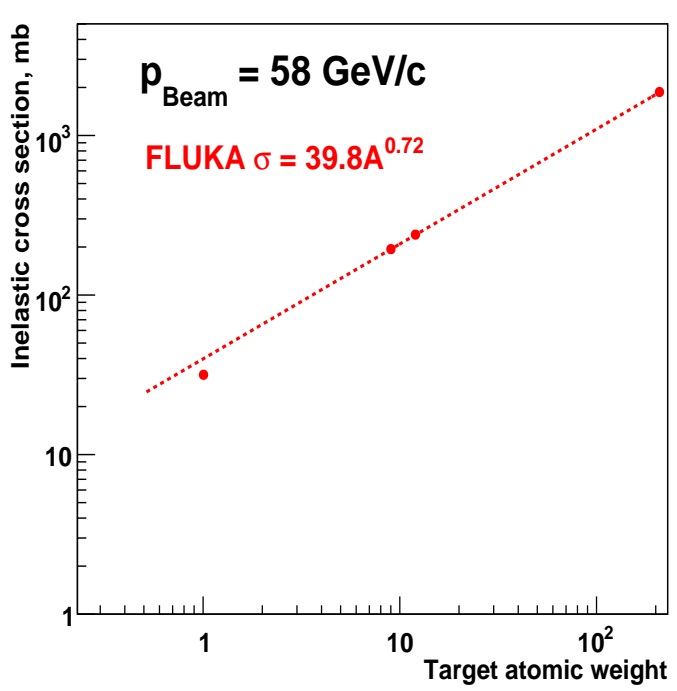

(a)

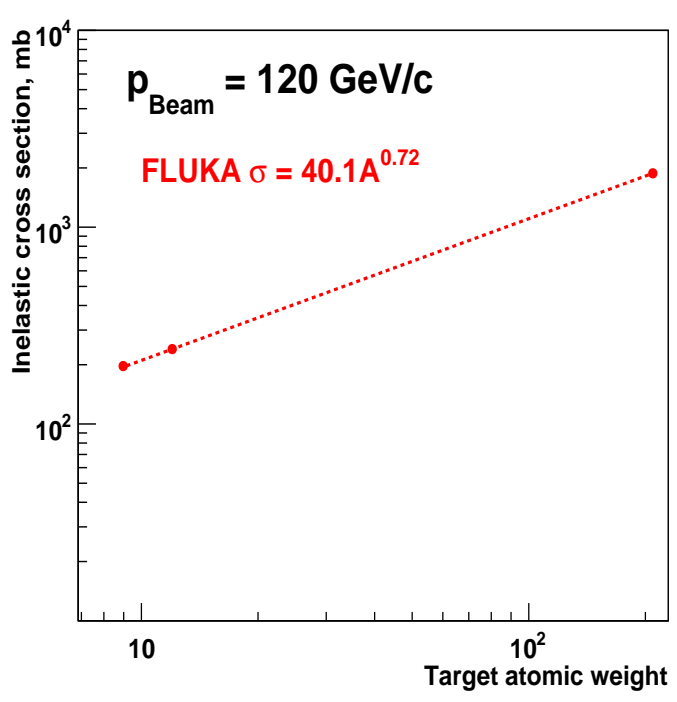

(b)

Figure 5.8: Inelastic cross sections vs target atomic weight for the MC using (a) 58 and (b) $120 \mathrm{GeV} / \mathrm{c}$ proton beams.

\begin{tabular}{|l|l|l|}
\hline $\begin{array}{l}\mathbf{p}_{\text {Beam }} \\
(\mathbf{G e V} / \mathbf{c})\end{array}$ & Coefficient & Power \\
\hline 58 & $40.9 \pm 3.69$ & $0.75 \pm 0.0274$ \\
\hline 120 & $42.3 \pm 3.64$ & $0.66 \pm 0.0252$ \\
\hline
\end{tabular}

Table 5.8: The fit parameters for the data using 58 and $120 \mathrm{GeV} / \mathrm{c}$ proton beams.

\begin{tabular}{|l|l|l|}
\hline $\begin{array}{l}\mathbf{p}_{\text {Beam }} \\
(\mathbf{G e V} / \mathbf{c})\end{array}$ & Coefficient & Power \\
\hline 58 & $39.8 \pm 0.23$ & $0.72 \pm 0.0011$ \\
\hline 120 & $40.1 \pm 0.23$ & $0.72 \pm 0.0011$ \\
\hline
\end{tabular}

Table 5.9: The fit parameters for the MC using 58 and $120 \mathrm{GeV} / \mathrm{c}$ proton beams. 


\section{$5.2 \quad \mathrm{LH}_{2}$ and carbon multiplicities}

We know that charged multiplicities should be even in case of $\mathrm{LH}_{2}$ target. Both even and odd multiplicities are observed in the data because of acceptances and reconstruction inefficiencies. We can unfold the data using the K-matrix (see section 3.5 from the Monte Carlo. Since discrepancies have been observed between the data and MC multiplicity shapes as described in section 3.5 , it has now been decided to use the KNO scaling function (see section 3.5.1) [55, 101] to get the true multiplicity probabilities for the data.

The KNO function for hydrogen target is written as:

$$
\psi\left(Z=\frac{n}{<n>}\right)=\left(3.79 Z+33.7 Z^{3}-6.64 Z^{5}+0.332 Z^{7}\right) e^{-3.04 Z}=<n>\times P(n) .
$$

$<\mathrm{n}>$ from our data is used. The value of $<\mathrm{n}>$ for $58 \mathrm{GeV} / \mathrm{c}$ beam is 5.859 and for $85 \mathrm{GeV} / \mathrm{c}$ beam, is 6.447 . The probabilities obtained from equation 5.2 are multiplied by the total inelastic cross section given in the table 5.2 to get the inelastic cross sections as a function of multiplicity.

The cross sections in bins of multiplicity for 58 and $85 \mathrm{GeV} / \mathrm{c} \mathrm{p}+\mathrm{p}$ interactions along with total combined errors are shown in figures 5.9(a) and 5.9(b). The cross sections from the PDG and MC (DPMJET) predictions are also superimposed. The statistical error is calculated using the number of events. For the systematics, the value of $<\mathrm{n}>$ is varied by $\pm 5 \%$ and the probabilities are re-calculated using the equation 5.2 . This variation is chosen on the basis of differences of the MIPP $<$ n $>$ 's and published data $<\mathrm{n}>$ 's (see section 3.5). These probabilities are superimposed on the nominal probabilities for 58 and $85 \mathrm{GeV} / \mathrm{c} \mathrm{p}+\mathrm{p}$ interactions in figure 5.10. The cross sections are recalculated using these new probabilities. The differences of these cross sections from the nominal cross sections give the systematic error $( \pm)$ in the inelastic cross sections as a function of multiplicity. The systematic error on the total inelastic cross section given in table 5.7 is also taken into account in the assignment of systematic errors on the inelastic cross sections in bins of multiplicity.

At $58 \mathrm{GeV} / \mathrm{c}$ (figure 5.9(a) discrepancies are found between the MIPP data and the PDG for 2-prongs and 4-prongs. For higher multiplicities the MIPP data is consistent, within error bars, with the PDG. At $85 \mathrm{GeV} / \mathrm{c}$ (figure 5.9(b)] the MIPP data is consistent, within error bars, with the PDG except for 8-prongs. The MC shape completely disagrees with the data for both the beam momenta.

Similarly cross sections are calculated for carbon target where both even and odd multiplicities are observed. The KNO function for carbon target is written as:

$$
\psi(Z)=0.5 \times 2.30(Z+0.142) \exp \left(-0.0586 Z-0.659 Z^{2}\right)=<n>\times P(n) .
$$




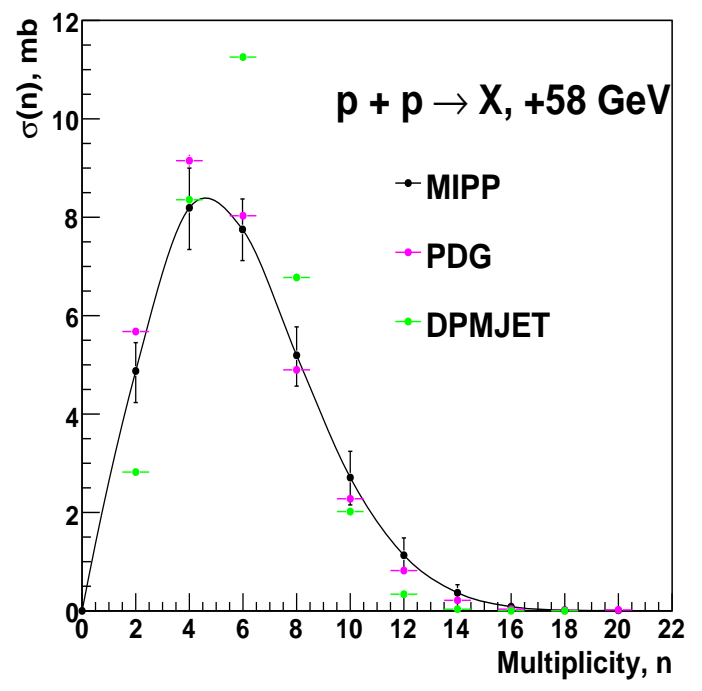

(a)

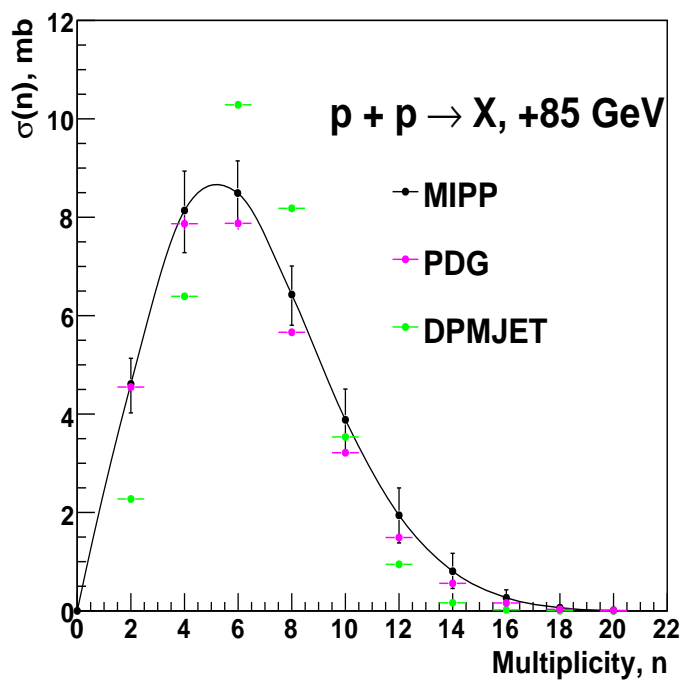

(b)

Figure 5.9: Comparison of the data and MC inelastic cross sections as a function of multiplicity for (a) 58 and (b) $85 \mathrm{GeV} / \mathrm{c}$ p+p interactions.

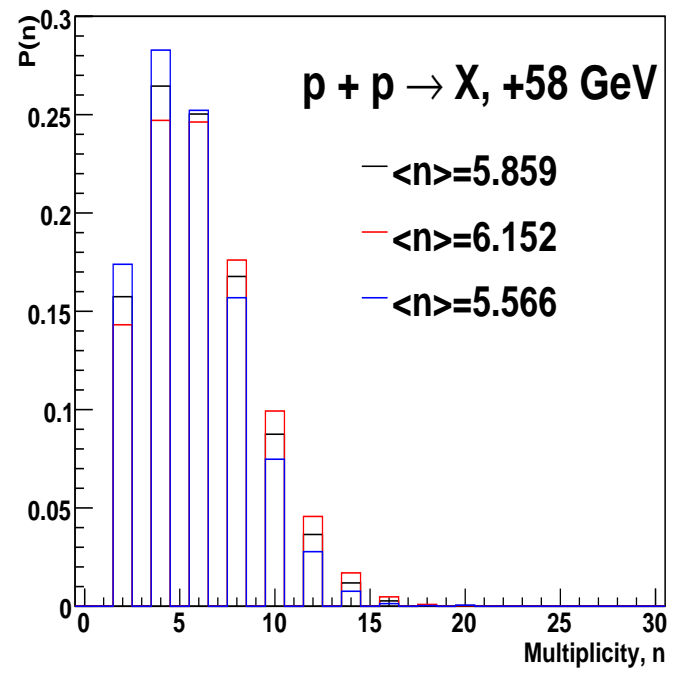

(a)

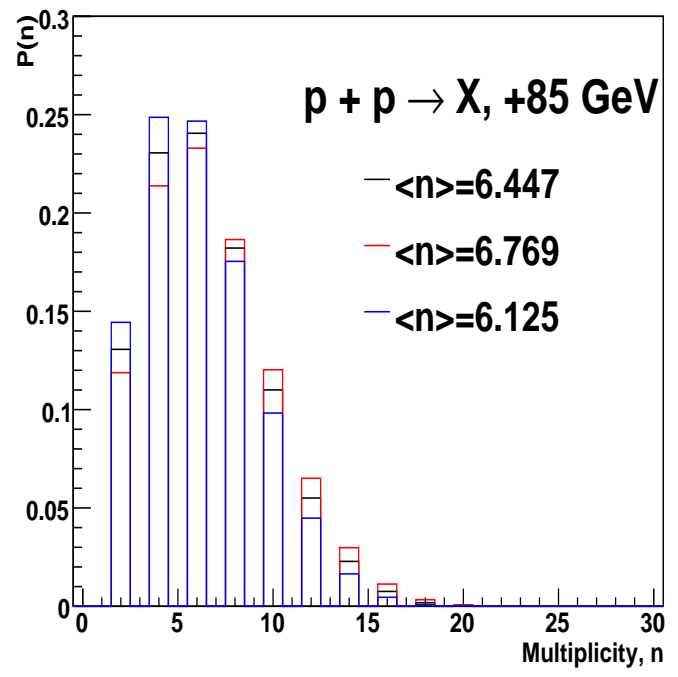

(b)

Figure 5.10: Systematics from average multiplcity $<\mathrm{n}>$ for (a) 58 and (b) $85 \mathrm{GeV} / \mathrm{c} \mathrm{p}+\mathrm{p}$ interactions. 
The value of $<\mathrm{n}>$ for $58 \mathrm{GeV} / \mathrm{c}$ beam is 7.182 and for $120 \mathrm{GeV} / \mathrm{c}$ beam, is 8.488. Just like hydrogen target, the probabilities obtained from the equation 5.3 are multiplied by the total inelastic cross section given in the table 5.3 to get the inelastic cross sections as a function of multiplicity.

The comparison of the MIPP data with the MC (FLUKA) predictions and previous measurements along with total combined errors for 58 and $120 \mathrm{GeV} / \mathrm{c} \mathrm{p}+\mathrm{C}$ interactions is shown in figures 5.11(a) and 5.11(b), respectively. The statistical error is calculated using the number of events. For the systematics, the value of $\langle\mathrm{n}\rangle$ is varied by $\pm 5 \%$ and the probabilities are re-calculated using the equation 5.3. These probabilities are superimposed on the nominal probabilities for 58 and $120 \mathrm{GeV} / \mathrm{c} \mathrm{p}+\mathrm{C}$ interactions in figure 5.12 . The cross sections are recalculated using these new probabilities. The differences of these cross sections from the nominal cross sections give the systematic error $( \pm)$ in the inelastic cross sections as a function of multiplicity. The systematic error on the total inelastic cross section given in table 5.7 is also taken into account in the assignment of systematic errors on the inelastic cross sections in bins of multiplicity.

At $58 \mathrm{GeV} / \mathrm{c}$ (figure 5.11(a) the MIPP data is consistent, within error bars, with the measurement of Denisov et al. [90], and consistent with the measurement of Carroll et al. 91] for multiplicity $>13$ only. The MC starts agreeing with the MIPP data for multiplicity $\geq 7$. At $120 \mathrm{GeV} / \mathrm{c}$ (figure 5.11(b)), there is no previous measurement available. The MC completely disagrees with the data.

\subsection{Charged particle production cross sections as a function of momentum}

The charged particle production cross sections are also measured in bins of momentum for 58 and $120 \mathrm{GeV} / \mathrm{c} \mathrm{p}+\mathrm{C}$ interactions as a cross check. All the event selection cuts described in the section 3.2 are required to select the inelastic interactions. All the charged particles from the primary vertex are selected. The cross sections are corrected for the inefficiencies due to the trigger and applied cuts, detector acceptances and reconstruction inefficiencies. All corrections are calculated in bins of momentum using the reconstructed MC as described in the section 3.6.

The track momentum distributions for 58 and $120 \mathrm{GeV} / \mathrm{c}$ proton interactions on carbon and empty targets are shown in figure 5.13 . The distributions after doing the empty target subtraction are also shown.

The cross section formula (equation 5.1) for getting the charged particle production 


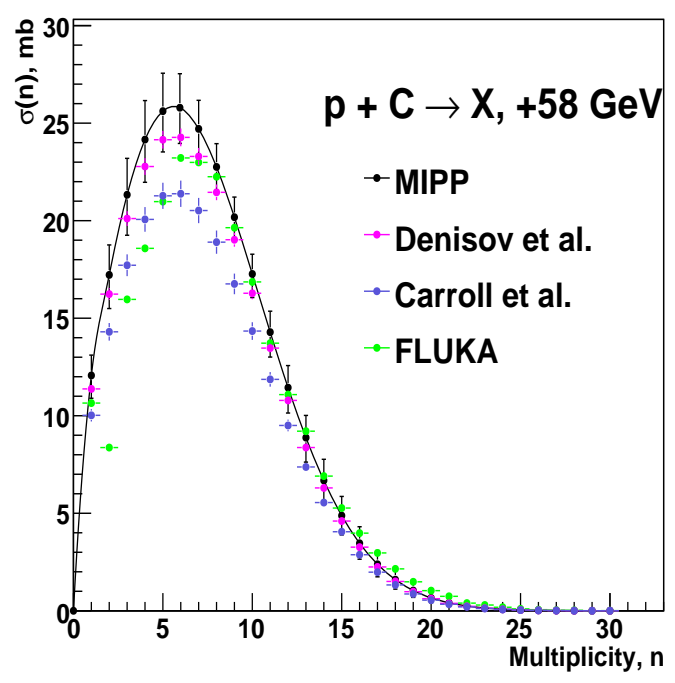

(a)

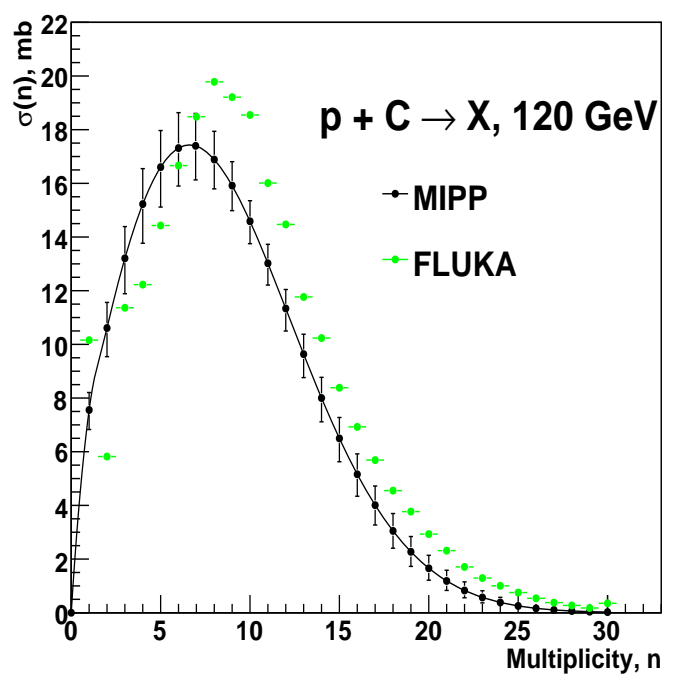

(b)

Figure 5.11: Inelastic cross sections as a function of multiplicity for (a) 58 and (b) 120 $\mathrm{GeV} / \mathrm{c} \mathrm{p}+\mathrm{C}$ interactions. The previous measurements and the $\mathrm{MC}$ predictions are also superimposed. No previous measurement is available at $120 \mathrm{GeV} / \mathrm{c}$ beam momentum.

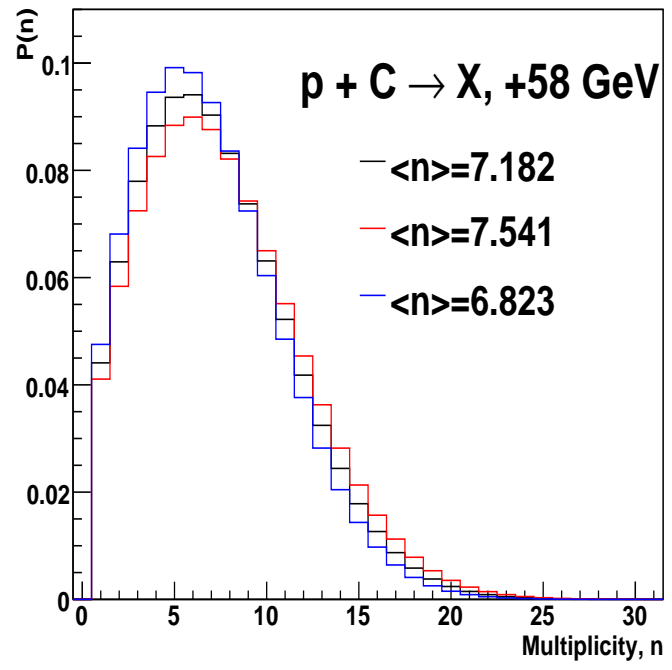

(a)

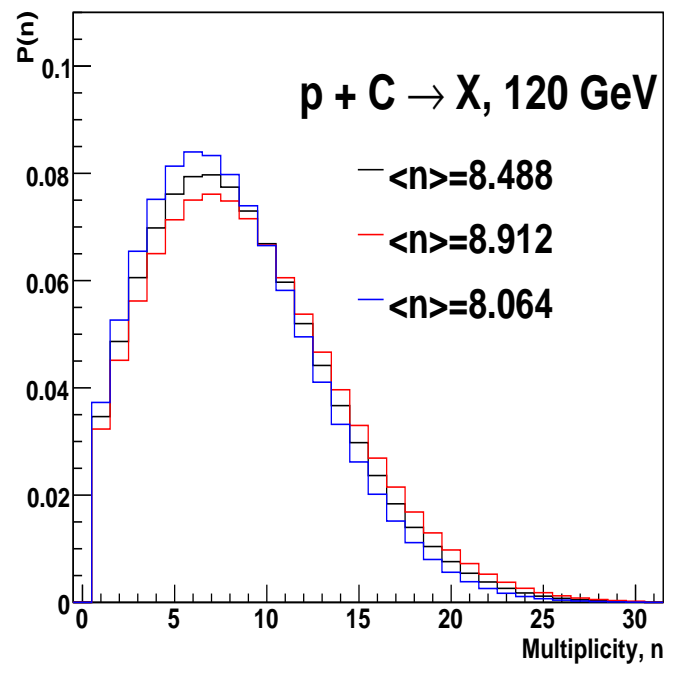

(b)

Figure 5.12: Systematics from average multiplcity $<\mathrm{n}>$ for (a) 58 and (b) $120 \mathrm{GeV} / \mathrm{c}$ p+C interactions. 


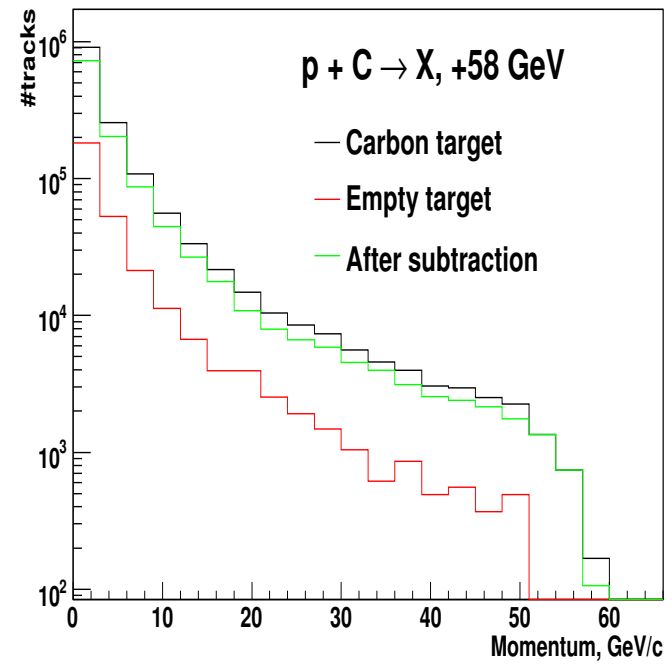

(a)

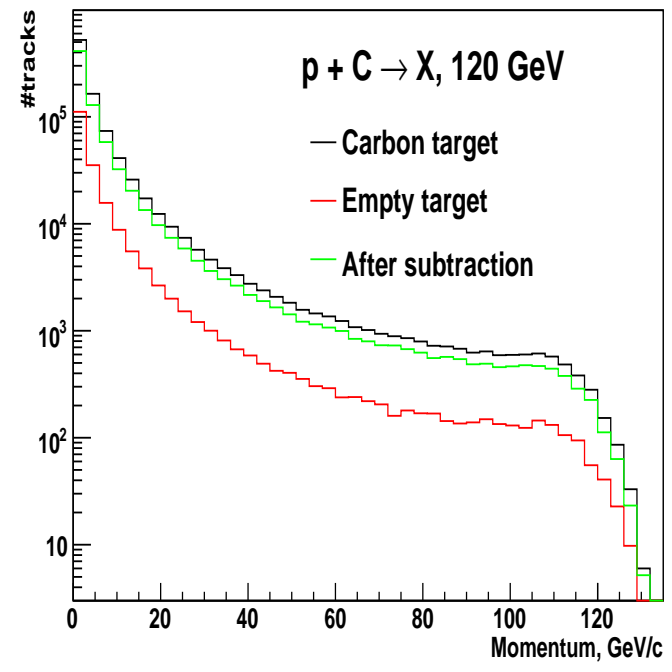

(b)

Figure 5.13: The track momentum distributions for (a) 58 and (b) $120 \mathrm{GeV} / \mathrm{c}$ proton interactions on carbon and empty targets. The distributions after empty target subtraction are also shown.

cross sections in bins of momentum can be written as:

$$
\frac{d \sigma}{d p}=\frac{N_{\text {tracks }}}{N_{\text {beam }} \times n_{t} \times \epsilon \times \Delta p},
$$

where $\mathrm{N}_{\text {tracks }}$ is the number of charged tracks after subtracting the empty target contribution from the carbon target, $\mathrm{N}_{\text {beam }}$ is the number of beam particles and $\Delta \mathrm{p}$ is the momentum bin width. The cross sections are measured in units of $\mathrm{mb} / \mathrm{GeV} / c$.

The uncorrected charged particle production cross sections in bins of momentum along with statistical errors for 58 and $120 \mathrm{GeV} / \mathrm{c}$ p $+\mathrm{C}$ interactions are shown in figure 5.14. The charged particle production cross sections in bins of momentum where all the corrections are applied are shown in figure 5.15. The total charged particle production cross section is calculated by adding all the momentum bins and is given on the plots along with the statistical error. The statistical error for $58 \mathrm{GeV} / \mathrm{c}$ data is $\sim 3 \%$ and for $120 \mathrm{GeV} / \mathrm{c}$ data, is $\sim 1 \%$.

The comparison of the data and MC charged particle production cross sections in bins of momentum for 58 and $120 \mathrm{GeV} / \mathrm{c}$ p $+\mathrm{C}$ interactions is shown in figure 5.16 . The data is shown as black points and the MC as red line. Discrepancies are observed between the data and MC cross sections. 


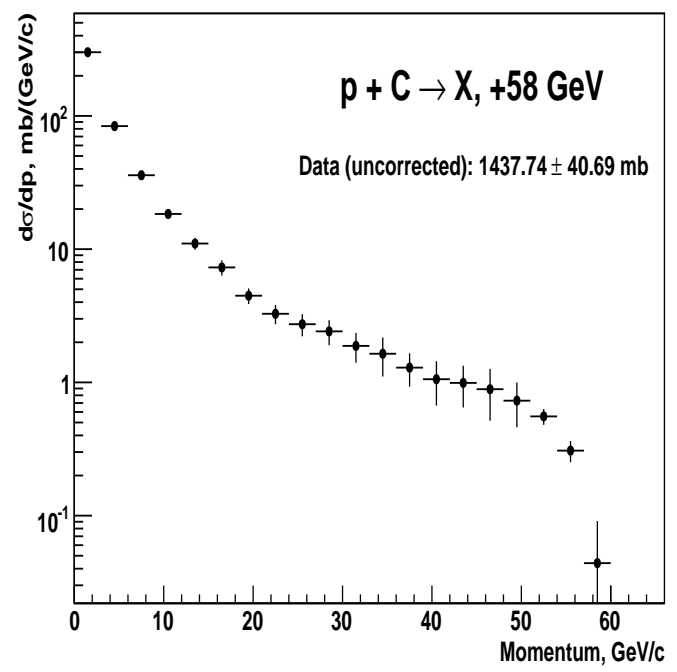

(a)

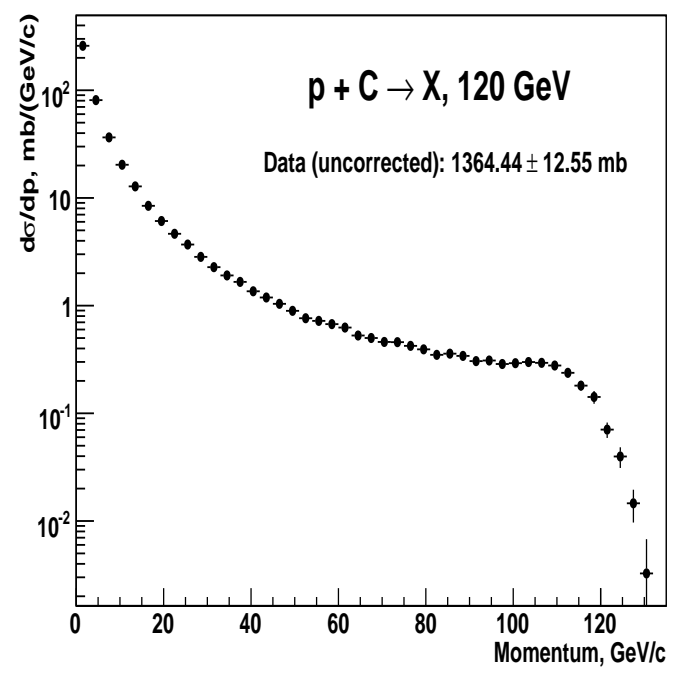

(b)

Figure 5.14: The uncorrected charged particle production cross sections in bins of momentum for (a) 58 and (b) $120 \mathrm{GeV} / \mathrm{c} \mathrm{p}+\mathrm{C}$ interactions.

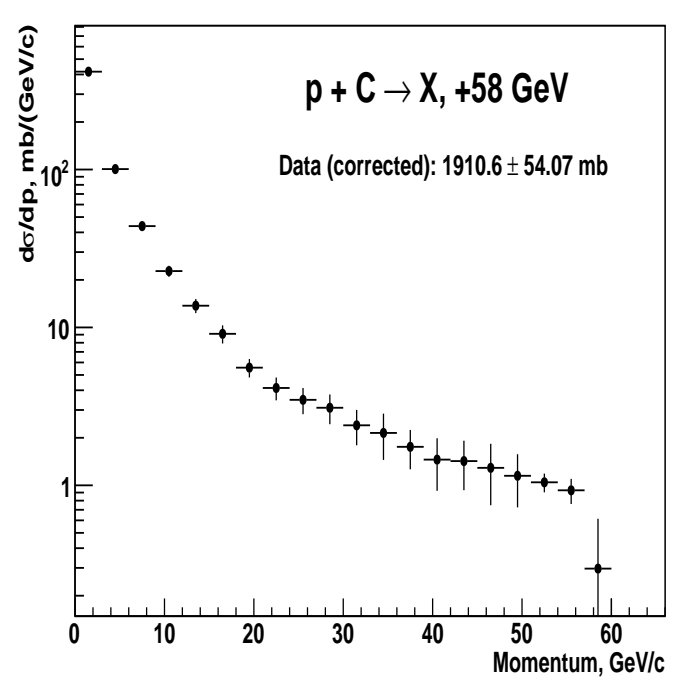

(a)

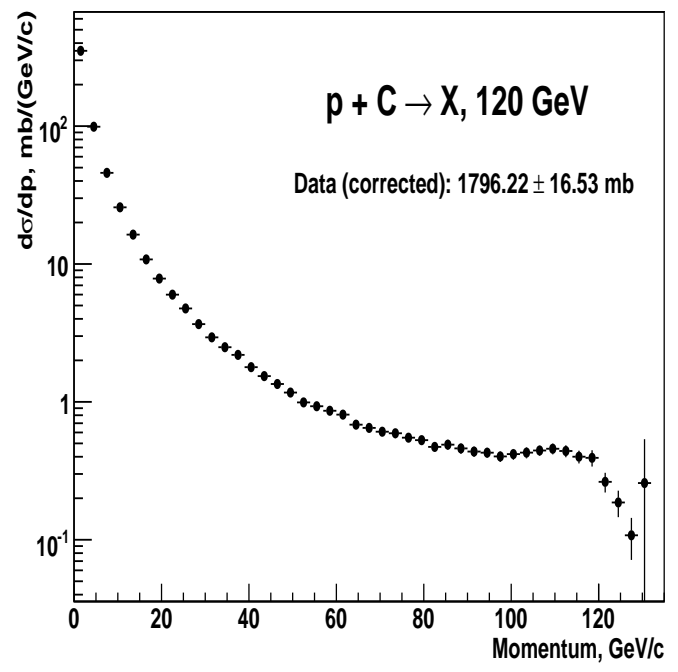

(b)

Figure 5.15: The corrected charged particle production cross sections in bins of momentum for (a) 58 and (b) $120 \mathrm{GeV} / \mathrm{c} \mathrm{p}+\mathrm{C}$ interactions. 
Systematic errors: The systematic errors are calculated in a similar way as discussed in

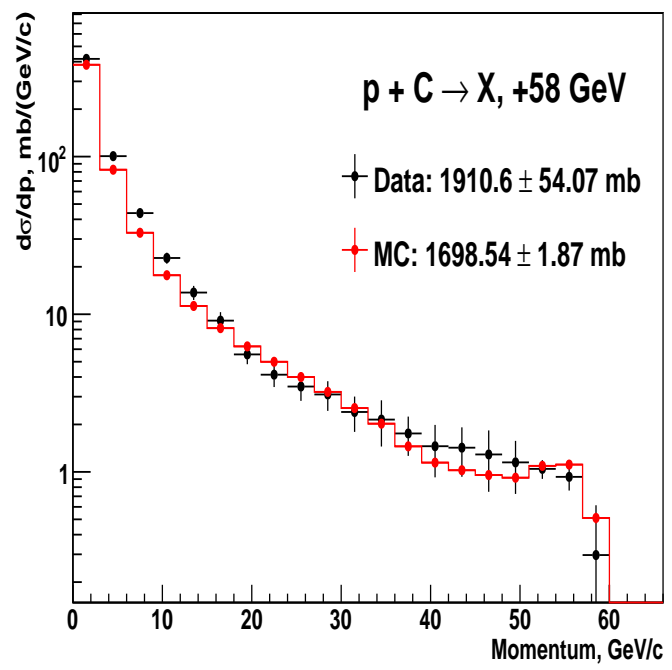

(a)

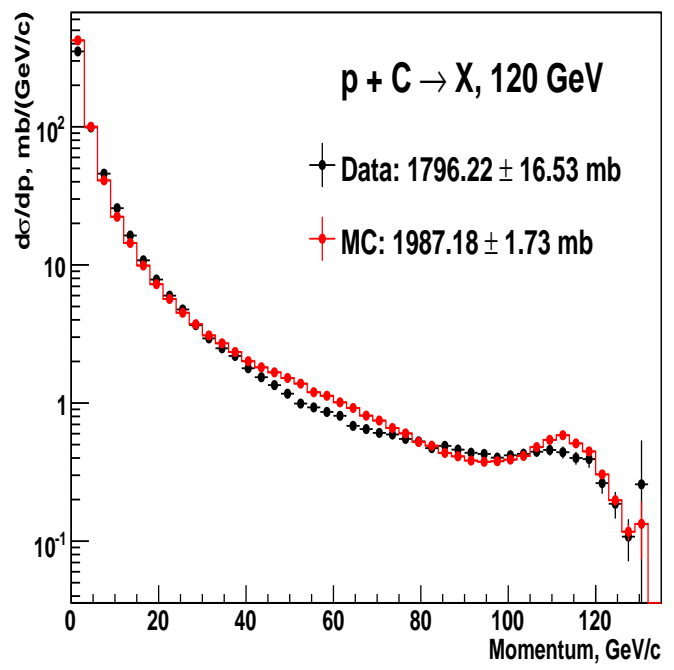

(b)

Figure 5.16: The comparison of the data and $\mathrm{MC}$ charged particle production cross sections in bins of momentum for (a) 58 and (b) $120 \mathrm{GeV} / \mathrm{c} \mathrm{p}+\mathrm{C}$ interactions.

the section 5.1 .4 for the total inelastic cross sections. The comparison of nominal $\mathcal{A} \epsilon_{\mathcal{R}}$ and $\mathcal{A} \epsilon_{\mathcal{R}}$ using the reweighted MC in bins of momentum for 58 and $120 \mathrm{GeV} / \mathrm{c} \mathrm{p}+\mathrm{C}$ interactions is shown in figure 5.17 .

In the case of charged particle production cross sections in bins of momentum, another systematics is considered and that is systematics from momentum scale i.e. the error on momentum. This is calculated using $120 \mathrm{GeV} / \mathrm{c}$ data. For the $120 \mathrm{GeV} / \mathrm{c}$ beam tracks (straight throughs), the mean value of momentum is $118.8 \mathrm{GeV} / \mathrm{c}$. For the $120 \mathrm{GeV} / \mathrm{c}$ Main Injector protons, the actual value of the momentum is known and that is $119.7 \mathrm{GeV} / \mathrm{c}$. Using these two values of momentum, a correction factor is obtained i.e. 119.7/118.8 = 1.00758. This correction factor is used to correct the momentum of the data tracks [114. The error on the correction factor is assumed to be $1 \%$. In this analysis, we have not applied the corrections to the track momentum but we need to assign a systematics from the momentum scale. This systematics is calculated as:

$$
\Delta \sigma(\text { nominal })=a b s(\sigma(\text { pscale }+\Delta \text { pscale })-\sigma(\text { pscale }-\Delta \text { pscale })) / 2,
$$

where pscale is 1.00758 and $\Delta$ pscale is $0.0100758 . \sigma($ pscale $+\Delta$ pscale $)$ and $\sigma($ pscale- $\Delta$ pscale $)$ 


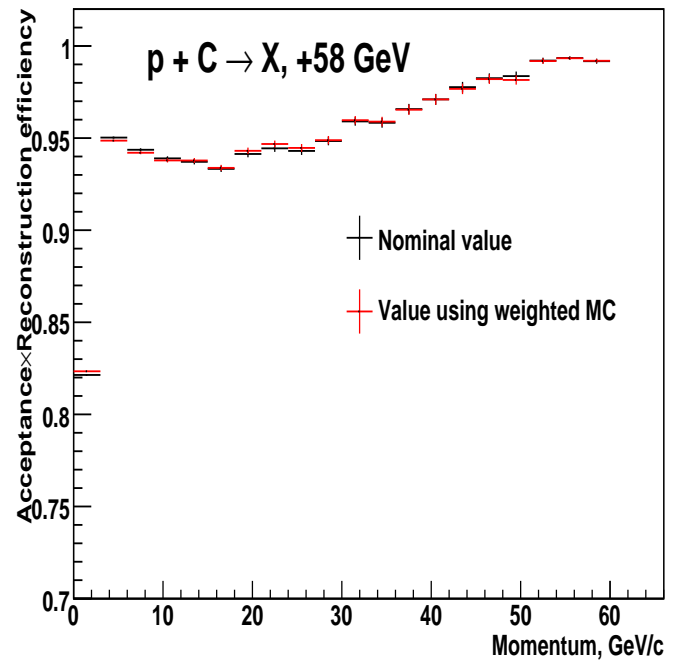

(a)

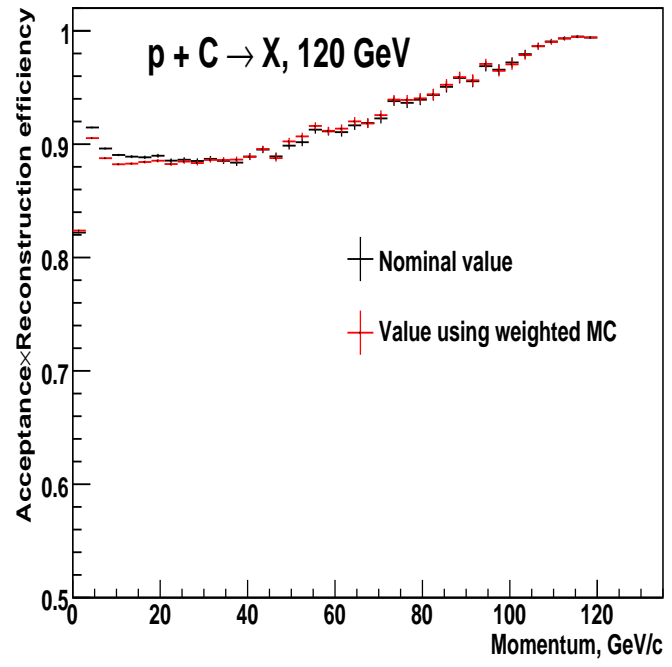

(b)

Figure 5.17: The comparison of nominal $\mathcal{A} \epsilon_{\mathcal{R}}$ and $\mathcal{A} \epsilon_{\mathcal{R}}$ using the reweighted MC in bins of momentum for (a) 58 and (b) $120 \mathrm{GeV} / \mathrm{c} \mathrm{p}+\mathrm{C}$ interactions.

are the cross sections obtained after taking into account the error on track momentum. $\Delta \sigma$ (nominal) is the difference of the nominal cross section from the one obtained after taking the momentum scale into account and gives the systematic error. All the systematic errors are listed in table 5.10 .

\begin{tabular}{|c|c|c|c|c|c|c|c|c|c|}
\hline \multirow[b]{2}{*}{ 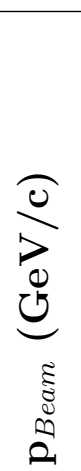 } & \multirow[b]{2}{*}{ 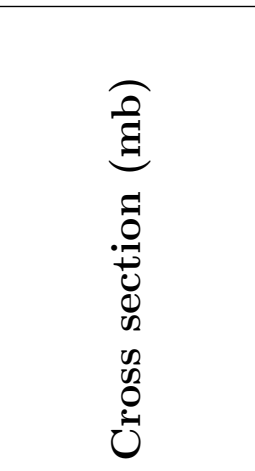 } & \multicolumn{8}{|c|}{ Errors (\%) } \\
\hline & & 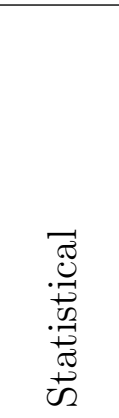 & 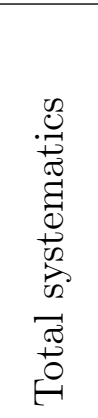 & 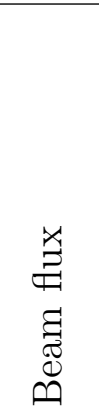 & 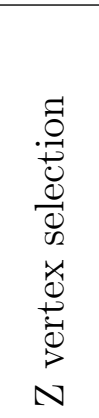 & $\begin{array}{l}\ddot{0} \\
\infty 0 \\
\infty 0 \\
.00 \\
\ddot{E}\end{array}$ & 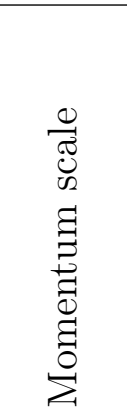 & 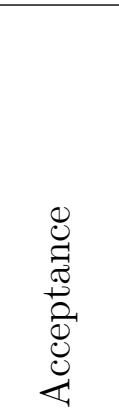 & 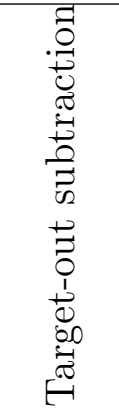 \\
\hline 58 & $1910.60_{-116.82}^{+108.61}$ & \pm 2.83 & $\begin{array}{r}+4.93 \\
-5.42 \\
\end{array}$ & $\begin{array}{l}+4.76 \\
-5.26\end{array}$ & $\begin{array}{l}+0.02 \\
-0.01\end{array}$ & \pm 0.20 & \pm 0.20 & \pm 0.12 & \pm 1.26 \\
\hline 120 & $1796.22_{-99.45}^{+90.43}$ & \pm 0.92 & $\begin{array}{l}+4.95 \\
{ }_{-5.46}\end{array}$ & $\begin{array}{l}+4.76 \\
{ }_{-5.26}\end{array}$ & $\begin{array}{l}+0.03 \\
{ }_{-0.51}\end{array}$ & \pm 0.13 & \pm 0.11 & \pm 0.19 & \pm 1.35 \\
\hline
\end{tabular}

Table 5.10: Total charged particle production cross sections for $\mathrm{p}+\mathrm{C}$ interactions at 58 and $120 \mathrm{GeV} / \mathrm{c}$.

In table 5.10, we have given the total charged particle production cross sections with 
total combined errors using 58 and $120 \mathrm{GeV} / \mathrm{c} \mathrm{p}+\mathrm{C}$ data. The statistical and total systematic errors are also given. All the systematic errors are added in quadrature to get the total systematic error. The dominating systematic error is from the beam flux and it is $\sim 5 \%$. The total systematic variations for the charged particle production cross sections in bins of momentum for 58 and $120 \mathrm{GeV} / \mathrm{c} \mathrm{p}+\mathrm{C}$ interactions are shown in figure 5.18 .

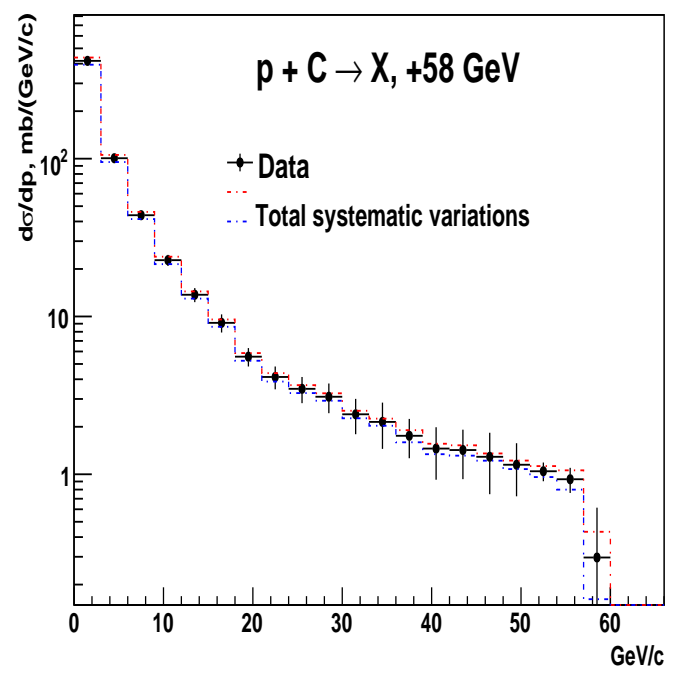

(a)

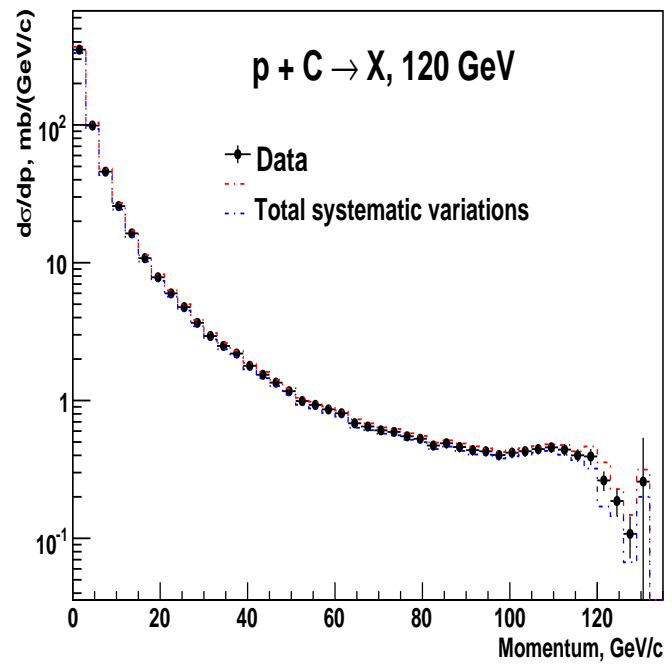

(b)

Figure 5.18: Total systematic variations for the charged particle production cross sections for (a) 58 and (b) $120 \mathrm{GeV} / \mathrm{c} \mathrm{p}+\mathrm{C}$ interactions.

The average production cross section should be equal to average multiplicity times the average inelastic cross section. The average multiplicity is $\sim 7$ and $\sim 8.5$ in case of 58 and $120 \mathrm{GeV} / \mathrm{c} \mathrm{p}+\mathrm{C}$ interactions, respectively. If we multiply it by the average inelastic cross section as presented in section 5.1, we get the numbers very close to the cross section numbers shown in table 5.10 .

\subsection{Inclusive charged pion production cross sections}

The inclusive charged pion $\left(\pi^{ \pm}\right)$production cross sections are calculated in bins of true momentum using the interaction triggers for 58 and $120 \mathrm{GeV} / \mathrm{c} \mathrm{p}+\mathrm{C}$ collisions. All event selection cuts described in chapter 3 are required during the selection of interactions. TPC and RICH are used to do the particle identification (PID) as discussed in chapter 4 . The cross section measurements are divided into two momentum ranges. The TPC is used to do 
the PID in momentum range $0.3-1 \mathrm{GeV} / \mathrm{c}$ and the $\mathrm{RICH}$, in momentum range $4.64-120$ $\mathrm{GeV} / \mathrm{c}$. The pion momentum spectrum from the data is unfolded using the MC as described in the sections 4.4 and 4.5 . This unfolded momentum spectrum is used in the cross section measurements. The formula used for the cross sections is given in the equation 5.4, where $\mathrm{N}_{\text {tracks }}$ is now the number of charged pions $\left(\pi^{ \pm}\right)$identified. The cross sections are corrected using the corrections in bins of true momentum from the $\mathrm{MC}$ as described in the section 4.6. The cross section measurements for these two momentum ranges using 58 and $120 \mathrm{GeV} / \mathrm{c}$ $\mathrm{p}+\mathrm{C}$ interactions are described as follows:

\subsubsection{Cross sections using $58 \mathrm{GeV} / \mathrm{c} \mathrm{p}+\mathrm{C}$ interactions}

\section{Cross sections using TPC}

The inclusive $\pi^{-}$and $\pi^{+}$production cross sections in bins of true momentum with no corrections applied for momentum range $0.3-1 \mathrm{GeV} / \mathrm{c}$ using TPC for $58 \mathrm{GeV} / \mathrm{c}$ p $+\mathrm{C}$ interactions are shown in figure 5.19. The cross sections with all the corrections applied are shown in figure 5.20. The errors shown are statistical only. The statistical error is totally based on the statistics i.e. total number of pion tracks. The total inclusive pion production cross section is calculated by adding all the momentum bins and is given on the plots along with the statistical error. The statistical error on the integral value of the inclusive pion production cross section is $\sim 2.4 \%$.

The comparison of the data inclusive $\pi^{-}$and $\pi^{+}$production cross sections with the MC (FLUKA) predictions is shown in figure 5.21. The data is shown as black points and the $\mathrm{MC}$ as red line. The errors shown are statistical only.

\section{Cross sections using RICH}

The inclusive $\pi^{-}$and $\pi^{+}$production cross sections in bins of true momentum with no corrections applied for momentum range $4.64-60 \mathrm{GeV} / \mathrm{c}$ using $\mathrm{RICH}$ for $58 \mathrm{GeV} / \mathrm{c} \mathrm{p}+\mathrm{C}$ interactions are shown in figure 5.22 , and with corrections applied, are shown in figure 5.23 . The total inclusive pion production cross section is calculated by adding all the momentum bins.

The comparison of the data inclusive $\pi^{-}$and $\pi^{+}$production cross sections with the MC predictions is shown in figure 5.24. The errors shown are statistical only. The statistical error on the integral value of the inclusive pion production cross section for the data is $\sim$ $2.5 \%$. 


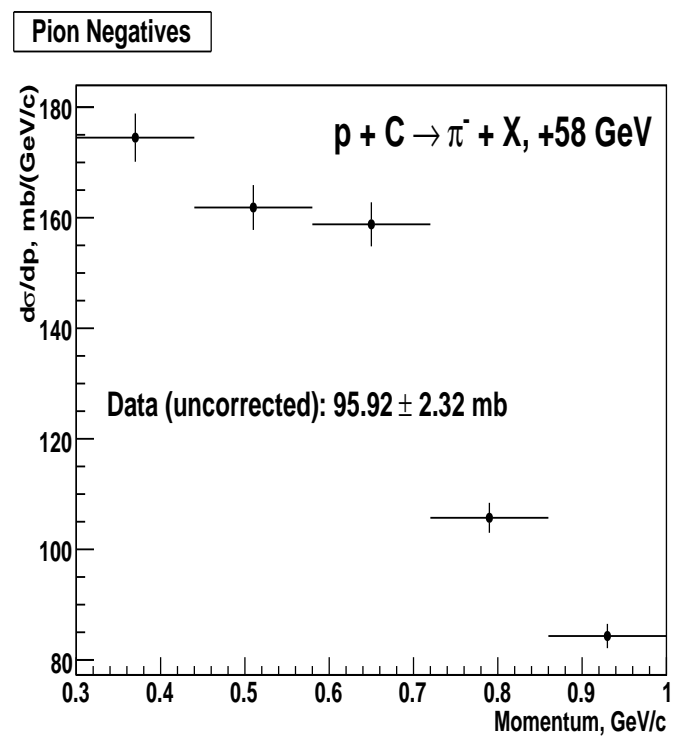

(a)

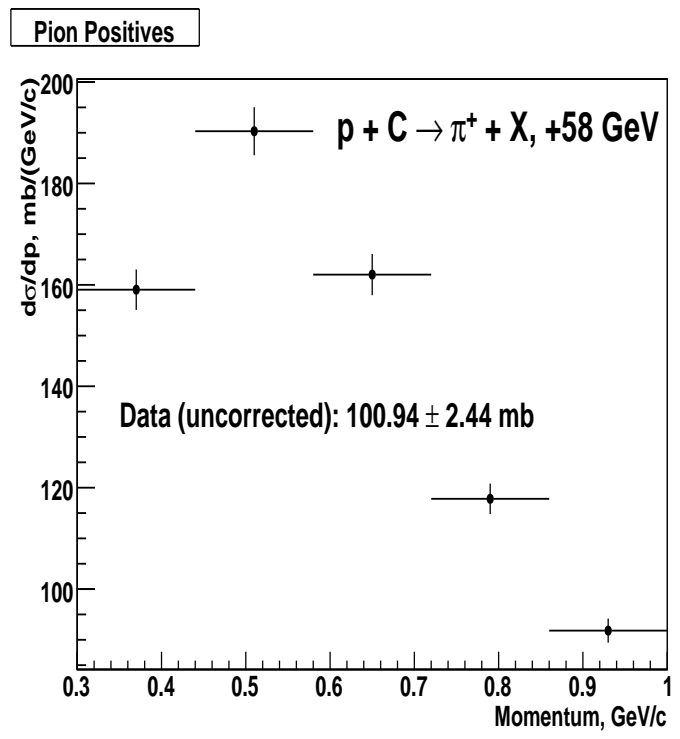

(b)

Figure 5.19: The inclusive (a) $\pi^{-}$and (b) $\pi^{+}$production cross sections in bins of true momentum for momentum range $0.3-1 \mathrm{GeV} / \mathrm{c}$ using $\mathrm{TPC}$ for $58 \mathrm{GeV} / \mathrm{c} \mathrm{p}+\mathrm{C}$ interactions. Corrections are not applied.

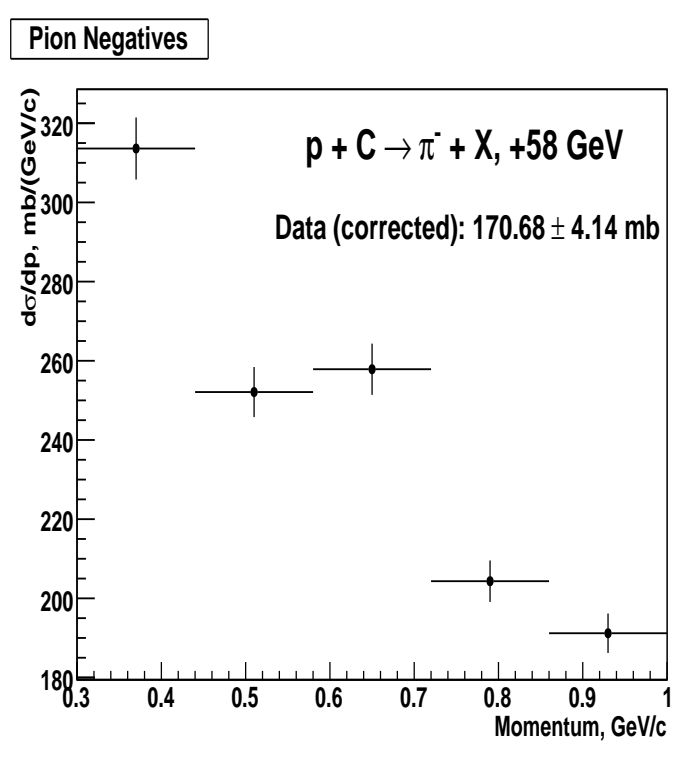

(a)

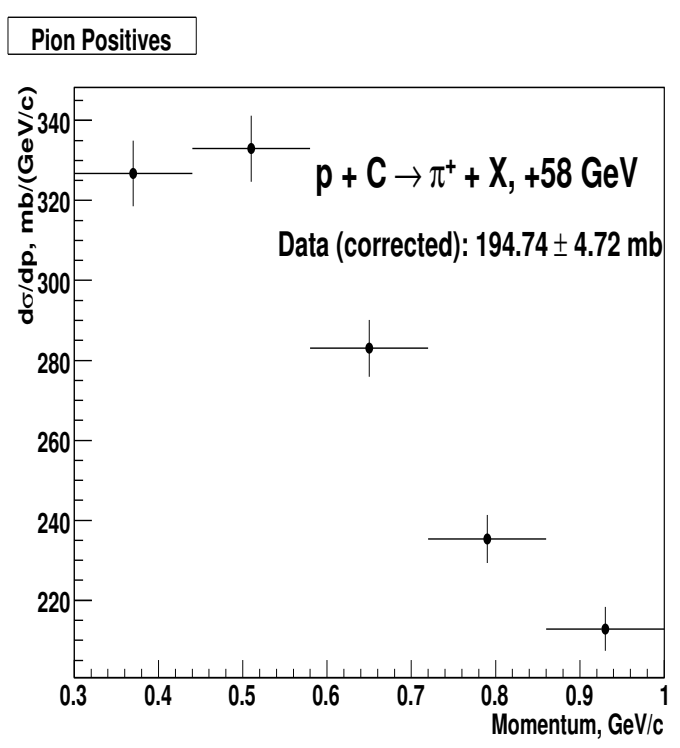

(b)

Figure 5.20: The inclusive (a) $\pi^{-}$and (b) $\pi^{+}$production cross sections in bins of true momentum for momentum range $0.3-1 \mathrm{GeV} / \mathrm{c}$ using $\mathrm{TPC}$ for $58 \mathrm{GeV} / \mathrm{c} \mathrm{p}+\mathrm{C}$ interactions. Corrections are applied. 


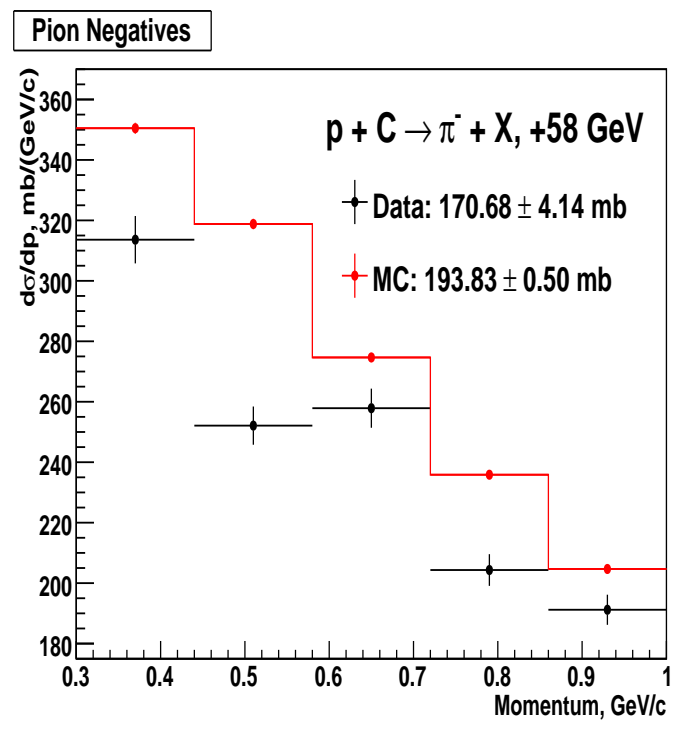

(a)

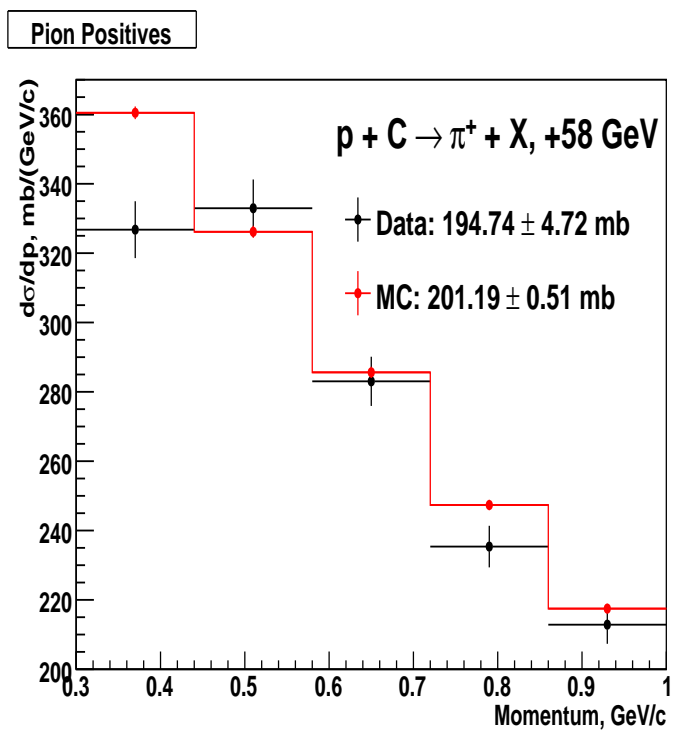

(b)

Figure 5.21: The comparison of the data inclusive (a) $\pi^{-}$and (b) $\pi^{+}$production cross sections with the MC predictions for momentum range $0.3-1 \mathrm{GeV} / \mathrm{c}$ using TPC for $58 \mathrm{GeV} / \mathrm{c} \mathrm{p}+\mathrm{C}$ interactions. Only statistical errors are shown.

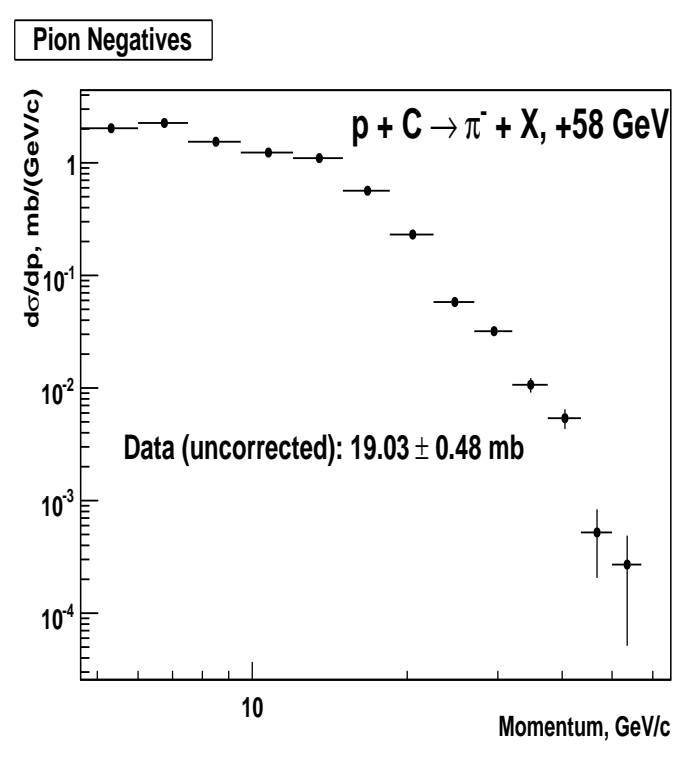

(a)

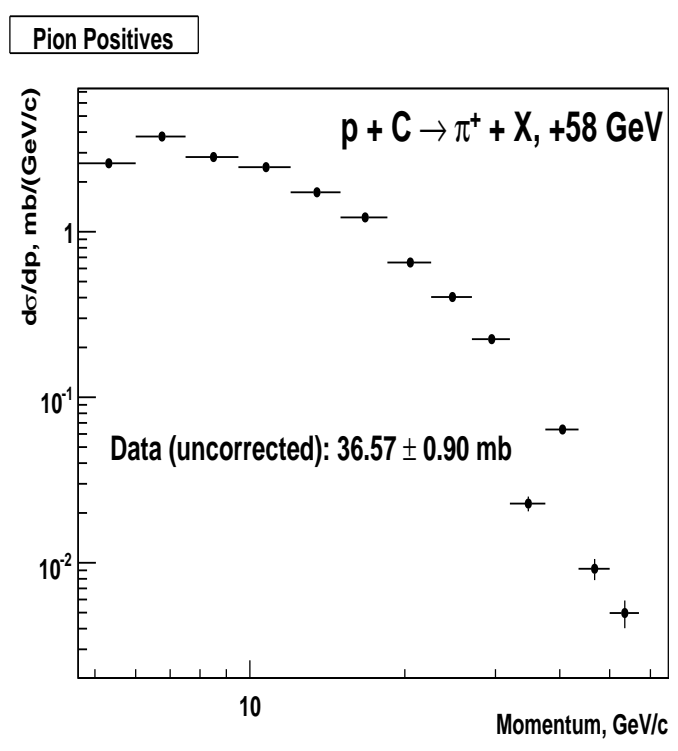

(b)

Figure 5.22: The inclusive (a) $\pi^{-}$and (b) $\pi^{+}$production cross sections in bins of true momentum for momentum range $4.64-60 \mathrm{GeV} / \mathrm{c}$ using RICH for $58 \mathrm{GeV} / \mathrm{c} \mathrm{p}+\mathrm{C}$ interactions. Corrections are not applied. 


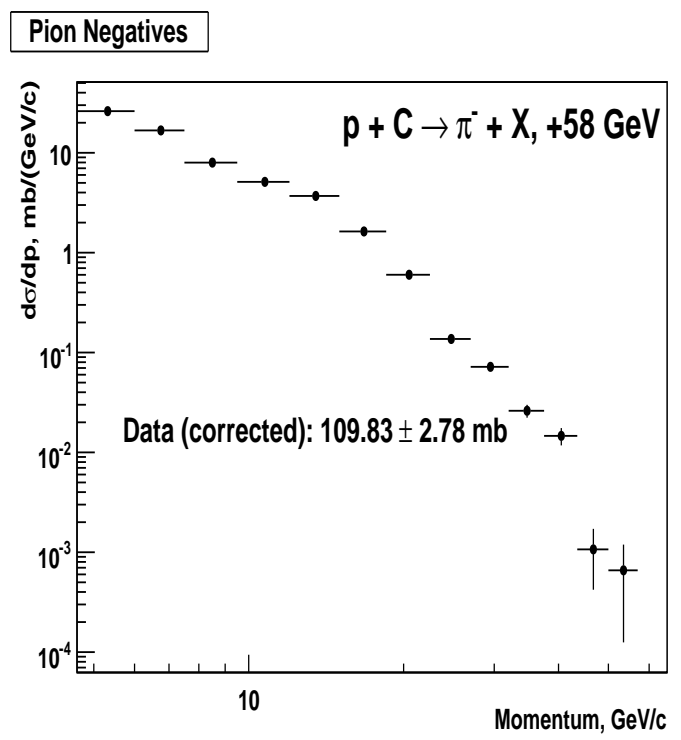

(a)

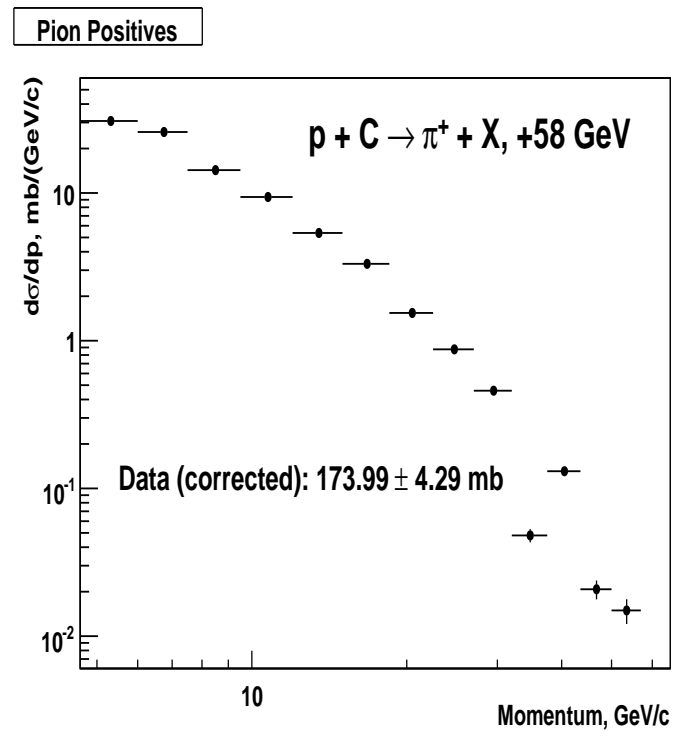

(b)

Figure 5.23: The inclusive (a) $\pi^{-}$and (b) $\pi^{+}$production cross sections in bins of true momentum for momentum range $4.64-60 \mathrm{GeV} / \mathrm{c}$ using $\mathrm{RICH}$ for $58 \mathrm{GeV} / \mathrm{c} \mathrm{p}+\mathrm{C}$ interactions. Corrections are applied.

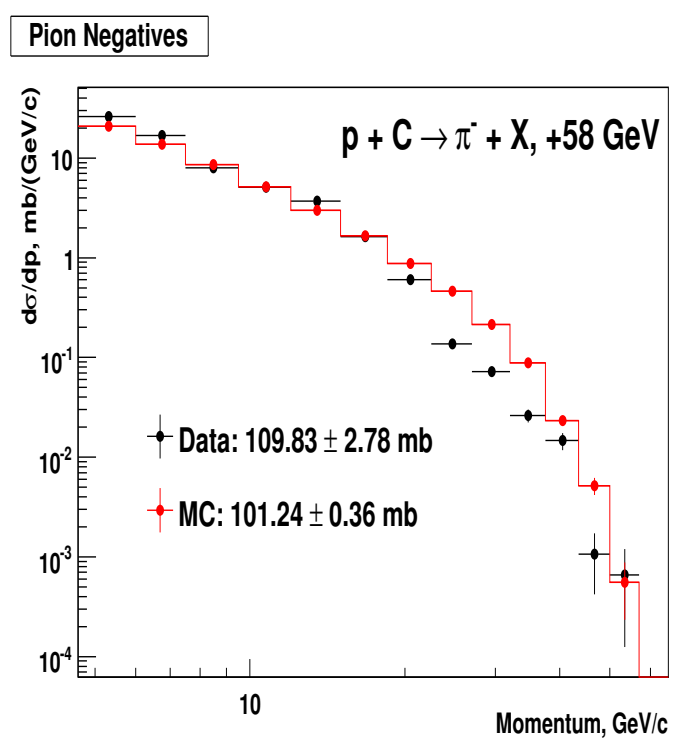

(a)

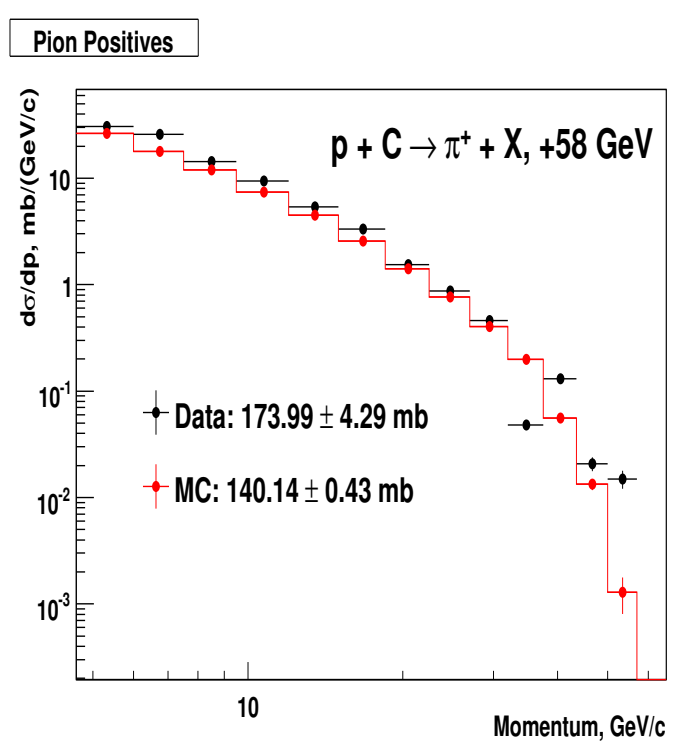

(b)

Figure 5.24: The comparison of the data inclusive (a) $\pi^{-}$and (b) $\pi^{+}$production cross sections with the MC predictions for momentum range $4.64-60 \mathrm{GeV} / \mathrm{c}$ using $\mathrm{RICH}$ for $58 \mathrm{GeV} / \mathrm{c}$ $\mathrm{p}+\mathrm{C}$ interactions. Only statistical errors are shown. 


\subsubsection{Cross sections using $120 \mathrm{GeV} / \mathrm{c} \mathrm{p}+\mathrm{C}$ interactions}

\section{Cross sections using TPC}

The inclusive $\pi^{-}$and $\pi^{+}$production cross sections in bins of true momentum with no corrections applied for momentum range $0.3-1 \mathrm{GeV} / \mathrm{c}$ using TPC for $120 \mathrm{GeV} / \mathrm{c} \mathrm{p}+\mathrm{C}$ interactions are shown in figure 5.25. The cross sections with all the corrections applied are shown in figure 5.26. The total inclusive pion production cross section is calculated by adding all the momentum bins and is given on the plots along with the statistical error. The statistical error on the integral value of the inclusive pion production cross section is $\sim 1 \%$.

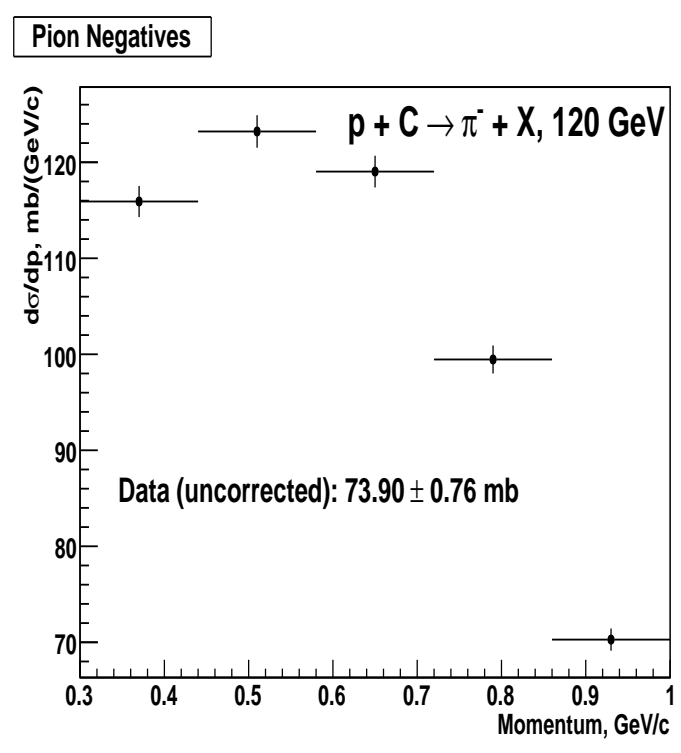

(a)

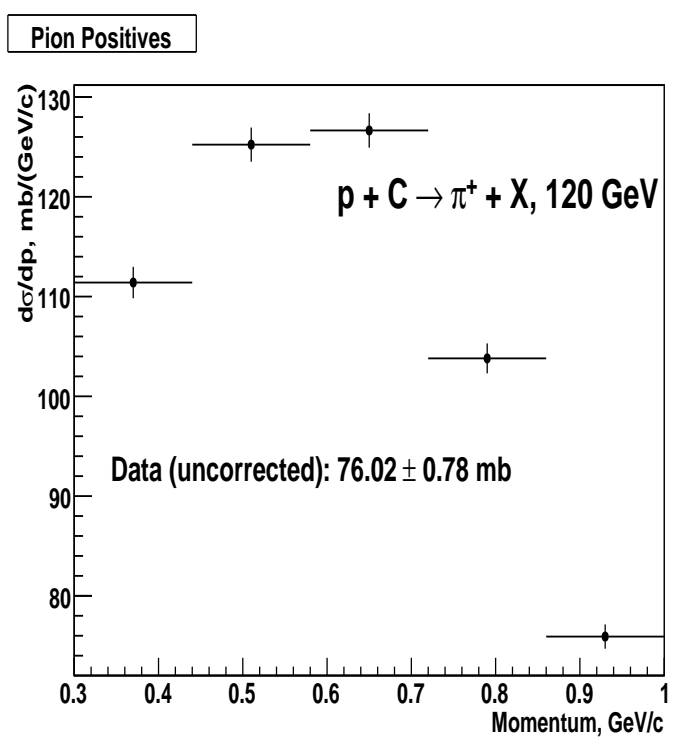

(b)

Figure 5.25: The inclusive (a) $\pi^{-}$and (b) $\pi^{+}$production cross sections in bins of true momentum for momentum range $0.3-1 \mathrm{GeV} / \mathrm{c}$ using TPC for $120 \mathrm{GeV} / \mathrm{c} \mathrm{p}+\mathrm{C}$ interactions. Corrections are not applied.

The comparison of the data inclusive $\pi^{-}$and $\pi^{+}$production cross sections with the MC predictions is shown in figure 5.27. The data is shown as black points and the MC as red line. The errors shown are statistical only. The data cross sections are much lower than the $\mathrm{MC}$ cross sections for both the pion negatives and positives.

\section{Cross sections using RICH}

The inclusive $\pi^{-}$and $\pi^{+}$production cross sections in bins of true momentum with no corrections applied for momentum range $4.64-120 \mathrm{GeV} / \mathrm{c}$ using RICH for $120 \mathrm{GeV} / \mathrm{c}$ p+C interactions are shown in figure 5.28, and with corrections applied, are shown in figure 5.29. 


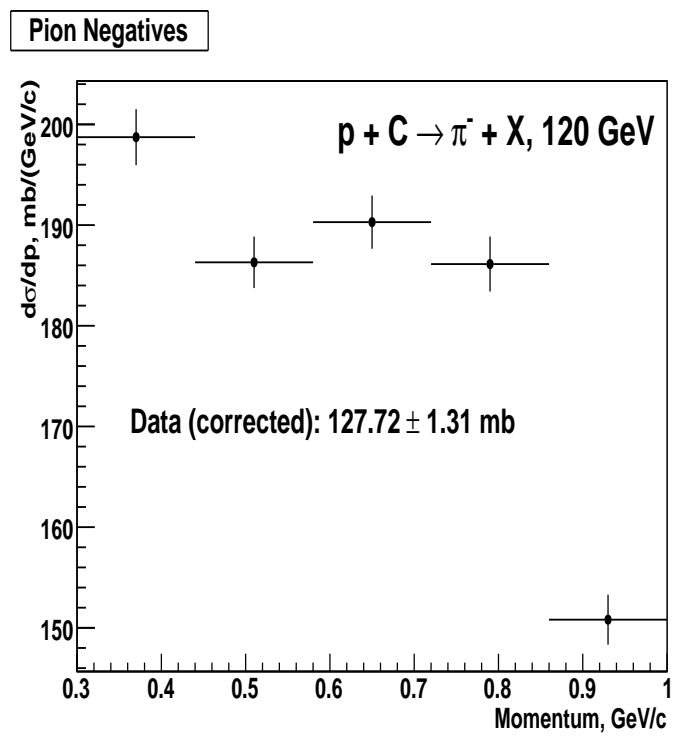

(a)

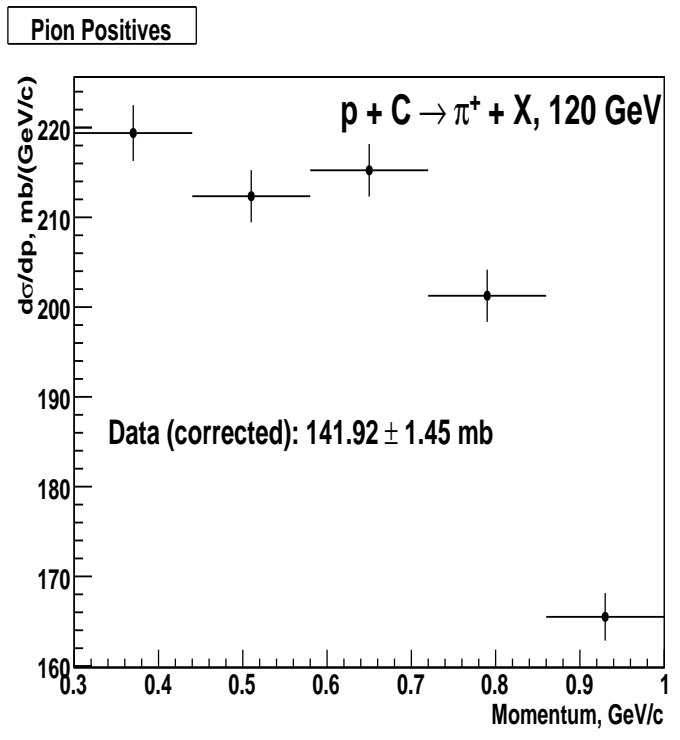

(b)

Figure 5.26: The inclusive (a) $\pi^{-}$and (b) $\pi^{+}$production cross sections in bins of true momentum for momentum range $0.3-1 \mathrm{GeV} / \mathrm{c}$ using TPC for $120 \mathrm{GeV} / \mathrm{c}$ p $+\mathrm{C}$ interactions. Corrections are applied.

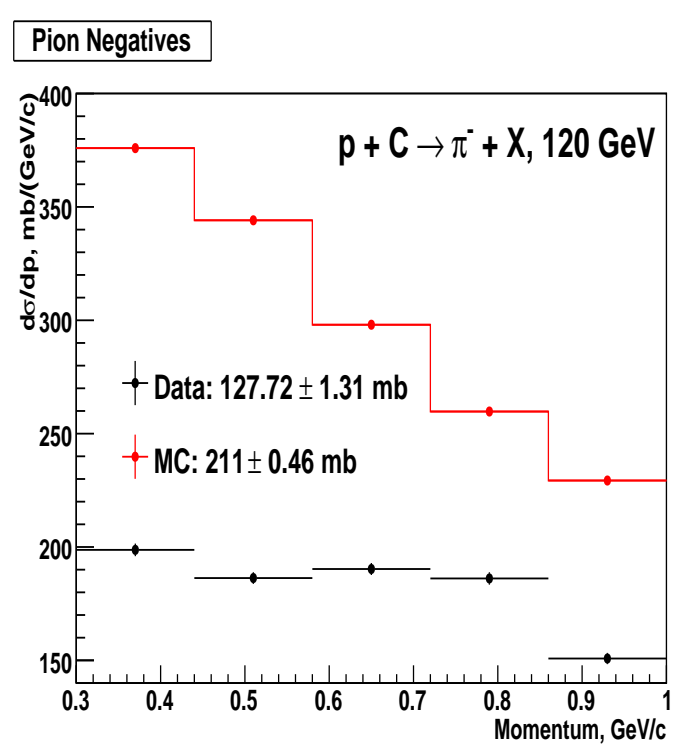

(a)

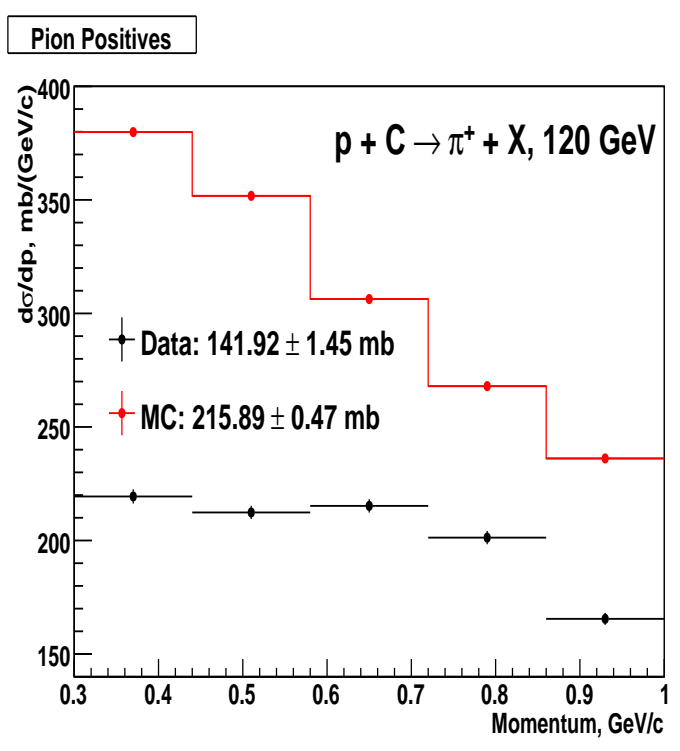

(b)

Figure 5.27: The comparison of the data inclusive (a) $\pi^{-}$and (b) $\pi^{+}$production cross sections with the MC predictions for momentum range $0.3-1 \mathrm{GeV} / \mathrm{c}$ using $\mathrm{TPC}$ for $120 \mathrm{GeV} / \mathrm{c} \mathrm{p}+\mathrm{C}$ interactions. Only statistical errors are shown. 
The total inclusive pion production cross section is calculated by adding all the momentum bins.

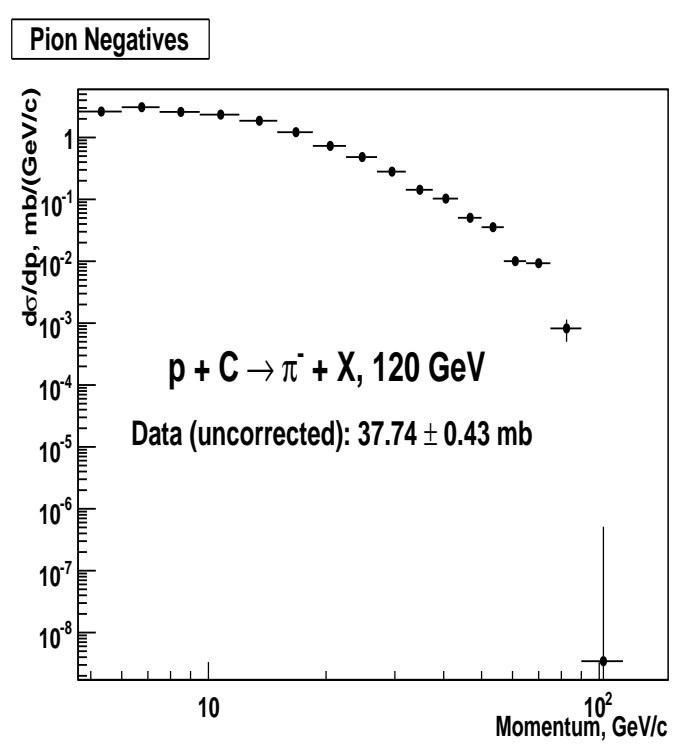

(a)

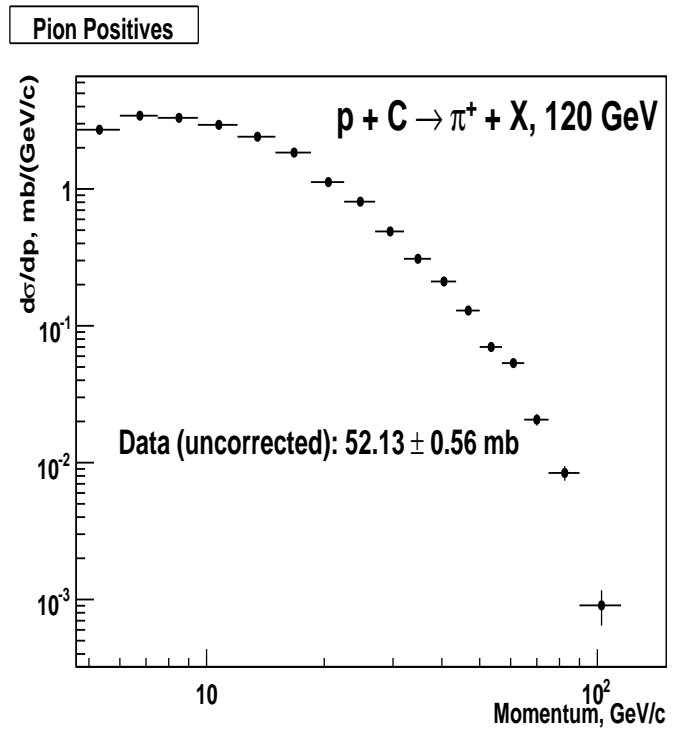

(b)

Figure 5.28: The inclusive (a) $\pi^{-}$and (b) $\pi^{+}$production cross sections in bins of true momentum for momentum range $4.64-120 \mathrm{GeV} / \mathrm{c}$ using $\mathrm{RICH}$ for $120 \mathrm{GeV} / \mathrm{c}$ p $+\mathrm{C}$ interactions. Corrections are not applied.

The comparison of the data inclusive $\pi^{-}$and $\pi^{+}$production cross sections with the MC predictions is shown in figure 5.30. The errors shown are statistical only. The data cross sections are lower than the MC cross sections for all the momentum bins for both the pion negatives and positives. The statistical error on the integral value of the inclusive pion production cross section for the data is $\sim 1.1 \%$.

\subsubsection{Systematic Errors}

The systematic errors are measured for the inclusive pion production cross sections for momentum ranges $0.3-1 \mathrm{GeV} / \mathrm{c}$ and $4.64-120 \mathrm{GeV} / \mathrm{c}$ using the TPC and RICH, respectively for both the 58 and $120 \mathrm{GeV} / \mathrm{c} \mathrm{p}+\mathrm{C}$ data. The systematics is calculated in a similar way as calculated for total inelastic cross sections and total charged particle production cross sections discussed in sections 5.1 and 5.3. The comparison of the nominal $\mathcal{A} \epsilon_{\mathcal{R}}$ and $\mathcal{A} \epsilon_{\mathcal{R}}$ using the reweighted $\mathrm{MC}$ in bins of true momentum using the negatively charged particles for the momentum ranges $0.3-1 \mathrm{GeV} / \mathrm{c}$ and $4.64-120 \mathrm{GeV} / \mathrm{c}$ using $120 \mathrm{GeV} / \mathrm{c}$ p $+\mathrm{C}$ interactions is shown in figure 5.31 . 


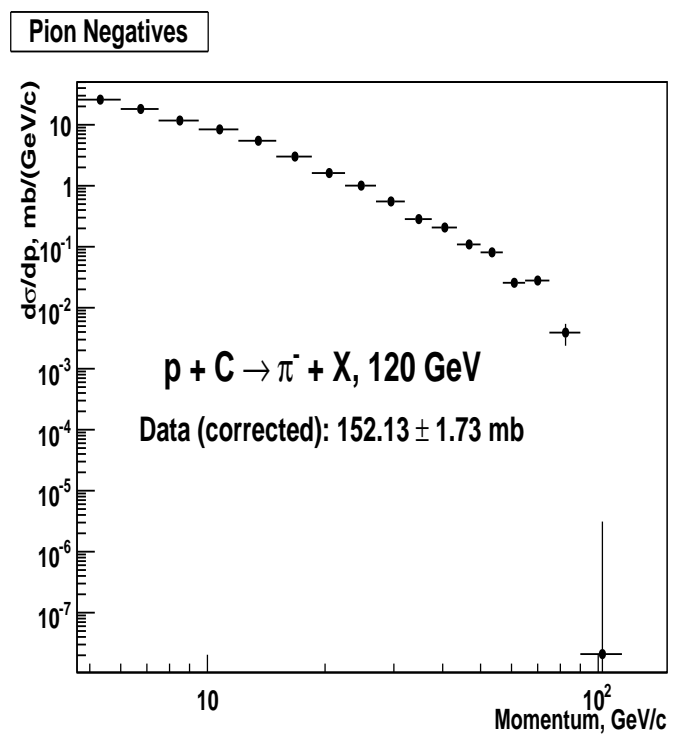

(a)

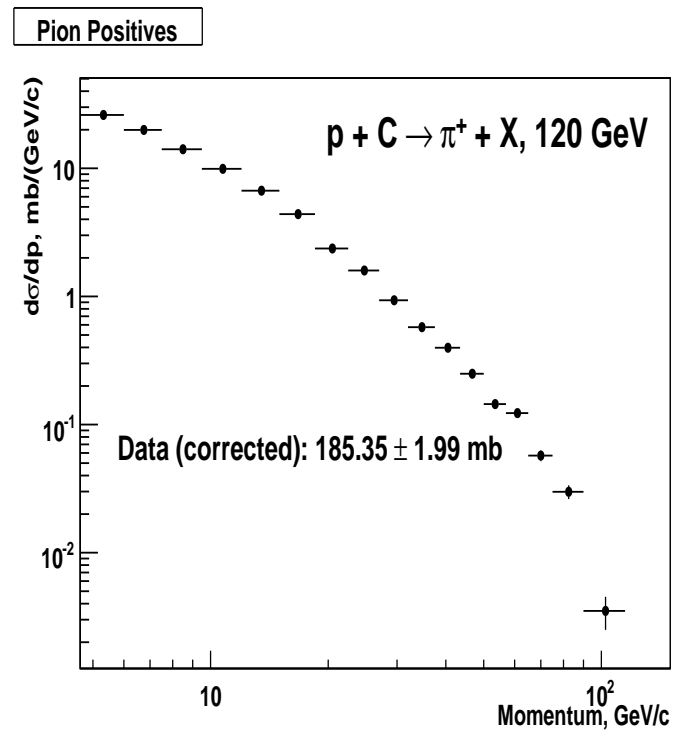

(b)

Figure 5.29: The inclusive (a) $\pi^{-}$and (b) $\pi^{+}$production cross sections in bins of true momentum for momentum range $4.64-120 \mathrm{GeV} / \mathrm{c}$ using $\mathrm{RICH}$ for $120 \mathrm{GeV} / \mathrm{c} \mathrm{p}+\mathrm{C}$ interactions. Corrections are applied.

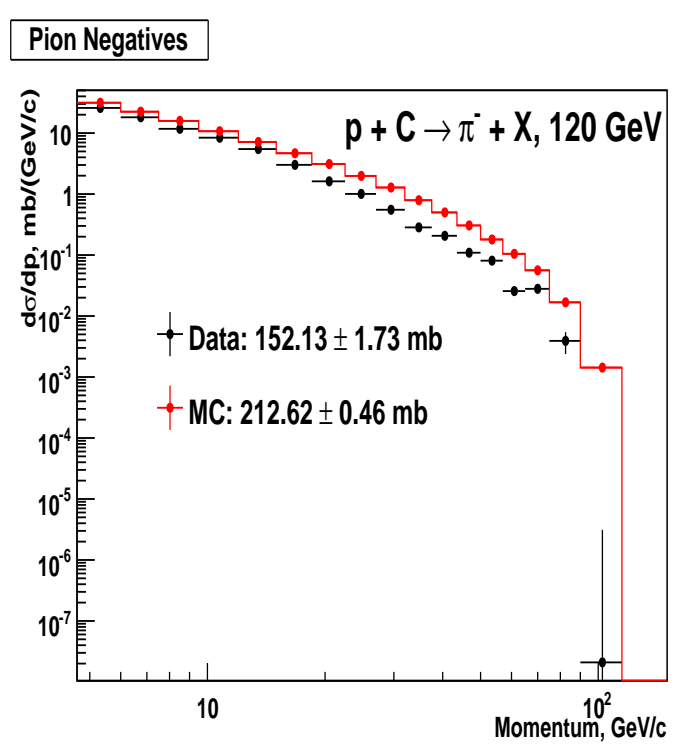

(a)

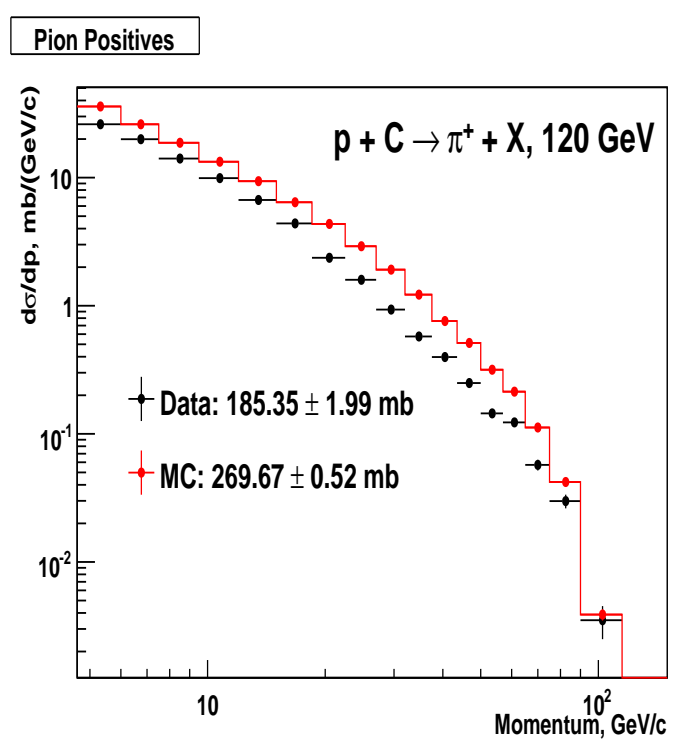

(b)

Figure 5.30: The comparison of the data inclusive (a) $\pi^{-}$and (b) $\pi^{+}$production cross sections with the MC predictions for momentum range $4.64-120 \mathrm{GeV} / \mathrm{c}$ using RICH for $120 \mathrm{GeV} / \mathrm{c}$ $\mathrm{p}+\mathrm{C}$ interactions. Only statistical errors are shown. 


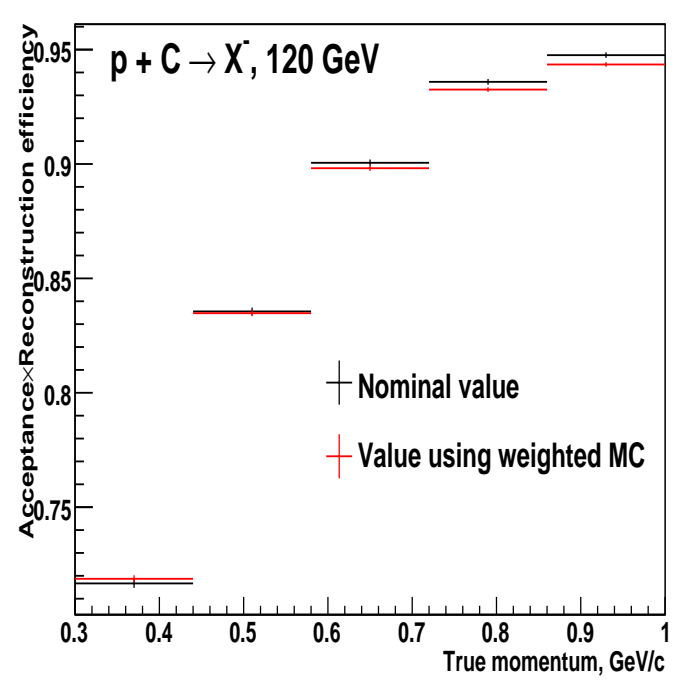

(a)

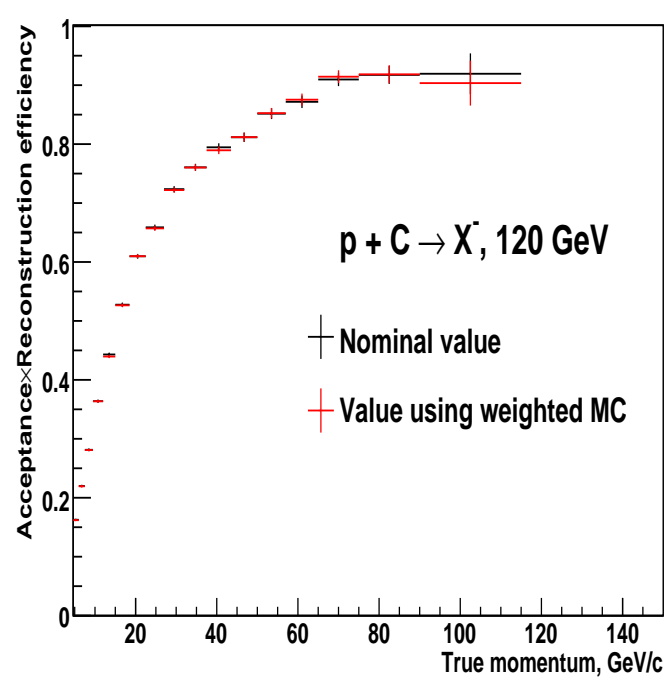

(b)

Figure 5.31: The comparison of nominal $\mathcal{A} \epsilon_{\mathcal{R}}$ and $\mathcal{A} \epsilon_{\mathcal{R}}$ using the reweighted MC in bins of true momentum using the negatively charged particles for momentum ranges (a) $0.3-1$ $\mathrm{GeV} / \mathrm{c}$ and (b) $4.64-120 \mathrm{GeV} / \mathrm{c}$ using $120 \mathrm{GeV} / \mathrm{c}$ p $+\mathrm{C}$ interactions.

Another source of systematics which is systematics from pion identification (PID) efficiency corrections, is also considered. The PID efficiencies are calculated using MC. To get an estimate of systematics from these corrections, the corrections are varied by $\pm 10 \%$, and the pion production cross sections are recalculated. Differences of these cross sections from the nominal cross section give the systematic errors $( \pm)$ from PID efficiency corrections.

Table 5.11 shows the inclusive charged pion $\left(\pi^{ \pm}\right)$production cross sections with total combined errors for the two momentum ranges i.e. $0.3-1 \mathrm{GeV} / \mathrm{c}$ and $4.64-120 \mathrm{GeV} / \mathrm{c}$ using 58 and $120 \mathrm{GeV} / \mathrm{c} \mathrm{p}+\mathrm{C}$ data. The statistical errors, systematic errors from various sources along with the total systematic errors obtained by adding all the systematic errors in quadrature are also given. The dominating systematic error is from the beam flux and it is $\sim 5 \%$. For the MC, uncertainties in the FLUKA predictions are of the order of $\sim 10-$ $12 \%$ [115.

For both the momentum ranges i.e. $0.3-1 \mathrm{GeV} / \mathrm{c}$ and $4.64-120 \mathrm{GeV} / \mathrm{c}$ using both the 58 and $120 \mathrm{GeV} / \mathrm{c}$ data, the production cross section for the pion positives is higher than that of the pion negatives, and that is expected because we have used the positive (proton) beam. 


\begin{tabular}{|c|c|c|c|c|c|c|c|c|c|c|}
\hline \multirow[b]{2}{*}{ 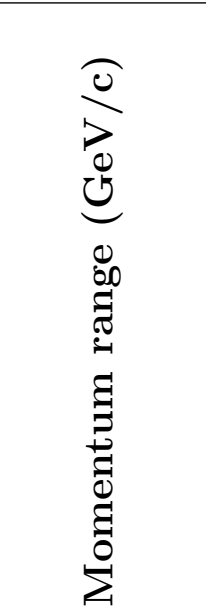 } & \multirow[b]{2}{*}{ 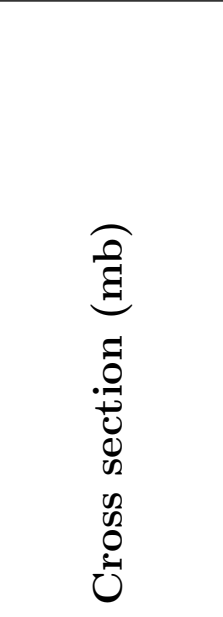 } & \multicolumn{9}{|c|}{ Errors (\%) } \\
\hline & & 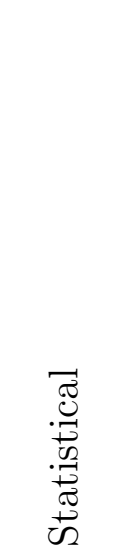 & 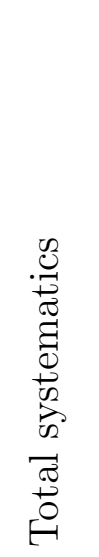 & 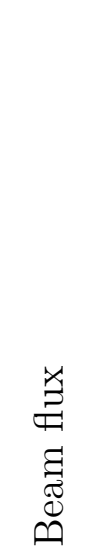 & 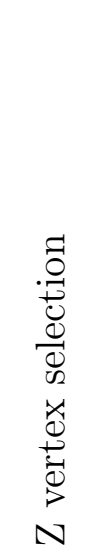 & 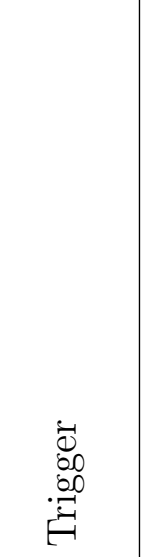 & 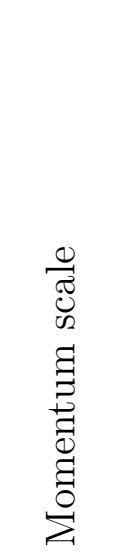 & 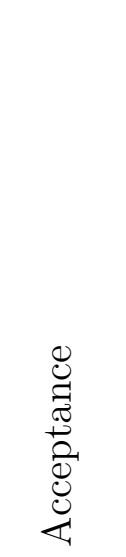 & 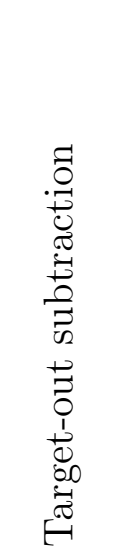 & $\hat{\theta}$ \\
\hline \multicolumn{11}{|c|}{$\mathrm{p}+\mathrm{C} \rightarrow \pi^{-}+\mathrm{X}, 58 \mathrm{GeV}$} \\
\hline $0.3-1$ & $170.68_{-10.92}^{+10.22}$ & \pm 2.42 & $\begin{array}{l}+5.47 \\
-5.92\end{array}$ & $\begin{array}{l}+4.76 \\
-5.26\end{array}$ & ${ }_{-0.31}^{+0.04}$ & \pm 0.19 & \pm 0.77 & \pm 0.16 & \pm 1.0 & \pm 2.36 \\
\hline $4.64-60$ & $109.83_{-7.08}^{+6.64}$ & \pm 2.53 & $\begin{array}{l}+5.49 \\
-5.93\end{array}$ & ${ }_{-5.26}^{+4.76}$ & ${ }_{-0.47}^{+0.37}$ & \pm 0.29 & \pm 0.65 & \pm 0.83 & \pm 1.42 & \pm 2.03 \\
\hline \multicolumn{11}{|c|}{$\mathrm{p}+\mathrm{C} \rightarrow \pi^{+}+\mathrm{X}, 58 \mathrm{GeV}$} \\
\hline $0.3-1$ & $194.74_{-12.44}^{+11.63}$ & \pm 2.42 & $\begin{array}{l}+5.46 \\
-5.91\end{array}$ & $\begin{array}{l}+4.76 \\
-5.26\end{array}$ & ${ }_{-0.27}^{+0.07}$ & \pm 0.27 & \pm 0.55 & \pm 0.49 & \pm 1.07 & \pm 2.33 \\
\hline $4.64-60$ & $173.99_{-11.09}^{+10.23}$ & \pm 2.47 & $\begin{array}{l}+5.34 \\
-5.88\end{array}$ & ${ }_{-5.26}^{+4.76}$ & $\begin{array}{l}+0.17 \\
-1.0\end{array}$ & \pm 0.40 & \pm 0.65 & \pm 1.09 & \pm 1.12 & \pm 1.69 \\
\hline \multicolumn{11}{|c|}{$\mathrm{p}+\mathrm{C} \rightarrow \pi^{-}+\mathrm{X}, 120 \mathrm{GeV}$} \\
\hline $0.3-1$ & $127.72_{-7.79}^{+7.21}$ & \pm 1.02 & $\begin{array}{l}+5.55 \\
-6.01\end{array}$ & $\begin{array}{l}+4.76 \\
-5.26\end{array}$ & $\begin{array}{l}+0.13 \\
-0.57\end{array}$ & \pm 0.13 & \pm 0.61 & \pm 0.16 & \pm 1.35 & \pm 2.42 \\
\hline $4.64-120$ & $152.13_{-8.87}^{+8.21}$ & \pm 1.14 & $\begin{array}{l}+5.28 \\
-5.72\end{array}$ & $\begin{array}{l}+4.76 \\
-5.26\end{array}$ & $\begin{array}{l}+0.39 \\
{ }_{-0.09}\end{array}$ & \pm 0.17 & \pm 0.56 & \pm 0.28 & \pm 1.38 & \pm 1.66 \\
\hline \multicolumn{11}{|c|}{$\mathrm{p}+\mathrm{C} \rightarrow \pi^{+}+\mathrm{X}, 120 \mathrm{GeV}$} \\
\hline $0.3-1$ & $141.92_{-8.69}^{+8.03}$ & \pm 1.02 & $\begin{array}{l}+5.57 \\
-6.04 \\
\end{array}$ & $\begin{array}{l}+4.76 \\
-5.26\end{array}$ & $\stackrel{-0}{+0.63}_{-0.06}$ & \pm 0.18 & \pm 0.69 & \pm 0.45 & \pm 1.40 & \pm 2.39 \\
\hline $4.64-120$ & $185.35_{-11.10}^{+10.31}$ & \pm 1.08 & $\begin{array}{l}+5.46 \\
-5.89\end{array}$ & $\begin{array}{l}+4.76 \\
{ }_{-5.26}\end{array}$ & $\begin{array}{l}+0.39 \\
-0.06\end{array}$ & \pm 0.26 & \pm 0.46 & \pm 1.42 & \pm 1.49 & \pm 1.58 \\
\hline
\end{tabular}

Table 5.11: Inclusive $\pi^{ \pm}$production cross sections for $\mathrm{p}+\mathrm{C}$ interactions at 58 and $120 \mathrm{GeV} / \mathrm{c}$. 


\subsubsection{Comparison of data with MC}

The comparison of the data inclusive $\pi^{-}$and $\pi^{+}$production cross sections with the MC predictions for momentum ranges $0.3-1 \mathrm{GeV} / \mathrm{c}$ and $4.64-60 \mathrm{GeV} / \mathrm{c}$ where both the statistical and systematic errors are shown for the data using $58 \mathrm{GeV} / \mathrm{c} \mathrm{p}+\mathrm{C}$ interactions, is shown in figures 5.32 and 5.33 , respectively. The data is shown as black points and the $\mathrm{MC}$ as red points. For momentum range $0.3-1 \mathrm{GeV} / \mathrm{c}$, the data cross sections are lower than the $\mathrm{MC}$ cross sections for the pion negatives, on the other hand, the data and $\mathrm{MC}$ cross sections are consistent within error bars except for first bin i.e. $0.3-0.44 \mathrm{GeV} / \mathrm{c}$ for the pion positives. For momentum range $4.64-60 \mathrm{GeV} / \mathrm{c}$, the data and $\mathrm{MC}$ cross sections are consistent within error bars up to $\sim 20 \mathrm{GeV} / \mathrm{c}$ for the pion negatives and up to $\sim 30 \mathrm{GeV} / \mathrm{c}$ for the pion positives, after that some discrepancies are observed.

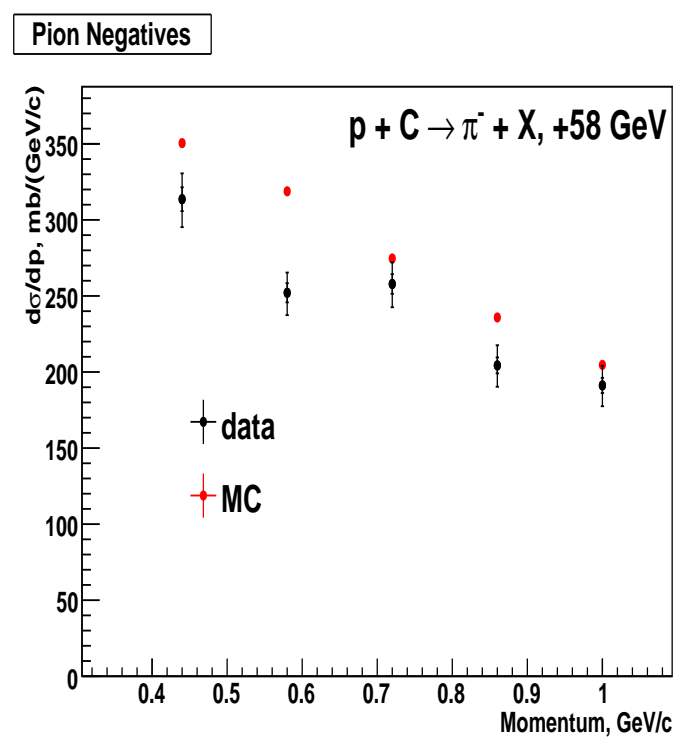

(a)

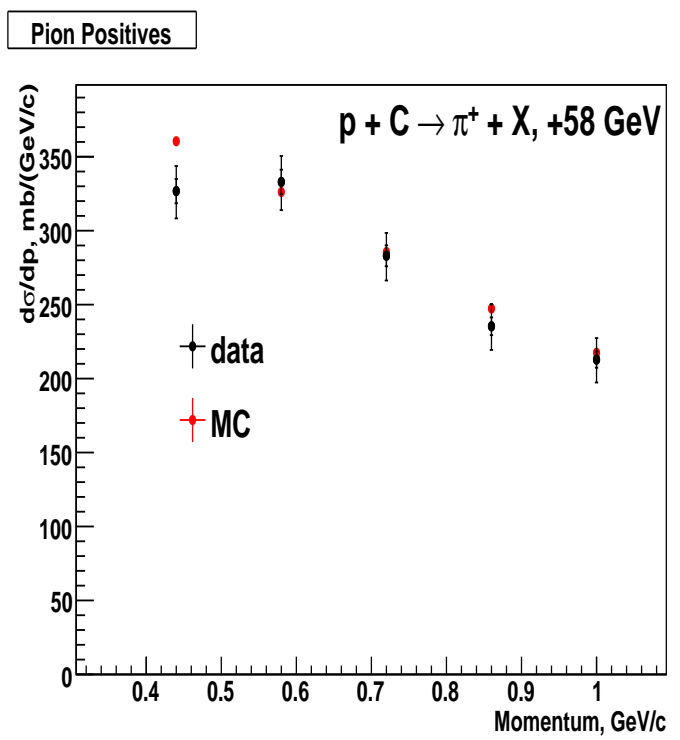

(b)

Figure 5.32: The comparison of the data inclusive (a) $\pi^{-}$and (b) $\pi^{+}$production cross sections with the MC predictions for momentum range $0.3-1 \mathrm{GeV} / \mathrm{c}$ using the TPC for $58 \mathrm{GeV} / \mathrm{c}$ $\mathrm{p}+\mathrm{C}$ interactions.

The data/MC comparison for momentum ranges $0.3-1 \mathrm{GeV} / \mathrm{c}$ and $4.64-120 \mathrm{GeV} / \mathrm{c}$ using $120 \mathrm{GeV} / \mathrm{c} \mathrm{p}+\mathrm{C}$ interactions is shown in figures 5.34 and 5.35, respectively. For momentum range $0.3-1 \mathrm{GeV} / \mathrm{c}$, the data cross sections are much lower than the $\mathrm{MC}$ cross sections for both the pion negatives and positives. For momentum range $4.64-120 \mathrm{GeV} / \mathrm{c}$, the data and $\mathrm{MC}$ cross sections are consistant within error bars up to $\sim 20 \mathrm{GeV} / \mathrm{c}$ and after that the data cross sections are lower than the MC cross sections for the pion negatives, 


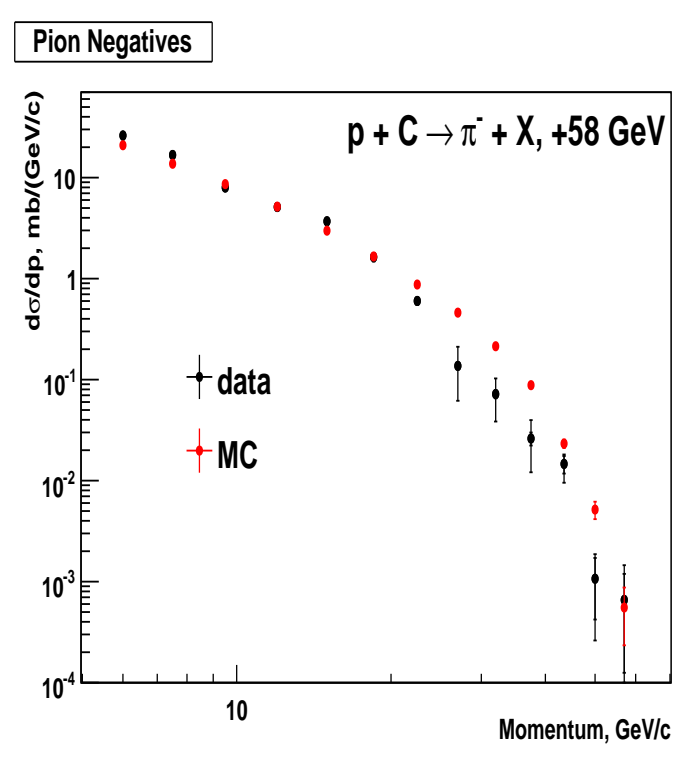

(a)

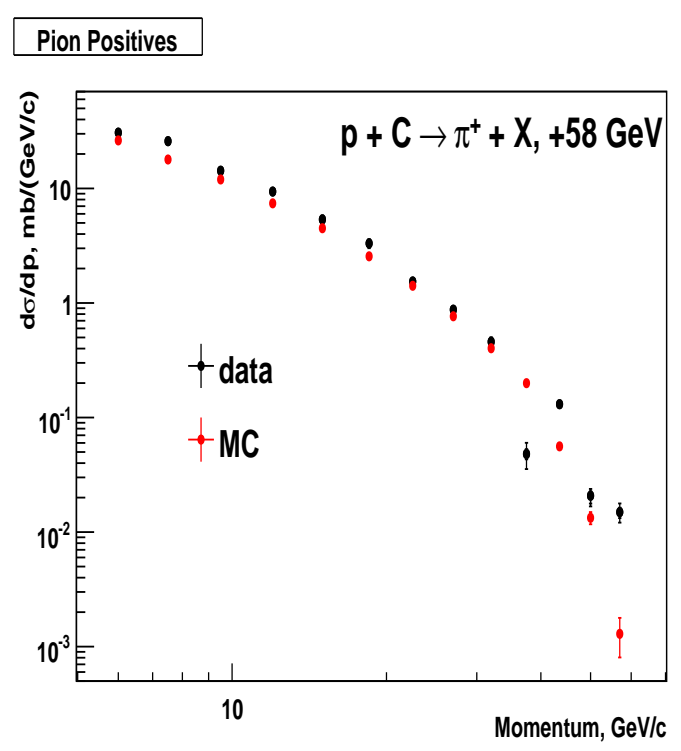

(b)

Figure 5.33: The comparison of the data inclusive (a) $\pi^{-}$and (b) $\pi^{+}$production cross sections with the MC predictions for momentum range $4.64-60 \mathrm{GeV} / \mathrm{c}$ using the $\mathrm{RICH}$ for $58 \mathrm{GeV} / \mathrm{c} \mathrm{p}+\mathrm{C}$ interactions.

on the other hand, the data cross sections are lower than the MC cross sections for all the momentum bins for the pion positives.

The comparison of the data and MC inclusive $\pi^{ \pm}$production cross sections for the momentum ranges $0.3-1 \mathrm{GeV} / \mathrm{c}$ and $4.64-120 \mathrm{GeV} / \mathrm{c}$ using 58 and $120 \mathrm{GeV} / \mathrm{c} \mathrm{p}+\mathrm{C}$ interactions, is shown in table 5.12 .

The comparison of the data and MC inclusive $\pi^{-}$and $\pi^{+}$production cross sections for both the momentum ranges i.e. $0.3-1 \mathrm{GeV} / \mathrm{c}$ and $4.64-60 \mathrm{GeV} / \mathrm{c}$ using $58 \mathrm{GeV} / \mathrm{c}$ $\mathrm{p}+\mathrm{C}$ interactions is shown on a single plot in figure 5.36. The data/MC comparison for both the momentum ranges i.e. $0.3-1 \mathrm{GeV} / \mathrm{c}$ and $4.64-120 \mathrm{GeV} / \mathrm{c}$ using $120 \mathrm{GeV} / \mathrm{c} \mathrm{p}+\mathrm{C}$ interactions is shown in figure 5.37. Both the statistical and systematic errors are also shown for the data.

\subsubsection{Comparison of 58 and $120 \mathrm{GeV} / \mathrm{c}$ data}

The inclusive $\pi^{-}$and $\pi^{+}$production cross sections for 58 and $120 \mathrm{GeV} / \mathrm{c} \mathrm{p}+\mathrm{C}$ data along with statistical and systematic errors are compared in figure 5.38 . The $120 \mathrm{GeV} / \mathrm{c}$ cross sections are lower than the $58 \mathrm{GeV} / \mathrm{c}$ cross sections at lower momenta (using TPC) but they start becoming higher than the $58 \mathrm{GeV} / \mathrm{c}$ cross sections at the higher momenta (using 


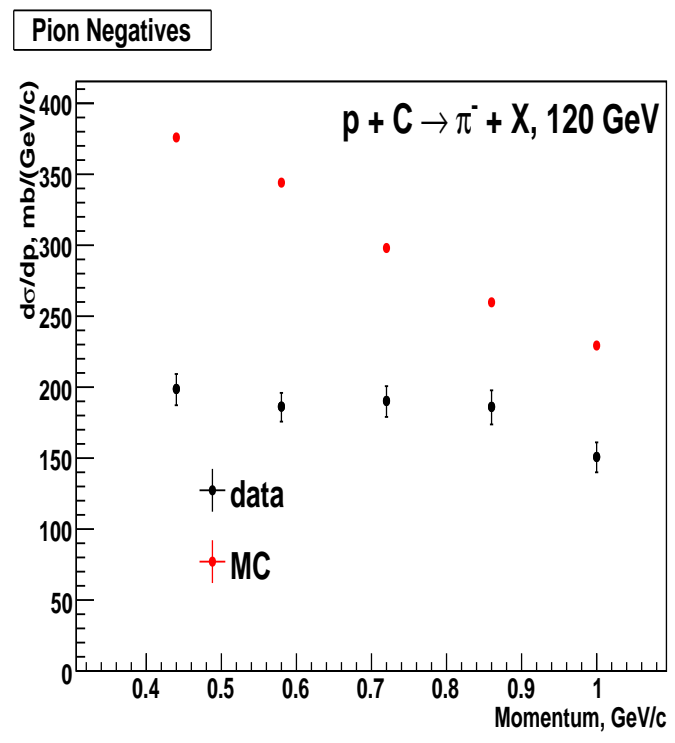

(a)

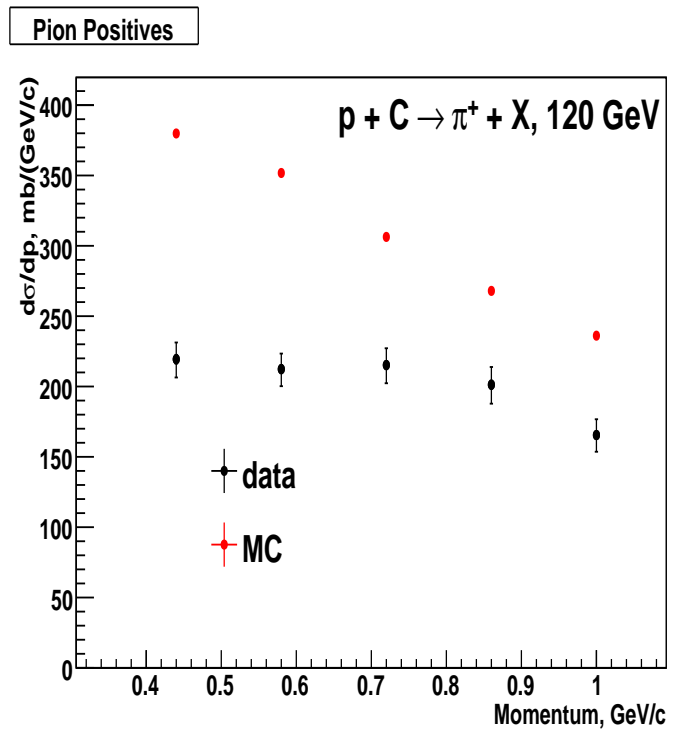

(b)

Figure 5.34: The comparison of the data inclusive (a) $\pi^{-}$and (b) $\pi^{+}$production cross sections with the MC predictions for momentum range $0.3-1 \mathrm{GeV} / \mathrm{c}$ using the TPC for $120 \mathrm{GeV} / \mathrm{c}$ $\mathrm{p}+\mathrm{C}$ interactions.

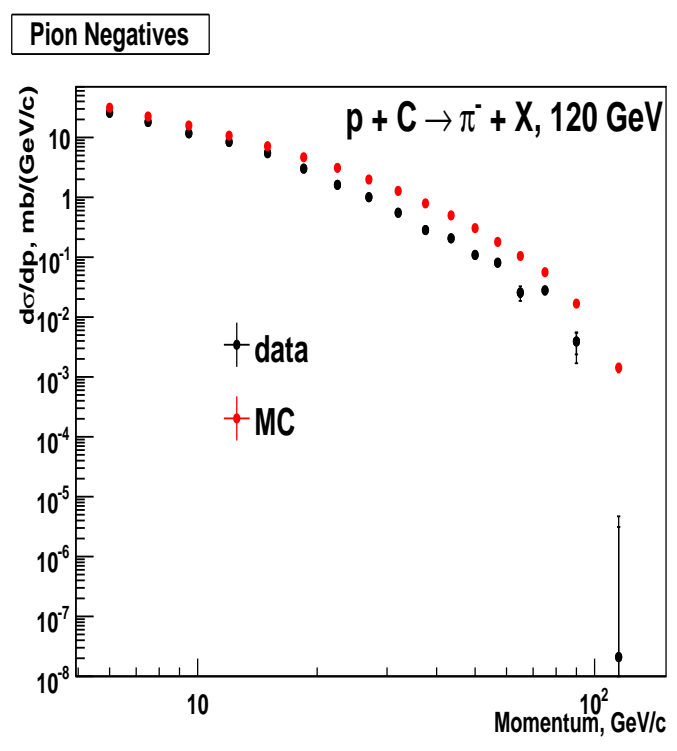

(a)

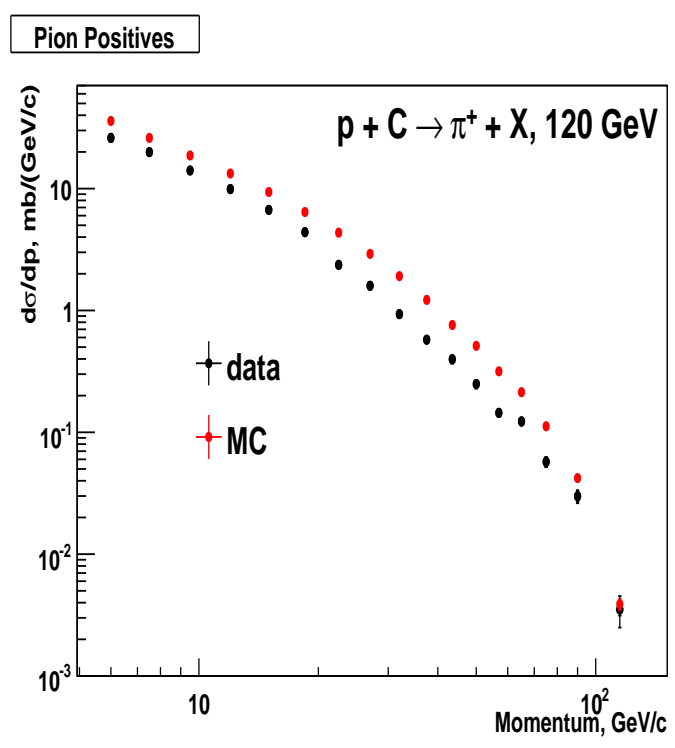

(b)

Figure 5.35: The comparison of the data inclusive (a) $\pi^{-}$and (b) $\pi^{+}$production cross sections with the MC predictions for momentum range $4.64-120 \mathrm{GeV} / \mathrm{c}$ using the RICH for 120 $\mathrm{GeV} / \mathrm{c} \mathrm{p}+\mathrm{C}$ interactions. 


\begin{tabular}{|c|c|c|}
\hline $\begin{array}{l}\text { Momentum } \\
\text { range } \\
(\mathrm{GeV} / \mathrm{c})\end{array}$ & Data $(\mathrm{mb})$ & $\mathrm{MC}(\mathrm{mb})$ \\
\hline \multicolumn{3}{|c|}{$\mathrm{p}+\mathrm{C} \rightarrow \pi^{-}+\mathrm{X}, 58 \mathrm{GeV}$} \\
\hline $0.3-1$ & $170.68 \pm 4.14(\text { stat })_{-10.10}^{+9.34}$ (syst) & $193.83 \pm 0.50$ (stat) \\
\hline $4.64-60$ & $109.83 \pm 2.78(\text { stat })_{-6.51}^{+6.03}$ (syst) & $101.24 \pm 0.36$ (stat) \\
\hline \multicolumn{3}{|c|}{$\mathrm{p}+\mathrm{C} \rightarrow \pi^{+}+\mathrm{X}, 58 \mathrm{GeV}$} \\
\hline $0.3-1$ & $194.74 \pm 4.72(\text { stat })_{-11.51}^{+10.63}$ (syst) & $201.19 \pm 0.51$ (stat) \\
\hline $4.64-60$ & $173.99 \pm 4.29(\text { stat })_{-10.23}^{+9.29}($ syst $)$ & $140.14 \pm 0.43$ (stat) \\
\hline \multicolumn{3}{|c|}{$\mathrm{p}+\mathrm{C} \rightarrow \pi^{-}+\mathrm{X}, 120 \mathrm{GeV}$} \\
\hline $0.3-1$ & $127.72 \pm 1.31$ (stat) $)_{-7.68}^{+7.09}$ (syst) & $211.0 \pm 0.46$ (stat) \\
\hline $4.64-120$ & $152.13 \pm 1.73(\text { stat })_{-8.70}^{+8.03}$ (syst) & $212.62 \pm 0.46$ (stat) \\
\hline \multicolumn{3}{|c|}{$\mathbf{p}+\mathrm{C} \rightarrow \pi^{+}+\mathrm{X}, 120 \mathrm{GeV}$} \\
\hline $0.3-1$ & $141.92 \pm 1.45(\text { stat })_{-8.57}^{+7.90}$ (syst) & $215.89 \pm 0.47$ (stat) \\
\hline $4.64-120$ & $185.35 \pm 1.99(\text { stat })_{-10.92}^{+10.12}($ syst $)$ & $269.67 \pm 0.52$ (stat) \\
\hline
\end{tabular}

Table 5.12: Comparison of the data and MC inclusive $\pi^{ \pm}$production cross sections for the two momentum ranges i.e. $0.3-1 \mathrm{GeV} / \mathrm{c}$ and $4.64-120 \mathrm{GeV} / \mathrm{c}$ using 58 and $120 \mathrm{GeV} / \mathrm{c}$ $\mathrm{p}+\mathrm{C}$ interactions. For the MC, the systematic uncertainties in the FLUKA predictions are of the order of $\sim 10-12 \%$. 


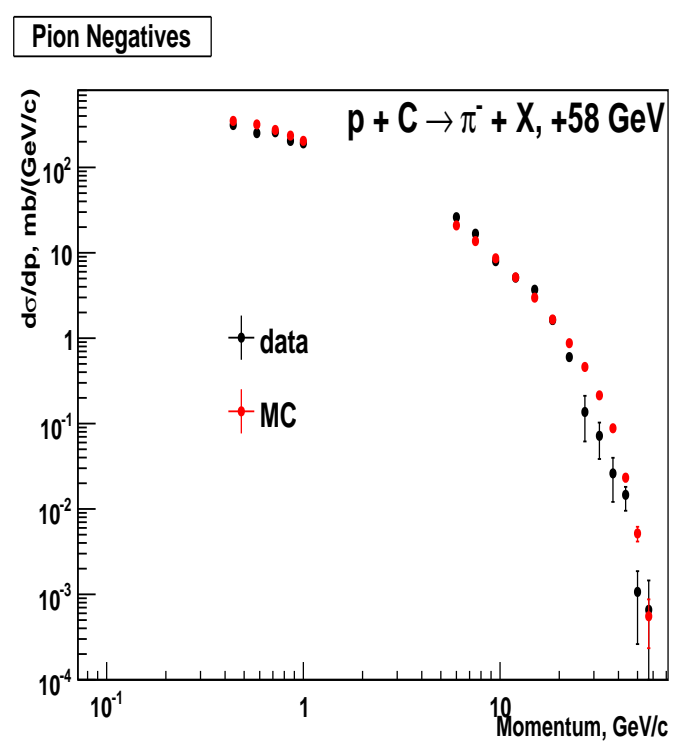

(a)

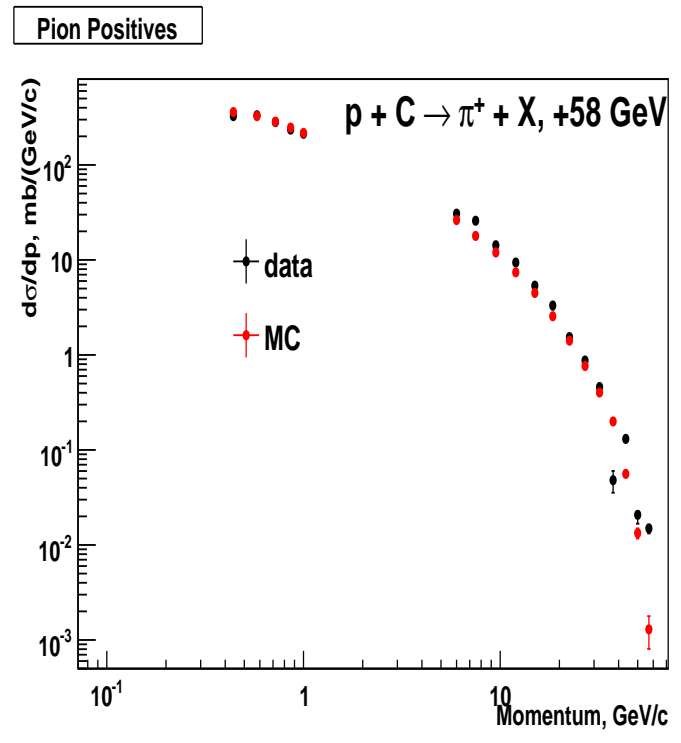

(b)

Figure 5.36: The comparison of the data and MC inclusive (a) $\pi^{-}$and (b) $\pi^{+}$production cross sections for momentum ranges $0.3-1 \mathrm{GeV} / \mathrm{c}$ and $4.64-60 \mathrm{GeV} / \mathrm{c}$ using the TPC and $\mathrm{RICH}$ shown on a single plot for $58 \mathrm{GeV} / \mathrm{c} \mathrm{p}+\mathrm{C}$ interactions.

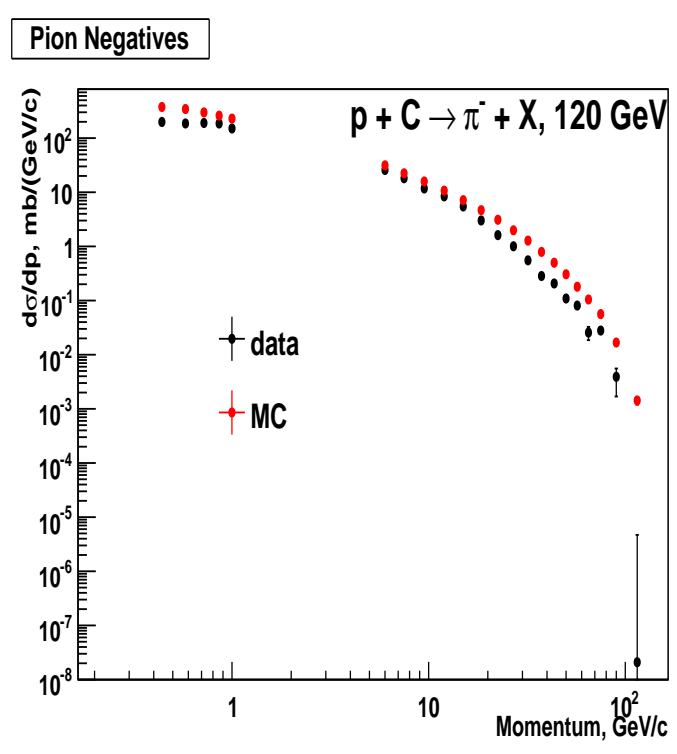

(a)

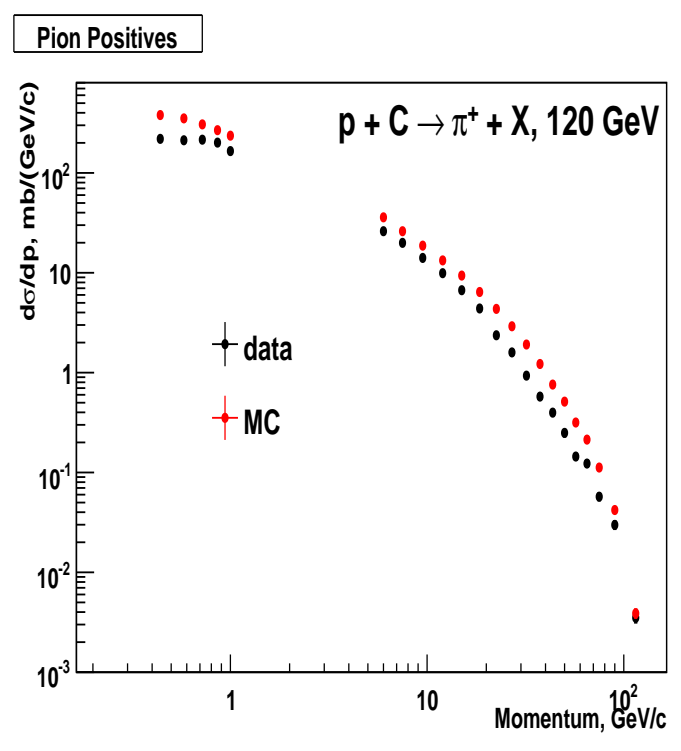

(b)

Figure 5.37: The comparison of the data and MC inclusive (a) $\pi^{-}$and (b) $\pi^{+}$production cross sections for momentum ranges $0.3-1 \mathrm{GeV} / \mathrm{c}$ and $4.64-120 \mathrm{GeV} / \mathrm{c}$ using the TPC and RICH shown on a single plot for $120 \mathrm{GeV} / \mathrm{c} \mathrm{p}+\mathrm{C}$ interactions. 
$\mathrm{RICH}$ ). Ideally the cross sections at $120 \mathrm{GeV} / \mathrm{c}$ beam momentum should be higher than those at $58 \mathrm{GeV} / \mathrm{c}$ beam momentum. The discrepancies observed in the lower momentum range are not understood.

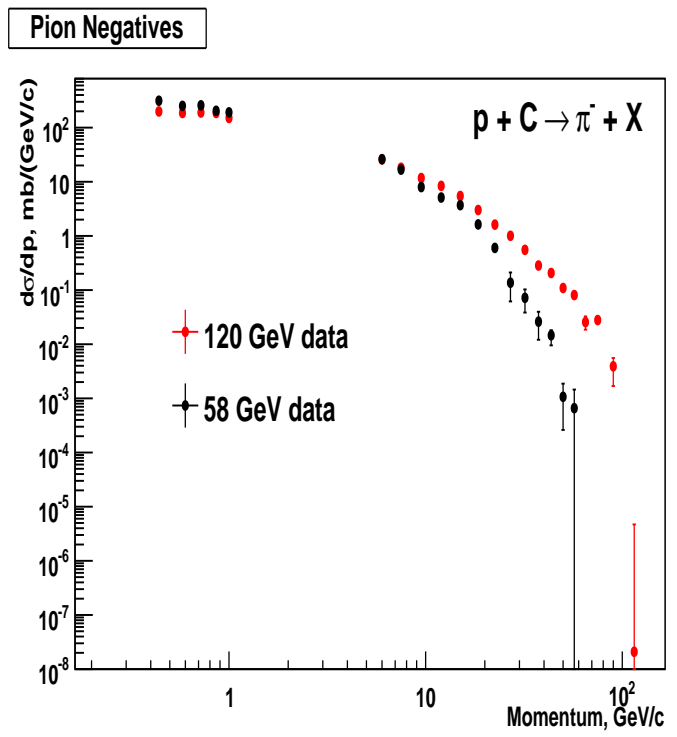

(a)

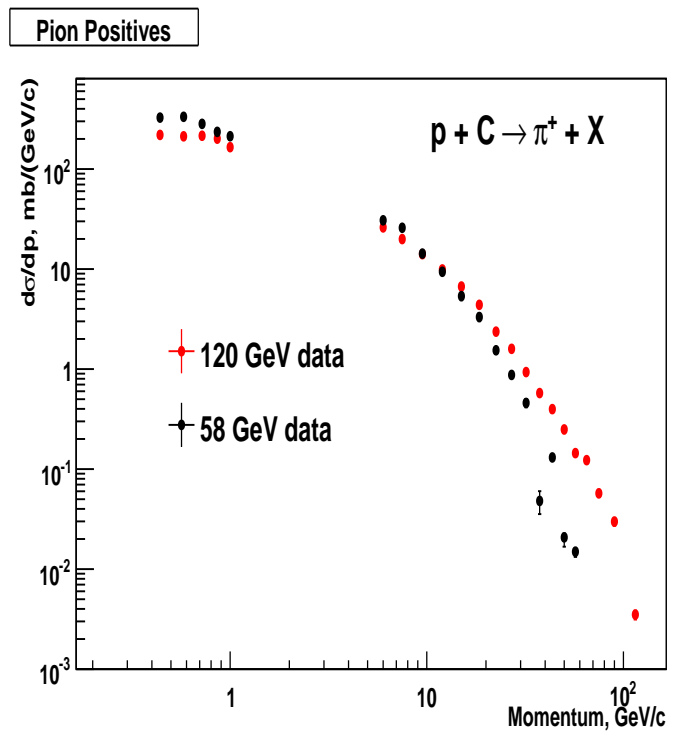

(b)

Figure 5.38: The comparison of inclusive (a) $\pi^{-}$and (b) $\pi^{+}$production cross sections for momentum ranges $0.3-1 \mathrm{GeV} / \mathrm{c}$ and $4.64-120 \mathrm{GeV} / \mathrm{c}$ using the TPC and RICH using 58 and $120 \mathrm{GeV} / \mathrm{c} \mathrm{p}+\mathrm{C}$ data. 


\section{Chapter 6}

\section{Summary and Outlook}

In this concluding chapter a brief summary of the results presented in the previous chapters is given.

\subsection{Experimental results}

The results presented in this thesis are summarized as follows:

- The total inelastic cross sections have been measured for 58 and $85 \mathrm{GeV} / \mathrm{c} \mathrm{p}+\mathrm{p}$ interactions, and 58 and $120 \mathrm{GeV} / \mathrm{c} \mathrm{p}+\mathrm{Be}, \mathrm{p}+\mathrm{C}$ and $\mathrm{p}+\mathrm{Bi}$ interactions using the MIPP experiment. These cross sections have been corrected for the interaction trigger inefficiencies, detector acceptances and the reconstruction inefficiencies. All these corrections have been estimated using the MIPP Monte Carlo (MC). Both the statistical and systematic errors have been measured. Total error is of the order of $6-7 \%$, dominating error being the systematic error from the beam flux which is $\sim 5 \%$.

- The inelastic cross sections using the MIPP data have been compared with the previous measurements and the MC predictions. For liquid hydrogen target, the MIPP measurement at $58 \mathrm{GeV} / \mathrm{c}$ is in a good agreement with the PDG and MC, while the measurement at $85 \mathrm{GeV} / \mathrm{c}$ is $\sim 10 \%$ higher than the PDG and MC. For beryllium target, the MIPP measurement at $58 \mathrm{GeV} / \mathrm{c}$ is consistent, within error bars, with the measurement of Denisov et al. [90] and the $\mathrm{MC}$ is $\sim 9 \%$ lower than our measurement, while at $120 \mathrm{GeV} / \mathrm{c}$, the $\mathrm{MC}$ is $\sim 6 \%$ higher than our measurement. For carbon target, the MIPP measurement at $58 \mathrm{GeV} / \mathrm{c}$ is consistent, within error bars, with the measurement of Denisov et al. and $\sim 20 \%$ higher than the measurement of Carroll et al. [91, and the $\mathrm{MC}$ is $\sim 10 \%$ lower than our measurement, while at $120 \mathrm{GeV} / \mathrm{c}$, the $\mathrm{MC}$ is $\sim 12 \%$ higher than our measurement. For bismuth target, the MC is $\sim 18 \%$ lower 
than our measurement at $58 \mathrm{GeV} / \mathrm{c}$, while $\sim 30 \%$ higher at $120 \mathrm{GeV} / \mathrm{c}$. No previous measurement is available at $58 \mathrm{GeV} / \mathrm{c}$ for the bismuth target and also, at $120 \mathrm{GeV} / \mathrm{c}$ for all the three thin targets i.e. Be, $\mathrm{C}$ and $\mathrm{Bi}$.

- We expect a little rise in the inelastic cross section value as we go higher in the beam momentum but we have observed that the MIPP measurement at $120 \mathrm{GeV} / \mathrm{c}$ is lower than that at $58 \mathrm{GeV} / \mathrm{c}$ for all the three thin targets. A similar feature was observed in the previous analyses [113. With the present study, it was not possible to figure out the exact reason for this discrepancy.

- We have also studied the inelastic cross section as a function of target atomic weight A. It is found that for $\mathrm{A}>1$, the inelastic cross section vary as $\mathrm{A}^{\alpha}$, where $\alpha$ is $0.75 \pm$ 0.03 for $58 \mathrm{GeV} / \mathrm{c}$ beam and $0.66 \pm 0.03$ for $120 \mathrm{GeV} / \mathrm{c}$ beam.

- A new method has been developed to account for the low multiplicity inefficiencies in the interaction trigger using KNO scaling [95, 101] for liquid hydrogen and carbon targets. This method treats the trigger efficiencies (as a function of the number of tracks going through the scintillator) as parameters to be determined by imposing the criterion that the true multiplicities are given by the KNO scaling relation. The inelastic cross sections have been cross checked using KNO-based trigger efficiencies and the cross sections are found to be consistent, within 10\%, with the cross sections using the MC efficiencies.

- Inelastic cross sections have also been presented as a function of multiplicity for $\mathrm{p}+\mathrm{p}$ and $\mathrm{p}+\mathrm{C}$ interactions at different beam momenta. The true multiplicities have been obtained from the KNO scaling relation using the average multiplicity $<\mathrm{n}>$ from MIPP rather than obtaining them from unfolding using the $\mathrm{MC}$, since large discrepancies have been observed between the MC and data multiplicity shapes. Again the inelastic cross sections using MIPP data have been compared with the MC predictions and the existing data. For $\mathrm{p}+\mathrm{p}$ at $58 \mathrm{GeV} / \mathrm{c}$, discrepancies have been found between the MIPP data and the PDG for 2-prongs and 4-prongs, while the MIPP data is consistent, within error bars, with the PDG for the higher multiplicities. For p+p at $85 \mathrm{GeV} / \mathrm{c}$, the MIPP data is consistent, within error bars, with the PDG except for 8-prongs. The $\mathrm{MC}$ shape completely disagrees with the data for both the beam momenta. For $\mathrm{p}+\mathrm{C}$ at $58 \mathrm{GeV} / \mathrm{c}$, the MIPP data is consistent, within error bars, with the measurement of Denisov et al. [90], and consistent with the measurement of Carroll et al. [91] for multiplicity $>13$ only. The MC starts agreeing with the MIPP data for multiplicity 
27. For $\mathrm{p}+\mathrm{C}$ at $120 \mathrm{GeV} / \mathrm{c}$, there is no previous measurement available, the $\mathrm{MC}$ completely disagrees with the data.

- The total charged particle production cross sections have also been measured in bins of momentum for both the data and MC as a cross check for 58 and $120 \mathrm{GeV} / \mathrm{c}$ $\mathrm{p}+\mathrm{C}$ interactions. The data and $\mathrm{MC}$ cross sections have been compared and some discrepancies have been found. The average production cross section has been found to be equal to average multiplicity $<\mathrm{n}>$ times the average inelastic cross section for both the data and MC, and that is expected.

- We have also presented the inclusive charged pion $\left(\pi^{ \pm}\right)$production cross sections in bins of true momentum using 58 and $120 \mathrm{GeV} / \mathrm{c} \mathrm{p}+\mathrm{C}$ data. The TPC and RICH detectors have been used independently to do the particle identification (PID) using the maximum likelihood technique. The TPC is used to identify the particles in lower momentum range i.e. $0.3-1 \mathrm{GeV} / \mathrm{c}$ and the $\mathrm{RICH}$ in higher momentum range i.e. $4.64-120 \mathrm{GeV} / \mathrm{c}$. Hence the momentum range of $1-4.64 \mathrm{GeV} / \mathrm{c}$ is not covered for the cross section measurements. The observed (data) pion momentum spectrum is unfolded using the MC. This unfolded momentum spectrum has been used for the cross section measurements. All the corrections including trigger efficiencies, detector acceptances, reconstruction efficiencies and PID efficiencies have been taken into account in the final cross section measurements. All the corrections have been calculated in bins of true momentum. The statistical and systematic errors have been measured. Total error is of the order of $6-7 \%$, dominating error being the systematic error from the beam flux which is $\sim 5 \%$.

- The inclusive pion production cross sections using the MIPP data have also been compared with the MC predictions. At $58 \mathrm{GeV} / \mathrm{c}$ beam momentum, the data and $\mathrm{MC}$ are consistent, within error bars, for most of the momenta except at the higher momenta ( $>\sim 20-30 \mathrm{GeV} / \mathrm{c}$ ) where some discrepancies have been observed. At 120 $\mathrm{GeV} / \mathrm{c}$, the data have been observed to be significantly lower than the MC in the lower momentum range i.e. $0.3-1 \mathrm{GeV} / \mathrm{c}$ for both the pion negatives and positives, and these discrepancies are not understood. For the higher momentum range, the MIPP data for the pion negatives is consistent, within error bars, with the MC up to $\sim 20$ $\mathrm{GeV} / \mathrm{c}$ and after that, the data is lower than the MC. For the pion positives, the MIPP data is lower than the MC for all the momentum bins. The inclusive pion production cross sections at 58 and $120 \mathrm{GeV} / \mathrm{c}$ have also been compared. The cross sections at 120 $\mathrm{GeV} / \mathrm{c}$ are lower than those at $58 \mathrm{GeV} / \mathrm{c}$ in the lower momentum range, but the 120 $\mathrm{GeV} / \mathrm{c}$ cross sections are higher than those at $58 \mathrm{GeV} / \mathrm{c}$ at the higher momenta, and 
this is expected due to the kinematics and available phase space. The discrepancies observed in the lower momentum range are not understood.

\subsection{Implications of these results}

As already discussed in this thesis, the MC simulation programs like GEANT4, MARS, FLUKA, etc. model hadronic interactions based on available data. All the data presented in this thesis could be used to improve the hadron shower simulations. These data could also be helpful for the neutrino experiments like MINER $\nu$ A, MINOS, NO $\nu$ A, ELBNF [46, 47, 45, 42, 43, 44] to minimize the systematic errors due to the neutrino flux predictions since MIPP used the same beamline as used by these experiments.

\subsection{Outlook}

In this thesis, we have presented the inclusive pion production cross sections for $\mathrm{p}+\mathrm{C}$ interactions at 58 and $120 \mathrm{GeV} / \mathrm{c}$. This work can be extended to the other thin nuclear targets such as $\mathrm{Be}, \mathrm{Bi}$ and $\mathrm{U}$, to check the cross section behaviour as a function of the target atomic weight. All these $\mathrm{p}+$ nucleus data can be helpful for the better modeling of hadronic showers and tuning of the $\mathrm{MC}$ models for the better neutrino flux predictions. 


\section{Bibliography}

[1] D. Griffiths, Introduction to Elementary Particles, John Wiley and Sons, Inc. (1987).

[2] D. H. Perkins, Introduction to High Energy Physics, Cambridge University Press (2000).

[3] M. Veltman, Facts and Mysteries in Elementary Particle Physics, World Scientific (2003).

[4] F. Halzen and A. Martin, Quarks and Leptons, John Wiley and Sons, Inc. (1984).

[5] M. Peskin, D. Schroeder, An Introduction to Quantum Field Theory, Westview Press (1995).

[6] http://www.physics.gla.ac.uk/ppt/research.htm.

[7] Particle Data Group (PDG) http://pdg.1bl.gov/2013/reviews/ rpp2013-rev-neutrino-mixing.pdf.

[8] K. A. Olive et al. (PDG), Chin. Phys. C 38, 090001 http://pdg.lbl.gov (2014).

[9] R. P. Feynman, QED: The Strange Theory of Light and matter, Princeton University Press (1985). 
[10] Glashow-Weinberg-Salam theory of electroweak interactions and the neutral currents, S. M. Bilenky, J. Hosek, Physics Reports (Review Section of Physics Letters) 90, No. 2, 73-157 (1982).

[11] http://pdg.lbl.gov/2013/reviews/rpp2013-rev-w-mass.pdf.

[12] J. Beringer et al. (PDG), Phys. Rev. D 86, 010001, doi:10.1103/PhysRevD.86.010001 (2012).

[13] ATLAS Experiment, http://atlas.ch/.

[14] Compact Muon Solenoid experiment at CERN's LHC, http://cms .web.cern.ch/.

[15] The European Organization for Nuclear Research, http://home.web.cern.ch/.

[16] The Large Hadron Collider (LHC) at CERN, http://home.web.cern.ch/topics/ large-hadron-collider.

[17] G. Aad et al., Observation of a new particle in the search for the Standard Model Higgs boson with the ATLAS detector at the LHC, Phy. Lett. B 716(1), 1-29 (2012).

[18] S. Chatrchyan et al., Observation of a new boson at a mass of $125 \mathrm{GeV}$ with the CMS experiment at the LHC, Phy. Lett. B 716(1), 30-61 (2012).

[19] "Englert-Brout-Higgs-Guralnik-Hagen-Kibble Mechanism on Scholarpedia". Scholarpedia.org. Retrieved 2012-06-16.

[20] K. Fialkowski and W. Kittel, Rep. Prog. Phys., 46, 1283 (1983). 
[21] D.S. Barton et al., Experimental study of the A-dependence of inclusive hadron fragmentation, Phys. Rev. D 27, 2580 (1983).

[22] SPY Collaboration, Eur. Phys. J. C 10, 605 (1999).

[23] H. W. Atherton et al., Precise measurements of particle production by $400 \mathrm{GeV} / \mathrm{c}$ protons on beryllium targets, CERN-80-07 (1980).

[24] D. Aschman et al., Production of pi0 pi0 and pi0 $\mathrm{n}$ in photon-photon collisions, Phys. Lett. B 107, 310-327 (1981).

[25] N. Mokhov, MARS code system, http://www-ap.fnal.gov/MARS/.

[26] A. Fassò, A. Ferrari, J. Ranft, and P. R. Sala, FLUKA: a multi-particle transport code, CERN-2005-010, INFN TC_05/11, SLAC-R-773 (2005).

[27] MINOS is the first experiment to utilize the NuMI beam. MINOS proposal may be found at "P-875: E. Ables et al., A long baseline neutrino oscillation experiment at Fermilab"; FERMILAB-PROPOSAL -P875 (1995).

[28] NuMI stands for Neutrinos at the Main Injector. NuMI neutrino beam is used by the MINOS, NO $\nu \mathrm{A}$ and the MINER $\nu \mathrm{A}$ detectors. http://www-numi.fnal.gov.

[29] M. G. Catanesi et al., Proposal for hadron production measurements using the NA49 detector for use in long-baseline and atmospheric neutrino flux calculations, CERN-SPSC/2001-017 (2001).

[30] D. G. Michael et al., Observation of muon neutrino disappearance with the MINOS detector in the NuMI neutrino beam, Phys. Rev. Lett. 97, 191801 (2006). 
[31] MINER $\nu$ A Collaboration, The MINER $\nu$ A technical design report (2006).

[32] The MiniBooNE Collaboration, A search for electron neutrino appearance at the $\Delta \mathrm{m}^{2} \sim 1 \mathrm{eV}^{2}$ scale, [arXiv:hep-ex/0704.1500] (2007).

[33] Y. Fukuda et al., Evidence for oscillation of atmospheric neutrinos, Phys. Rev. Lett. 81, 1562 (1998).

[34] R. Brun, F. Bruyant, A. C. McPherson and P. Zanarini, CERN Data handling Division, (GEANT3), DD/EE/84-1, 1987; CERN Program Library, W5103 (1994). M. Bonesini, A. Marchionni, F. Pietropaolo and T. Tabarelli de Fatis, (BMPT), Eur. Phys. J. C 20, 13-27, doi:10.1007/S100520100656 [arXiv:hep-ph/0101163] (2001).

N. V. Mokhov and S. I. Striganov, "Model for Pion Production in Proton-Nucleus Interaction", AIP Conf. Proc. 435, 543 (1997). N. V. Mokhov, The MARS Code System Users Guide, version 13(95), Report Fermilab-FN-628 (1995); http://www-ap.fnal.gov/MARS. A. J. Malensek, Fermilab-FN-341 (1981).

[35] Sin Man Seun, Measurement of $\pi$-K Ratios from the NuMI Target, Ph.D. thesis, Harvard University, Cambridge, MA, July (2007).

[36] J. M. Paley et al., Measurement of Charged Pion Production Yields off the NuMI Target, Phys. Rev. D 90, 032001, [arXiv:hep-ex/1404.5882] (2014).

[37] MIPP Experiment, http://ppd.fnal.gov/experiments/e907/.

[38] C. Alt et al., Inclusive production of charged pions in $\mathrm{p}+\mathrm{C}$ collisions at $158 \mathrm{GeV} / \mathrm{c}$ beam momentum, Eur. Phys. J. C 49, 897-917, [arXiv:hep-ex/0606028] (2007).

[39] Rajendran Raja, The Main Injector Particle Physics Experiment (MIPP FNAL E-907) at Fermilab - status and plans, [arxiv:hep-ex/0612007] (2006). 
[40] Fermilab is America's premier Particle Physics Laboratory, http://www .fnal.gov/.

[41] J. Whitmore, Experiment results on strong interactions in the NAL hydrogen bubble chamber, PHYSICS REPORTS (Section C of Physics Letters) 10, No. 5, 273-373 (1974).

[42] NO $\nu$ A Collaboration, The $\mathrm{NO} \nu \mathrm{A}$ technical design report (2007).

[43] C. Adams et al., The Long-Baseline Neutrino Experiment: Exploring Fundamental Symmetries of the Universe, FERMILAB-PUB-14-022, [arXiv:hep-ex/1307.7335] (2013).

[44] An Experimental Program in Neutrino Physics, Nucleon Decay, and Astroparticle Physics Enabled by the Fermilab Long-Baseline Neutrino Facility http://www. fnal.gov/directorate/program_planning/Jan2015Public/LOI-LBNF.pdf https://indico.fnal.gov/getFile.py/access?resId=0\&materialId= O\&conf $I d=9209$,

[45] P. Adamson et al. (MINOS Collaboration), Neutrino and antineutrino inclusive charged-current cross section measurements with the MINOS near detector, Phy. Rev. D 81, 072002 (2010).

[46] L. Fields et al. (MINER $\nu$ A Collaboration), Measurement of Muon Antineutrino Quasi-Elastic Scattering on a Hydrocarbon Target at E 3.5 GeV, Phys. Rev. Lett. 111, 022501 (2013).

[47] G. A. Fiorentini et al. (MINER $\nu$ A Collaboration), Measurement of Muon Neutrino Quasi-Elastic Scattering on a Hydrocarbon Target at E 3.5 GeV, Phys. Rev. Lett. 111, 022502 (2013). 
[48] M. H. Ahn et al., Measurement of Neutrino Oscillation by the K2K Experiment, Phys. Rev. D 74, 072003, [arXiv:hep-ex/0606032] (2006).

[49] K. Abe et al., First muon-neutrino disappearance study with an off-axis beam, Phys. Rev. D 85, 031103(R) (2012).

[50] K. Abe et al., Measurement of Neutrino Oscillation Parameters from Muon Neutrino Disappearance with an Off-axis Beam, Phys. Rev. Lett. 111, 211803, [arXiv:hep-ex/1308.0465] (2013).

[51] R. Raja, personal communication.

[52] Information on the Hadronic Shower Simulation Workshop can be found at http://conferences.fnal.gov/hss06/. See talk by S. Striganov on "Grand Validation" and references therein.

[53] Taizo Muta, Foundations of Quantum Chromodynamics, World Scientific Lectures Notes in Physics - Vol. 5 (1987).

[54] R. Raja, Observation of a new regularity in hadronic spectra, Phys. Rev. D 18, 204 (1978).

[55] R. Raja, Estimation of the annihilation component in pp interaction, Phys. Rev. D 16, $142(1977)$.

[56] S. E. Kopp, Accelerator neutrino beams, Phys. Rept. 439, 101-159, [arXiv:physics/0609129] (2007).

[57] M. G. Catanesi et al., The HARP detector at the CERN PS, Nucl. Inst. and Meth. A 571, 527-561 (2007). 
[58] M. G. Catanesi et al., Measurement of the production cross section of positive pions in the collision of $8.9 \mathrm{GeV} / \mathrm{c}$ protons on beryllium, Eur. Phys. J. C 52, 29-53, [arXiv:hep-ex/0702024] (2007).

[59] B. A. Popov, HARP and NA61 (SHINE) hadron production experiments, AIP Conf. Proc. 1189, 97-104 (2009).

[60] NA61/SHINE Experiment, na61.web.cern.ch/.

[61] Abgrall N et al., Measurements of Cross Sections and Charged Pion Spectra in Proton-Carbon Interactions at $31 \mathrm{GeV} / \mathrm{c}$, Phys. Rev. C 84, 034604 (2011).

[62] Tevatron at Fermilab, http://www.fnal.gov/pub/tevatron/.

[63] D. C. Christian et al., High rate drift chambers, Nucl. Inst. and Meth. A 345, 62-71 (1994).

[64] M. De Palma et al., A system of large multiwire proportional chambers for a high intensity experiment, Nucl. Inst. and Meth. 217, 135 (1983).

[65] J. B. Lindsay, C. Millerin, J. C. Tarlé, H. Verveij, and H. Wendler, A fast and flexible data acquisition system for multiwire proportional chambers and other detectors, Nucl. Inst. and Meth. 156, 329-333 (1978).

[66] Fred Bieser, Ron Jones, and Charles McFarland, Data links for the EOS TPC, IEEE Trans. Nucl. Sc. 38, 335-336 (1991).

[67] K. L. Lee et al., Analog-to-digital conversion using custom CMOS analog memory for the EOS time projection chamber, IEEE Trans. Nucl. Sc. 38, 344-347 (1991). 
[68] G. Rai et al., A TPC detector for the study of high multiplicity heavy ion collisions, IEEE Trans. Nucl. Sc. 37, 56-64 (1990).

[69] Stuart A. Kleinfelder, A 4096 cell switched capacitor analog waveform storage integrated circuit, IEEE Trans. Nucl. Sc. 37, 1230-1236 (1990).

[70] M. Nakamura, D.A. Landis, and G. Rai, Low power shaper amplifier for the EOS TPC detector, IEEE Trans. Nucl. Sc. 38, 50-52 (1991).

[71] H. Wieman and the EOS Collaboration, Heavy ion reaction measurements with the EOS TPC (looking for central collisions with missing energy), in Proceedings of the Tenth Winter Workshop on Nuclear Dynamics (Snowbird, Utah), January 14-21 1994.

[72] H. Wieman et al., A TPC detector for the study of high multiplicity heavy ion collisions, Nucl. Phys. A 525, 617-620 (1991).

[73] J. Engelfried et al., The E781 (SELEX) RICH detector, Nucl. Inst. and Meth. A 409, 439-442 (1998).

[74] J. Engelfried et al., The SELEX phototube RICH detector. Nucl. Inst. and Meth. A 431, 53-69 (1999).

[75] J. Engelfried et al., The RICH detector of the SELEX experiment, Nucl. Inst. and Meth. A 433, 149-152 (1999).

[76] R. A. Burnstein et al., HyperCP: A high-rate spectrometer for the study of charged hyperon and kaon decays, Nucl. Inst. and Meth. A 541, 516-565 (2005).

[77] A. Abramov et al., Dynamic Stress Calculations for ME and LE Targets and Results of Prototyping for the LE Target, MINOS-doc-675 (2000). 
[78] NuMI Technical Design Handbook (2004).

[79] J. Paley, M. D. Messier, C. Bower, MIPP NuMI-Target Run Plan, MIPP Note, MIPP-doc-302 (2007).

[80] Andrey V. Lebedev, Ratio of Pion Kaon Production in Proton Carbon Interactions, Ph.D. thesis, Harvard University, Cambridge, MA, May (2007).

[81] C. Bower, N. Graf, M. D. Messier and J. Paley, The Indiana trigger counter, MIPP Note, MIPP-doc-55 (2005).

[82] https://root.cern.ch/root/html534/guides/users-guide/

ROOTUsersGuideA4.pdf.

[83] Xihong Yang, Centrality dependence of strangeness production in proton-nucleus collisions at AGS energies, Ph.D. thesis, Columbia University (2000).

[84] J. Paley, R. Soltz, P. D. Barnes, M. Heffner, J. Klay, and D. Miller, MIPP TPC reconstruction, MIPP Note, MIPP-doc-129 (2006).

[85] http://root.cern.ch/root/html/TMinuit.html.

[86] CERN, Geneva, Switzerland. GEANT - Detector Description and Simulation Tool (1993).

[87] Stefan Roesler, Ralph Engel, Johannes Ranft, The Monte Carlo Event Generator DPMJET-III, in Proceedings of the Monte Carlo 2000 Conference, SLAC-PUB8740, [arXiv:hep-ph/0012252v1] (2000).

[88] A. Fassò, A. Ferrari, S. Roesler, P. R. Sala, G. Battistoni, F. Cerutti, E. Gadioli, M. V. Garzelli, F. Ballarini, A. Ottolenghi, A. Empl, and J. Ranft, The Physics 
models of FLUKA: status and recent developments, Computing in High Energy and Nuclear Physics Conference (2003).

[89] G. Bellettini et al., Nucl. Phys. 79, 609 (1966).

[90] S. P. Denisov et al., Nucl. Phys. B 61, 62 (1973).

[91] A. S. Carroll et al., Phys. Lett. B 80, 319 (1979).

[92] Sonam Mahajan, Rajendran Raja, Vipin Bhatnagar, Brajesh C. Choudhary, Durga Rajaram and Holger Meyer, Inelastic Cross section Analysis in MIPP Experiment at Fermilab, MIPP Note, MIPP-doc-1267-v5 (2015).

[93] Z. Koba, H. B. Nielsen, and P. Olesen, Nucl. Phys. B40, 317 (1972).

[94] Sàndor Hegyi, KNO scaling 30 years later, [arXiv:hep-ph/0011301v1] (2000).

[95] Evidence for the Onset of Semi-inclusive Scaling in Proton-Proton Collisions in the 50-300 GeV/c Momentum Range, P. Slattery, Phys. Rev. Lett. 29, 1624-1627 (1972).

[96] The PDG files for the total and elastic cross section data for pp interaction are available at http://pdg.lbl.gov/2011/hadronic-xsections/pp_total.dat and http://pdg.lbl.gov/2011/hadronic-xsections/pp_elastic.dat.

[97] The plots of total and elastic cross section vs $\mathrm{p}_{L a b}$ for pp interaction are available at http://pdg.lbl.gov/2011/hadronic-xsections/hadronicrpp_page11.pdf.

[98] J. W. Martin, J. R. Florian, L. D. Kirkpatrick, and J. J. Lord, Nuovo Cimento 25A, 447 (1975). 
[99] J. Hébert et al., Batavia-Belgrade-Lund-Lyon-Montreal-Nancy-Ottawa-Paris-RomeStrasbourg-Valencia Collaboration, in Proceedings of the fourteenth International Conference on Cosmic Rays, Munich, 1975 edited by Klaus Pinkau (Max-PlanckInstitut, Munich), Vol. 7, 2248 (1975).

[100] A. J. Buras, J. Dias De Deus, and R. Moller, Phys. Lett. B 47, 251 (1973).

[101] V. H. Areti, C. J. D. Hébert, and J. Hébert, Phys. Rev. D 15, 7 (1977).

[102] G. Calucci, R. Jengo, and A. Pignotti, Phys. Rev. D 10, 1468 (1974).

[103] P. J. Camillo, P. M. Fishbane, and J. S. Trefil, Phys. Rev. Lett. 34, 622 (1975).

[104] Sonam Mahajan, Rajendran Raja, Vipin Bhatnagar, Brajesh C. Choudhary, Durga Rajaram and Holger Meyer, Inclusive Pion Production Analysis in MIPP Experiment at Fermilab, MIPP Note, MIPP-doc-1343-v6 (2015).

[105] http://pdg.lbl.gov/2004/reviews/passagerpp.pdf.

[106] Richard C. Fernow, Introduction to experimental particle physics, Cambridge University Press (1986).

[107] Holger Meyer, Normalized Log-Likelihood Particle ID from MIPP PID detectors, MIPP Note, MIPP-doc-936-v1 (2009).

[108] Holger Meyer, Particle Production Results from the MIPP Experiment, Fermilab Joint Experimental and Theoretical Seminar, MIPP-doc-1024-v2 (2010).

[109] Sonam Mahajan and Rajendran Raja, Particle Production Measurements using the MIPP Detector at Fermilab, [arXiv:hep-ex/1311.2258] (2013). 
[110] K Nakamura (PDG), J. Phys. G: Nucl. Part. Phys. 37, 075021, doi:10.1088/09543899/37/7A/075021 (2010).

[111] H. J. Möhring, Hadron-nucleus inelastic cross sections for use in hadron cascade calculations at high energies CERN TIS-RP/116 (1983).

[112] Qing-biao Shen, Systematics of intermediate energy proton nonelastic and neutron total cross section, IAEA Report INDC(CPR)-020 (1991).

[113] Turgun Nigmanov et al., Inclusive Neutron Analysis in MIPP Experiment, MIPP Note, MIPP-doc-989-v2 (2010).

[114] Sonam Mahajan and Rajendran Raja, Momentum corrections for the data and the Monte Carlo, MIPP Note, MIPP-doc-1122-v1 (2011).

[115] T. S. Nigmanov et al., Forward neutron production at the Fermilab Main Injector, Phys. Rev. D 83, 012002, doi:10.1103/PhysRevD.83.012002 (2011). 


\section{List of Publications}

\section{Papers Published}

1. J. M. Paley et al., "Measurement of Charged Pion Production Yields off the NuMI Target", Phys. Rev. D 90, 032001, [arXiv:hep-ex/1404.5882] (2014).

2. Sonam Mahajan and Rajendran Raja, "Particle Production Measurements using the MIPP Detector at Fermilab", [arXiv:hep-ex/1311.2258], FERMILAB-CONF-13-461-E (2013).

3. Sonam Mahajan, Ashok Kumar and Rajendran Raja, "Main Injector Particle Production Experiment (MIPP) at Fermilab", Pramana J. Phys. 79(5), 1243-1246, doi:10.1007/s12043-012-0432-0 (2012).

4. Sonam Mahajan, "Main Injector Particle Production Experiment (MIPP) at Fermilab", AIP Conf. Proc. 1382, 256-258, doi:10.1063/1.3644327 (2011).

\section{MIPP Internal Notes}

1. Sonam Mahajan, Rajendran Raja, Vipin Bhatnagar, Brajesh C. Choudhary, Durga Rajaram and Holger Meyer, "Inclusive Pion Production Analysis in MIPP Experiment at Fermilab", MIPP-doc-1343-v6 (2015).

2. Sonam Mahajan, Rajendran Raja, Vipin Bhatnagar, Brajesh C. Choudhary, Durga Rajaram and Holger Meyer, "Inelastic Cross section Analysis in MIPP Experiment at Fermilab", MIPP-doc-1267-v5 (2015).

3. Sonam Mahajan, Rajendran Raja, Durga Rajaram and Turgun Nigmanov, "Fitting for Scintillator Interaction trigger (SCIHI) efficiency using Liquid Hydrogen data and known KNO true multiplicity distributions", MIPP-doc-1149-v2 (2012).

4. Sonam Mahajan and Rajendran Raja, "Momentum corrections for the data and the Monte Carlo", MIPP-doc-1122-v1 (2011). 
5. Sonam Mahajan, Rajendran Raja and Holger Meyer, "Changes to the DPMJET and FLUKA packages", MIPP-doc-1121-v2 (2011).

\section{Conferences/Workshops/Schools Attended}

1. " $21^{\text {st }}$ DAE-BRNS HEP Symposium 2014", December 8 - 12, 2014, IIT Guwahati, India.

2. "Workshop on Contemporary Trends in High-Energy Physics and Experimentation", March 10 - 11, 2014, Department of Physics, Panjab University, Chandigarh, India.

3. "Science Colloquium", March 6 - 7, 2014, DAV College, Jalandhar, India.

4. "8th Chandigarh Science Congress (CHASCON)", February 26 - 28, 2014, Panjab University, Chandigarh, India.

5. "Neutrino Workshop", January 23 - 24, 2014, Department of Physics, Panjab University, Chandigarh, India.

6. "DPF Meeting", August 13 - 17, 2013, University of California, Santa Cruz, USA.

7. "New Perspectives Conference $2013-46^{\text {th }}$ Annual Fermilab Users Meeting", June 10 - 11, 2013, Fermilab, USA.

8. "APS April Meeting", April 13 - 16, 2013, Denver, Colorado, USA.

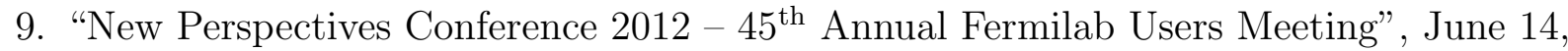
2012, Fermilab, USA.

10. "12th International Workshop on Neutrino Factories, Superbeams, and Betabeams (NuFact10)", October 20 - 25, 2010, TIFR, Mumbai, India.

11. "7th SERC SCHOOL on Experimental High energy Physics", February 9 - 28, 2009, IIT Bombay, India (conducted by the Department of Science and Technology, Govt. of India).

\section{List of Talks}

1. "Particle Production Measurements using the MIPP detector at Fermilab", $21^{\text {st }}$ DAEBRNS HEP Symposium 2014", December 8 - 12, 2014, IIT Guwahati, India.

2. "Particle Production Measurements using the MIPP Detector at Fermilab", $8^{\text {th }}$ Chandigarh Science Congress (CHASCON) 2014, February 26 - 28, 2014, Panjab University, Chandigarh, India. 
3. "Main Injector Particle Production (MIPP) Experiment at Fermilab", Neutrino Workshop, January 23 - 24, 2014, Department of Physics, Panjab University, Chandigarh, India.

4. "Particle Production Measurements using the MIPP Detector at Fermilab", DPF Meeting, August 13 - 17, 2013, University of California, Santa Cruz, USA.

5. "Particle Production Measurements using the MIPP Detector at Fermilab", New Perspectives Conference, June 10 - 11, 2013, Fermilab, USA.

6. "Particle Production Measurements using the MIPP Detector at Fermilab", APS April meeting, April 13 - 16, 2013, Denver, Colorado, USA.

7. "Particle Production Measurements using the MIPP Detector at Fermilab", 3rd IIFCnuP meeting, February 7 - 10, 2013, IIT Guwahati, India.

8. "Particle Production Measurements using the MIPP Detector at Fermilab", IIFC meeting, November 26 - 27, 2012, Fermilab, USA.

9. "Particle Production Measurements using the MIPP Detector at Fermilab", $2^{\text {nd }}$ IndoUS Neutrino Collaboration Meeting, August 13 - 14, 2012, Department of Physics, Panjab University, Chandigarh, India.

10. "Particle Production Measurements using the MIPP Detector at Fermilab", New Perspectives Conference, June 14, 2012, Fermilab, USA.

11. "Track Finding Using Hough Transform", 7th SERC SCHOOL, February 9 - 28, 2009, IIT Bombay, India.

\section{$\underline{\text { List of Posters }}$}

1. Sonam Mahajan, Vipin Bhatnagar, Brajesh C. Choudhary and Rajendran Raja, "Particle Production Measurements using the MIPP Detector at Fermilab", Workshop on Contemporary Trends in High-Energy Physics and Experimentation, March 10 - 11, 2014, Department of Physics, Panjab University, Chandigarh, India.

2. Sonam Mahajan, Vipin Bhatnagar, Brajesh C. Choudhary and Rajendran Raja, "Particle Production Measurements using the MIPP Detector at Fermilab", Science Colloquium, March 6-7, 2014, DAV College, Jalandhar, India. 
3. Sonam Mahajan and Rajendran Raja, "Particle Production Measurements using the MIPP Detector at Fermilab", DPF Meeting, August 13 - 17, 2013, University of California, Santa Cruz, USA.

4. Sonam Mahajan and Rajendran Raja, "Particle Production Measurements using the MIPP Detector at Fermilab", 46 ${ }^{\text {th }}$ Annual Fermilab Users' meeting, June 12 - 13, 2013, Fermilab, USA.

5. Sonam Mahajan and Rajendran Raja, "Particle Production Measurements using the MIPP Detector at Fermilab", 45 ${ }^{\text {th }}$ Annual Fermilab Users' meeting, June 12 - 13, 2012, Fermilab, USA.

6. Sonam Mahajan, Ashok Kumar and Rajendran Raja, "Main Injector Particle Production Experiment at Fermilab", XXV International Symposium on Lepton Photon Interactions at high energies (Lepton Photon 2011), August 22 - 27, 2011, TIFR, Mumbai, India.

7. Sonam Mahajan, "Main Injector Particle Production Experiment at Fermilab", 44" Annual Fermilab Users' meeting, June 1 - 2, 2011, Fermilab, USA.

8. Sonam Mahajan, "Main Injector Particle Production Experiment at Fermilab", 12" International Workshop on Neutrino Factories, Superbeams, and Beta beams (NuFact10), October 20 - 25, 2010, TIFR, Mumbai, India. 